\title{
Dynamic Behaviour of Short-Term Floating Bridges
}

\author{
by \\ Osama El-Desouky Mohamed Ibrahim, B.Sc., M.Sc.
}

A thesis submitted to the Faculty of Graduate and Postdoctoral Affairs in partial fulfillment of the requirements for the degree of

\section{Doctor of Philosophy in Civil Engineering}

Department of Civil and Environmental Engineering Carleton University

Ottawa-Carleton Institute of Civil and Environmental Engineering Ottawa, Ontario, Canada

June, 2011

(C) 2011, Osama El-Desouky Mohamed Ibrahim 


$\begin{array}{ll}\begin{array}{l}\text { Library and Archives } \\ \text { Canada }\end{array} & \begin{array}{l}\text { Bibliothèque et } \\ \text { Archives Canada }\end{array} \\ \begin{array}{l}\text { Published Heritage } \\ \text { Branch }\end{array} & \begin{array}{l}\text { Direction du } \\ \text { Patrimoine de l'édition }\end{array} \\ \begin{array}{l}\text { 395 Wellington Street } \\ \text { Ottawa ON K1A ON4 }\end{array} & \begin{array}{l}\text { 395, rue Wellington } \\ \text { Canada }\end{array} \\ \text { Canada ON K1A ON4 }\end{array}$

Your file Votre référence

ISBN: 978-0-494-83232-5

Our file Notre référence

ISBN: $978-0-494-83232-5$

NOTICE:

AVIS:

The author has granted a nonexclusive license allowing Library and Archives Canada to reproduce, publish, archive, preserve, conserve, communicate to the public by telecommunication or on the Internet, loan, distribute and sell theses worldwide, for commercial or noncommercial purposes, in microform, paper, electronic and/or any other formats.

The author retains copyright ownership and moral rights in this thesis. Neither the thesis nor substantial extracts from it may be printed or otherwise reproduced without the author's permission.

L'auteur a accordé une licence non exclusive permettant à la Bibliothèque et Archives Canada de reproduire, publier, archiver, sauvegarder, conserver, transmettre au public par télécommunication ou par l'Internet, prêter, distribuer et vendre des thèses partout dans le monde, à des fins commerciales ou autres, sur support microforme, papier, électronique et/ou autres formats.

L'auteur conserve la propriété du droit d'auteur et des droits moraux qui protège cette thèse. $\mathrm{Ni}$ la thèse ni des extraits substantiels de celle-ci ne doivent être imprimés ou autrement reproduits sans son autorisation.
In compliance with the Canadian Privacy Act some supporting forms may have been removed from this thesis.

While these forms may be included in the document page count, their removal does not represent any loss of content from the thesis.
Conformément à la loi canadienne sur la protection de la vie privée, quelques formulaires secondaires ont été enlevés de cette thèse.

Bien que ces formulaires aient inclus dans la pagination, il n'y aura aucun contenu manquant.

\section{Canadä}


This thesis is dedicated to the soul of my dear father 


\section{Abstract}

Rapid deployment floating bridges are temporary structures built for use in emergency situations or in time of war. Therefore very little, or no, information is available in the literature about their design or analysis. Due to the evolution of new generation of heavier and faster military vehicles and equipments coupled with sudden increasing demand for their use in natural disasters, new requirements are needed for their design and analysis. This thesis considers the complex three-dimensional vehicle-bridge-fluid interactions associated with rapid deployment floating bridges.

A finite element (FE) numerical technique was used to develop a robust program to incorporate the dynamic behaviour of the vehicle-bridge-fluid system. The developed FE program was utilized to study several parameters such as vehicle types and their weights and speeds, spacing between successive vehicles, types of rapid deployment floating bridges in addition to considering pontoons made of steel or aluminum.

The results of utilizing the numerical technique showed the potential of developed FE program to study and analyze possible loading and vehicle speed combinations leading to optimization of the transportation capacity of the examined bridges. Also, the research results showed that the developed FE program is a simple and easy to use tool for analyzing and understanding the many facets of the complexity associated with rapid deployment floating bridges. 


\section{Acknowledgements}

\section{Thank God for all the strength and guidance}

I would like to express my deepest gratitude and debt to The Egyptian Government for its financial sponsorship and moral support during my $\mathrm{PhD}$ study period at Carleton University. Also, I would like to express my deepest thanks to my co-supervisors Professor Abd El Halim O. Abd El Halim and Professor Abass Braimah for their time and efforts, valuable guidance, and advice throughout my research work. The assistance and support invested by my co-supervisors are highly appreciated.

In addition, I would like to extend my sincere appreciation and thanks to my professors and fellow students in Civil and Environmental Engineering Department at Carleton University for their kind help and support. Many thanks are due to Professor Jagmohan L. Humar for his valuable advice and guidance throughout my research work.

Special thanks are due to my beloved mother and family for their spiritual support and prayers for successful completion of my studies. I would like to remember and mention my late father whose love, help and unlimited support since my birth led me to do my best.

Finally, I would like to acknowledge and offer special thanks to my lovely wife and two young princesses Jana and Judi who suffered the many nights waiting for my late arrival to assure me that my hard work is worth the sacrifice for better future. 


\section{Table of Contents}

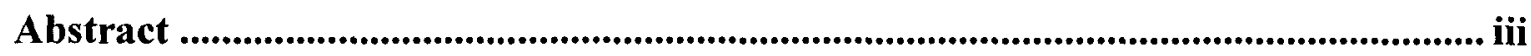

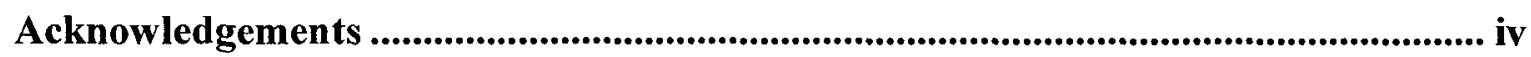

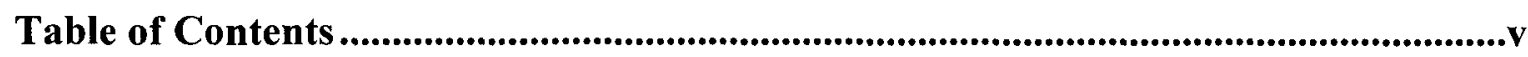

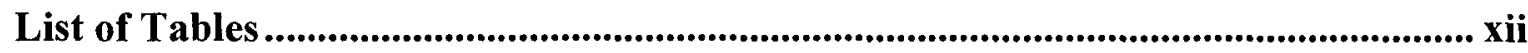

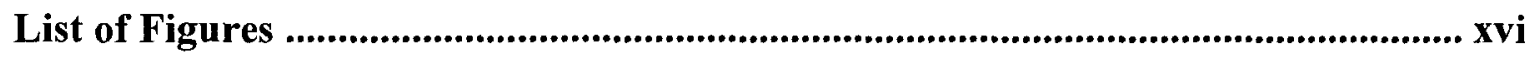

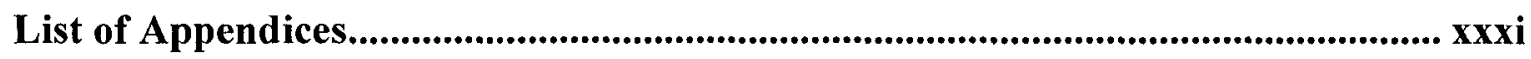

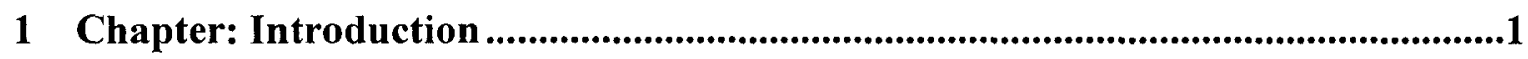

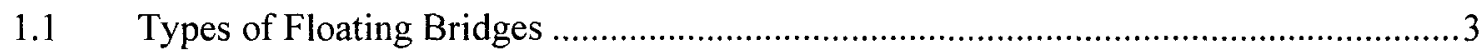

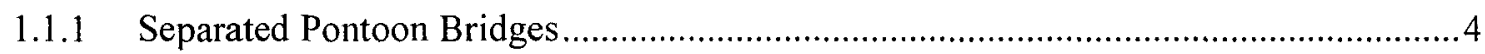

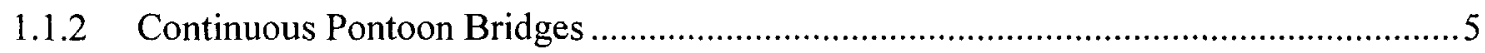

1.1.3 Rapid Deployment Floating Bridges (Ribbon Bridges) .......................................

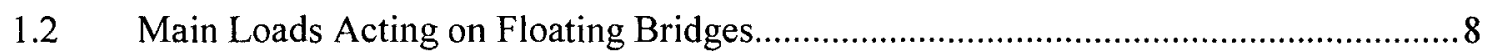

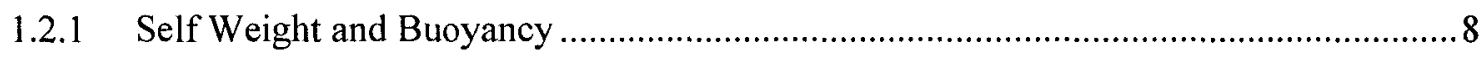

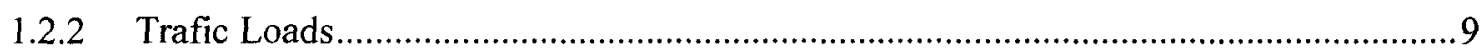

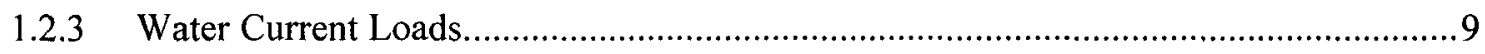

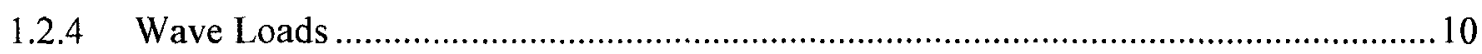

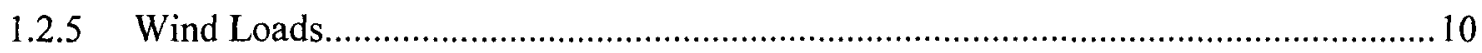

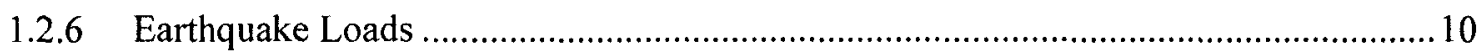

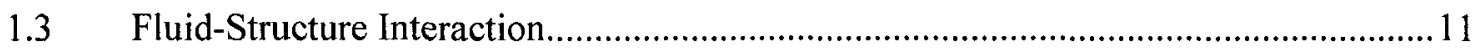

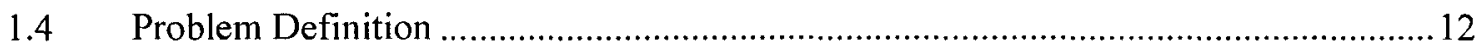

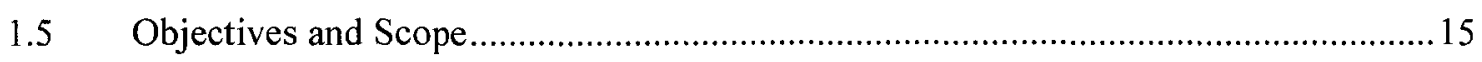

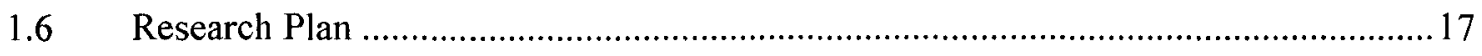

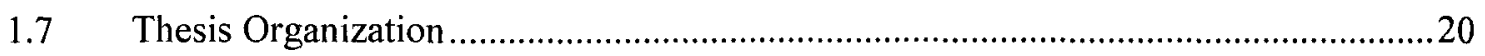


2 Chapter: Literature Review.

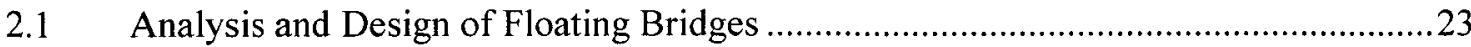

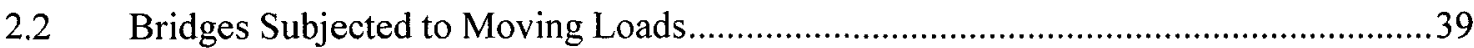

2.3 Buoyancy, Water Current and Wave Loads Acting on Floating Bridges.....................55

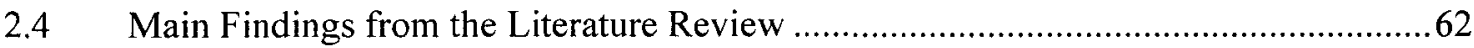

3 Chapter: Analytical Approach and Model Development ..........................................65

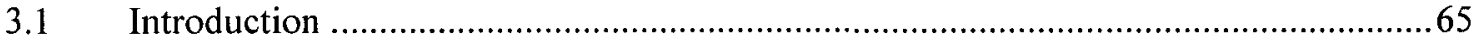

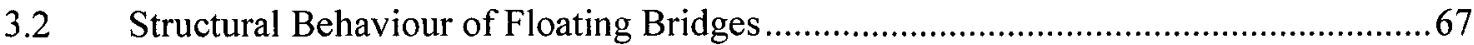

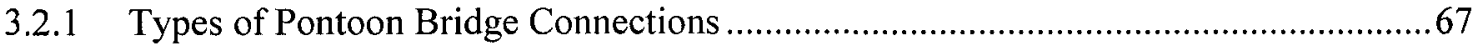

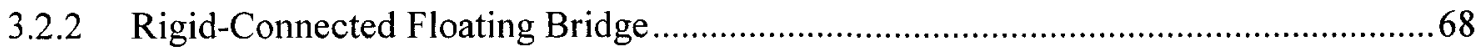

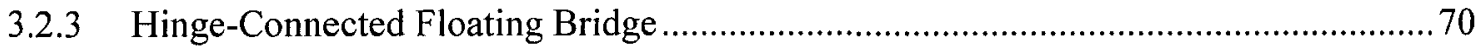

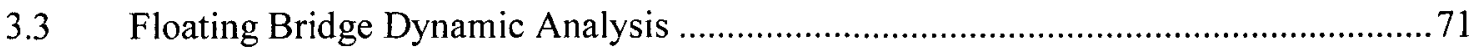

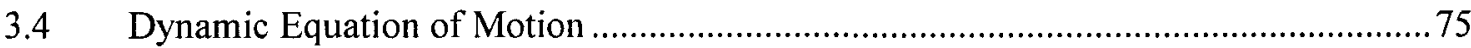

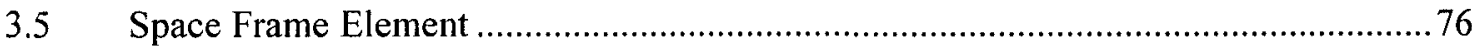

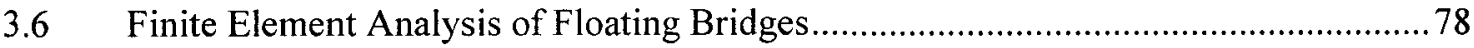

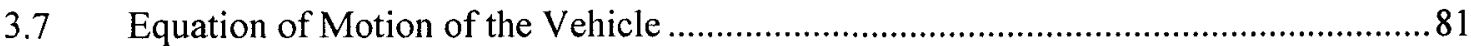

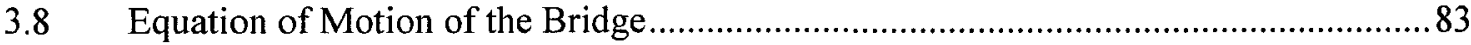

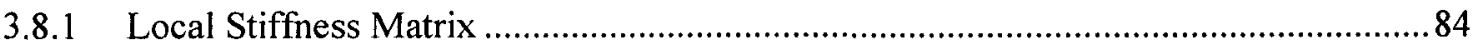

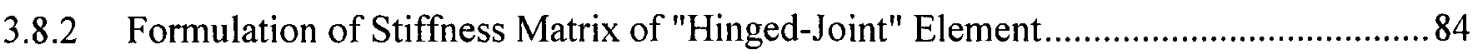

3.8.3 Formulation of Stiffness Matrix Due to Surrounding Water .....................................86

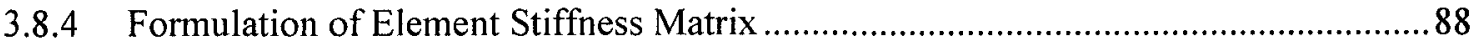

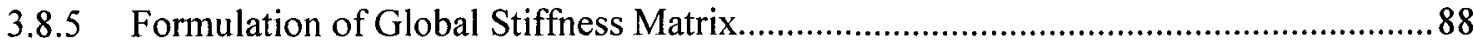

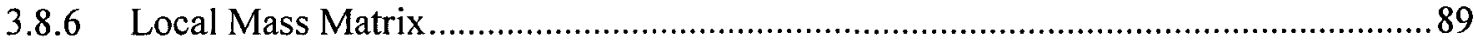

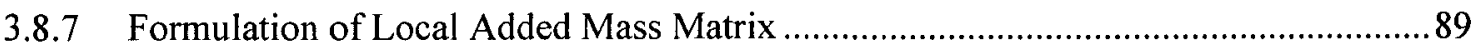

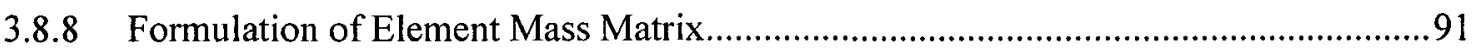

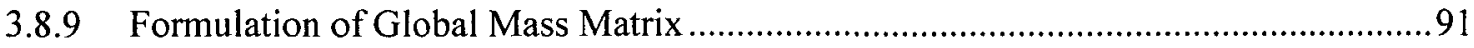


3.8.10 Formulation of Global Damping Matrix of the Bridge.............................................

3.8.11 Formulation of the Local Force Vector ............................................................... 93

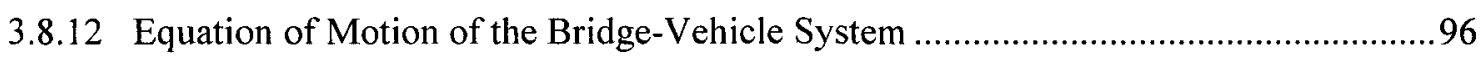

3.9 Dynamic Response of Floating Bridges to Two-Axle Vehicle Loads..........................97

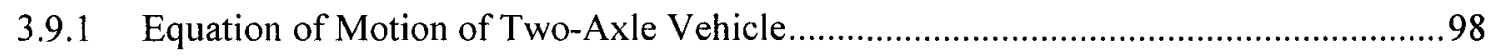

3.9.2 Formulation of the Local Force Vector ............................................................. 100

3.9.3 Equation of Motion of the Bridge-Vehicle System for Two-Axle Vehicle................ 103

3.10 Dynamic Response of Floating Bridges to Successive Two-Axle Vehicle Loads ..... 105

3.10.1 Equation of Motion of the Bridge-Vehicle System for Two Successive Two-Axle

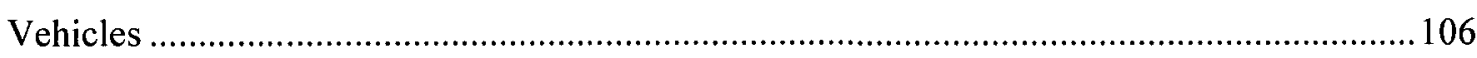

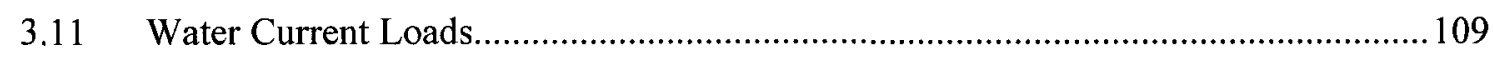

3.11.1 Basic Hypotheses for Open Channel Flow ........................................................ 110

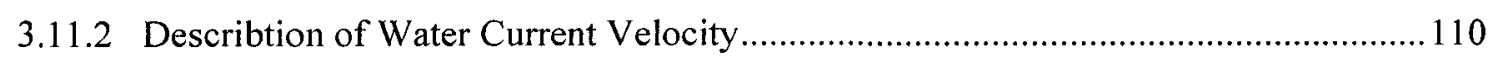

3.11.3 Drag Forces Acting on Floating Bridges Due to Water Current ............................ 112

3.11.4 Formulation of the Local Force Vector Due to Water Current.................................114

3.12 Determining the Floating Bridge Dynamic Response ...................................... 118

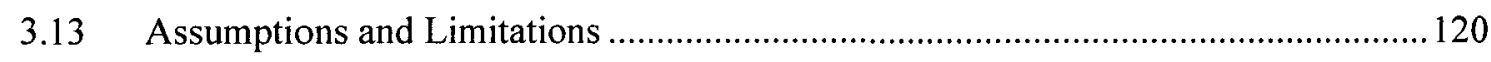

\section{Chapter: Parametric Studies for the Dynamic Behaviour and Transport}

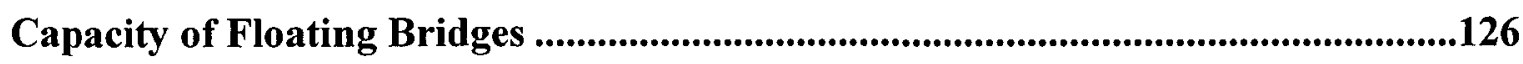

4.1 Parametric Study for the Dynamic Response of Floating Bridges to Single-Axle

Vehicle Loads

4.1.1 Dynamic Response of Rigid-Connected Floating Bridge to Single-Axle Vehicle

Loads.

4.1.1.1 Discussion of Results. 
4.1.2 Dynamic Response of Hinge-Connected Floating Bridge to Single-Axle Vehicle

Loads.

4.1.2.1 Discussion of Results

4.2 Parametric Study for the Dynamic Response of Floating Bridges to Two-Axle Vehicle

Loads

4.2.1 Dynamic Response of Rigid-Connected Floating Bridge to Two-Axle Vehicle

Loads.

4.2.1.1 Discussion of Results

4.2.2 Dynamic Response of Hinge-Connected Floating Bridge to Two-Axle Heavy Vehicle

Loads

4.2.2.1 Discussion of Results

4.3 Comparison of the Dynamic Response to Single-Axle and Two-Axle Vehicle

Loads.

4.3.1 Dynamic Response of Rigid-Connected Floating Bridge to Single- and Two-Axle

Vehicle Loads

4.3.1.1 Discussion of Results

4.3.2 Comparison of the Dynamic Response of Hinge-Connected Floating Bridge to Singleand Two-Axle Vehicle Loads 164

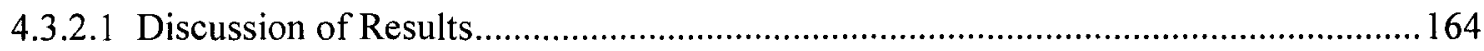

4.4 Dynamic Response of Floating Bridges to Eccentric Moving Vehicle Loads ........... 169

4.5 Dynamic Response of Light-Weight Floating Bridges to Moving Vehicle Loads....171

4.5.1 Dynamic Response of Rigid-Connected Aluminum Floating Bridge to Two-Axle

Vehicle Loads

4.5.1.1 Discussion of Results

4.5.2 Dynamic Response of Hinged-Connected Aluminum Floating Bridge to Two-Axle Vehicle Loads 
4.6 Parametric Study for the Transport Capacity and Dynamic Response of Floating

Bridges to Successive Vehicle Loads

4.6.1 Dynamic Response of Rigid-Connected Floating Bridge to Two successive Vehicle

Loads

4.6.1.1 Discussion of Results

4.6.2 Dynamic Response of Hinge-Connected Floating Bridge to Two successive Vehicle

Loads

4.6.2.1 Discussion of Results.

4.7 Effect of the Passage of Multiple Vehicles.

4.7.1 Dynamic Response of Rigid-Connected Floating Bridge to Single and Two Vehicle Loads...

4.7.2 Dynamic Response of Hinge-Connected Floating Bridge to Single and Two Vehicle

Loads... 216

4.8 Parametric Study for the Three-Dimensional Interactive Dynamic Response of

Floating Bridges to Moving Vehicle Loads and Dynamic Water Current Loads

4.8.1 Three-Dimensional Dynamic Response of Rigid-Connected Floating Bridge to

Eccentric Two-Axle Moving Vehicle Loads and Dynamic Water Current Loads....

4.8.1.1 Discussion of Results

4.8.2 Three-Dimensional Dynamic Response of Hinge-Connected Floating Bridge to

Eccentric Two-Axle Moving Vehicle Loads and Dynamic Water Current Loads....... 228

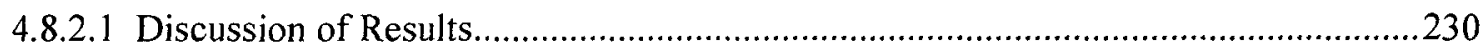

4.9 Summary

\section{Chapter: Verification of the Developed Analytical Approach and the Developed}

Finite Element Program ......................................................................................................235

5.1 Simply Supported Bridge Subjected to Moving Vehicle Loads...............................235 
5.2 Simply Supported Bridge Subjected to Moving Two-Axle Vehicle Loads

5.3 Hinge-Connected Floating Bridge Subjected to Moving Load with Different

Speeds. 240

5.4 Dynamic Analysis of Floating Bridges Using ANSYS Software Package ...............242

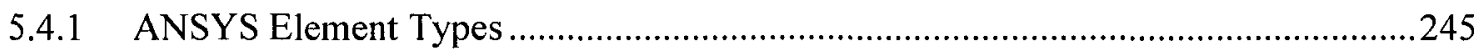

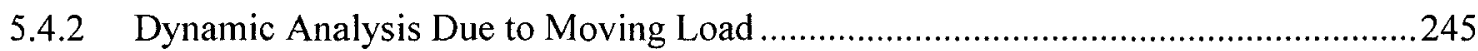

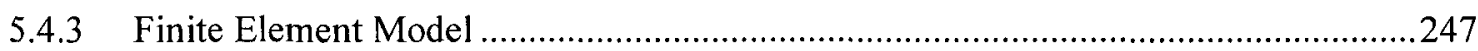

5.4.4 Dynamic Response of Rigid-Connected Floating Bridge to Single-Axle Vehicle Loads

Using ANSYS Software Package. 248

5.4.5 Dynamic Response of Hinge-Connected Floating Bridge to Single-Axle Vehicle Loads

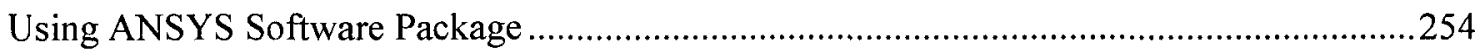

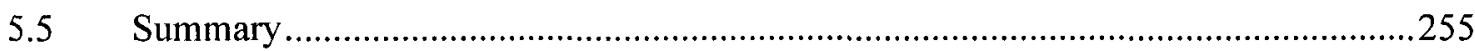

6 Chapter: Summary, Conclusions and Recommendations for Future Work .....261

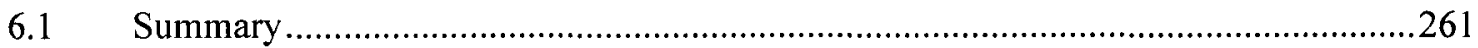

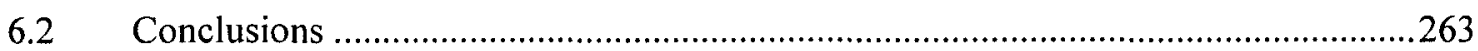

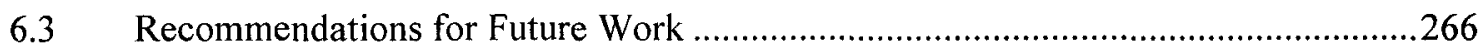

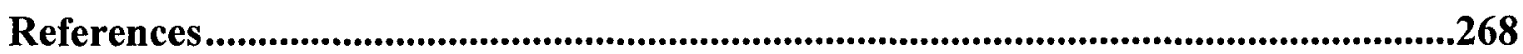

Appendices.......................................................................................................276

Appendix A: Parametric Study for the Structural Behaviour of Floating Bridges Subjected to

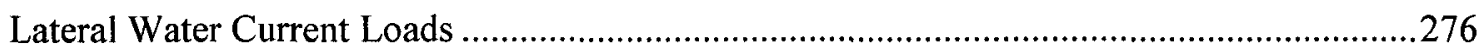

A.1 Structural Behaviour of Floating Bridge under Water Current Loads......................276

A.2 Effect of Supporting Boat Force on the Structural Behaviour of Floating Bridge

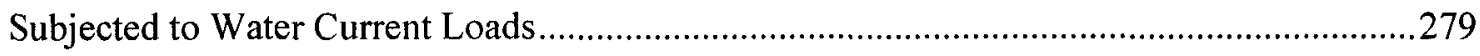

A.3 Structural Behaviour of Floating Bridge under Water Current Loads and Supporting

Boat Forces 


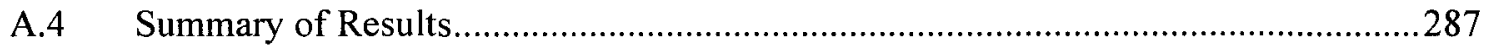

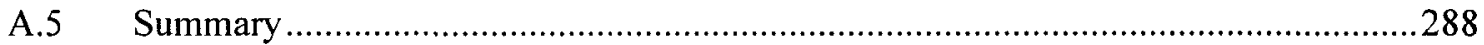

Appendix B: Ribbon Bridge Composition, Construction and Operation ..............................289

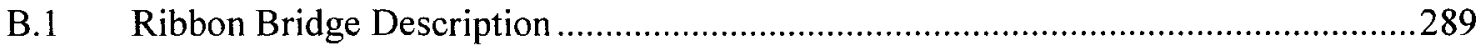

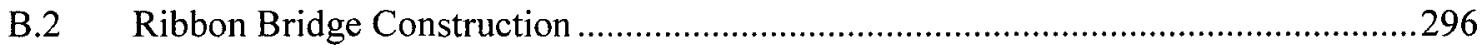

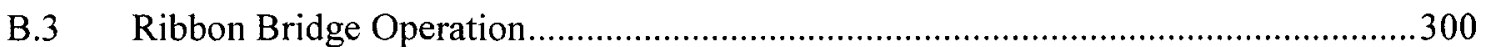




\section{List of Tables}

Table 1.1 Vehicle design crossing speeds (Rosenthal et al. 1996) ..................................15

Table 4.1 Input parameters for parametric studies ............................................ 127

Table 4.2 Static and dynamic response of rigid-connected bridge to 50-tonne singleaxle vehicle loads.

Table 4.3 Static and dynamic response of rigid-connected bridge to 60-tonne single-

axle vehicle loads.

Table 4.4 Static and dynamic response of rigid-connected bridge to 70 -tonne single-

axle vehicle loads.

Table 4.5 Static and dynamic response of hinge-connected bridge to 50-tonne single-

axle vehicle loads.

Table 4.6 Static and dynamic response of hinge-connected bridge to 60 -tonne single-

axle vehicle loads.

Table 4.7 Static and dynamic response of hinge-connected bridge to 70-tonne single-

axle vehicle loads.

Table 4.8 Static and dynamic response of rigid-connected bridge to 50-tonne two-axle

vehicle loads

Table 4.9 Static and dynamic response of rigid-connected bridge to 60 -tonne two-axle

vehicle loads

Table 4.10 Static and dynamic response of rigid-connected bridge to 70-tonne two-axle vehicle loads 
Table 4.11 Static and dynamic response of hinge-connected bridge to 50-tonne twoaxle vehicle loads.

Table 4.12 Static and dynamic response of hinge-connected bridge to 60-tonne twoaxle vehicle loads.

Table 4.13 Static and dynamic response of hinge-connected bridge to 50-tonne twoaxle vehicle loads.

Table 4.14 Dynamic response of rigid-connected bridge to 50-tonne two-axle eccentric vehicle loads

Table 4.15 Dynamic response of hinge-connected bridge to 50-tonne two-axle eccentric vehicle loads

Table 4.16 Dynamic response of rigid-connected aluminum bridge to 50-tonne two-axle eccentric vehicle loads 173

Table 4.17 Dynamic response of hinge-connected aluminum bridge to 50-tonne twoaxle eccentric vehicle loads 176

Table 4.18 Dynamic response of rigid-connected bridge to two successive 50-tonne two-axle vehicle loads (spacing $50 \mathrm{~m}$ )

Table 4.19 Dynamic response of rigid-connected bridge to two successive 60 -tonne two-axle vehicle loads (spacing $50 \mathrm{~m}$ ) 181

Table 4.20 Dynamic response of rigid-connected bridge to two successive 70-tonne two-axle vehicle loads (spacing $50 \mathrm{~m}$ ) 182

Table 4.21 Dynamic response of rigid-connected bridge to two successive 50-tonne two-axle vehicle loads (spacing $40 \mathrm{~m}$ ) 182 
Table 4.22 Dynamic response of rigid-connected bridge to two successive 60-tonne two-axle vehicle loads (spacing $40 \mathrm{~m}$ )

Table 4.23 Dynamic response of rigid-connected bridge to two successive 70-tonne two-axle vehicle loads (spacing $40 \mathrm{~m}$ )

Table 4.24 Dynamic response of rigid-connected bridge to two successive 50-tonne two-axle vehicle loads (spacing $30 \mathrm{~m}$ )

Table 4.25 Dynamic response of rigid-connected bridge to two successive 60 -tonne two-axle vehicle loads (spacing $30 \mathrm{~m}$ )

Table 4.26 Dynamic response of rigid-connected bridge to two successive 70 -tonne two-axle vehicle loads (spacing $30 \mathrm{~m}$ )

Table 4.27 Dynamic response of hinge-connected bridge to two successive 50-tonne two-axle vehicle loads (spacing $50 \mathrm{~m}$ )

Table 4.28 Dynamic response of hinge-connected bridge to two successive 60-tonne two-axle vehicle loads (spacing $50 \mathrm{~m}$ ) 196

Table 4.29 Dynamic response of hinge-connected bridge to two successive 70-tonne two-axle vehicle loads (spacing $50 \mathrm{~m}$ ) 196

Table 4.30 Dynamic response of hinge-connected bridge to two successive 50-tonne two-axle vehicle loads (spacing $40 \mathrm{~m}$ )

Table 4.31 Dynamic response of hinge-connected bridge to two successive 60-tonne two-axle vehicle loads (spacing $40 \mathrm{~m}$ )

Table 4.32 Dynamic response of hinge-connected bridge to two successive 70-tonne two-axle vehicle loads (spacing $40 \mathrm{~m}$ ) 198 
Table 4.33 Dynamic response of hinge-connected bridge to two successive 50-tonne two-axle vehicle loads (spacing $30 \mathrm{~m}$ )

Table 4.34 Dynamic response of hinge-connected bridge to two successive 60-tonne two-axle vehicle loads (spacing $30 \mathrm{~m}$ )

Table 4.35 Dynamic response of hinge-connected bridge to two successive 70-tonne two-axle vehicle loads (spacing $30 \mathrm{~m}$ )

Table 4.36 3-D dynamic response of rigid-connected bridge to 50-tonne two-axle eccentric vehicle loads and water current loads with current velocity $1 \mathrm{~m} / \mathrm{s}$

Table 4.37 3-D dynamic response of rigid-connected bridge to 50-tonne two-axle eccentric vehicle loads and water current loads with current velocity $2 \mathrm{~m} / \mathrm{s}$

Table 4.38 3-D dynamic response of hinge-connected bridge to 50-tonne two-axle eccentric vehicle loads and water current loads with current velocity $1 \mathrm{~m} / \mathrm{s}$

Table 4.39 3-D dynamic response of hinge-connected bridge to 50-tonne two-axle eccentric vehicle loads and water current loads with current velocity $2 \mathrm{~m} / \mathrm{s}$ 


\section{List of Figures}

Figure 1.1 Types of floating bridges (Watanabe and Utsunomiya 2003) ......................4

Figure 1.2 Bergsoysund pontoon bridge, Norway (Watanabe 2003) .............................5

Figure 1.3 Lacey V. Murrow Bridge, Seattle, US (commons.wikimedia.org)................6

Figure 1.4 Ribbon floating bridge (army-technology.com) ......................................

Figure 1.5 Sckematic cross-section of the pontoon and its components ......................8

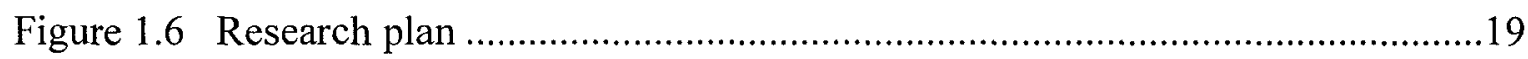

Figure 2.1 Model of the idealized floating ferry (Wu and Sheu 1996) .......................25

Figure 2.2 The relationship between the moving-load position and (a) the heave displacement and (b) the pitch angle for different moving-load speeds (Wu and Sheu 1996)

Figure 2.3 The influence of the moving-load speed on (a) the max heave displacement and (b) the max pitch angle for different moving-load values (Wu and Sheu 1996) .......26 Figure 2.4 Mathematical model for the dynamic analysis of floating bridge (Wu and Shih 1998)

Figure 2.5 The relationship between the moving-load position and the central vertical displacement in the hinged-connected floating bridge (Wu and Shih 1998). 29

Figure 2.6 Discrete-pontoon floating bridge model (Seif and Inoue 1998) ...................30 Figure 2.7 System and loads of the floating bridge (a) longitudinal section (b) crosssection (c) model (Fleischer and Park 2004)

Figure 2.8 Variation of peak values of dynamic amplification in deflection with traveling speed, for different spring stiffness values (Thambiratnam and Zhuge 1996)...33 
Figure 2.9 Time histories of maximum deflections due to one- and two-wheel loads

(Thambiratnam and Zhuge 1996) 34

Figure 2.10 Plan view of the proposed floating bridge (Seif and Koulaei 2005) .35

Figure 2.11 Cut-away of typical pontoon of Lacey V. Murrow Floating Bridge

(Dusenberry et al. 1995) 38

Figure 2.12 Structure arrangement of pontoon-type very large floating structure

(Fujikubo and Tetsuya 2001)

Figure 2.13 Modeling of bridge-vehicle system using single-axle vehicle model (Humar and Kashif 1993)

Figure 2.14 Two-axle vehicle model (Humar and Kashif 1993)

Figure 2.15 Dynamic response of the bridge to single- and two-axle vehicle loads

(Humar and Kashif 1993)

Figure 2.16 Normalized midpoint deflection in the bridge represented by beam and plate element under central vehicle load (Humar and Kashif 1995) .44

Figure 2.17 Train-bridge system (Yang and Yau 1997). .46

Figure 2.18 Vehicle-bridge interaction element (Yang and Yau 1997) .46

Figure 2.19 Beam with moving sprung mass neglecting damping (Yang and Yau 1997)

Figure 2.20 Midpoint vertical deflection in beam (Yang and Yau 1997)....... 47

Figure 2.21 Dynamic analysis procedures for bridge and vehicle interaction (Henchi et al. 1998) 48

Figure 2.22 Schematic illustration of vehicle-bridge interaction (Yang and Wu 2001)..50 
Figure 2.23 Vehicle-bridge interaction models: (a) moving load, (b) moving mass, (c) sprung mass, (d) suspended rigid beam or two-axle vehicle (Yang and $\mathrm{Wu} 2001$ ).........50

Figure 2.24 Time history of midpoint displacement in a simply supported bridge traversed by single-axle vehicle loads (Yang and Wu 2001)

Figure 2.25 Time history of midpoint displacement in a simply supported bridge traversed by a two-axle vehicle (Yang and Wu 2001) .52

Figure 2.26 General structural model for a moored floating bridge (Georgiadis 1984)..56

Figure 2.27 Buoyancy forces acting on VLFS (ISSC, 2006d) .56

Figure 2.28 Sign convections for motion response of a floating body in regular waves

(Das and Das 2005) .61

Figure 3.1 Hinged connection used for ribbon floating bridges ..............................68

Figure 3.2 Experimental loading for rigid ribbon floating bridge (Harre 2002) ...........69

Figure 3.3 Ribbon bridge traversed by a tank conccentric along the bridge centre line (defenselink.com) .72

Figure 3.4 Floating bridge simply supported at shores and resting on water along its span (army-technology.com) 74

Figure 3.5 Space frame element with twelve degrees of freedom (Rao 2004)..............76

Figure 3.6 Detailed degrees of freedom of a space frame element (Rao 2004) ............77

Figure 3.7 Idealization of bridge-vehicle system

Figure 3.8 Free body diagram of a floating bridge traversed by a moving vehicle (modified from Humar and Kashif 1993).

Figure 3.9 Free body diagram of a floating bridge traversed by a moving two-axle vehicle (modified from Humar and Kashif 1993) .98 
Figure 3.10 Cartesian coordinate system used for open channel flow

Figure 3.11 Drag forces acting on floating bridge profile due to water current

Figure 3.12 Flowchart of the analytical algorithm and the developed finite element program for the dynamic analysis of floating bridges under moving vehicle loads and dynamic water current loads

Figure 4.1 Time history of midpoint displacements in rigid-connected bridge due to 50tonne single-axle vehicle

Figure 4.2 Time history of midpoint acceleration in rigid-connected bridge due to 50tonne single-axle vehicle

Figure 4.3 Effect of vehicle speed and weight on maximum deflection in rigidconnected bridge due to single-axle vehicle

Figure 4.4 Effect of vehicle speed and weight on maximum bending moment in rigidconnected bridge due to single-axle vehicle 134

Figure 4.5 DAFs for rigid-connected bridge due to 50-tonne single-axle vehicle 135

Figure 4.6 DAFs for deflection in rigid-connected bridge due to single-axle vehicle with different weights

Figure 4.7 DAFs for bending moment in rigid-connected bridge due to single-axle vehicle with different weights

Figure 4.8 Time history of midpoint displacements in hinge-connected bridge due to 50tonne single-axle vehicle

Figure 4.9 Time history of midpoint accelerations in hinge-connected bridge due to 50 tonne single-axle vehicle 
Figure 4.10 Effect of vehicle speed and weight on maximum displacement in hingeconnected bridge due to 50-tonne single-axle vehicle

Figure 4.11 Effect of vehicle speed and weight on maximum bending moment in hingeconnected bridge due to 50 -tonne single-axle vehicle

Figure 4.12 DAFs in hinge-connected bridge due to 50-tonne single-axle vehicle ......143 Figure 4.13 DAFs for displacement in hinge-connected bridge due to 50-tonne singleaxle vehicle

Figure 4.14 DAFs for bending moment in hinge-connected bridge due to 50-tonne single-axle vehicle

Figure 4.15 Time history of midpoint displacement in rigid-connected bridge due to 50tonne two-axle vehicle $(\mathrm{v}=15 \mathrm{~km} / \mathrm{h}$, twice the traversing time $)$ 148

Figure 4.16 Time history of midpoint acceleration in rigid-connected bridge due to 50tonne two-axle vehicle $(\mathrm{v}=15 \mathrm{~km} / \mathrm{h}$, twice the traversing time $)$

Figure 4.17 Effect of vehicle speed and weight on maximum deflection in rigidconnected bridge due to two-axle vehicle

Figure 4.18 Effect of vehicle speed and weight on maximum bending moment in rigidconnected bridge due to two-axle vehicle

Figure 4.19 DAFs in rigid-connected bridge due to 50-tonne two-axle vehicle 150 Figure 4.20 DAFs for deflection in rigid-connected bridge due to 50-tonne two-axle vehicle 151

Figure 4.21 DAFs for bending moment in rigid-connected bridge due to 50-tonne twoaxle vehicle 151 
Figure 4.22 Time history of midpoint displacement in hinge-connected bridge due to 50tonne two-axle vehicle $(\mathrm{v}=15 \mathrm{~km} / \mathrm{h}$, twice the traversing time)

Figure 4.23 Time history of midpoint displacement in hinge-connected bridge due to 50tonne two-axle vehicle $(\mathrm{v}=15 \mathrm{~km} / \mathrm{h}$, twice the traversing time)

Figure 4.24 Effect of vehicle speed and weight on maximum displacement in hingeconnected bridge due to 50-tonne two-axle vehicle

Figure 4.25 Effect of vehicle speed and weight on maximum bending moment in hingeconnected bridge due to 50-tonne two-axle vehicle 157

Figure 4.26 DAFs in hinge-connected bridge due to 50-tonne two-axle vehicle 157 Figure 4.27 DAFs for displacement in hinge-connected bridge due to 50-tonne two-axle vehicle 158

Figure 4.28 DAFs for bending moment in hinge-connected bridge due to 50-tonne twoaxle vehicle 158

Figure 4.29 Time history of midpoint displacements in rigid-connected bridge due to 50-tonne single- and two-axle vehicle $(\mathrm{v}=10 \mathrm{~km} / \mathrm{h})$

Figure 4.30 Time history of midpoint displacements in rigid-connected bridge due to 50-tonne single- and two-axle vehicle $(\mathrm{v}=15 \mathrm{~km} / \mathrm{h})$ 162

Figure 4.31 Maximum deflections in rigid-connected bridge due to single- and two-axle vehicle 162

Figure 4.32 Maximum bending moments in rigid-connected bridge due to single- and two-axle vehicle 163

Figure 4.33 DAFs for deflection in rigid-connected bridge due to 50-tonne single- and two-axle vehicle 163 
Figure 4.34 DAFs for bending moment in rigid-connected bridge due to 50-tonne single- and two-axle vehicle

Figure 4.35 Time history of midpoint displacements in hinge-connected bridge due to 50-tonne single- and two-axle vehicle $(\mathrm{v}=10 \mathrm{~km} / \mathrm{h})$ .166

Figure 4.36 Time history of midpoint displacements in hinge-connected bridge due to 50-tonne single- and two-axle vehicle $(\mathrm{v}=15 \mathrm{~km} / \mathrm{h})$ 167

Figure 4.37 Maximum displacements in hinge-connected bridge due to single- and twoaxle vehicle 167

Figure 4.38 Maximum bending moments in hinge-connected bridge due to single- and two-axle vehicle 168

Figure 4.39 DAFs for displacement in hinge-connected bridge due to 50-tonne singleand two-axle vehicle 168

Figure 4.40 DAFs for bending moments in hinge-connected bridge due to 50-tonne single- and two-axle vehicle

Figure 4.41 Effect of vehicle speed on maximum torsional moment due to 50 -tonne twoaxle eccentric vehicle loads

Figure 4.42 Time history of midpoint displacements in rigid-connected steel and aluminum bridges due to 50 -tonne two-axle vehicle loads $(\mathrm{v}=10 \mathrm{~km} / \mathrm{h})$

Figure 4.43 Time history of midpoint displacements in rigid-connected steel and aluminum bridges due to 50-tonne two-axle vehicle loads $(\mathrm{v}=15 \mathrm{~km} / \mathrm{h})$ 174

Figure 4.44 Effect of vehicle speed on maximum deflection in rigid-connected steel and aluminum bridges due to 50 -tonne two-axle vehicle loads 175 
Figure 4.45 Effect of vehicle speed on maximum bending moment in rigid-connected steel and aluminum bridges due to 50-tonne two-axle vehicle loads

Figure 4.46 Time history of midpoint displacements in hinge-connected steel and aluminum bridges due to 50 -tonne two-axle vehicle loads $(\mathrm{v}=10 \mathrm{~km} / \mathrm{h})$

Figure 4.47 Time history of midpoint displacements in hinge-connected steel and aluminum bridges due to 50 -tonne two-axle vehicle loads $(\mathrm{v}=15 \mathrm{~km} / \mathrm{h})$

Figure 4.48 Effect of vehicle speed on maximum displacement in hinge-connected steel and aluminum bridges due to 50-tonne two-axle vehicle loads.

Figure 4.49 Effect of vehicle speed on maximum bending moment in hinge-connected steel and aluminum bridges due to 50-tonne two-axle vehicle loads

Figure 4.50 Effect of vehicle speed and weight on maximum deflection in rigid-

connected bridge (two successive vehicles with spacing $50 \mathrm{~m}$ )

Figure 4.51 Effect of vehicle speed and weight on maximum bending moment in rigidconnected bridge (two successive vehicles with spacing $50 \mathrm{~m}$ ) 188 Figure 4.52 DAFs in rigid-connected bridge due to two successive 50-tonne vehicle loads with spacing $50 \mathrm{~m}$ 188 Figure 4.53 DAFs for deflection in rigid-connected bridge due to two successive vehicles with spacing $50 \mathrm{~m}$ 189 Figure 4.54 DAFs for bending moment in rigid-connected bridge due to two successive vehicles with spacing $50 \mathrm{~m}$ 189

Figure 4.55 Effect of vehicle speed and weight on maximum deflection in rigidconnected bridge (two successive vehicles with spacing $40 \mathrm{~m}$ ) 190 
Figure 4.56 Effect of vehicle speed and weight on maximum bending moment in rigid-

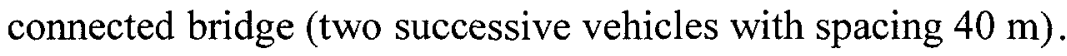
190

Figure 4.57 DAFs in rigid-connected bridge due to two successive 50-tonne vehicle loads with spacing $40 \mathrm{~m}$

Figure 4.58 DAFs for deflection in rigid-connected bridge due to two successive vehicles with spacing $40 \mathrm{~m}$

Figure 4.59 DAFs for bending moment in rigid-connected bridge due to two successive vehicles with spacing $40 \mathrm{~m}$

Figure 4.60 Effect of vehicle speed and weight on maximum deflection in rigid-

connected bridge (two successive vehicles with spacing $30 \mathrm{~m}$ )

Figure 4.61 Effect of vehicle speed and weight on maximum bending moment in rigidconnected bridge (two successive vehicles with spacing $30 \mathrm{~m}$ )

Figure 4.62 DAFs in rigid-connected bridge due to two successive 50-tonne vehicle loads with spacing $30 \mathrm{~m}$

Figure 4.63 DAFs for deflection in rigid-connected bridge due to two successive vehicles with spacing $30 \mathrm{~m}$ 194

Figure 4.64 DAFs for bending moment in rigid-connected bridge due to two successive vehicles with spacing $30 \mathrm{~m}$ 194

Figure 4.65 Effect of vehicle speed and weight on maximum displacement in hingeconnected bridge (two successive vehicles with spacing $50 \mathrm{~m}$ ) .202 Figure 4.66 Effect of vehicle speed and weight on maximum bending moment in hingeconnected bridge (two successive vehicles with spacing $50 \mathrm{~m}$ ) 202 
Figure 4.67 DAFs in hinge-connected bridge due to two successive 50-tonne vehicle loads with spacing $50 \mathrm{~m}$ 203

Figure 4.68 DAFs for displacement in hinge-connected bridge due to two successive vehicles with spacing $50 \mathrm{~m}$ 203

Figure 4.69 DAFs for bending moment in hinge-connected bridge due to two successive vehicles with spacing $50 \mathrm{~m}$ 204

Figure 4.70 Effect of vehicle speed and weight on maximum displacement in hingeconnected bridge (two successive vehicles with spacing $40 \mathrm{~m}$ ).

Figure 4.71 Effect of vehicle speed and weight on maximum bending moment in hingeconnected bridge (two successive vehicles with spacing $40 \mathrm{~m}$ ). 205

Figure 4.72 DAFs in hinge-connected bridge due to two successive 50-tonne vehicle loads with spacing $40 \mathrm{~m}$ .205

Figure 4.73 DAFs for displacement in hinge-connected bridge due to two successive vehicles with spacing $40 \mathrm{~m}$ 206

Figure 4.74 DAFs for bending moment in hinge-connected bridge due to two successive vehicles with spacing $40 \mathrm{~m}$ .206

Figure 4.75 Effect of vehicle speed and weight on maximum displacement in hingeconnected bridge (two successive vehicles with spacing $30 \mathrm{~m}$ )

Figure 4.76 Effect of vehicle speed and weight on maximum bending moment in hingeconnected bridge (two successive vehicles with spacing $30 \mathrm{~m}$ ) 207

Figure 4.77 DAFs in hinge-connected bridge due to two successive 50-tonne vehicle loads with spacing $30 \mathrm{~m}$ 208 
Figure 4.78 DAFs for displacement in hinge-connected bridge due to two successive vehicles with spacing 30 .208

Figure 4.79 DAFs for bending moment in hinge-connected bridge due to two successive vehicles with spacing 30 .209

Figure 4.80 Time history of midpoint displacements in rigid-connected bridge due to two successive 50 -tonne vehicle loads with different spacing $(\mathrm{v}=10 \mathrm{~km} / \mathrm{h})$ .212 Figure 4.81 Time history of midpoint displacements in rigid-connected bridge due to two successive 50 -tonne vehicle loads with different spacing $(\mathrm{v}=15 \mathrm{~km} / \mathrm{h})$ .212 Figure 4.82 Time history of midpoint accelerations in rigid-connected bridge due to two successive 50-tonne vehicle loads with different spacing $(\mathrm{v}=10 \mathrm{~km} / \mathrm{h})$ 213 Figure 4.83 Time history of midpoint accelerations in rigid-connected bridge due to two successive 50 -tonne vehicle loads with different spacing $(\mathrm{v}=15 \mathrm{~km} / \mathrm{h})$ 213 Figure 4.84 Maximum deflections in rigid-connected bridge due to 50-tonne vehicle loads. 214

Figure 4.85 Maximum bending moments in rigid-connected bridge due to 50-tonne vehicle loads .214

Figure 4.86 DAFs for deflection in rigid-connected bridge due to 50-tonne vehicle loads .215

Figure 4.87 DAFs for bending moment in rigid-connected bridge due to 50-tonne vehicle loads .215

Figure 4.88 Time history of midpoint displacements in hinge-connected bridge due to two successive 50 -tonne vehicle loads with different spacing $(\mathrm{v}=10 \mathrm{~km} / \mathrm{h}$ ) 
Figure 4.89 Time history of midpoint displacements in hinge-connected bridge due to two successive 50-tonne vehicle loads with different spacing $(\mathrm{v}=15 \mathrm{~km} / \mathrm{h})$

Figure 4.90 Time history of midpoint acceleration in hinge-connected bridge due to two successive 50-tonne vehicle loads with different spacing $(\mathrm{v}=10 \mathrm{~km} / \mathrm{h})$ .220

Figure 4.91 Time history of midpoint acceleration in hinge-connected bridge due to two successive 50-tonne vehicle loads with different spacing $(\mathrm{v}=15 \mathrm{~km} / \mathrm{h})$ .220

Figure 4.92 Maximum displacements in hinge-connected bridge due to 50-tonne vehicle loads .221

Figure 4.93 Maximum bending moments in hinge-connected bridge due to 50-tonne vehicle loads 221

Figure 4.94 DAFs for displacement in hinge-connected bridge due to 50-tonne vehicle loads .222

Figure 4.95 DAFs for bending moment in hinge-connected bridge due to 50-tonne vehicle loads .222

Figure 4.96 Maximum vertical displacements in rigid-connected bridge 226

Figure 4.97 Maximum bending moments in rigid-connected bridge .226 Figure 4.98 Maximum lateral displacements in rigid-connected bridge (current velocity $1 \mathrm{~m} / \mathrm{s})$ .227

Figure 4.99 Maximum lateral bending moments in rigid-connected bridge (current velocity $1 \mathrm{~m} / \mathrm{s})$ .227

Figure 4.100 Maximum torsional moments in rigid-connected bridge (current velocity 1 $\mathrm{m} / \mathrm{s})$ 228

Figure 4.101 Maximum vertical displacements in hinge-connected bridge .230 
Figure 4.102 Maximum bending moments in hinge-connected bridge

Figure 4.103 Maximum lateral displacements in hinge-connected bridge (current velocity $1 \mathrm{~m} / \mathrm{s}$ )

Figure 4.104 Maximum lateral bending moment in hinge-connected bridge (current velocity $1 \mathrm{~m} / \mathrm{s}$ )

Figure 4.105 Maximum torsional moment in hinge-connected bridge (current velocity 1 $\mathrm{m} / \mathrm{s})$ .232

Figure 5.1 A simply supported bridge traversed by a moving vehicle load (Humar and Kashif 1993)

Figure 5.2 Time history of midpoint deflection in a simply supported bridge traversed by a moving vehicle load obtained by different approaches .237

Figure 5.3 Time history of midpoint dimensionless deflection in a simply supported bridge traversed by a moving vehicle load obtained by different approaches. .237

Figure 5.4 Simply supported bridge traversed by a two-axle vehicle (Yang and Wu 2001)

Figure 5.5 Time history of midpoint displacement in a simply supported bridge traversed by a two-axle vehicle (Yang and $\mathrm{Wu} 2001$ ) .239

Figure 5.6 Time history of midpoint displacement in a simply supported bridge traversed by a two-axle vehicle (developed FE program) .239

Figure 5.7 Mathematical model of the floating bridge studied by Wu and Shih (1998) 
Figure 5.8 The relationship between the moving load position and the central vertical displacement in the hinged-connected bridge $\left(P_{c}=50 \mathrm{kN}, e_{P}=9 \mathrm{~m}, v_{P}=26 \mathrm{~m} / \mathrm{s}\right)$ (Wu and Shih 1998)

Figure 5.9 The relationship between the moving load position and the central vertical displacement in the hinged-connected bridge $\left(P_{c}=50 \mathrm{kN}, e_{P}=9 \mathrm{~m}, v_{P}=26 \mathrm{~m} / \mathrm{s}\right)$ (developed FE program)

Figure 5.10 The relationship between the moving load position and the central vertical displacement in the hinged-connected bridge $\left(P_{c}=50 \mathrm{kN}, e_{P}=9 \mathrm{~m}, v_{P}=40 \mathrm{~m} / \mathrm{s}\right)$ (Wu and Shih 1998)

Figure 5.11 The relationship between the moving load position and the central vertical displacement in the hinged-connected bridge $\left(P_{c}=50 \mathrm{kN}, e_{P}=9 \mathrm{~m}, v_{P}=40 \mathrm{~m} / \mathrm{s}\right)$ (developed FE program)

Figure 5.12 Enlarged view of the left end of the floating bridge finite element model

Figure 5.13 Load-step file for the vehicle load moving along the bridge 250

Figure 5.14 Deflection in rigid-connected floating bridge for different position of the vehicle load along the bridge using ANSYS

Figure 5.15 Time history for midpoint displacement in rigid-connected floating bridge due to 50 tonne single-axle vehicle loads using ANSYS software package 253 Figure 5.16 Time history for midpoint displacements in rigid-connected floating bridge due to 50 tonne single-axle vehicle loads using ANSYS and the developed FE program 
Figure 5.17 Bridge with three intermediate hinges at one fourth, half and three fourth of the bridge length .256

Figure 5.18 Enlarged view of part of the floating bridge including an intermediate hinge .257

Figure 5.19 Vertical displacement in hinged-connected floating bridge for different position of the vehicle load along the bridge using ANSYS .259

Figure 5.20 Time history for midpoint displacement in hinged-connected floating bridge due to 50 tonne single-axle vehicle loads using ANSYS software package .259 Figure 5.21 Time history for midpoint displacement in hinged-connected floating bridge due to 50 tonne single-axle vehicle loads using ANSYS and the developed FE program 


\section{List of Appendices}

Appendix A: Parametric Study for the Structural Behaviour of Floating Bridges

Subjected to Lateral Water Current Loads ...... .276 Appendix B: Ribbon Bridge Composition, Construction and Operation .....................289 


\section{Chapter: Introduction}

Bridges are one of the most important transportation infrastructures worldwide because they provide mobility of people and goods across natural and/or man-made obstacles such as water channels, rivers and ravines, and alleviate traffic congestion in urban areas. Bridges are a common feature in many modes of transportation, and they become an important factor in the economic development and cultural exchange among people. In addition to the traditional use of bridge structures for day-to-day normal operational services, bridges play a strategic role during emergencies and war times, where they are essential for providing a safe path for evacuating crowds and injured people, transporting supplies, and other relevant movements.

Generally, bridges are designed and built to last for long periods and therefore they are built as fixed structures when crossing over land and water bodies. However, under certain conditions, such as emergencies or a state of war, fixed bridges may be supplemented or replaced by floating bridges which float directly on water, relying mainly on the buoyancy to support the structure. Among the main characteristics of the floating bridges are their relative light weight, higher mobility and shorter time for construction. Most floating bridges are made of light concrete, steel, aluminum alloys and composite materials (Seif and Inoue, 1998; Seif and Koulaei, 2005).

Under certain conditions, floating bridges may have several advantages over conventional fixed bridges. Due to their temporary nature, they have been designed to take advantage of the buoyancy force generated by floating over water instead of just considering water as a barrier. They offer the most economic and practical solution for 
civilian water crossing in deep waters or in case of soft soil conditions of a river bed. Clearly, for short-term use the economics are expected to make them viable economic alternatives when the cost of building a fixed structure exceeds several times the economic return to be gained at that location. Under certain conditions, a floating bridge is estimated to cost three to five times less than a conventional long-span bridge (Watanabe and Utsunomiya 2003). Floating bridges that are permanent floating structures may require a minimum head clearance under the bridge deck or a movable section to allow ships to pass in navigable rivers or channels. Those permanent floating bridges are mainly used for civilian water crossings.

Temporary floating bridges are the main means for civilian water crossing for evacuation in case of emergencies and natural disasters. For example, severe earthquakes can lead to damage of conventional fixed bridges. In this case, temporary floating bridges can be quickly constructed across rivers and/or canals, and used for water crossing having the advantage of being less affected by earthquakes. Temporary floating bridges can be used in many other applications as needed, for example to get access to some areas that are surrounded by water, whether they are industrial areas or even historical or entertainment areas.

Floating bridges are also the main means for moving of troops, heavy equipment and supplies across water during military operations. Military floating bridges are mobile, movable and quickly installable regardless of water depth or type of soil of the water bed. Damaged units are easily replaced when required. These bridges are temporary structures 
that can be easily dismantled after use and transported to be used at other locations in further crossing operations and to prevent hostile forces from taking them over.

Military floating bridges should be designed and constructed to have certain characteristics different than those of civilian floating bridges. They are expected to have higher loading capacities, higher limits of deflections, be lightweight, have lower service life time, have the ability of being easily transported and have shorter installation time. Also, military floating bridges are usually constructed to serve traffic in one-way direction and not two-way traffic as in the case of civilian bridges. The following section discusses the main types of floating bridges and their major characteristics.

\subsection{Types of Floating Bridges}

Similar to conventional bridges, there are several types that structurally describe the majority of floating bridges. The most common types of floating bridges are as follows (Watanabe and Utsunomiya 2003; Chen and Duan 1999; Oka et al. 2000):

- Separated pontoon floating bridges where the bridge is supported by separated or discrete pontoon foundations (Figure 1.1(a)).

- Continuous pontoon floating bridges (Figure 1.1(b)). This type of floating bridges has full surface contact with water and is also called pontoon girders floating bridges.

- Rapid deployment floating bridges or Ribbon floating bridges (Figure 1.4) are a special type of continuous pontoon floating bridges. A rapid deployment bridge is 
a modular asset consisting of a number of pontoons connected together in the field in a short time for water crossing.

Separated pontoon floating bridges and continuous pontoon floating bridges are the most commonly used types of floating bridges for civilian water crossing (Oka et al. 2000; Chen and Duan 1999). In military and emergency operations, the rapid deployment floating bridges are the main means for water crossing and this makes them of great importance regarding safety issues and national defense. For these reasons, the rapid deployment floating bridge will form the focus of the present study. The most commonly used types of floating bridges are briefly discussed below.

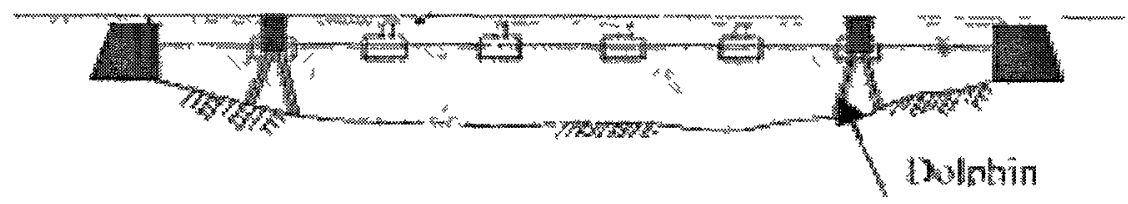

(a) Separated pontoon bridge

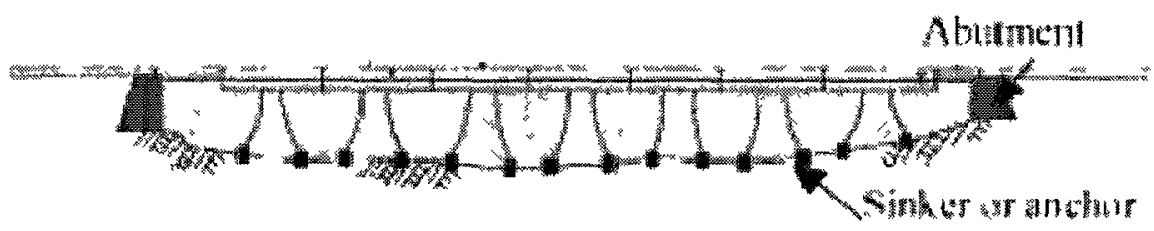

(b) Continues pontoon bridge

Figure 1.1 Types of Floating Bridges (Watanabe and Utsunomiya 2003)

\subsubsection{Separated Pontoon Bridges}

A separated pontoon bridge is a floating structure supported by discrete floating units called pontoons with sufficient buoyancy to support the bridge and the loads (both static and dynamic) acting on it. Pontoons are hollow floating units that can be made of 
lightweight reinforced concrete, prestressed concrete, steel, aluminum or composite lightweight materials. The bridge has a superstructure that is supported by these floating pontoons; this superstructure is designed to be able to carry the traffic loads safely. Bergsoysund Floating Bridge at Bergsoyfjord near Kristiansund (Figure 1.2) and Nordhordland Floating Bridge at Salhus near Bergen, Norway are two examples of separated pontoon floating bridges.

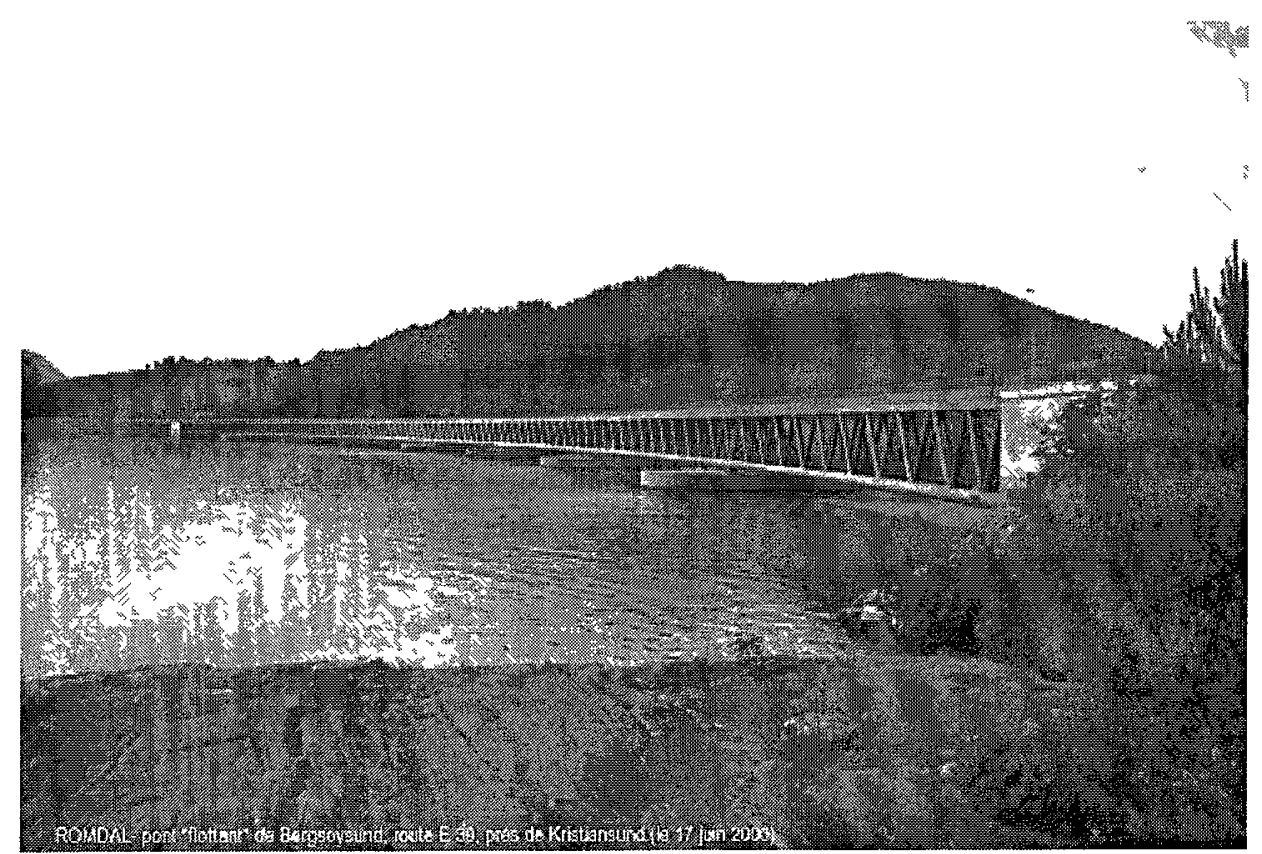

Figure 1.2 Bergsoysund pontoon bridge, Norway (Watanabe 2003)

\subsubsection{Continuous Pontoon Bridges}

A continuous pontoon floating bridge has full surface contact with water as the pontoons supporting the bridge are continuous. Continuous pontoon floating bridge can have a superstructure on top of the pontoons to carry the traffic loads; also the top of the pontoons can be used as roadway. The Lacey V. Murrow Bridge in Seattle (Figure 1.3), the Hood Canal Bridge, the Evergreen Point Bridge and the Homer Hadley Bridge are 
examples of continuous pontoon floating bridges constructed across Washington Lake, USA. This type of floating bridge can allow for navigation of ships at some elevated sections. The drag forces acting on a continuous pontoon floating bridge due to water current are greater than those acting on a separated pontoon floating bridge. In the analysis of this type of floating bridge, water current loads are one of the major loads acting on the bridge. At the same time, the buoyancy force offered by water and supporting this type of bridge is greater than that offered to a separated pontoon bridge because of the large contact surface with water and the large volume of the fluid displaced by the continuous pontoon bridge.

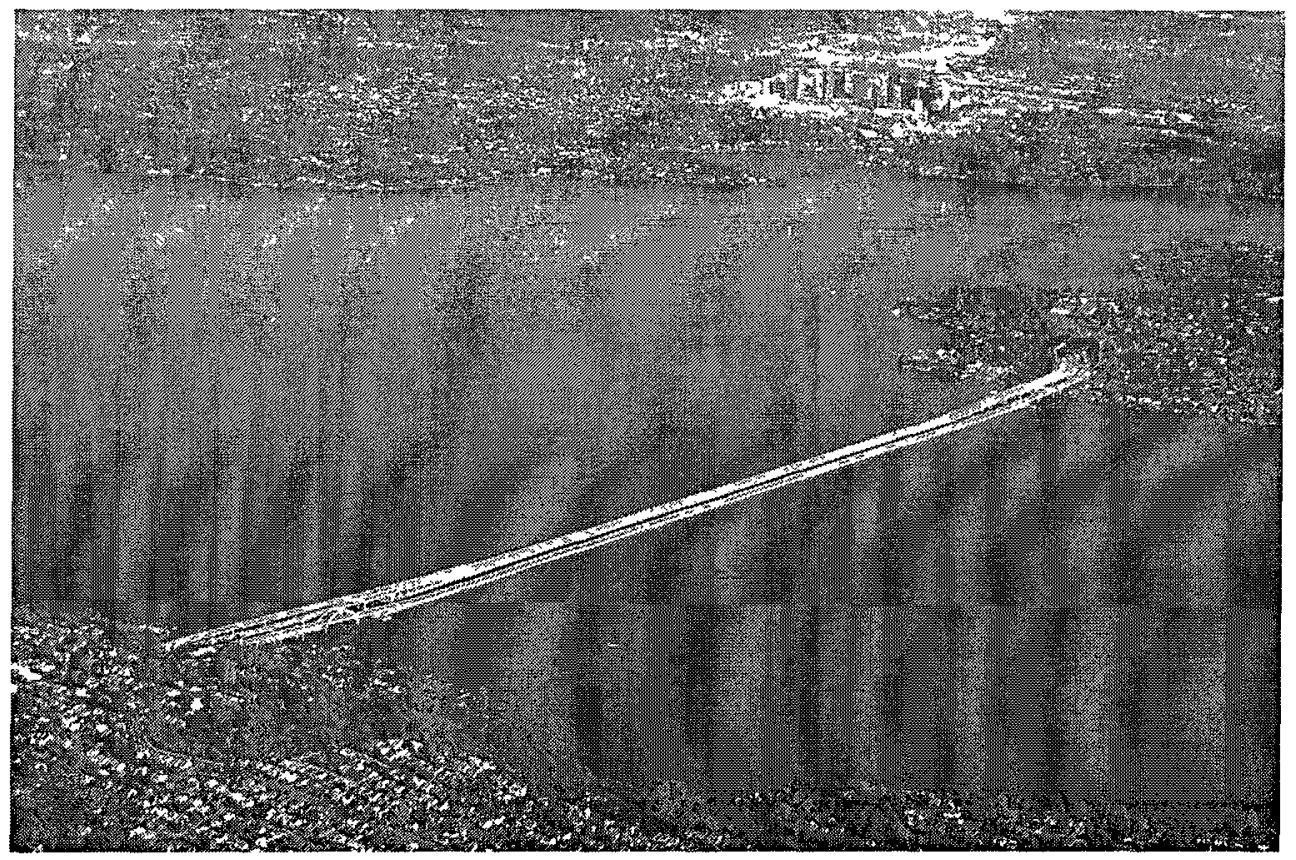

Figure 1.3 Lacey V. Murrow Bridge, Seattle, US (commons.wikimedia.org)

\subsubsection{Rapid Deployment Floating Bridges (Ribbon Bridges)}

Rapid deployment floating bridges or Ribbon bridges (Figure 1.4) are a special type of continuous pontoon floating bridges. A rapid deployment bridge is a modular asset 
consisting of a number of pontoons connected together in the field with special connections to form the bridge. These connections can be hinged or rigid connections and they enable the bridge to be constructed and/or disassembled in a short period of time. The pontoon is a closed-shape section (Figure 1.5) that has the ability to float while carrying the imposed loads safely. The closed-shape pontoon consists of an upper deck that carries the traffic loads, a lower deck that resists the hydrostatic pressure induced from water and side stiffening which connects the upper and lower decks as well as resists the lateral loads such as water current, waves and wind loadings in addition to the reactions induced from supporting boats.

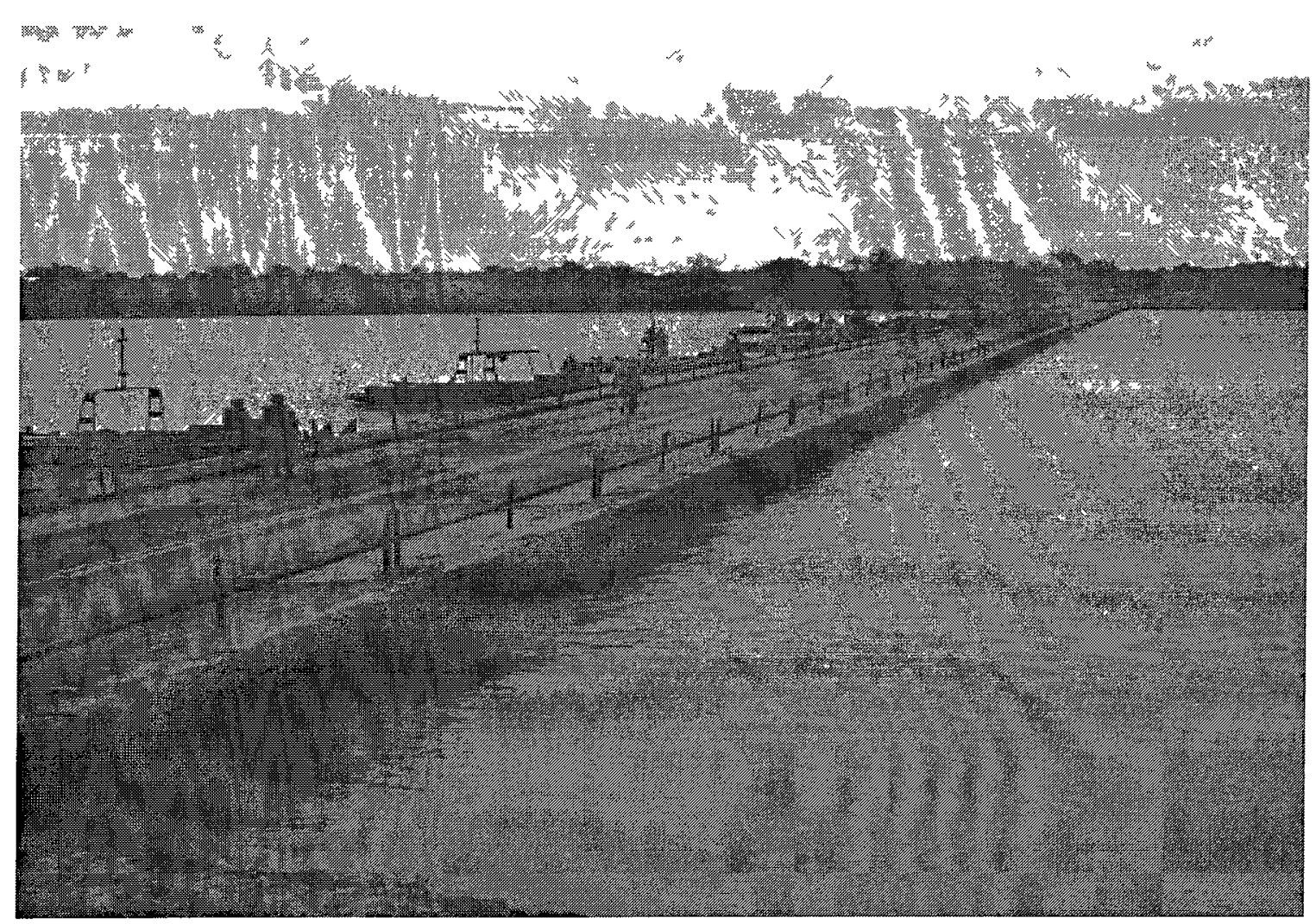

Figure 1.4 Ribbon floating bridge (army-technology.com). 
Rapid deployment bridges have been used to a great advantage in many battles throughout the history of wars as they provide a fast means for crossing rivers and waters channels. The famous crossings in the Second World War, October war and Iraq invasion are examples of the strategic significance of these bridges.

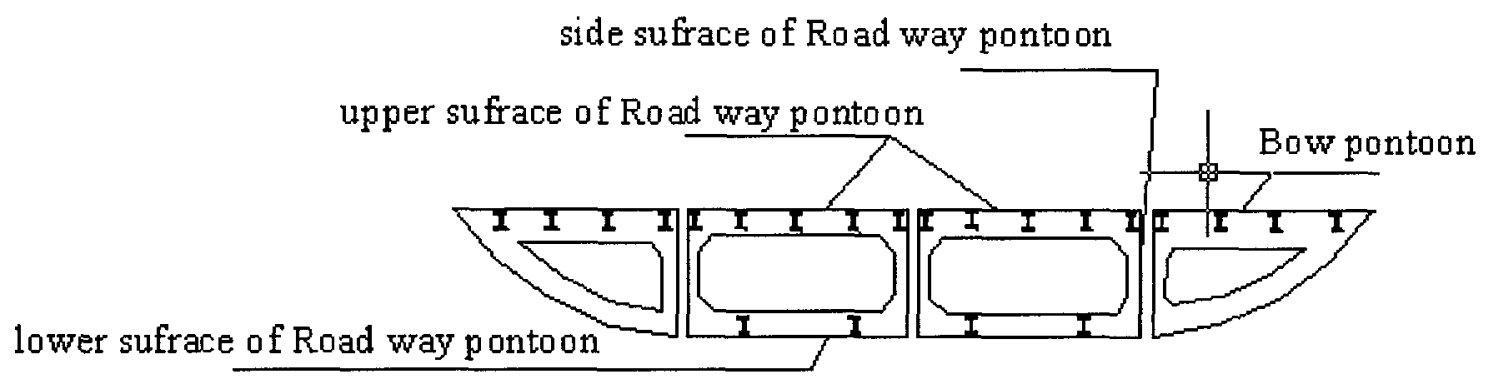

Figure 1.5 Schematic cross-section of the pontoon and its components

\subsection{Main Loads Acting on Floating Bridges}

Floating bridge analysis requires careful study of all loads acting on them. The most important loads that should be taken into account during the different stages of the design and analysis of the global behaviour of the bridge are mentioned briefly below.

\subsubsection{Self Weight and Buoyancy}

In structural engineering the self-weight of the structure is often a major contributor to the overall load. In case of floating bridges, another major force, the buoyancy force, has to be considered with the self-weight of the bridge. The buoyancy force is calculated as the integration of the static pressure over the undisturbed wetted surface of the floating bridge, and is equal to the weight of the water displaced by the floating bridge. The 
buoyancy force is an upward force which is equilibrated with the self-weight of the floating bridge.

\subsubsection{Traffic Loads}

The main objective for constructing a floating bridge is to pass the traffic from one side of a water channel to the other side. The vehicles passing a floating bridge can induce both static and dynamic loading on the bridge. Vibration of the bridge due to passing vehicles is a very important consideration in the analysis and design of bridges. Owing to the evolution of heavier and faster vehicles, the dynamic response of bridges to moving vehicle loads has become a very important factor in bridge design. The displacements and straining actions developed in the bridge can be magnified due to the interaction between the vehicle and the bridge. The bridge must be designed to withstand the induced straining actions and to be stiff enough to limit the displacements and keep them within the acceptable limits.

\subsubsection{Water Current Loads}

The transverse loading induced by the water current is one of the major loads acting on a floating bridge, especially in the case of continuous pontoon floating bridges, and it is a major source of out-of-plane deformations and straining actions developed in a floating bridge. In practice, a significant reduction in the transverse deformations and straining actions is achieved by providing transverse supports for the bridge at different locations along its length. A good understanding of the hydraulic characteristics of water flow is necessary for accurate floating bridge design. 


\subsubsection{Wave Loads}

Wave loads are of great importance in the global dynamic analysis of floating structures constructed in open seas. Winds in an ocean, open sea, or large lake generate waves on the surface of the ocean or sea; these waves are a basic feature of coastal regions. In the design of floating structures, it is very important to determine the forces these waves can generate on such floating structures. In case of floating bridges constructed across small rivers or narrow water channels, wave loads are small and have minor effects on the bridge and therefore can be neglected.

\subsubsection{Wind Loads}

Wind loads acting on rapid deployment floating bridge are typically small as a great part of the bridge is immersed in water. Forces due to wind on such a floating bridge can only arise from the fetch, the clear height over the water, which is usually small. Based on the local wind conditions in a particular site, wind loads can be considered or neglected in the design of a floating bridge.

\subsubsection{Earthquake Loads}

Floating bridges are not affected directly by ground shaking due to earthquakes like landsupported bridges. Earthquakes can cause minor surface waves in the water channel. In ocean or open seas, strong earthquakes can cause tsunamis or harbour waves that affect floating structures constructed there. In the case of floating bridges supported with anchors located on a soft soil, an earthquake can cause the anchors to slip or move creating bending stresses in the floating bridge. In the case of ribbon floating bridges 
supported laterally with boats like those used for military operations, the effect of earthquakes is negligible and often ignored in the design.

\subsection{Fluid-Structure Interaction}

Fluid-structure interaction is one of the most important considerations in the design of floating bridges. In fluid-structure interaction problems, separate structural modeling and hydrodynamic modeling can be combined to obtain the system response. In this case, the structural properties and movements will affect the fluid and vice versa. The oscillating body in the fluid will raise the movement of surrounding fluid; this increased movement of the surrounding fluid will in turn affect the oscillations of the floating body. The inertial forces of the fluid will induce reaction forces to the wetted surface of the body.

The fluid forces acting upon a structure moving in fluid may have terms proportional to acceleration, velocity and displacement. Terms proportional to the structural acceleration are defined as mass terms, terms proportional to the velocity are defined as damping terms and terms proportional to the displacement are defined as stiffness terms. For example, the added mass or virtual mass is the inertial force added to a structural system because an accelerating or decelerating body in a fluid must move some volume of surrounding fluid as it moves through it, since the object and fluid cannot occupy the same physical space simultaneously. For simplicity this can be modeled as some volume of fluid moving with the object, although in reality all the fluid will be accelerated to various degrees. The interactive bridge-vehicle dynamic analysis is extended to the field of floating bridges by incorporating the water hydrostatic and hydrodynamic forces 
including the buoyancy forces and the added mass and damping caused by the movement of the surrounding fluid into these complex models for bridge-vehicle interaction.

\subsection{Problem Definition}

Rapid deployment floating bridges are of strategic significance regarding safety of civilian and military personnel. Therefore, it is very important to develop reliable and high-performance rapid deployment floating bridges; this can be achieved through further investigation of the transport capacity and dynamic response of these bridges to the main loads of moving vehicles and water current.

The displacements and straining actions at some point in a floating bridge due to the passage of a vehicle differ from those induced by a static vehicle load acting at the same point. This different behaviour makes the dynamic response of bridges to moving vehicle loads a major factor in bridge design. The time-dependant forces caused by moving vehicles and induced vibrations on the bridge can lead to additional effects on the bridge, increasing the displacements and straining actions developed in the bridge.

Vehicle-bridge interaction, considering the dynamic characteristics of the vehicle, can increase the displacements and straining actions developed in the bridge and this should be considered in the bridge design. Therefore, the vehicle, the bridge and the surrounding fluid cannot be treated separately and should be coupled together in one dynamic system to get the dynamic response of the floating bridge. The interaction between the vehicle, the bridge and the fluid results in dynamic equations of motions composed of high order 
matrices, and this makes the dynamic analysis of the integrated vehicle-bridge-fluid system a complicated mathematical problem.

"In practice, any bridge is assigned a class number representing the load it can carry. Vehicles are also assigned a number indicating the minimum class of bridge they are authorized to use" (DOD Dictionary of Military Terms). This standard system of class numbering is referred to as Military Load Classification (MLC). Many existing rapid deployment floating bridges were designed and classified in accordance with MLC60, which means that the bridge can safely carry vehicles of weight up to 60 tonnes. The recent ones are MLC70 that can carry vehicles up to 70 tonnes with limitations on their traversing speed and the minimum distances separating them. This means that the transport capacity of floating bridges needs further investigation in terms of the parameters controlling the rate of flow of vehicles on the bridge; namely the vehicle weight, vehicle speed, number of vehicles traversing the bridge at the same time and the separation distance between successive vehicles.

Due to the evolution of new generations of heavier and faster vehicles and tanks, new requirements for bridge design must be considered in terms of capacities, stability and dynamic behaviour. This means that there is an urgent need to further investigate the dynamic behaviour of floating bridges interactively with the moving vehicles and underlying water upthrust forces. Therefore, there is a need to establish new tools for the dynamic analysis, assessment and optimum design of bridges.

Rapid deployment floating bridges differ from conventional bridges in many aspects. They lack the rigid intermediate supports and have an alternative support provided by 
water uplift forces as they use buoyancy force as a part of the load-resisting system. These bridges usually have long spans constructed piece by piece to cover the entire width of a water channel. This makes the bridge highly flexible and subject to high deformations and straining actions due to vertical vehicle loads as well as to lateral water current loads. While vertical vehicle loads may affect only a few bays in the vicinity of the loaded bay, water current loads act on the whole bridge length producing high transverse straining actions and displacements.

Water current loads depend on the depth of submerged part of the bridge; this submerged depth is not constant along the bridge length, but varies from the unloaded pontoons to the loaded ones according to the position of the vehicle along the bridge. This submerged depth also changes with time as the vehicle position changes with time inducing different submerged depth at each node along the bridge. For accurate analysis of floating bridges, water current loads need to be treated as dynamic loads that change with both time and space coordinates. Therefore, it is important to investigate the three-dimensional interactive dynamic response of rapid deployment floating bridges to moving vehicle loads and dynamic water current loads.

Some of the main problems encountered in the analysis, design and field use of rapid deployment floating bridges are summarized as follows:

- The inability of existing rapid deployment bridges to carry higher traffic loads, corresponding to the evolution of new generations of heavier vehicles.

- The limitations on the speed of the vehicles traversing the bridge (see Table 1.1). This means that a longer time is needed for the passage of vehicles. It is clear that 
the traversing time is a major factor for evacuation in case of emergencies and natural disasters and also in case of military operations, which may lead to success or failure of a mission.

- The limitations on the minimum distance between successive vehicles traversing the bridge at the same time which affects the traversing time as well. The minimum distance between the wheel contact points of two successive vehicles should be greater than $30.5 \mathrm{~m}$ (100 ft), (Rosenthal et al. 1996).

- The transport capacity and dynamic response of rapid deployment floating bridges need more investigation, especially for successive vehicles traversing the bridge at different speeds and with different separation distances.

- Investigating the three-dimensional interactive dynamic response of rapid deployment floating bridges to moving vehicle loads and dynamic water current loads.

Table 1.1: Vehicle design crossing speeds (Rosenthal et al. 1996)

\begin{tabular}{|c|c|c|}
\hline Vehicle Design Speed & Up to MLC 30 & Above MLC 30 \\
\hline Essential* & $25 \mathrm{~km} / \mathrm{h}(15 \mathrm{mi} / \mathrm{h})$ & $16 \mathrm{~km} / \mathrm{h}(10 \mathrm{mi} / \mathrm{h})$ \\
\hline Desirable & $40 \mathrm{~km} / \mathrm{h}(25 \mathrm{mi} / \mathrm{h})$ & $25 \mathrm{~km} / \mathrm{h}(15 \mathrm{mi} / \mathrm{h})$ \\
\hline
\end{tabular}

*Essential speeds are the maximum speeds under normal field conditions and the speeds up to which bridges must be tested.

\subsection{Objectives and Scope}

The present research deals with the dynamic response of rapid deployment floating bridge to moving vehicle loads and water current loads. An analytical technique for the dynamic analysis of the vehicle-bridge-fluid integrated system is developed based on 
finite element method of analysis. The bridge is modeled using space frame elements and the vehicle is represented by different models including single- and multiple-axle models. Fluid-structure interaction is considered by adding the hydrostatic and hydrodynamic forces acting on the bridge due to the surrounding fluid. This analytical technique is implemented in a finite element program developed by the author for the formulation of the dynamic equations of motion of vehicle-bridge-fluid system, as well as conducting the dynamic analysis and determining the floating bridge dynamic response.

The dynamic response of rigid-connected and hinge-connected floating bridges to moving vehicle loads is investigated for the passage of single and multiple vehicles considering different vehicle weights, speeds and separation distances between successive vehicles. A practical bridge assignment is presented by investigating the dynamic response of rapid deployment bridges consisting of hinge-connected typical bays; each typical bay consists of three pontoons rigidly connected to each other.

The structural behaviour of floating bridges subjected to lateral water current loads is also investigated. The effects of water current velocity, number of supporting boats and their locations and the magnitude of force they exert on the bridge are investigated (Appendix A). Finally, the three-dimensional interactive dynamic behaviour of floating bridges due to the combined actions of moving vehicle loads and dynamic water current loads along with possible eccentricities of these loads is investigated. Water current loads are treated as dynamic loads that change with both time and space coordinates and determined based on the actual dynamic response of the bridge profile, and are incorporated into the dynamic equations of vehicle-bridge-fluid system. 
The objectives of the present research can be summarized as follows:

- To develop an analytical technique and implement it into a finite element program as a useful tool for the dynamic analysis and assessment of floating bridges.

- To investigate the dynamic response of rigid-connected and hinge-connected rapid deployment floating bridges to the passage of single and multiple vehicles considering different vehicle weights, speeds and separation distances between successive vehicles.

- To optimize the transport capacity of rapid deployment floating bridges in terms of the parameters controlling the rate of flow of vehicles on the bridge, namely; the vehicle weight, the vehicle speed and the separation distances between successive vehicles.

- To investigate the dynamic response of light-weight floating bridges to moving vehicle loads, and the use of light-weight materials for improving the global dynamic response of floating bridges.

- To investigate the three-dimensional interactive dynamic behaviour of floating bridges due to the combined actions of moving vehicle loads and dynamic water current loads along with possible eccentricities of these loads.

\subsection{Research Plan}

As discussed above, one of the main objectives of the present thesis is to optimize the transport capacity of rapid deployment floating bridges in terms of the parameters controlling the rate of flow of vehicles on the bridge, namely; the vehicle weight, the vehicle speed and the separation distances between successive vehicles. The results of 
this thesis are expected to provide recommendations and guidelines for the optimum use of the bridge to support a certain group of vehicles. In order to meet this objective, a research plan is adopted consisting of several phases as shown in Figure 1.6.

The first phase includes two tasks. The first task is to conduct a comprehensive literature review and summarize its main findings. The second task deals with identifying the problem and defining the main parameters contributing to it. The second phase consists of three tasks. The first task includes the details of the bridge and vehicle models and the development of an analytical approach for the dynamic analysis of floating bridges under the effects of moving vehicle loads and water current loads. The second task is the implementation of this analytical approach. The third task includes the development of a finite element program as a useful tool for the dynamic analysis and assessment of floating bridges. The third phase deals with the parametric studies for the transport capacity of floating bridges and it has three tasks. The first is to determine the parameters controlling the dynamic response and transport capacity of the bridge. The second is to investigate the dynamic response of the bridge for different scenarios of loading conditions. The third includes the analysis and discussion of results and comparison between the results of different loading conditions and different vehicle models. The fourth phase describes the verification of the analytical approach and the developed finite element program. It also describes the dynamic analysis of floating bridges using ANSYS software package after adding a subroutine to it to fit the problem in hand. Based on the results of the analytical investigation, the fifth phase is completed by providing conclusions and recommendation. 


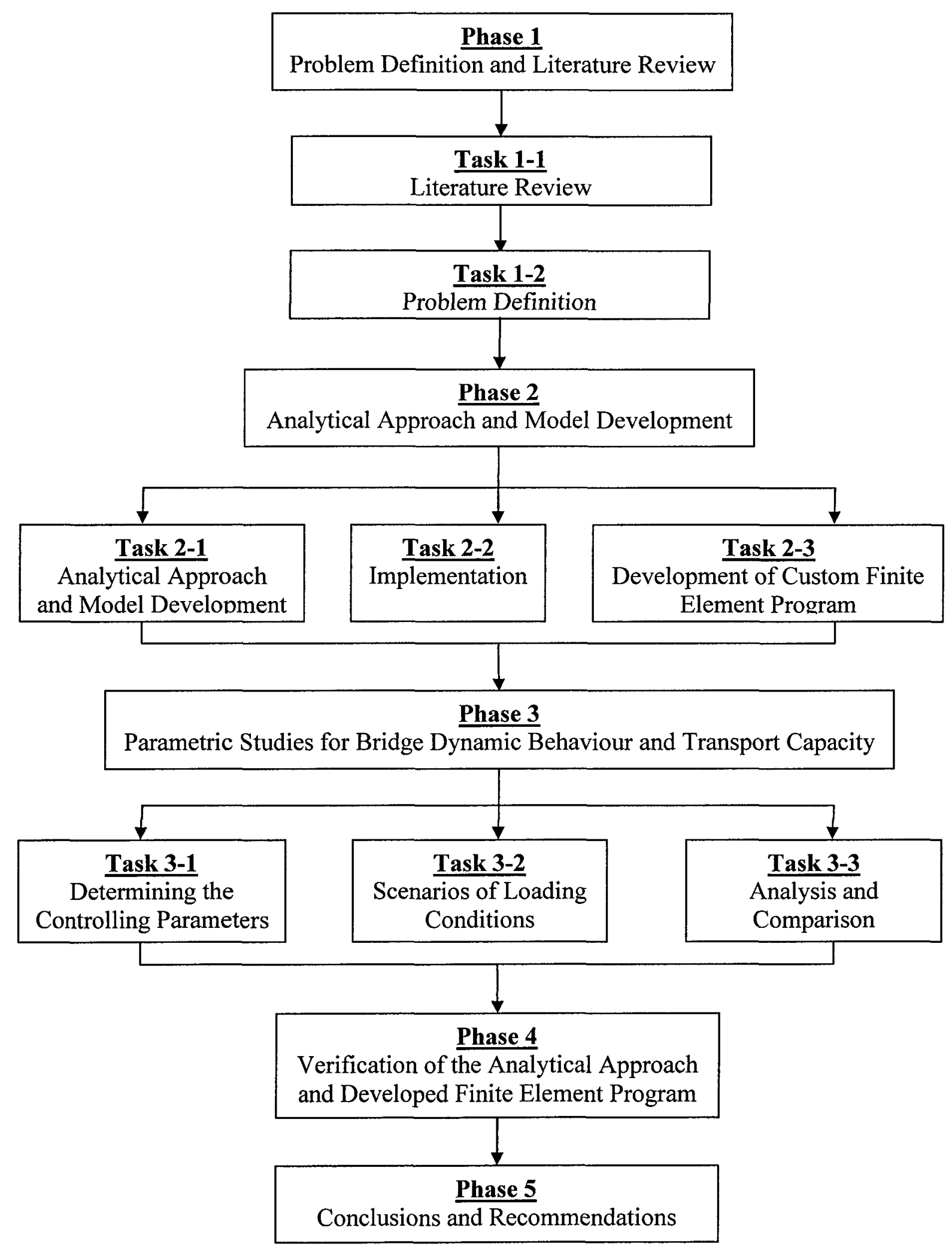

Figure 1.6 Research Plan 


\subsection{Thesis Organization}

The thesis is structured in six chapters. The first chapter provides a general introduction to floating bridges, the problem definition, objectives and scope, the research plan and organization of the thesis. The second chapter presents a comprehensive literature review including that on the design and analysis of floating bridges and their behaviour to moving loads, dynamic response analysis of conventional bridges to moving loads and vehicle-bridge interaction, and analysis of floating bridges response to water current and wave loads. Based on the main findings from the literature review, the proposed technique to be used in the next chapters for the dynamic analysis of floating bridges is illustrated.

In the third chapter, an analytical technique for vehicle-bridge-fluid system is developed and implemented into a finite element program for the dynamic analysis of floating bridges under the effect of moving vehicle loads and water current loads. The equations of motion of the vehicle-bridge-fluid system are derived for different vehicle models including single-axle and multiple-axle models, and for the passage of single and multiple vehicles traversing the bridge.

In the fourth chapter, the transport capacity and dynamic response of rigid-connected and hinge-connected rapid deployment floating bridges to the passage of single and multiple vehicles are investigated considering different vehicle weights, speeds and separation distances between successive vehicles and considering different vehicle models. The effect of vehicle eccentricity on the dynamic response of floating bridges is also investigated. The dynamic response of light-weight floating bridges to moving vehicle 
loads and the use of light-weight materials for improving the global dynamic response of floating bridges are investigated. Finally, the three-dimensional interactive dynamic behaviour of floating bridges due to the combined actions of moving vehicle loads and dynamic water current loads along with possible eccentricities of these loads is investigated. Water current loads are represented as dynamic loads that change with both time and space coordinates and are determined based on the actual dynamic response of the bridge profile, and are incorporated into the dynamic equations of vehicle-bridgefluid system.

In the fifth chapter, verification of the developed analytical approach and finite element program for the dynamic analysis of floating bridges is described through several examples from the literature. This chapter also describes the dynamic analysis of floating bridges subjected to moving vehicle loads using ANSYS software package after adding a subroutine to it to fit the problem in hand.

The sixth chapter presents summary of the completed research work, the main findings and conclusions, and recommendations for future work.

In Appendix A, the structural behaviour of floating bridges subjected to lateral water current loads is investigated. The effects of water current velocity, number of supporting boats and their locations and the magnitude of force they exert on the bridge are investigated. Appendix B presents the global description of the ribbon bridge including its composition, construction and operation. 


\section{Chapter: Literature Review}

Floating bridges may be chosen as the best alternative among different structural systems of bridges based on economic, technical and construction reasons. The reasons for which a floating bridge alternative may be the most practical and economical solution for water crossing are summarized as follows (Seif and Koulaei 2005):

- At locations with deep waters, where the construction of fixed foundations is difficult and expensive.

- At locations with very soft soils at the sea/river bed, where the actual bearing capacity of the sea/river bed soil can be too small to support fixed foundations with reasonable dimensions.

- For temporary projects in general, where the bridge is needed only for a short time and the cost of constructing a fixed bridge is much more than the gain at this location.

- During military operation, a floating bridge can be the optimum means for water crossing, where the bridge has to be constructed in a very short time, and the bridge is temporary and is only needed for a short duration.

- In regions with high earthquake risk, where a severe earthquake can lead to damage of a fixed bridge, while a floating bridge is far less affected by earthquakes.

The following sections address some of the concepts and previous research work relevant to the analysis and design of floating bridges. 


\subsection{Analysis and Design of Floating Bridges}

Different from conventional fixed bridges, there is no unified code of practice for the analysis and design of floating bridges and there is no unified method for determining their dynamic behaviour (Seif and Inoue 1998). This is due to the fact that the structural system of a floating bridge may differ from a location to another one according to many factors such as the width of the water channel, water current and wave forces, and the navigation operation requirements at that location. In addition to the complexity of the dynamic behaviour of bridges under moving vehicle loads.

Many studies for the analysis of floating bridges have been carried out based on representing the floating bridge by a beam resting on elastic foundations represented by elastic springs, (Georgiadis 1984; Wu and Sheu 1996; Wu and Shih 1998). Another study for the analysis of separated pontoon floating bridges has been conducted based on representing the floating bridge by a beam resting on multiple floating bodies representing the separated pontoons (Seif and Inoue 1998). In other studies, the continuous floating bridge was represented by a simply supported beam floating in still water (Fleischer and Park 2004). Also some studies have been carried out for the dynamic analysis of beams on an elastic foundation, subjected to moving point loads (Thambiratnam and Zhuge 1996).

Wu and Sheu (1996) studied the coupled heave and pitch motions of a simplified nonuniform ship hull floating on a still water surface and subjected to a moving load. This ship hull can be an aircraft carrier, a car ferry or a floating pontoon bridge. The authors treated the ship hull as a rigid body supported by an elastic foundation represented by 
distributed springs and dampers. They derived the closed form of the equations of motion of the center of gravity of the ship hull subjected to a moving load with constant speed. The analytical approach developed by the authors can be applied to the analysis of floating bridge to moving loads. However, the equations of motion were derived for the heave and pitch motions of the center of gravity of the ship hull assuming the whole structure to have only two degrees of freedom and limiting the study to the rigid body motion only and neglecting the elastic deformations of the ship hull or the floating structure. Also, the moving load was represented by a moving force neglecting the dynamic characteristics of the vehicle that represent its stiffness and damping properties and neglecting the vehicle-bridge interaction which has a significant effect on the dynamic response of the floating structure.

The authors provided a numerical approach for the cases of constant and varying velocity, and carried out a simple model test to check the results obtained. The equations of motion for the free vibration analysis as well as the forced vibration analysis due to a moving load were derived, and numerical results were provided for the dynamic response of a car ferry to a moving load traveling the ferry at different speeds. The model used for the ferry is shown in Figure 2.1 and the dimensions of the ferry were assumed to be 132 $\mathrm{m}$ in length, $22 \mathrm{~m}$ in width and $7 \mathrm{~m}$ in depth.

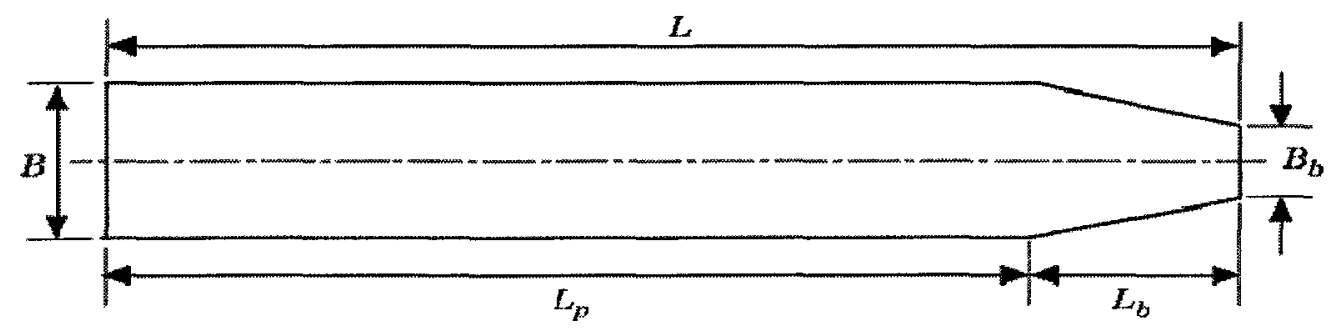

(a) Top view 


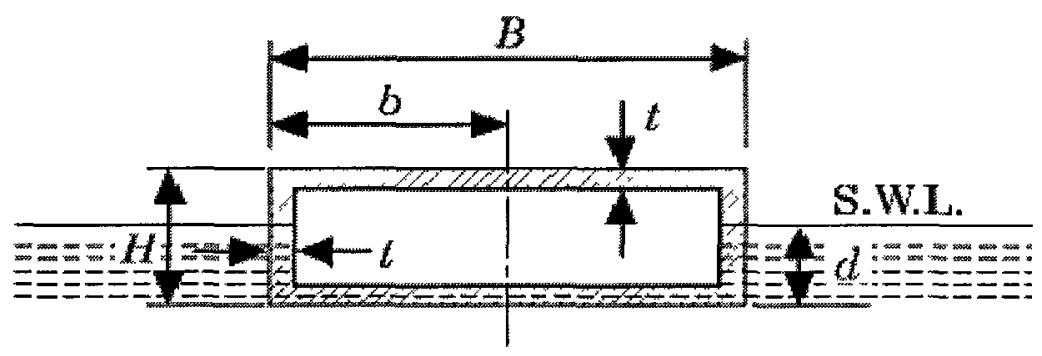

(b) Cross section of the parallel part (enlarged)

Figure 2.1 Model of the idealized floating ferry (Wu and Sheu 1996)
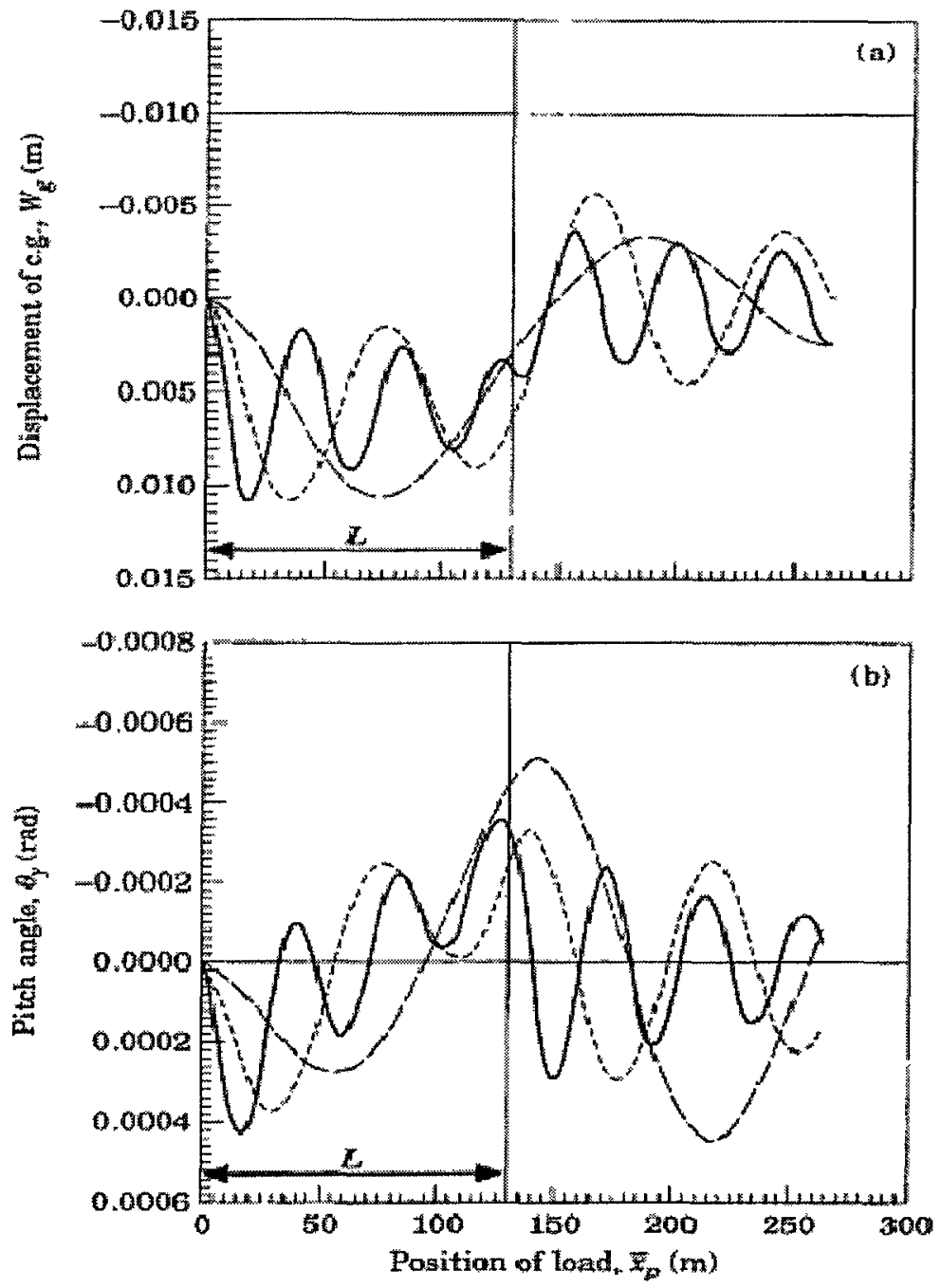

Figure 2.2 The relationship between the moving-load position and (a) the heave displacement and (b) the pitch angle for different moving-load speeds (Wu and Sheu 1996) 

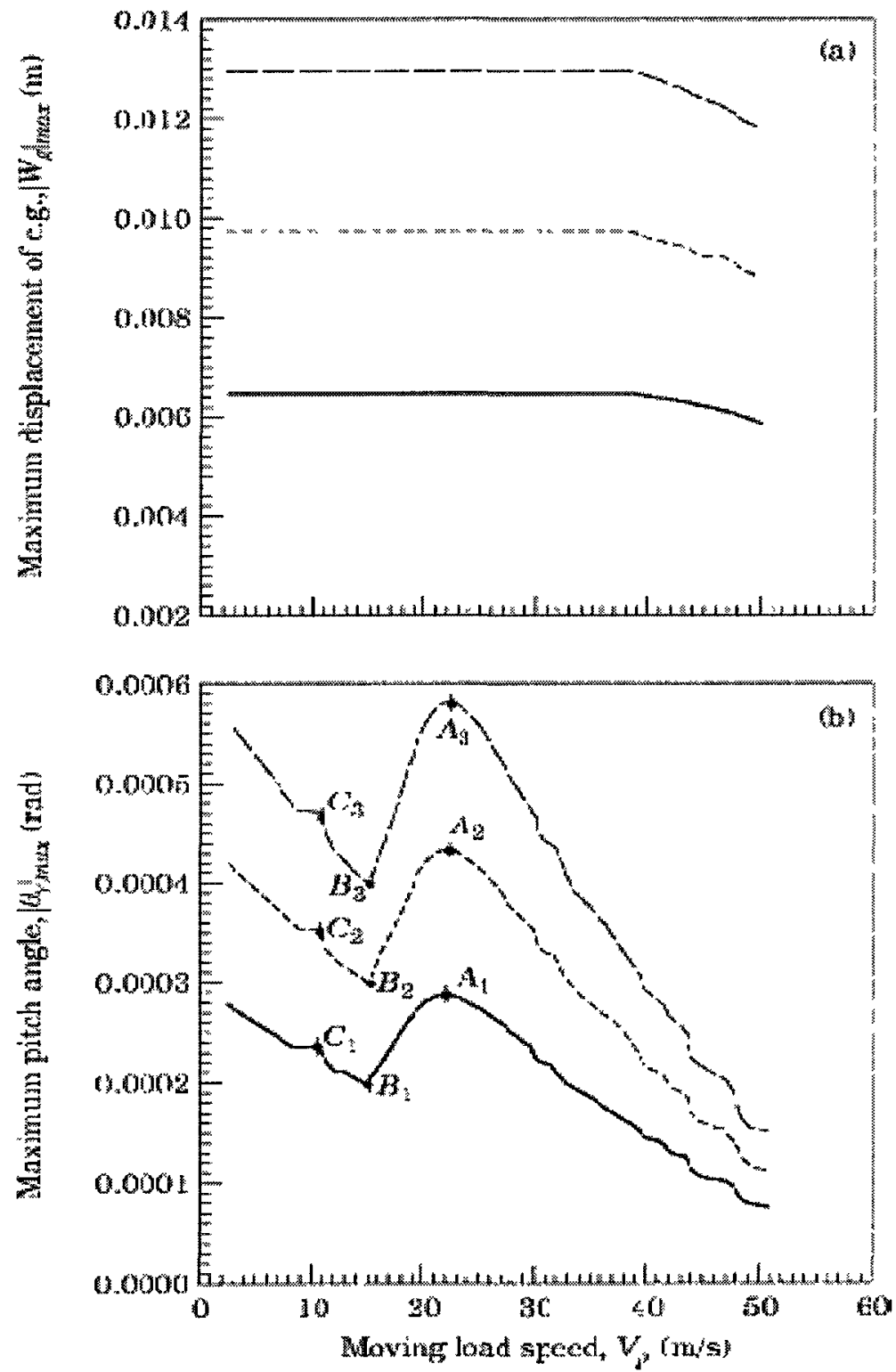

Figure 2.3 The influence of the moving-load speed on (a) the maximum heave displacement and (b) the maximum pitch angle for different moving-load values (Wu and Sheu 1996)

The results show that the moving load speed has a great effect on the dynamic response of the ferry. The heave displacement and pitch angle of the center of gravity of the ferry due to a $150 \mathrm{KN}$ moving load traveling on the ferry at different speed of $6 \mathrm{~m} / \mathrm{s}, 11 \mathrm{~m} / \mathrm{s}$ and $22 \mathrm{~m} / \mathrm{s}$ are shown in Figure 2.2 and are represented by solid, dotted and dashed lines, 
respectively. The effect of the moving-load speed on the maximum heave displacement and the maximum pitch angle of the center of gravity of the same ferry was investigated for different values of moving-load of $100 \mathrm{KN}, 150 \mathrm{KN}$ and $200 \mathrm{KN}$ and the results are shown in Figure 2.3 and represented by solid, dotted and dashed lines, respectively. As shown in Figure 2.3, the maximum heave displacement have the same trend for different values of the moving-load, however, for any specific speed of the moving-load, the maximum heave displacement increases with increasing moving-load magnitude. The same trend was observed for maximum pitch angle.

The elastic vibration of a floating bridge moored by partial-catenary mooring lines and subjected to a moving load was studied by $\mathrm{Wu}$ and Shih (1998). The study was carried out for still water and considering the entire pontoon as a slender beam resting on an elastic foundation represented by uniform elastic springs. The mooring lines were also represented by concentrated springs at the positions of the mooring cables. The mathematical model used by the authors for the dynamic analysis of the floating bridge is shown in Figure 2.4.

The effect of hydrodynamic forces was considered as a constant added mass. The stiffness and mass matrices for rigid-rigid and rigid-hinged beam elements were derived based on the finite element method of analysis and the dynamic response of both rigidconnected and hinge-connected floating bridges to moving load was investigated. In this study, the moving load was represented by a constant value neglecting the dynamic characteristics of the vehicle that represent its stiffness and damping properties and have a significant effect on the dynamic response of the floating bridge. The authors applied 
their analysis to one of the floating bridges on Washington Lake where the bridge was simplified by a beam resting on elastic foundation and the superstructure of the bridge was represented by a uniformly distributed mass. The authors provided some numerical results for the dynamic response of the floating bridge on Washington Lake to a moving load traveling on the bridge at different speeds. The results showed that the moving load speed has a significant effect on the dynamic response of the bridge. The midpoint displacements due to a moving load of constant value of $50 \mathrm{KN}$ with eccentricity of $9 \mathrm{~m}$ traveling the bridge at different speed of $10 \mathrm{~m} / \mathrm{s}, 26 \mathrm{~m} / \mathrm{s}$ and $40 \mathrm{~m} / \mathrm{s}$ are shown in Figure 2.5 .

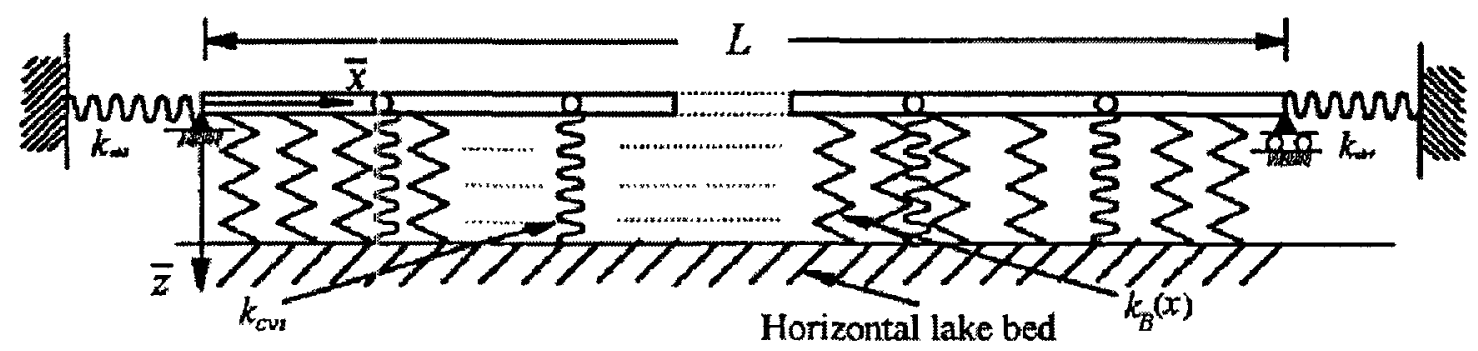

(a) Side view

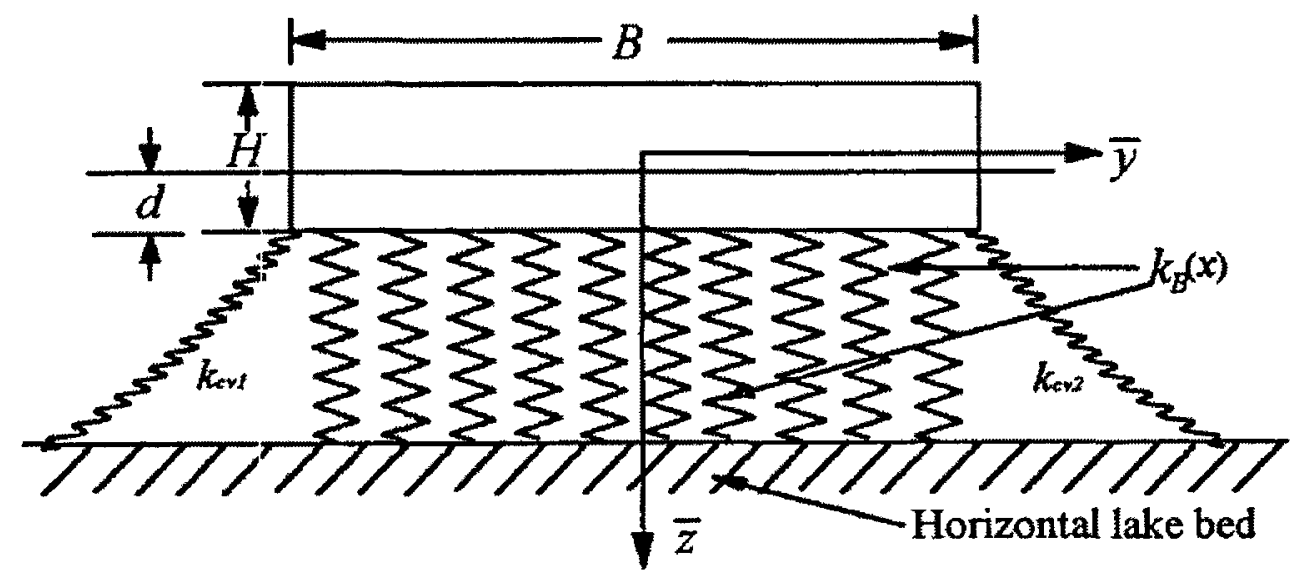

(b) Left end view (enlarged)

Figure 2.4 Mathematical model for the dynamic analysis of floating bridge (Wu and Shih 1998) 


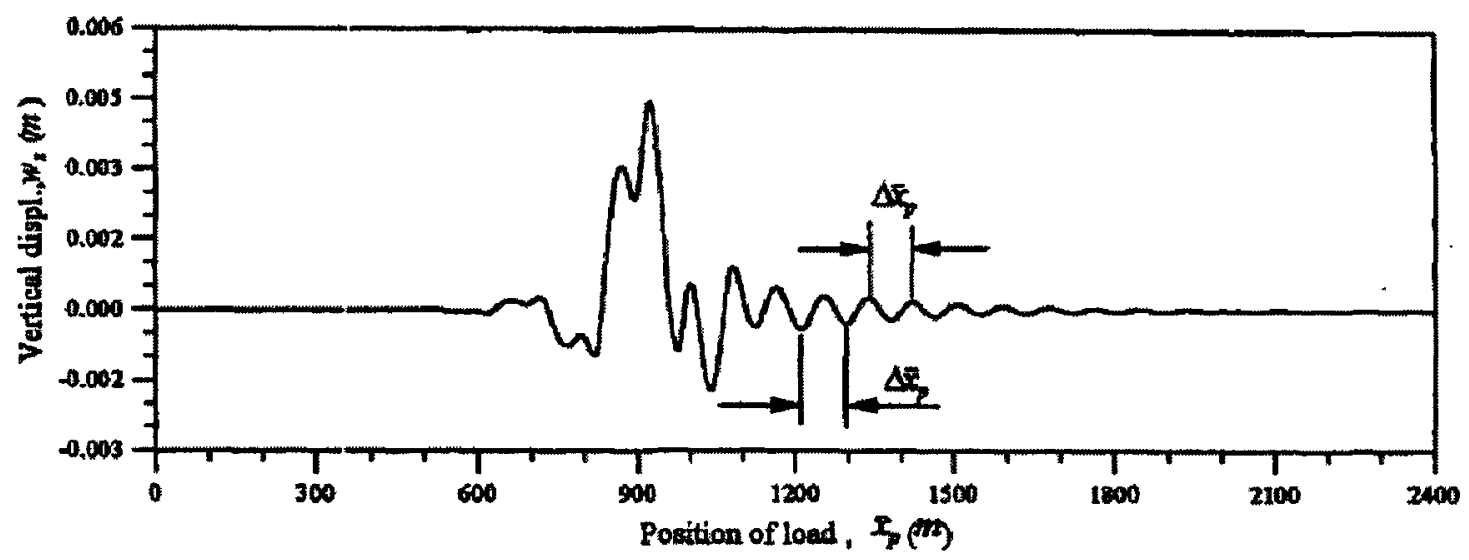

(a) Moving-load speed $\mathrm{v}=10 \mathrm{~m} / \mathrm{s}$

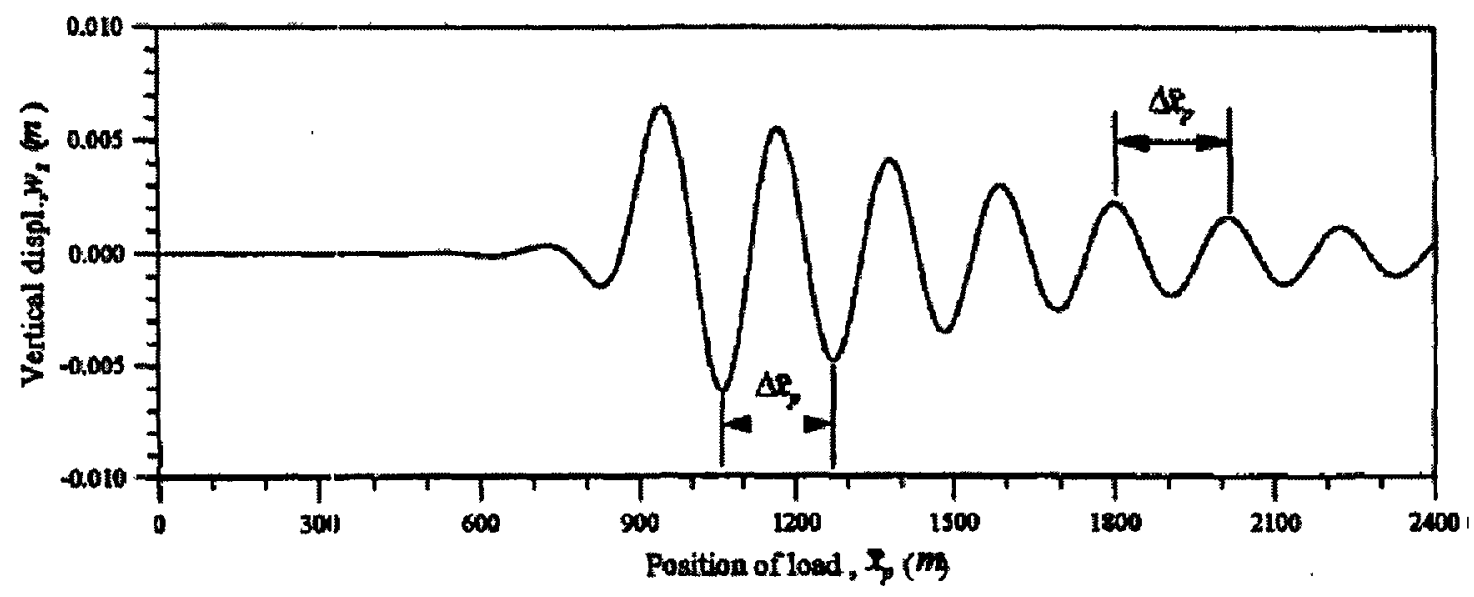

(b) Moving-load speed $\mathrm{v}=26 \mathrm{~m} / \mathrm{s}$

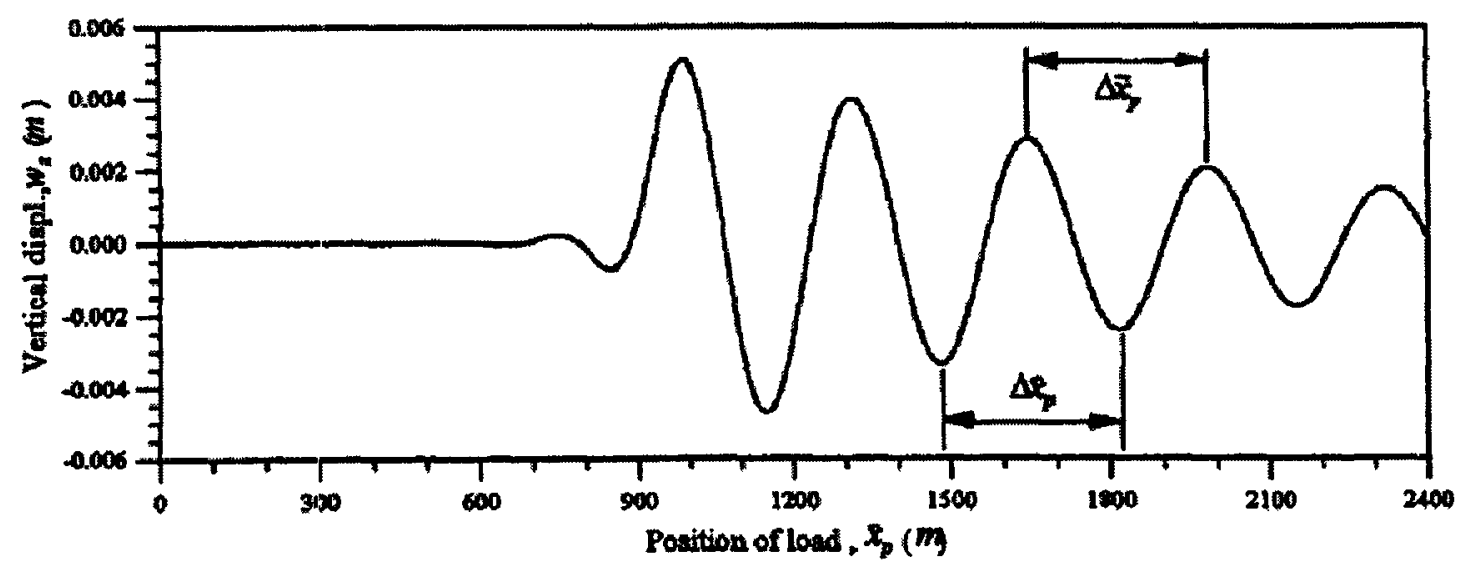

(c) Moving-load speed $v=40 \mathrm{~m} / \mathrm{s}$

Figure 2.5 The relationship between the moving-load position and the central vertical displacement in the hinge-connected floating bridge (Wu and Shih 1998) 
The dynamic analysis of floating bridges was studied by Seif and Inoue (1998). The authors studied mainly the analysis of the separated pontoon floating bridge with special emphasis to wave loads acting on the bridge and effect of the wave parameters on the bridge response. They studied the behaviour of a group of a number of three-dimensional bodies of random shapes floating in water of uniform depth to evaluate the water wave velocity and the forces acting on the floating body.

The finite element method was used for determining the stiffness and mass matrices of the bridge deck. The bridge deck was considered as a beam element supported by the pontoons (floating bodies) and connecting them together as shown in Figure 2.6 for the bridge model. The stiffness and mass matrices of the bridge deck were determined considering six degrees of freedom at each node of the beam element and then transformed to the coordinate systems passing through the center of gravity of the pontoons to get the global stiffness and mass matrices of the whole structure. The effect of wave conditions on the dynamic behaviour of the bridge was investigated and the heave motions of different pontoons were calculated for different wave lengths.

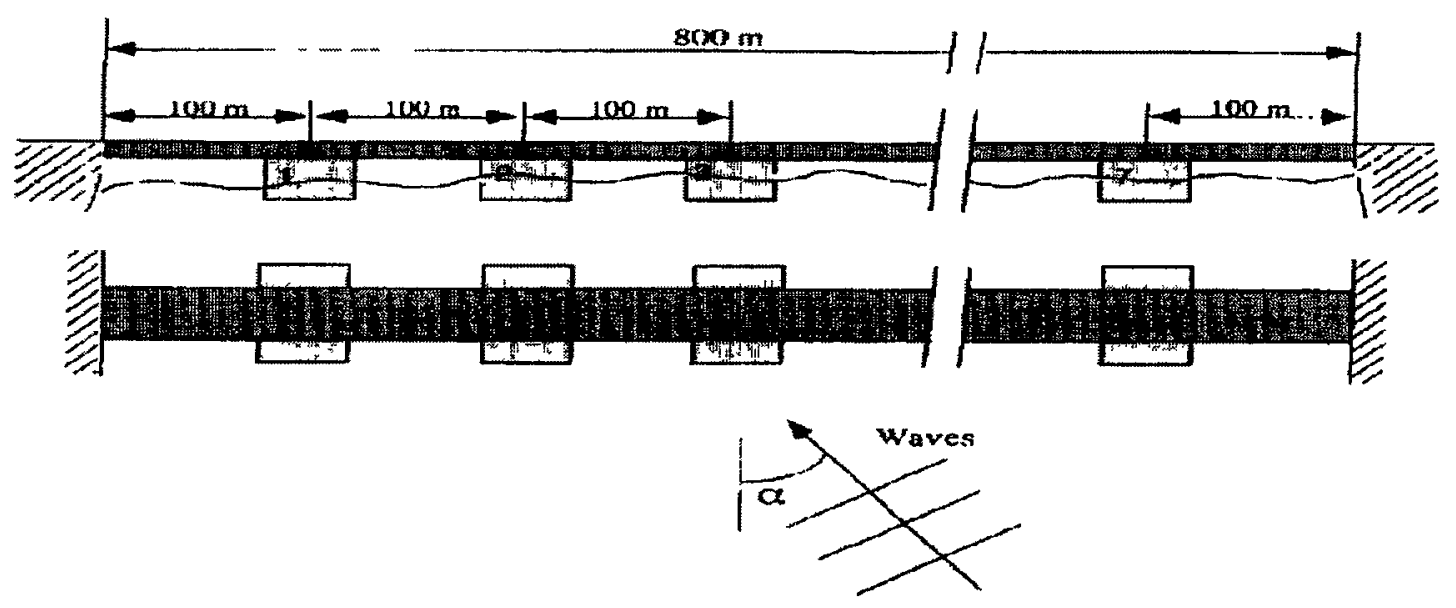

Figure 2.6 Discrete-pontoon floating bridge model (Seif and Inoue 1998) 
Fleischer and Park (2004) analyzed the hydroelastic vibrations of a beam with rectangular cross-section under the effect of a uniformly moving single axle vehicle using modal analysis. The floating bridge was represented by a simply supported beam with uniform rectangular cross-section (see Figure 2.7). The authors pointed out that this representation of the floating bridge is appropriate for continuous floating bridges with pontoons closely connected and ramps pressed to the banks. The riverbed was considered as a rectangular cross-section with rigid boundaries containing still water. This representation of the bridge and underlaying water is considered as a special case of a homogeneous beam resting on the surface of a water-filled prismatic bowl. The normal modes of the bridge were determined taking into account surface waves in the beam direction and considering the volume of the enclosed fluid. Numerical examples were provided assuming different water depths. The response of the bridge system was analyzed under the effect of mass moving with constant speed, and the vertical acceleration of this mass representing the vehicle was determined.

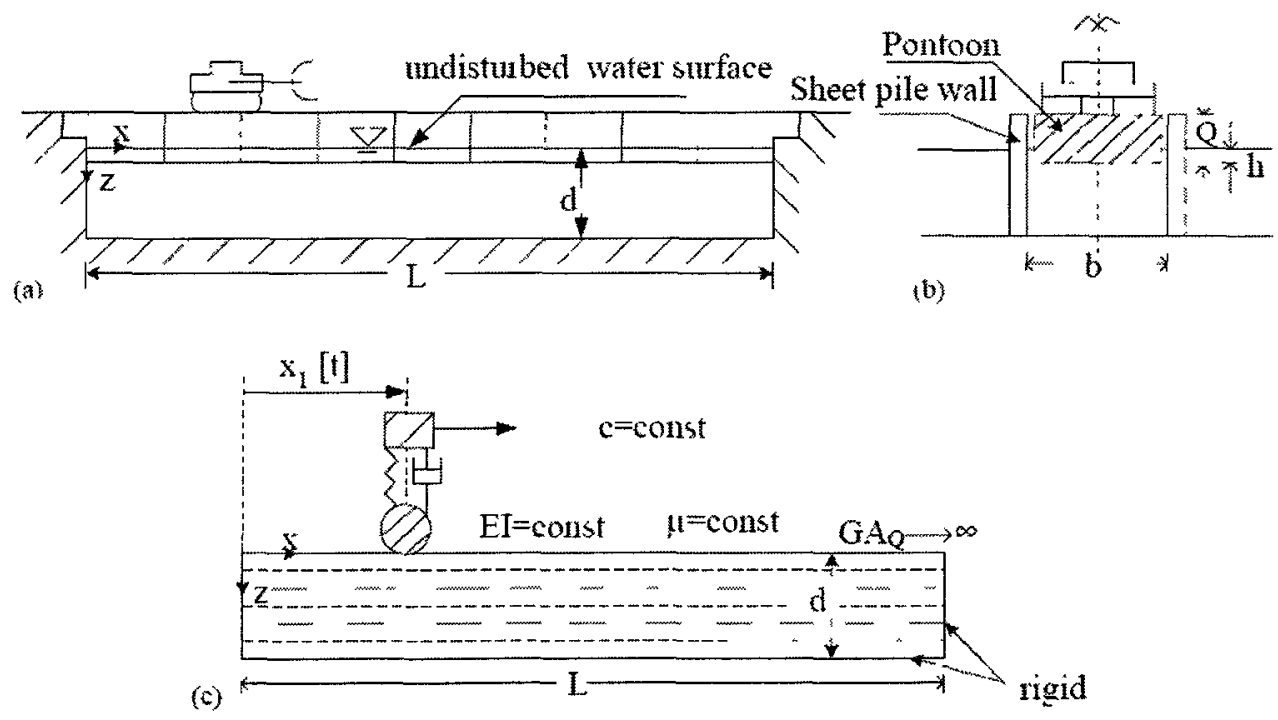

Figure 2.7 System and loads of the floating bridge (a) longitudinal section (b) crosssection (c) model (Fleischer and Park 2004) 
Thambiratnam and Zhuge (1996) studied the dynamic analysis of beams on an elastic foundation, subjected to moving point loads. The authors represented the elastic foundation by linear elastic springs and used a finite element method to develop a simple procedure for the dynamic analysis of the beam. Different factors were considered in their study including the effect of the beam length, speed of the moving load and foundation stiffness on the dynamic response of the beam. The authors programmed the developed procedure to run fast on a computer, and they provided some numerical examples for different stiffness values of the elastic springs and for different speeds of the moving load. The effect of the moving-load speed on the peak values of dynamic amplification of deflection is shown in Figure 2.8 for different values of the elastic spring stiffness of $1.14 \mathrm{e}+2,1.14 \mathrm{e}+4,1.14 \mathrm{e}+5$ and $1.14 \mathrm{e}+7 \mathrm{~N} / \mathrm{m}^{2}$ from bottom to top, respectively. It was noted that for smaller values of foundation stiffness, the dynamic amplification in deflection increases with traveling speed. The same trend was also observed for stresses where for smaller values of foundation stiffness, the dynamic amplification in stress increases with traveling speed. The equivalent stiffness of water is less than the typical values of the equivalent stiffness for different soils. Therefore, for a floating bridge represented by a beam resting on elastic springs representing the underlying water and subjected to a moving load, it is expected that the dynamic amplifications in both deflection and stress increase more with increasing the traveling speed of the moving load.

This procedure was applied to the analysis and design of railway track structures where the study was extended by modeling the moving load as a sprung mass considering the effect of the spring stiffness of the moving load and neglecting damping and the mass of 
the wheel. The maximum vertical deflection occurring at mid-span of a beam of $10 \mathrm{~m}$ length resting on elastic foundation having a stiffness of $1.14 \mathrm{e}+7 \mathrm{~N} / \mathrm{m}^{2}$ and subjected to two wheel loads with separation distance of $3.2 \mathrm{~m}$ and moving with a speed of $120 \mathrm{~km} / \mathrm{h}$ is shown in Figure 2.9 along with the maximum vertical deflection due to one wheel load with the same magnitude and moving with the same speed. The two curves are the same up to the time corresponding to the separation distance between the two wheel loads and before the second load act on the beam. The same trend was observed for different speeds of the moving load.

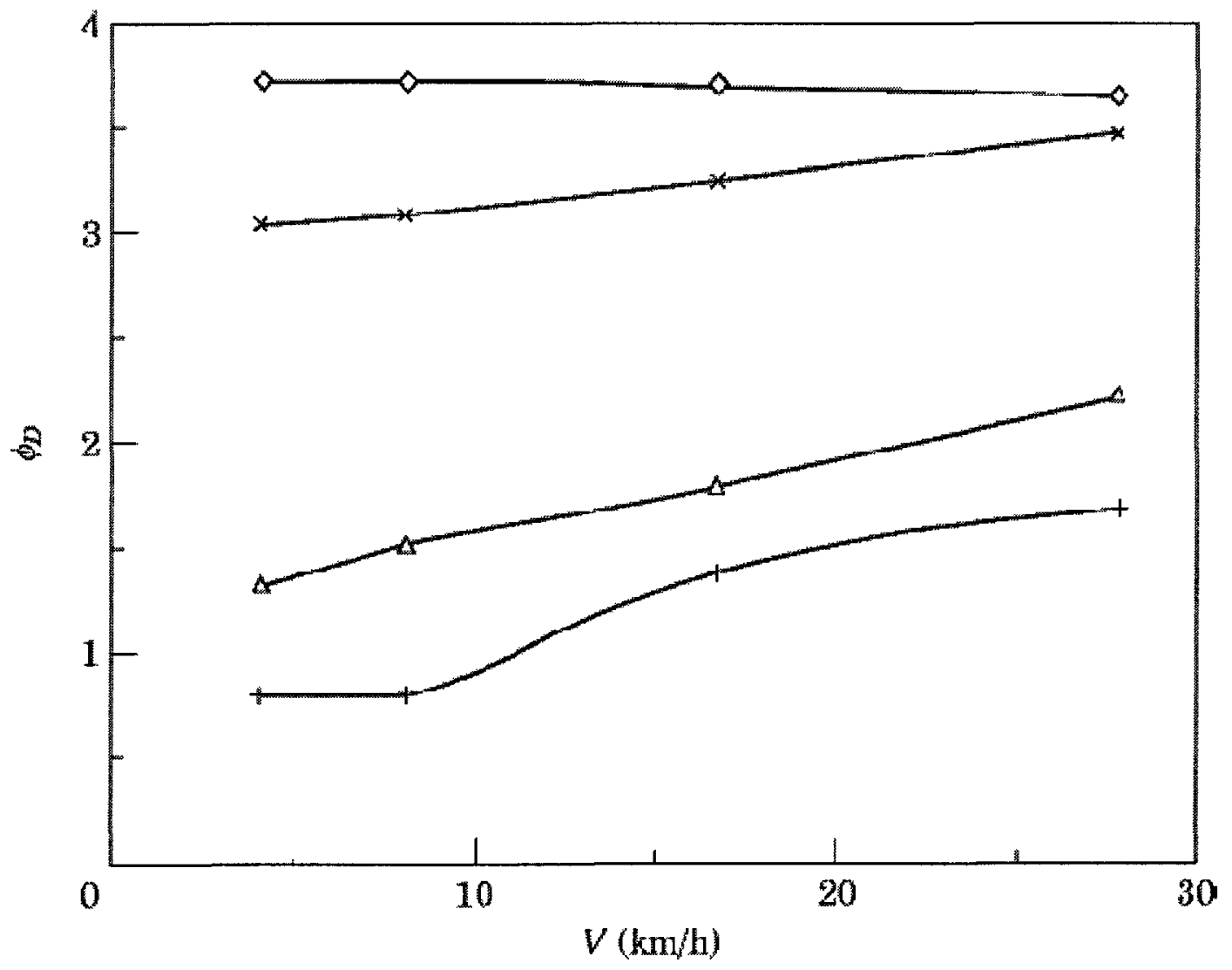

Figure 2.8 Variation of peak values of dynamic amplification in deflection with traveling speed, for different spring stiffness values (spring stiffness is $1.14 \mathrm{e}+2,1.14 \mathrm{e}+4,1.14 \mathrm{e}+5$ and $1.14 \mathrm{e}+7 \mathrm{~N} / \mathrm{m}^{2}$ from bottom to top, respectively), (Thambiratnam and Zhuge 1996) 


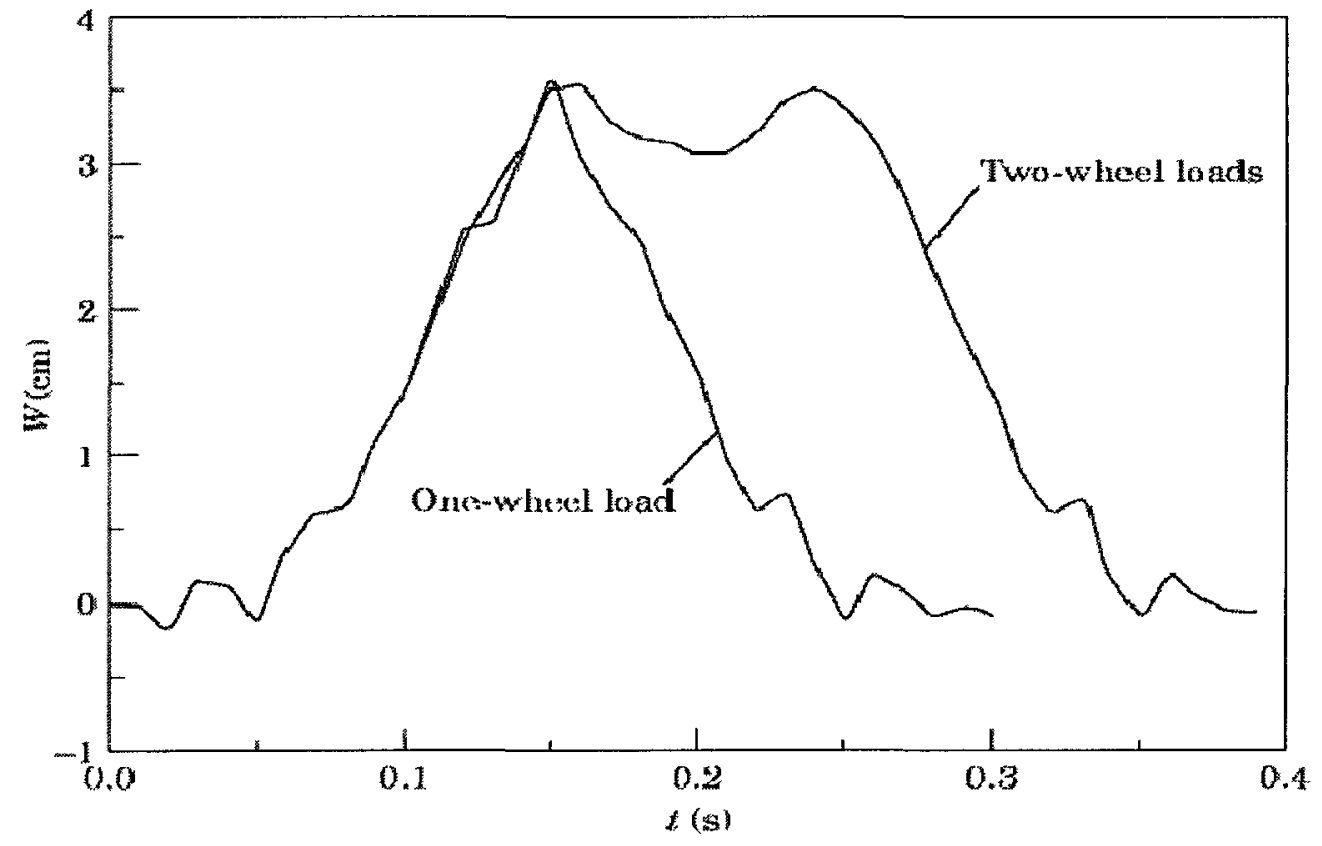

Figure 2.9 Time histories of maximum deflections due to one- and two-wheel loads (Thambiratnam and Zhuge 1996)

The finite element method is one of the most practical and efficient numerical methods for structural analysis. It has been used in many studies to model the idealized structures of floating bridges, (Seif and Inoue 1998; Seif and Koulaei 2005; Shixiao et al. 2005; Zhang et al. 2008).

Seif and Koulaei (2005) discussed the different alternatives for bridge configurations over Urmia Lake; three types of bridge designs were proposed, namely fixed, floating and innovational bridge design. The authors discussed five different proposals for fixed bridge design and two alternatives for innovational bridge design in addition to the floating bridge alternative. The floating bridge proposal was selected to be the most feasible according to technical and economical reasons. It was found that at this particular site of Urima Lake, a floating bridge will cost less than one-half of the cost of a fixed bridge in addition to the shorter time of construction for the floating bridge. The proposed 
floating bridge was a continuous pontoon bridge with a total length of $1330 \mathrm{~m}$, and consisted of 19 hollow reinforced concrete pontoons each of $70 \mathrm{~m}$ length, $16 \mathrm{~m}$ width and $5 \mathrm{~m}$ height, as shown in Figure 2.10.

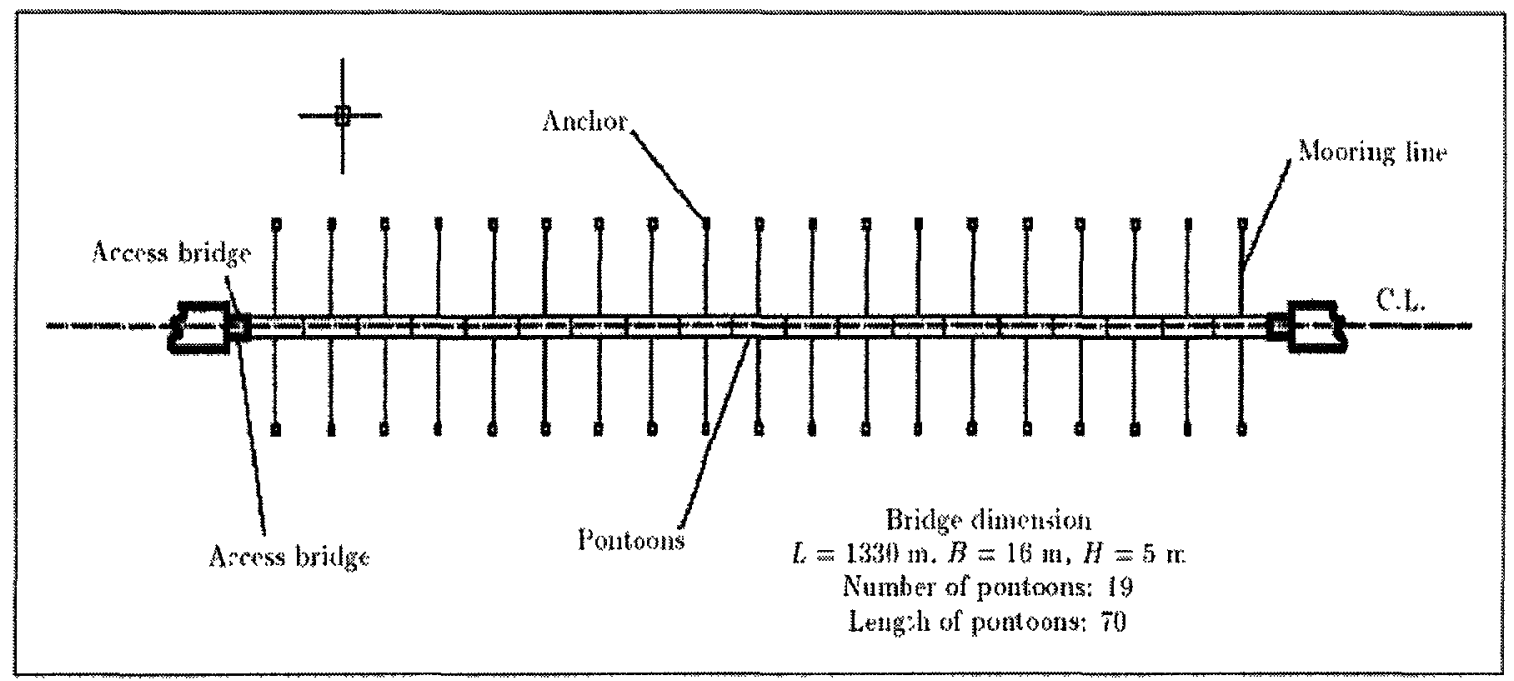

Figure 2.10 Plan view of the proposed floating bridge (Seif and Koulaei 2005)

For water current analysis, the modeling was accomplished in a two-dimensional form, since the length of the bridge is large compared to its cross-section dimensions and the current is almost perpendicular to the bridge. The fluid velocity was assumed as $1.14 \mathrm{~m} / \mathrm{s}$ and no air current was assumed. The finite element method was used to model the bridge structure using ANSYS software package and using shell elements for the pontoons, linear spring elements for the buoyancy forces of water and nonlinear spring elements for the mooring lines. The analysis and design of the floating bridge under the effect of static traffic loads and different environmental loads were discussed; however, the study was limited to static traffic loads and no dynamic analysis for the bridge response to moving traffic loads was conducted. 
Another important point of interest in this field is the inter-connecting joints between bridge pontoons. These connection joints are usually subjected to bi-directional loading, and are susceptible to damage due to excessive stresses developed in them. In a study presented by Shixiao et al. (2005), the equations of motion for the nonlinearly connected floating bridge were derived using the finite element method and based on local separation of variables, taking into account the nonlinear properties of connectors. The nonlinear behaviour of the connectors was represented by a tension-only or compressiononly nonlinear truss element with an initial gap. The resulting equations of motion usually comprise large number of unknowns and can be put in the form of high order matrices. To reduce the large memory and analysis time requirements, the authors implemented the super-element method to compress the whole calculation scale. Then, the condensed equations of motion were solved by applying direct integration and Newton-Raphson iteration methods. The authors conducted analytical and numerical studies to evaluate the dynamic displacement and the connection forces of a floating bridge with nonlinear connectors, under the effect of moving loads, using modal and static analysis. The super-element method was found to provide a reasonable accuracy for the stiffness and mass properties of the floating bridge. However, condensation of matrices by eliminating the degrees of freedom of the vehicle and condensing them to the corresponding degrees of freedom of the bridge element acted upon by the vehicle is not adequate for calculating the accurate vehicle response indicating the riding comfortability, because of the approximation made in relating the degrees of freedom of the vehicle to those of the bridge (Yang and $\mathrm{Wu} 2001$ ). The results showed that nonlinearity and the initial gap of the connectors should be considered for accurate 
hydroelastic response of a nonlinearly connected floating bridge. However, in their study, the moving loads were represented by uniformly distributed loads of constant value over the area loaded by the vehicle, neglecting the dynamic characteristics of the vehicle that represent its stiffness and damping properties and which have a significant effect on the dynamic response of the floating bridge.

The effect of water depth on floating bridges carrying moving loads was studied by Zhang et al. (2008). Analytical models of both separated pontoon and continuous pontoon floating bridges were used to study the dynamic responses with hydrodynamic influence coefficients for different water depths. The continuous floating bridge was modeled as a simply supported beam resting on uniform equally-spaced elastic springs representing the stiffness of the underlying water, while the separated floating bridge was modeled as a simply supported beam resting on separate elastic springs representing the carrying pontoons. The hydrodynamic coefficients were evaluated by the boundary element method and the dynamic responses of bridges were evaluated using the Galerkin method of weighted residuals. The hydrodynamic frequencies were computed using an iteration method. The authors concluded that the water depth can be neglected during bridge design, as it has little influence on the dynamic responses of both bridge forms. In this study, the moving load was represented by a constant value neglecting the dynamic characteristics of the vehicle that represent the stiffness and damping properties and have a significant effect on the dynamic response of the floating bridge.

Floating bridges are water tight structures that make use of the buoyancy force offered by water and therefore they have to be lightweight to increase its floating ability. In general, 
floating bridges are composed of lightweight hollow sections made of impermeable materials with high precautions on its water tightness. Water getting inside the units of the floating bridge can cause the bridge to fail or sink. Dusenberry et al. (1995) studied the failure of The Lacey V. Murrow Floating Bridge which was built in 1940. This floating bridge consists of 22 hollow concrete pontoons; each pontoon was divided into compartments by interior walls. A cut-away of typical pontoon of Lacey V. Murrow Floating Bridge is shown in Figure 2.11. The authors discovered that the cause of the sinking of Lacey V. Murrow Floating Bridge was the accumulation of water in the bridge during maintenance and restoration. Water accumulation was due to rain, wave splash and hydro-demolition, through the construction openings.

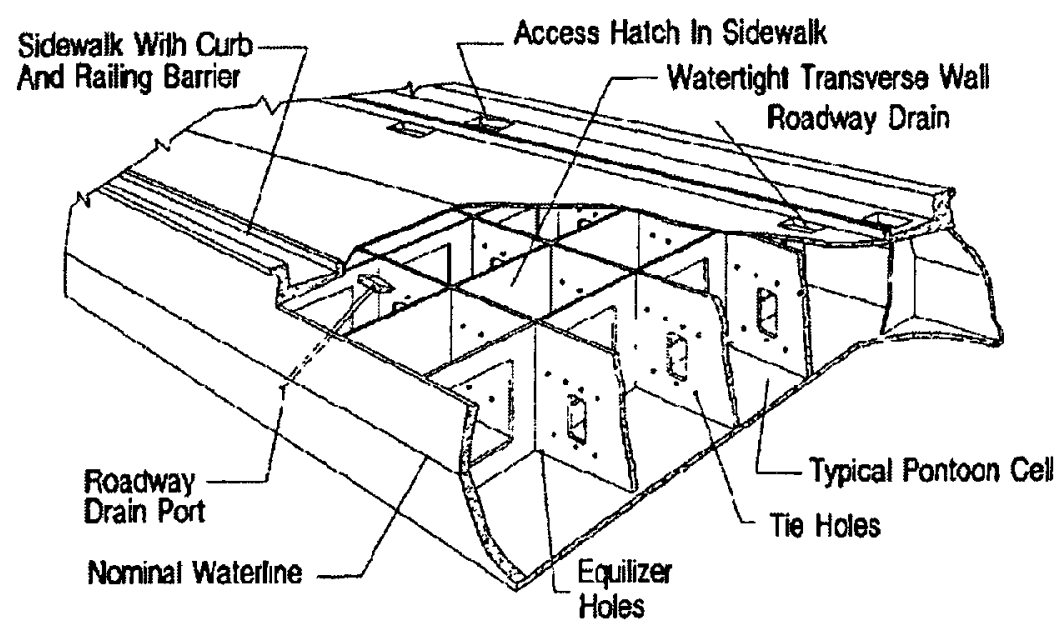

Figure 2.11 Cut-away of typical pontoon of Lacey V. Murrow Floating Bridge (Dusenberry et al. 1995)

Fujikubo and Tetsuya (2001) introduced a model for the structural design of Very Large Floating Structures (VLFS) of several thousand meters long by developing a hierarchical system of structural analysis. The structural analysis for such huge floating structures using the normal procedure of the finite element method encompasses a large number of 
structural nodes required for reasonable accuracy of the analysis. This large number of nodes results in an enormous number of degrees of freedom and equations to be solved for the structural response, which makes the calculation process very expensive and time consuming. For this reason, the hierarchical system of structural analysis is needed for the analysis of VLFS, in which the global response analysis can be conducted by using an idealized structural modeling while the local structural response is investigated using a zooming technique. This concept can be applied for the global dynamic response of floating bridges using simple idealized structural modeling, while the stresses at any specific section within the bridge can be investigated using zooming technique. Figure 2.12 shows a typical structural arrangement of a pontoon-type VLFS.

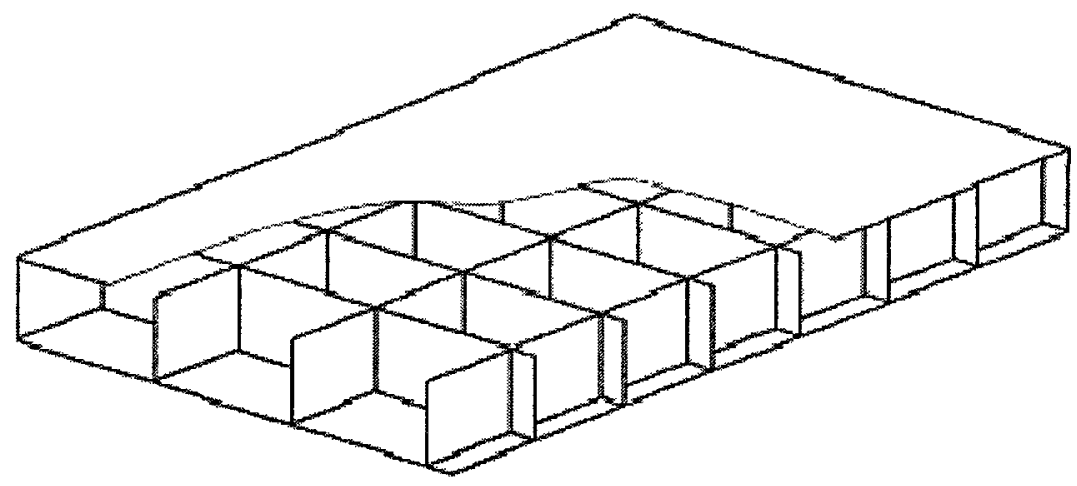

Figure 2.12 Structure arrangement of pontoon-type very large floating structure (Fujikubo and Tetsuya 2001)

\subsection{Bridges Subjected to Moving Loads}

Some previous studies pointed out that in most cases, sophisticated analytical models of bridges are not capable of determining the factors affecting the dynamic behaviour of the bridge. By using a simplified model, the governing parameters controlling the bridge 
response can be determined and their effects can be thoroughly examined, and therefore design procedures can be developed, (Humar and Kashif 1993).

Many studies have been done for the analysis of bridges under the effect of moving vehicles using finite element method, representing the bridge by beam element and the vehicle by moving load or moving mass attached to a spring and damper system (Humar and Kashif 1993; Yang and Yau 1997; Henchi et al. 1998; Farad et al. 1998; Yang and Wu 2001; Qiu 2007; Qiu 2009). It was concluded that the vehicle model represented by moving mass attached to a spring and damper system is more accurate than the moving load model in predicting both the bridge and the vehicle dynamic responses. Although still more sophisticated models can be devised for the vehicles, the efficiency of solution of the vehicle-bridge interaction system becomes an issue of great concern, especially when there are number of vehicles traversing the bridge. The vehicle-bridge interaction problem in this regard is complicated in that it is time-dependent and there are multiple contact points (Yang and $\mathrm{Wu} 2001$ ).

Humar and Kashif (1993) studied the dynamic response of a simply supported clear span bridge traversed by moving vehicles. Parameters that affect the dynamic response of a bridge were determined by developing a simple model for the bridge-vehicle interaction; these parameters are the speed parameter representing the vehicle speed, the ratio of the mass of vehicle to the mass of bridge and the ratio of the vehicle frequency to the bridge frequency. The bridge was modeled by a beam and the vehicle was modeled by a singleaxle or two-axle model using sprung and unsprung masses, connected through springs and viscous dashpots (see Figures 2.13 and 2.14). 


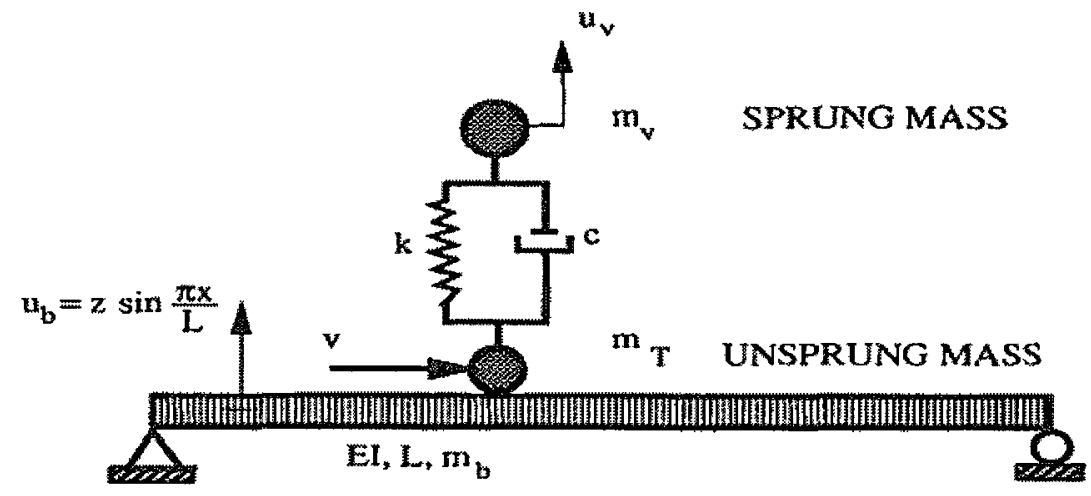

BEAM WITH MASS

Figure 2.13 Modeling of bridge-vehicle system using single-axle vehicle model (Humar and Kashif 1993)

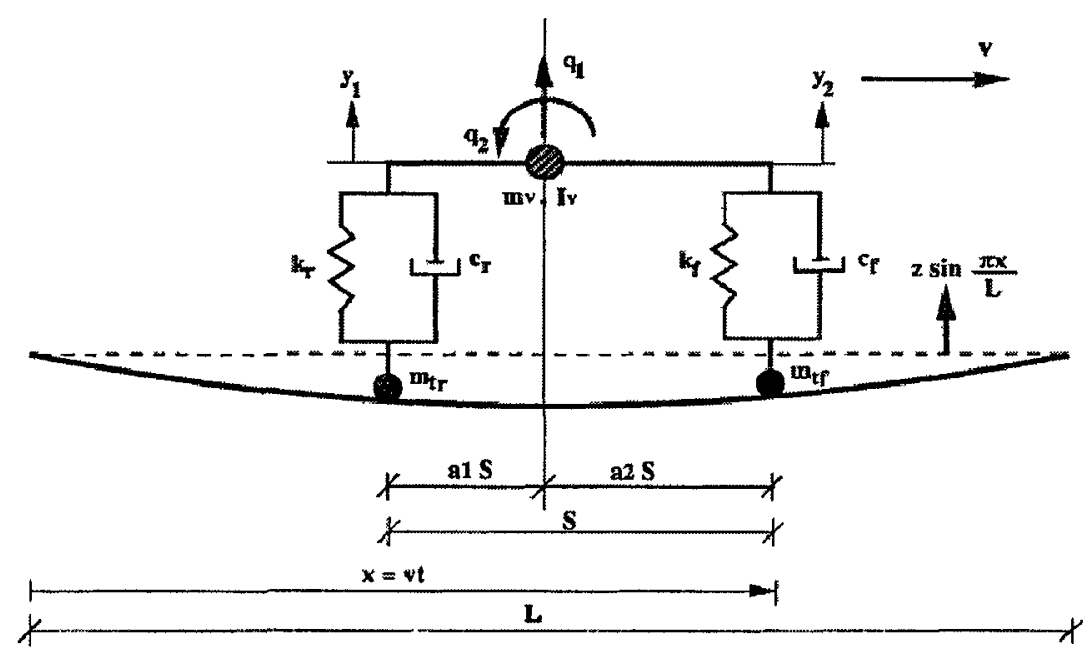

Figure 2.14 Two-axle vehicle model (Humar and Kashif 1993)

The single-axle spring mass model, with the entire vehicle mass lumped over the axle, was found to provide accurate results of the bridge response if the axle spacing is very small relative to the bridge span. For larger ratios of axle spacing to the bridge span $(\mathrm{S} / \mathrm{L})$, the two-axle spring mass model was found to produce a smaller response and more accurate results (see Figure 2.15). For the two-axle vehicle model, additional parameters were found to control the bridge response; these parameters are the axle spacing, the location of the mass center of the vehicle and the mass moment of inertia of the vehicle 
which has a little effect on the dynamic response of the bridge. From Figure 2.15, it is clear that the dynamic response of the bridge to two-axle vehicle is smaller than that response to single-axle vehicle, where the amplification factor (D) is the dynamic deflection divided by the static deflection of the bridge. The ratio of axle spacing to the bridge span (S/L) is affecting the bridge dynamic response where, in general, the bridge response is smaller for larger ratios of axle spacing to the bridge span.
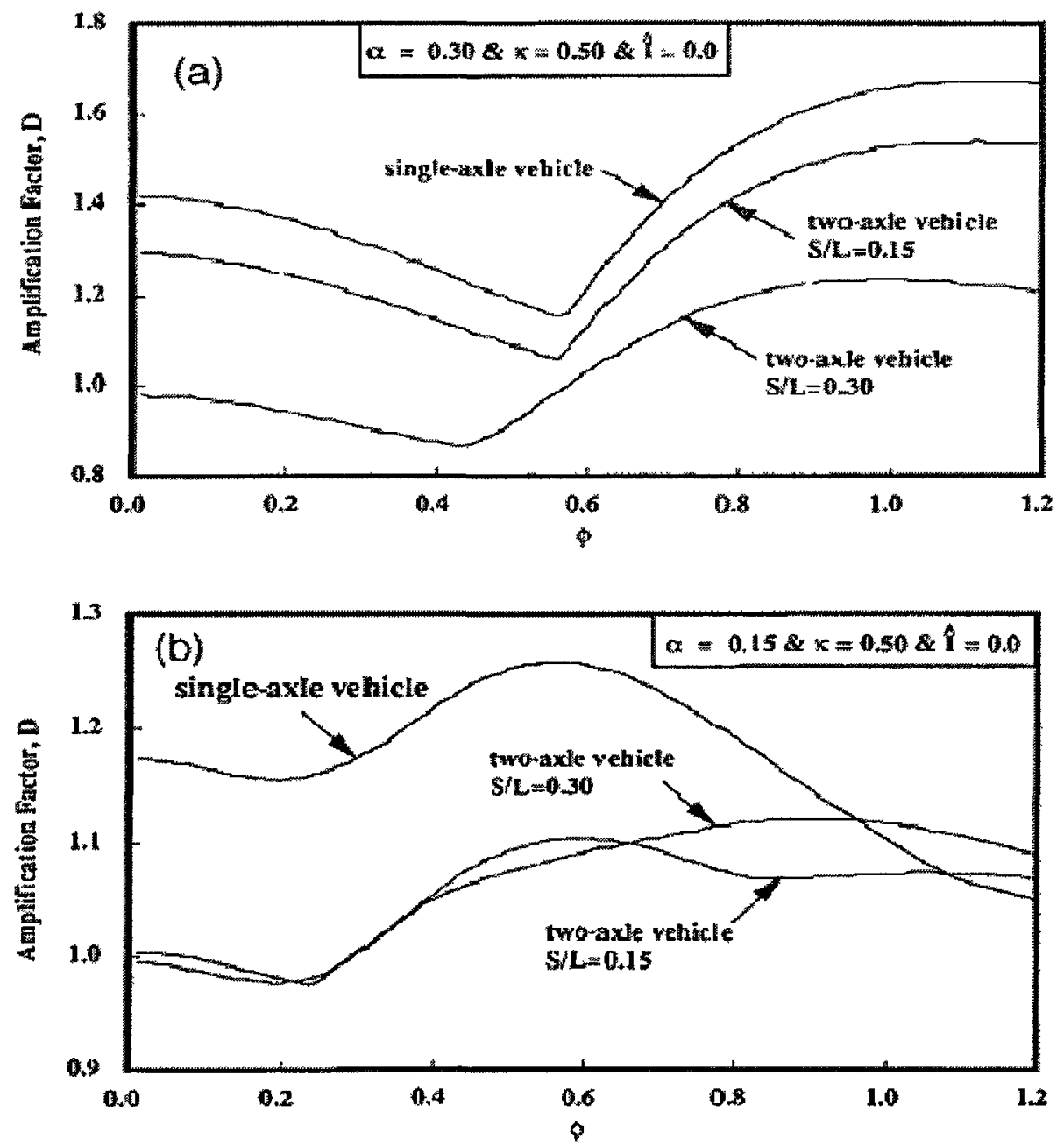

Figure 2.15 Dynamic response of the bridge to single- and two-axle vehicle loads (Humar and Kashif 1993) 
The authors also compared the dynamic response of the bridge to a moving force without interaction between the vehicle and the bridge to that response to a sprung mass vehicle model, and it was recognized that the interaction of the vehicle with the bridge has a significant effect on the dynamic response of the bridge. Therefore, a moving force model is quite inadequate in predicting the dynamic response of the bridge to moving vehicle loads.

In their study, the damping of the bridge was neglected. In the case of a floating bridge, the damping of the bridge is expected to be more effective on the dynamic response of the bridge and should be taken into account. The underlying water helps the dynamic response of the floating bridge to damp rapidly.

The previous study was extended by Humar and Kashif (1995) by modeling the bridge as a rectangular isotropic or orthotropic plate and using the same modeling for the vehicle. The nonlinear equations of motion of the bridge-vehicle system were solved by step-bystep numerical integration to determine the dynamic response of the bridge. The aspect ratio and the geometry and natural properties of the plate were found to be governing parameters of the bridge dynamic response. The dynamic response of bridge represented by orthotropic plate elements to central moving vehicle load is close to the response of bridge represented by beam elements as shown in Figure 2.16, where plate A has $20 \mathrm{~m}$ length, $10 \mathrm{~m}$ width and $1 \mathrm{~m}$ depth, and plate $B$ has $10 \mathrm{~m}$ length, $5 \mathrm{~m}$ width and $0.25 \mathrm{~m}$ depth. 

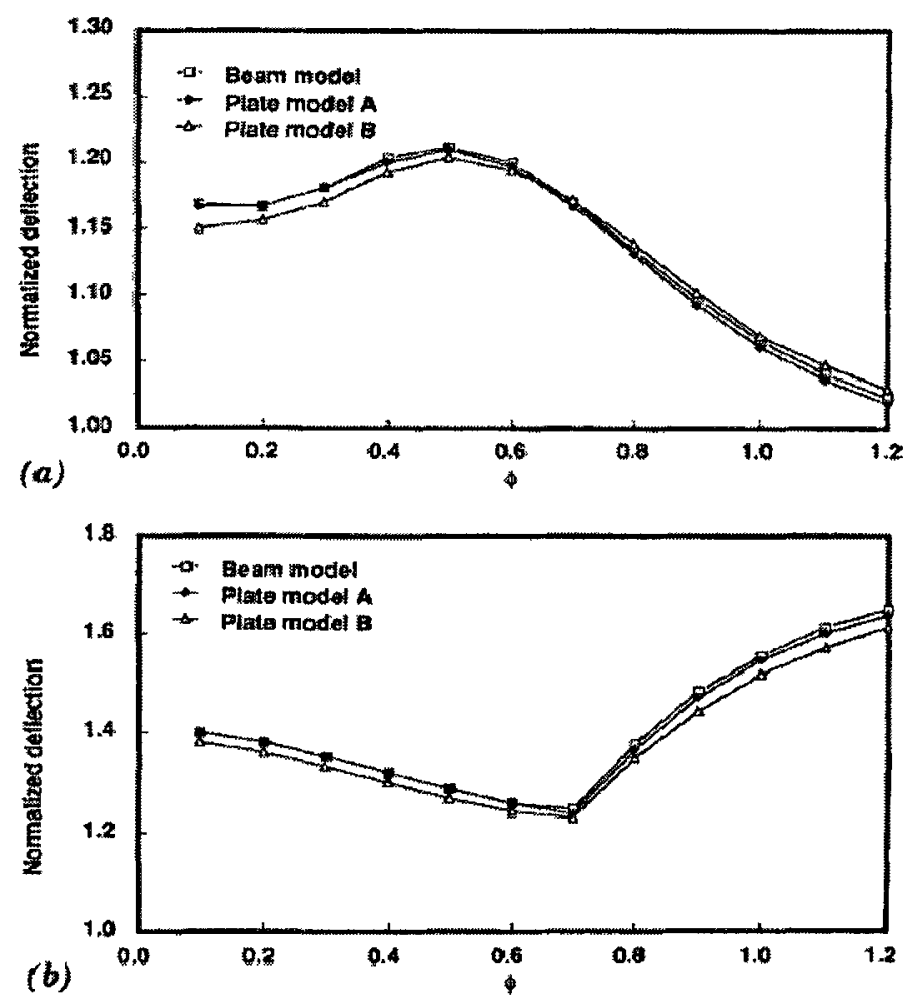

Figure 2.16 Normalized midpoint deflections in the bridge represented by beam and plate element under central vehicle load (Humar and Kashif 1995)

Paultre et al. (1995) reviewed most of the analytical and experimental work related to bridge dynamics, and stated that there are three alternatives to obtain the bridge dynamic response, namely; applying codes of practice, dynamic analysis, and full scale dynamic tests. The authors discussed a lot of previous experimental research relevant to the dynamic response of bridges. They also conducted many field loading tests on three bridges in the province of Quebec, Canada. The truck load used for field testing of the bridges was 24 tonnes and the trailer load ranged from 13 to 18 tonnes. They found that light vehicles compared with the design loads have a tendency to incorrectly shift the Dynamic Amplification Factor (DAF) to the higher side. In this respect, data from light vehicles should not be used in determining DAF. They also found that sometimes DAFs at points away from the load can be larger than those at points under the load. 
Yang and Yau (1997) developed an element for modeling the vehicle-bridge interaction in the analysis of railway bridges carrying high-speed trains consisting of cars in connection. The railway bridge was modeled by a beam resting on elastic springs representing the stiffness of the underlying ballast and the train was modeled by a series of lumped masses supported by the suspension systems represented by the springs and dashpots as shown in Figure 2.17. The track irregularities were also considered in the proposed vehicle-bridge interaction element as shown in Figure 2.18. The equations of motion for the bridge and each of the sprung masses are derived; these equations of motion are coupled equations and not easy to solve. The equation of motion for the sprung mass is first discretized using Newmark's finite difference formulas and then compressed into the bridge equation to cope with coupling more easily. The developed element is referred to as the vehicle-bridge interaction element and has the same number of degrees of freedom as the original element, and also has the properties of symmetry and bandedness in element matrices. Equations of motion for the whole vehicle-bridge system were obtained by using a direct assembly process. However, condensation of matrices by eliminating the degrees of freedom of the vehicle and condensing them to the corresponding degrees of freedom of the bridge element acted upon by the vehicle has some disadvantages where it is not adequate for determining the accurate vehicle response indicating the riding comfortability (Yang and $\mathrm{Wu} 2001$ ).

Some numerical examples were provided to verify the applicability of the vehicle-bridge interaction element and the solution procedure presented by the authors. The dynamic response solved by the authors' procedure for a simply supported bridge subjected to a moving sprung mass was compared to that dynamic response obtained by solving the 
equations of motion of the vehicle and the bridge given by Biggs (1964), Fryba (1972) and Humar (2002) for the system shown in Figure 2.19 where the damping of the sprung mass is neglected and considering the contribution of the first mode of vibration. The dynamic responses of the midpoint displacements in the beam subjected to the moving load and the sprung mass are shown in Figure 2.20. As shown in the figure, the response obtained by the authors' procedure based on the sprung mass model agrees well with that response obtained in the previous studies.

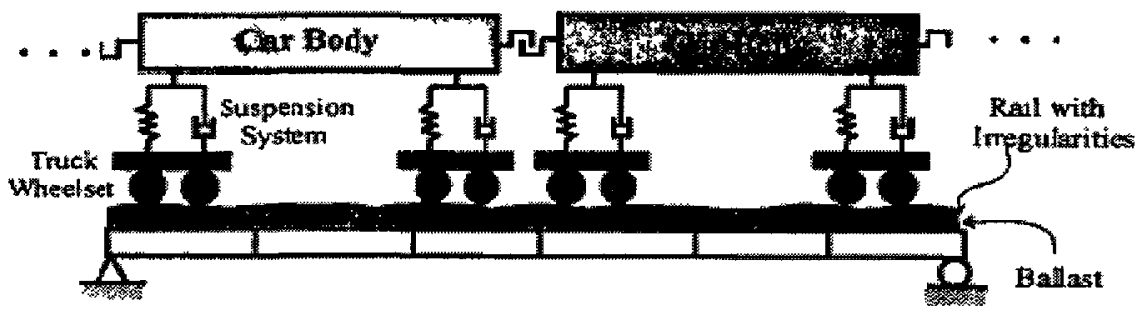

(a) General model

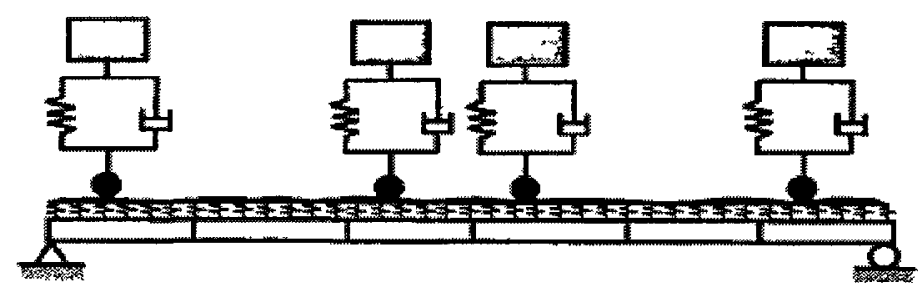

(b) Sprung model

Figure 2.17 Train-bridge system (Yang and Yau 1997)

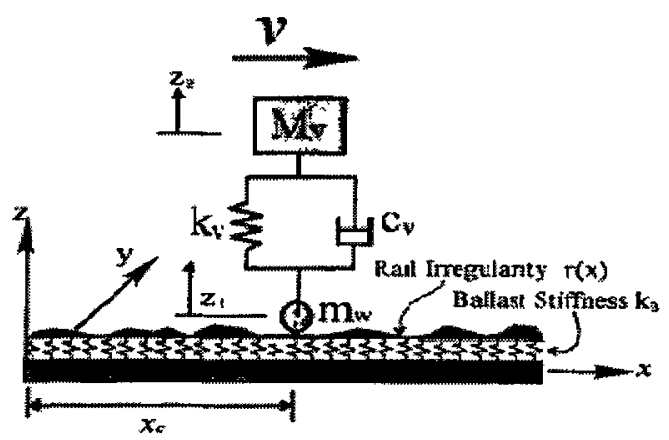

Figure 2.18 Vehicle-bridge interaction element (Yang and Yau 1997) 


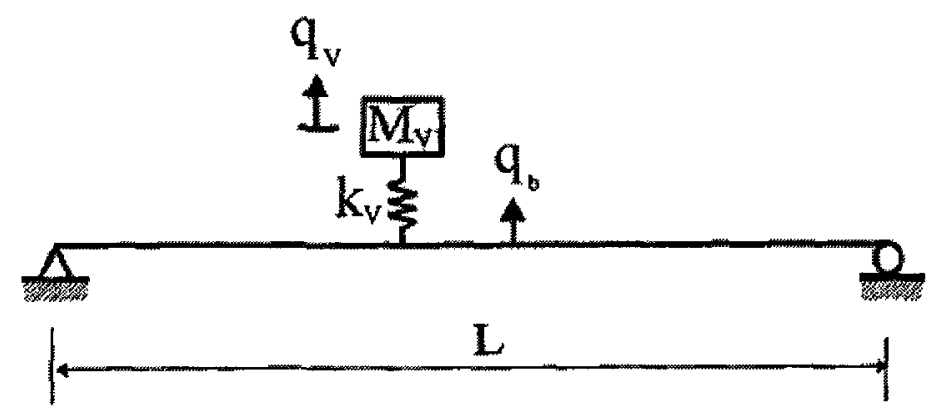

Figure 2.19 Beam with moving sprung mass neglecting damping (Yang and Yau 1997)

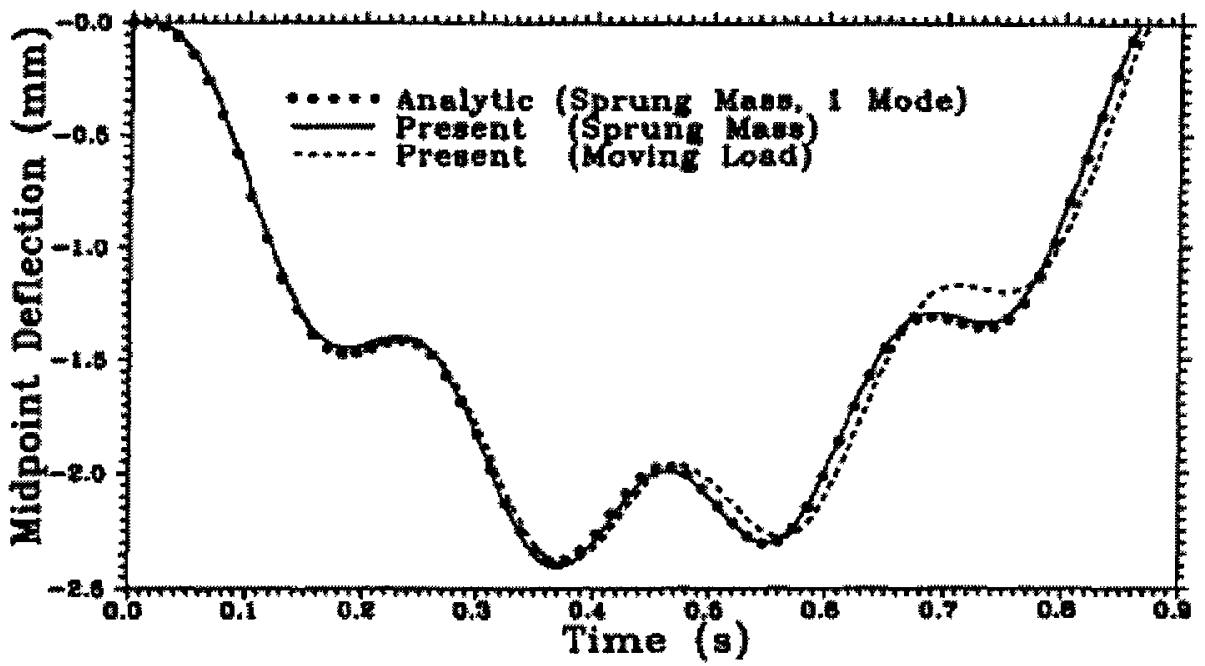

Figure 2.20 Midpoint deflection in beam (Yang and Yau 1997)

Henchi et al. (1998) presented an efficient algorithm for the dynamic analysis of bridges under moving vehicles using a coupled modal and physical components approach. The degrees of freedom of the bridge model were referred to as the modal components and the degrees of freedom of the vehicle were referred to as the physical components. The bridge was modeled using a finite element method and the vehicle was represented by mass attached to spring-damper system. The authors pointed out that there are two ways to simulate the dynamic interaction between bridge and vehicles as shown in Figure 2.21 however other methods for solving the equations other than those illustrated in the figure are available. The first way is based on the uncoupled iteration method in which each 
system of the bridge and the vehicles is solved separately and an iterative process in each time step is performed to find the equilibrium between the bridge and vehicle tires. The other way to simulate the dynamic interaction between bridge and vehicle is solving the super system fully coupled and the solution is given at each time step without any iteration. The second method of solving the fully coupled equations of bridge-vehicle system has some advantages over the former as following; the CPU time is reduced in comparison with the uncoupled iterative method, easy and compact numerical implementation, reduced computer memory storage and no iteration in the computational process.

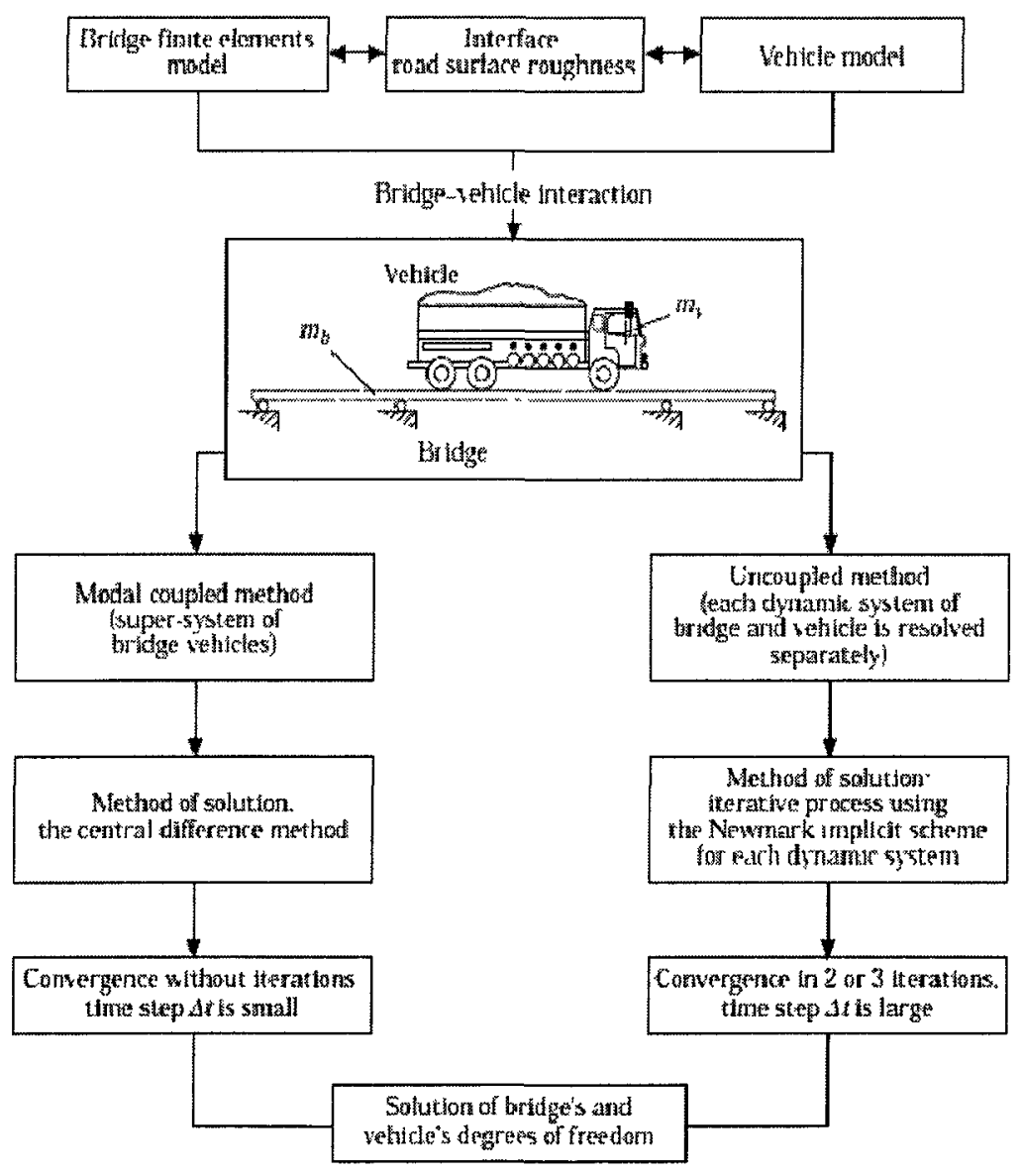

Figure 2.21 Dynamic analysis procedures for bridge and vehicle interaction (Henchi et al. 1998) 
Farad et al. (1998) investigated the effect of dynamic loads on DAFs of an existing continuous bridge. The model of the vehicle was a three-dimensional analytical model, where the bridge was modeled with finite elements. The Ministry of Transportation of Quebec performed an experimental test program for the same existing bridge. The authors provided a comparison between the solutions acquired from their presented models and the experimental results for different vehicle speeds. The results showed that DAFs calculated for the displacements are less than those computed from strains and reactions. Therefore, the authors recommended that the codes of practice should encompass three types of DAFs; one for displacement, one for moment and a third for shear. The authors deduced that DAFs presented in the codes of practice are underestimated for long span continuous bridges.

A computational procedure was developed by Yang and $\mathrm{Wu}(2001)$ to analyze vehiclebridge interaction response using finite element method. This procedure was used to simulate the dynamic response of general vehicle-bridge system. The bridge was modeled by beam elements while the vehicle was represented by models of various complexities ranging from the moving load, moving mass, sprung mass supported by spring and dashpot unit, to suspended rigid bar (Figures 2.22 and 2.23). An interaction element was defined to consist of a bridge element and the suspension units of the vehicle resting on the element. The composition of an interaction element and the parts of car bodies in contact was regarded as a substructure. The vehicle-bridge interaction element has the same number of degrees of freedom as the original bridge element and keeping the advantage of symmetry. By the dynamic condensation method, this procedure eliminated all the degrees of freedom associated with the car bodies existing within each 
substructure. As the condensation has been performed on the element level, a conventional assembly process can be applied to form the structural equations including both the ordinary bridge elements and the vehicle-bridge interaction elements. The used technique can be extended for the simulation of bridges traversed by a train represented by a series of lumped masses supported by springs and dashpots moving on the bridge. Some numerical examples were provided to verify the applicability of the vehicle-bridge interaction element and the solution procedure presented by the authors.

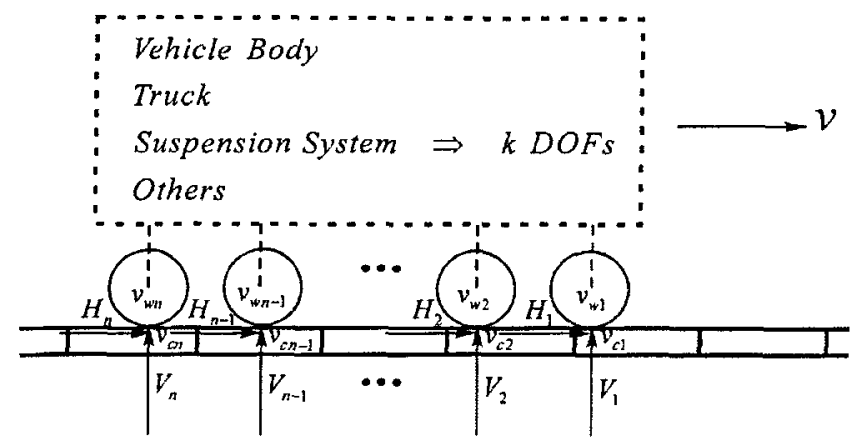

Figure 2.22 Schematic illustration of vehicle-bridge interaction (Yang and $\mathrm{Wu} 2001$ )

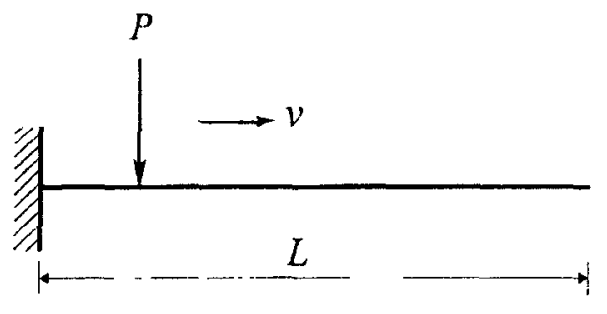

(a)

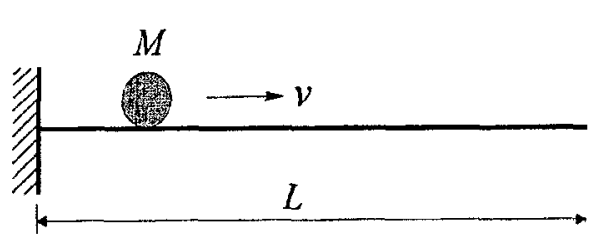

(b)

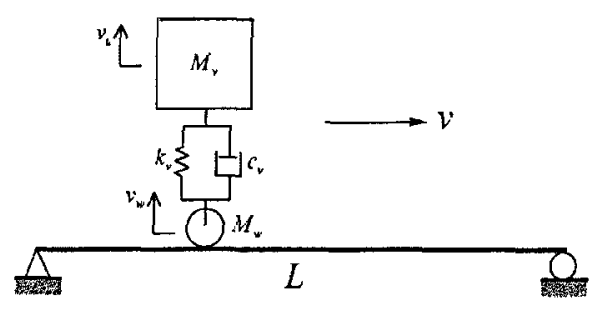

(c)

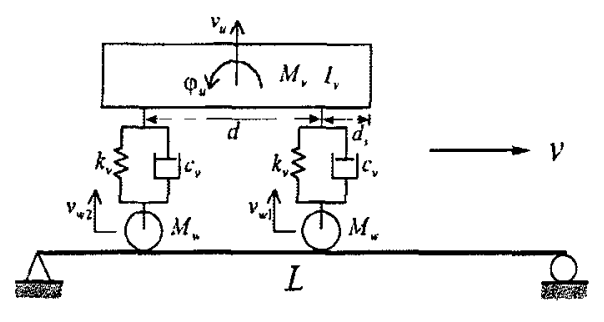

(d)

Figure 2.23 Vehicle-bridge interaction models: (a) moving load, (b) moving mass, (c) sprung mass, (d) suspended rigid beam or two-axle vehicle (Yang and $\mathrm{Wu}$ 2001) 
The dynamic response solved by the authors' procedure for a simply supported bridge subjected to a moving sprung mass was compared to that dynamic response obtained by solving the equations of motion of the vehicle-bridge system given by Biggs (1964), Fryba (1972) and Humar (2002) for the system shown in Figure 2.23(c) where the mass of the wheel and the damping of the sprung mass are neglected and considering the contribution of the first mode of vibration. The time history of midpoint displacements in the bridge subjected to moving load and moving sprung mass are shown in Figure 2.24.

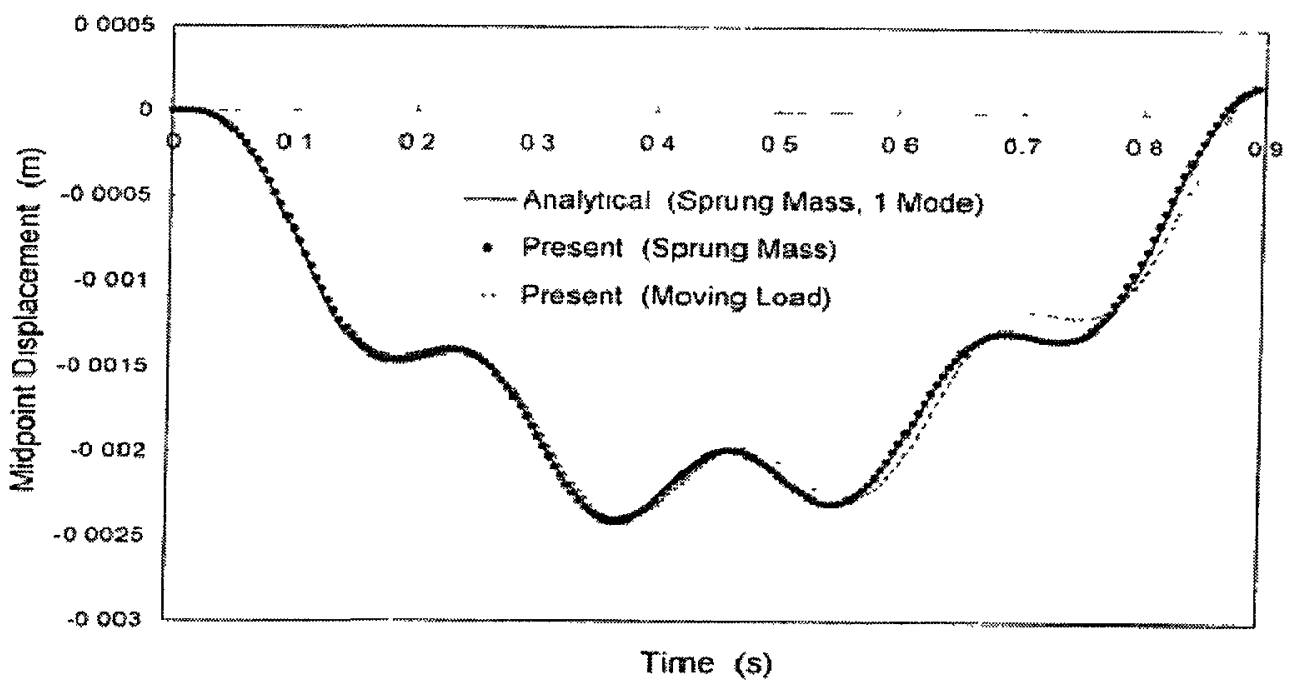

Figure 2.24 Time history of midpoint displacement in a simply supported bridge traversed by single-axle vehicle loads (Yang and Wu 2001)

As shown in the figure, the response obtained by the authors' procedure based on the sprung mass model agrees well with that response obtained in the previous studies. Another example of a simply supported bridge subjected to moving two-axle vehicle loads represented by a rigid bar supported by two spring-dashpot units as shown in Figure 2.23(d) is solved using the author's approach and compared to the results in the literature obtained by Yau and Yang (1998). The time history of midpoint displacements in the 
bridge subjected to moving two-axle vehicle loads are shown in Figure 2.25, where the response obtained by the authors' procedure agrees well with that response obtained by Yau and Yang (1998).

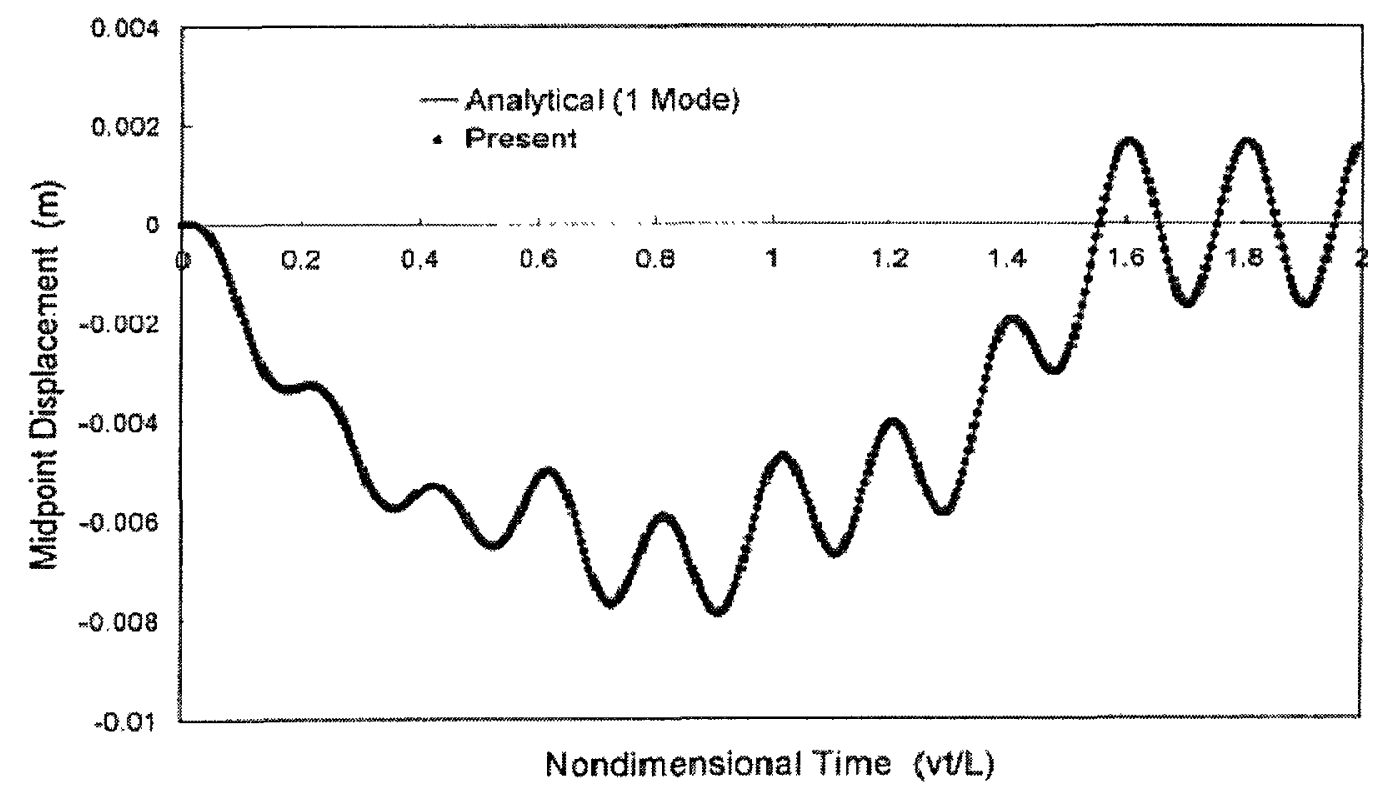

Figure 2.25 Time history of midpoint displacement in a simply supported bridge traversed by a two-axle vehicle (Yang and $\mathrm{Wu} 2001$ )

Kim et al. (2005) proposed a method of analysis of the bridge-vehicle interaction to investigate the dynamic responses of steel girder bridges and vehicles. The Lagrange equation of motion was used to derive the equations of motion of the three-dimensional bridge-vehicle system. The vehicle was modeled by two-axle model with seven degrees of freedom or three-axle model with eight degrees of freedom while the bridge was modeled using beam elements for the girders, cross beams and guard rails of the bridge and using a flat plate element for the bridge deck. The lumped mass formulation was used for the mass matrix of the bridge model. Newmark's $\beta$ method was used to solve the coupled equations of the bridge-vehicle interaction system. The authors considered a 
cargo truck, dump truck and steel girder bridge in their numerical models and the roadway roughness profile was measured and used for analysis. The authors compared the dynamic wheel loads and dynamic responses of the vehicles and the bridge obtained from the analytical procedure with data from field tests, and they demonstrated that their proposed procedure is valid and of acceptable accuracy in calculating the dynamic wheel loads and the bridge response to moving loads.

Jia and Ulfvarson (2005) studied the static and dynamic behaviour of a high tensile steel deck designed with trapezoidal stiffeners. The deck structure was modeled using a finite element method taking into account the effect of support condition for the girders and the contact area between the vehicle tire and panel. The modal analysis of the structure was performed and the results were provided for different loading conditions of loaded and unloaded deck and for different load types of car loads and truck loads. The results obtained showed that the locations and numbers of cars occupying the deck strongly affect the dynamic response of the structure. The dynamic characteristics of the vehicle, namely the stiffness and damping, were found to considerably affect the dynamic response of the deck structure and the results obtained using this representation of the vehicle were more accurate than those obtained from a moving mass model of the vehicle.

Among the miscellaneous uses and applications of VLFS are the floating airports and runways which have very large length and width with relatively small stiffness to bending deformations. Due to these configurations, a floating airport or runway can be represented by a thin elastic plate. However, if the length of this plate is very large 
relative to its width, another simplification can be made by representing this plate by a beam. Qiu (2007) proposed a numerical simulation of transient hydroelastic response of a floating beam induced by landing loads. The author represented the floating platform by a flexible beam, floating in an infinite water domain which is assumed to be compressible and inviscid. The governing equations of motion were formulated in terms of the beam deflection and hydrodynamic pressure. Finite element method was used to model both the platform and the fluid domain. The platform was modeled by a free-free beam floating in an open water domain and subjected to a mass moving with constant speed. The author developed a time-domain finite element model, to analyze the fluid-structure interactive dynamic system, which results in a system of coupled equations of motion. Some numerical examples were provided and the numerical results were compared to experimental test data, and it was illustrated that the method presented by the author is valid. However, the moving load was represented by a moving mass, neglecting the dynamic characteristics of the vehicle that represent its stiffness and damping properties and that have a significant effect on the dynamic response of the floating structure.

Qiu (2009) extended his work by presenting modeling and simulations of transient responses of a flexible beam floating in finite depth water under moving loads. The finite element method was used to develop the equations of motion for the transient behaviour of the elastic beam under the effect of a moving force with constant speed. The effect of some factors on the dynamic responses of the floating beam was studied, including the length of the beam, the travelling speed and the water depth. 


\subsection{Buoyancy, Water Current and Wave Loads Acting on Floating Bridges}

Buoyancy is an upward force acting on an object immersed in a fluid, enabling it to float. According to Archimedes' Principle of Floatation, the buoyancy force on a floating body is equal to the weight of the fluid displaced by the body. The buoyancy offered to a floating structure can be represented by elastic springs supporting the floating structure along its length.

According to many authors, a floating bridge can be idealized as a beam or long structure resting on water represented by elastic springs of equal stiffness (Georgiadis 1984; Wu and Sheu 1996; Wu and Shih 1998; Seif and Koulaei, 2005). Georgiadis (1984) presented a general structural model for the floating bridge as shown in Figure 2.26 where the bridge has a constant rectangular cross-section with stiffness $E I$, width $B$ and total length $L$. The bridge is moored using anchor cables with cable stiffness $k_{j}$ to resist the lateral water loads. For the heave and roll motion, the bridge behaves as a beam on elastic foundation with foundation modulus equal to the buoyancy stiffness. For the heave motion, the buoyancy stiffness is given as $k_{w}=\rho_{w} g B$. For the roll motion, the buoyancy stiffness is given as $k_{w}=\rho_{w} g B^{3} / 12$, where $\rho_{w}$ is the density of water and $g$ is the gravitational acceleration.

Moreover, the buoyancy force is a function of the submerged depth of the floating structure and proportional to its displacement into water as shown in Figure 2.27. Seif and Koulaei (2005) discussed the modeling and analysis of the continuous pontoon floating bridge over Urmia Lake shown in Figure 2.10. The buoyancy force acting on the floating bridge was modeled by equivalent spring elements. Seif and Koulaei concluded 
that the hydrodynamic analysis of a floating bridge can be well predicted by using linear spring elements for the buoyancy stiffness and nonlinear spring elements for anchor stiffness. This conclusion implies that the buoyancy force can be modeled to be linearly proportional to the bridge displacement into water, and such representation is appropriate to predict the hydrodynamic behaviour of the floating bridge.

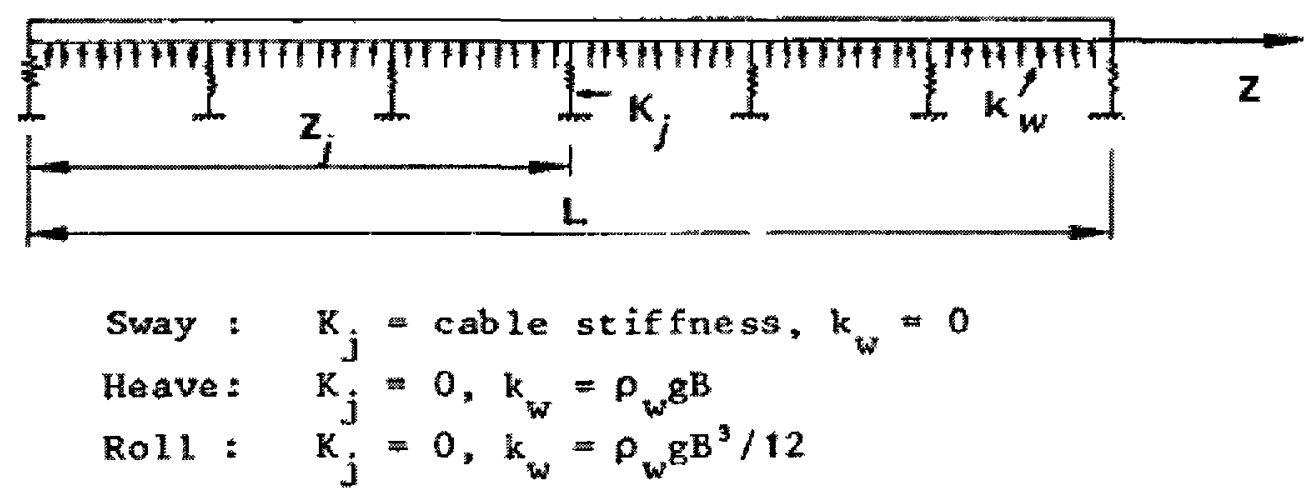

Figure 2.26 General structural model for a moored floating bridge (Georgiadis 1984)

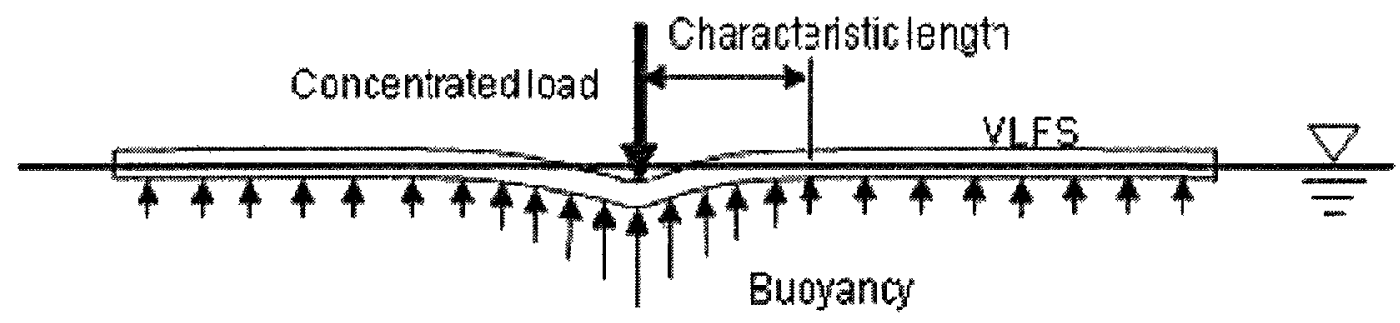

Figure 2.27 Buoyancy forces acting on VLFS (ISSC, 2006d)

Ali and Anan (2009) investigated the hydrodynamic interactions between the elements of an array of free floating three dimensional rectangular bodies in regular waves. The rectangular bodies were identical, and equally spaced along the array. The hydrodynamic interaction effect was found to be strong for surge exciting forces and surge motions, while it was found to be very weak for heave exciting forces and heave and pitch 
motions. Such an array of three dimensional rectangular floating boxes is somehow similar to a rapid deployment floating bridge, except that the pontoons of the floating bridge are connected to each other and the whole bridge is supported at shores. In the case of a rapid deployment floating bridge under the effect of moving vehicle loads, the heave and pitch motions represent the dynamic response of the bridge. In such a case, the effect of the hydrodynamic interaction between the bridge pontoons is expected to be very week on the dynamic response of the bridge in terms of the heave and roll motions of the bridge.

Floating bridges are subjected to different hydraulic forces such as water current and wave forces, in addition to wind, traffic loads and other loads. Therefore, floating bridges must be designed to withstand the expected current and wave loads safely. Mooring systems are required for floating bridges to resist water current and wave loads. They act as lateral supports and serve to stabilize the bridge against lateral movement and to reduce the straining actions developed in the bridge structure due to water current and wave loads. There are many types of mooring systems such as catenary and limited mooring cables, etc. Motorized boats are also used as lateral supports for rapid deployment temporary floating bridges.

In Urmia Lake, another study was done by Daghigh et al. (2002) who studied the mooring system design and optimization for floating bridges. Mooring lines were categorized into two types; the catenary type settling on the sea floor and the limited type which has no dead-length on the sea floor. For a simple stiffness analysis of mooring lines, the mooring lines are represented by separated horizontal and vertical springs. For 
the same loading conditions, the stiffness of the limited type mooring line was much greater than that of the catenary type. The response of the moored body was studied for different mooring types and the limited type mooring lines were found more suitable for floating bridges.

Insufficient mooring systems for floating bridges can cause the bridge to become dislodged or even sank. Previous studies have been done to figure out the reasons of some disasters that took place with some floating bridges and to assess the risk involved in the field use of floating bridges in severe environmental conditions.

The loads acting on the mooring cables supporting the Evergreen Point Floating Bridge were investigated by Peterson et al. (2003). The bridge is the longest floating bridge in the world with a total length of $2310 \mathrm{~m}$ which was completed in 1963 . The bridge is a continuous pontoon floating bridge composed of concrete hollow pontoons. It was subjected to a storm in January 1993 which led to damage at two mooring cables at the bridge ends, and damage at several other locations. It was deduced that the more stiff cables near the bridge ends were subjected to much higher loads than longer and more flexible cables near the bridge midspan; the higher loads during the storm were the reason behind the failure of the two cables.

Sannasiraj et al. (1998) studied the effect of both regular and random waves on the response of pontoon-type floating breakwaters. Finite element method was implemented to study the behaviour of breakwaters in waves, where the mooring lines were represented by linear springs. Different mooring configurations were considered for determining the hydrodynamic coefficients and wave exciting forces on the floating 
structure, namely, mooring at water level, mooring at base bottom, and cross-mooring at base bottom level. The mooring forces were found significantly affected by the mooring line configurations. The moorings at water level and at base bottom yield significantly smaller mooring forces than that with crossed moorings.

Georgiadis (1984) presented a formula for obtaining the nodal loads due to wave forces acting on the floating bridge using a finite element analysis. Georgiadis stated that all the general finite element programs were usually used to calculate the response but they don't calculate the nodal loading in the case of continuous harmonic oblique loading along the structure. The method Georgiadis developed took into account the amplitude and phase variations of the loading between nodal points, resulting in response accuracy.

The dynamic response of floating bridges in waves is normally estimated on the assumption that the floating body is rigid. Some recent studies revealed that the consideration of the influence of structural elasticity on the dynamic response of floating bridge to waves is important and leads to results that more closely approximate the experimental data (Oka et al. 2000; Ikegami et al. 2001)

VLFS have a wide range of applications among which is the floating runway. Floating runways are mat-type floating structures with a very large area. Due to the very large area, the mat-type floating structure has a similar behaviour to that of elastic thin plate. These large structures are constructed of modules assembled together on site and then welded. During the welding process, the welded joints perform as vertical and rotational springs connecting adjacent modules, with stiffness varying from zero for separate modules to infinity for finally welded modules. In order to study the behaviour of the 
whole floating structure, the relative motions between successive modules and the bending moment and shear force on the joint due to waves should be evaluated. Xia et al. (2000) investigated the hydroelastic behaviour of two-dimensional articulated plates in regular waves using analytical approach, based on linear theory. The two successive plates are connected together by a rotational spring and a vertical spring where the displacement and the slope at the two connected ends are discontinuous but the shear force and bending moment are continuous. The authors implemented this approach to solve for the response of a multi-module articulated plate to wave loads. They concluded that the hydroelastic response of articulated plates to waves is strongly dependent on the stiffness of the connectors and incoming wave frequency.

Das and Das (2005) presented a semi-analytical approach for the calculation of coupled roll and yaw motions of a floating body in the time domain under small amplitude unidirectional regular waves. The sign convections for motion response of a floating body are as shown in Figure 2.28. For each ship section, two-dimensional added mass and damping are first determined; then, the sectional added mass and damping are integrated over the length of the ship. In order to compute floating body motions, the governing equations are derived from balancing between the hydrodynamic forces and the external exciting forces. Criteria for the system stability and incidence of roll resonance were derived and numerical computations were used to get the time histories of coupled motions. 


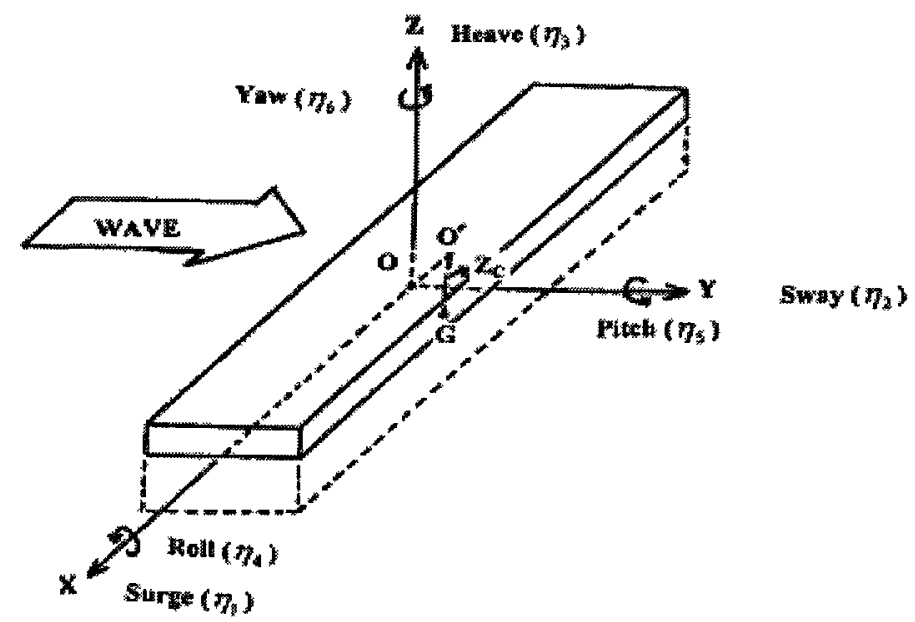

Figure 2.28 Sign convections for motion response of a floating body in regular waves (Das and Das 2005)

Wang et al. (2009) studied the hydroelastic performances of a ribbon bridge under wave action. The bridge was modeled using finite element method, and the mode superposition method and the three dimensional hydroelasticity theory were implemented to analyze the hydroelastic behaviour of the bridge. Experimental field tests were conducted using a tenth-scale elastic model in the ocean bowl. Numerical results were compared to experimental data and the results were in good agreement.

Ertekin et al. (2000) analyzed the hydroelastic static responses of the mechanically interconnected military floating bridge under the combined effects of the stationary live and/or dead loads, water current loads and loads from mooring cables. The bridge was modeled using finite element method where beam elements with six degrees of freedom at each node were used to discretize the bridge structure. The equations of small deformation beam theory were utilized to form the main governing equations of the bridge response, and the finite element method was implemented for solving these 
equations for calculation of the global forces, bending moments and the resulting displacements in the bridge. The results obtained were compared to model test data for verification and the results indicated that the model proposed by the authors can predict the bridge response fairly well.

\subsection{Main Findings from the Literature Review}

Unlike conventional fixed bridges, there is no unified code of practice for the analysis and design of floating bridges nor is there a unified method for determining their dynamic behaviour, as the structural system of a floating bridge may differ from one location to another in accordance with many factors. Most of the research work done regarding vehicle-bridge interaction is dedicated to ordinary conventional bridges, and only scant information is available regarding the vehicle interaction with floating bridges. In most cases, sophisticated analytical models of bridges are not capable of determining the factors affecting the dynamic behaviour of the bridge. By using a simplified model, the governing parameters controlling the bridge response can be determined and their effects can be thoroughly examined, leading to design procedures development.

The analysis of bridges under the effect of moving vehicles was accomplished by representing the bridge with a beam and the vehicle with a moving load or moving mass attached to a spring and damper system. The interaction of the vehicle with the bridge has a significant effect on the dynamic response of the bridge. Therefore, a moving force model is quite inadequate in predicting the dynamic response of the bridge to moving vehicle loads. The vehicle model represented by moving mass attached to a spring and damper system is more accurate than the moving load model in predicting both the bridge 
and the vehicle dynamic responses. The single-axle spring mass model, with the entire vehicle mass lumped over the axle, was found to provide accurate results of the bridge response if the axle spacing is very small relative to the bridge span. For larger ratios of axle spacing to the bridge span, the two-axle spring mass model was found to produce more accurate results. The two-axle spring mass model was found to produce a smaller response. Although more sophisticated models can be devised for the vehicles, the efficiency of solution of the vehicle-bridge interaction system becomes an issue of great concern, especially when there are multiple vehicles traversing the bridge. The vehiclebridge interaction problem in this regard is complicated in that it is time-dependent and there are multiple contact points.

In many previous studies, the dynamic characteristics of the vehicle that represent its stiffness and damping properties were neglected. The dynamic characteristics of the vehicle considerably affect the dynamic response of the bridge and the results obtained using this representation of the vehicle are more accurate than those obtained from a moving mass model of the vehicle. Also in some studies, the damping of the bridge was neglected; the damping of the bridge is expected to be more effective on the dynamic response of floating bridge and should be taken into account.

The locations and numbers of cars occupying the bridge deck strongly affect the dynamic response of the bridge. Also, the moving-load speed has a great effect on the dynamic response of the bridge, where the dynamic amplifications in both deflection and stresses increase with traveling speed. DAFs for the displacements were found to be less than those for strains and reactions. Therefore, it was recommended that the codes of practice 
should encompass three types of DAFs; one for displacement, one for moment and a third for shear. DAFs presented in the codes of practice were found to be underestimated for long span continuous bridges.

The analysis of floating bridges was accomplished by representing the floating bridge by a beam resting on elastic foundations represented by elastic springs. A hierarchical system of structural analysis was used for the analysis of VLFS, in which the global response analysis can be conducted by using an idealized structural model while the local structural response is investigated using a zooming technique. This concept can be applied to the global dynamic response of floating bridges. Most of the research work done regarding lateral hydraulic loads acting on floating bridges was devoted to wave loads. Limited literature is available regarding water current loads although water current load may be the most dominant hydraulic load acting on floating bridges constructed on narrow rivers and water channels in calm wind regions.

The comprehensive literature review presented in this chapter shows an urgent need to further investigate the dynamic response of floating bridges to the combined actions of moving vehicle loads and water current loads. In addition to the transport capacity of floating bridges in terms of the parameters controlling the rate of flow of vehicles on the bridge, namely; the vehicle weight, the vehicle speed and the separation distances between successive vehicles. This will form the focus of the present research. 


\section{Chapter: Analytical Approach and Model Development}

\subsection{Introduction}

Field testing of the dynamic response of floating bridges to moving vehicle loads and water current loads is very expensive. Also, model testing can be used for the assessment of the dynamic behaviour of floating bridges; however, it is not feasible for parametric studies and is not capable of including all possible loading conditions. Moreover, due to specific loading conditions and environmental requirements, the floating bridge design will always differ from one site to another. Therefore, a reliable analytical tool is of vital importance for designers and analysts of floating bridges and can be of great advantage for optimizing the structural behaviour and design of these bridges. Analytical models can be used to investigate and predict the dynamic response of these bridges to moving vehicle loads and other loading conditions. These models can simulate the vehicles, the bridge and the fluid-structure interaction in an analytical environment which greatly reduces the high cost of field testing. Therefore, they are used prior to more expensive field tests to maximize the benefits of both approaches. Field testing may be required at the final stage to examine the behaviour of the final product after investigating and predicting this behaviour using reliable analytical methods.

Although a lot of studies have been done to investigate the behaviour of floating bridges under static vehicle loads, fewer studies have been done to investigate the dynamic response of floating bridges to moving vehicle loads. Some of the previous studies have modeled the vehicle loads by axle forces with constant values; this simplification neglects the vehicle dynamic characteristics and therefore neglects the vehicle-bridge interaction 
which affects the dynamic response of the bridge. The interaction between the vehicles and the bridge increases the deflections and stresses induced in the bridge; bridges therefore should be designed to withstand these increased stresses, and to keep the deflections within the safe limits. More convenient and reliable analytical models are needed to investigate the dynamic response of floating bridges to moving vehicle loads and to serve as a useful tool to establish new concepts for the analysis and design of these floating bridges.

The objective of the present chapter is to develop an analytical approach and a finite element program as a reliable tool for the dynamic analysis of floating bridges under moving vehicle loads and water current loads. This analytical approach will be used to optimize the transport capacity of rapid deployment floating bridges in terms of the parameters controlling the rate of flow of vehicles on the bridge, namely; the vehicle weight, the vehicle speed and the separation distances between successive vehicles. Based on the results of parametric studies conducted, recommendations and guidelines will be provided to improve the behaviour of floating bridges in order to acquire additional carrying capacity to withstand the recent increased vehicle loads, and to achieve increased speed for the passing vehicles.

There are many methods to study the behaviour of floating bridges, such as, the differential equations for beam on elastic foundations, the dynamic analysis using the dynamic equations of motion, and the finite element method. In the present study, the dynamic equations of motion of the integrated vehicle-bridge-fluid system are derived based on the finite element method, and implemented into a finite element program 
developed by the author to achieve simple and reliable solutions for the dynamic response of floating bridges to moving vehicle loads and water current loads.

\subsection{Structural Behaviour of Floating Bridges}

In the analysis of pontoon floating bridges under the effect of vertical loads, the vertical movements of the bridge correspond to the passage of vehicles on its surface, causing high deformations and straining actions in the bridge. The type of connection between the consecutive bridge pontoons is a major factor affecting the bridge's overall structural behaviour. The following is a brief description of the structural behaviour of the two main types of floating bridges, classified according to the type of their connecting joints.

\subsubsection{Types of Pontoon Bridge Connections}

Two types of connections are commonly used in rapid deployment floating bridges; namely the hinged connection and the rigid connection. The hinged connection shown in Figure 3.1 consists of two flat plates with circular openings welded to each pontoon. When the pontoons are assembled, the flat plates overlap with the circular openings lined up. A circular steel shaft is inserted through the circular openings and bolted to hold the pontoons in place. The diameter of the shaft is approximately the same as the diameter of the openings, with a small tolerance to facilitate field erection of the bridge. This connection exists in two horizontal locations at the same vertical level near the top of the pontoon and symmetric about the longitudinal centre line of the pontoon. The other type of connection is the rigid connection where the hinged connection near the top of the 
pontoon is repeated near the bottom of the pontoon forming a rigid connection that can transfer bending moment.
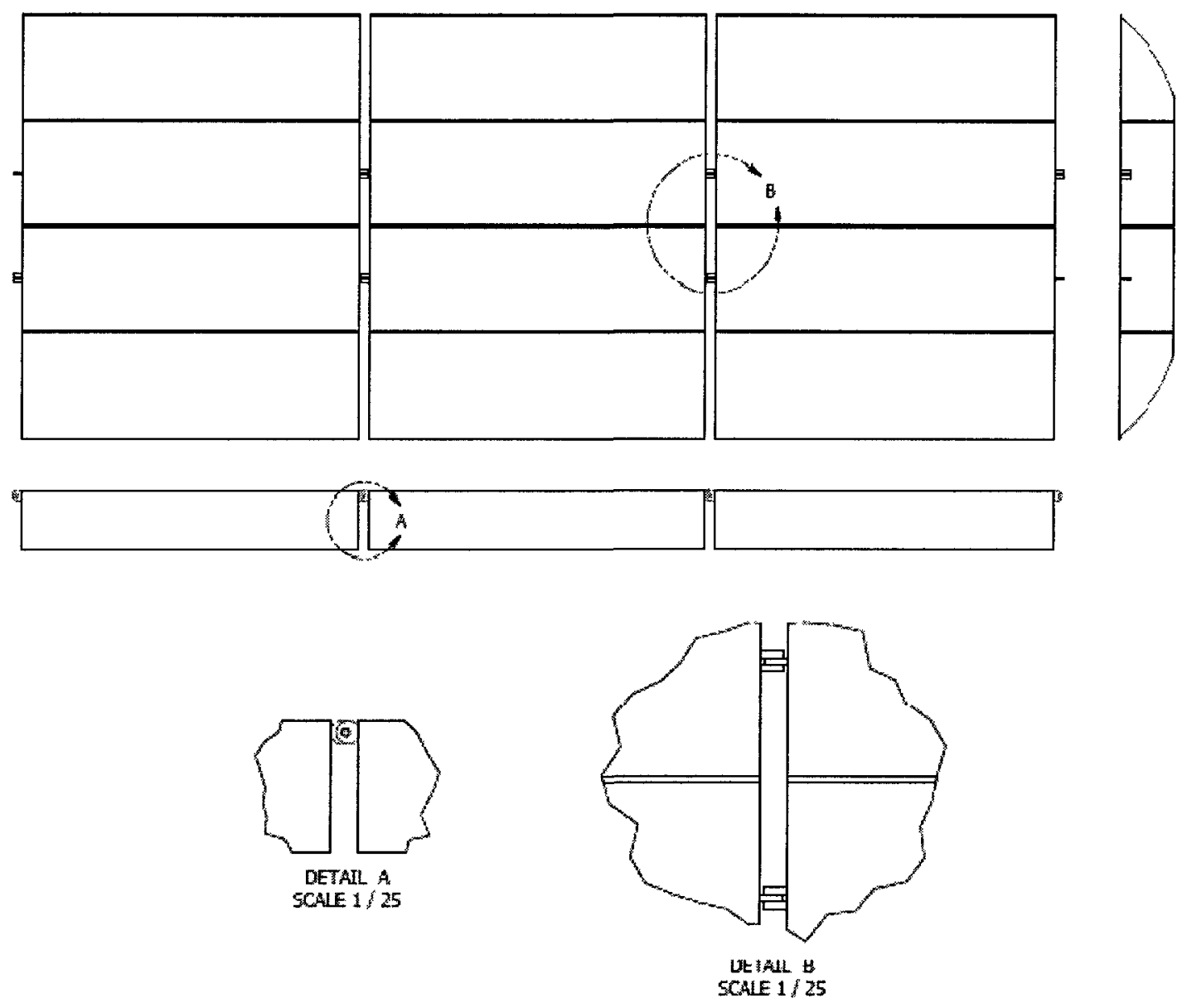

Figure 3.1 Hinged connection used for ribbon floating bridges

\subsubsection{Rigid-Connected Floating Bridge}

The bridge is called rigid-connected if all of its pontoons are joined together by rigid joints. In this type of bridge, the joints are designed and constructed to be able to resist bending moments caused by moving vehicles along the bridge at the pontoon connection joints. 
Rigid joints are designed and constructed to transfer bending moments in the longitudinal direction, from one pontoon to the next. An experimental program for the testing of bridge pontoons of the rigid-connected type was presented by Harre (2002). Figure 3.2 from that study shows a typical bending moment diagram for such type of floating bridge construction. The diagram illustrates the structural behaviour of this type of bridge, since the bridge acts as a double-cantilever system, where distributed water upthrust forces act upward along the bridge length and the vehicle load acts downward at the position of the vehicle, and a large number of pontoons jointly act to carry the vehicle load. However, the long cantilever arm causes significantly high bending moment values along the bridge in the longitudinal direction.

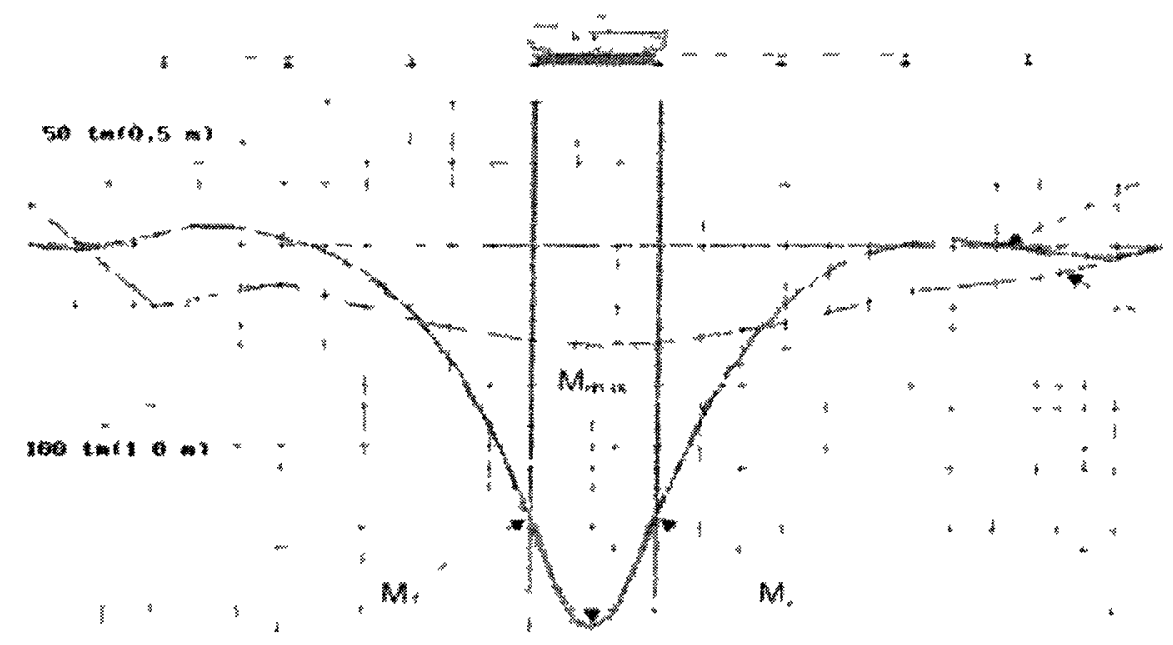

Figure 3.2 Experimental loading for rigid ribbon floating bridge (Harre 2002)

The advantages of this type of connection can be summarized as follows:

- The upward thrust of water acts on a large number of pontoons simultaneously, thus reducing the required flotation volume of a single pontoon. 
- The relatively high stiffness of the bridge structure, due to the fixity at the joints, reduces the bridge's vertical deflections and the vibration experienced by the drivers of traversing vehicles.

- For a pontoon of a specific flotation volume, rigid-joints increase the overall bridge load-carrying capacity.

However, this type of connection also results in some effects which can be considered as disadvantages as follows:

- The high bending moment values produced along the bridge in the longitudinal direction affect the bridge pontoons and their connecting joints.

- The design of the bridge pontoons and connecting joints needs to satisfy the high longitudinal bending moment values, combined with other straining actions produced by lateral loads.

\subsubsection{Hinge-Connected Floating Bridge}

This type of floating bridges is frequently used for fast and temporary passage across waterways. Only shear forces are transferred through these hinged joints connecting adjacent pontoons. For a floating bridge with hinged connections, each pontoon supports the moving vehicle almost in isolation with a small percentage of the load carried by the rest of the bridge pontoons.

The major advantage of this type of connection is that the connecting joints are subjected to only shear forces, and are therefore far less affected by the longitudinal loading of the bridge than in case of bridges with rigid connections. Also, the bridge pontoons are 
subjected to far less bending moment values compared to the case of rigid-connected bridge. While a significant reduction in the longitudinal moments acting on the bridge pontoons is achieved in case of hinged joints, it should be noted that this affects the overall load-carrying capacity of the bridge negatively due to the subsequent reduction in floating volume available to balance the vertical static and dynamic loads exerted by the passing vehicles.

In practice, three -or more- pontoons can be rigidly connected together to form a typical unit of the bridge; these typical units are connected to each other using hinged connections. The idea behind this form of hinge-connected rapid deployment bridge is to take advantage of both the rigid- and hinge-connected types while overcoming their disadvantages. The study presented in next chapters is concerned with the structural behaviour of the rigid-connected rapid deployment floating bridges as well as the practical form of hinge-connected (hybrid) rapid deployment floating bridges combining both types of connections in the same bridge.

Both the rigid-connected and hinge-connected floating bridge behave as rigidly connected bridge in the lateral direction because the hinged connection used exists in two horizontal positions at the same vertical level having a moment arm between them to resist lateral bending moment.

\subsection{Floating Bridge Dynamic Analysis}

The nature of use of rapid deployment floating bridges differs from that of conventional fixed bridges constructed over land or water. Rapid deployment floating bridges are 
usually constructed to serve traffic in one-way direction and not two-way traffic as in case of civilian bridges. The typical bay of the ribbon bridge is a four-pontoon folding module consisting of two roadway pontoons and two bow pontoons. While the total width of the ribbon bridge is about $8 \mathrm{~m}$, the roadway is only $4 \mathrm{~m}$ in width (see Appendix B for detailed description of the ribbon bridge). This means that only a few centimeters tolerance is left for eccentricity of the vehicle or tank. In addition, personnel standing on many points along the longitudinal centre line of the bridge provide guidance to vehicles and/or tanks crossing the bridge to move concentric along the bridge (see Figure 3.3). This means that there is a low probability of a vehicle or tank crossing the bridge being off centre line, and if there is any eccentricity, although it would be very small, it can be considered in the dynamic analysis of the floating bridge under moving vehicle loads.

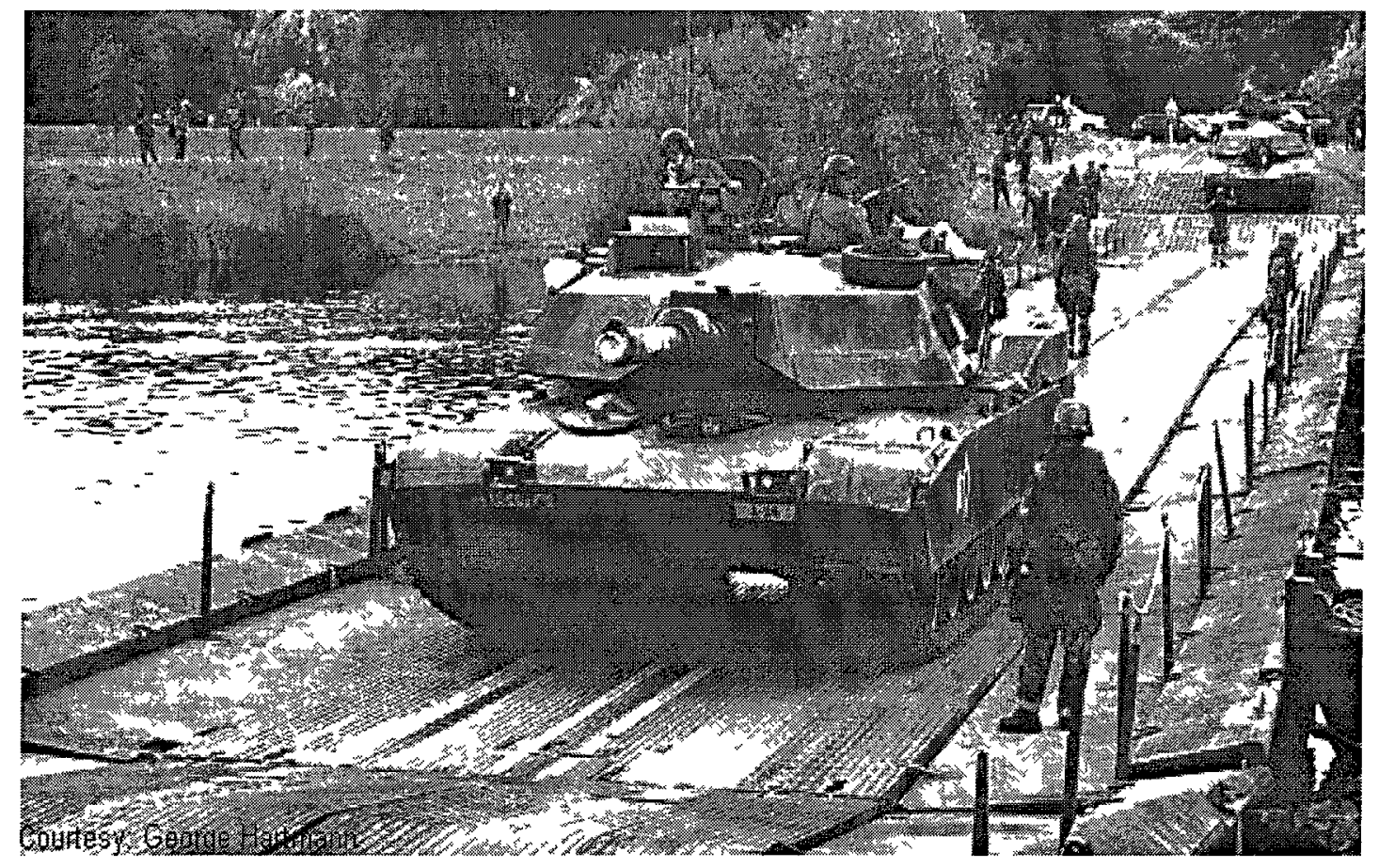

Figure 3.3 Ribbon bridge traversed by a tank concentric along the bridge centre line (defenselink.com) 
For accurate stress distribution analysis of the bridge cross-section, it is necessary to model the bridge using three-dimensional finite elements to investigate the stress distribution within each element. However, the use of three-dimensional finite elements to model the floating bridge increases the scale of the problem much more compared to the use of two-dimensional or one-dimensional finite elements because of the larger number of degrees of freedom for three-dimensional element. Also, the use of twodimensional finite elements to model the floating bridge enlarges the scale of the problem compared to the use of one-dimensional finite elements. For example, the use of threedimensional cubic element having eight nodes with six degrees of freedom at each node results in 48 degrees of freedom for each element compared to 24 degrees of freedom for two-dimensional plate element and 12 degrees of freedom for one-dimensional bar element. This means that the use of three- or two-dimensional finite elements to model the floating bridge would require more time and more computation memory and processing capabilities for the analysis runs because a large number of unknowns (degrees of freedom) need to be determined, specially for the analysis of the global behaviour of the whole bridge with the total bridge length involving a large number of elements.

Dynamic analysis requires more time and more computation memory and processing capabilities, where the problem under consideration is solved at each time step within the time domain of the problem; sometimes the time of the problem is divided into thousands of small time steps. Moreover, parametric studies involving many parameters require a large number of analysis runs. This can be not feasible in case of dynamic analysis of floating bridges using three-dimensional elements. 
For the global dynamic response of the rapid deployment floating bridge to moving vehicle loads, it is more appropriate to simplify the bridge model using one-dimensional finite elements where the dimensions of the bridge cross-section are relatively very small compared to the bridge length (see Figure 3.4). Modeling the bridge using onedimensional finite elements is more feasible especially in case of optimizing the transport capacity or the rate of flow of vehicles on the bridge which require performing parametric studies involving many parameters such as vehicle weight, vehicle speed, number of vehicles traversing the bridge at the same time, separation distance between successive vehicles and type of floating bridge whether it is rigid-connected or hinge-connected bridge.

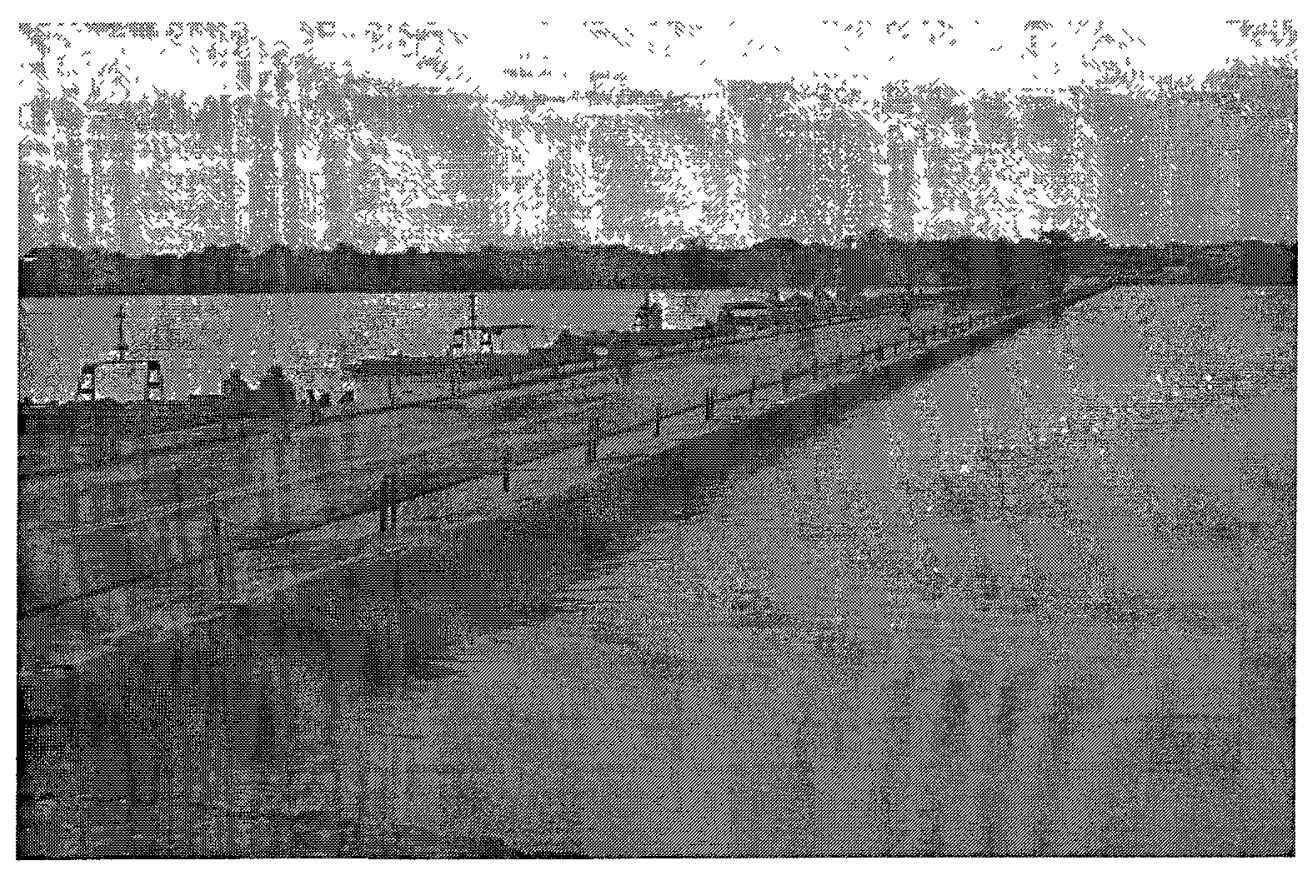

Figure 3.4 Floating bridge simply supported at shores and resting on water along its span (army-technology.com).

The dynamic behaviour of floating bridges will be investigated by modeling the bridge using space frame elements with three-dimensional degrees of freedom. Two main 
loadings act on floating bridges; moving vehicle loads along with upward floatation loads and lateral water current loads. First, the dynamic response of floating bridges to moving vehicle loads will be investigated. Then, the structural behaviour of floating bridges under the effects of water current loads and supporting boat forces is investigated. Finally, the three-dimensional interactive dynamic behaviour of floating bridges due to the combined actions of moving vehicle loads and dynamic water current loads along with possible eccentricities of these loads will be investigated. The three-dimensional dynamic analysis can be achieved using space frame finite elements with twelve degrees of freedom which can take into account applied loads and their eccentricities in the three dimensions and give the bridge response to applied loads in the three dimensions.

\subsection{Dynamic Equation of Motion}

The general equation of motion or the equation of dynamic equilibrium of any system can be written as follows;

$F_{I}+F_{D}+F_{S}=F$

In this equation, four types of forces are considered:

- The inertia force $F_{I}$ represents the inertial characteristic of the system.

- The damping force $F_{D}$ represents the energy loss due to friction in the system.

- The spring force $F_{S}$ represents the elastic restoring force of the system.

- The external force $\mathrm{F}$ represents a time-varying force applied to the system externally. 


\subsection{Space Frame Element}

Considering the three-dimensional global dynamic response of rapid deployment floating bridges to travelling vehicles and water current loads, the bridge section can be idealized by a space frame element. "A space frame element is a straight bar of uniform cross section which is capable of resisting axial forces, shear forces, bending moments about the two principal axes in the plane of its cross section and twisting moment about its centroidal axis" (Rao 2004). The space frame element has six degrees of freedom at each node; three translational degrees of freedom in $\mathrm{x}, \mathrm{y}$ and $\mathrm{z}$ directions, and three rotational degrees of freedom about $\mathrm{x}, \mathrm{y}$, and $\mathrm{z}$ axes as shown in Figure 3.5. This makes the space frame element able to consider acting loads as well as displacements and straining actions in the three dimensions. The twelve degrees of freedom for the space frame element are shown in details in Figure 3.6 for axial behaviour, torsion behaviour and bending behaviour in both the vertical and horizontal planes.

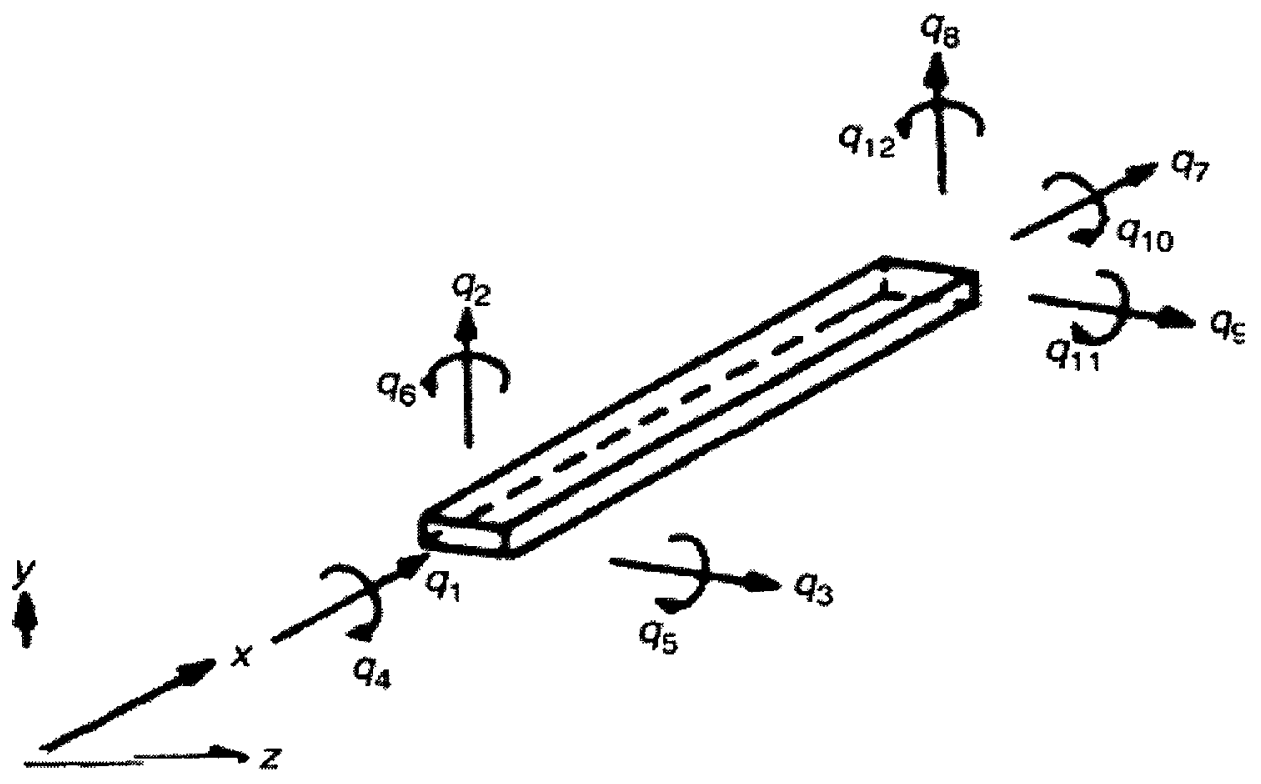

Figure 3.5 Space frame element with twelve degrees of freedom (Rao 2004) 


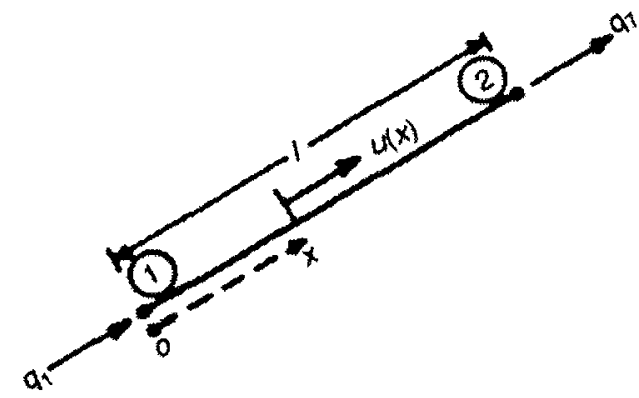

(a) Axial degrees of freedom

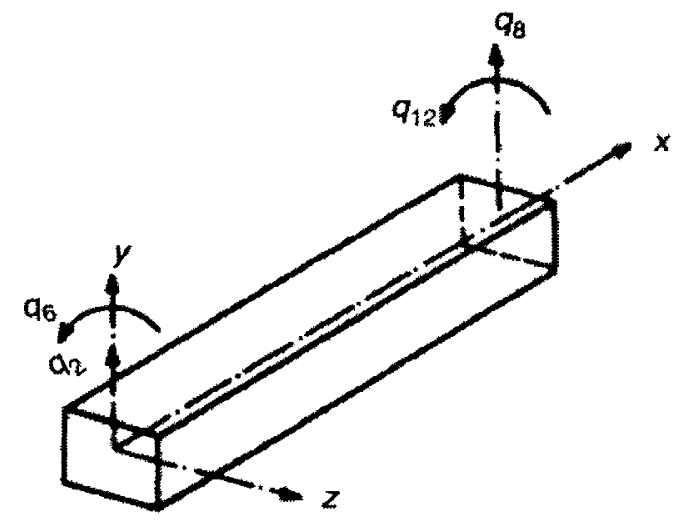

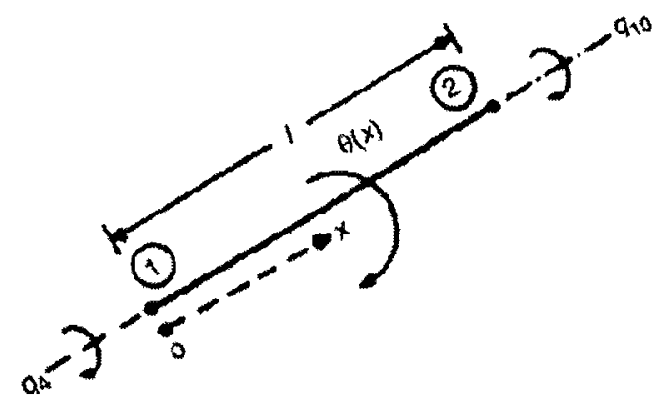

(b) Torsional degrees of freedom

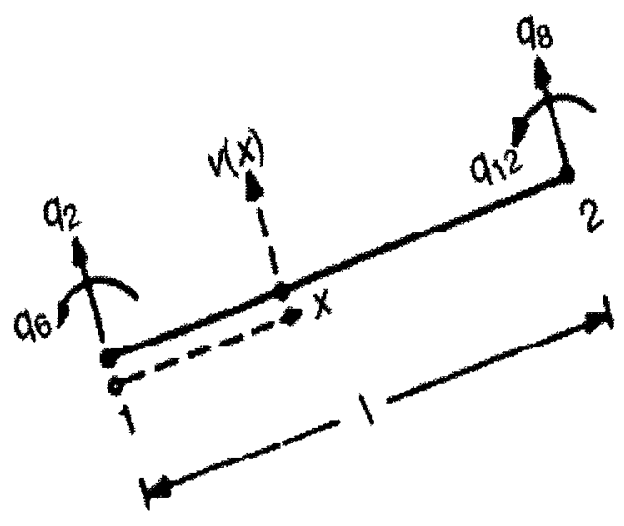

(c) Bending degrees of freedom in $\mathrm{x}-\mathrm{y}$ plane
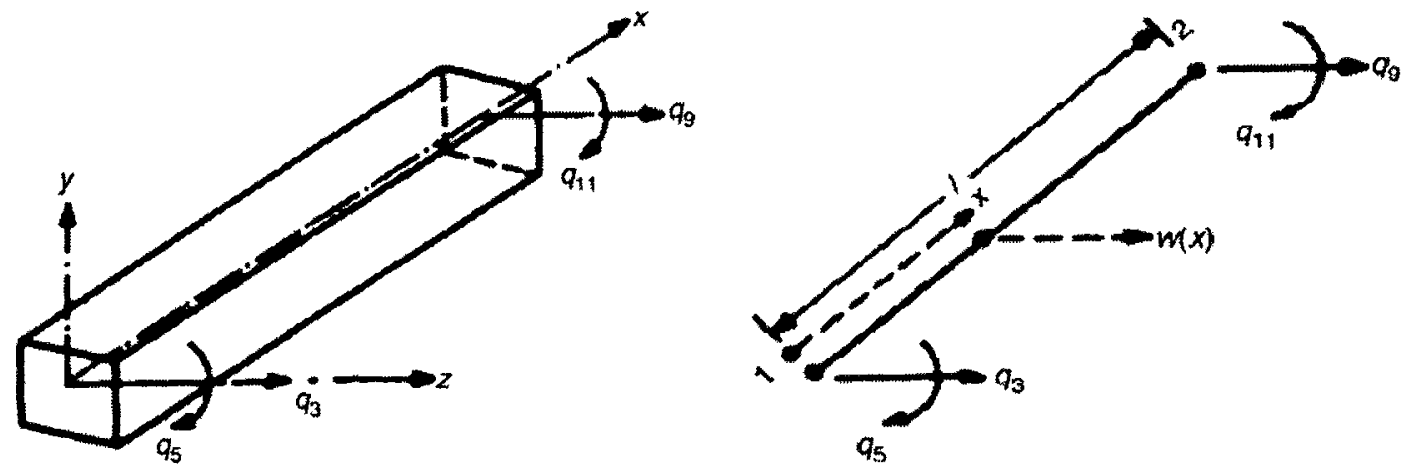

(d) Bending degrees of freedom in $\mathrm{x}-\mathrm{z}$ plane

Figure 3.6 Detailed degrees of freedom of a space frame element (Rao 2004) 


\subsection{Finite Element Analysis of Floating Bridges}

Solution of the governing equations of motion of simple bridge-vehicle system is sometimes performed using closed-form solutions. However, for the real problem of a rapid deployment floating bridge under the effect of both dynamic vehicle loads and the fluid forces, the problem is more suited towards a numerical analysis approach. Finite element method is one of the most convenient and reliable numerical methods that can be used for the analysis of floating bridges.

Considering the global dynamic response of a rapid deployment floating bridge to travelling vehicles and water current loads, the bridge section is idealized by a space frame element that has a uniform cross section with cross-sectional area $(A)$, moment of inertia about the z-axis $\left(I_{z}\right)$, moment of inertia about the $y$-axis $\left(I_{y}\right)$, polar moment of inertia about x-axis $(J)$, material modulus of elasticity $(E)$ and shear modulus $(G)$. The bridge has a span $L_{b}$, uniform mass $\bar{m}$ per unit length, flexural rigidity $(E I)$, and torsional rigidity $(G J)$. The bridge is simply supported at the two ends (the two shores) and resting on water represented by continuous elastic springs with stiffness $\bar{k}_{w}$ per unit length. A vehicle traveling across the bridge deck is represented by an unsprung mass $m_{t}$ connected to a sprung mass $m_{v}$ through a spring of stiffness $k$ and a damper of damping coefficient $c$ as shown in Figure 3.7. This model of the vehicle exists in the literature and is considered suitable for representing the dynamic characteristics of the vehicle, (Humar and Kashif 1993). 


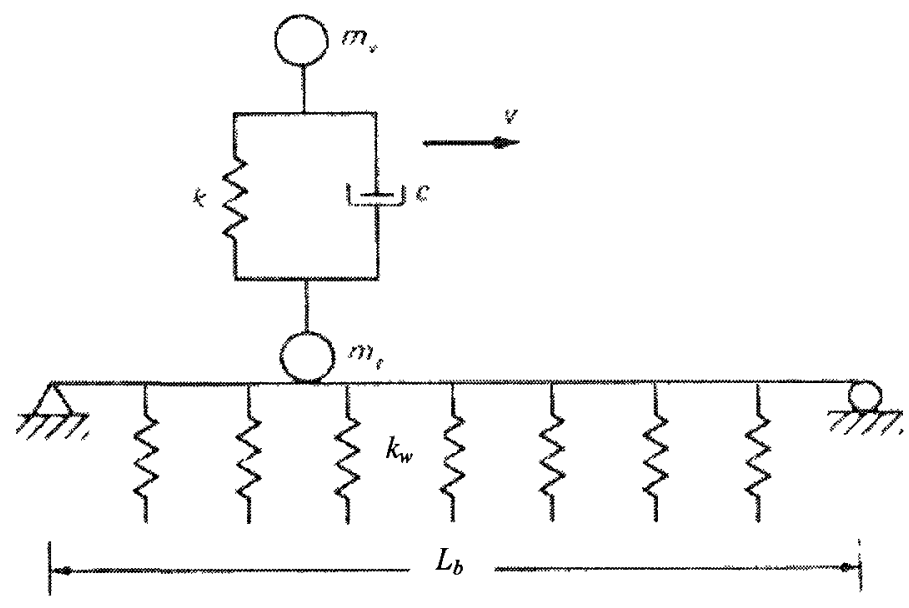

Figure 3.7 Idealization of bridge-vehicle system

In finite element analysis, the structure is discretized into a number of small elements. The displacements within each element are expressed by the superposition of a number of shape functions; each shape function is multiplied by a generalized coordinate. For the space frame element used in the present study to model the bridge, the generalized coordinates are the deflections and their first derivatives in both $x-y$ and $x-z$ planes as well as the axial displacement along the $\mathrm{x}$-axis and the rotation about the $\mathrm{x}$-axis (torsion), at the interconnecting nodes between elements.

The four shape functions for the bending behaviour of a space frame element in both $x-y$ plane and $\mathrm{x}-\mathrm{z}$ plane (vertical and horizontal directions) are given as follows where $L$ is the element length (Humar 2002; Rao 2004);

$$
\begin{aligned}
& \psi_{1}=1-3 \frac{x^{2}}{L^{2}}+2 \frac{x^{3}}{L^{3}} \\
& \psi_{2}=x-2 \frac{x^{2}}{L}+\frac{x^{3}}{L^{2}}
\end{aligned}
$$




$$
\begin{aligned}
& \psi_{3}=3 \frac{x^{2}}{L^{2}}-2 \frac{x^{3}}{L^{3}} \\
& \psi_{4}=-\frac{x^{2}}{L}+\frac{x^{3}}{L^{2}}
\end{aligned}
$$

In x-y plane (vertical direction), the shape functions $\left[N_{v}\right]$ can be written as follows;

$$
\left[N_{v}\right]=\left[\begin{array}{llll}
\psi_{1}(x) & \psi_{2}(x) & \psi_{3}(x) & \psi_{4}(x)
\end{array}\right]
$$

In X-z plane (horizontal direction), the shape functions $\left[N_{h}\right]$ can be written as follows;

$$
\left[N_{h}\right]=\left[\begin{array}{llll}
\psi_{1}(x) & -\psi_{2}(x) & \psi_{3}(x) & -\psi_{4}(x)
\end{array}\right]
$$

The two shape functions for the torsional behaviour as well as the axial behaviour of a space frame element are given as follows;

$$
\begin{aligned}
& \psi_{t_{1}}=1-\frac{x}{L} \\
& \psi_{t_{2}}=\frac{x}{L}
\end{aligned}
$$

The shape functions for the torsional behaviour $\left[N_{t}\right]$ and the axial behaviour $\left[N_{a}\right]$ can be written in matrix form as follows;

$$
\left[N_{t}\right]=\left[N_{a}\right]=\left[\begin{array}{ll}
\psi_{t_{1}}(x) & \psi_{t_{2}}(x)
\end{array}\right]
$$


Within each element, the deflected shape of the bridge in the vertical direction is expressed as the superposition of four shape functions multiplied by the corresponding degrees of freedom as follows;

$u(x, t)=\Delta_{1 y}(t) \psi_{1}(x)+\theta_{1 z}(t) \psi_{2}(x)+\Delta_{2 y}(t) \psi_{3}(x)+\theta_{2 z} \psi_{4}(x)$

where $\Delta_{1 y}$ and $\Delta_{2 y}$ are the nodal vertical displacements along y-axis, and $\theta_{1 z}$ and $\theta_{2 z}$ are the nodal rotation about $\mathrm{z}$-axis at the two nodes of the space frame element.

\subsection{Equation of Motion of the Vehicle}

The vertical displacement of the point of contact between the vehicle and the bridge deck $\eta$ is given by;

$\eta=\Delta_{1 y}(t) \psi_{1}(x)+\theta_{1 z}(t) \psi_{2}(x)+\Delta_{2 y}(t) \psi_{3}(x)+\theta_{2 z}(t) \psi_{4}(x)$

In case of constant speed, the spatial coordinate $x$ can be expressed in terms of time as follows;

$x=v t$

The first and second derivatives of $\eta$ with respect to time $\dot{\eta}$ and $\ddot{\eta}$ are given by;

$\dot{\eta}=\Delta_{1 y} \dot{\psi}_{1}+\dot{\Delta}_{1 y} \psi_{1}+\theta_{1 z} \dot{\psi}_{2}+\dot{\theta}_{1 z} \psi_{2}+\Delta_{2 y} \dot{\psi}_{3}+\dot{\Delta}_{2 y} \psi_{3}+\theta_{2 z} \dot{\psi}_{4}+\dot{\theta}_{2 z} \psi_{4}$

$\ddot{\eta}=\Delta_{1 y} \ddot{\psi}_{1}+2 \dot{\Delta}_{1 y} \dot{\psi}_{1}+\ddot{\Delta}_{1 y} \psi_{1}+\theta_{1 z} \ddot{\psi}_{2}+2 \dot{\theta}_{1 z} \dot{\psi}_{2}+\ddot{\theta}_{1 z} \psi_{2}+\Delta_{2 y} \ddot{\psi}_{3}+2 \dot{\Delta}_{2 y} \dot{\psi}_{3}+$

$\ddot{\Delta}_{2 y} \psi_{3}+\theta_{2 z} \ddot{\psi}_{4}+2 \dot{\theta}_{2 z} \dot{\psi}_{4}+\ddot{\theta}_{2 z} \psi_{4}$ 
From the free body diagram of the sprung mass of the vehicle system (Figure 3.8), the equation of motion of the vehicle can be written as;

$$
m_{v} \ddot{u}_{v}+c\left(\dot{u}_{v}-\dot{\eta}\right)+k\left(u_{v}-\eta\right)=0
$$

Substituting the expressions of $\eta$ and $\dot{\eta}$, the equation of motion of the vehicle is given as follows;

$$
\begin{gathered}
m_{v} \ddot{u}_{v}+c\left(\dot{u}_{v}-\Delta_{1 y} \dot{\psi}_{1}-\dot{\Delta}_{1 y} \psi_{1}-\theta_{1 z} \dot{\psi}_{2}-\dot{\theta}_{1 z} \psi_{2}-\Delta_{2 y} \dot{\psi}_{3}-\dot{\Delta}_{2 y} \psi_{3}-\theta_{2 z} \dot{\psi}_{4}\right. \\
\left.-\dot{\theta}_{2 z} \psi_{4}\right)+k\left(u_{v}-\Delta_{1 y} \psi_{1}-\theta_{1 z} \psi_{2}-\Delta_{2 y} \psi_{3}-\theta_{2 z} \psi_{4}\right)=0
\end{gathered}
$$

where

$m_{v}$ is the sprung mass of the vehicle.

$m_{t}$ is the unsprung mass of the vehicle.

$k$ is the stiffness of the vehicle spring.

$c$ is the damping constant of the vehicle model.

$u_{v}$ is the vertical displacement of the sprung mass of the vehicle relative to static displacement under gravity.

$\dot{u}_{v}$ is the vertical velocity of the sprung mass of the vehicle.

$\ddot{u}_{v}$ is the vertical acceleration of the sprung mass of the vehicle. 


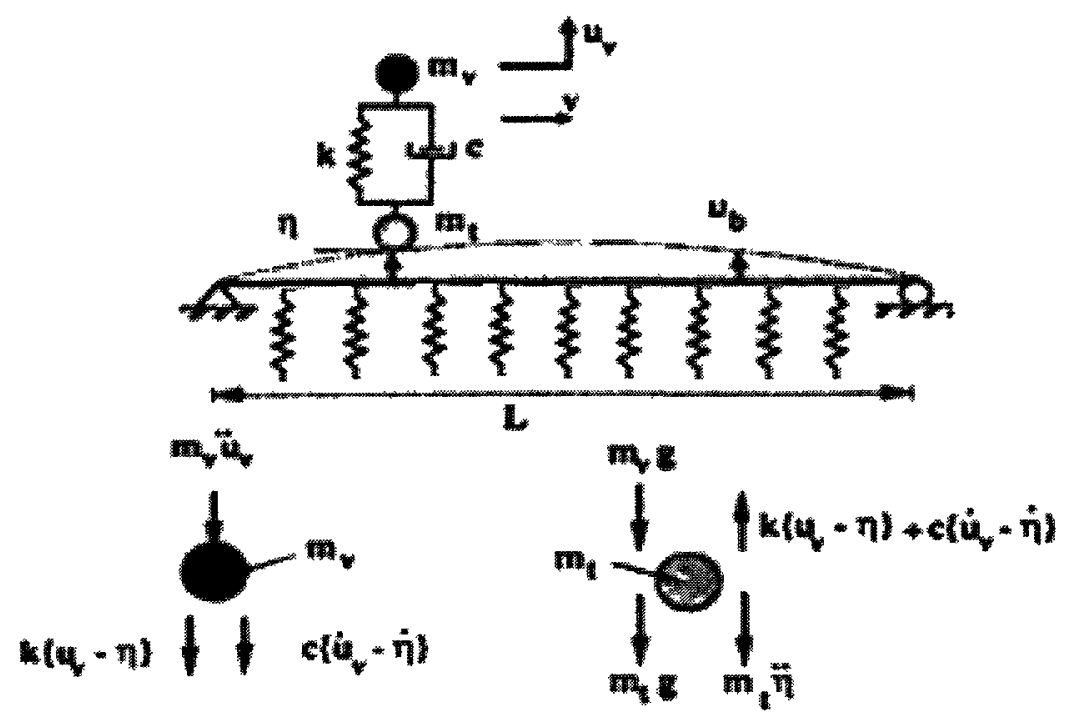

Figure 3.8 Free body diagram of a floating bridge traversed by a moving vehicle (modified from Humar and Kashif 1993)

\subsection{Equation of Motion of the Bridge}

The equation of motion of the floating bridge is given by;

$\left[M_{G}\right]\{\ddot{D}\}+\left[C_{G}\right]\{\dot{D}\}+\left[K_{G}\right]\{D\}=\{P\}$

where

$\{D\}$ is the global displacement vector of the bridge.

$\{\dot{D}\}$ is the first time derivative of the global displacement vector of the bridge.

$\{\ddot{D}\}$ is the second time derivative of the global displacement vector of the bridge.

$\left[M_{G}\right]$ is the global mass matrix of the bridge.

$\left[C_{G}\right]$ is the global damping matrix of the bridge. 
$\left[K_{G}\right]$ is the global stiffness matrix of the bridge.

$\{P\}$ is the global load vector acting on the bridge.

\subsubsection{Local Stiffness Matrix}

The local stiffness matrix of the space frame element is given as follows (Rao 2004);

$[K]=\left[\begin{array}{cccccccccccc}\frac{E A}{L} & 0 & 0 & 0 & 0 & 0 & -\frac{E A}{L} & 0 & 0 & 0 & 0 & 0 \\ 0 & \frac{12 E I_{z}}{L^{3}} & 0 & 0 & 0 & \frac{6 E I_{z}}{L^{2}} & 0 & -\frac{12 E I_{z}}{L^{3}} & 0 & 0 & 0 & \frac{6 E I_{z}}{L^{2}} \\ 0 & 0 & \frac{12 E I_{y}}{L^{3}} & 0 & -\frac{6 E I_{y}}{L^{2}} & 0 & 0 & 0 & -\frac{12 E I_{y}}{L^{3}} & 0 & -\frac{6 E I_{y}}{L^{2}} & 0 \\ 0 & 0 & 0 & \frac{G J}{L} & 0 & 0 & 0 & 0 & 0 & -\frac{G J}{L} & 0 & 0 \\ 0 & 0 & -\frac{6 E I_{y}}{L^{2}} & 0 & \frac{4 E I_{y}}{L} & 0 & 0 & 0 & \frac{6 E I_{y}}{L^{2}} & 0 & \frac{2 E I_{y}}{L} & 0 \\ 0 & \frac{6 E I_{z}}{L^{2}} & 0 & 0 & 0 & \frac{4 E I_{z}}{L} & 0 & -\frac{6 E I_{z}}{L^{2}} & 0 & 0 & 0 & \frac{2 E I_{z}}{L} \\ -\frac{E A}{L} & 0 & 0 & 0 & 0 & 0 & \frac{E A}{L} & 0 & 0 & 0 & 0 & 0 \\ 0 & -\frac{12 E I_{z}}{L^{3}} & 0 & 0 & 0 & -\frac{6 E I_{z}}{L^{2}} & 0 & \frac{12 E I_{z}}{L^{3}} & 0 & 0 & 0 & -\frac{6 E I_{z}}{L^{2}} \\ 0 & 0 & -\frac{12 E I_{y}}{L^{3}} & 0 & \frac{6 E I_{y}}{L^{2}} & 0 & 0 & 0 & \frac{12 E I_{y}}{L^{3}} & 0 & \frac{6 E I_{y}}{L^{2}} & 0 \\ 0 & 0 & 0 & -\frac{G J}{L} & 0 & 0 & 0 & 0 & 0 & \frac{G J}{L} & 0 & 0 \\ 0 & 0 & -\frac{6 E I_{y}}{L^{2}} & 0 & \frac{2 E I_{y}}{L} & 0 & 0 & 0 & \frac{6 E I_{y}}{L^{2}} & 0 & \frac{4 E I_{y}}{L} & 0 \\ 0 & \frac{6 E I_{z}}{L^{2}} & 0 & 0 & 0 & \frac{2 E I_{z}}{L} & 0 & -\frac{6 E I_{z}}{L^{2}} & 0 & 0 & 0 & \frac{4 E I_{z}}{L}\end{array}\right]$

\subsubsection{Formulation of Stiffness Matrix of "Hinged-Joint" Element}

The hinge-connected floating bridge will be represented by a beam with a number of internal hinges at the connections between successive bays. In general, an internal hinge causes a discontinuity in the slope of the deflection curve at the hinge. Also the bending moment is zero at the hinge. This special condition can be treated by starting with the generalized unreleased stiffness matrix of the space frame element and eliminate the 
known zero moment. This results in a modified stiffness matrix with the moment at the hinge equal to zero and the corresponding rotational degree of freedom eliminated. The internal hinge can be represented by considering one of the following three alternatives;

- considering the element before the internal hinge as a space frame element with nodal hinge at its right.

- considering the element after the internal hinge as a space frame element with nodal hinge at its left end.

- combining both the first and the second alternatives; namely, considering the element before the internal hinge as a space frame element with nodal hinge at its right end and the element after the internal hinge as a space frame element with nodal hinge at its left end.

The third alternative results in eliminating the rotational degree of freedom of the hinge from the global equations of the whole structure. This elimination is necessary to avoid having a singular global stiffness matrix for the whole structure.

Considering the first alternative for a space frame element with a nodal hinge at its right end, the moment $M_{2 z}$ is zero. The stiffness matrix is partitioned to eliminate the corresponding rotational degree of freedom $\theta_{2 z}$. The stiffness coefficients $k_{i j}{ }^{\prime}$ of the modified stiffness matrix of this special element are calculated using the stiffness coefficients $k_{i j}$ of the standard stiffness matrix of element with rigid nodes at its two ends using the following equation:

$k_{i j}{ }^{\prime}=k_{i j} \frac{k_{i 6}}{k_{66}} k_{6 j} \quad(i, j=1: 11)$ 
The modified stiffness matrix is expanded to include the rotational degree of freedom $\theta_{2 z}$ which is not zero in general. The local stiffness equation of space frame element with a nodal hinge at its right end is obtained as shown in Equation 3.17. However $\theta_{2 z}$ calculated from the global equation of the whole structure belongs to the first node of the element after the hinge, which is in general different from the rotational degree of freedom of the second node of the element before the hinge.

$$
\left\{\begin{array}{l}
F_{1 x} \\
F_{1 y} \\
F_{1 z} \\
M_{1 x} \\
M_{1 y} \\
M_{1 z} \\
F_{2 x} \\
F_{2 y} \\
F_{2 z} \\
M_{2 x} \\
M_{2 y} \\
M_{2 z}
\end{array}\right\}=\left[\begin{array}{cccccccccccc}
\frac{E A}{L} & 0 & 0 & 0 & 0 & 0 & -\frac{E A}{L} & 0 & 0 & 0 & 0 & 0 \\
0 & \frac{3 E I_{z}}{L^{3}} & 0 & 0 & 0 & \frac{3 E I_{z}}{L^{2}} & 0 & -\frac{3 E I_{z}}{L^{3}} & 0 & 0 & 0 & 0 \\
0 & 0 & \frac{12 E I_{y}}{L^{3}} & 0 & -\frac{6 E I_{y}}{L^{2}} & 0 & 0 & 0 & -\frac{12 E I_{y}}{L^{3}} & 0 & -\frac{6 E I_{y}}{L^{2}} & 0 \\
0 & 0 & 0 & \frac{G J}{L} & 0 & 0 & 0 & 0 & 0 & -\frac{G J}{L} & 0 & 0 \\
0 & 0 & -\frac{6 E I_{y}}{L^{2}} & 0 & \frac{4 E I_{y}}{L} & 0 & 0 & 0 & \frac{6 E I_{y}}{L^{2}} & 0 & \frac{2 E I_{y}}{L} & 0 \\
0 & \frac{3 E I_{z}}{L^{2}} & 0 & 0 & 0 & \frac{3 E I_{z}}{L} & 0 & -\frac{3 E I_{z}}{L^{2}} & 0 & 0 & 0 & 0 \\
-\frac{E A}{L} & 0 & 0 & 0 & 0 & 0 & \frac{E A}{L} & 0 & 0 & 0 & 0 & 0 \\
0 & -\frac{3 E I_{z}}{L^{3}} & 0 & 0 & 0 & -\frac{3 E I_{z}}{L^{2}} & 0 & \frac{3 E I_{z}}{L^{3}} & 0 & 0 & 0 & 0 \\
0 & 0 & -\frac{12 E I_{y}}{L^{3}} & 0 & \frac{6 E I_{y}}{L^{2}} & 0 & 0 & 0 & \frac{12 E I_{y}}{L^{3}} & 0 & \frac{6 E I_{y}}{L^{2}} & 0 \\
0 & 0 & 0 & -\frac{G J}{L} & 0 & 0 & 0 & 0 & 0 & \frac{G J}{L} & 0 & 0 \\
0 & 0 & -\frac{6 E I_{y}}{L^{2}} & 0 & \frac{2 E I_{y}}{L} & 0 & 0 & 0 & \frac{6 E I_{y}}{L^{2}} & 0 & \frac{4 E I_{y}}{L} & 0 \\
0 & 0 & 0 & 0 & 0 & 0 & 0 & 0 & 0 & 0 & 0 & 0
\end{array}\right]\left\{\begin{array}{l}
\Delta_{1 x} \\
\Delta_{1 y} \\
\theta_{1 z} \\
\theta_{1 x} \\
\theta_{1 z} \\
\theta_{2 x} \\
\Delta_{2 y} \\
\Delta_{2 z} \\
\theta_{2 x} \\
\theta_{2 y} \\
\theta_{2 z}
\end{array}\right\}
$$

\subsubsection{Formulation of Stiffness Matrix Due to Surrounding Water}

The buoyancy offered to a floating structure can be represented by elastic springs supporting the floating structure along its length. The buoyancy force acting on a floating structure is equal to the weight of the fluid displaced by the structure. This buoyancy force is a function of the submerged depth of the floating structure and proportional to its displacement into water. For a floating bridge traversed by moving vehicle loads, the submerged depth of the bridge is not constant along the bridge length, but varies from 
unloaded pontoons to loaded ones according to the position of the vehicle along the bridge. This submerged depth also changes with time as the vehicle position changes with time inducing different submerged depth at each node along the bridge, meaning that buoyancy forces should be treated as dynamic loads. In the present study, the buoyancy forces offered to a floating bridge are represented as dynamic loads that changes with both space and time coordinates.

The floating bridge can be idealized as a beam resting on water represented by elastic springs of equal stiffness. The bridge is considered as a beam on elastic foundation with foundation modulus equal to the buoyancy stiffness. The bending stiffness coefficients $k_{i j}$ and the torsional stiffness coefficients $k_{i j_{t}}$ due to surrounding water are given as follows (Humar 2002; Georgiadis 1984);

$k_{i j}=\int_{0}^{L} \bar{k}_{w} \psi_{i}(x) \psi_{j}(x) d x$

$k_{i j_{t}}=\int_{0}^{L} \bar{k}_{w_{t}} \psi_{t_{i}}(x) \psi_{t_{j}}(x) d x$

where $\bar{k}_{w}$ is the buoyancy stiffness per unit length of the bridge for the heave motion and $\bar{k}_{w_{t}}$ is the buoyancy stiffness per unit length of the bridge for the roll motion, and they are calculated as follows;

$\bar{k}_{w}=\rho_{w} b g$

$\bar{k}_{w_{t}}=\rho_{w} g b^{3} / 12$

where $\rho_{w}$ is the density of water, $g$ is the gravitational acceleration and $b$ is the width of the bridge. 
Linear relation between the buoyancy forces and the bridge displacement into water is considered in the present study. The consistent stiffness matrix due to surrounding water is derived as follows;

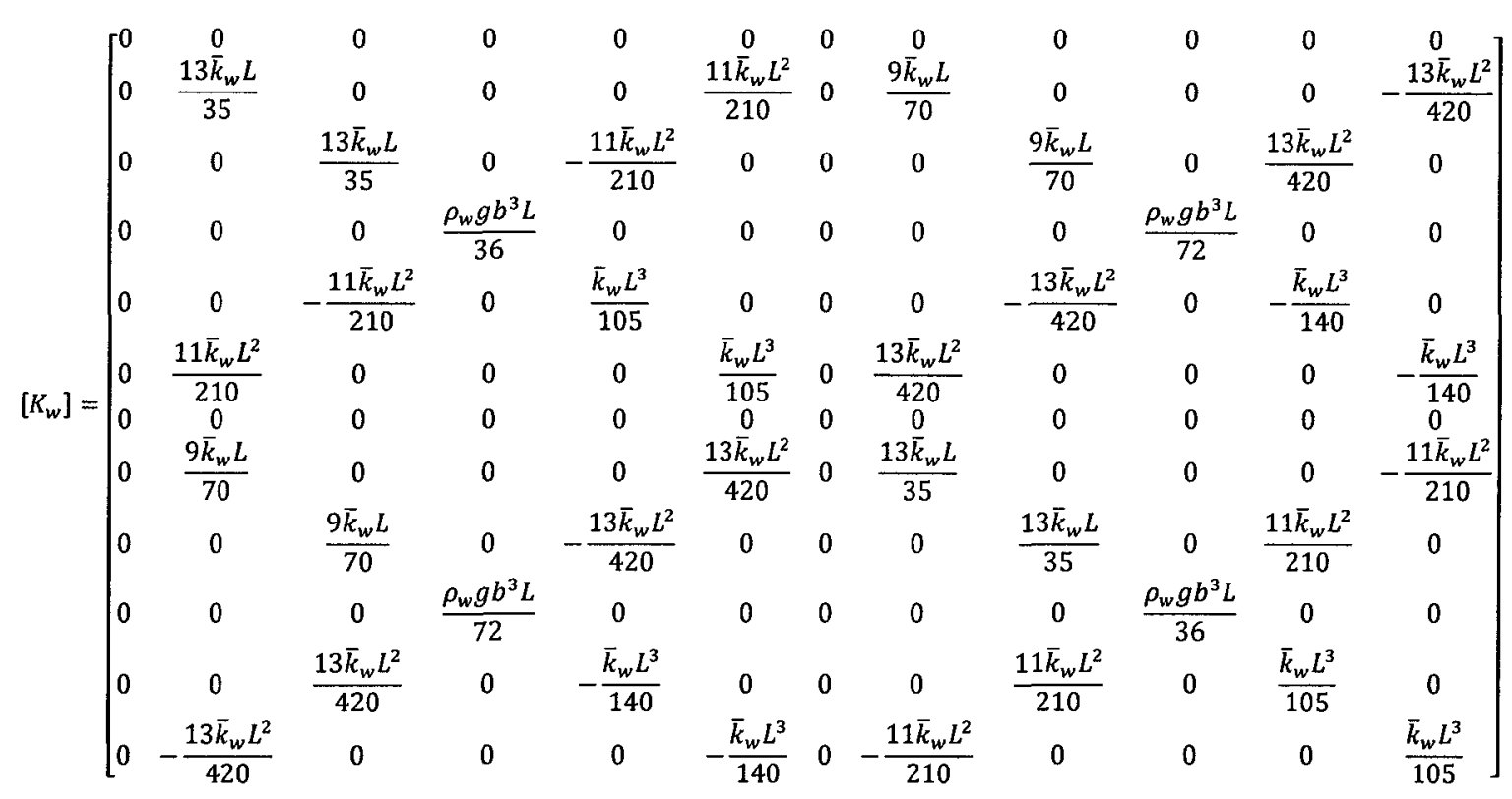

\subsubsection{Formulation of Element Stiffness Matrix}

The element stiffness matrix is obtained as the sum of the element stiffness and stiffness of the water;

$$
\left[K_{i}\right]=[K]+\left[K_{w}\right]
$$

\subsubsection{Formulation of the Global Stiffness Matrix}

The global stiffness matrix of the whole bridge is obtained using the standard finite element assembly technique and this involves combining the local stiffness matrices for 
each element including elements with nodal hinge at one end, if needed, into a global stiffness matrix.

\subsubsection{Local Mass Matrix}

The consistent local mass matrix for a space frame element of length $L$, distributed mass per unit length $\bar{m}$ and polar moment of inertia $J$ is given as follows (Rao 2004);

$$
[M]=\left[\begin{array}{cccccccccccc}
\frac{\bar{m} L}{3} & 0 & 0 & 0 & 0 & 0 & \frac{\bar{m} L}{6} & 0 & 0 & 0 & 0 & 0 \\
0 & \frac{156 \bar{m} L}{420} & 0 & 0 & 0 & \frac{22 \bar{m} L^{2}}{420} & 0 & \frac{54 \bar{m} L}{420} & 0 & 0 & 0 & -\frac{13 \bar{m} L^{2}}{420} \\
0 & 0 & \frac{156 \bar{m} L}{420} & 0 & -\frac{22 \bar{m} L^{2}}{420} & 0 & 0 & 0 & \frac{54 \bar{m} L}{420} & 0 & \frac{13 \bar{m} L^{2}}{420} & 0 \\
0 & 0 & 0 & \frac{\rho J L}{3} & 0 & 0 & 0 & 0 & 0 & \frac{\rho J L}{6} & 0 & 0 \\
0 & 0 & -\frac{22 \bar{m} L^{2}}{420} & 0 & \frac{4 \bar{m} L^{3}}{420} & 0 & 0 & 0 & -\frac{13 \bar{m} L^{2}}{420} & 0 & -\frac{3 \bar{m} L^{3}}{420} & 0 \\
0 & \frac{22 \bar{m} L^{2}}{420} & 0 & 0 & 0 & \frac{4 \bar{m} L^{3}}{420} & 0 & \frac{13 \bar{m} L^{2}}{420} & 0 & 0 & 0 & -\frac{3 \bar{m} L^{3}}{420} \\
\frac{m}{m} L & 0 & 0 & 0 & 0 & 0 & \frac{\bar{m} L}{3} & 0 & 0 & 0 & 0 & 0 \\
0 & \frac{54 \bar{m} L}{420} & 0 & 0 & 0 & \frac{13 \bar{m} L^{2}}{420} & 0 & \frac{156 \bar{m} L}{420} & 0 & 0 & 0 & -\frac{22 \bar{m} L^{2}}{420} \\
0 & 0 & \frac{54 \bar{m} L}{420} & 0 & -\frac{13 \bar{m} L^{2}}{420} & 0 & 0 & 0 & \frac{156 \bar{m} L}{420} & 0 & \frac{22 \bar{m} L^{2}}{420} & 0 \\
0 & 0 & 0 & \frac{\rho J L}{6} & 0 & 0 & 0 & 0 & 0 & \frac{\rho J L}{3} & 0 & 0 \\
0 & 0 & \frac{13 \bar{m} L^{2}}{420} & 0 & -\frac{3 \bar{m} L^{3}}{420} & 0 & 0 & 0 & \frac{22 \bar{m} L^{2}}{420} & 0 & \frac{4 \bar{m} L^{3}}{420} & 0 \\
0 & -\frac{13 \bar{m} L^{2}}{420} & 0 & 0 & 0 & -\frac{3 \bar{m} L^{3}}{420} & 0 & -\frac{22 \bar{m} L^{2}}{420} & 0 & 0 & 0 & \frac{4 \bar{m} L^{3}}{420}
\end{array}\right]
$$

\subsubsection{Formulation of Local Added Mass Matrix}

For unsteady flow around objects or unsteady motion of bodies in water, the additional force resulting from the fluid acting on the structure must be considered when formulating the equations of motion of the system encompassing the structure and the surrounding fluid; this additional force is called the added mass. This added mass is the 
weight added to a system due to the fact that an accelerating or decelerating body must move some volume of surrounding fluid as it moves. The added mass force opposes the objects motion and contributes to the equations of motion of the system.

$\bar{m}_{a}$ is the added mass per unit length in the vertical direction and $\bar{I}_{a}$ is the added mass moment of inertia per unit length in the torsional direction due to surrounding water and can be calculated as follows (Newmans 1977; Chung and Chen 1984; Slater 1984; Brennen 1982);

$\bar{m}_{a}=c_{m} \pi \rho_{w}\left(\frac{b}{2}\right)^{2}$

$\bar{I}_{a}=\frac{8}{3} \rho_{w} \frac{b}{2} \frac{d}{2}\left\{\left(\frac{b}{2}\right)^{2}+\left(\frac{d}{2}\right)^{2}\right\} c_{I}$

where $b$ is the width of the bridge, $d$ is the depth of the bridge cross-section, and $c_{m}$ and $c_{I}$ are the hydrodynamic added mass coefficient and the hydrodynamic added mass moment of inertia coefficient, respectively which can be determined according to the geometry of the bridge cross-section.

The added mass coefficients are given by the following equation;

$$
m_{a_{22}}=m_{a_{88}}=c_{m} \pi \rho_{w}\left(\frac{b}{2}\right)^{2} \frac{L}{2}=\frac{1}{8} c_{m} \pi \rho_{w} b^{2} L
$$

The added mass moment of inertia coefficients are given by the following equation;

$$
m_{I_{44}}=m_{I_{10,10}}=\frac{8}{3} \rho_{w} \frac{b}{2} \frac{d}{2}\left\{\left(\frac{b}{2}\right)^{2}+\left(\frac{d}{2}\right)^{2}\right\} c_{I} \frac{L}{2}=\frac{1}{12} \rho_{w} b d\left(b^{2}+d^{2}\right) c_{I} L
$$


The added mass matrix for the space frame element is given by the following equation;

$\left[M_{a}\right]$
$=\left[\begin{array}{cccccccccccc}0 & 0 & 0 & 0 & 0 & 0 & 0 & 0 & 0 & 0 & 0 & 0 \\ 0 & \frac{1}{8} c_{m} \pi \rho b^{2} L & 0 & 0 & 0 & 0 & 0 & 0 & 0 & 0 & 0 & 0 \\ 0 & 0 & 0 & 0 & 0 & 0 & 0 & 0 & 0 & 0 & 0 & 0 \\ 0 & 0 & 0 & \frac{1}{12} \rho b d\left(b^{2}+d^{2}\right) L & 0 & 0 & 0 & 0 & 0 & 0 & 0 & 0 \\ 0 & 0 & 0 & 0 & 0 & 0 & 0 & 0 & 0 & 0 & 0 & 0 \\ 0 & 0 & 0 & 0 & 0 & 0 & 0 & 0 & 0 & 0 & 0 & 0 \\ 0 & 0 & 0 & 0 & 0 & 0 & 0 & 0 & 0 & 0 & 0 & 0 \\ 0 & 0 & 0 & 0 & 0 & 0 & 0 & \frac{1}{8} c_{m} \pi \rho b^{2} L & 0 & 0 & 0 & 0 \\ 0 & 0 & 0 & 0 & 0 & 0 & 0 & 0 & 0 & 0 & 0 & 0 \\ 0 & 0 & 0 & 0 & 0 & 0 & 0 & 0 & 0 & \frac{1}{12} \rho b d\left(b^{2}+d^{2}\right) L & 0 & 0 \\ 0 & 0 & 0 & 0 & 0 & 0 & 0 & 0 & 0 & 0 & 0 & 0 \\ 0 & 0 & 0 & 0 & 0 & 0 & 0 & 0 & 0 & 0 & 0 & 0\end{array}\right]$

\subsubsection{Formulation of Element Mass Matrix}

The element mass matrix can be obtained by the following equation;

$$
\left[M_{i}\right]=[M]+\left[M_{a}\right]
$$

\subsubsection{Formulation of the Global Mass Matrix}

The global mass matrix of the whole bridge is formulated using the same standard finite element assembly procedure.

\subsubsection{Formulation of the Global Damping Matrix of the Bridge}

The damping forces represent the energy loss due to friction in the system and can be written as follows; 
$\left\{F_{D}\right\}=\left[C_{G}\right]\{\dot{D}\}$

where $\left[C_{G}\right]$ is the global damping matrix of the bridge.

The exact magnitude of the damping coefficient $(\xi)$ can be determined only from experimental tests on the real structure. For a dynamic system with low values of damping, viscous damping can be used in the mathematical modeling where the amplitude of free vibration decays exponentially. In practical structural dynamic problems, linear damping can be simplified as viscous damping, which is easy to deal with mathematically. Rayleigh or Proportional damping is used in the present study to form the damping matrix as a linear combination of the stiffness matrix and the mass matrix of the structure; this damping matrix is given as follows (Humar 2002);

$\left[C_{G}\right]=\alpha\left[K_{G}\right]+\beta\left[M_{G}\right]$

where $\alpha$ and $\beta$ are the stiffness and mass proportional damping constants, respectively. These constants are related to the fraction of critical damping $\xi$ by the following equation (Humar 2002);

$\xi=0.5(\alpha \omega+\beta / \omega)$

The constants $\alpha$ and $\beta$ are determined by choosing the fractions of critical damping $\xi_{1}$ and $\xi_{2}$ at two different frequencies $\omega_{1}$ and $\omega_{2}$, and using the following equations (Humar 2002):

$\alpha=\frac{2\left(\xi_{2} \omega_{2}-\xi_{1} \omega_{1}\right)}{\left(\omega_{2}^{2}-\omega_{1}^{2}\right)}$ 
$\beta=\frac{2 \omega_{1} \omega_{2}\left(\xi_{1} \omega_{2}-\xi_{2} \omega_{1}\right)}{\left(\omega_{2}^{2}-\omega_{1}^{2}\right)}$

In the present study, the Rayleigh damping factors $\alpha$ and $\beta$ are computed using the first and second bending modes of the floating bridge, and the critical damping $\xi$ is assumed to be $5 \%$.

\subsubsection{Formulation of the Local Force Vector}

From the free body diagram (Figure 3.8), the load acting on the bridge is given as follows;

$p=k\left(u_{v}-\eta\right)+c\left(\dot{u}_{v}-\dot{\eta}\right)-m_{t} \ddot{\eta}-m_{t} g-m_{v} g$

Substituting the values of $\eta, \dot{\eta}$, and $\ddot{\eta}$, the expression of the load $p$ can be written as;

$$
\begin{aligned}
p=k\left(u_{v}-\Delta_{1 y}\right. & \left.\psi_{1}-\theta_{1 z} \psi_{2}-\Delta_{2 y} \psi_{3}-\theta_{2 z} \psi_{4}\right) \\
& +c\left(\dot{u}_{v}-\Delta_{1 y} \dot{\psi}_{1}-\dot{\Delta}_{1 y} \psi_{1}-\theta_{1 z} \dot{\psi}_{2}-\dot{\theta}_{1 z} \psi_{2}-\Delta_{2 y} \dot{\psi}_{3}-\dot{\Delta}_{2 y} \psi_{3}-\theta_{2 z} \dot{\psi}_{4}\right. \\
& \left.-\dot{\theta}_{2 z} \psi_{4}\right) \\
& -m_{t}\left(\Delta_{1 y} \ddot{\psi}_{1}+2 \dot{\Delta}_{1 y} \dot{\psi}_{1}+\ddot{\Delta}_{1 y} \psi_{1}+\theta_{1 z} \ddot{\psi}_{2}+2 \dot{\theta}_{1 z} \dot{\psi}_{2}+\ddot{\theta}_{1 z} \psi_{2}+\Delta_{2 y} \ddot{\psi}_{3}\right. \\
& \left.+2 \dot{\Delta}_{2 y} \dot{\psi}_{3}+\ddot{\Delta}_{2 y} \psi_{3}+\theta_{2 z} \ddot{\psi}_{4}+2 \dot{\theta}_{2 z} \dot{\psi}_{4}+\ddot{\theta}_{2 z} \psi_{4}\right)-\left(m_{t}+m_{v}\right) g
\end{aligned}
$$

The torsional moment acting on the bridge due to eccentricity $\left(e_{p}\right)$ of vehicle load off the longitudinal centre line of the bridge, is given as follows; 


$$
\begin{aligned}
t=e_{p}\left\{k \left(u_{v}-\right.\right. & \left.\Delta_{1 y} \psi_{1}-\theta_{1 z} \psi_{2}-\Delta_{2 y} \psi_{3}-\theta_{2 z} \psi_{4}\right) \\
& +c\left(\dot{u}_{v}-\Delta_{1 y} \dot{\psi}_{1}-\dot{\Delta}_{1 y} \psi_{1}-\theta_{1 z} \dot{\psi}_{2}-\dot{\theta}_{1 z} \psi_{2}-\Delta_{2 y} \dot{\psi}_{3}-\dot{\Delta}_{2 y} \psi_{3}-\theta_{2 z} \dot{\psi}_{4}\right. \\
& \left.-\dot{\theta}_{2 z} \psi_{4}\right) \\
& -m_{t}\left(\Delta_{1 y} \ddot{\psi}_{1}+2 \dot{\Delta}_{1 y} \dot{\psi}_{1}+\ddot{\Delta}_{1 y} \psi_{1}+\theta_{1 z} \ddot{\psi}_{2}+2 \dot{\theta}_{1 z} \dot{\psi}_{2}+\ddot{\theta}_{1 z} \psi_{2}+\Delta_{2 y} \ddot{\psi}_{3}\right. \\
& \left.\left.+2 \dot{\Delta}_{2 y} \dot{\psi}_{3}+\ddot{\Delta}_{2 y} \psi_{3}+\theta_{2 z} \ddot{\psi}_{4}+2 \dot{\theta}_{2 z} \dot{\psi}_{4}+\ddot{\theta}_{2 z} \psi_{4}\right)-\left(m_{t}+m_{v}\right) g\right\}
\end{aligned}
$$

In case of concentrated load and torsional moment, the load coefficients $p_{i}$ are given by;

$$
p_{i}=\sum_{k} p_{k} \psi_{i}\left(x_{k}\right)+\sum_{k} t_{k} \psi_{t_{i}}\left(x_{k}\right)
$$

For single-axle load and torsional moment, the load coefficients $p_{i}$ are given by;

$$
\begin{aligned}
& p_{i}=p \psi_{i}(x)+ t \psi_{t_{i}}(x) \\
& p_{i}=\left\{k\left(u_{v}-\Delta_{1 y} \psi_{1}-\theta_{1 z} \psi_{2}-\Delta_{2 y} \psi_{3}-\theta_{2 z} \psi_{4}\right)\right. \\
&+c\left(\dot{u}_{v}-\Delta_{1 y} \dot{\psi}_{1}-\dot{\Delta}_{1 y} \psi_{1}-\theta_{1 z} \dot{\psi}_{2}-\dot{\theta}_{1 z} \psi_{2}-\Delta_{2 y} \dot{\psi}_{3}-\dot{\Delta}_{2 y} \psi_{3}-\theta_{2 z} \dot{\psi}_{4}\right. \\
&\left.-\dot{\theta}_{2 z} \psi_{4}\right) \\
&-m_{t}\left(\Delta_{1 y} \ddot{\psi}_{1}+2 \dot{\Delta}_{1 y} \dot{\psi}_{1}+\ddot{\Delta}_{1 y} \psi_{1}+\theta_{1 z} \ddot{\psi}_{2}+2 \dot{\theta}_{1 z} \dot{\psi}_{2}+\ddot{\theta}_{1 z} \psi_{2}+\Delta_{2 y} \ddot{\psi}_{3}\right. \\
&\left.\left.+2 \dot{\Delta}_{2 y} \dot{\psi}_{3}+\ddot{\Delta}_{2 y} \psi_{3}+\theta_{2 z} \ddot{\psi}_{4}+2 \dot{\theta}_{2 z} \dot{\psi}_{4}+\ddot{\theta}_{2 z} \psi_{4}\right)-\left(m_{t}+m_{v}\right) g\right\} \psi_{i}(x) \\
&+e_{p}\left\{k\left(u_{v}-\Delta_{1 y} \psi_{1}-\theta_{1 z} \psi_{2}-\Delta_{2 y} \psi_{3}-\theta_{2 z} \psi_{4}\right)\right. \\
&+c\left(\dot{u}_{v}-\Delta_{1 y} \dot{\psi}_{1}-\dot{\Delta}_{1 y} \psi_{1}-\theta_{1 z} \dot{\psi}_{2}-\dot{\theta}_{1 z} \psi_{2}-\Delta_{2 y} \dot{\psi}_{3}-\dot{\Delta}_{2 y} \psi_{3}-\theta_{2 z} \dot{\psi}_{4}\right. \\
&\left.-\dot{\theta}_{2 z} \psi_{4}\right) \\
&-m_{t}\left(\Delta_{1 y} \ddot{\psi}_{1}+2 \dot{\Delta}_{1 y} \dot{\psi}_{1}+\ddot{\Delta}_{1 y} \psi_{1}+\theta_{1 z} \ddot{\psi}_{2}+2 \dot{\theta}_{1 z} \dot{\psi}_{2}+\ddot{\theta}_{1 z} \psi_{2}+\Delta_{2 y} \ddot{\psi}_{3}\right. \\
&\left.\left.+2 \dot{\Delta}_{2 y} \dot{\psi}_{3}+\ddot{\Delta}_{2 y} \psi_{3}+\theta_{2 z} \ddot{\psi}_{4}+2 \dot{\theta}_{2 z} \dot{\psi}_{4}+\ddot{\theta}_{2 z} \psi_{4}\right)-\left(m_{t}+m_{v}\right) g\right\} \psi_{t_{i}}(x)
\end{aligned}
$$


Substituting for $i=1$ to 12 , the load coefficients $p_{1}$ to $p_{12}$ are determined and the load vector $\{p\}$ is derived as follows;

$$
\begin{aligned}
\{p\}=\left\{k\left(u_{v}-\Delta_{1 y} \psi_{1}-\theta_{1 z} \psi_{2}-\Delta_{2 y} \psi_{3}-\theta_{2 z} \psi_{4}\right)\right. & \\
& +c\left(\dot{u}_{v}-\Delta_{1 y} \dot{\psi}_{1}-\dot{\Delta}_{1 y} \psi_{1}-\theta_{1 z} \dot{\psi}_{2}-\dot{\theta}_{1 z} \psi_{2}-\Delta_{2 y} \dot{\psi}_{3}-\dot{\Delta}_{2 y} \psi_{3}-\theta_{2 z} \dot{\psi}_{4}\right. \\
& \left.-\dot{\theta}_{2 z} \psi_{4}\right) \\
& -m_{t}\left(\Delta_{1 y} \ddot{\psi}_{1}+2 \dot{\Delta}_{1 y} \dot{\psi}_{1}+\ddot{\Delta}_{1 y} \psi_{1}+\theta_{1 z} \ddot{\psi}_{2}+2 \dot{\theta}_{1 z} \dot{\psi}_{2}+\ddot{\theta}_{1 z} \psi_{2}+\Delta_{2 y} \ddot{\psi}_{3}\right.
\end{aligned}
$$


The equation of motion of the bridge can be written in the following matrix form:

$\left[M+m^{*}\right]\{\ddot{D}\}+\left[C+c^{*}\right]\{\dot{D}\}+\left[K+k^{*}\right]\{D\}=\left\{p^{*}\right\}$

\subsubsection{Equation of Motion of the Bridge-Vehicle System}

Combining the two equations of motion of the vehicle and the bridge (Equations 3.13 and

3.41), the equation of motion of the bridge-vehicle system can be expressed as;

$$
\left[\begin{array}{cc}
m_{v} & 0 \\
0 & M+m^{*}
\end{array}\right]\left\{\begin{array}{c}
\ddot{u}_{v} \\
\ddot{D}
\end{array}\right\}+\left[\begin{array}{cc}
c & c_{h} \\
c_{v} & C+c^{*}
\end{array}\right]\left\{\begin{array}{c}
\dot{u}_{v} \\
\dot{D}
\end{array}\right\}+\left[\begin{array}{cc}
k & k_{h} \\
k_{v} & K+k^{*}
\end{array}\right]\left\{\begin{array}{c}
u_{v} \\
D
\end{array}\right\}=\left\{\begin{array}{c}
0 \\
p^{*}
\end{array}\right\}
$$

where

$$
\begin{aligned}
& m^{*}=m_{t} N^{T} N+e_{p} m_{t} N_{t}^{T} N \\
& c^{*}=2 m_{t} v N^{T} N_{, x}+c N^{T} N+2 e_{p} m_{t} v N_{t}{ }^{T} N_{, x}+e_{p} c N_{t}^{T} N \\
& k^{*}=m_{t} v^{2} N^{T} N_{, x x}+c v N^{T} N_{, x}+k N^{T} N+e_{p} m_{t} v^{2} N_{t}^{T} N_{, x x}+e_{p} c v N_{t}{ }^{T} N_{, x}+e_{p} k N_{t}{ }^{T} N \\
& c_{h}=-c N \\
& c_{v}=-c N^{T}-e_{p} c N_{t}{ }^{T} \\
& k_{h}=-k N-c v N_{, x} \\
& k_{v}=-k N^{T}-e_{p} k N_{t}^{T} \\
& p^{*}=-\left(m_{v}+m_{t}\right) g N^{T}-e_{p}\left(m_{v}+m_{t}\right) g N_{t}^{T}
\end{aligned}
$$




$$
\begin{aligned}
& N=\left[\begin{array}{llllllllllll}
0 & \psi_{1}(x) & 0 & 0 & 0 & \psi_{2}(x) & 0 & \psi_{3}(x) & 0 & 0 & 0 & \psi_{4}(x)
\end{array}\right] \\
& N_{, x}=\left[\begin{array}{llllllllllll}
0 & \frac{d}{d x} \psi_{1}(x) & 0 & 0 & 0 & \frac{d}{d x} \psi_{2}(x) & 0 & \frac{d}{d x} \psi_{3}(x) & 0 & 0 & 0 & \frac{d}{d x} \psi_{4}(x)
\end{array}\right]
\end{aligned}
$$

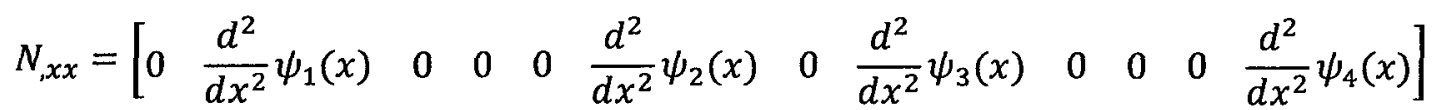

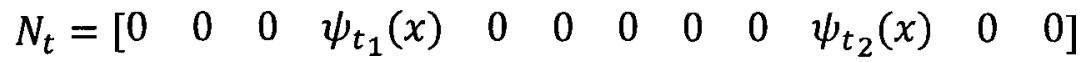

$$
\begin{aligned}
& \dot{\psi}(x)=\frac{d}{d t} \psi(x)=\frac{d}{d x} \psi(x) \frac{d x}{d t}=v \frac{d}{d x} \psi(x) \\
& \ddot{\psi}(x)=\frac{d^{2}}{d t^{2}} \psi(x)=\frac{d}{d t} \dot{\psi}(x)=\frac{d^{2}}{d x^{2}} \psi(x)\left(\frac{d x}{d t}\right)^{2}=v^{2} \frac{d^{2}}{d x^{2}} \psi(x)
\end{aligned}
$$

\subsection{Dynamic Response of Floating Bridges to Two-Axle Vehicle Loads}

The idealization of the vehicle by a single sprung mass model can provide a reasonably accurate estimate of the bridge response when the axle spacing for the vehicle is relatively small compared to the bridge span. In reality, the weight of the vehicle is distributed on two or more axles. It is important to consider the multiple-axle idealization of the vehicle for large ratios of axle spacing to the bridge span. The response produced by a two-axle vehicle model is smaller than that produced by an equivalent single-axle vehicle model of the same mass (Humar and Kashif 1993).

In next sections, the dynamic response of floating bridges traversed by a two-axle vehicle is investigated. The vehicle is represented by a two-axle sprung mass model as shown in Figure 3.9. The vehicle has two degrees of freedom: $q_{1}$ representing the bounce of the 
vehicle and $q_{2}$ representing the pitching motion of the vehicle about lateral axis perpendicular to the longitudinal axis of the bridge, while the roll motion of the vehicle about an axis parallel to the longitudinal axis of the bridge is ignored.

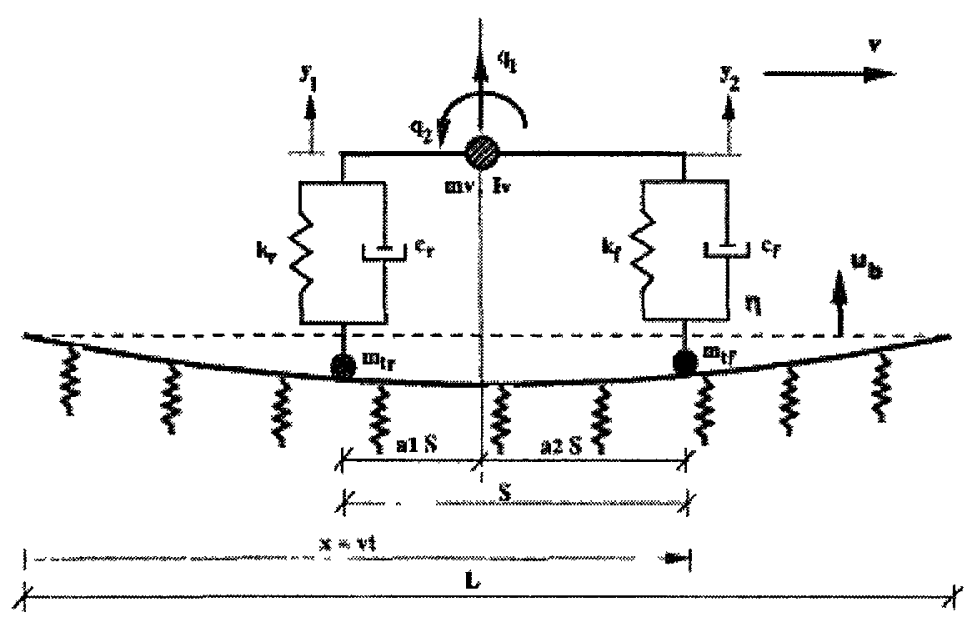

Figure 3.9 Free body diagram of a floating bridge traversed by a moving two-axle vehicle (modified from Humar and Kashif 1993)

\subsubsection{Equation of Motion of Two-Axle Vehicle}

The equation of motion of the two-axle vehicle is given by Equation 3.44 .

where

$k_{r}$ is the stiffness of the rear axle spring.

$k_{f}$ is the stiffness of the front axle spring.

$c_{r}$ is the damping constant of the rear axle.

$c_{f}$ is the damping constant of the front axle.

$m_{v}$ is the sprung mass of the vehicle.

$m_{t r}$ is the unsprung mass of the rear axle. 
$m_{t f}$ is the unsprung mass of the front axle.

$a_{1} S$ is the distance of the rear axle from the mass centre of vehicle body

$a_{2} S$ is the distance of the front axle from the mass centre of vehicle body.

$I_{v}$ is the mass moment of inertia of the vehicle body.

$$
\begin{aligned}
& d_{1}=q_{1} \\
& d_{2}=S q_{2}
\end{aligned}
$$

$$
\begin{aligned}
{\left[\begin{array}{cc}
m_{v} & 0 \\
0 & \frac{I_{v}}{S^{2}}
\end{array}\right]\left\{\begin{array}{l}
\ddot{d}_{1} \\
\ddot{d}_{2}
\end{array}\right\} } & +\left[\begin{array}{cc}
c_{r}+c_{f} & -a_{1} c_{r}+a_{2} c_{f} \\
-a_{1} c_{r}+a_{2} c_{f} & a_{1}^{2} c_{r}+a_{2}^{2} c_{f}
\end{array}\right]\left\{\begin{array}{l}
\dot{d}_{1} \\
\dot{d}_{2}
\end{array}\right\} \\
& +\left[\begin{array}{cc}
k_{r}+k_{f} & -a_{1} k_{r}+a_{2} k_{f} \\
-a_{1} k_{r}+a_{2} k_{f} & a_{1}^{2} k_{r}+a_{2}^{2} k_{f}
\end{array}\right]\left\{\begin{array}{l}
d_{1} \\
d_{2}
\end{array}\right\} \\
& +\left\{\begin{array}{c}
-c_{r} \\
a_{1} c_{r}
\end{array}\right\}\left\{\Delta_{1 y} \dot{\psi}_{1}(x-S)+\dot{\Delta}_{1 y} \psi_{1}(x-S)+\theta_{1 z} \dot{\psi}_{2}(x-S)+\dot{\theta}_{1 z} \psi_{2}(x\right. \\
& \left.-S)+\Delta_{2 y} \dot{\psi}_{3}(x-S)+\dot{\Delta}_{2 y} \psi_{3}(x-S)+\theta_{2 z} \dot{\psi}_{4}(x-S)+\dot{\theta}_{2 z} \psi_{4}(x-S)\right\} \\
& +\left\{\begin{array}{c}
-c_{f} \\
\left.-a_{2} c_{f}\right\}
\end{array}\right\}\left\{\Delta_{1 y} \dot{\psi}_{1}(x)+\dot{\Delta}_{1 y} \psi_{1}(x)+\theta_{1 z} \dot{\psi}_{2}(x)+\dot{\theta}_{1 z} \psi_{2}(x)+\Delta_{2 y} \dot{\psi}_{3}(x)\right. \\
& \left.+\dot{\Delta}_{2 y} \psi_{3}(x)+\theta_{2 z} \dot{\psi}_{4}+\dot{\theta}_{2 z} \psi_{4}(x)\right\} \\
& +\left\{\begin{array}{c}
-k_{r} \\
a_{1} k_{r}
\end{array}\right\}\left\{\Delta_{1 y} \psi_{1}(x-S)+\theta_{1 z} \psi_{2}(x-S)+\Delta_{2 y} \psi_{3}(x-S)+\theta_{2 z} \psi_{4}(x\right. \\
& -S)\}+\left\{\begin{array}{c}
-k_{f} \\
-a_{2} k_{f}
\end{array}\right\}\left\{\Delta_{1 y} \psi_{1}(x)+\theta_{1 z} \psi_{2}(x)+\Delta_{2 y} \psi_{3}(x)+\theta_{2 z} \psi_{4}(x)\right\}=0
\end{aligned}
$$




\subsubsection{Formulation of the Local Force Vector}

The equation of motion of the bridge is similar to that for the case of single-axle vehicle and takes the same form shown in Equation 3.41 except for the load imposed from the vehicle on the bridge.

The expression of the load vector $\{p\}$ due to two-axle vehicle can be derived as follows;

$$
\begin{aligned}
& \{p\}=\left\{\left[\begin{array}{ll}
k_{r} & -a_{1} k_{r}
\end{array}\right]\left\{\begin{array}{l}
d_{1} \\
d_{2}
\end{array}\right\}+\left[\begin{array}{ll}
c_{r} & -a_{1} c_{r}
\end{array}\right]\left\{\begin{array}{l}
\dot{d}_{1} \\
\dot{d}_{2}
\end{array}\right\}\right. \\
& -k_{r}\left\{\Delta_{1 y} \psi_{1}(x-S)+\theta_{1 z} \psi_{2}(x-S)+\Delta_{2 y} \psi_{3}(x-S)+\theta_{2 z} \psi_{4}(x-S)\right\} \\
& -c_{r}\left\{\Delta_{1 y} \dot{\psi}_{1}(x-S)+\dot{\Delta}_{1 y} \psi_{1}(x-S)+\theta_{1 z} \dot{\psi}_{2}(x-S)+\dot{\theta}_{1 z} \psi_{2}(x-S)\right. \\
& \left.+\Delta_{2 y} \dot{\psi}_{3}(x-S)+\dot{\Delta}_{2 y} \psi_{3}(x-S)+\theta_{2 z} \dot{\psi}_{4}(x-S)+\dot{\theta}_{2 z} \psi_{4}(x-S)\right\} \\
& -m_{t r}\left\{\Delta_{1 y} \ddot{\psi}_{1}(x-S)+2 \dot{\Delta}_{1 y} \dot{\psi}_{1}(x-S)+\ddot{\Delta}_{1 y} \psi_{1}(x-S)+\theta_{1 z} \ddot{\psi}_{2}(x-S)\right. \\
& +2 \dot{\theta}_{1 z} \dot{\psi}_{2}(x-S)+\ddot{\theta}_{1 z} \psi_{2}(x-S)+\Delta_{2 y} \ddot{\psi}_{3}(x-S)+2 \dot{\Delta}_{2 y} \dot{\psi}_{3}(x-S) \\
& \left.+\ddot{\Delta}_{2 y} \psi_{3}(x-S)+\theta_{2 z} \ddot{\psi}_{4}(x-S)+2 \dot{\theta}_{2 z} \dot{\psi}_{4}(x-S)+\ddot{\theta}_{2 z} \psi_{4}(x-S)\right\} \\
& \left.-\left(a_{2} m_{v}+m_{t r}\right) g\right\}\left\{\begin{array}{c}
0 \\
\psi_{1}(x-S) \\
0 \\
0 \\
0 \\
\psi_{2}(x-S) \\
0 \\
\psi_{3}(x-S) \\
0 \\
0 \\
0 \\
\psi_{4}(x-S)
\end{array}\right\}
\end{aligned}
$$




$$
\begin{aligned}
+\left\{\begin{array}{ll}
k_{f} & a_{2} k_{f}
\end{array}\right]\left\{\begin{array}{l}
d_{1} \\
d_{2}
\end{array}\right\}+\left[\begin{array}{ll}
c_{f} & a_{2} c_{f}
\end{array}\right]\left\{\begin{array}{l}
\dot{d}_{1} \\
\dot{d}_{2}
\end{array}\right\} \\
+k_{f}\left\{\Delta_{1 y} \psi_{1}(x)+\theta_{1 z} \psi_{2}(x)+\Delta_{2 y} \psi_{3}(x)+\theta_{2 z} \psi_{4}(x)\right\} \\
+c_{f}\left\{\Delta_{1 y} \dot{\psi}_{1}(x)+\dot{\Delta}_{1 y} \psi_{1}(x)+\theta_{1 z} \dot{\psi}_{2}(x)+\dot{\theta}_{1 z} \psi_{2}(x)+\Delta_{2 y} \dot{\psi}_{3}(x)\right. \\
\left.+\dot{\Delta}_{2 y} \psi_{3}(x)+\theta_{2 z} \dot{\psi}_{4}(x)+\dot{\theta}_{2 z} \psi_{4}(x)\right\} \\
+m_{t f}\left\{\Delta_{1 y} \ddot{\psi}_{1}(x)+2 \dot{\Delta}_{1 y} \dot{\psi}_{1}(x)+\ddot{\Delta}_{1 y} \psi_{1}(x)+\theta_{1 z} \ddot{\psi}_{2}(x)+2 \dot{\theta}_{1 z} \dot{\psi}_{2}(x)\right. \\
+\ddot{\theta}_{1 z} \psi_{2}(x)+\Delta_{2 y} \ddot{\psi}_{3}(x)+2 \dot{\Delta}_{2 y} \dot{\psi}_{3}(x)+\ddot{\Delta}_{2 y} \psi_{3}(x)+\theta_{2 z} \ddot{\psi}_{4}(x) \\
\left.\left.+2 \dot{\theta}_{2 z} \dot{\psi}_{4}(x)+\ddot{\theta}_{2 z} \psi_{4}(x)\right\}-\left(a_{1} m_{v}+m_{t f}\right) g\right\}\left\{\begin{array}{c}
0 \\
\psi_{1}(x) \\
0 \\
0 \\
0 \\
\psi_{2}(x) \\
0 \\
\psi_{3}(x) \\
0 \\
0 \\
0 \\
\psi_{4}(x)
\end{array}\right\}
\end{aligned}
$$




$$
\begin{aligned}
& +e_{p}\left\{\left[\begin{array}{ll}
k_{r} & -a_{1} k_{r}
\end{array}\right]\left\{\begin{array}{l}
d_{1} \\
d_{2}
\end{array}\right\}+\left[\begin{array}{ll}
c_{r} & -a_{1} c_{r}
\end{array}\right]\left\{\begin{array}{l}
\dot{d}_{1} \\
\dot{d}_{2}
\end{array}\right\}\right. \\
& -k_{r}\left\{\Delta_{1 y} \psi_{1}(x-S)+\theta_{1 z} \psi_{2}(x-S)+\Delta_{2 y} \psi_{3}(x-S)+\theta_{2 z} \psi_{4}(x-S)\right\} \\
& -c_{r}\left\{\Delta_{1 y} \dot{\psi}_{1}(x-S)+\dot{\Delta}_{1 y} \psi_{1}(x-S)+\theta_{1 z} \dot{\psi}_{2}(x-S)+\dot{\theta}_{1 z} \psi_{2}(x-S)\right. \\
& \left.+\Delta_{2 y} \dot{\psi}_{3}(x-S)+\dot{\Delta}_{2 y} \psi_{3}(x-S)+\theta_{2 z} \dot{\psi}_{4}(x-S)+\dot{\theta}_{2 z} \psi_{4}(x-S)\right\} \\
& -m_{t r}\left\{\Delta_{1 y} \ddot{\psi}_{1}(x-S)+2 \dot{\Delta}_{1 y} \dot{\psi}_{1}(x-S)+\ddot{\Delta}_{1 y} \psi_{1}(x-S)+\theta_{1 z} \ddot{\psi}_{2}(x-S)\right. \\
& +2 \dot{\theta}_{1 z} \dot{\psi}_{2}(x-S)+\ddot{\theta}_{1 z} \psi_{2}(x-S)+\Delta_{2 y} \ddot{\psi}_{3}(x-S)+2 \dot{\Delta}_{2 y} \dot{\psi}_{3}(x-S) \\
& \left.+\ddot{\Delta}_{2 y} \psi_{3}(x-S)+\theta_{2 z} \ddot{\psi}_{4}(x-S)+2 \dot{\theta}_{2 z} \dot{\psi}_{4}(x-S)+\ddot{\theta}_{2 z} \psi_{4}(x-S)\right\} \\
& \left.-\left(a_{2} m_{v}+m_{t r}\right) g\right\}\left\{\begin{array}{c}
0 \\
0 \\
0 \\
\psi_{t_{1}}(x-S) \\
0 \\
0 \\
0 \\
0 \\
0 \\
\psi_{t_{2}}(x-S) \\
0 \\
0
\end{array}\right\}
\end{aligned}
$$




$$
\begin{aligned}
& +e_{p}\left\{\left[\begin{array}{ll}
k_{f} & a_{2} k_{f}
\end{array}\right]\left\{\begin{array}{l}
d_{1} \\
d_{2}
\end{array}\right\}+\left[\begin{array}{ll}
c_{f} & a_{2} c_{f}
\end{array}\right]\left\{\begin{array}{l}
\dot{d}_{1} \\
\dot{d}_{2}
\end{array}\right\}\right. \\
& -k_{f}\left\{\Delta_{1 y} \psi_{1}(x)+\theta_{1 z} \psi_{2}(x)+\Delta_{2 y} \psi_{3}(x)+\theta_{2 z} \psi_{4}(x)\right\} \\
& -c_{f}\left\{\Delta_{1 y} \dot{\psi}_{1}(x)+\dot{\Delta}_{1 y} \psi_{1}(x)+\theta_{1 z} \dot{\psi}_{2}(x)+\dot{\theta}_{1 z} \psi_{2}(x)+\Delta_{2 y} \dot{\psi}_{3}(x)\right. \\
& \left.+\dot{\Delta}_{2 y} \psi_{3}(x)+\theta_{2 z} \dot{\psi}_{4}(x)+\dot{\theta}_{2 z} \psi_{4}(x)\right\} \\
& -m_{t f}\left\{\Delta_{1 y} \ddot{\psi}_{1}(x)+2 \dot{\Delta}_{1 y} \dot{\psi}_{1}(x)+\ddot{\Delta}_{1 y} \psi_{1}(x)+\theta_{1 z} \ddot{\psi}_{2}(x)+2 \dot{\theta}_{1 z} \dot{\psi}_{2}(x)\right. \\
& +\ddot{\theta}_{1 z} \psi_{2}(x)+\Delta_{2 y} \ddot{\psi}_{3}(x)+2 \dot{\Delta}_{2 y} \dot{\psi}_{3}(x)+\ddot{\Delta}_{2 y} \psi_{3}(x)+\theta_{2 z} \ddot{\psi}_{4}(x) \\
& \left.\left.+2 \dot{\theta}_{2 z} \dot{\psi}_{4}(x)+\ddot{\theta}_{2 z} \psi_{4}(x)\right\}-\left(a_{1} m_{v}+m_{t f}\right) g\right\}\left\{\begin{array}{c}
0 \\
0 \\
0 \\
\psi_{t_{1}}(x) \\
0 \\
0 \\
0 \\
0 \\
0 \\
\psi_{t_{2}}(x) \\
0 \\
0
\end{array}\right\}
\end{aligned}
$$

\subsubsection{Equation of Motion of the Bridge-Vehicle System for Two-Axle Vehicle}

Combining the two equations of motion of the vehicle and the bridge (Equations 3.44 and 3.41), the equations of motion of the bridge-vehicle system can be expressed as follows; 


$$
\begin{array}{r}
{\left[\begin{array}{ccc}
m_{v} & 0 & 0 \\
0 & \frac{I_{v}}{S^{2}} & 0 \\
0 & 0 & M+m^{*}
\end{array}\right]\left\{\begin{array}{l}
\ddot{d}_{1} \\
\ddot{d}_{2} \\
\ddot{D}
\end{array}\right\}+\left[\begin{array}{ccc}
c_{r}+c_{f} & -a_{1} c_{r}+a_{2} c_{f} & c_{h 1} \\
-a_{1} c_{r}+a_{2} c_{f} & a_{1}^{2} c_{r}+a_{2}^{2} c_{f} & c_{h 2} \\
c_{v 1} & c_{v 2} & C+c^{*}
\end{array}\right]\left\{\begin{array}{c}
\dot{d}_{1} \\
\dot{d}_{2} \\
\dot{D}
\end{array}\right\}} \\
+\left[\begin{array}{ccc}
k_{r}+k_{f} & -a_{1} k_{r}+a_{2} k_{f} & k_{h 1} \\
-a_{1} k_{r}+a_{2} k_{f} & a_{1}^{2} k_{r}+a_{2}^{2} k_{f} & k_{h 2} \\
k_{v 1} & k_{v 2} & K+k^{*}
\end{array}\right]\left\{\begin{array}{c}
d_{1} \\
d_{2} \\
D
\end{array}\right\}=\left\{\begin{array}{c}
0 \\
0 \\
p^{*}
\end{array}\right\}
\end{array}
$$

where

$$
\begin{aligned}
& m^{*}=m_{t r} N_{r}{ }^{T} N_{r}+m_{t f} N^{T} N+e_{p} m_{t r} N_{t_{r}}{ }^{T} N_{r}+e_{p} m_{t f} N_{t}^{T} N \\
& c^{*}=2 m_{t r} v N_{r}{ }^{T} N_{r, x}+2 m_{t f} v N^{T} N_{, x}+c_{r} N_{r}{ }^{T} N_{r}+c_{f} N^{T} N+2 e_{p} m_{t r} v N_{t_{r}}{ }^{T} N_{r, x} \\
& +2 e_{p} m_{t f} v N_{t}{ }^{T} N_{, x}+e_{p} c_{r} N_{t_{r}}{ }^{T} N_{r}+e_{p} c_{f} N_{t}{ }^{T} N \\
& k^{*}=m_{t r} v^{2} N_{r}{ }^{T} N_{r, x x}+m_{t f} v^{2} N^{T} N_{, x x}+c_{r} v N_{r}{ }^{T} N_{r, x}+c_{f} v N^{T} N_{, x}+k_{r} N_{r}{ }^{T} N_{r}+k_{f} N^{T} N \\
& +e_{p} m_{t r} v^{2} N_{t_{r}}{ }^{T} N_{r_{, x x}}+e_{p} m_{t f} v^{2} N_{t}^{T} N_{, x x}+e_{p} c_{r} v N_{t_{r}}{ }^{T} N_{r, x} \\
& +e_{p} c_{f} v N_{t}{ }^{T} N_{, x}+e_{p} k_{r} N_{t_{r}}{ }^{T} N_{r}+e_{p} k_{f} N_{t}{ }^{T} N \\
& c_{h 1}=-c_{r} N_{r}-c_{f} N \\
& c_{h 2}=a_{1} c_{r} N_{r}-a_{2} c_{f} N \\
& c_{v 1}=-c_{r} N_{r}^{T}-c_{f} N^{T}-e_{p} c_{r} N_{t_{r}}{ }^{T}-e_{p} c_{f} N_{t}^{T} \\
& c_{v 2}=a_{1} c_{r} N_{r}^{T}-a_{2} c_{f} N^{T}+e_{p} a_{1} c_{r} N_{t_{r}}^{T}-e_{p} a_{2} c_{f} N_{t}^{T} \\
& k_{h 1}=-c_{r} v N_{r, x}-c_{f} v N_{, x}-k_{r} N_{r}-k_{f} N
\end{aligned}
$$




$$
\begin{aligned}
& k_{h 2}=a_{1} c_{r} v N_{r, x}-a_{2} c_{f} v N_{, x}+a_{1} k_{r} N_{r}-a_{2} k_{f} N \\
& k_{v 1}=-k_{r} N_{r}^{T}-k_{f} N^{T}-e_{p} k_{r} N_{t_{r}}^{T}-e_{p} k_{f} N_{t}^{T} \\
& k_{v 2}=a_{1} k_{r} N_{r}^{T}-a_{2} k_{f} N^{T}+e_{p} a_{1} k_{r} N_{t_{r}}{ }^{T}-e_{p} a_{2} k_{f} N_{t}^{T} \\
& p^{*}=-\left(a_{2} m_{v}+m_{t r}\right) g N_{r}{ }^{T}-\left(a_{1} m_{v}+m_{t f}\right) N^{T}-\left(a_{2} m_{v}+m_{t r}\right) g N_{t r}{ }^{T} \\
& -\left(a_{1} m_{v}+m_{t f}\right) N_{t}^{T} \\
& N_{r}=\left[\begin{array}{llllllllllll}
0 & \psi_{1}(x-S) & 0 & 0 & 0 & \psi_{2}(x-S) & 0 & \psi_{3}(x-S) & 0 & 0 & 0 & \psi_{4}(x-S)
\end{array}\right] \\
& N_{r, x} \\
& =\left[\begin{array}{llllllllllll}
0 & \frac{d}{d x} \psi_{1}(x-S) & 0 & 0 & 0 & \frac{d}{d x} \psi_{2}(x-S) & 0 & \frac{d}{d x} \psi_{3}(x-S) & 0 & 0 & 0 & \frac{d}{d x} \psi_{4}(x-S)
\end{array}\right] \\
& N_{r, x x}
\end{aligned}
$$

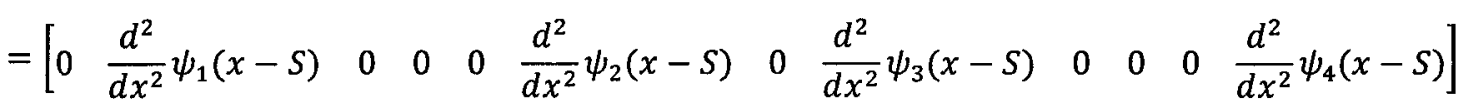

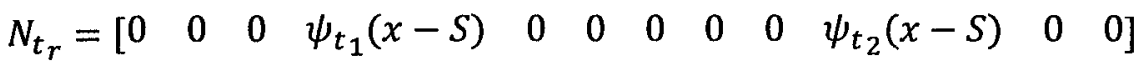

\subsection{Dynamic Response of Floating Bridges to Successive Two-Axle Vehicle Loads}

For large bridges, it is possible for the bridge to be traversed by more than one vehicle traveling in sequence. The separation distance between successive vehicles $\left(S_{v}\right)$ is a very important factor that can significantly affect the dynamic response of the bridge to moving vehicle loads. At the same time, this separation distance is a major factor 
affecting the transport capacity and the rate of flow of vehicles on the bridge. In the next sections, the equations of motion of the bridge-vehicle system are formulated where the floating bridge is traversed by two successive two-axle vehicles.

\subsubsection{Equation of Motion of the Bridge-Vehicle System for Two Successive Two-} Axle Vehicles

The equations of motions of each of the two successive vehicles and the bridge are derived. On combining the three equations of motion, those for the two vehicles and one for the bridge, the equations of motion of the bridge-vehicle system can be expressed as follows;

$$
\begin{aligned}
& {\left[\begin{array}{ccccc}
m_{v} & 0 & 0 & 0 & 0 \\
0 & \frac{I_{v}}{S^{2}} & 0 & 0 & 0 \\
0 & 0 & m_{v} & 0 & 0 \\
0 & 0 & 0 & \frac{I_{v}}{S^{2}} & 0 \\
0 & 0 & 0 & 0 & M+m^{*}
\end{array}\right]\left\{\begin{array}{c}
\ddot{d}_{1} \\
\ddot{d}_{2} \\
\ddot{d}_{3} \\
\ddot{d}_{4} \\
\ddot{D}
\end{array}\right\}} \\
& +\left[\begin{array}{ccccc}
c_{r}+c_{f} & -a_{1} c_{r}+a_{2} c_{f} & 0 & 0 & c_{h 1} \\
-a_{1} c_{r}+a_{2} c_{f} & a_{1}^{2} c_{r}+a_{2}^{2} c_{f} & 0 & 0 & c_{h 1 r} \\
0 & 0 & c_{r}+c_{f} & -a_{1} c_{r}+a_{2} c_{f} & c_{h 2} \\
0 & 0 & -a_{1} c_{r}+a_{2} c_{f} & a_{1}^{2} c_{r}+a_{2}^{2} c_{f} & c_{h 2 r} \\
c_{v 1} & c_{v 1 r} & c_{v 2} & c_{v 2 r} & C+c^{*}
\end{array}\right]\left\{\begin{array}{c}
\dot{d}_{1} \\
\dot{d}_{2} \\
\dot{d}_{3} \\
\dot{d}_{4} \\
\dot{D}
\end{array}\right\} \\
& +\left[\begin{array}{ccccc}
k_{r}+k_{f} & -a_{1} k_{r}+a_{2} k_{f} & 0 & 0 & k_{h 1} \\
-a_{1} k_{r}+a_{2} k_{f} & a_{1}^{2} k_{r}+a_{2}^{2} k_{f} & 0 & 0 & k_{h 1 r} \\
0 & 0 & k_{r}+k_{f} & -a_{1} k_{r}+a_{2} k_{f} & k_{h 2} \\
0 & 0 & -a_{1} k_{r}+a_{2} k_{f} & a_{1}^{2} k_{r}+a_{2}^{2} k_{f} & k_{h 2 r} \\
k_{v 1} & k_{v 1 r} & k_{v 2} & k_{v 2 r} & K+k^{*}
\end{array}\right]\left\{\begin{array}{c}
d_{1} \\
d_{2} \\
d_{3} \\
d_{4} \\
D
\end{array}\right\} \\
& =\left\{\begin{array}{c}
0 \\
0 \\
0 \\
0 \\
p^{*}
\end{array}\right\}
\end{aligned}
$$


where

$$
\begin{aligned}
& m^{*}=m_{t r} N_{r}^{T} N_{r}+m_{t f} N^{T} N+e_{p} m_{t r} N_{t_{r}}{ }^{T} N_{r}+e_{p} m_{t f} N_{t}^{T} N+m_{t r} N_{2 r}^{T} N_{2 r}+m_{t f} N_{2}^{T} N_{2} \\
& +e_{p} m_{t r} N_{t_{2 r}}{ }^{T} N_{r}+e_{p} m_{t f} N_{t_{2}}{ }^{T} N \\
& c^{*}=2 m_{t r} v N_{r}^{T} N_{r, x}+2 m_{t f} v N^{T} N_{, x}+c_{r} N_{r}^{T} N_{r}+c_{f} N^{T} N+2 e_{p} m_{t r} v N_{t_{r}}{ }^{T} N_{r, x} \\
& +2 e_{p} m_{t f} v N_{t}^{T} N_{, x}+e_{p} c_{r} N_{t_{r}}{ }^{T} N_{r}+e_{p} c_{f} N_{t}^{T} N+2 m_{t r} v N_{2 r}^{T} N_{2 r_{, x}} \\
& +2 m_{t f} v N_{2}^{T} N_{2, x}+c_{r} N_{2 r}^{T} N_{2 r}+c_{f} N_{2}^{T} N_{2}+2 e_{p} m_{t r} v N_{t_{2 r}}{ }^{T} N_{r, x} \\
& +2 e_{p} m_{t f} v N_{t_{2}}{ }^{T} N_{, x}+e_{p} c_{r} N_{t_{2 r}}{ }^{T} N_{r}+e_{p} c_{f} N_{t_{2}}{ }^{T} N \\
& k^{*}=m_{t r} v^{2} N_{r}^{T} N_{r, x x}+m_{t f} v^{2} N^{T} N_{, x x}+c_{r} v N_{r}^{T} N_{r, x}+c_{f} v N^{T} N_{, x}+k_{r} N_{r}^{T} N_{r}+k_{f} N^{T} N \\
& +e_{p} m_{t r} v^{2} N_{t_{r}}{ }^{T} N_{r, x x}+e_{p} m_{t f} v^{2} N_{t}{ }^{T} N_{, x x}+e_{p} c_{r} v N_{t_{r}}{ }^{T} N_{r, x} \\
& +e_{p} c_{f} v N_{t}{ }^{T} N_{, x}+e_{p} k_{r} N_{t_{r}}{ }^{T} N_{r}+e_{p} k_{f} N_{t}{ }^{T} N+m_{t r} v^{2} N_{2 r}^{T} N_{2 r, x x} \\
& +m_{t f} v^{2} N_{2}^{T} N_{2, x x}+c_{r} v N_{2 r}^{T} N_{2 r_{, x}}+c_{f} v N_{2}^{T} N_{2, x}+k_{r} N_{2 r}^{T} N_{2 r}+k_{f} N_{2}^{T} N_{2} \\
& +e_{p} m_{t r} v^{2} N_{t_{2 r}}{ }^{T} N_{r, x x}+e_{p} m_{t f} v^{2} N_{t_{2}}{ }^{T} N_{, x x}+e_{p} c_{r} v N_{t_{2 r}}{ }^{T} N_{r, x} \\
& +e_{p} c_{f} v N_{t_{2}}{ }^{T} N_{, x}+e_{p} k_{r} N_{t_{2 r}}{ }^{T} N_{r}+e_{p} k_{f} N_{t_{2}}{ }^{T} N \\
& c_{h 1}=-c_{r} N_{r}-c_{f} N \\
& c_{h 1 r}=a_{1} c_{r} N_{r}-a_{2} c_{f} N \\
& c_{h 2}=-c_{r} N_{2 r}-c_{f} N_{2} \\
& c_{h 2 r}=a_{1} c_{r} N_{2 r}-a_{2} c_{f} N_{2} \\
& c_{v 1}=-c_{r} N_{r}^{T}-c_{f} N^{T}-e_{p} c_{r} N_{t_{r}}{ }^{T}-e_{p} c_{f} N_{t}^{T} \\
& c_{v 1 r}=a_{1} c_{r} N_{r}^{T}-a_{2} c_{f} N^{T}+e_{p} a_{1} c_{r} N_{t_{r}}{ }^{T}-e_{p} a_{2} c_{f} N_{t}{ }^{T} \\
& c_{v 2}=-c_{r} N_{2 r}^{T}-c_{f} N_{2}^{T}-e_{p} c_{r} N_{t_{2 r}}^{T}-e_{p} c_{f} N_{t_{2}}^{T}
\end{aligned}
$$




$$
\begin{aligned}
& c_{v 2 r}=a_{1} c_{r} N_{2 r}^{T}-a_{2} c_{f} N_{2}^{T}+e_{p} a_{1} c_{r} N_{t_{2 r}}^{T}-e_{p} a_{2} c_{f} N_{t_{2}}^{T} \\
& k_{h 1}=-c_{r} v N_{r, x}-c_{f} v N_{, x}-k_{r} N_{r}-k_{f} N \\
& k_{h 1 r}=a_{1} c_{r} v N_{r, x}-a_{2} c_{f} v N_{, x}+a_{1} k_{r} N_{r}-a_{2} k_{f} N \\
& k_{h 2}=-c_{r} v N_{2 r, x}-c_{f} v N_{2, x}-k_{r} N_{2 r}-k_{f} N_{2} \\
& k_{h 2 r}=a_{1} c_{r} v N_{2 r_{,} x}-a_{2} c_{f} v N_{2, x}+a_{1} k_{r} N_{2 r}-a_{2} k_{f} N_{2} \\
& k_{v 1}=-k_{r} N_{r}^{T}-k_{f} N^{T}-e_{p} k_{r} N_{t_{r}}{ }^{T}-e_{p} k_{f} N_{t}^{T} \\
& k_{v 1 r}=a_{1} k_{r} N_{r}^{T}-a_{2} k_{f} N^{T}+e_{p} a_{1} k_{r} N_{t_{r}}^{T}-e_{p} a_{2} k_{f} N_{t}^{T} \\
& k_{v 2}=-k_{r} N_{2 r}^{T}-k_{f} N_{2}^{T}-e_{p} k_{r} N_{t_{2 r}}^{T}-e_{p} k_{f} N_{t 2}^{T} \\
& k_{v 2 r}=a_{1} k_{r} N_{2 r}^{T}-a_{2} k_{f} N_{2}^{T}+e_{p} a_{1} k_{r} N_{t 2 r}^{T}-e_{p} a_{2} k_{f} N_{t 2}^{T} \\
& p^{*}=-\left(a_{2} m_{v}+m_{t r}\right) g N_{r}^{T}-\left(a_{1} m_{v}+m_{t f}\right) N^{T}-\left(a_{2} m_{v}+m_{t r}\right) g N_{t_{r}}{ }^{T} \\
& \quad-\left(a_{1} m_{v}+m_{t f}\right) N_{t}^{T}-\left(a_{2} m_{v}+m_{t r}\right) g N_{2 r}^{T}-\left(a_{1} m_{v}+m_{t f}\right) N_{2}^{T} \\
& \quad-\left(a_{2} m_{v}+m_{t r}\right) g N_{t}{ }_{2 r}^{T}-\left(a_{1} m_{v}+m_{t f}\right) N_{t 2}^{T}
\end{aligned}
$$

$N_{2}$

$=\left[\begin{array}{llllllllllll}0 & \psi_{1}(x-S v) & 0 & 0 & 0 & \psi_{2}(x-S v) & 0 & \psi_{3}(x-S v) & 0 & 0 & 0 & \psi_{4}(x-S v)\end{array}\right]$

$N_{2 r}$

$=\left[\begin{array}{llllllllllll}0 & \psi_{1}(x-S v-S) & 0 & 0 & 0 & \psi_{2}(x-S v-S) & 0 & \psi_{3}(x-S v-S) & 0 & 0 & 0 & \psi_{4}(x-S v-S)\end{array}\right]$

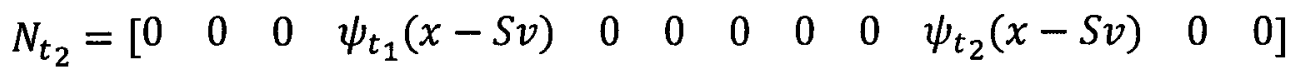

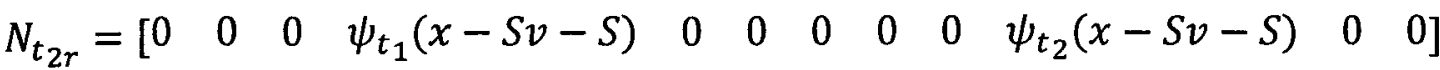




\subsection{Water Current Loads}

The total loads acting on the floating bridge comprise the contribution of two main loads acting on the floating bridge; lateral loads and torsional moments due to water current and vertical dynamic loads due to moving vehicles. The vertical dynamic loads due to moving vehicles act at a specific location of the bridge according to the vehicle position on the bridge at a certain time, while the distributed lateral loads and torsional moments due to water current act on the whole bridge according to the depth of the submerged part along the bridge profile at this time.

Water current loads depend on the depth of submerged part of the bridge; this submerged depth is not constant along the bridge length, but varies from unloaded pontoons to loaded ones according to the position of the vehicle along the bridge. This submerged depth also changes with time as the vehicle position changes with time inducing different submerged depth at each node along the bridge. This means that water current loads should be treated as dynamic loads that depend on both time and space coordinates. In the present study, water current loads are treated as dynamic loads that changes with both time and space coordinates, and are incorporated into the dynamic equations of vehiclebridge-fluid system. At each time step, water current loads are calculated based on the actual dynamic response of the bridge profile and updated in the global load vector for the next time step to investigate the interactive structural behaviour of floating bridges due to the combined actions of moving vehicle loads and dynamic water current loads more accurately. 
The transverse loading induced by the water current is a major source of out-of-plane deformations and straining actions on a floating bridge. In order to resist lateral water current loads, motorized supporting boats are used to support the rapid deployment floating bridge at different points along its length.

Among the objectives of the present research is to investigate the deformations and straining actions developed in the floating bridge due to water current loads and to investigate the optimum use of supporting boats to control and minimize these deformations and straining actions. Also, to investigate the three-dimensional interactive dynamic response of rapid deployment floating bridges to the combined actions of moving vehicle loads and dynamic water current loads along with possible eccentricities of these loads.

\subsubsection{Basic Hypotheses for Open Channel Flow}

The Cartesian coordinate system is oriented such that the z-axis along the longitudinal direction is parallel to the average channel bed slope; the $\mathrm{x}$-axis along the transverse direction and the y-axis along the depth and normal to the channel bottom (Figure 3.10). Water current loads are derived assuming the main velocity component $u$ is along the zaxis and is a function of $\mathrm{x}$ and $\mathrm{y}$ and the velocity components along the $\mathrm{x}$-axis and the $\mathrm{y}$ axis are zero.

\subsubsection{Description of Water Current Velocity}

Water current velocity is not constant all over the water channel and it varies in both the horizontal and vertical directions where it has functional shapes in both the horizontal 
and the vertical planes. These functional shapes depend on the type of flow whether it is laminar or turbulent according to the Reynolds number. Based on water current velocity at the vertical level of the submerged part of the floating bridge, the current velocity functional shape in the horizontal plane ( $\mathrm{x}-\mathrm{z}$ plane) is approximately expressed as follows (Olson and Wright 1990);

$$
\begin{array}{ll}
u=u_{m}\left(1-\frac{r}{R}\right)^{\frac{1}{7}}=u_{m}\left(\frac{x}{R}\right)^{\frac{1}{7}} & \text { for turbulent flow } \\
u=u_{m}\left[1-\left(\frac{r}{R}\right)^{2}\right] & \text { for laminar flow }
\end{array}
$$

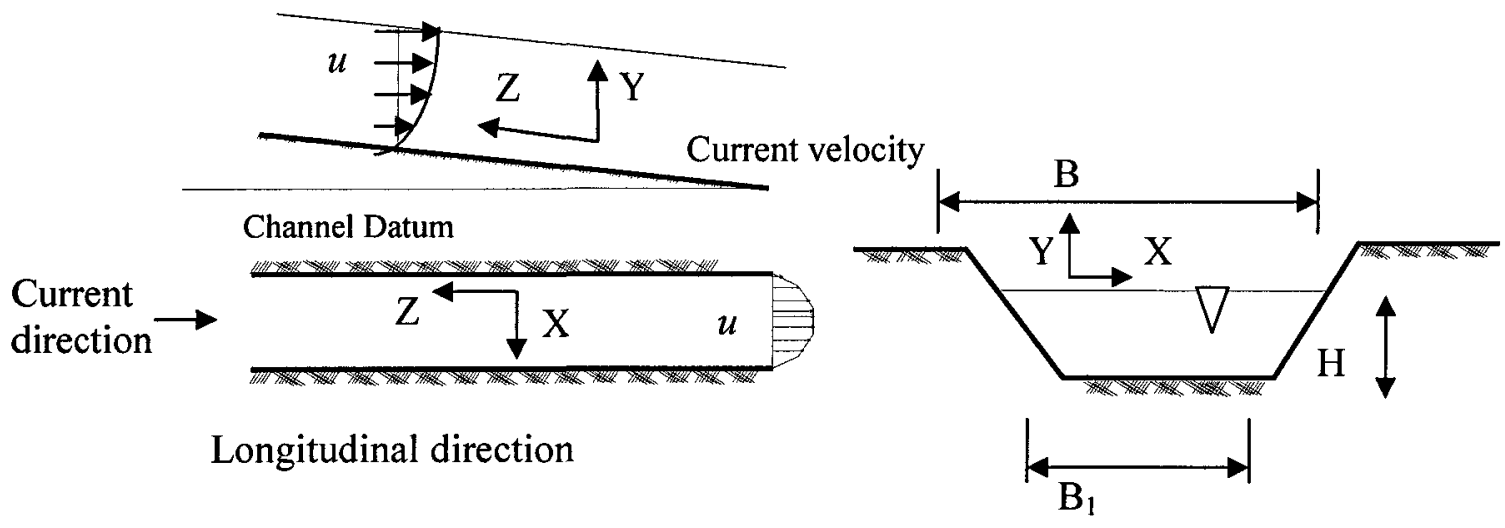

Cross section of channel

Figure 3.10 Cartesian coordinate system used for open channel flow

where:

$u$ is the water current velocity at any horizontal distance $x$ from the channel wall.

$u_{m}$ is the maximum water current velocity at the centerline of the water channel. 
$x$ is the horizontal distance from the channel wall.

$R$ is the distance from the centerline to the edge of the water channel.

$r=R-x$ where $x$ is the horizontal distance from the channel wall.

\subsubsection{Drag Forces Acting on Floating Bridges Due to Water Current}

The drag forces acting on floating bridges due to water current comprise two main forces; form drag forces and friction drag forces. The drag forces act on the curved surfaces and the bottom of the floating bridge profile as shown in Figure 3.11; this curved area can be resolved into vertical projection area and horizontal projection area. The form drag forces are calculated using the submerged vertical projected area, and the friction drag forces are calculated using the wetted surface area comprising the submerged horizontal projected area of the curved surface and the surface area of the bridge bottom.

The form drag forces are calculated as follows (Olson and Wright 1990);

$F_{D}=c_{D} \frac{\rho_{w} u^{2}}{2} A$

where:

$F_{D}$ is the form drag forces

$c_{D}$ is the form drag coefficient

$\rho_{w}$ is the water density

$u$ is the water current velocity 
$A$ is the vertical projection of the wetted area

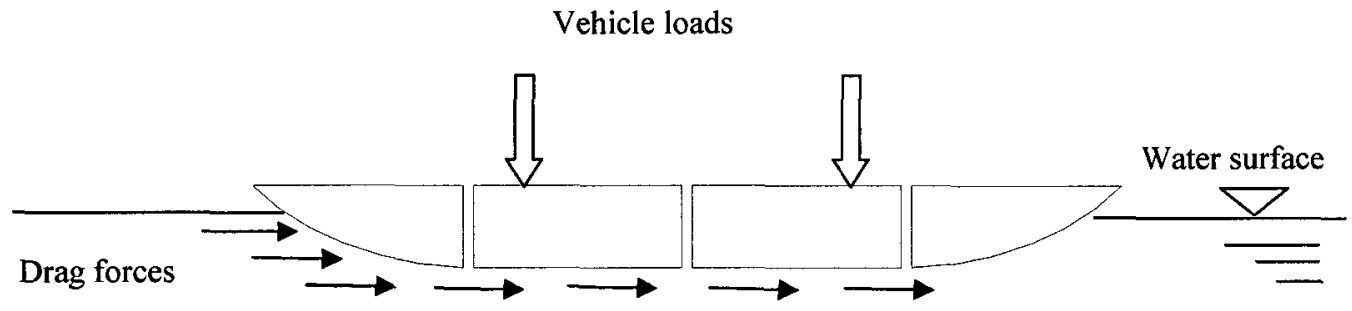

Current direction

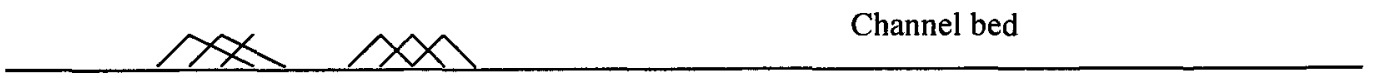

Figure 3.11 Drag forces acting on floating bridge profile due to water current

The form drag coefficient for a specific body depends on the shape of that body and can be determined experimentally. Approximate values for the form drag coefficient for some standard shapes are given based on experimental studies.

The friction drag forces are calculated as follows (Olson and Wright 1990);

$F_{F}=c_{F} \frac{\rho_{w} u^{2}}{2} A_{W}$

where:

$F_{F}$ is the friction drag forces

$c_{F}$ is the friction drag coefficient

$A_{W}$ is the wetted area parallel to the water flow 
The friction drag coefficient can be approximately obtained from theoretical and empirical formulae.

\subsubsection{Formulation of the Local Force Vector Due to Water Current}

The distributed load acting on each element of the bridge due to water current loads has to be converted to equivalent nodal forces using load coefficients using the following equation (Humar 2002);

$p_{i}=\int_{0}^{L} \bar{p} \psi_{i}(x) d x$

where $\bar{p}$ is the intensity of the distributed load. The load coefficients $p_{l_{i}}$ and $p_{t_{i}}$ for the distributed load and torsional moment, respectively are given as follows;

$p_{l_{i}}=\int_{0}^{L} \bar{p}\left[\begin{array}{c}1-3 \frac{x^{2}}{L^{2}}+2 \frac{x^{3}}{L^{3}} \\ x-2 \frac{x^{2}}{L}+\frac{x^{3}}{L^{2}} \\ 3 \frac{x^{2}}{L^{2}}-2 \frac{x^{3}}{L^{3}} \\ -\frac{x^{2}}{L}+\frac{x^{3}}{L^{2}}\end{array}\right] d x$

$p_{t_{i}}=\int_{0}^{L} \overline{p_{t}}\left[\begin{array}{c}1-\frac{x}{L} \\ \frac{x}{L}\end{array}\right] d x$

Where $\overline{p_{l}}$ and $\overline{p_{t}}$ are the intensities of the distributed load and distributed torsional moment, respectively that depend on the depth of submerged part of the bridge at each time step corresponding to the position of the vehicle on the bridge and including the vertical displacement under self weight of the bridge $h_{o w}$. The intensities of form drag 
forces and friction drag forces as well as torsional moments acting on each element in the floating bridge are calculated as follows;

Form drag forces per unit length:

for turbulent flow

$\overline{p_{D}}(t+h)=\frac{1}{2} C_{D} \rho_{w}\left[h_{o w}+0.5\left\{\Delta_{n y}(t)+\Delta_{(n+1) y}(t)\right\}\right] u_{m}^{2}\left(\frac{x}{R}\right)^{\frac{2}{7}}$

for laminar flow

$\overline{p_{D}}(t+h)=\frac{1}{2} C_{D} \rho_{w}\left[h_{o w}+0.5\left\{\Delta_{n y}(t)+\Delta_{(n+1) y}(t)\right\}\right] u_{m}^{2}\left[1-\left(1-\frac{x}{R}\right)^{2}\right]$

where

$t$ is the current time.

$h$ is the time step used in integration.

$n$ is the element number.

$h_{o w}$ is the static vertical displacement under self weight of the bridge.

$\Delta_{n y}(t)$ is the dynamic vertical displacement at the first node of element $n$ at time $t$.

$\Delta_{(n+1) y}(t)$ is the dynamic vertical displacement at the second node of element $n$ at time $t$ due to moving vehicle loads. 
Friction drag forces per unit length:

$\overline{p_{F}}(t+h)=\frac{1}{2} C_{F} \gamma_{w} L_{w e t}(t) u_{m}^{2}\left(\frac{x}{R}\right)^{\frac{2}{7}}$

for turbulent flow

$\overline{p_{F}}(t+h)=\frac{1}{2} C_{F} \gamma_{w} L_{w e t}(t) u_{m}^{2}\left[1-\left(\frac{r}{R}\right)^{2}\right]$

for laminar flow

where $L_{w e t}$ is the wetted length in the direction of flow.

Total drag forces per unit length:

$\overline{p_{l}}(t+h)=\overline{p_{D}}(t+1)+\overline{p_{F}}(t+1)$

Torsional moment per unit length:

$\overline{p_{t}}(t+h)=\overline{p_{D}}(t+1)\left[\frac{d}{2}-\left(h_{o w}+\frac{\Delta_{n y}(t)+\Delta_{(n+1) y}(t)}{2}\right)\right]+\overline{p_{F}}(t+1)\left[\frac{d}{2}\right]$

Having calculated the intensity of the drag forces, the local force vector for each element due to water current loads $p_{w c_{i}}$ can be written as follows; 


$$
p_{w c_{i}}(t+h)=\left[\begin{array}{c}
0 \\
0 \\
\overline{p_{l}}(t+1) \frac{L}{2} \\
\overline{p_{t}}(t+1) \frac{L}{2} \\
\overline{p_{l}}(t+1) \frac{L^{2}}{12} \\
0 \\
0 \\
0 \\
\overline{p_{l}}(t+1) \frac{L}{2} \\
\overline{p_{t}}(t+1) \frac{L}{2} \\
-\overline{p_{l}}(t+1) \frac{L^{2}}{12} \\
0
\end{array}\right]
$$

Wherever the bridge experiences an upward displacement equal to or greater than the downward displacement under self weight, there are no lateral forces due to water current at those positions. This consideration was taken into account in the present study.

The global force vector of the whole bridge due to water current loads can be obtained by adding the element load coefficients appropriately using the standard finite element assembly procedure. Assembling the global force vector involves combining the local load coefficients for each element within the bridge and including the boat forces acting on the bridge in the opposite direction of water current according to the number of boats, their positions and the magnitude of force each boat exerts on the bridge. This global force vector due to water current loads is used as the initial value of the global force vector at each time step, where the local force vector due to moving vehicle loads is incorporated at specific locations into the global force vector according to the vehicle position on the bridge resulting in the global force vector due to both water current loads and moving vehicle loads at each time step in the time domain of the dynamic problem. 


\subsection{Determining the Floating Bridge Dynamic Response}

A program based on finite element method of analysis and utilizing the MATLAB software package was developed by the author for the formulation of the equations of motion of the vehicle-bridge-fluid integrated system, as well as conducting the dynamic analysis and determining the floating bridge dynamic response. As mentioned before, after forming the local stiffness and mass matrices for each element of the bridge model, it is necessary to form the global stiffness, mass and damping matrices of the whole floating bridge model. This can be very difficult to accomplish manually, because of the large number of finite elements needed for reasonable accuracy of the bridge response. Based on the shape functions of the space frame element, the developed program can form the local stiffness and mass matrices of each element including the contribution of the fluid-structure interaction in the form of added mass and stiffness. Then, based on the number of elements, the program constructs the global stiffness and mass matrices of the bridge model using the finite element assembly technique. The second step is to conduct the free vibration analysis of the bridge to calculate the natural frequencies and natural periods of the bridge such that they can be used in calculating the global damping matrix of the bridge based on Rayleigh formulation. Then the equation of motion of the vehicle is incorporated into the global matrices to form the equations of motion of the vehiclebridge-fluid system.

The equations of motion of the bridge-vehicle-fluid system represent a set of nonlinear time-dependent differential equations that can be solved only by a process of numerical integration. The Newmark's $\beta$ average acceleration method was used to conduct the 
dynamic analysis for the bridge-vehicle system by time marching from an initial time at which the displacements are known to the next time where the new displacements are calculated. These new displacements are used as initial values for the next time step and so on. The formulation of the matrices composing the equations of motion is different at each time step and needs to be updated as the vehicle load changes its position at each time step based on the velocity of the vehicle and changes its magnitude due to the vehicle-bridge interaction, and this was taken into account while developing the finite element program used in this thesis.

Integration is carried out with a time step that is a reasonably small fraction of the natural period of the bridge and at the same time a small fraction of the traversing time for the vehicle along the bridge $\tau$, and satisfying the requirements to avoid the common types of errors involved in numerical integration methods such as divergence of results and numerical oscillations.

Newmark's $\beta$ average acceleration method is based on the following equations (Humar 2002);

$$
\begin{aligned}
& \left(\frac{4 m}{h^{2}}+\frac{2 c}{h}+k\right) u_{n+1}=p_{n+1}+m\left(\frac{4}{h^{2}} u_{n}+\frac{4}{h} \dot{u}_{n}+\ddot{u}_{n}\right)+c\left(\frac{2}{h} u_{n}+\dot{u}_{n}\right) \\
& \dot{u}_{n+1}=-\dot{u}_{n}+\frac{2}{h}\left(u_{n+1}-u_{n}\right) \\
& \ddot{u}_{n+1}=\frac{4}{h^{2}}\left(u_{n+1}-u_{n}-h \dot{u}_{n}\right)-\ddot{u}_{n}
\end{aligned}
$$


In the present application, $m, c$ and $k$ are matrices and $p, u, \dot{u}$ and $\ddot{u}$ are vectors and $h$ is the time step used in integration.

These equations of Newmark's $\beta$ average acceleration method are incorporated into the program to conduct the dynamic analysis of the vehicle-bridge system and obtain the dynamic response of the bridge. The whole procedure for the developed finite element program is illustrated in the following flowchart (Figure 3.12);

\subsection{Assumptions and Limitations}

The following assumptions and limitations are considered in the present study:

- The vehicle is assumed to travel across the bridge at constant speed $v$, where the constant speed represents the practical case for field use of rapid deployment floating bridges.

- No braking or accelerating forces acting on the bridge (longitudinal or transverse) are assumed.

- The unsprung mass is assumed to remain in contact with the deck, while the bridge is assumed to remain in contact with water throughout the analysis.

- The initial vertical displacement, velocity and acceleration of the vehicle on entry to the bridge are assumed to be zero.

- The effect of large deflections in the bridge on the bridge response is not considered in the present study. 


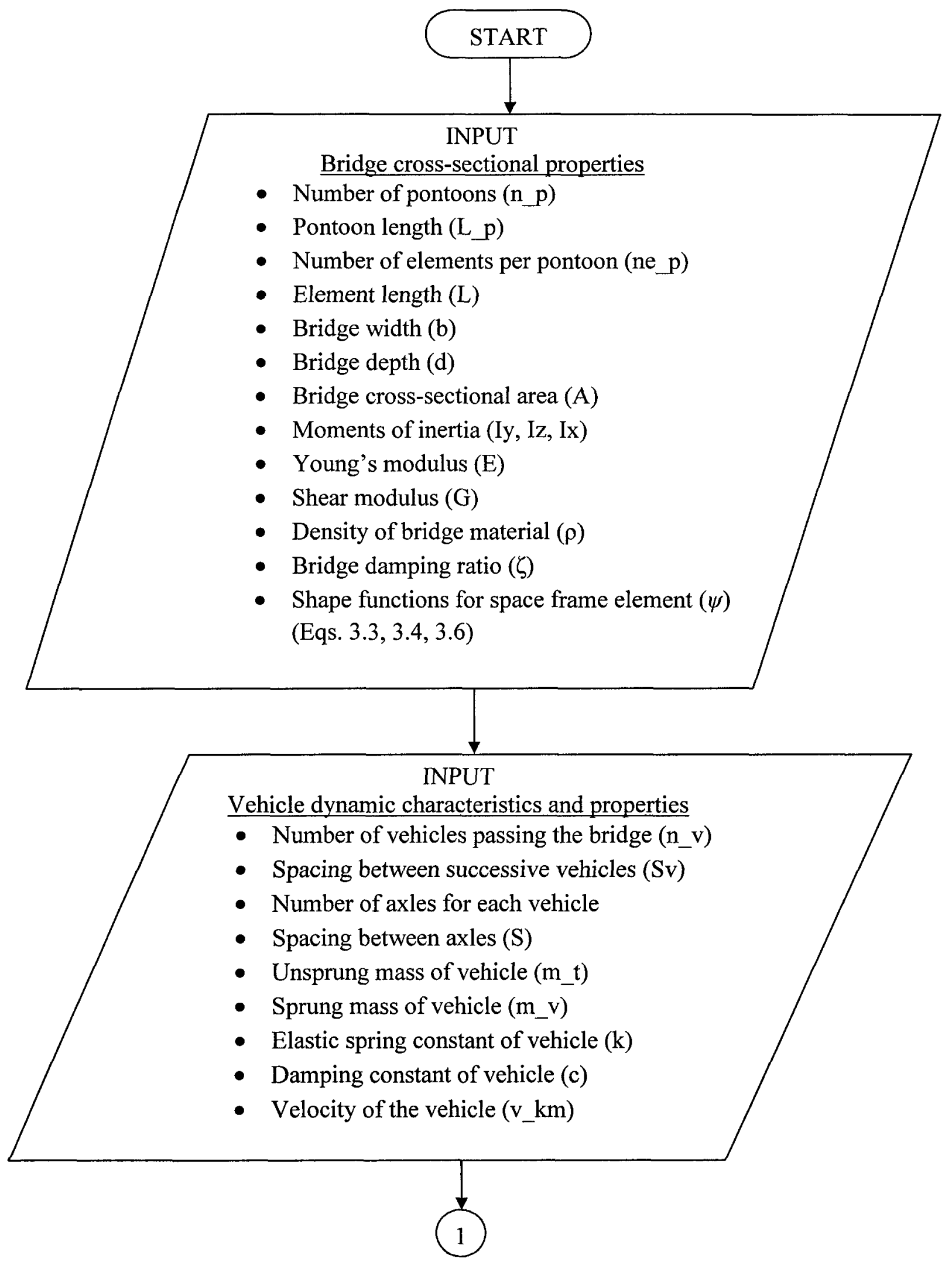




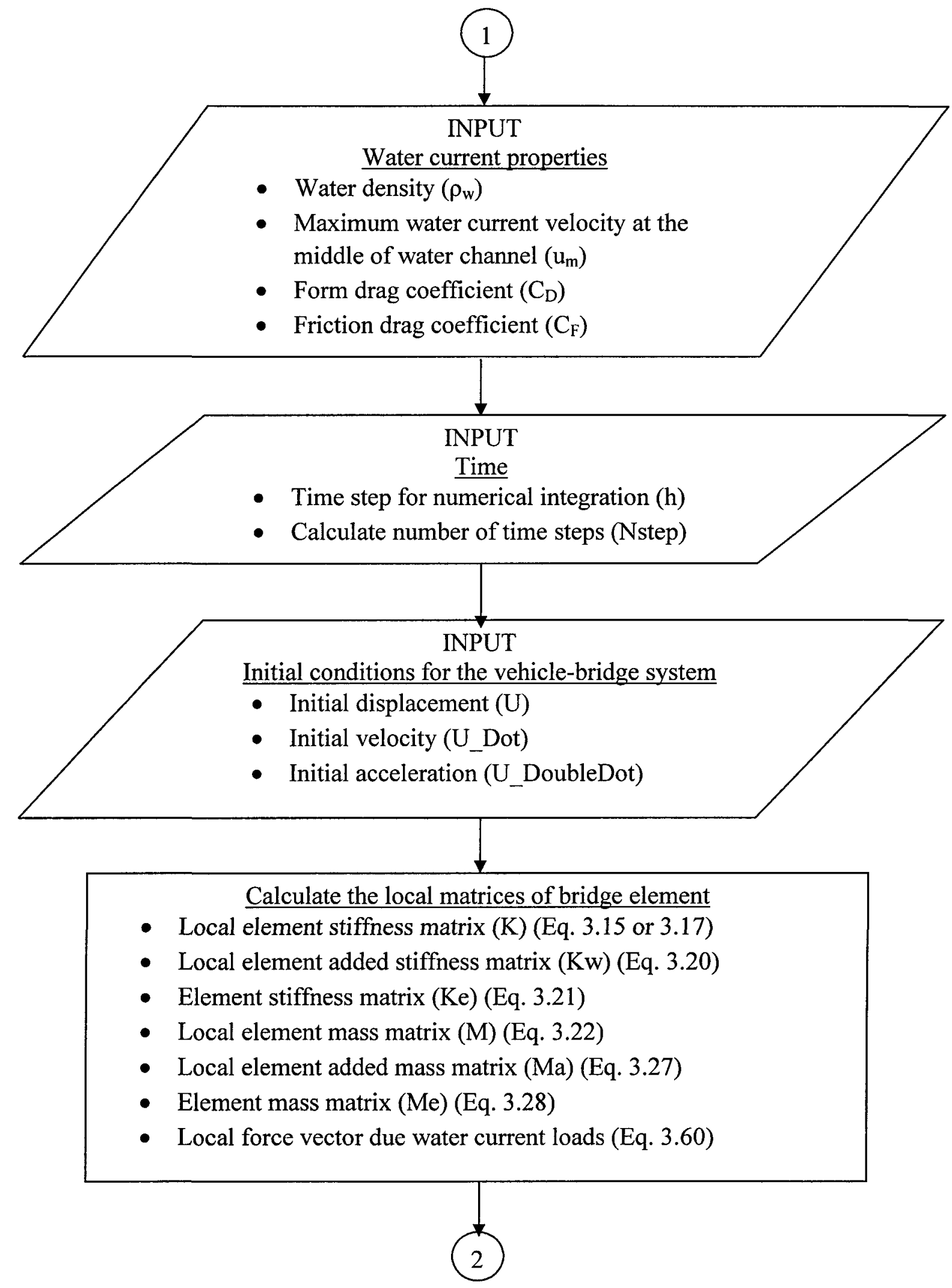


Construct and calculate the global matrices of the bridge

- Global mass matrix (M_Gb)

- Global stiffness matrix (K_Gb)

- Global force vector due to water current loads

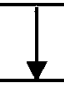

Free vibration analysis of the bridge

- Solve the undamped free vibration equation of the bridge

- Calculate the eigenvectors and eigenvalues of the bridge

- Calculate the natural frequencies of the bridge

Construct and calculate the global damping matrix of the bridge

- First and second natural frequencies of the bridge $(\omega 1, \omega 2)$

- Calculate the global damping matrix of the bridge using Rayleigh Damping (C_Gb) (Eq. 3.30)

\section{Construct matrices for the vehicle-bridge system}

- Equation of motion of each vehicle (Eq. 3.13 or 3.44)

- Equation of motion of the bridge (Eq. 3.41)

- Combine the equations of motions of the vehicles and the bridge to get the equation of motion of the bridge-vehicle system (Eq. 3.42, 3.46 or 3.48)

- Global mass matrix of the bridge-vehicle system (M_Gs)

- Global stiffness matrix of the bridge-vehicle system (K_Gs)

- Global damping matrix of the bridge-vehicle system (C_Gs)

- Initial global force vector due to water current loads 
1

Time Marching Finite Element Analysis

Calculation loop

- Start time $\left(\mathrm{t}_{0}\right)$

- Start calculation loop

\section{5}

\section{Time Marching Finite Element Analysis}

At each time step

- Update time

- Calculate the location of each axle in each vehicle (x1, $\mathrm{x} 1 \mathrm{r}$, $\mathrm{x} 2, \mathrm{x} 2 \mathrm{r}, \ldots)$

- Calculate the location of each axle in each vehicle with respect to the element local coordinates (x)

- Calculate the load vectors from each axle in each vehicle based on the element local coordinates (p1, p1r, p2, p2r, ..) (Eq. 3.40 or 3.45 for each vehicle)

- Separate the mass, stiffness and damping terms from the load vector and move them to the left hand side of the dynamic equation of bridge-vehicle system

- Allocate all the load terms to the corresponding elements

- Update the mass, stiffness and damping matrices and the load vector of the bridge-vehicle system (Eq. 3.43, 3.47 or 3.49)

- Calculate the global force vector due to water current loads according to the actual submerged depth from the dynamic response of the bridge profile (Eq. 3.60) 


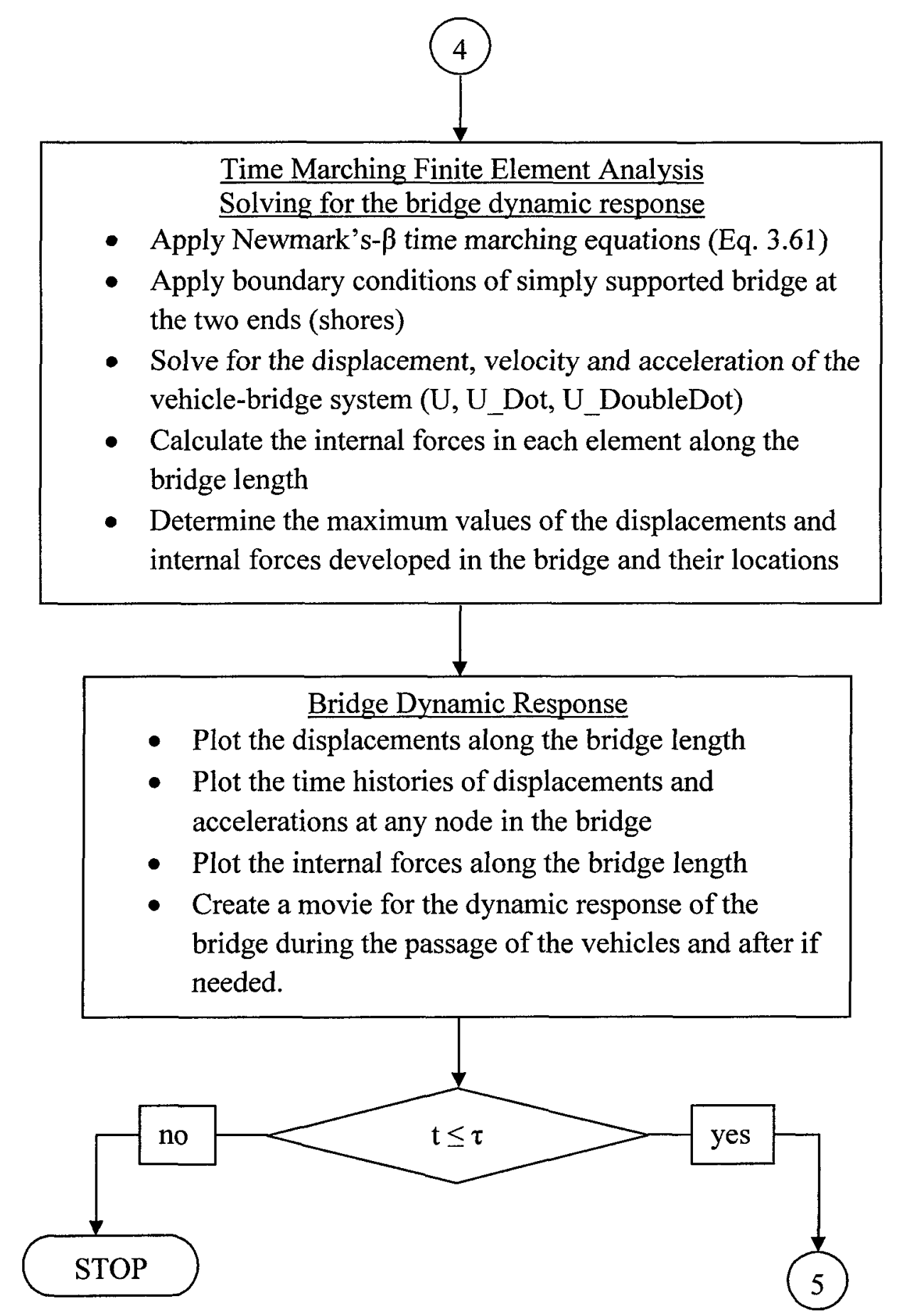

Figure 3.12 Flowchart of the analytical algorithm and the developed finite element program for the dynamic analysis of floating bridges under moving vehicle loads and dynamic water current loads 


\section{Chapter: Parametric Studies for the Dynamic Behaviour and Transport Capacity of Floating Bridges}

The dynamic behaviour and transport capacity of rapid deployment floating bridges is investigated in terms of the passage of single and multiple vehicles considering different vehicle weights, speeds and separation distances between successive vehicles. The transport capacity of rigid-connected as well as hinge-connected floating bridges is investigated considering different models for the vehicles including single-axle and twoaxle models. Before the parametric studies are conducted, several finite element analysis runs of the floating bridge were performed to determine the appropriate element size and the time increment (step) to achieve convergence and accuracy of the results.

The input parameters shown in Table 4.1 were used for the parametric studies conducted throughout the present chapter (see Appendix B for cross-section and detailed description of the ribbon bridge). The rigid-connected bridge studied consists of twelve pontoons each of length $7 \mathrm{~m}$ and rigidly connected to each other, to form a bridge of total length 84 $\mathrm{m}$. The hinge-connected bridge (hybrid bridge) on the other hand consists of twelve pontoons each of length $7 \mathrm{~m}$, each three pontoons are rigidly connected to each other, to form a typical bay of length $21 \mathrm{~m}$, these typical bays are hinge-connected to each other to form a bridge of total length $84 \mathrm{~m}$. The vehicle models used in the analyses have a sprung mass equal to $90 \%$ of the vehicle total mass while the sum of the unsprung masses of the vehicle is $10 \%$ of the vehicle total mass.

The results reported in this parametric study present the static and dynamic responses of the bridge to vehicle loads relative to the equilibrium position of the bridge under self 
weight. The maximum static deflection in the rigid-connected bridge under self weight is $-0.113 \mathrm{~m}$, while the maximum static displacement in the hinge-connected bridge under self weight is $-0.127 \mathrm{~m}$. The sign convention adopted for displacement (deflection) throughout the thesis is positive upward displacement. For safe operation of rapid deployment floating bridges, the maximum deflection in the bridge is limited to $80 \%$ of the total bridge depth. This leaves a free board of $20 \%$ of the total depth of the bridge as safety margin or reserve buoyancy (Rosenthal et al. 1996). The floating bridge studied in this thesis has a total depth of $1.12 \mathrm{~m}$; meaning the maximum total deflection in the bridge including its deflection under self weight should not exceed $0.896 \mathrm{~m}$. For a rigidconnected bridge, subtracting the deflection under self weight, the maximum deflection under vehicle loads should not exceed $0.783 \mathrm{~m}$. Similarly, the maximum displacement in a hinge-connected bridge under vehicle loads should not exceed $0.769 \mathrm{~m}$.

Table 4.1: Input parameters for parametric studies

\begin{tabular}{|c|c|c|c|}
\hline Parameter & Value & Parameter & Value \\
\hline Bridge length & $84 \mathrm{~m}$ & Shear modulus of steel & $0.793 \mathrm{E} 11 \mathrm{~N} / \mathrm{m}^{2}$ \\
\hline Element length & $0.5 \mathrm{~m}$ & Density of steel & $7.85 \mathrm{E} 3 \mathrm{~kg} / \mathrm{m}^{3}$ \\
\hline Bridge width & $8.13 \mathrm{~m}$ & Bridge mass per unit length & $812 \mathrm{~kg}$ \\
\hline Bridge depth & $1.12 \mathrm{~m}$ & Modulus of elasticity of aluminum & $7 \mathrm{E} 10 \mathrm{~N} / \mathrm{m}^{2}$ \\
\hline Cross-sectional area & $0.103 \mathrm{~m}^{2}$ & Shear modulus of aluminum & $2.7 \mathrm{E} 10 \mathrm{~N} / \mathrm{m}^{2}$ \\
\hline Moment of inertia (Iz) & $0.015 \mathrm{~m}^{4}$ & Density of aluminum & $2.7 \mathrm{E} 3 \mathrm{~kg} / \mathrm{m}^{3}$ \\
\hline Moment of inertia (Iy) & $0.048 \mathrm{~m}^{4}$ & Axle spacing & $6 \mathrm{~m}$ \\
\hline Polar moment of inertia (J) & $0.063 \mathrm{~m}^{4}$ & Damping coefficient & $5 \%$ \\
\hline Modulus of elasticity of steel & $2 \mathrm{E} 11 \mathrm{~N}^{2} \mathrm{~m}^{2}$ & & \\
\hline
\end{tabular}


The dynamic response (displacement and acceleration) along the bridge length are calculated at each position of the vehicle while traversing the bridge. Once the displacements are determined, the internal forces in each finite element along the bridge length are calculated by multiplying the stiffness matrix by the nodal displacement vector for each element. Then, the maximum values for the displacements and internal forces are determined. Also, the maximum static displacements and internal forces are calculated, and then the Dynamic Amplification Factors (DAFs) for both the displacement and bending moment are calculated using the following equation:

$$
D A F=\frac{u_{d}}{u_{s}}
$$

where $u_{d}$ is the maximum dynamic response of the bridge relative to its position of equilibrium under self weight, and $u_{s}$ is the maximum static response of the bridge relative to its position of equilibrium under self weight.

\subsection{Parametric Study for the Dynamic Response of Floating Bridges to Single-}

\section{Axle Vehicle Loads}

The objective of the parametric study presented in this section is to investigate the effect of increasing the vehicle speed and/or weight on the dynamic response of rigid-connected and hinge-connected floating bridge. The vehicle is represented by a single-axle model and has different weights of 50,60 and 70 tonnes. The single-axle model of the vehicle used in this analysis consists of an unsprung mass connected to a sprung mass through a spring and damper unit that represent the dynamic characteristics of the vehicle (see 
Figure 3.8). This single-axle model of the vehicle is less complex, more conservative and faster in predicting the dynamic response of the bridge.

\subsubsection{Dynamic Response of Rigid-Connected Floating Bridge to Single-Axle}

\section{Vehicle Loads}

The dynamic response of rigid-connected floating bridge to heavy single-axle vehicle loads is investigated for different vehicle speeds varying from $5 \mathrm{~km} / \mathrm{h}$ to $45 \mathrm{~km} / \mathrm{h}$, knowing that the maximum allowable speed is $16 \mathrm{~km} / \mathrm{h}$ (see Table 1.1). The bridge consists of twelve rigidly connected pontoons with a total length of $84 \mathrm{~m}$. The details of the performed analyses and the results for the static and dynamic response of the bridge (deflection and bending moment) are summarized in Tables 4.2, 4.3 and 4.4, where B.M. is the bending moment.

Table 4.2: Static and dynamic response of rigid-connected bridge to 50-tonne single-axle vehicle loads

\begin{tabular}{|c|c|c|c|c|c|c|c|c|}
\hline Span & $\begin{array}{c}\text { Vehicle } \\
\text { Weight } \\
(\mathbf{m})\end{array}$ & $\begin{array}{c}\text { Vehicle } \\
\mathbf{\text { speed }} \\
(\mathbf{k m} / \mathbf{h})\end{array}$ & $\begin{array}{c}\text { Max } \\
\mathbf{d e f l} . \\
(\mathbf{m})\end{array}$ & $\begin{array}{c}\text { Static } \\
\text { defl. } \\
(\mathbf{m})\end{array}$ & $\begin{array}{c}\text { DAF } \\
\text { for defl. }\end{array}$ & $\begin{array}{c}\text { Max } \\
\text { B.M. } \\
(\mathbf{K N} . \mathbf{m})\end{array}$ & $\begin{array}{c}\text { Static } \\
\text { B.M. } \\
(\text { KN.m) }\end{array}$ & $\begin{array}{c}\text { DAF for } \\
\text { B.M. }\end{array}$ \\
\hline 84 & 50 & 5 & -0.165 & -0.162 & 1.02 & -2950 & -2382 & 1.24 \\
\hline 84 & 50 & 10 & -0.170 & -0.162 & 1.05 & -2971 & -2382 & 1.25 \\
\hline 84 & 50 & 15 & -0.176 & -0.162 & 1.09 & -3096 & -2382 & 1.30 \\
\hline 84 & 50 & 20 & -0.185 & -0.162 & 1.14 & -3191 & -2382 & 1.34 \\
\hline 84 & 50 & 25 & -0.195 & -0.162 & 1.20 & -3226 & -2382 & 1.35 \\
\hline 84 & 50 & 30 & -0.210 & -0.162 & 1.30 & -3382 & -2382 & 1.42 \\
\hline 84 & 50 & 45 & -0.272 & -0.162 & 1.68 & -3963 & -2382 & 1.66 \\
\hline
\end{tabular}


Table 4.3: Static and dynamic response of rigid-connected bridge to 60 -tonne single-axle vehicle loads

\begin{tabular}{|c|c|c|c|c|c|c|c|c|}
\hline $\begin{array}{l}\text { Span } \\
(\mathrm{m}) \\
\end{array}$ & $\begin{array}{c}\text { Vehicle } \\
\text { Weight } \\
\text { (t) }\end{array}$ & $\begin{array}{c}\text { Vehicle } \\
\text { speed } \\
(\mathbf{k m} / \mathbf{h})\end{array}$ & $\begin{array}{l}\text { Max } \\
\text { defl. } \\
(\mathrm{m})\end{array}$ & $\begin{array}{c}\text { Static } \\
\text { defl. } \\
\text { (m) }\end{array}$ & $\begin{array}{c}\text { DAF } \\
\text { for defl. }\end{array}$ & $\begin{array}{c}\text { Max } \\
\text { B.M. } \\
\text { (KN.m) }\end{array}$ & $\begin{array}{c}\text { Static } \\
\text { B.M. } \\
\text { (KN.m) }\end{array}$ & $\begin{array}{c}\text { DAF for } \\
\text { B.M. }\end{array}$ \\
\hline 84 & 60 & 5 & -0.198 & -0.194 & 1.02 & -3540 & -2858 & 1.24 \\
\hline 84 & 60 & 10 & -0.205 & -0.194 & 1.05 & -3565 & -2858 & 1.25 \\
\hline 84 & 60 & 15 & -0.211 & -0.194 & 1.09 & -3717 & -2858 & 1.30 \\
\hline 84 & 60 & 20 & -0.222 & -0.194 & 1.14 & -3832 & -2858 & 1.34 \\
\hline 84 & 60 & 25 & -0.234 & -0.194 & 1.20 & -3874 & -2858 & 1.36 \\
\hline 84 & 60 & 30 & -0.253 & -0.194 & 1.30 & -4063 & -2858 & 1.42 \\
\hline 84 & 60 & 45 & -0.327 & -0.194 & 1.68 & -4766 & -2858 & 1.67 \\
\hline
\end{tabular}

Table 4.4: Static and dynamic response of rigid-connected bridge to 70-tonne single-axle vehicle loads

\begin{tabular}{|c|c|c|c|c|c|c|c|c|}
\hline Span & $\begin{array}{c}\text { Vehicle } \\
\text { Weight } \\
(\mathbf{m})\end{array}$ & $\begin{array}{c}\text { Vehicle } \\
\text { speed }\end{array}$ & $\begin{array}{c}\text { Max } \\
\text { defl. } \\
(\mathbf{k m} / \mathbf{h})\end{array}$ & $\begin{array}{c}\text { Static } \\
\mathbf{d} \text { defl. }\end{array}$ & $\begin{array}{c}\text { DAF } \\
\text { for } \mathbf{\text { defl. }}\end{array}$ & $\begin{array}{c}\text { Max } \\
\text { B.M. } \\
(\mathbf{K N . m})\end{array}$ & $\begin{array}{c}\text { Static } \\
\text { B.M. } \\
(\mathbf{K N} . \mathbf{m})\end{array}$ & $\begin{array}{c}\text { DAF for } \\
\text { B.M. }\end{array}$ \\
\hline 84 & 70 & 5 & -0.230 & -0.227 & 1.02 & -4131 & -3335 & 1.24 \\
\hline 84 & 70 & 10 & -0.239 & -0.227 & 1.05 & -4160 & -3335 & 1.25 \\
\hline 84 & 70 & 15 & -0.246 & -0.227 & 1.09 & -4340 & -3335 & 1.30 \\
\hline 84 & 70 & 20 & -0.259 & -0.227 & 1.14 & -4473 & -3335 & 1.34 \\
\hline 84 & 70 & 25 & -0.274 & -0.227 & 1.21 & -4525 & -3335 & 1.36 \\
\hline 84 & 70 & 45 & -0.381 & -0.227 & 1.68 & -5572 & -3335 & 1.67 \\
\hline
\end{tabular}




\subsubsection{Discussion of Results}

The results obtained from the analysis described in the previous section are used to explain the dynamic response of rigid-connected floating bridges to single-axle vehicle loads, and the effect of increasing vehicle weight and speed on the bridge dynamic response.

Figure 4.1 shows the midpoint displacement during the passage of 50-tonne single-axle vehicle along the bridge length at different speeds. The time needed for the vehicle to traverse the bridge differs according to the vehicle speed. In order to have the same distance on $\mathrm{x}$-axis, representing the position of the vehicle on the bridge, for different vehicle speeds, a normalized distance was plot on $\mathrm{x}$-axis. The value 1 on $\mathrm{x}$-axis represents the position of the vehicle at the end of the bridge for all vehicle speeds. The midpoint displacement, in general, increases with vehicle speed. The same trend was observed for the time history of midpoint acceleration as shown in Figure 4.2.

Figure 4.3 shows the maximum deflection in the bridge due to moving vehicle loads. The maximum deflection in the bridge increases with increasing vehicle weight and speed. The maximum deflection in the bridge is within the allowable limit $(0.783 \mathrm{~m})$ for 50,60 and 70 tonnes vehicle weights and for all the vehicle speeds from $5 \mathrm{~km} / \mathrm{h}$ to $45 \mathrm{~km} / \mathrm{h}$. It can be seen from Figure 4.3 that the maximum deflection in the bridge has the same trend for different vehicle weights however the maximum deflection increases as the vehicle weight increases. The maximum bending moments developed in the bridge are presented in Figure 4.4 where the maximum bending moment increases with vehicle weight and/or speed. The maximum bending moment in the bridge has the same trend for different 
vehicle weights however the maximum bending moment increases as the vehicle weight increases as shown in Figure 4.4.

Figure 4.5 presents the DAF for both deflection and bending moment in the bridge due to 50-tonne single-axle vehicle loads travelling the bridge at different speeds, however similar figures can be produced for 60- and 70-tonne vehicles. DAF for deflection increases with vehicle speed ranging from 1.02 at $5 \mathrm{~km} / \mathrm{h}$ to 1.68 at $45 \mathrm{~km} / \mathrm{h}$. DAF for bending moment also increases with vehicle speed ranging from 1.24 at $5 \mathrm{~km} / \mathrm{h}$ to 1.66 at $45 \mathrm{~km} / \mathrm{h}$. The values of DAF for bending moment are greater than those for deflection, while the rate of increase of DAF for deflection with increasing the vehicle speed is higher than that for bending moment.

Figure 4.6 shows DAF for deflection for different vehicle weights 50, 60 and 70 tonnes. DAFs for deflection are the same for different vehicle weights, however, the deflections increase as the vehicle weight increases. The same trend was observed for DAFs for bending moment shown in Figure 4.7. This means that the DAFs for both deflection and bending moment are independent of vehicle weight. As a result, vehicle weight can be increased without increasing DAF for either the deflection or the bending moment as long as the allowable limit for total deflection in the bridge is not exceeded. This is not, however true for an increase in vehicle speed. 


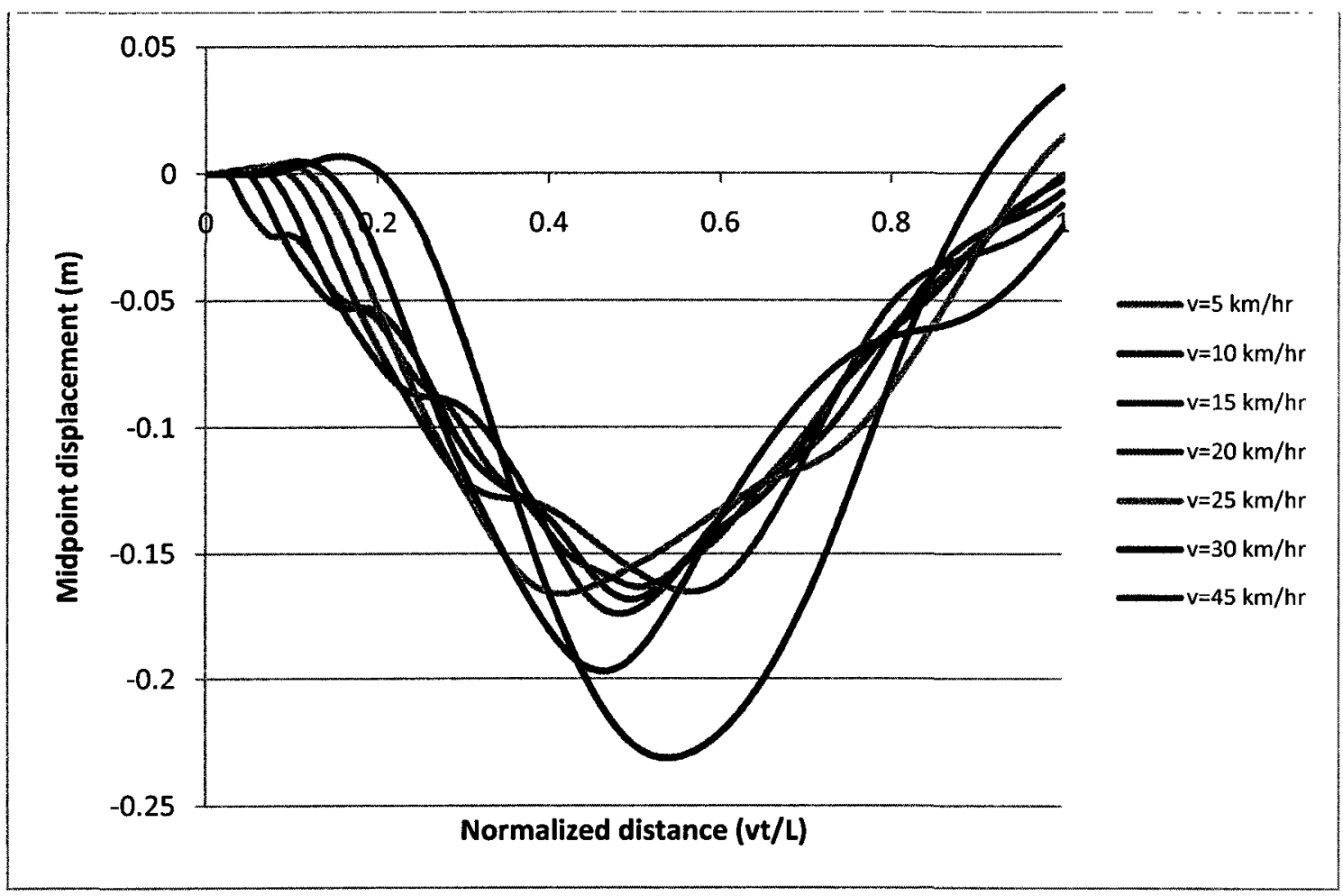

Figure 4.1: Time history of midpoint displacements in rigid-connected bridge due to 50tonne single-axle vehicle

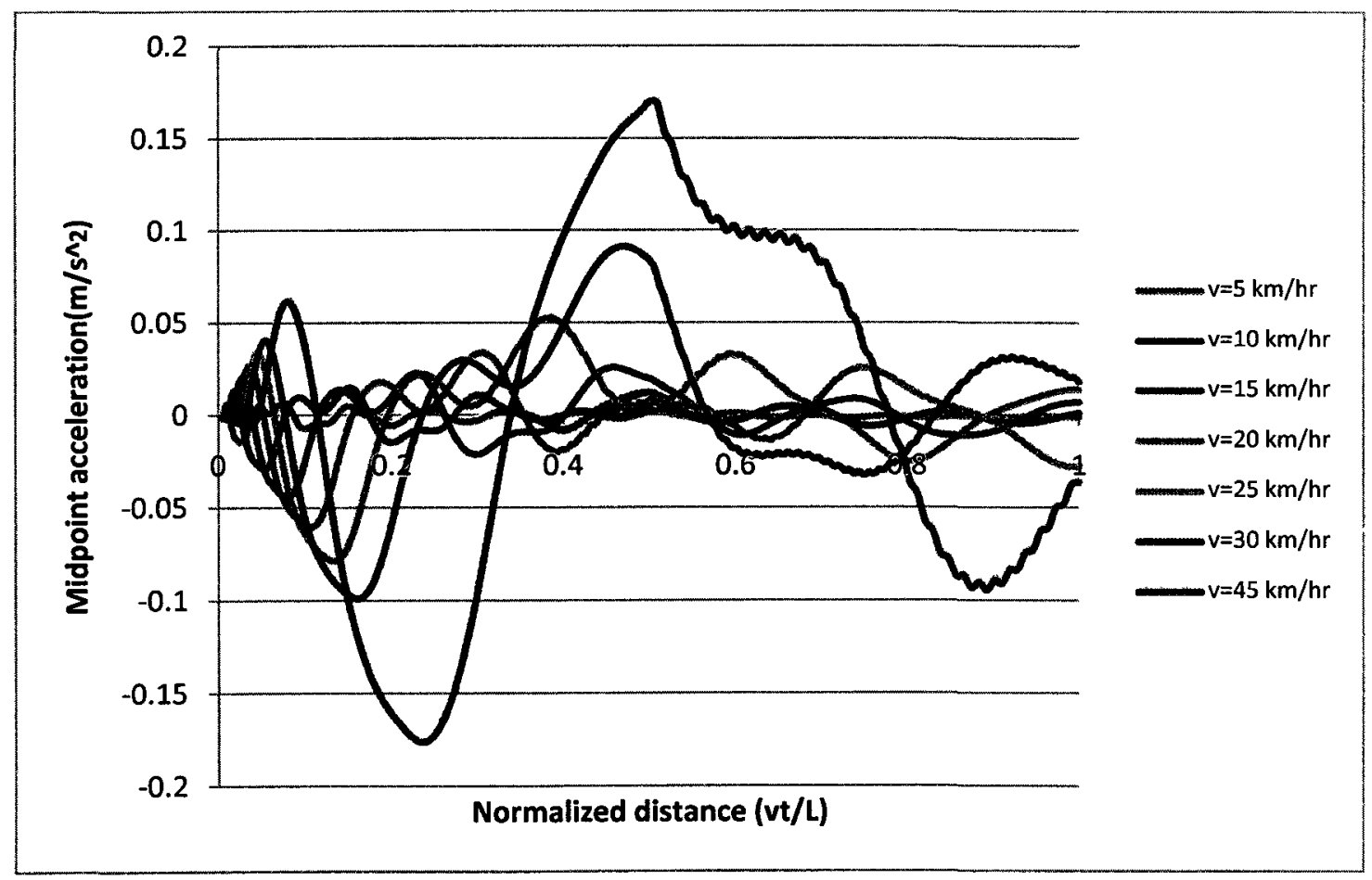

Figure 4.2: Time history of midpoint acceleration in rigid-connected bridge due to 50tonne single-axle vehicle 


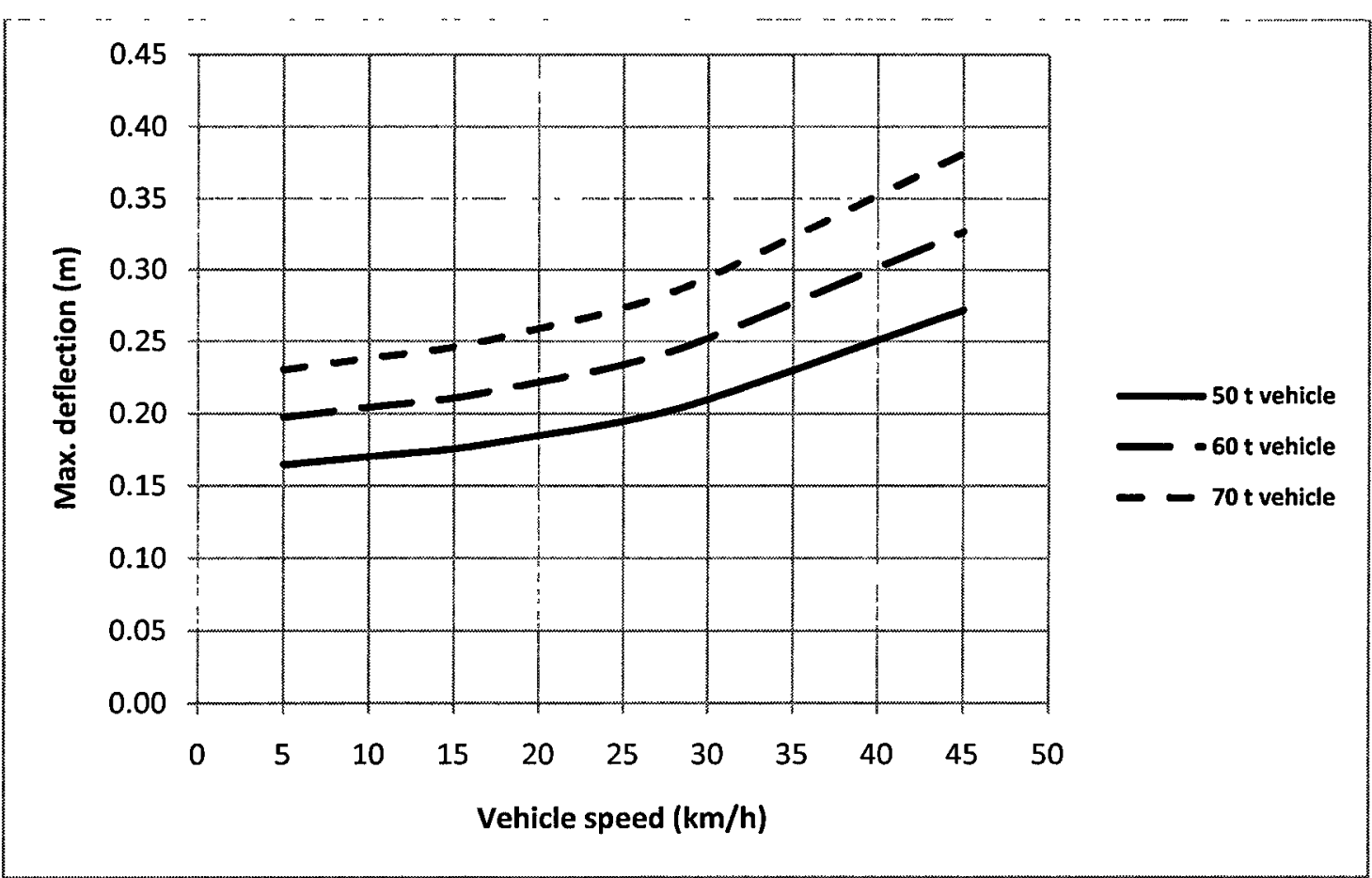

Figure 4.3: Effect of vehicle speed and weight on maximum deflection in rigid-connected bridge due to single-axle vehicle

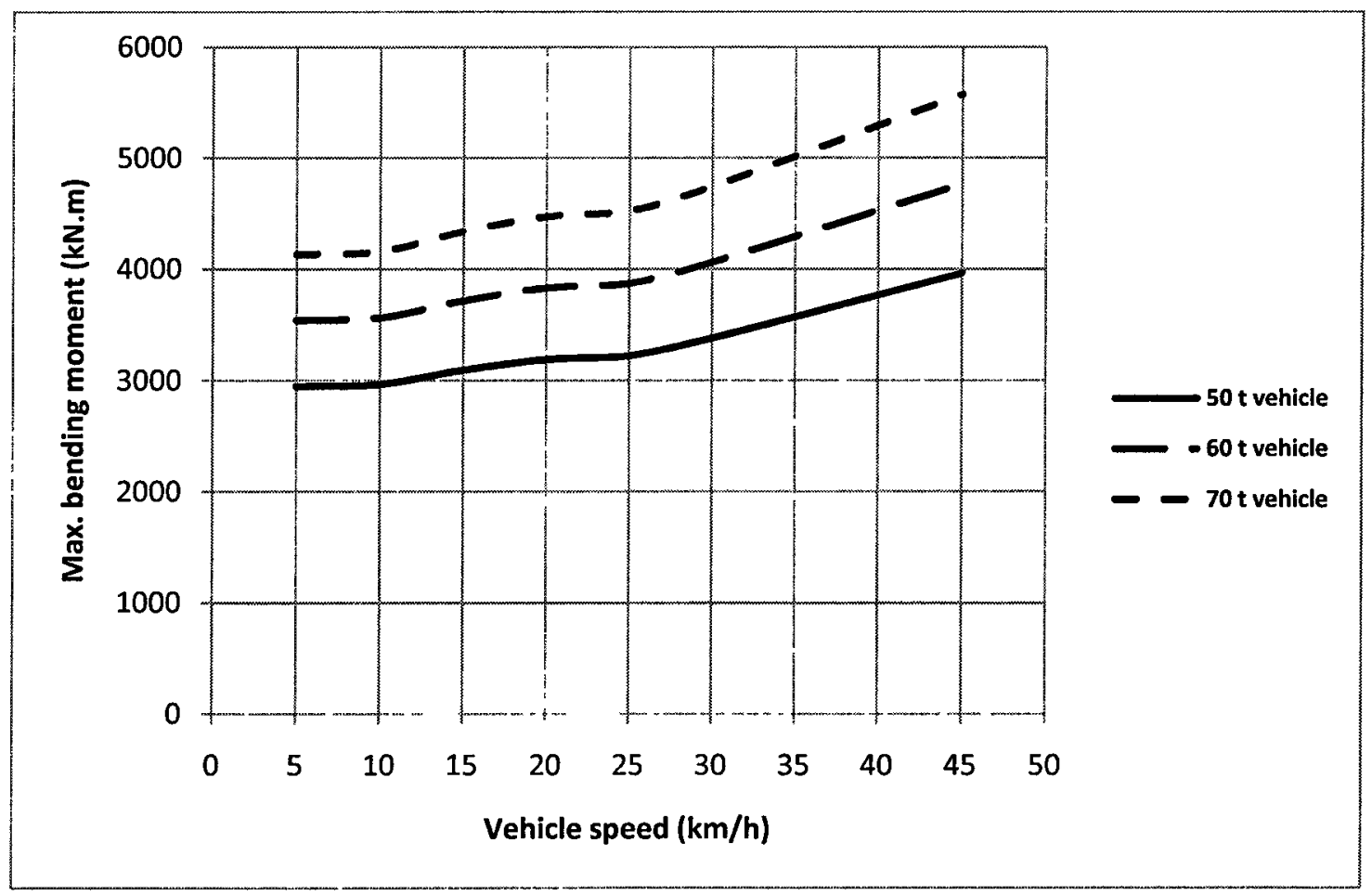

Figure 4.4: Effect of vehicle speed and weight on maximum bending moment in rigidconnected bridge due to single-axle vehicle 


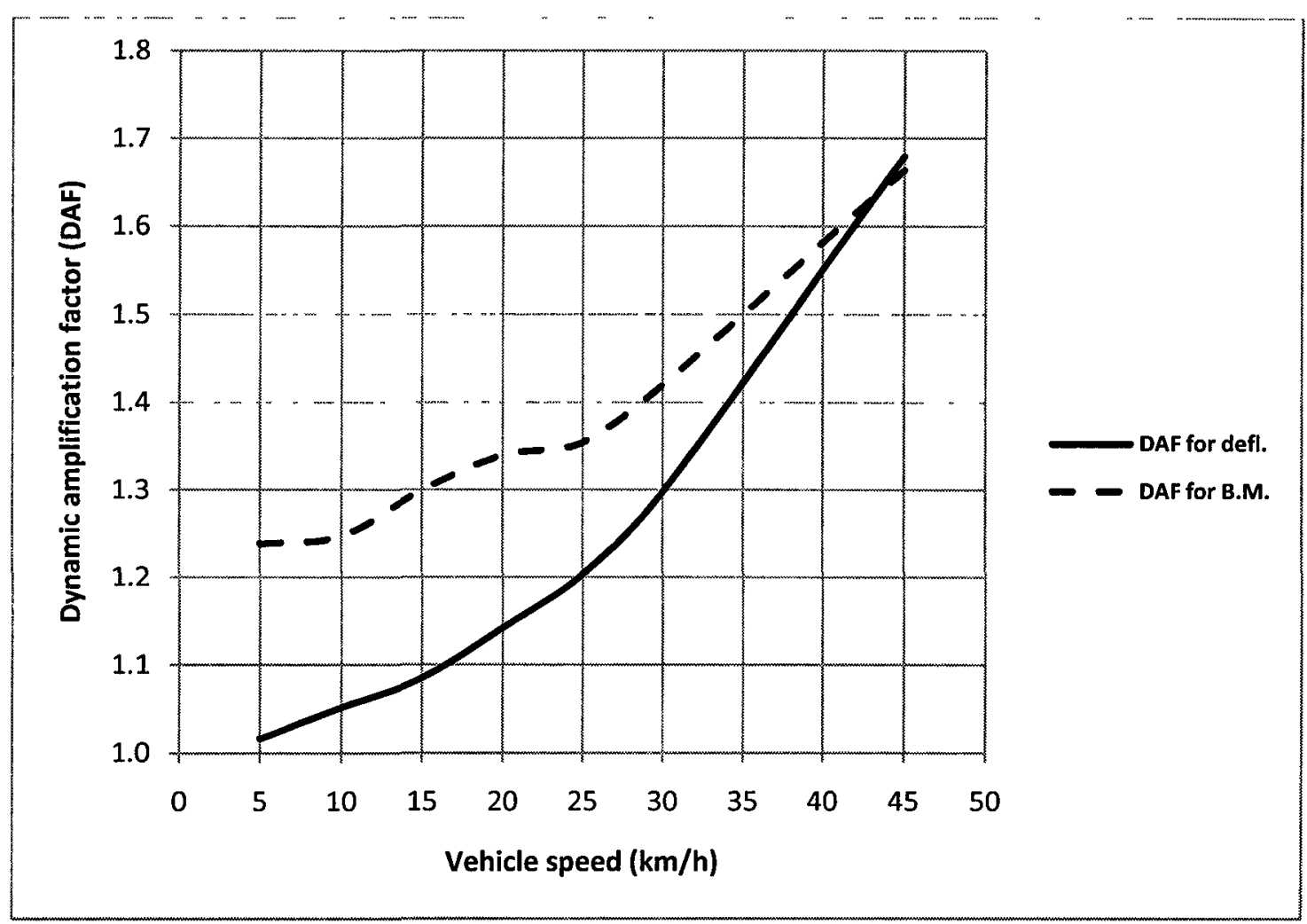

Figure 4.5: DAFs for rigid-connected bridge due to 50-tonne single-axle vehicle

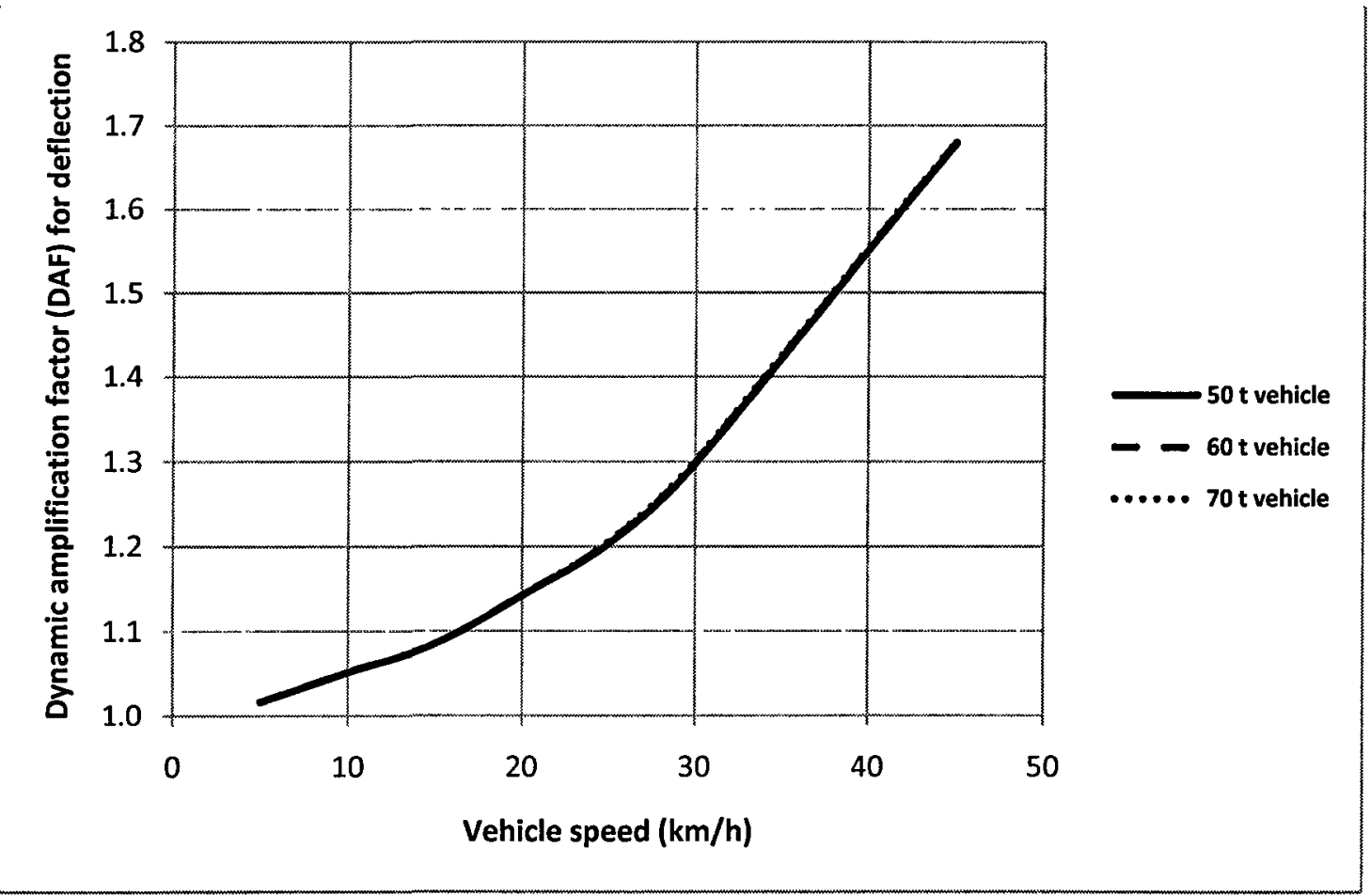

Figure 4.6: DAFs for deflection in rigid-connected bridge due to single-axle vehicle with different weights 


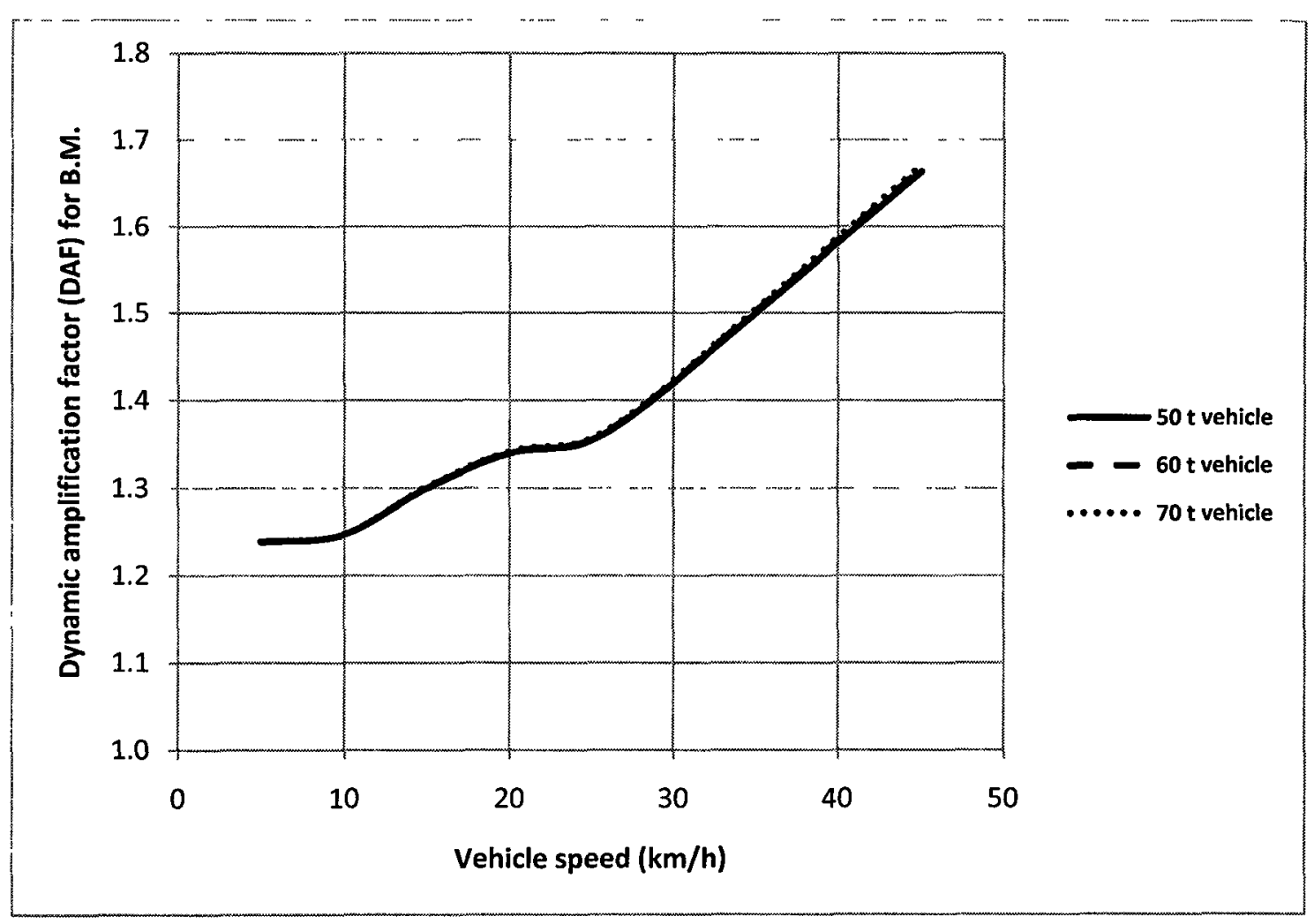

Figure 4.7: DAFs for bending moment in rigid-connected bridge due to single-axle vehicle with different weights

\subsubsection{Dynamic Response of Hinge-Connected Floating Bridge to Single-Axle}

\section{Vehicle Loads}

The dynamic response of hinge-connected floating bridge to heavy single-axle vehicle loads is investigated for different vehicle speeds varying from $5 \mathrm{~km} / \mathrm{h}$ to $30 \mathrm{~km} / \mathrm{h}$. The bridge consists of four typical bays each of length $21 \mathrm{~m}$; these typical bays are hingeconnected to each other to form a bridge of total length $84 \mathrm{~m}$. The performed analyses and the results for the static and dynamic response of the bridge are summarized in Tables 4.5, 4.6 and 4.7. 
Table 4.5: Static and dynamic response of hinge-connected bridge to 50-tonne single-axle vehicle loads

\begin{tabular}{|c|c|c|c|c|c|c|c|c|}
\hline Span & $\begin{array}{c}\text { Vehicle } \\
\text { Weight } \\
(\mathbf{m})\end{array}$ & $\begin{array}{c}\text { Vehicle } \\
\text { speed } \\
(\mathbf{k m} / \mathbf{h})\end{array}$ & $\begin{array}{c}\text { Max } \\
\mathbf{d i s p l} \\
\mathbf{( m )}\end{array}$ & $\begin{array}{c}\text { Static } \\
\mathbf{d i s p l .} \\
(\mathbf{m})\end{array}$ & $\begin{array}{c}\text { DAF for } \\
\text { displ. }\end{array}$ & $\begin{array}{c}\text { Max } \\
\text { B.M. } \\
(\text { KN.m) }\end{array}$ & $\begin{array}{c}\text { Static } \\
\text { B.M. } \\
(\mathbf{K N} . \mathbf{m})\end{array}$ & $\begin{array}{c}\text { DAF for } \\
\text { B.M. }\end{array}$ \\
\hline $\mathbf{8 4}$ & 50 & 5 & -0.552 & -0.514 & 1.07 & -2049 & -1998 & 1.03 \\
\hline 84 & 50 & 10 & -0.570 & -0.514 & 1.11 & -2052 & -1998 & 1.03 \\
\hline 84 & 50 & 15 & -0.567 & -0.514 & 1.10 & -2056 & -1998 & 1.03 \\
\hline 84 & 50 & 20 & -0.732 & -0.514 & 1.42 & -2063 & -1998 & 1.03 \\
\hline 84 & 50 & 25 & -0.805 & -0.514 & 1.57 & -2103 & -1998 & 1.05 \\
\hline 84 & 50 & 30 & -0.813 & -0.514 & 1.58 & -2150 & -1998 & 1.08 \\
\hline
\end{tabular}

Table 4.6: Static and dynamic response of hinge-connected bridge to 60-tonne single-axle vehicle loads

\begin{tabular}{|c|c|c|c|c|c|c|c|c|}
\hline $\begin{array}{c}\text { Span } \\
(\mathrm{m})\end{array}$ & $\begin{array}{c}\text { Vehicle } \\
\text { Weight } \\
(t)\end{array}$ & $\begin{array}{c}\text { Vehicle } \\
\text { speed } \\
(\mathrm{km} / \mathrm{h})\end{array}$ & $\begin{array}{c}\text { Max } \\
\text { displ. } \\
(\mathbf{m})\end{array}$ & $\begin{array}{c}\text { Static } \\
\text { displ. } \\
\text { (m) }\end{array}$ & $\begin{array}{l}\text { DAF for } \\
\text { displ. }\end{array}$ & $\begin{array}{c}\text { Max } \\
\text { B.M. } \\
\text { (KN.m) }\end{array}$ & $\begin{array}{c}\text { Static } \\
\text { B.M. } \\
\text { (KN.m) }\end{array}$ & $\begin{array}{c}\text { DAF for } \\
\text { B.M. }\end{array}$ \\
\hline 84 & 60 & 5 & -0.664 & -0.616 & 1.08 & -2459 & -2398 & 1.03 \\
\hline 84 & 60 & 10 & -0.686 & -0.616 & 1.11 & -2463 & -2398 & 1.03 \\
\hline 84 & 60 & 15 & -0.682 & -0.616 & 1.11 & -2465 & -2398 & 1.03 \\
\hline 84 & 60 & 20 & -0.882 & -0.616 & 1.43 & -2469 & -2398 & 1.03 \\
\hline 84 & 60 & 25 & -0.965 & -0.616 & 1.57 & -2499 & -2398 & 1.04 \\
\hline 84 & 60 & 30 & -0.976 & -0.616 & 1.58 & -2580 & -2398 & 1.08 \\
\hline
\end{tabular}


Table 4.7: Static and dynamic response of hinge-connected bridge to 70-tonne single-axle vehicle loads

\begin{tabular}{|c|c|c|c|c|c|c|c|c|}
\hline $\begin{array}{c}\text { Span } \\
(\mathbf{m})\end{array}$ & $\begin{array}{c}\text { Vehicle } \\
\text { Weight } \\
(\mathbf{t})\end{array}$ & $\begin{array}{c}\text { Vehicle } \\
\mathbf{\text { speed }}(\mathbf{k m} / \mathbf{h})\end{array}$ & $\begin{array}{c}\text { Max } \\
\mathbf{d i s p l .} \\
(\mathbf{m})\end{array}$ & $\begin{array}{c}\text { Static } \\
\mathbf{d i s p l} \\
(\mathbf{m})\end{array}$ & $\begin{array}{c}\text { DAF for } \\
\mathbf{d i s p l .}\end{array}$ & $\begin{array}{c}\text { Max } \\
\mathbf{B . M} . \\
(\mathbf{K N . m})\end{array}$ & $\begin{array}{c}\text { Static } \\
\text { B.M. } \\
(\mathbf{K N} . \mathbf{m})\end{array}$ & $\begin{array}{c}\text { DAF for } \\
\text { B.M. }\end{array}$ \\
\hline 84 & 70 & 5 & -0.776 & -0.719 & 1.08 & -2868 & -2797 & 1.03 \\
\hline 84 & 70 & 10 & -0.803 & -0.719 & 1.12 & -2873 & -2797 & 1.03 \\
\hline 84 & 70 & 15 & -0.798 & -0.719 & 1.11 & -2873 & -2797 & 1.03 \\
\hline 84 & 70 & 20 & -1.033 & -0.719 & 1.44 & -2911 & -2797 & 1.04 \\
\hline 84 & 70 & 25 & -1.127 & -0.719 & 1.57 & -2957 & -2797 & 1.06 \\
\hline 84 & 70 & 30 & -1.139 & -0.719 & 1.58 & -3024 & -2797 & 1.08 \\
\hline
\end{tabular}

\subsubsection{Discussion of Results}

The results obtained from the analysis are used to explain the dynamic response of hingeconnected floating bridges to single-axle vehicle loads, and the effect of increasing the vehicle weight and speed on that dynamic response.

Figures 4.8 and 4.9 show the time history of midpoint displacement and acceleration in the hinge-connected bridge, respectively due to the passage of 50-tonne single-axle vehicle at speeds up to $15 \mathrm{~km} / \mathrm{h}$. At higher vehicle speeds, the bridge displacement violates the allowable limit $(0.769 \mathrm{~m})$. Figure 4.10 shows the maximum displacement in the bridge due to moving vehicle loads. The maximum displacement in the bridge is almost constant up to $15 \mathrm{~km} / \mathrm{h}$, and increases with vehicle speed $20 \mathrm{~km} / \mathrm{h}$ and up. It can be seen from Figure 4.10 that the maximum displacement in the bridge has the same trend for different vehicle weights however it increases as the vehicle weight increases. 
The maximum displacement in the bridge is within the allowable limit $(0.769 \mathrm{~m})$ for a 50-tonne vehicle traversing the bridge at a speed from $5 \mathrm{~km} / \mathrm{h}$ to $20 \mathrm{~km} / \mathrm{h}$ but exceeds the allowable limit for higher speeds. For the 60 -tonne vehicle, the maximum displacement in the bridge is within the allowable limit for speeds from $5 \mathrm{~km} / \mathrm{h}$ to $15 \mathrm{~km} / \mathrm{h}$ but exceeds the allowable limit for higher speeds. While for the 70-tonne vehicle, only the speed of 5 $\mathrm{km} / \mathrm{h}$ results in an accepted displacement in the bridge. This means that the hingeconnected bridge can support a 50-tonne vehicle load travelling at a speed up to $20 \mathrm{~km} / \mathrm{h}$, or 60-tonne vehicle loads travelling at a speed up to $15 \mathrm{~km} / \mathrm{h}$, or 70 -tonne vehicle loads travelling at a speed of $5 \mathrm{~km} / \mathrm{h}$.

The maximum bending moment developed in the bridge is presented in Figure 4.11 which shows that the maximum bending moment is almost constant with increasing vehicle speed. Only minor increase in the maximum bending moment is notices with increasing vehicle speed. As shown in Figure 4.11, the maximum bending moment in the bridge has the same trend for different vehicle weights however it increases as the vehicle weight increases.

Figure 4.12 shows the DAF for both displacement and bending moment in the bridge due to a 50-tonne single-axle vehicle load travelling the bridge at different speeds from 5 $\mathrm{km} / \mathrm{h}$ to $30 \mathrm{~km} / \mathrm{h}$; however similar figures can be produced for 60 - and 70 -tonne vehicles. The DAF for displacement increases with vehicle speed, from 1.07 at $5 \mathrm{~km} / \mathrm{h}$ to 1.58 at $30 \mathrm{~km} / \mathrm{h}$. However, it should be noted that the bridge can only support a 50 -tonne vehicle up to a speed of $20 \mathrm{~km} / \mathrm{h}$ where the maximum bridge displacements are within the allowable limit. On the other hand, the DAF for bending moment is almost constant with 
increasing vehicle speed. Only minor increase is noticed in the DAF for bending moment as the vehicle speed increases, from 1.03 at $5 \mathrm{~km} / \mathrm{h}$ to 1.08 at $30 \mathrm{~km} / \mathrm{h}$. These results imply that increasing vehicle speed almost does not affect the DAF for bending moment, and the bending moments in the hinge-connected floating bridge are not magnified due the dynamic interaction of the vehicle with the bridge. This is because of the existence of the intermediate hinges where the bending moment is equal to zero that result in reducing the length of the bridge in which the bending moment is developed.

Figure 4.13 shows the DAF for displacement for different vehicle weights 50,60 and 70 tonnes. The DAFs for displacement are the same for different vehicle weights, however, the displacement values increase as the vehicle weight increases. The same trend was observed for DAFs for bending moment shown in Figure 4.14, with only minor differences at vehicle speeds of $20 \mathrm{~km} / \mathrm{h}$ or more. This means that increasing the vehicle weight has almost no effect on the DAFs for both displacement and bending moment. As a result, vehicle weight can be increased without increasing the DAF for either the displacement or the bending moment as long as the bridge displacement is within the allowable limit for safe operation of the bridge.

On comparing Figures 4.3 and 4.10 , it is seen that the maximum displacements in the hinge-connected floating bridge are much higher than those in the rigid-connected floating bridge. At the same time, comparison of Figures 4.4 and 4.11 shows that the maximum bending moments in the hinge-connected floating bridge are much lower than those in the rigid-connected floating bridge because of the existence of the intermediate hinges where the bending moment is equal to zero. 


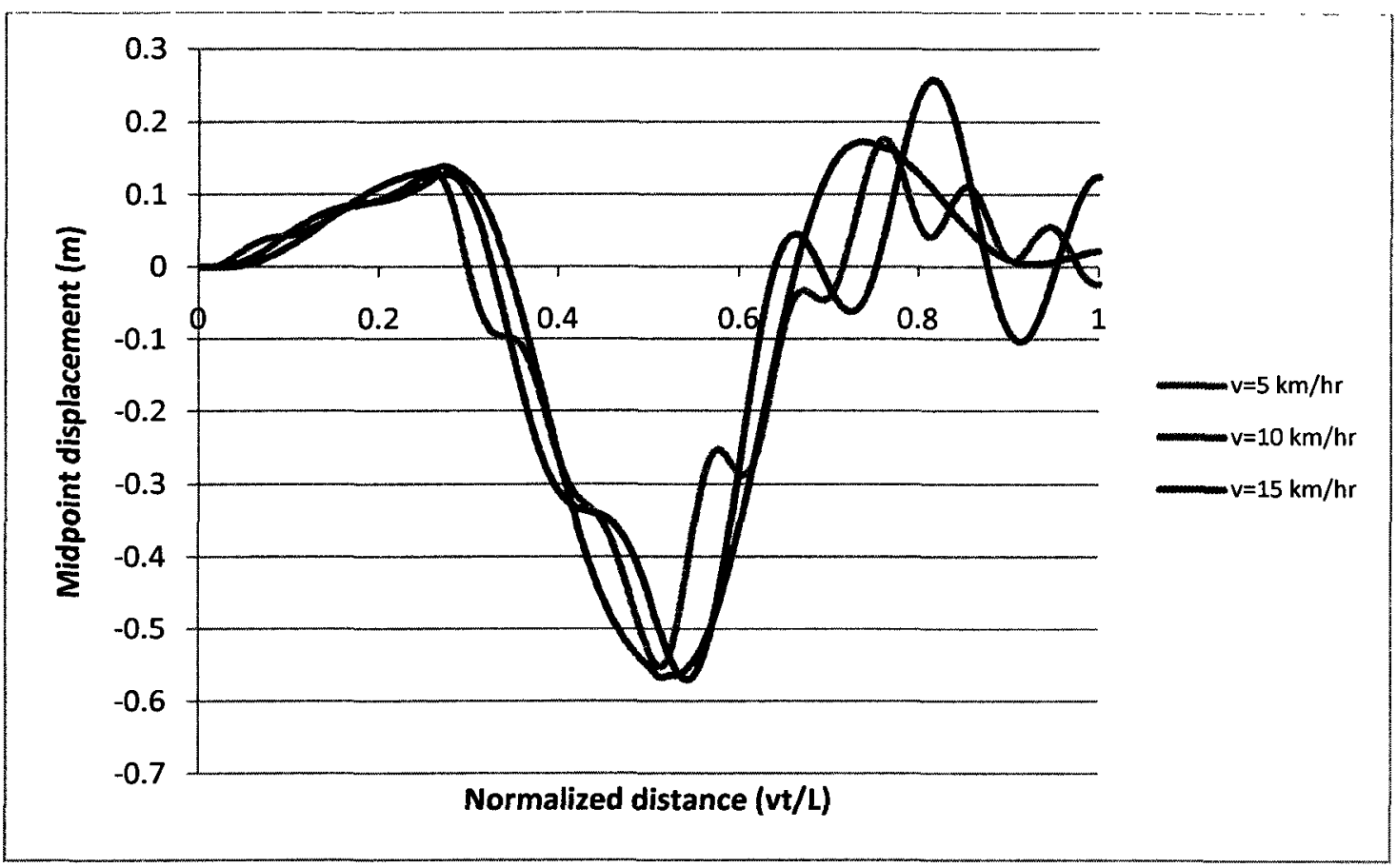

Figure 4.8: Time history of midpoint displacements in hinge-connected bridge due to 50tonne single-axle vehicle

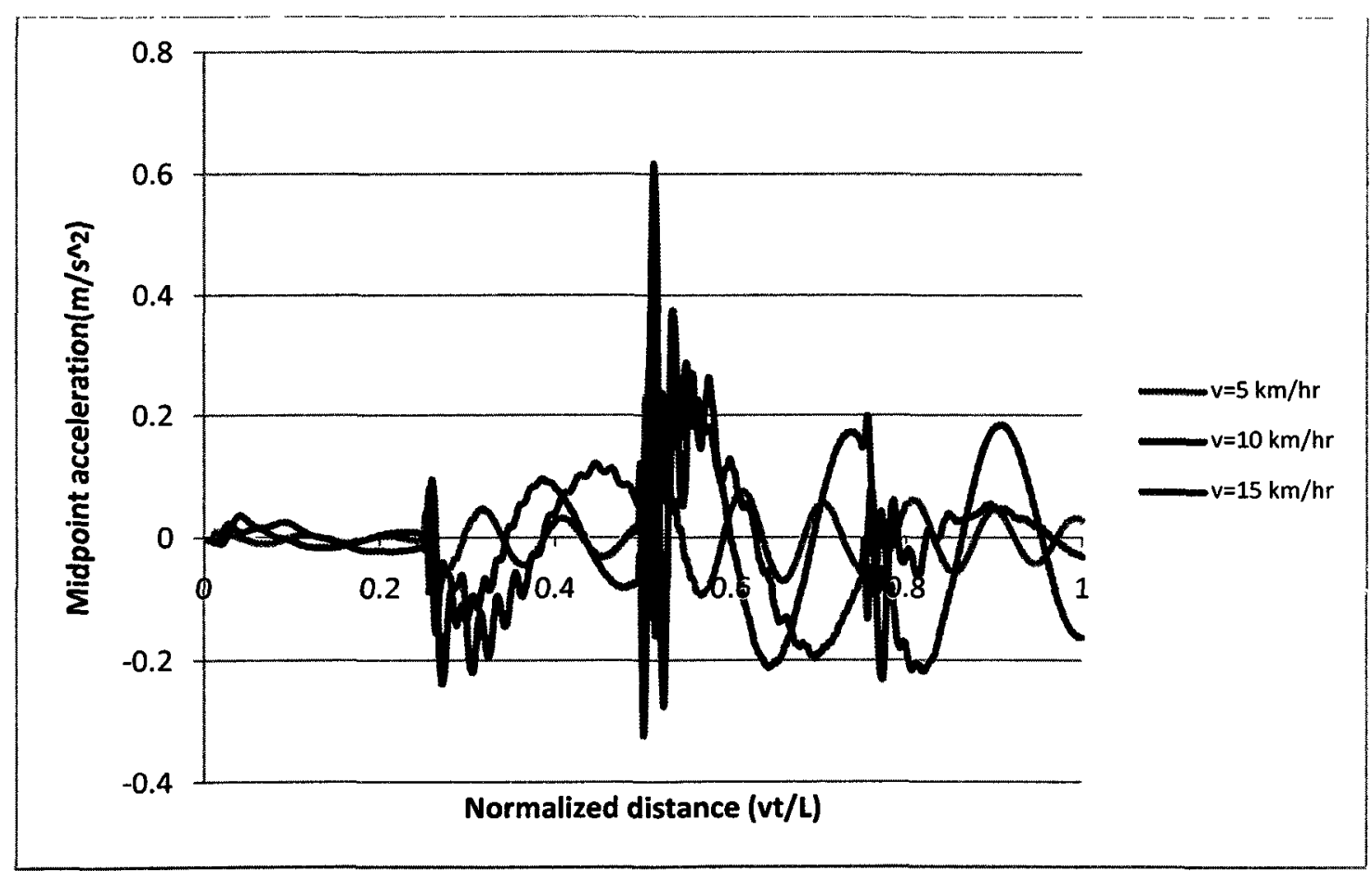

Figure 4.9: Time history of midpoint accelerations in hinge-connected bridge due to 50tonne single-axle vehicle 


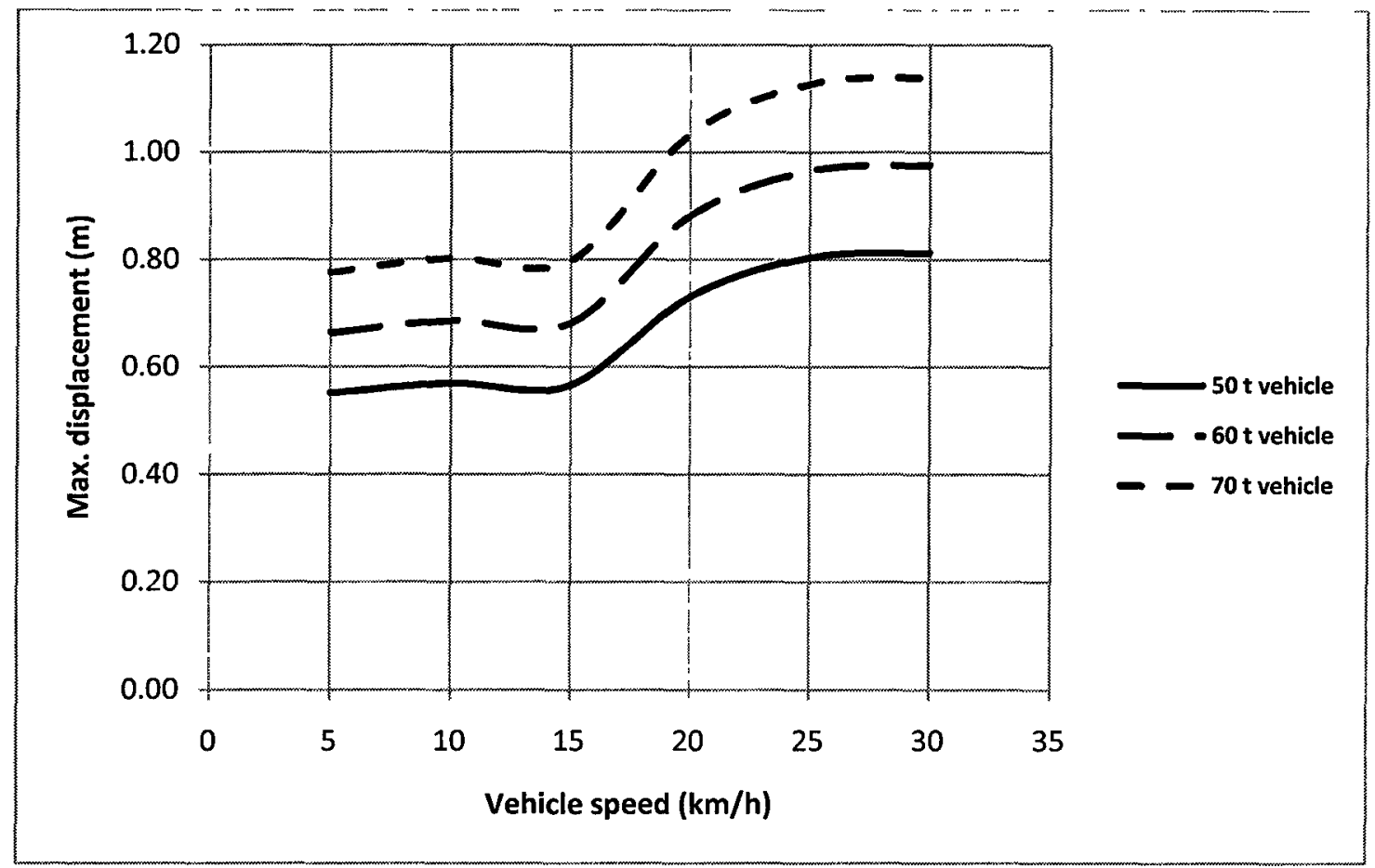

Figure 4.10: Effect of vehicle speed and weight on maximum displacement in hingeconnected bridge due to 50-tonne single-axle vehicle

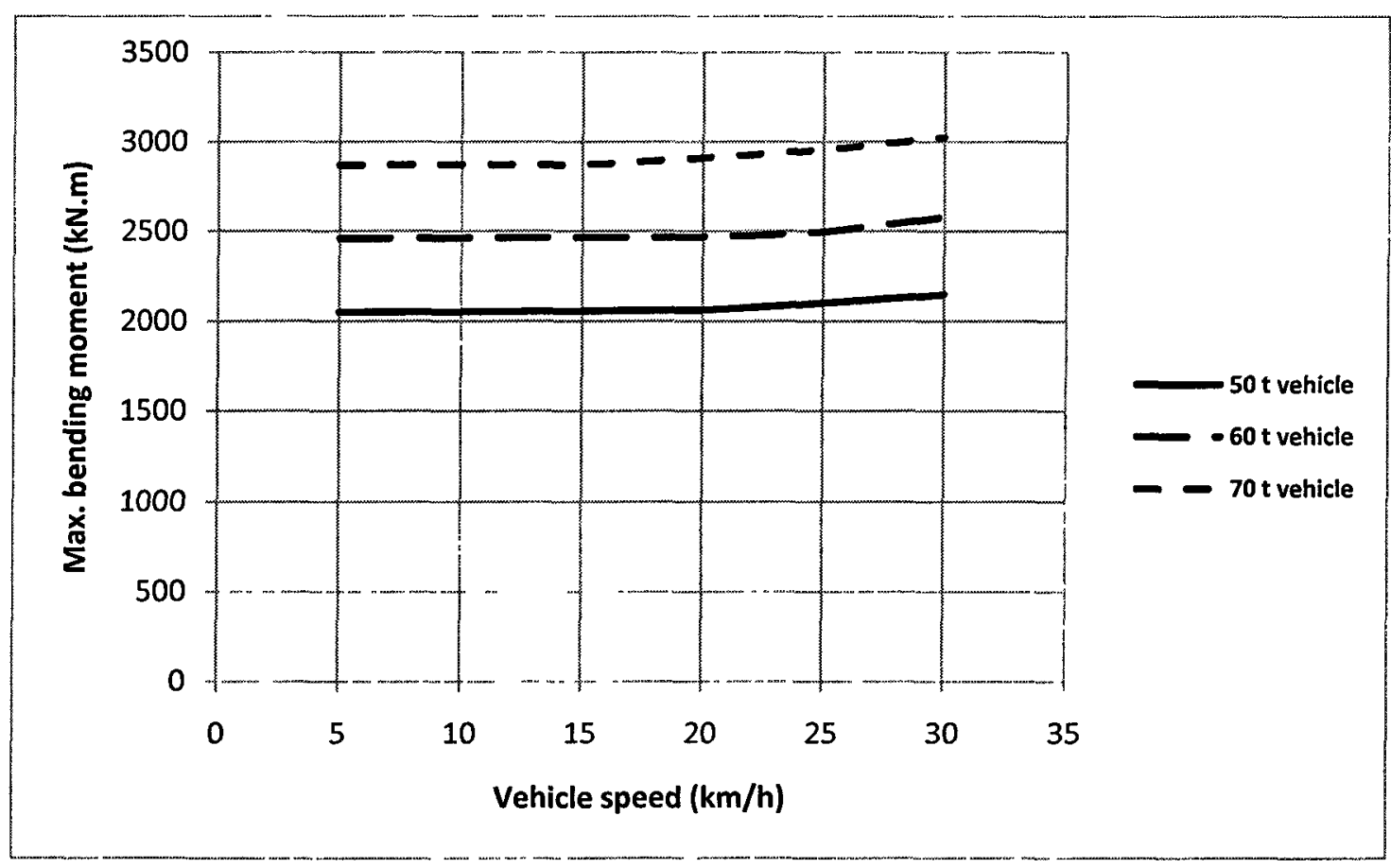

Figure 4.11: Effect of vehicle speed and weight on maximum bending moment in hingeconnected bridge due to 50-tonne single-axle vehicle 


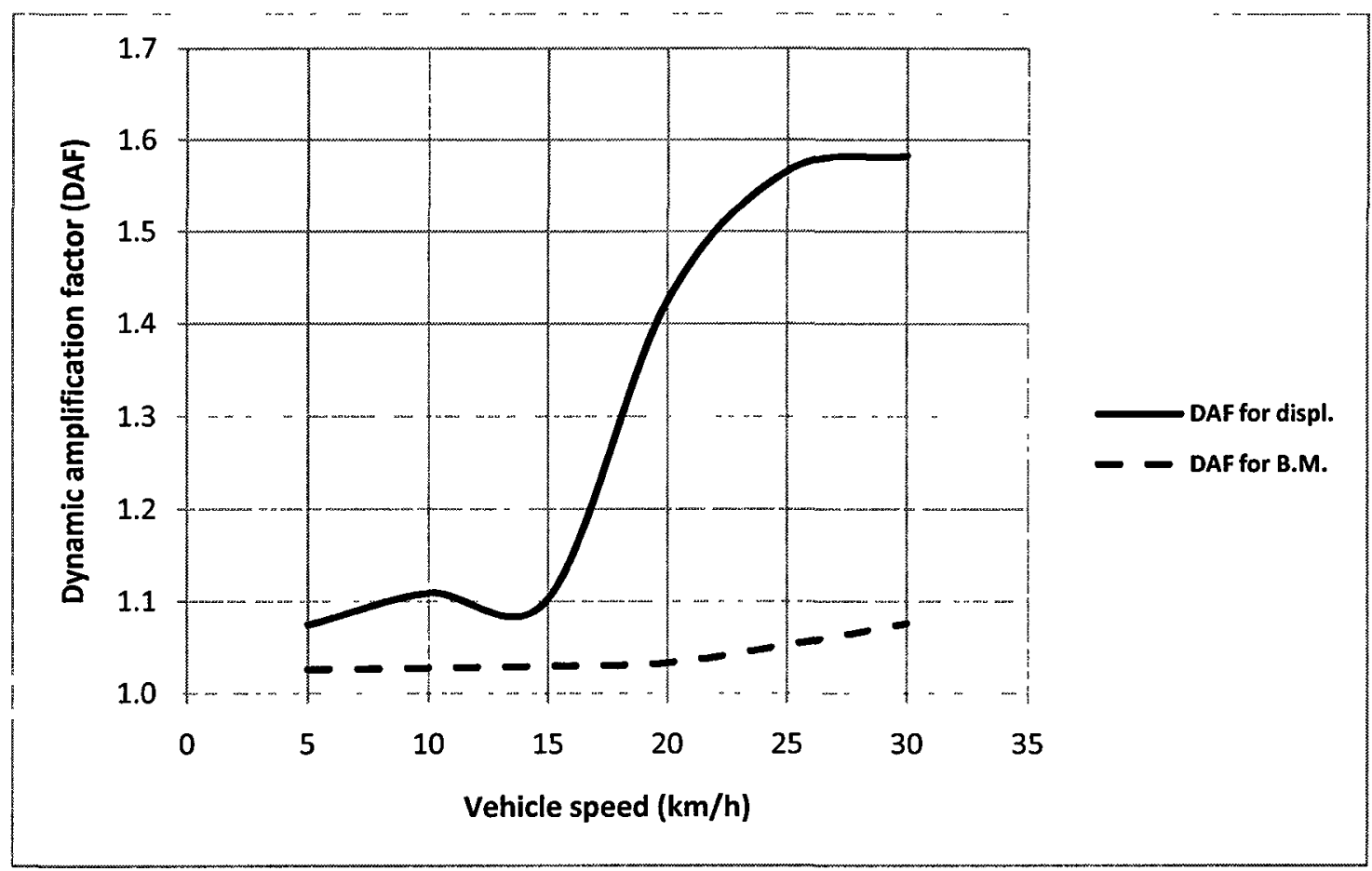

Figure 4.12: DAFs in hinge-connected bridge due to 50-tonne single-axle vehicle

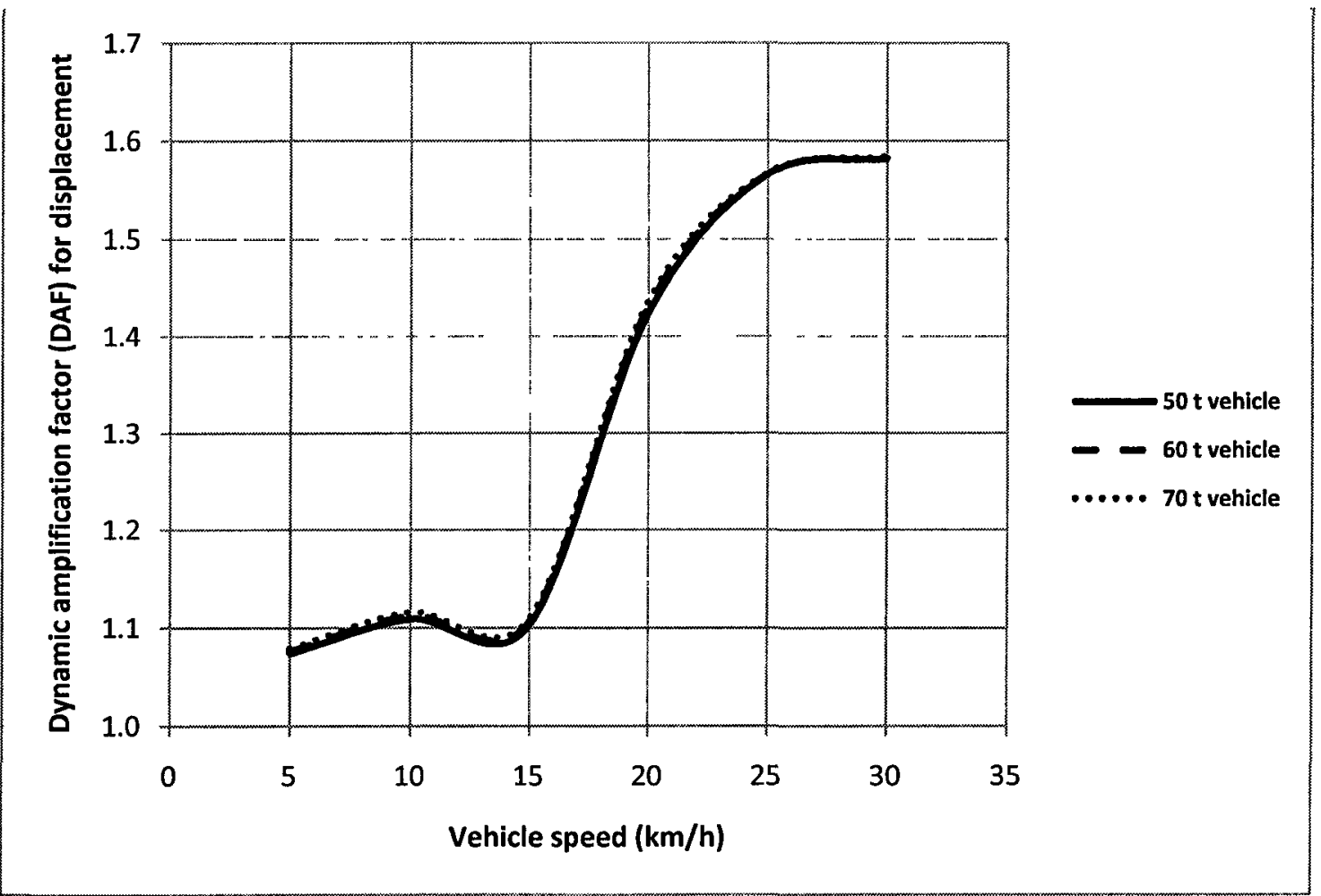

Figure 4.13: DAFs for displacement in hinge-connected bridge due to 50-tonne singleaxle vehicle 


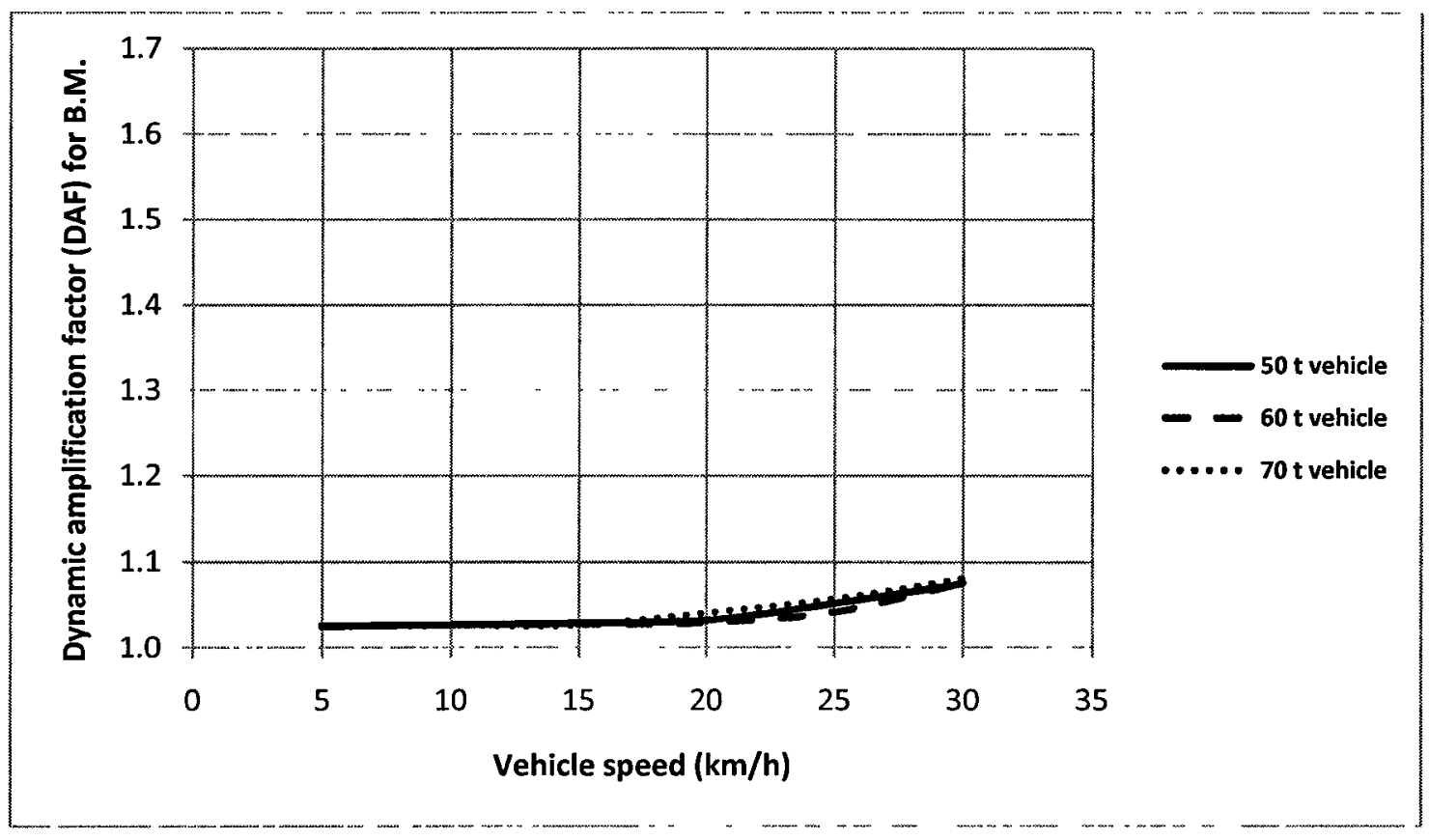

Figure 4.14: DAFs for bending moment in hinge-connected bridge due to 50 -tonne single-axle vehicle

\subsection{Parametric Study for the Dynamic Response of Floating Bridges to Two-Axle}

\section{Vehicle Loads}

The objective of the parametric study presented in this section is to investigate the effect of increasing vehicle weight and speed on DAFs and the dynamic response of floating bridges to two-axle vehicle loads. Another objective is to compare the dynamic response of floating bridges to single-axle and two-axle vehicle loads. The two-axle model of the vehicle used in this analysis consists of two unsprung mass connected to a sprung mass through two spring and damper units that represent the dynamic characteristics of the vehicle (see Figure 3.9). A vehicle with two-axle represents a more accurate model of the vehicle. 


\subsubsection{Dynamic Response of Rigid-Connected Floating Bridge to Two-Axle Vehicle}

\section{Loads}

In this section, the effect of distributing the vehicle weight on two axles instead of one axle on the dynamic response of rigid-connected bridge is investigated. The performed analyses and the results are summarized in Tables 4.8, 4.9 and 4.10.

Table 4.8: Static and dynamic response of rigid-connected bridge to 50-tonne two-axle vehicle loads

\begin{tabular}{|c|c|c|c|c|c|c|c|c|}
\hline $\begin{array}{c}\text { Span } \\
(\mathrm{m}) \\
\end{array}$ & $\begin{array}{c}\text { Vehicle } \\
\text { Weight } \\
(t) \\
\end{array}$ & $\begin{array}{c}\text { Vehicle } \\
\text { speed } \\
(\mathbf{k m} / \mathbf{h}) \\
\end{array}$ & $\begin{array}{l}\text { Max } \\
\text { defl. } \\
(\mathrm{m}) \\
\end{array}$ & $\begin{array}{c}\begin{array}{c}\text { Static } \\
\text { defl. } \\
(\mathrm{m})\end{array} \\
\end{array}$ & $\begin{array}{c}\text { DAF } \\
\text { for defl. }\end{array}$ & $\begin{array}{c}\text { Max } \\
\text { B.M. } \\
\text { (KN.m) }\end{array}$ & $\begin{array}{c}\text { Static } \\
\text { B.M. } \\
\text { (KN.m) }\end{array}$ & $\begin{array}{c}\text { DAF for } \\
\text { B.M. }\end{array}$ \\
\hline 84 & 50 & 5 & -0.161 & -0.159 & 1.02 & -2322 & -1778 & 1.31 \\
\hline 84 & 50 & 10 & -0.162 & -0.159 & 1.02 & -2345 & -1778 & 1.32 \\
\hline 84 & 50 & 15 & -0.169 & -0.159 & 1.06 & -2384 & -1778 & 1.34 \\
\hline 84 & 50 & 20 & -0.178 & -0.159 & 1.12 & -2514 & -1778 & 1.41 \\
\hline 84 & 50 & 25 & -0.188 & -0.159 & 1.18 & -2618 & -1778 & 1.47 \\
\hline 84 & 50 & 30 & -0.203 & -0.159 & 1.28 & -2756 & -1778 & 1.55 \\
\hline 84 & 50 & 45 & -0.264 & -0.159 & 1.66 & -3329 & -1778 & 1.87 \\
\hline
\end{tabular}


Table 4.9: Static and dynamic response of rigid-connected bridge to 60-tonne two-axle vehicle loads

\begin{tabular}{|c|c|c|c|c|c|c|c|c|}
\hline Span & $\begin{array}{c}\text { Vehicle } \\
\text { Weight } \\
(\mathbf{m})\end{array}$ & $\begin{array}{c}\text { Vehicle } \\
\text { speed } \\
(\mathbf{k m} / \mathbf{h})\end{array}$ & $\begin{array}{c}\text { Max } \\
\mathbf{d e f l} \\
(\mathbf{m})\end{array}$ & $\begin{array}{c}\text { Static } \\
\text { defl. } \\
(\mathbf{m})\end{array}$ & $\begin{array}{c}\text { DAF } \\
\text { for defl. }\end{array}$ & $\begin{array}{c}\text { Max } \\
\text { B.M. } \\
(\mathbf{K N . m})\end{array}$ & $\begin{array}{c}\text { Static } \\
\text { B.M. } \\
(\mathbf{K N . m})\end{array}$ & $\begin{array}{c}\text { DAF for } \\
\text { B.M. }\end{array}$ \\
\hline 84 & 60 & 5 & -0.194 & -0.191 & 1.01 & -2787 & -2134 & 1.31 \\
\hline 84 & 60 & 10 & -0.194 & -0.191 & 1.02 & -2815 & -2134 & 1.32 \\
\hline 84 & 60 & 15 & -0.203 & -0.191 & 1.06 & -2862 & -2134 & 1.34 \\
\hline 84 & 60 & 20 & -0.214 & -0.191 & 1.12 & -3019 & -2134 & 1.42 \\
\hline 84 & 60 & 25 & -0.225 & -0.191 & 1.18 & -3142 & -2134 & 1.47 \\
\hline 84 & 60 & 30 & -0.244 & -0.191 & 1.28 & -3311 & -2134 & 1.55 \\
\hline 84 & 60 & 45 & -0.317 & -0.191 & 1.66 & -4002 & -2134 & 1.88 \\
\hline
\end{tabular}

Table 4.10: Static and dynamic response of rigid-connected bridge to 70-tonne two-axle vehicle loads

\begin{tabular}{|c|c|c|c|c|c|c|c|c|}
\hline Span & $\begin{array}{c}\text { Vehicle } \\
\text { Weight } \\
(\mathbf{m})\end{array}$ & $\begin{array}{c}\text { Vehicle } \\
\mathbf{\text { speed }} \\
(\mathbf{k m} / \mathbf{h})\end{array}$ & $\begin{array}{c}\text { Max } \\
\mathbf{d e f l} . \\
(\mathbf{m})\end{array}$ & $\begin{array}{c}\text { Static } \\
\text { deff. } \\
(\mathbf{m})\end{array}$ & $\begin{array}{c}\text { DAF } \\
\text { for defl. }\end{array}$ & $\begin{array}{c}\text { Max } \\
\mathbf{B . M} . \\
(\mathbf{K N . m})\end{array}$ & $\begin{array}{c}\text { Static } \\
\text { B.M. } \\
(\mathbf{K N} . \mathbf{m})\end{array}$ & $\begin{array}{c}\text { DAF for } \\
\text { B.M. }\end{array}$ \\
\hline 84 & 70 & 5 & -0.226 & -0.223 & 1.01 & -3251 & -2489 & 1.31 \\
\hline 84 & 70 & 10 & -0.226 & -0.223 & 1.02 & -3285 & -2489 & 1.32 \\
\hline 84 & 70 & 15 & -0.236 & -0.223 & 1.06 & -3340 & -2489 & 1.34 \\
\hline 84 & 70 & 20 & -0.249 & -0.223 & 1.12 & -3525 & -2489 & 1.42 \\
\hline 84 & 70 & 25 & -0.263 & -0.223 & 1.18 & -3670 & -2489 & 1.47 \\
\hline 84 & 70 & 30 & -0.285 & -0.223 & 1.28 & -3868 & -2489 & 1.55 \\
\hline 84 & 70 & 45 & -0.370 & -0.223 & 1.66 & -4677 & -2489 & 1.88 \\
\hline
\end{tabular}




\subsubsection{Discussion of Results}

The midpoint displacement and acceleration in the bridge increase as the vehicle speed increases. Figure 4.15 shows a typical time history of midpoint displacement due to the passage of 50-tonne two-axle vehicle at $15 \mathrm{~km} / \mathrm{h}$, while Figure 4.16 shows a typical time history of midpoint acceleration due to 50-tonne two-axle vehicle travelling the bridge at the same speed. The time $t$ is measured with respect to the time the first vehicle-axle enter the bridge.

Figure 4.17 shows the maximum deflection in the bridge due to moving two-axle vehicle loads. The maximum deflection in the bridge increases with vehicle weight and speed. The maximum deflection in the bridge is within the allowable limit $(0.783 \mathrm{~m})$ for 50,60 and 70 tonne vehicle weights and for all speeds from $5 \mathrm{~km} / \mathrm{h}$ to $45 \mathrm{~km} / \mathrm{h}$. The maximum bending moment developed in the bridge is presented in Figure 4.18, which shows that the maximum bending moment increases with vehicle weight and speed.

Figure 4.19 shows the DAFs for both deflection and bending moment in the bridge due to 50-tonne two-axle vehicle loads travelling the bridge at different speeds; however similar figures can be produced for 60- and 70-tonne vehicles. The DAF for deflection increases with vehicle speed ranging from 1.02 at $5 \mathrm{~km} / \mathrm{h}$ to 1.66 at $45 \mathrm{~km} / \mathrm{h}$. The DAF for bending moment also increases with vehicle speed ranging from 1.31 at $5 \mathrm{~km} / \mathrm{h}$ to 1.88 at 45 $\mathrm{km} / \mathrm{h}$. This means that an increase in the vehicle speed has a strong effect on the increase in DAFs for both deflection and bending moment. The values of DAF for bending moment are greater than those for deflection. 
Figure 4.20 shows the DAF for deflection for different vehicle weights. The DAFs for deflection are the same for different vehicle weights, however, the deflections increase as the vehicle weight increases. The same trend is observed for DAFs for bending moment, as shown in Figure 4.21. This means that the DAFs for both deflection and bending moment are independent of vehicle weight as long as the maximum deflection in the bridge is within the allowable limit.

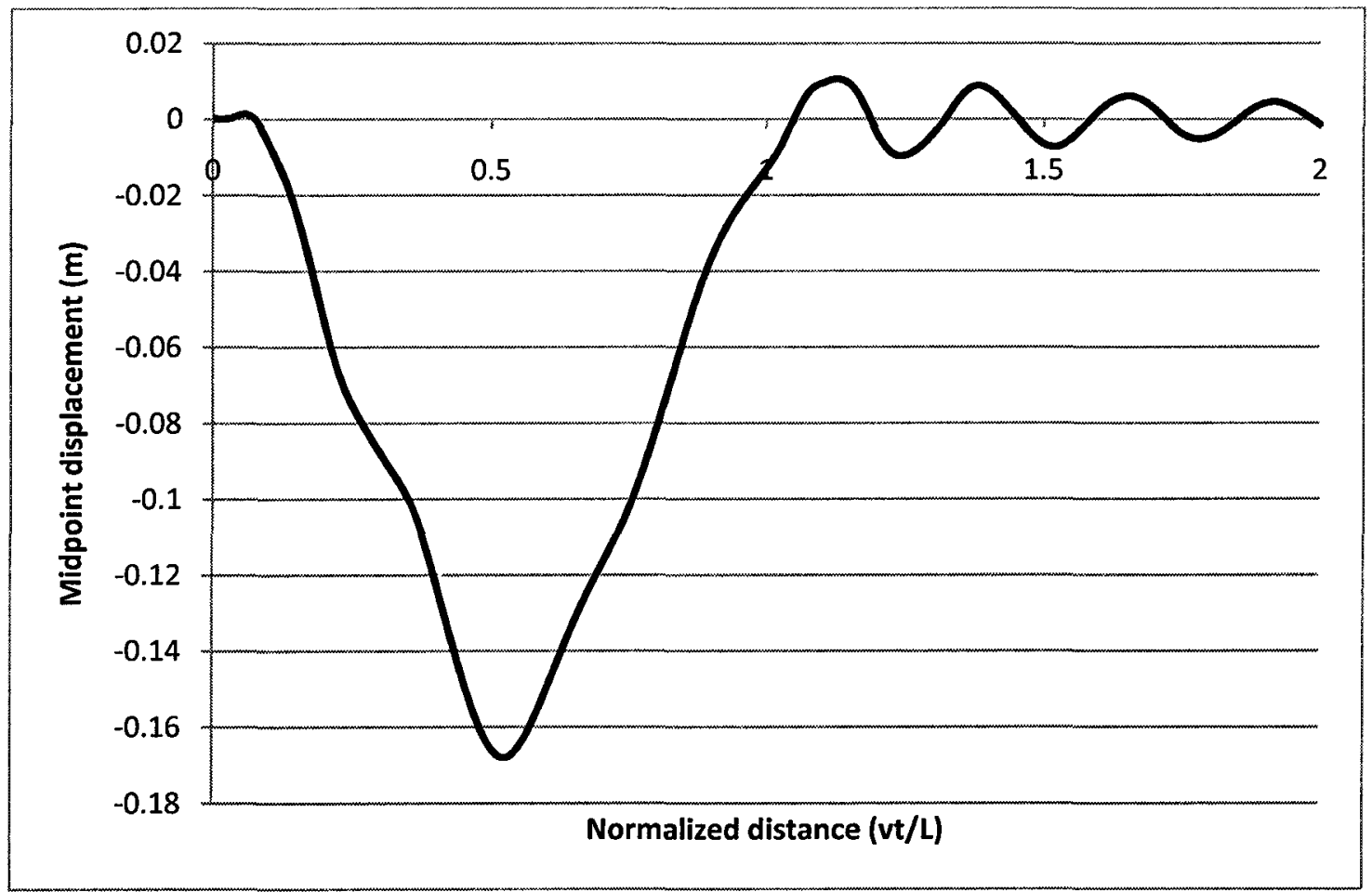

Figure 4.15: Time history of midpoint displacement in rigid-connected bridge due to 50tonne two-axle vehicle $(\mathrm{v}=15 \mathrm{~km} / \mathrm{h}$, twice the traversing time $)$ 


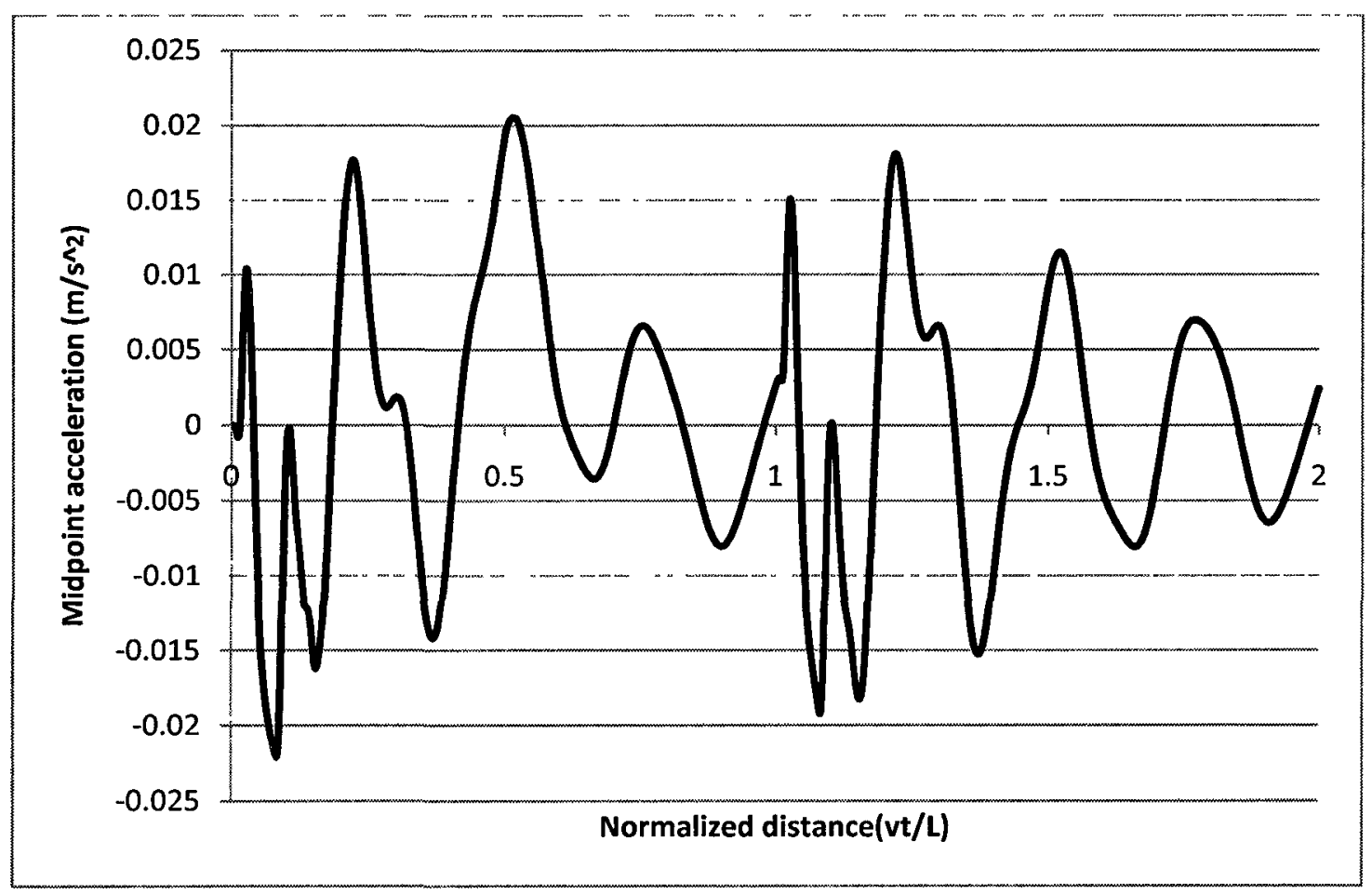

Figure 4.16: Time history of midpoint acceleration in rigid-connected bridge due to 50tonne two-axle vehicle $(\mathrm{v}=15 \mathrm{~km} / \mathrm{h}$, twice the traversing time)

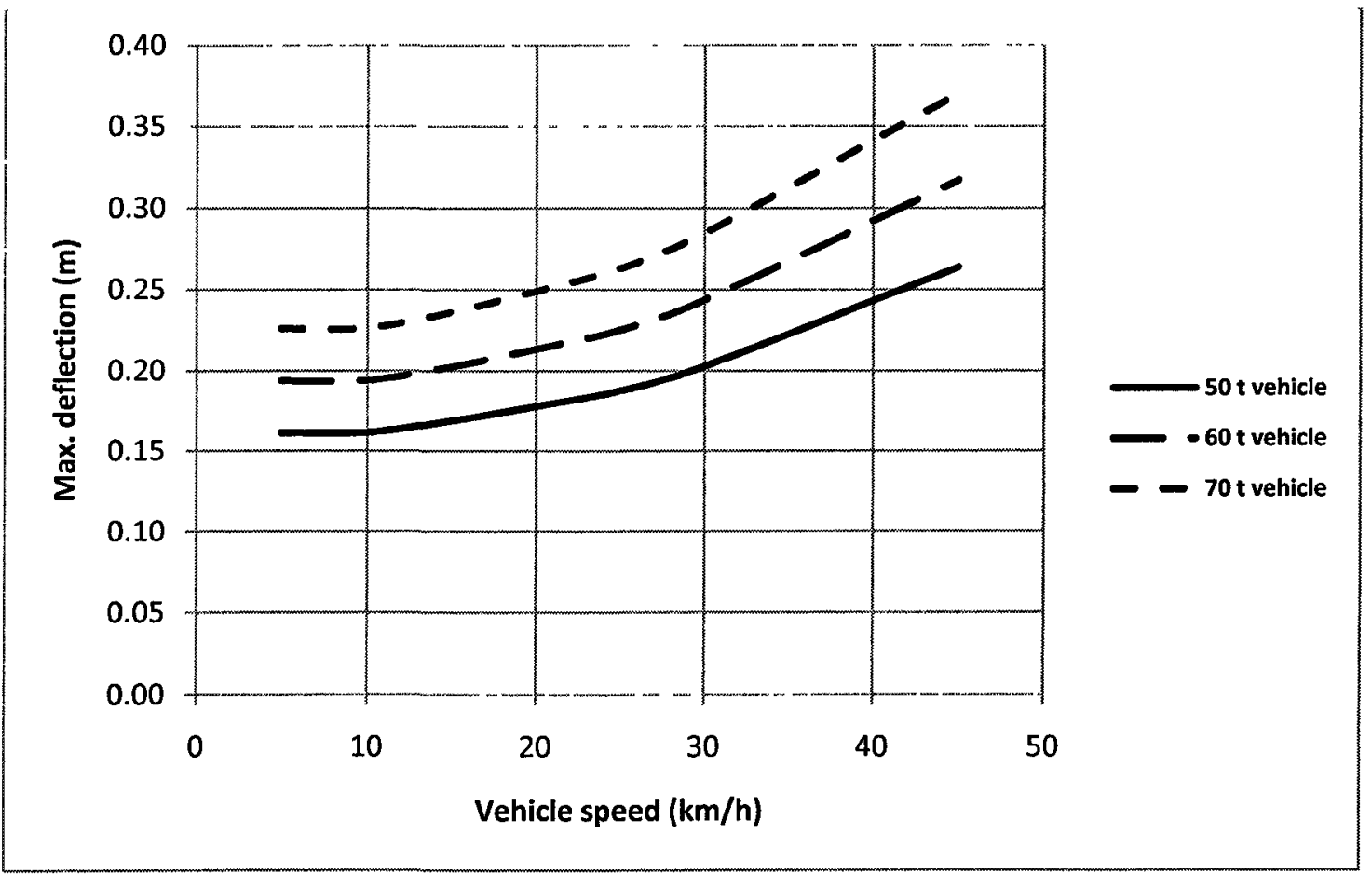

Figure 4.17: Effect of vehicle speed and weight on maximum deflection in rigidconnected bridge due to two-axle vehicle 


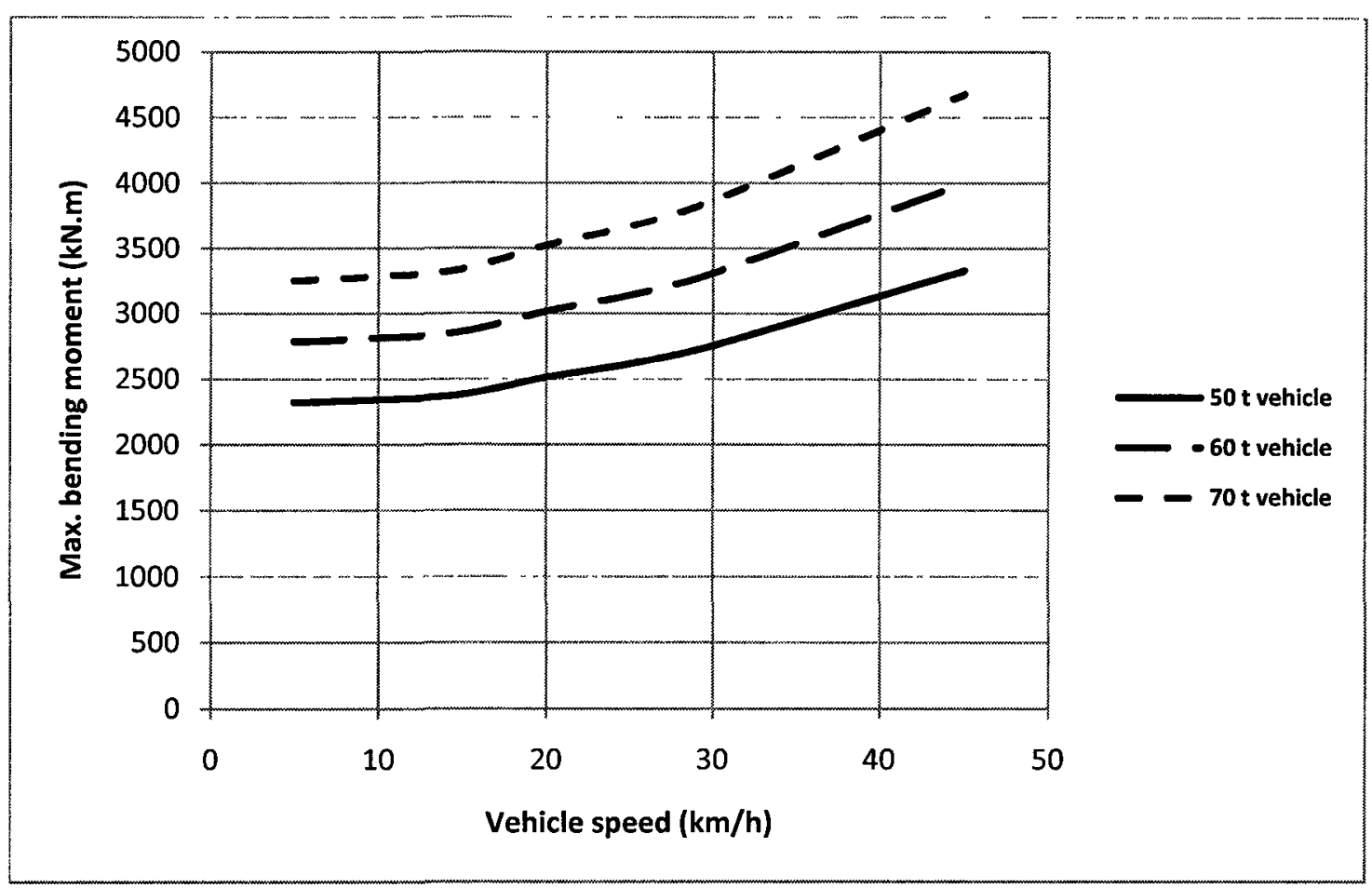

Figure 4.18: Effect of vehicle speed and weight on maximum bending moment in rigidconnected bridge due to two-axle vehicle

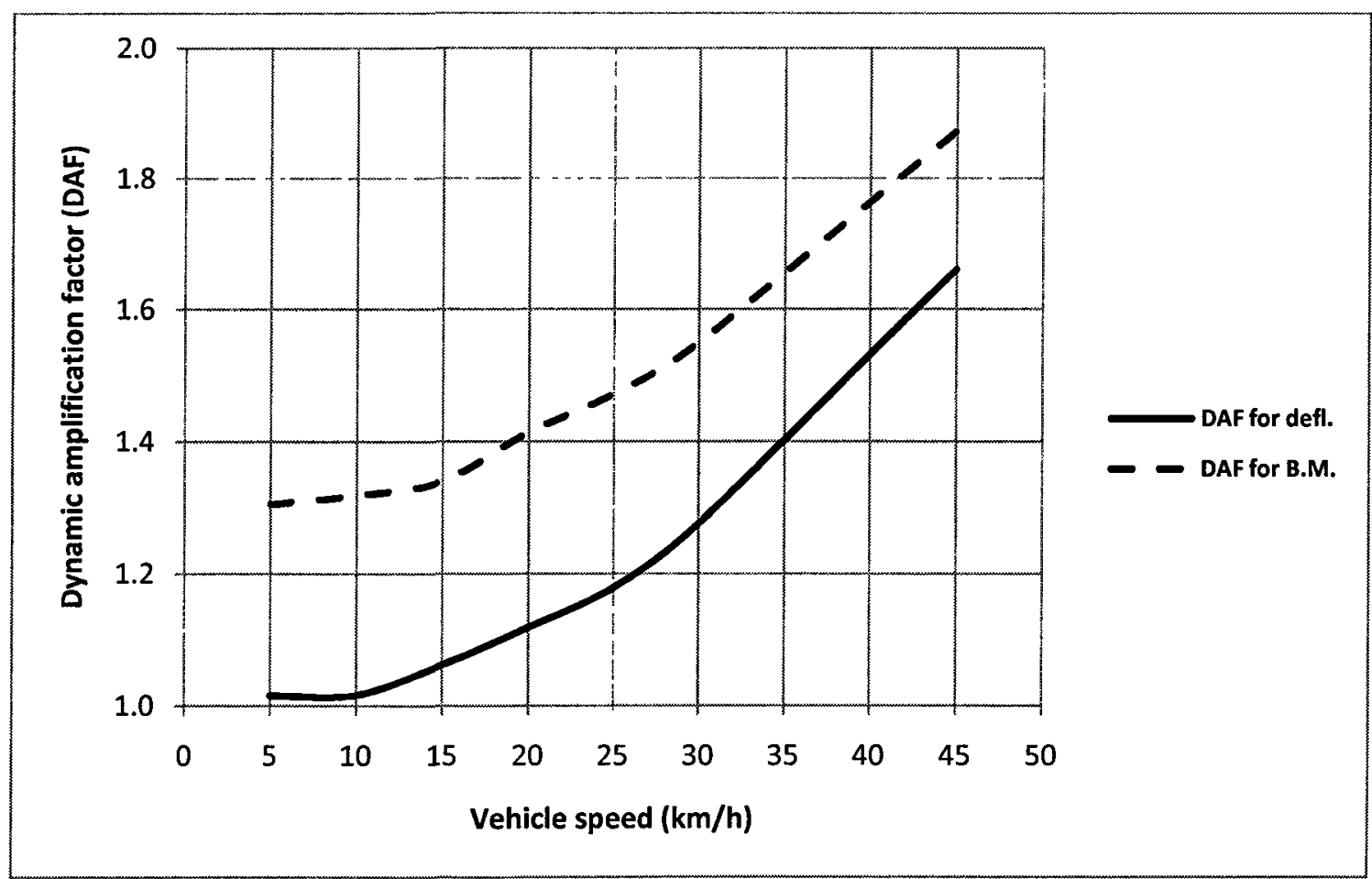

Figure 4.19: DAFs in rigid-connected bridge due to 50-tonne two-axle vehicle 


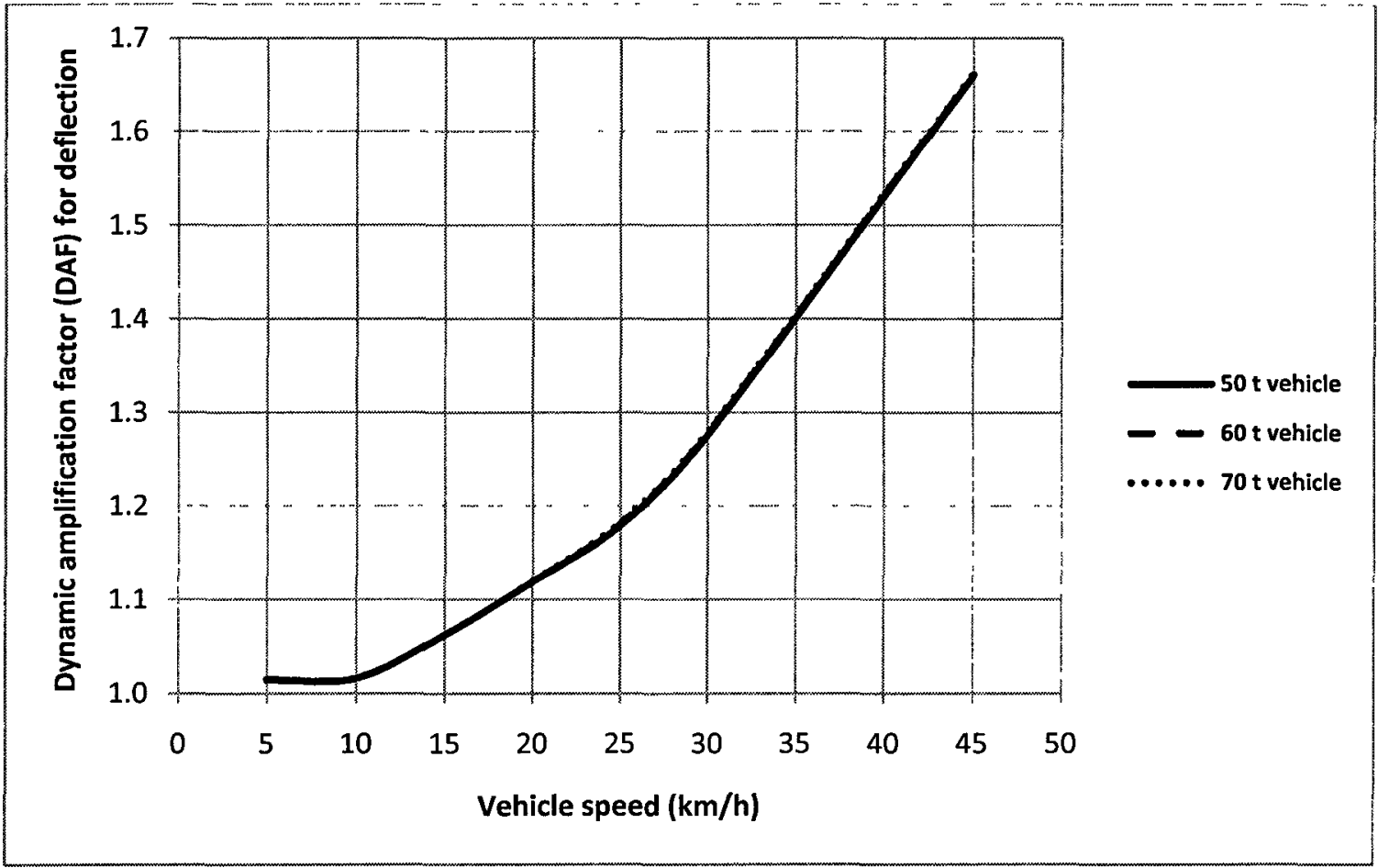

Figure 4.20: DAFs for deflection in rigid-connected bridge due to 50-tonne two-axle vehicle

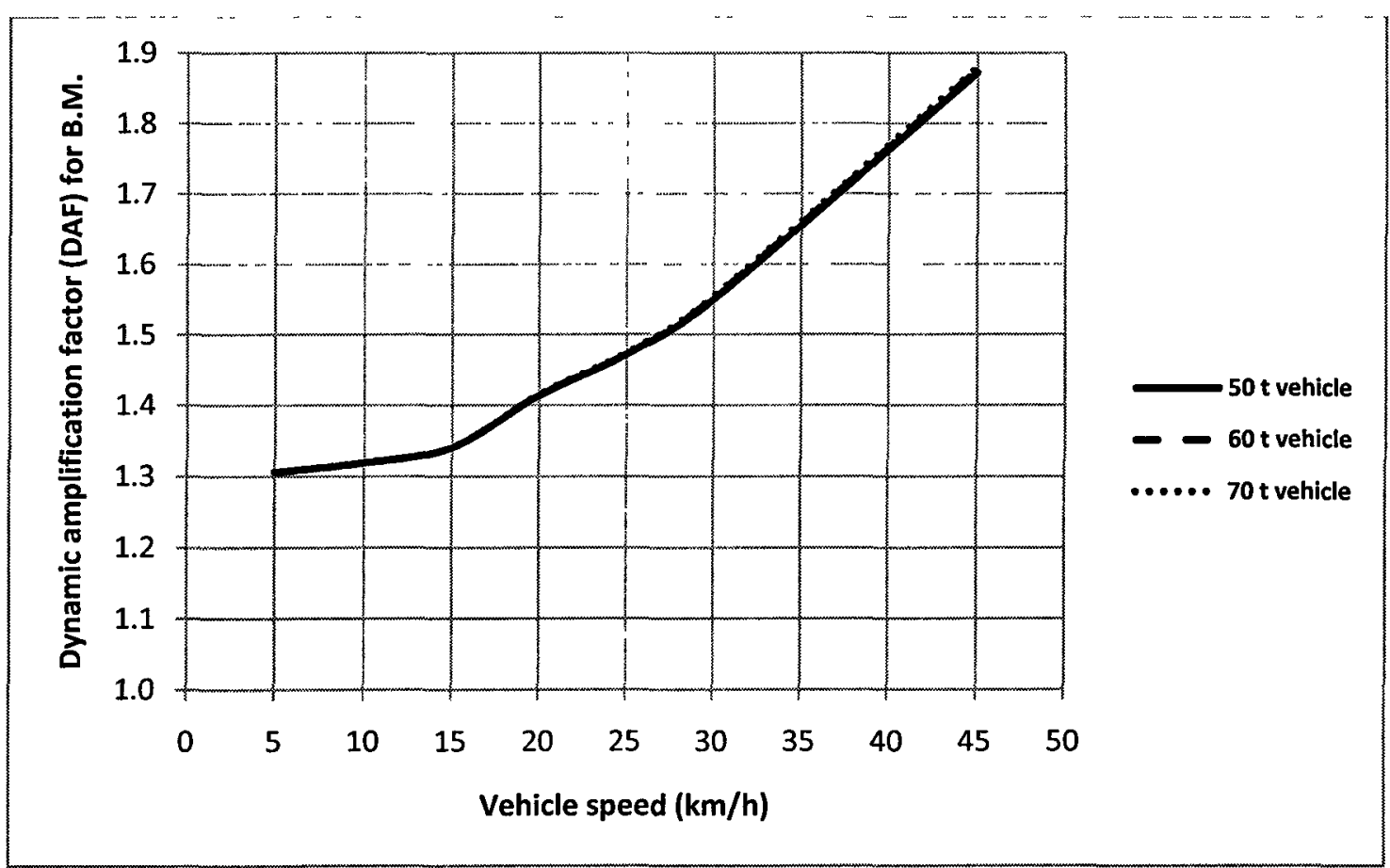

Figure 4.21: DAFs for bending moment in rigid-connected bridge due to 50-tonne twoaxle vehicle 


\subsubsection{Dynamic Response of Hinge-Connected Floating Bridge to Two-Axle Vehicle}

Loads

The dynamic response of hinge-connected floating bridge to heavy vehicle loads is investigated for different vehicle speeds varying from $5 \mathrm{~km} / \mathrm{h}$ to $30 \mathrm{~km} / \mathrm{h}$. The vehicle is represented by a two-axle model and has different weights of 50, 60 and 70 tonnes. The performed analysis runs and the results are summarized in Tables $4.11,4.12$ and 4.13.

Table 4.11: Static and dynamic response of hinge-connected bridge to 50-tonne two-axle vehicle loads

\begin{tabular}{|c|c|c|c|c|c|c|c|c|}
\hline $\begin{array}{l}\text { Span } \\
\text { (m) } \\
\end{array}$ & $\begin{array}{c}\text { Vehicle } \\
\text { Weight } \\
(t)\end{array}$ & $\begin{array}{c}\text { Vehicle } \\
\text { speed } \\
(\mathrm{km} / \mathrm{h}) \\
\end{array}$ & $\begin{array}{c}\text { Max } \\
\text { displ. } \\
(\mathrm{m}) \\
\end{array}$ & $\begin{array}{c}\text { Static } \\
\text { displ. } \\
\text { (m) } \\
\end{array}$ & $\begin{array}{l}\text { DAF for } \\
\text { displ. }\end{array}$ & $\begin{array}{c}\text { Max } \\
\text { B.M. } \\
\text { (KN.m) }\end{array}$ & $\begin{array}{c}\text { Static } \\
\text { B.M. } \\
\text { (KN.m) }\end{array}$ & $\begin{array}{l}\text { DAF for } \\
\text { B.M. }\end{array}$ \\
\hline 84 & 50 & 5 & -0.447 & -0.417 & 1.07 & -1430 & -1361 & 1.05 \\
\hline 84 & 50 & 10 & -0.476 & -0.417 & 1.14 & -1425 & -1361 & 1.05 \\
\hline 84 & 50 & 15 & -0.531 & -0.417 & 1.27 & -1443 & -1361 & 1.06 \\
\hline 84 & 50 & 20 & -0.671 & -0.417 & 1.61 & -1493 & -1361 & 1.10 \\
\hline 84 & 50 & 25 & -0.747 & -0.417 & 1.79 & -1470 & -1361 & 1.08 \\
\hline 84 & 50 & 30 & -0.762 & -0.417 & 1.83 & -1554 & -1361 & 1.14 \\
\hline
\end{tabular}


Table 4.12: Static and dynamic response of hinge-connected bridge to 60-tonne two-axle vehicle loads

\begin{tabular}{|c|c|c|c|c|c|c|c|c|}
\hline Span & $\begin{array}{c}\text { Vehicle } \\
\text { Weight } \\
(\mathbf{m})\end{array}$ & $\begin{array}{c}\text { Vehicle } \\
\mathbf{\text { speed }} \\
(\mathbf{k m} / \mathbf{h})\end{array}$ & $\begin{array}{c}\text { Max } \\
\mathbf{d i s p l} . \\
(\mathbf{m})\end{array}$ & $\begin{array}{c}\text { Static } \\
\mathbf{d i s p l .} \\
(\mathbf{m})\end{array}$ & $\begin{array}{c}\text { DAF for } \\
\mathbf{d i s p l .}\end{array}$ & $\begin{array}{c}\text { Max } \\
\text { B.M. } \\
(\mathbf{K N} . \mathbf{m})\end{array}$ & $\begin{array}{c}\text { Static } \\
\text { B.M. } \\
(\mathbf{K N} . \mathbf{m})\end{array}$ & $\begin{array}{c}\text { DAF for } \\
\text { B.M. }\end{array}$ \\
\hline 84 & 60 & 5 & -0.538 & -0.501 & 1.07 & -1716 & -1633 & 1.05 \\
\hline 84 & 60 & 10 & -0.572 & -0.501 & 1.14 & -1710 & -1633 & 1.05 \\
\hline 84 & 60 & 15 & -0.638 & -0.501 & 1.27 & -1732 & -1633 & 1.06 \\
\hline 84 & 60 & 20 & -0.807 & -0.501 & 1.61 & -1787 & -1633 & 1.09 \\
\hline 84 & 60 & 25 & -0.897 & -0.501 & 1.79 & -1757 & -1633 & 1.08 \\
\hline 84 & 60 & 30 & -0.916 & -0.501 & 1.83 & -1913 & -1633 & 1.17 \\
\hline
\end{tabular}

Table 4.13: Static and dynamic response of hinge-connected bridge to 70-tonne two-axle vehicle loads

\begin{tabular}{|c|c|c|c|c|c|c|c|c|}
\hline Span & $\begin{array}{c}\text { Vehicle } \\
\text { Weight } \\
(\mathbf{m})\end{array}$ & $\begin{array}{c}\text { Vehicle } \\
\text { speed } \\
(\mathbf{k m} / \mathbf{h})\end{array}$ & $\begin{array}{c}\text { Max } \\
\text { displ. } \\
(\mathbf{m})\end{array}$ & $\begin{array}{c}\text { Static } \\
\text { displ. } \\
(\mathbf{m})\end{array}$ & $\begin{array}{c}\text { DAF for } \\
\text { displ. }\end{array}$ & $\begin{array}{c}\text { Max } \\
\text { B.M. } \\
(\mathbf{K N . m})\end{array}$ & $\begin{array}{c}\text { Static } \\
\text { B.M. } \\
(\text { KN.m) }\end{array}$ & $\begin{array}{c}\text { DAF for } \\
\text { B.M. }\end{array}$ \\
\hline 84 & 70 & 5 & -0.628 & -0.584 & 1.07 & -2002 & -1906 & 1.05 \\
\hline 84 & 70 & 10 & -0.669 & -0.584 & 1.14 & -1992 & -1906 & 1.05 \\
\hline 84 & 70 & 15 & -0.746 & -0.584 & 1.28 & -2019 & -1906 & 1.06 \\
\hline 84 & 70 & 20 & -0.945 & -0.584 & 1.62 & -2080 & -1906 & 1.09 \\
\hline 84 & 70 & 25 & -1.047 & -0.584 & 1.79 & -2093 & -1906 & 1.10 \\
\hline 84 & 70 & 30 & -1.070 & -0.584 & 1.83 & -2268 & -1906 & 1.19 \\
\hline
\end{tabular}




\subsubsection{Discussion of Results}

Figure 4.22 and 4.23 show typical time histories of midpoint displacement and acceleration due to the passage of 50-tonne two-axle vehicle at a speed of $15 \mathrm{~km} / \mathrm{h}$, respectively. Figure 4.24 shows the maximum displacement in the bridge due to two-axle moving vehicle loads. The maximum displacement in the bridge increases with vehicle weight and speed. The maximum displacement in the bridge is within the allowable limit $(0.769 \mathrm{~m})$ for 50 tonne vehicle weight and for speeds from 5 up to $30 \mathrm{~km} / \mathrm{h}$. For the 60 tonne and 70-tonne vehicles, the maximum displacement in the bridge is within the allowable limit for speeds from $5 \mathrm{~km} / \mathrm{h}$ to $15 \mathrm{~km} / \mathrm{h}$ but exceeds the allowable limit for higher speeds. This means that the hinge-connected bridge can support 50 -tonne vehicle loads travelling at a speed up to $30 \mathrm{~km} / \mathrm{h}, 60$-tonne or 70 -tonne vehicle traveling at speeds up to $15 \mathrm{~km} / \mathrm{h}$. The maximum bending moment developed in the bridge is presented in Figure 4.25, which shows that the maximum bending moment increases with vehicle weight and slightly increases with vehicle speed.

Figure 4.26 shows the DAFs for both displacement and bending moment in the bridge due to 50-tonne two-axle vehicle loads traveling the bridge at different speeds from 5 to $30 \mathrm{~km} / \mathrm{h}$. The DAF for displacement increases with vehicle speed ranging from 1.07 at 5 $\mathrm{km} / \mathrm{h}$ to 1.83 at $30 \mathrm{~km} / \mathrm{h}$. Only minor increase is observed in the DAF for bending moment with increasing vehicle speed from 1.05 at $5 \mathrm{~km} / \mathrm{h}$ to 1.14 at $30 \mathrm{~km} / \mathrm{h}$. Figure 4.27 shows the DAF for displacement for different vehicle weights. The DAFs for displacement are the same for different vehicle weights, however, the displacements increase as the vehicle weight increases. The same trend is observed for the DAFs for 
bending moment as shown in Figure 4.28 with only minor changes with increasing vehicle weight. This means that an increase in the vehicle weight does not affect the DAFs for either the displacement or the bending moment.

On comparing Figures 4.17 and 4.24 , it is observed that the maximum displacements in the hinge-connected floating bridge due to two-axle vehicle loads are much higher than those in the rigid-connected floating bridge. At the same time, comparison of Figures 4.18 and 4.25 shows that the maximum bending moments in the hinge-connected floating bridge are much lower than those in the rigid-connected floating bridge.

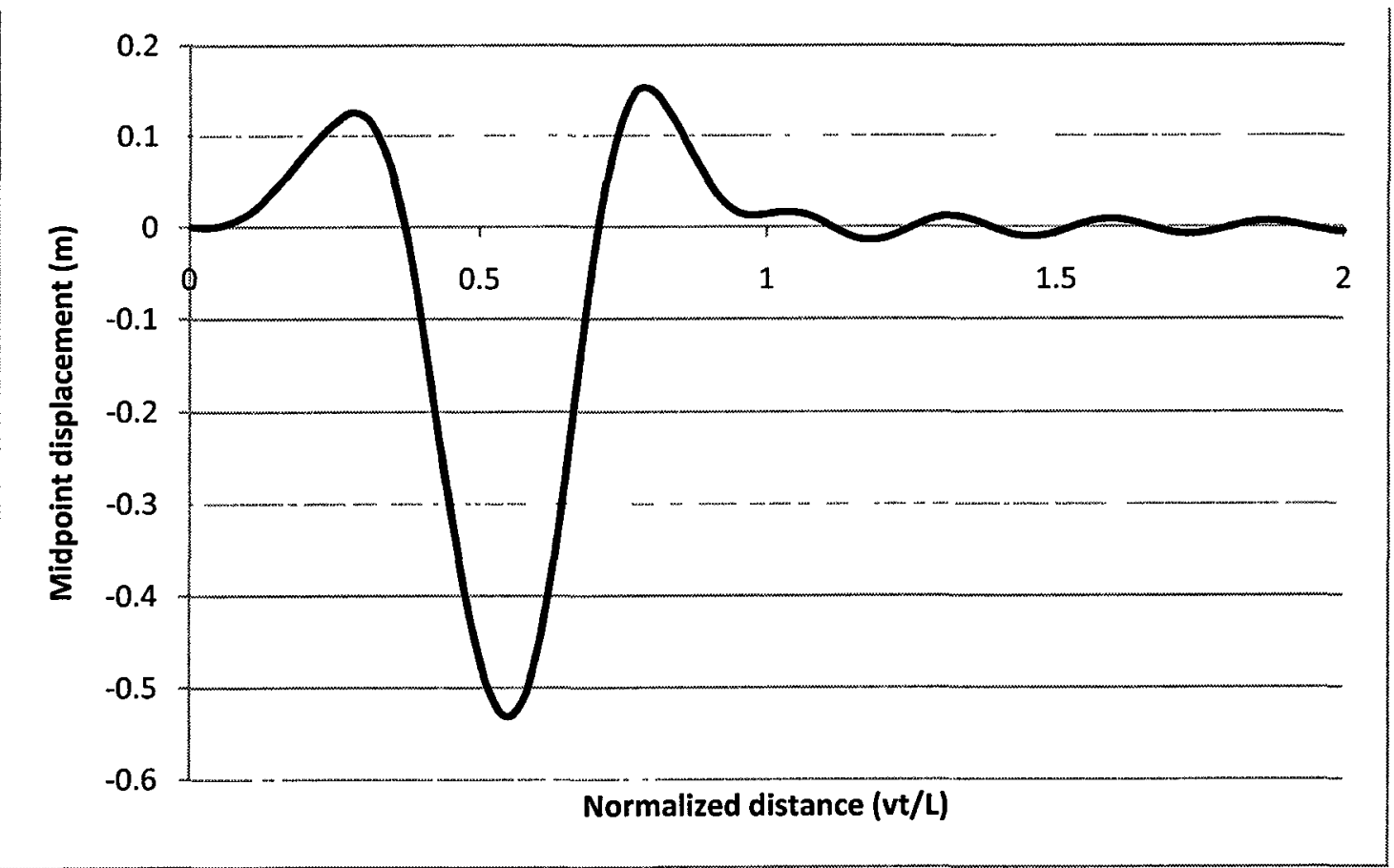

Figure 4.22: Time history of midpoint displacement in hinge-connected bridge due to 50tonne two-axle vehicle $(\mathrm{v}=15 \mathrm{~km} / \mathrm{h}$, twice the traversing time) 


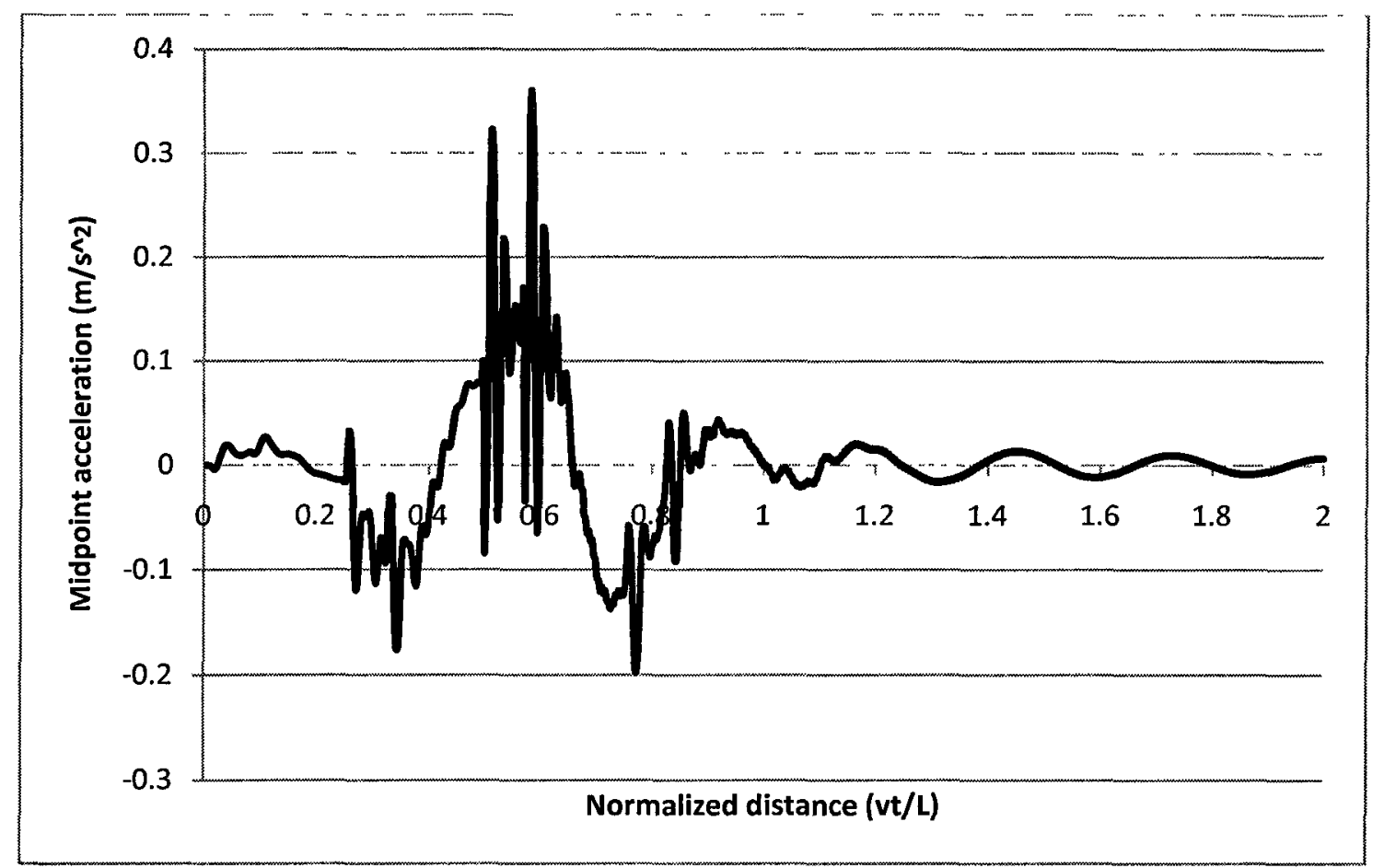

Figure 4.23: Time history of midpoint displacement in hinge-connected bridge due to 50tonne two-axle vehicle $(\mathrm{v}=15 \mathrm{~km} / \mathrm{h}$, twice the traversing time)

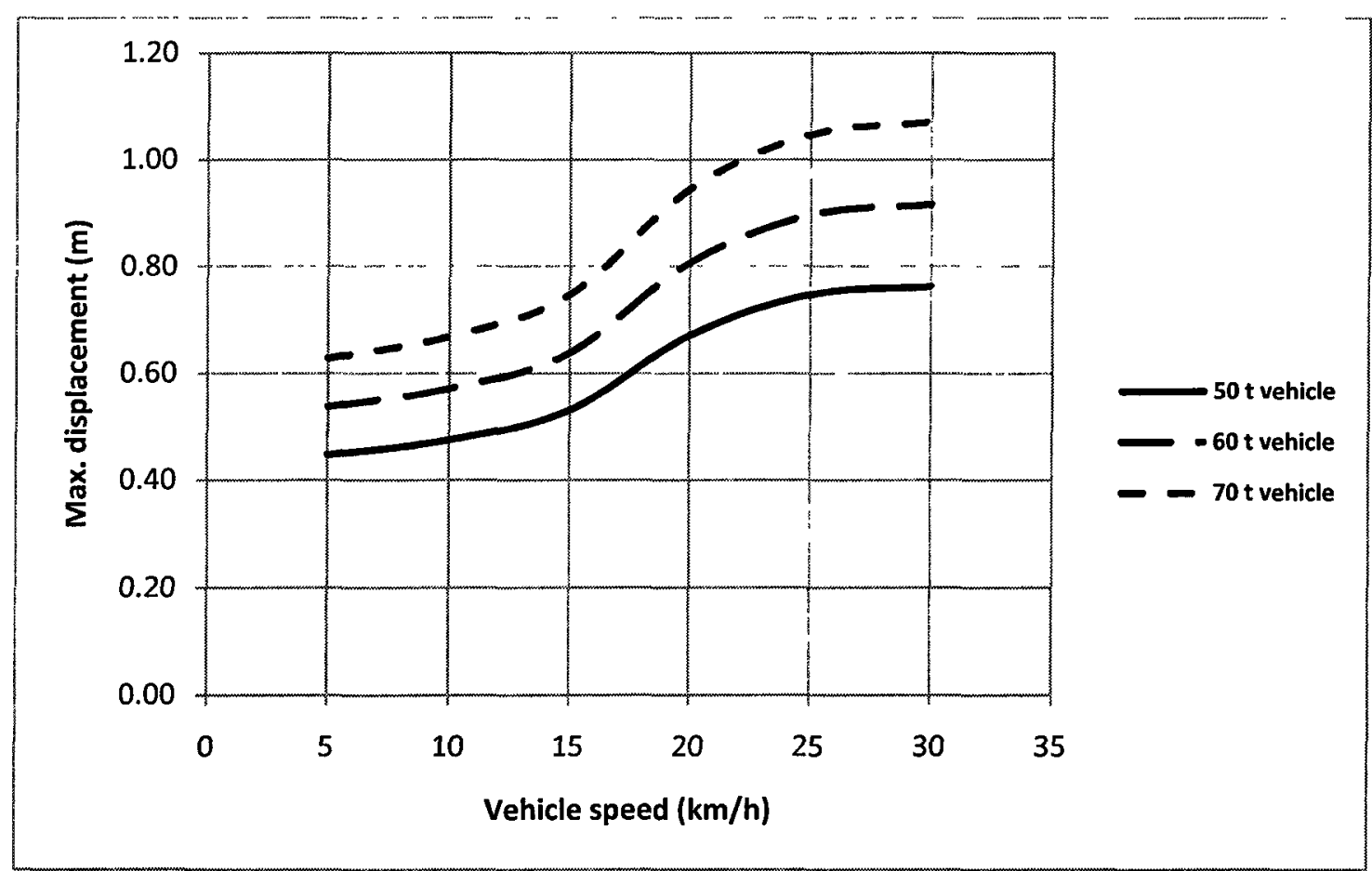

Figure 4.24: Effect of vehicle speed and weight on maximum displacement in hingeconnected bridge due to 50-tonne two-axle vehicle 


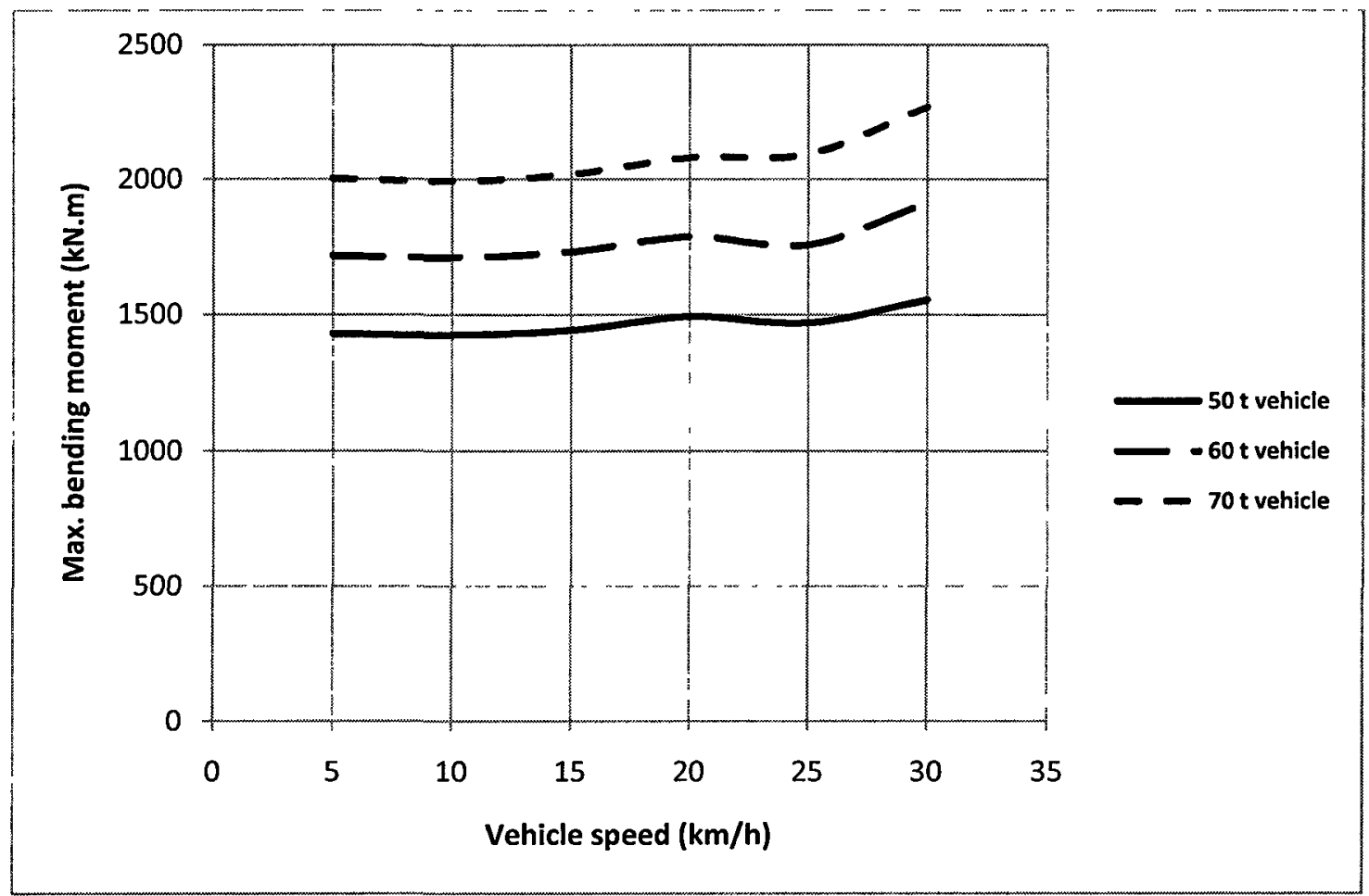

Figure 4.25: Effect of vehicle speed and weight on maximum bending moment in hingeconnected bridge due to 50-tonne two-axle vehicle

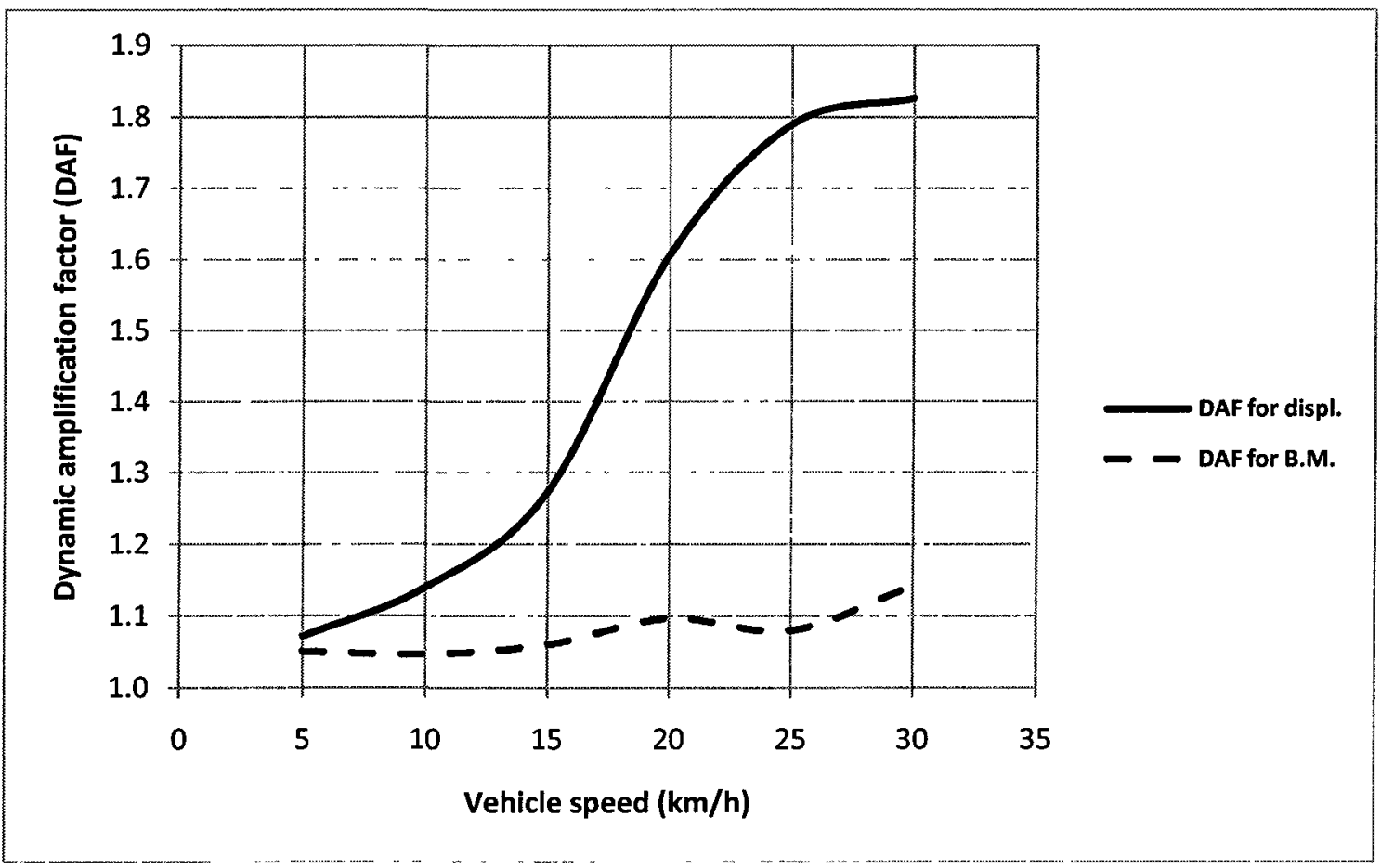

Figure 4.26: DAFs in hinge-connected bridge due to 50 -tonne two-axle vehicle 


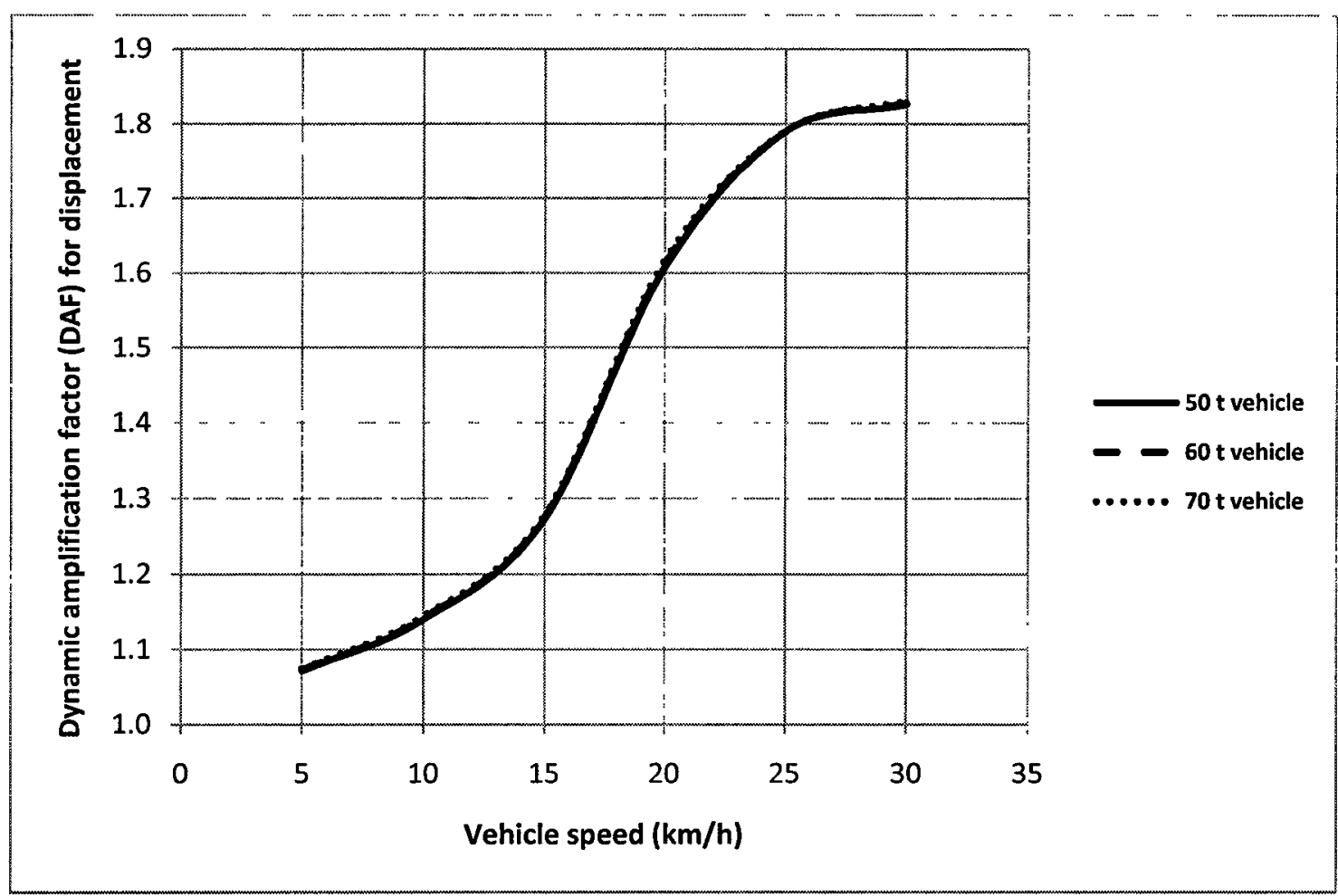

Figure 4.27: DAFs for displacement in hinge-connected bridge due to 50-tonne two-axle vehicle

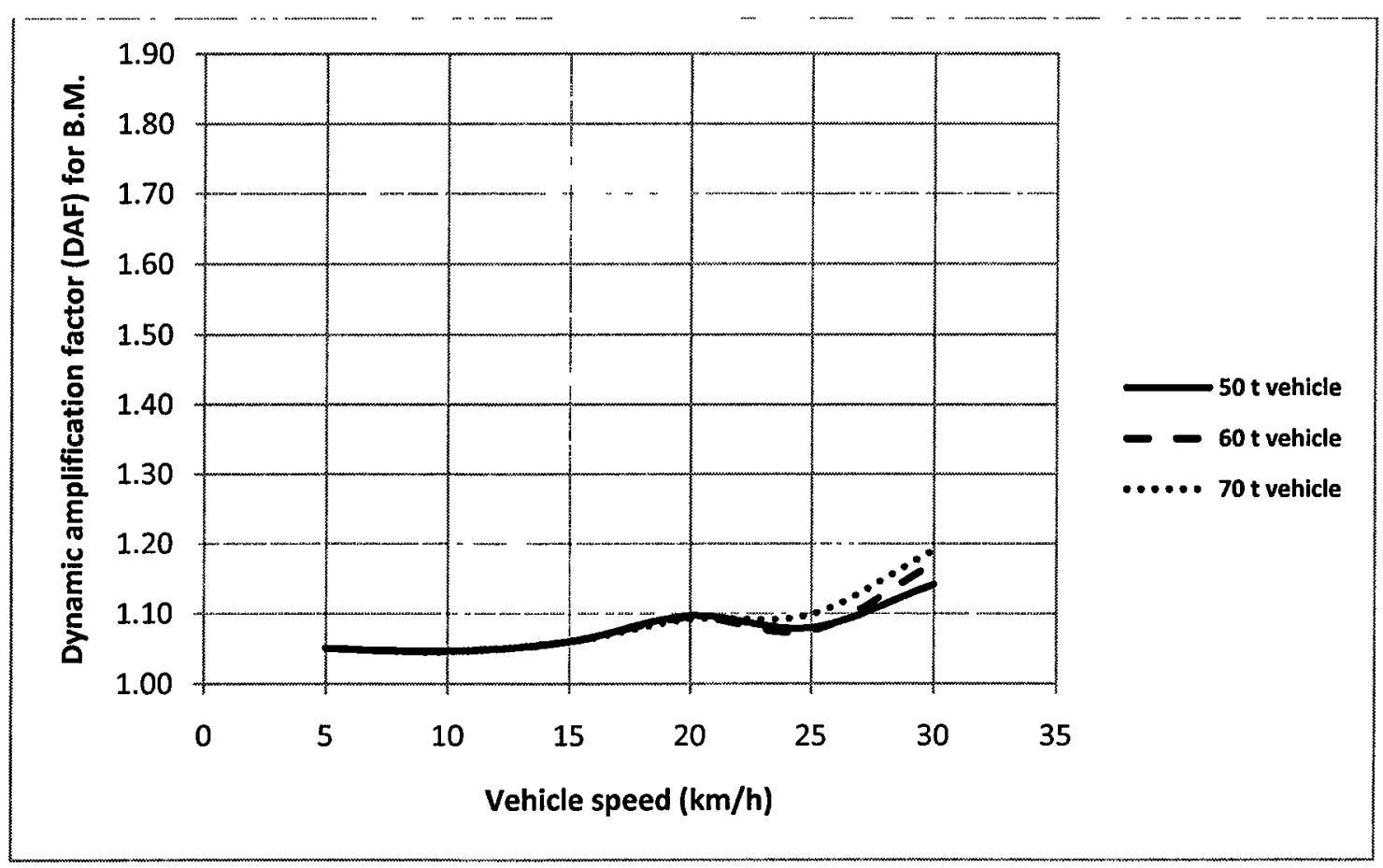

Figure 4.28: DAFs for bending moment in hinge-connected bridge due to 50 -tonne twoaxle vehicle 


\subsection{Comparison of the Dynamic Response to Single-Axle and Two-Axle Vehicle}

\section{Loads}

The objective of this section is to compare the dynamic response of floating bridges to single-axle and two-axle vehicle loads, and to investigate the effect of the vehicle model on the dynamic response of floating bridges.

\subsubsection{Dynamic Response of Rigid-Connected Floating Bridge to Single- and Two- Axle Vehicle Loads}

The dynamic response of rigid-connected floating bridge to single-axle vehicle and corresponding two-axle vehicle are compared to illustrate the effect of simplifying the vehicle model on the accuracy of the bridge dynamic response. Depending on the results, the single-axle representation of the vehicle which is less complex and less in computational time can be used to quickly obtain the response of the bridge.

\subsubsection{Discussion of Results}

The midpoint displacements due to two-axle vehicle loads are slightly smaller than those due to single-axle vehicle loads for all vehicle speeds from $5 \mathrm{~km} / \mathrm{h}$ to $45 \mathrm{~km} / \mathrm{h}$. Figures 4.29 and 4.30 show examples of the time history of midpoint displacement due to 50tonne single- and two-axle vehicle for vehicle speed of $10 \mathrm{~km} / \mathrm{h}$ and $15 \mathrm{~km} / \mathrm{h}$, respectively. Figure 4.31 shows the maximum deflection in the bridge due to single- and two-axle moving vehicle loads. In general, the maximum deflections in the bridge due to two-axle vehicle loads are slightly less than those due to single-axle vehicle loads for all vehicle weights and speeds. For example, the maximum deflection due to 50-tonne two- 
axle vehicle traversing the bridge at $5 \mathrm{~km} / \mathrm{h}$ is $98 \%$ of that due to 50 -tonne single-axle vehicle traversing the bridge at the same speed. The maximum bending moments in the bridge due to two-axle vehicle loads are also less than those due to single-axle vehicle loads as shown in Figure 4.32. For example, the maximum bending moment due to 50tonne two-axle vehicle traversing the bridge at $5 \mathrm{~km} / \mathrm{h}$ is $79 \%$ of that due to 50 -tonne single-axle vehicle traversing the bridge at the same speed. This response is expected as the weight is distributed to two axles $6 \mathrm{~m}$ apart compared to single-axle load.

Figure 4.33 shows the DAF for deflection due to 50-tonne single- and two-axle vehicle loads travelling the bridge at different speeds. The DAF for deflection due to two-axle vehicle loads are slightly smaller than those due to single-axle vehicle loads for all vehicle speeds. For example, the DAF for deflection due to 50-tonne two-axle vehicle travelling the bridge at $15 \mathrm{~km} / \mathrm{h}$ is $97 \%$ of that due to single-axle vehicle. The same trend was observed for 60 and 70 -tonne vehicle loads. Figure 4.34 shows the DAF for bending moment due to 50-tonne single- and two-axle vehicle loads travelling the bridge at different speeds. Although the maximum bending moments in the bridge due to two-axle vehicle are smaller than those due to single-axle vehicle, the DAF for bending moment due to two-axle vehicle are higher than those due to single-axle vehicle for all speeds. This is because the static bending moments developed in the bridge due to two-axle vehicle are much smaller than those due to single-axle vehicle. For example, the DAF for bending moment due to 50 -tonne two-axle vehicle travelling the bridge at $15 \mathrm{~km} / \mathrm{h}$ is $103 \%$ of that due to single-axle vehicle. The same trend was observed for 60 and 70 tonne vehicle loads. 
Representing the vehicle by two-axle model is more realistic and can represent the real vehicle more accurately than the single-axle model. The results show that in general, the dynamic response of rigid-connected floating bridge to two-axle vehicle loads is smaller than that due to single-axle vehicle loads.

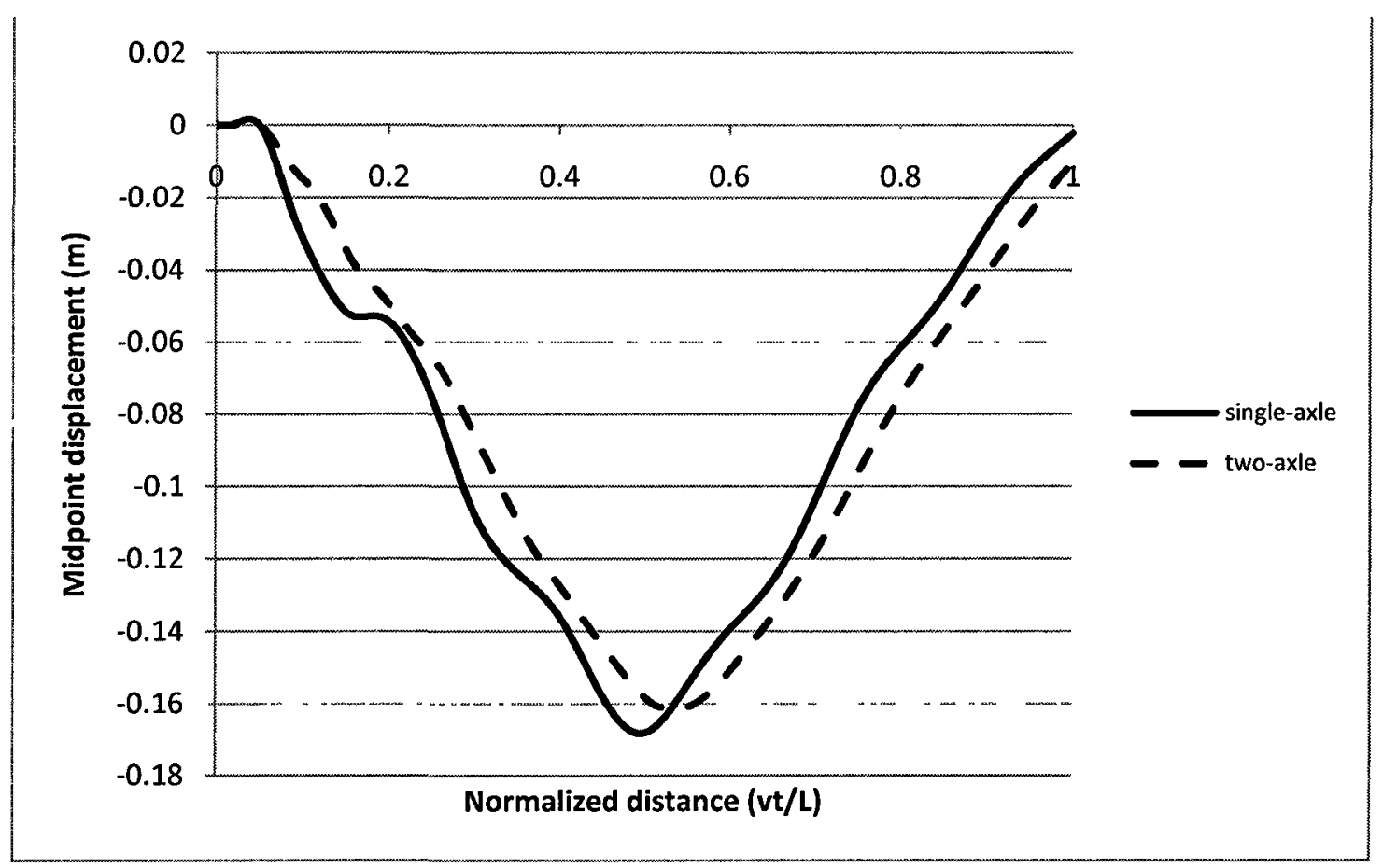

Figure 4.29: Time history of midpoint displacements in rigid-connected bridge due to 50tonne single- and two-axle vehicle $(\mathrm{v}=10 \mathrm{~km} / \mathrm{h})$ 


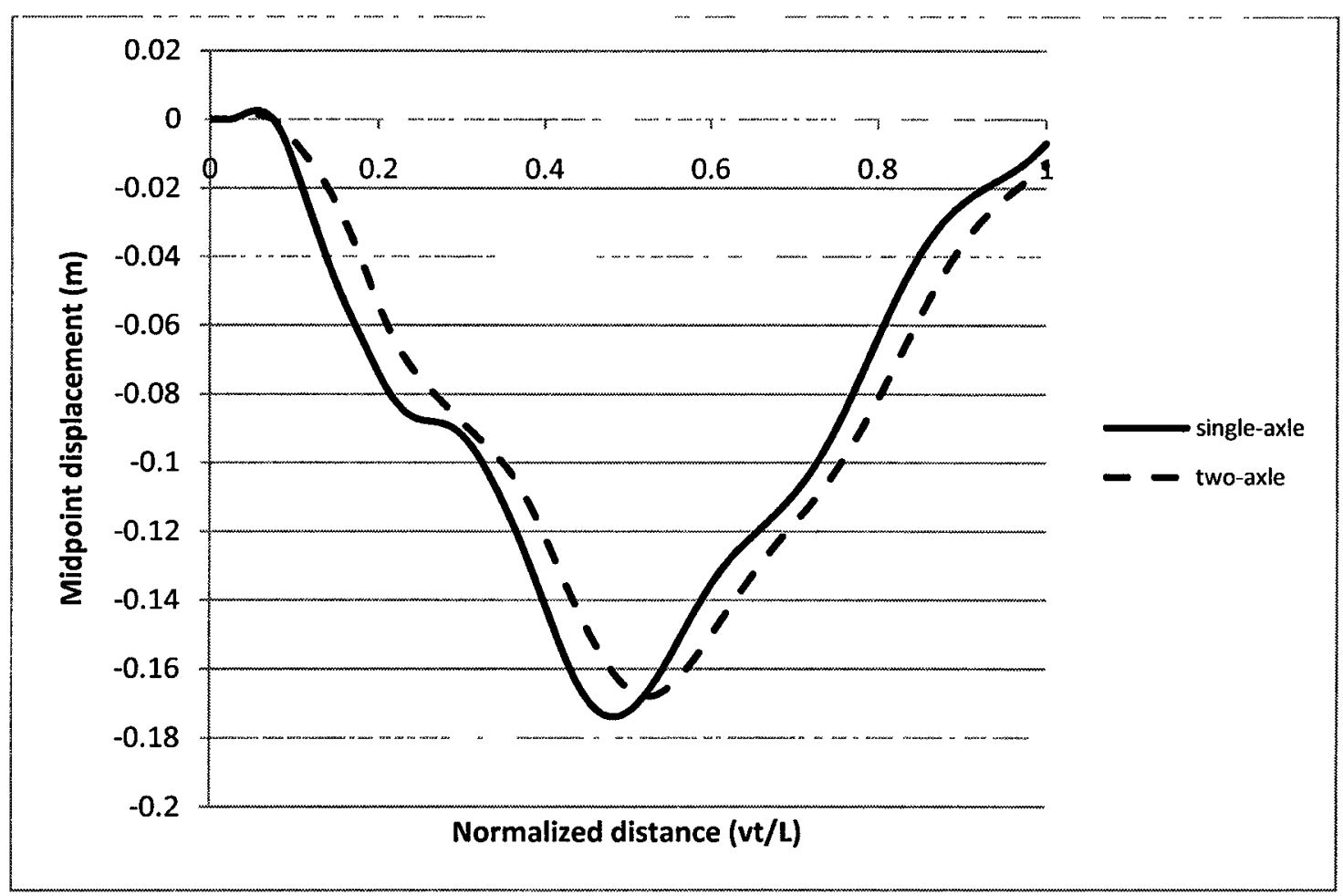

Figure 4.30: Time history of midpoint displacements in rigid-connected bridge due to 50tonne single- and two-axle vehicle $(\mathrm{v}=15 \mathrm{~km} / \mathrm{h})$

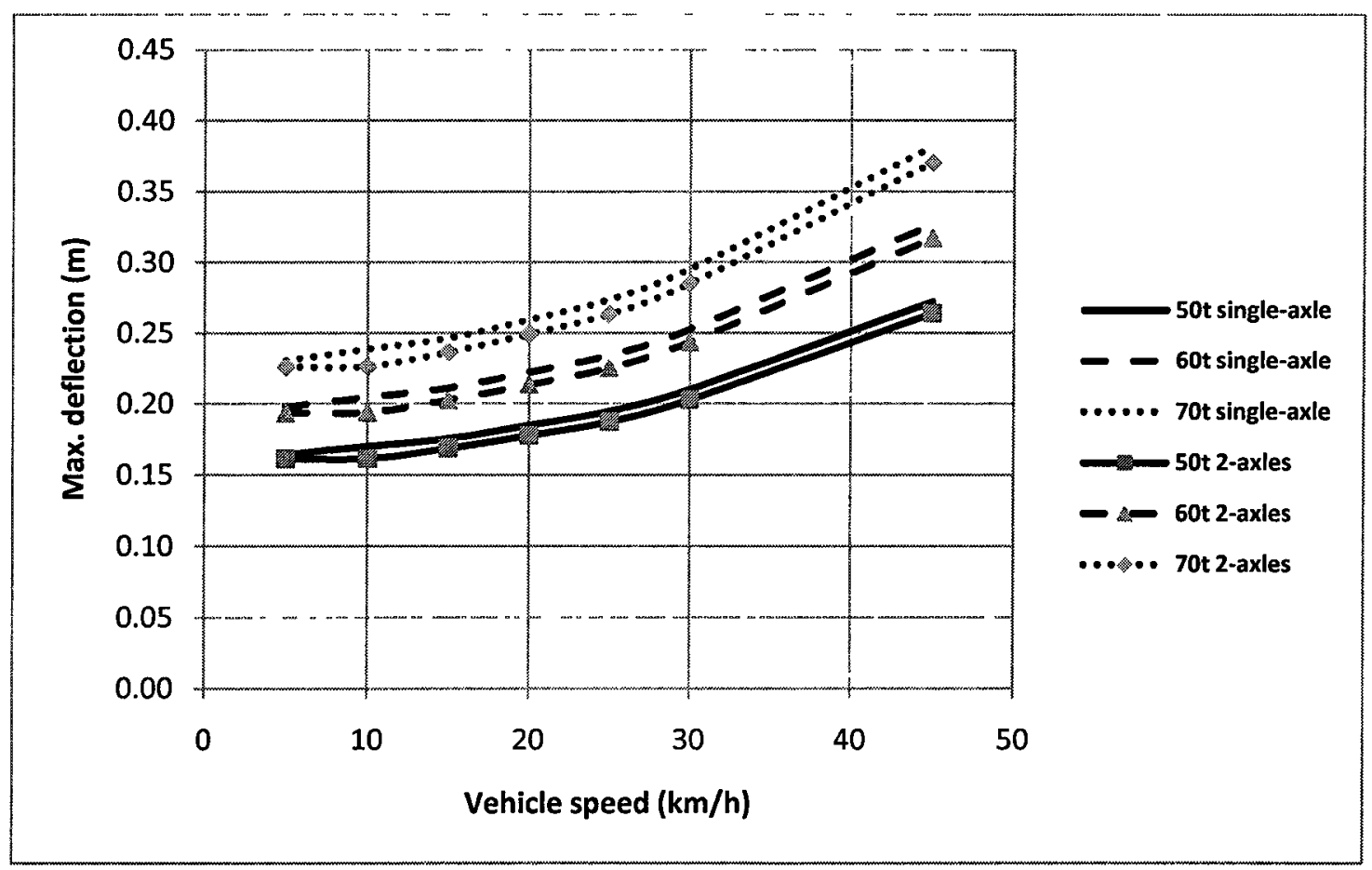

Figure 4.31: Maximum deflections in rigid-connected bridge due to single- and two-axle vehicle 


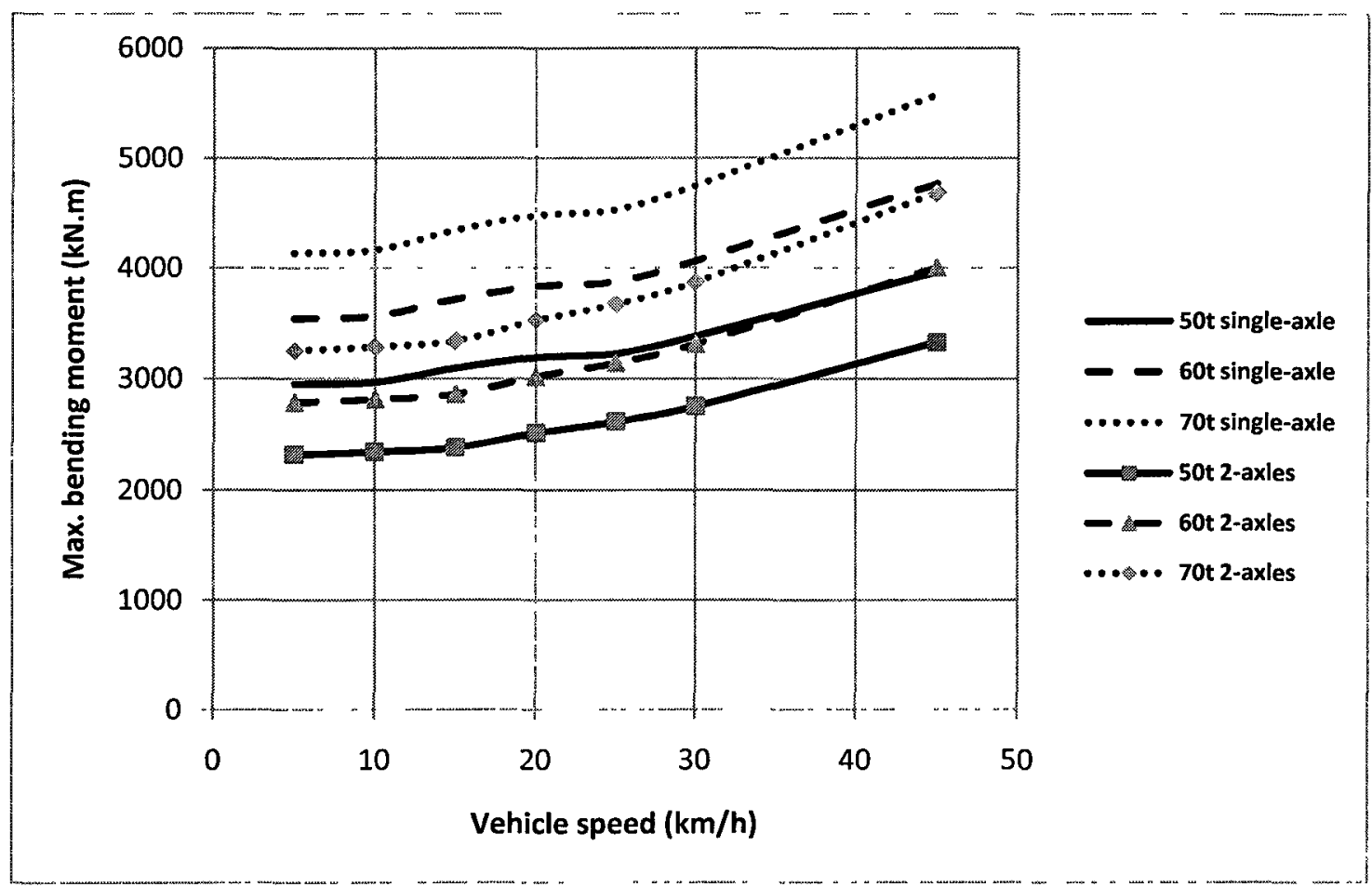

Figure 4.32: Maximum bending moments in rigid-connected bridge due to single- and two-axle vehicle

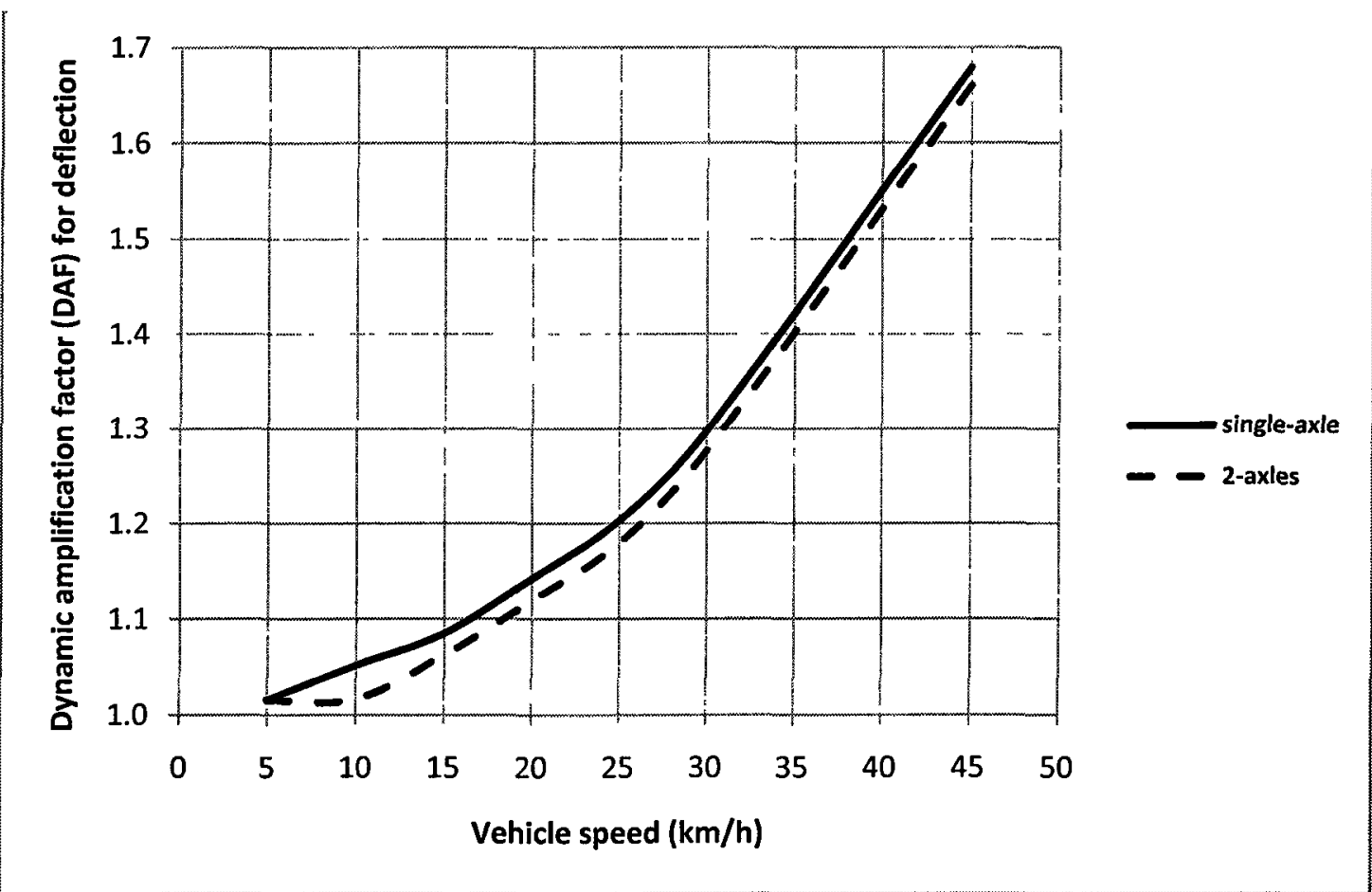

Figure 4.33: DAFs for deflection in rigid-connected bridge due to 50-tonne single- and two-axle vehicle 


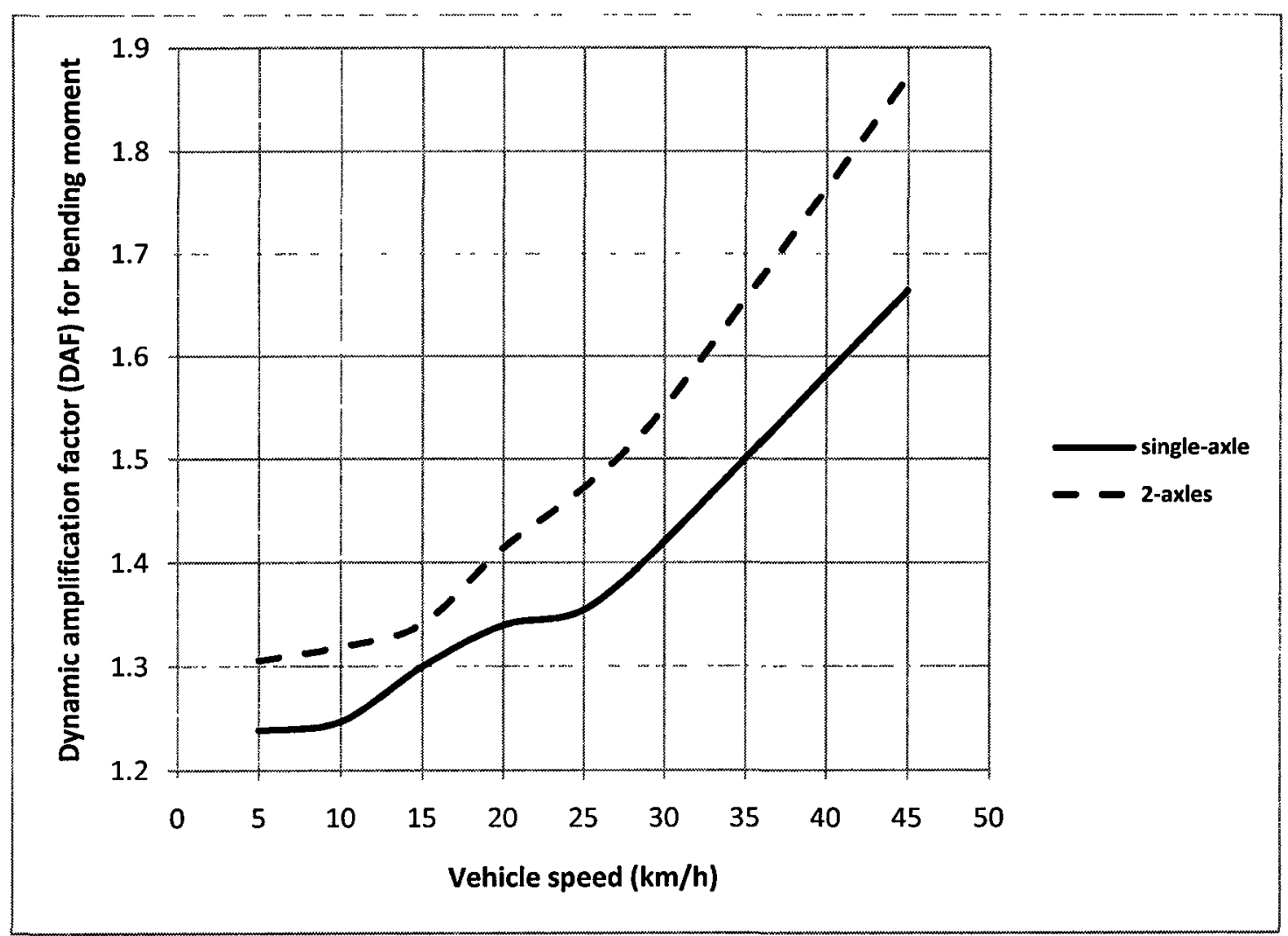

Figure 4.34: DAFs for bending moment in rigid-connected bridge due to 50-tonne singleand two-axle vehicle

\subsubsection{Dynamic Response of Hinge-Connected Floating Bridge to Single- and Two-}

\section{Axle Vehicle Loads}

The dynamic response of hinge-connected floating bridge to single-axle vehicle and twoaxle vehicle are compared to illustrate the effect of simplifying the vehicle model on the accuracy of the bridge dynamic response.

\subsubsection{Discussion of Results}

The midpoint displacements due to two-axle vehicle loads are smaller than those due to single-axle vehicle loads for all vehicle speeds from $5 \mathrm{~km} / \mathrm{h}$ to $30 \mathrm{~km} / \mathrm{h}$. Figures 4.35 and 
4.36 show typical time histories of midpoint displacement due to 50-tonne single- and two-axle vehicle loads speeds of $10 \mathrm{~km} / \mathrm{h}$ and $15 \mathrm{~km} / \mathrm{h}$, respectively.

Figure 4.37 shows the maximum displacements in the bridge due to single- and two-axle moving vehicle loads. The maximum displacements in the bridge due to two-axle vehicle loads are less than those due to single-axle vehicle loads for all vehicle weights and speeds. For example, the maximum displacement due to 50-tonne two-axle vehicle traversing the bridge at $5 \mathrm{~km} / \mathrm{h}$ is $81 \%$ of that due to 50 -tonne single-axle vehicle traversing the bridge at the same speed. The maximum bending moments of the bridge due to two-axle vehicle loads are much less than those due to single-axle vehicle loads as shown in Figure 4.38. For example, the maximum bending moment due to 50 -tonne twoaxle vehicle traversing the bridge at $5 \mathrm{~km} / \mathrm{h}$ is $70 \%$ of that due to 50 -tonne single-axle vehicle traversing the bridge at the same speed.

Figure 4.39 shows the DAF for displacement due to 50-tonne single- and two-axle vehicle loads travelling the bridge at different speeds. Although the maximum displacements in the bridge for two-axle vehicle loads are smaller than those for singleaxle vehicle loads, the DAF for displacement due to two-axle vehicle loads are higher than those due to single-axle vehicle loads for all vehicle speeds. This is because the static displacements in the bridge due to two-axle vehicle loads are much smaller than those due to single-axle vehicle loads. For example, the DAF for displacement due to 50tonne two-axle vehicle travelling the bridge at $15 \mathrm{~km} / \mathrm{h}$ is $115 \%$ of that due to single-axle vehicle. The DAF for bending moment due to two-axle vehicle loads are slightly higher than those due to single-axle vehicle loads for all vehicle speeds as shown in Figure 4.40. 
For example, the DAF for bending moment due to 50-tonne two-axle vehicle travelling the bridge at $15 \mathrm{~km} / \mathrm{h}$ is $103 \%$ of that due to single-axle vehicle. The same trends were observed for 60 and 70-tonne vehicle loads.

The results show that in general, the dynamic response of hinge-connected floating bridge to two-axle vehicle loads is smaller than that response to single-axle vehicle loads. A vehicle with two or more axles better represents the dynamic interaction of vehiclebridge-fluid system. Therefore, in the rest of the present research throughout next chapters, the vehicle is represented by a two-axle model.

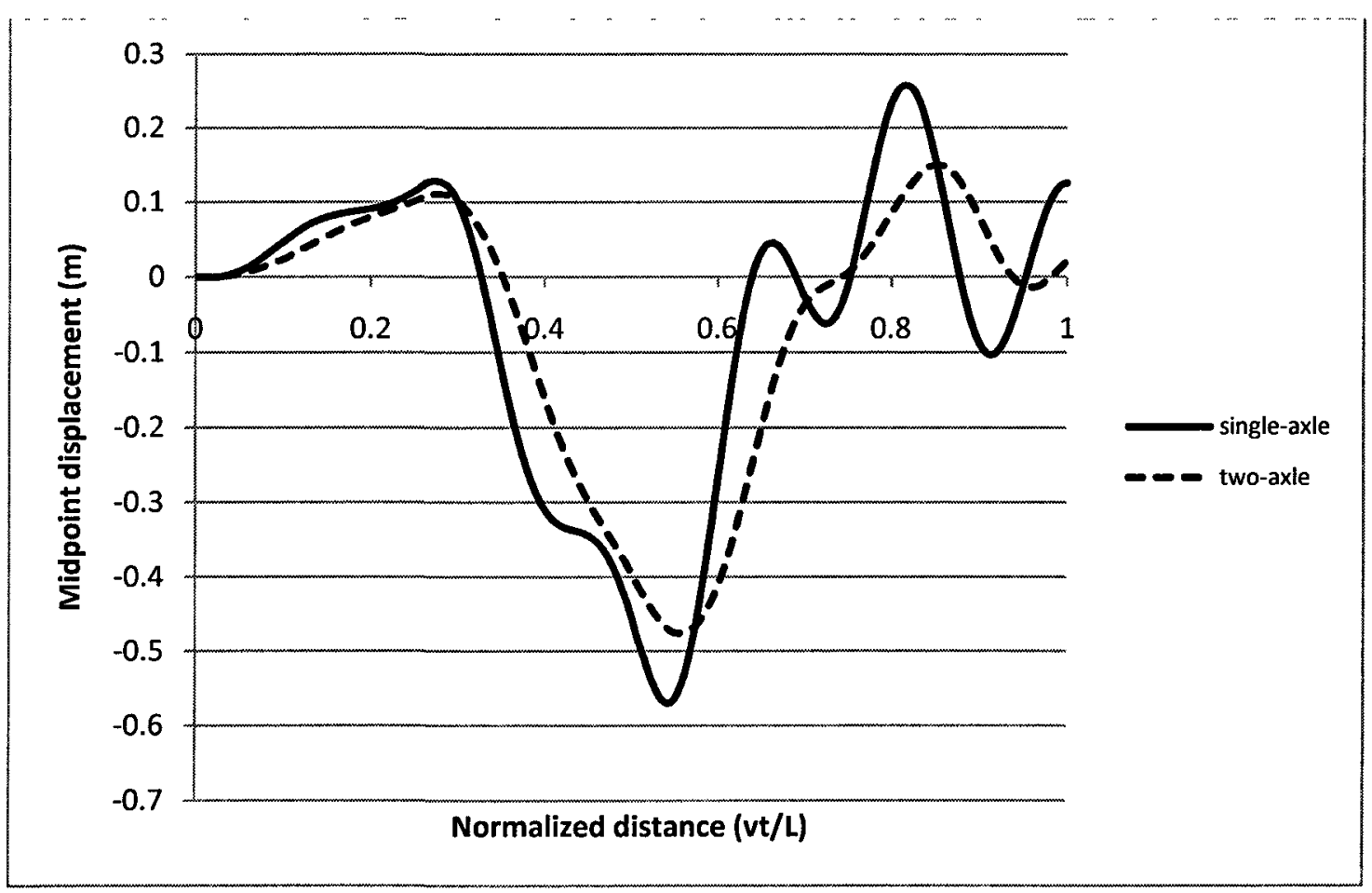

Figure 4.35: Time history of midpoint displacements in hinge-connected bridge due to 50 -tonne single- and two-axle vehicle $(\mathrm{v}=10 \mathrm{~km} / \mathrm{h})$ 


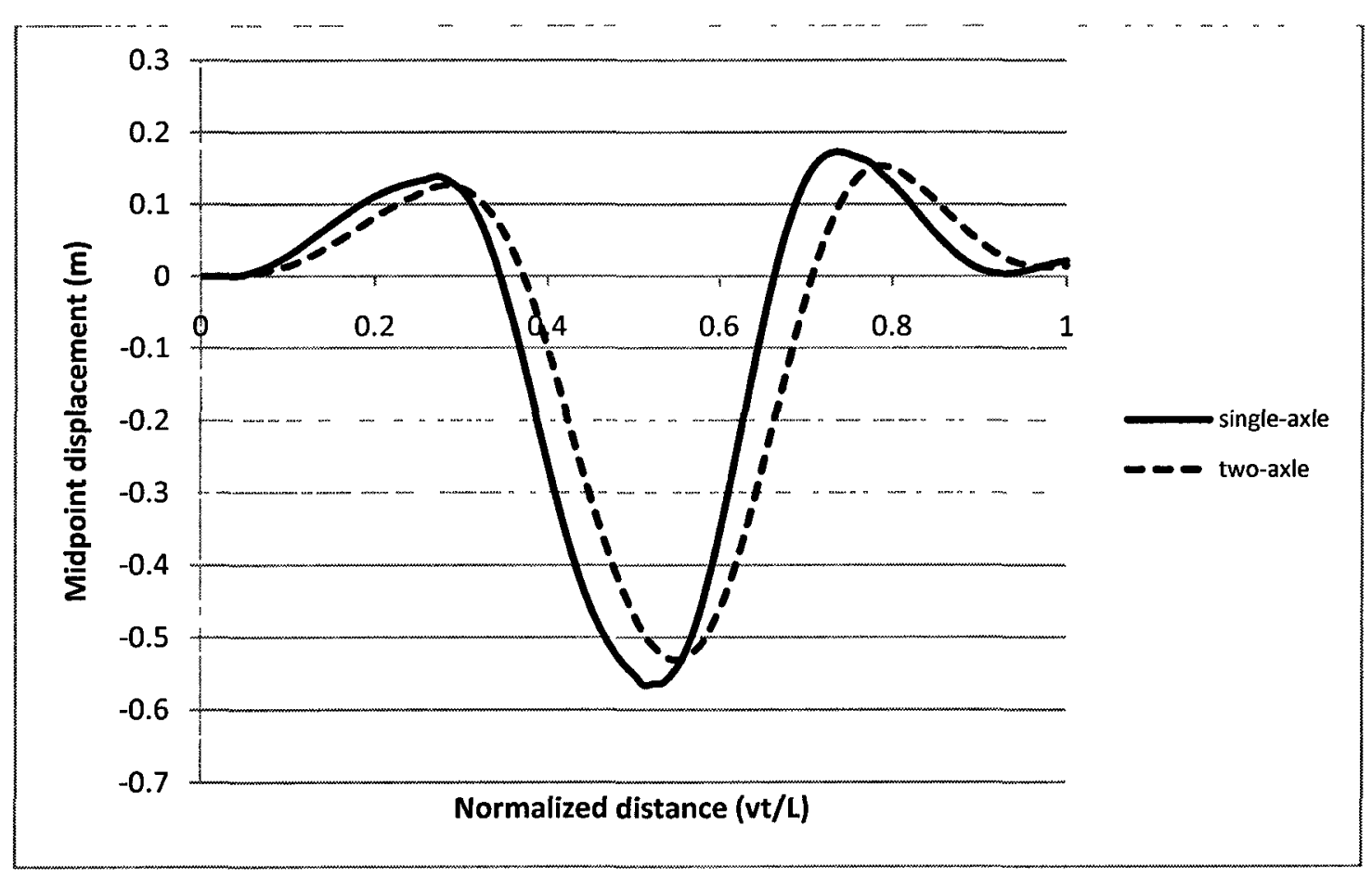

Figure 4.36: Time history of midpoint displacements in hinge-connected bridge due to 50 -tonne single- and two-axle vehicle $(\mathrm{v}=15 \mathrm{~km} / \mathrm{h})$

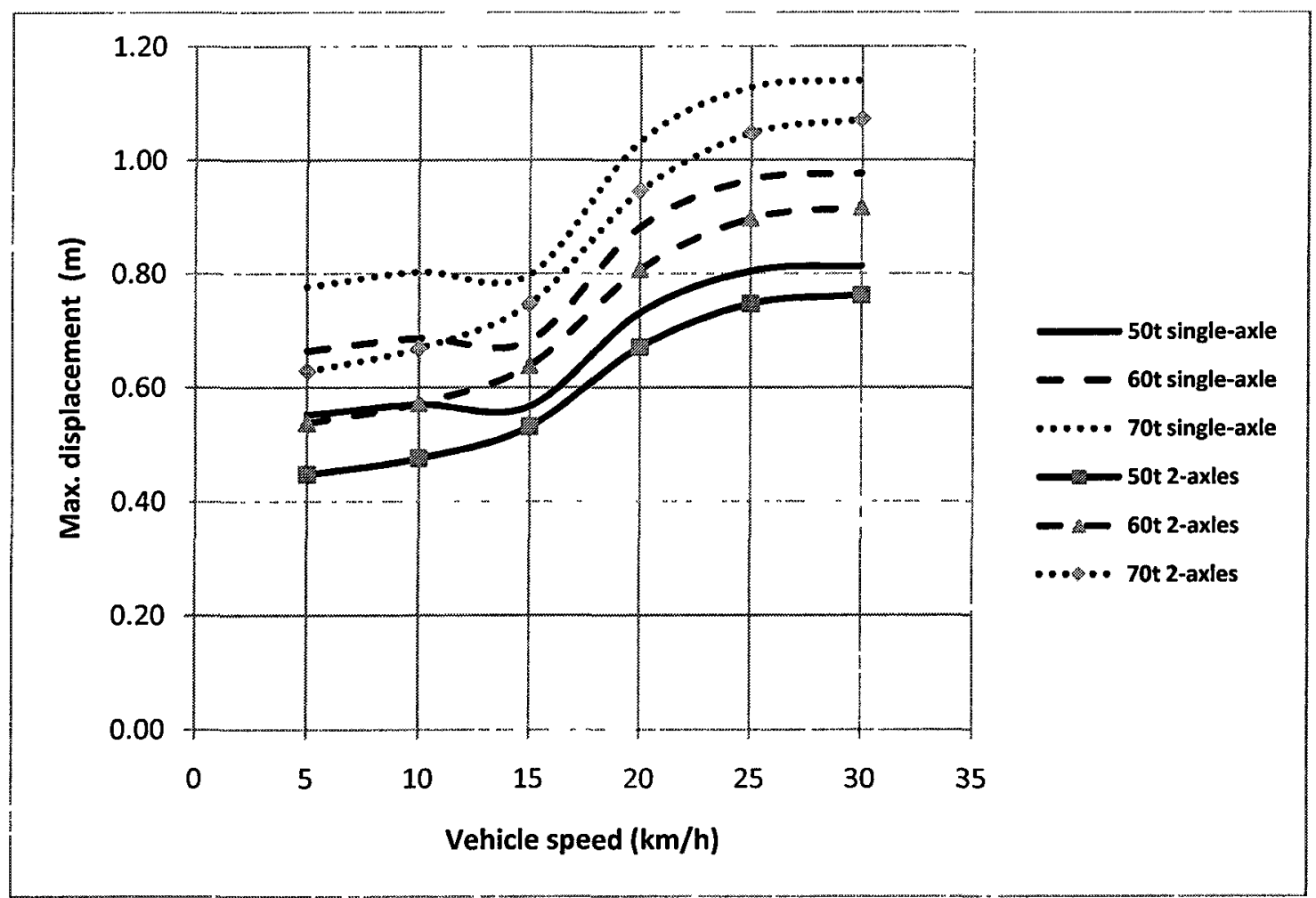

Figure 4.37: Maximum displacements in hinge-connected bridge due to single- and twoaxle vehicle 


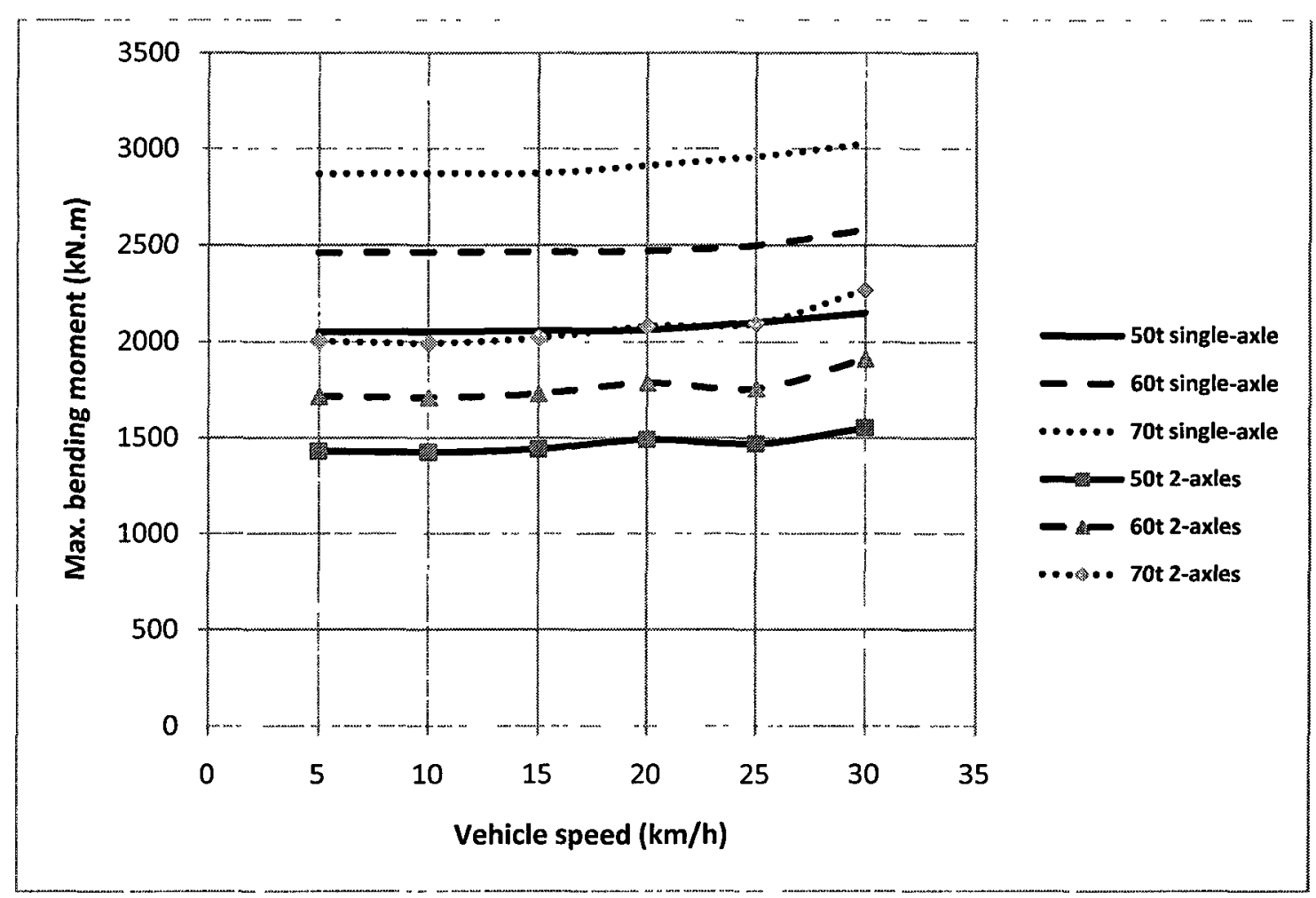

Figure 4.38: Maximum bending moments in hinge-connected bridge due to single- and two-axle vehicle

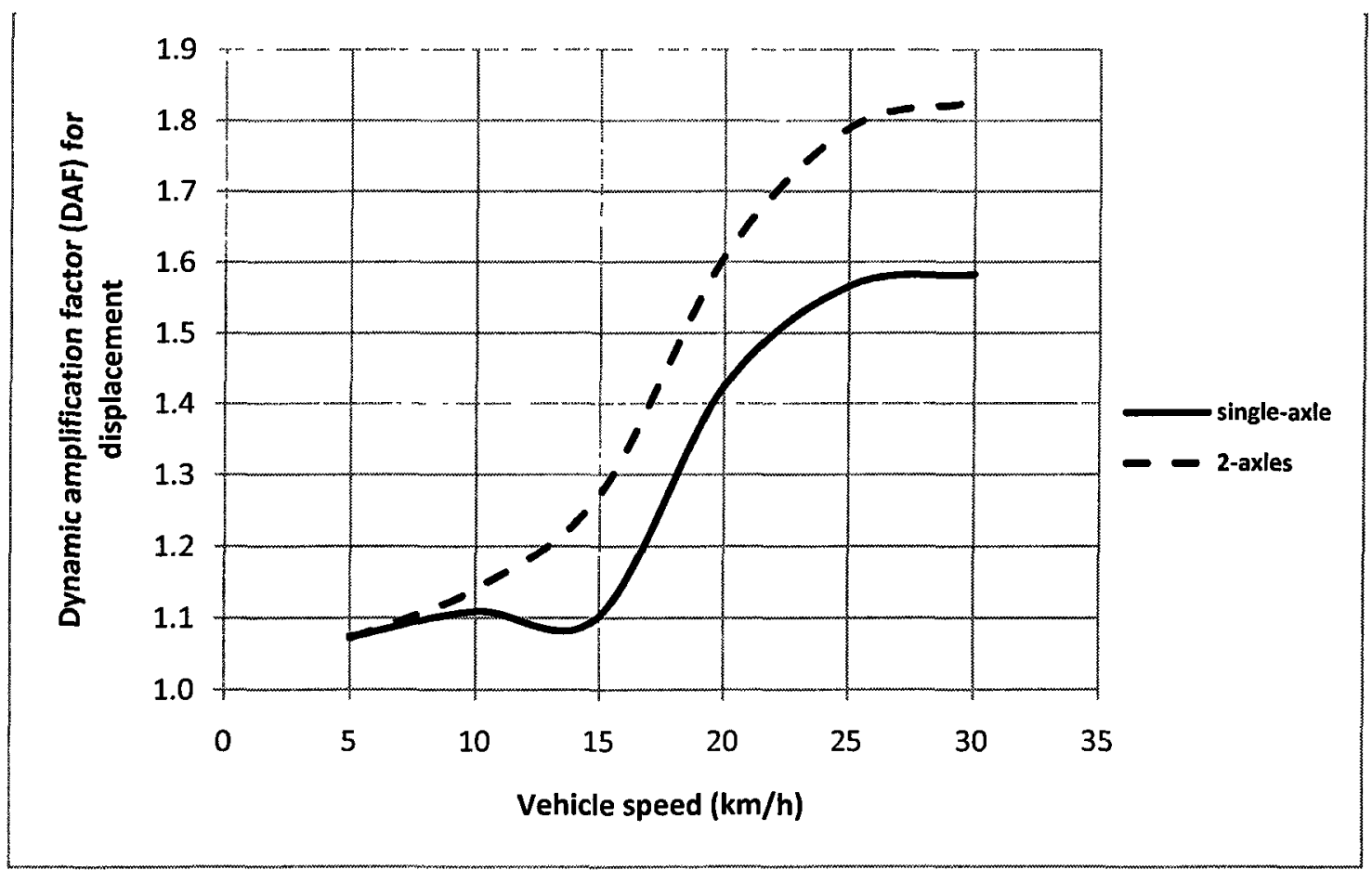

Figure 4.39: DAFs for displacement in hinge-connected bridge due to 50-tonne singleand two-axle vehicle 


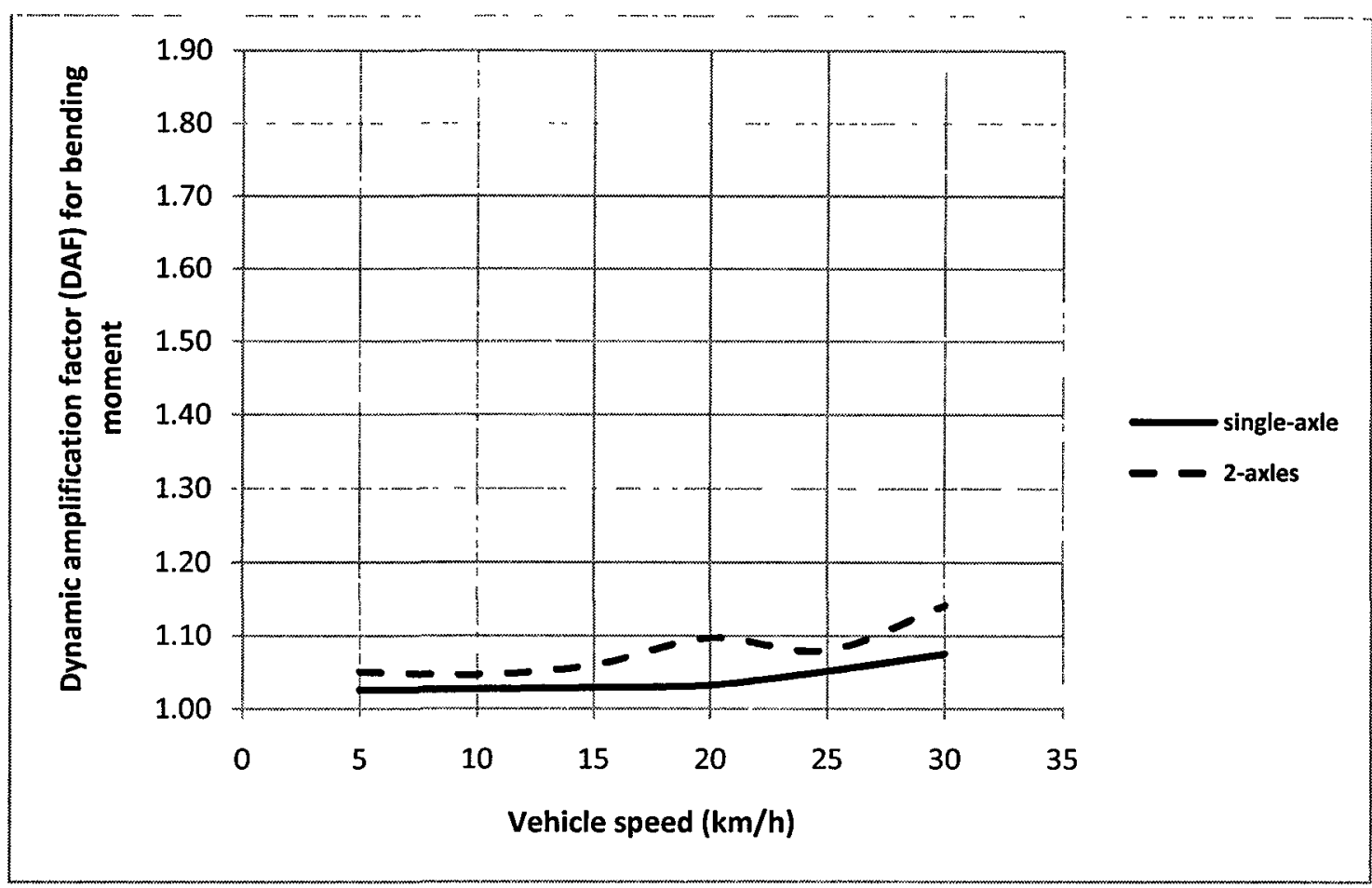

Figure 4.40: DAFs for bending moments in hinge-connected bridge due to 50-tonne single- and two-axle vehicle

\subsection{Dynamic Response of Floating Bridges to Eccentric Moving Vehicle Loads}

The objective of the parametric study presented in this section is to investigate the effect of vehicle eccentricity with respect to the longitudinal centre line of the bridge on the dynamic response of floating bridge to two-axle vehicle loads. The performed analyses and the results are summarized in Tables 4.14 and 4.15 for rigid-connected and hingeconnected floating bridges, respectively.

Figure 4.41 shows the maximum torsional moments in the bridge due to 50 -tonne twoaxle vehicle loads traversing the bridge at different speeds with an eccentricity of $0.3 \mathrm{~m}$ with respect to the longitudinal axis of the bridge. It is seen from Figure 4.41 that the maximum torsional moment in the bridge slightly changes with increasing vehicle speed. 
Table 4.14: Dynamic response of rigid-connected bridge to 50-tonne two-axle eccentric vehicle loads

\begin{tabular}{|c|c|c|c|c|c|c|}
\hline Span & $\begin{array}{c}\text { Vehicle } \\
\text { weight } \\
(\mathbf{t})\end{array}$ & $\begin{array}{c}\text { Vehicle } \\
\text { speed } \\
(\mathbf{k m} / \mathbf{h})\end{array}$ & $\begin{array}{c}\text { Vehicle } \\
\text { eccentricity } \\
(\mathbf{m})\end{array}$ & $\begin{array}{c}\text { Max } \\
\text { deflection } \\
(\mathbf{m})\end{array}$ & $\begin{array}{c}\text { Max torsional } \\
\text { moment } \\
(\mathbf{K N} . \mathbf{m})\end{array}$ & $\begin{array}{c}\text { Max } \\
\text { B.M. } \\
(\mathbf{K N} . \mathbf{m})\end{array}$ \\
\hline 84 & 50 & 5 & 0.3 & -0.161 & 139.8 & -2322 \\
\hline 84 & 50 & 10 & 0.3 & -0.162 & 139.5 & -2345 \\
\hline 84 & 50 & 15 & 0.3 & -0.169 & 139.4 & -2384 \\
\hline 84 & 50 & 20 & 0.3 & -0.178 & 138.8 & -2514 \\
\hline 84 & 50 & 25 & 0.3 & -0.188 & 138.5 & -2618 \\
\hline 84 & 50 & 30 & 0.3 & -0.203 & 138.0 & -2756 \\
\hline
\end{tabular}

Table 4.15: Dynamic response of hinge-connected bridge to 50-tonne two-axle eccentric vehicle loads

\begin{tabular}{|c|c|c|c|c|c|c|}
\hline Span & $\begin{array}{c}\text { Vehicle } \\
\text { weight } \\
(\mathbf{m})\end{array}$ & $\begin{array}{c}\text { Vehicle } \\
\text { speed } \\
(\mathbf{k m} / \mathbf{h})\end{array}$ & $\begin{array}{c}\text { Vehicle } \\
\text { eccentricity } \\
(\mathbf{m})\end{array}$ & $\begin{array}{c}\text { Max } \\
\text { displacement } \\
(\mathbf{m})\end{array}$ & $\begin{array}{c}\text { Max torsional } \\
\text { moment } \\
(\mathbf{K N} . \mathbf{m})\end{array}$ & $\begin{array}{c}\text { Max } \\
\text { B.M. } \\
(\mathbf{K N} . \mathbf{m})\end{array}$ \\
\hline 84 & 50 & 5 & 0.3 & -0.447 & 139.7 & -1430 \\
\hline 84 & 50 & 10 & 0.3 & -0.476 & 139.8 & -1425 \\
\hline 84 & 50 & 15 & 0.3 & -0.531 & 138.6 & -1443 \\
\hline 84 & 50 & 20 & 0.3 & -0.671 & 141.4 & -1493 \\
\hline 84 & 50 & 25 & 0.3 & -0.747 & 146.2 & -1470 \\
\hline 84 & 50 & 30 & 0.3 & -0.762 & 145.9 & -1554 \\
\hline
\end{tabular}




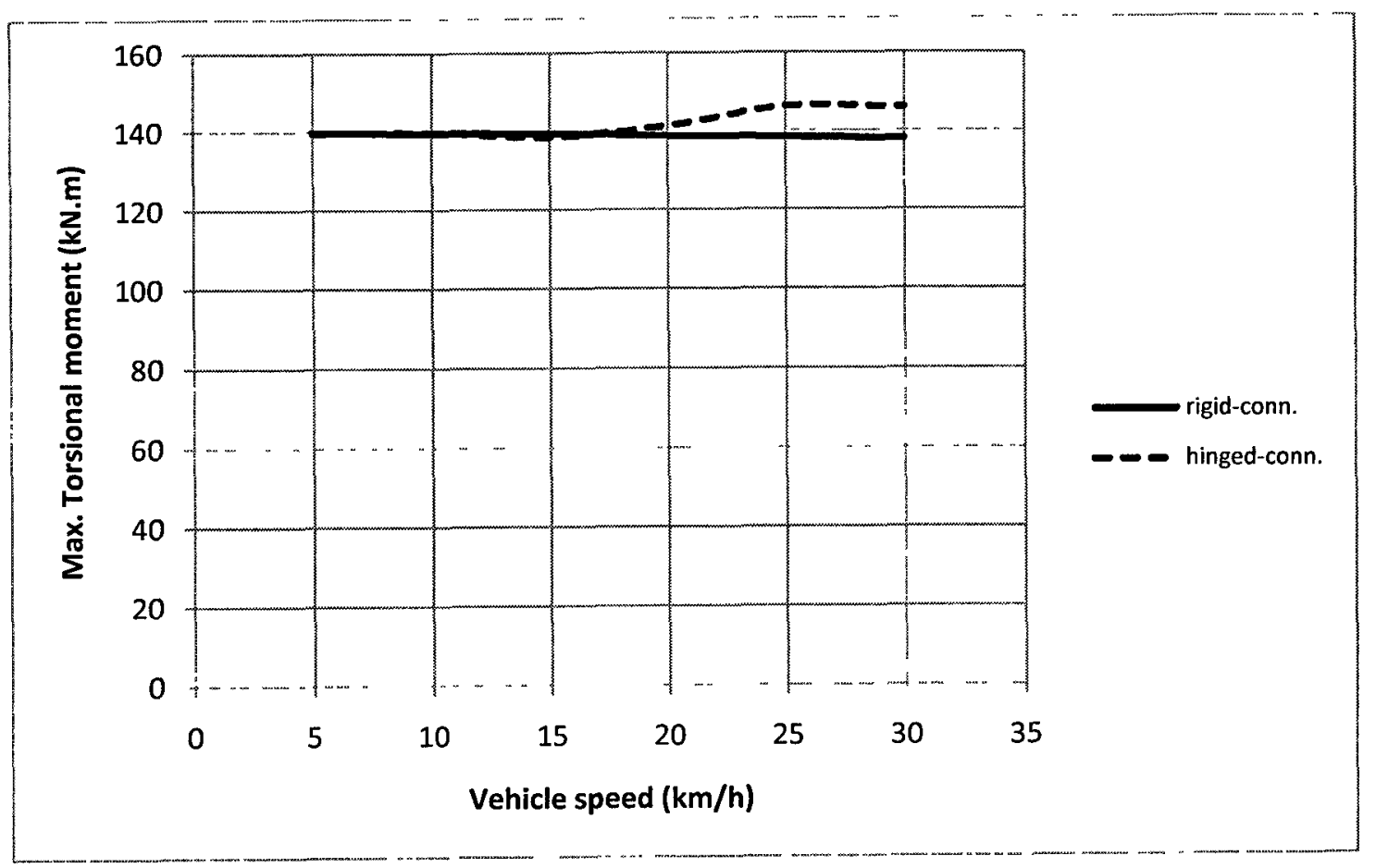

Figure 4.41: Effect of vehicle speed on maximum torsional moment due to 50-tonne twoaxle eccentric vehicle loads

\subsection{Dynamic Response of Light-Weight Floating Bridges to Moving Vehicle Loads}

The objective of the parametric study presented in this section is to investigate the dynamic response of floating bridges composed of light-weight materials like aluminum to two-axle eccentric vehicle loads, and to compare the dynamic response of the aluminum bridge to that of the steel bridge. The analyses performed present the dynamic response of the bridge to moving vehicle loads from the static equilibrium position under the self weight of the bridge. 


\subsubsection{Dynamic Response of Rigid-Connected Aluminum Floating Bridge to Two-}

Axle Vehicle Loads

The objective of this section is to study the potential of using light-weight materials for improving the global dynamic behaviour of floating bridges. The dynamic response of rigid-connected aluminum floating bridge to two-axle vehicle loads is investigated for different vehicle speeds from $5 \mathrm{~km} / \mathrm{h}$ to $45 \mathrm{~km} / \mathrm{h}$. The details of the analyses and the results are summarized in Table 4.16.

\subsubsection{Discussion of Results}

The maximum midpoint displacements in the aluminum bridge are higher than those in the steel bridge for all vehicle speeds from $5 \mathrm{~km} / \mathrm{h}$ to $45 \mathrm{~km} / \mathrm{h}$. Figures 4.42 and 4.43 show time history plots of midpoint displacement in the aluminum and steel bridges at speeds of $10 \mathrm{~km} / \mathrm{h}$ and $15 \mathrm{~km} / \mathrm{h}$, respectively.

Figure 4.44 shows the maximum deflection in the aluminum and steel bridges due to 50tonne two-axle vehicle loads traversing the bridge at different speeds. The maximum deflections in the aluminum bridge are higher than those in the steel bridge for all vehicle speeds; this is because of the lower modulus of elasticity of aluminum compared to that of steel. The maximum bending moments in the aluminum bridge are less than those in the steel bridge as shown in Figure 4.45.

While the maximum static deflection in the steel bridge under self weight is $-0.113 \mathrm{~m}$, the maximum static deflection in the aluminum bridge under self weight is $-0.038 \mathrm{~m}$ which is 
about one third of that in the steel bridge in accordance with the ratio of aluminum to steel density.

The deflection in the rigid-connected aluminum floating bridge is higher than that in the steel bridge; however the weight of the aluminum bridge is about one third of the weight of the steel bridge according to the ratio of the density of each of them. This means that another aluminum bridge cross-section having the same weight as that of the steel crosssection can give a better global dynamic response of the bridge.

Table 4.16: Dynamic response of rigid-connected aluminum bridge to 50-tonne two-axle vehicle loads

\begin{tabular}{|c|c|c|c|c|}
\hline $\begin{array}{c}\text { Span } \\
(\mathbf{m})\end{array}$ & $\begin{array}{c}\text { Vehicle } \\
\text { Weight } \\
(\mathbf{t})\end{array}$ & $\begin{array}{c}\text { Vehicle } \\
\text { speed } \\
(\mathbf{k m} / \mathbf{h})\end{array}$ & $\begin{array}{c}\text { Max } \\
\text { deflection } \\
(\mathbf{m})\end{array}$ & $\begin{array}{c}\text { Max } \\
\text { B.M. } \\
(\text { KN.m) }\end{array}$ \\
\hline 84 & 50 & 5 & -0.205 & -1672 \\
\hline 84 & 50 & 10 & -0.210 & -1701 \\
\hline 84 & 50 & 15 & -0.221 & -1755 \\
\hline 84 & 50 & 20 & -0.248 & -1866 \\
\hline 84 & 50 & 25 & -0.272 & -1939 \\
\hline 84 & 50 & 30 & -0.291 & -2025 \\
\hline 84 & 50 & 45 & -0.424 & -3617 \\
\hline
\end{tabular}




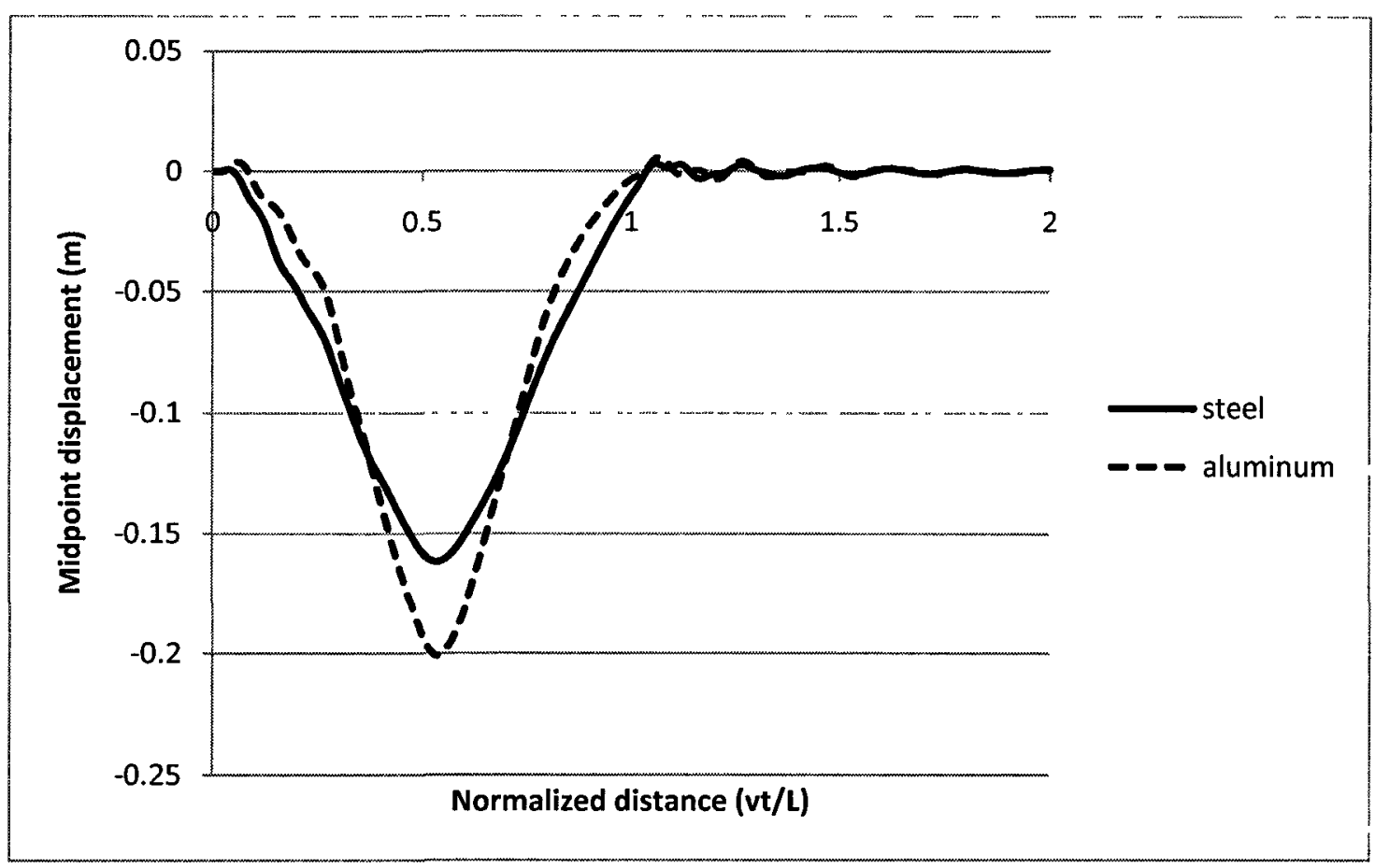

Figure 4.42: Time history of midpoint displacements in rigid-connected steel and aluminum bridges due to 50 -tonne two-axle vehicle loads $(\mathrm{v}=10 \mathrm{~km} / \mathrm{h})$

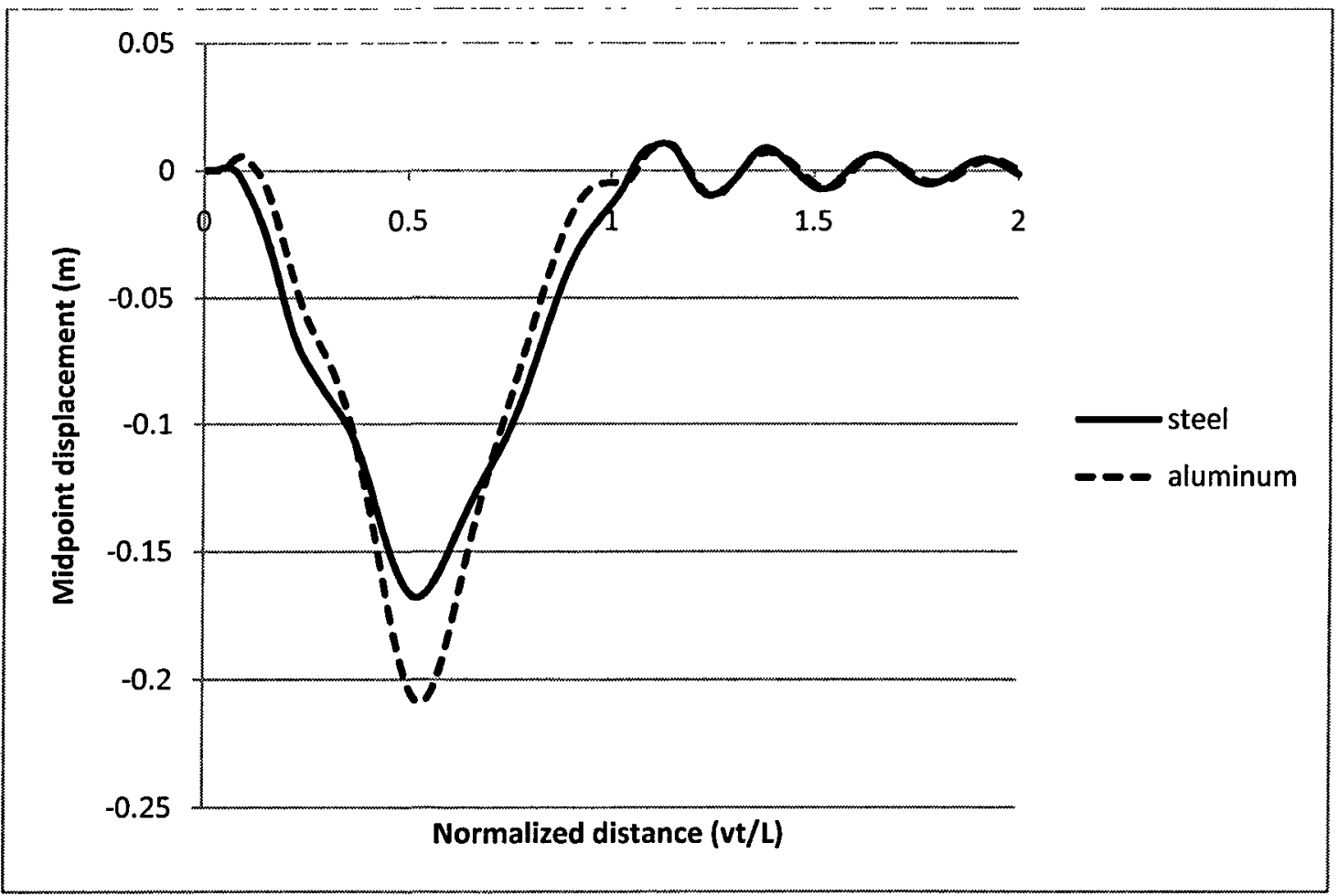

Figure 4.43: Time history of midpoint displacements in rigid-connected steel and aluminum bridges due to 50 -tonne two-axle vehicle loads $(\mathrm{v}=15 \mathrm{~km} / \mathrm{h})$ 


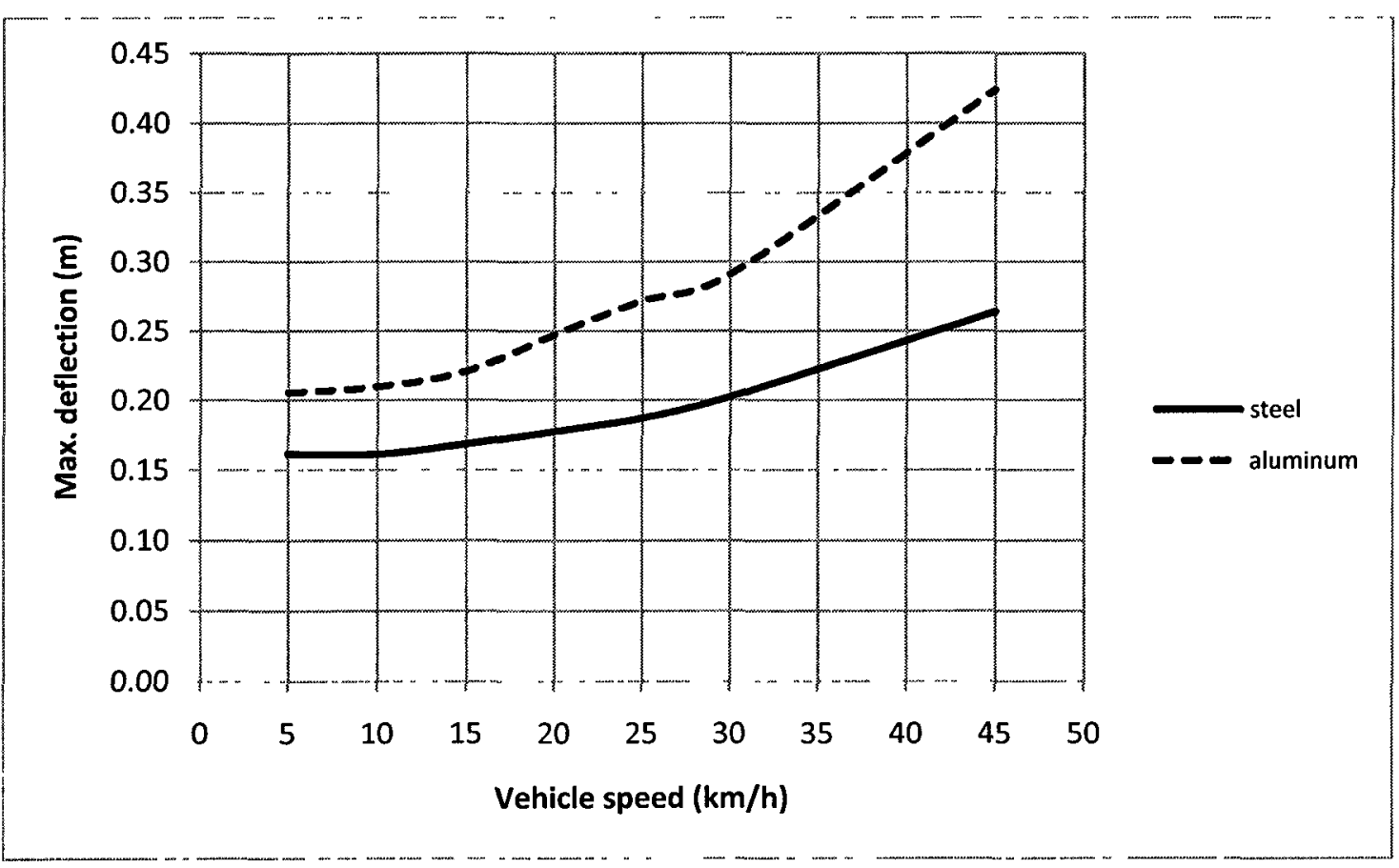

Figure 4.44: Effect of vehicle speed on maximum deflection in rigid-connected steel and aluminum bridges due to 50 -tonne two-axle vehicle loads

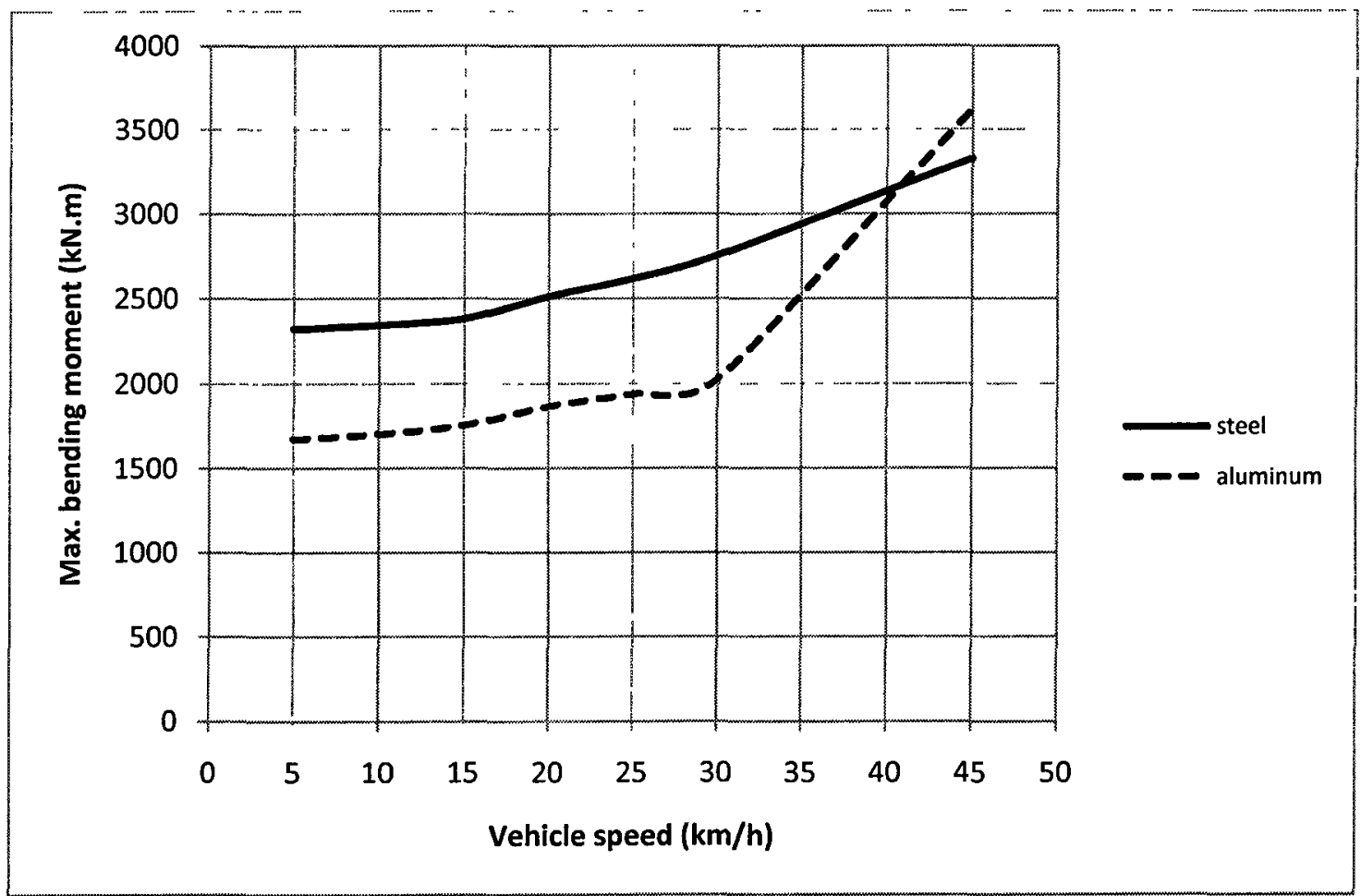

Figure 4.45: Effect of vehicle speed on maximum bending moment in rigid-connected steel and aluminum bridges due to 50-tonne two-axle vehicle loads 


\subsubsection{Dynamic Response of Hinge-Connected Aluminum Floating Bridge to Two- Axle Vehicle Loads}

The dynamic response of hinge-connected aluminum floating bridge to eccentric twoaxle vehicle loads is investigated for different vehicle speeds from $5 \mathrm{~km} / \mathrm{h}$ to $30 \mathrm{~km} / \mathrm{h}$. The details of the analyses and the results are summarized in Table 4.17.

Table 4.17: Dynamic response of hinge-connected aluminum bridge to 50-tonne two-axle vehicle loads

\begin{tabular}{|c|c|c|c|c|}
\hline Span & $\begin{array}{c}\text { Vehicle } \\
\text { Weight } \\
(\mathbf{m})\end{array}$ & $\begin{array}{c}\text { Vehicle } \\
\text { speed } \\
(\mathbf{k m} / \mathbf{h})\end{array}$ & $\begin{array}{c}\text { Max } \\
\text { displacement } \\
(\mathbf{m})\end{array}$ & $\begin{array}{c}\text { Max } \\
\text { B.M. } \\
(\text { KN.m })\end{array}$ \\
\hline 84 & 50 & 5 & -0.454 & -1350 \\
\hline 84 & 50 & 10 & -0.483 & -1396 \\
\hline 84 & 50 & 15 & -0.536 & -1412 \\
\hline 84 & 50 & 20 & -0.676 & -1420 \\
\hline 84 & 50 & 25 & -0.747 & -1609 \\
\hline 84 & 50 & 30 & -0.748 & -1779 \\
\hline
\end{tabular}

\subsubsection{Discussion of Results}

The midpoint displacements in the aluminum bridge are the same as those in the steel bridge for all vehicle speeds from $5 \mathrm{~km} / \mathrm{h}$ to $30 \mathrm{~km} / \mathrm{h}$, with very little differences caused by the elastic deformations in each typical bay of the bridge. Figure 4.46 and 4.47 show typical time history plots of midpoint displacement in the aluminum and steel bridges at speeds of $10 \mathrm{~km} / \mathrm{h}$ and $15 \mathrm{~km} / \mathrm{h}$, respectively. 
While the maximum static displacement in the steel bridge under self weight is $-0.127 \mathrm{~m}$, the maximum static displacement in the aluminum bridge under self weight is $-0.043 \mathrm{~m}$ which is about one third of that in the steel bridge in accordance with the ratio of aluminum to steel density.

Figure 4.48 shows the maximum displacement in both aluminum and steel hingeconnected bridges for different vehicle speeds. The maximum displacements in the aluminum bridge are the same as those in the steel bridge for all vehicle speeds with very little differences due to the elastic deformations in each typical bay of the bridge between the two intermediate hinges; these elastic deformations are slightly higher in the case of an aluminum bridge compared to that elastic deformations in the steel bridge. The elastic deformation in each bridge bay is much less than the rigid-body displacement so that the significant displacement in the entire hinge-connected bridge is due to rotation at the hinged nodes and not due to deformation at the rigid nodes. In this case, the water buoyancy provides most of the effective stiffness of the bridge and light-weight materials such as aluminum can be utilized decreasing the weight of the bridge significantly without affecting the bridge dynamic response.

The maximum bending moments in the aluminum bridge are less than those in the steel bridge for low vehicle speeds and higher than those in the steel bridge for high vehicle speeds as shown in Figure 4.49. 


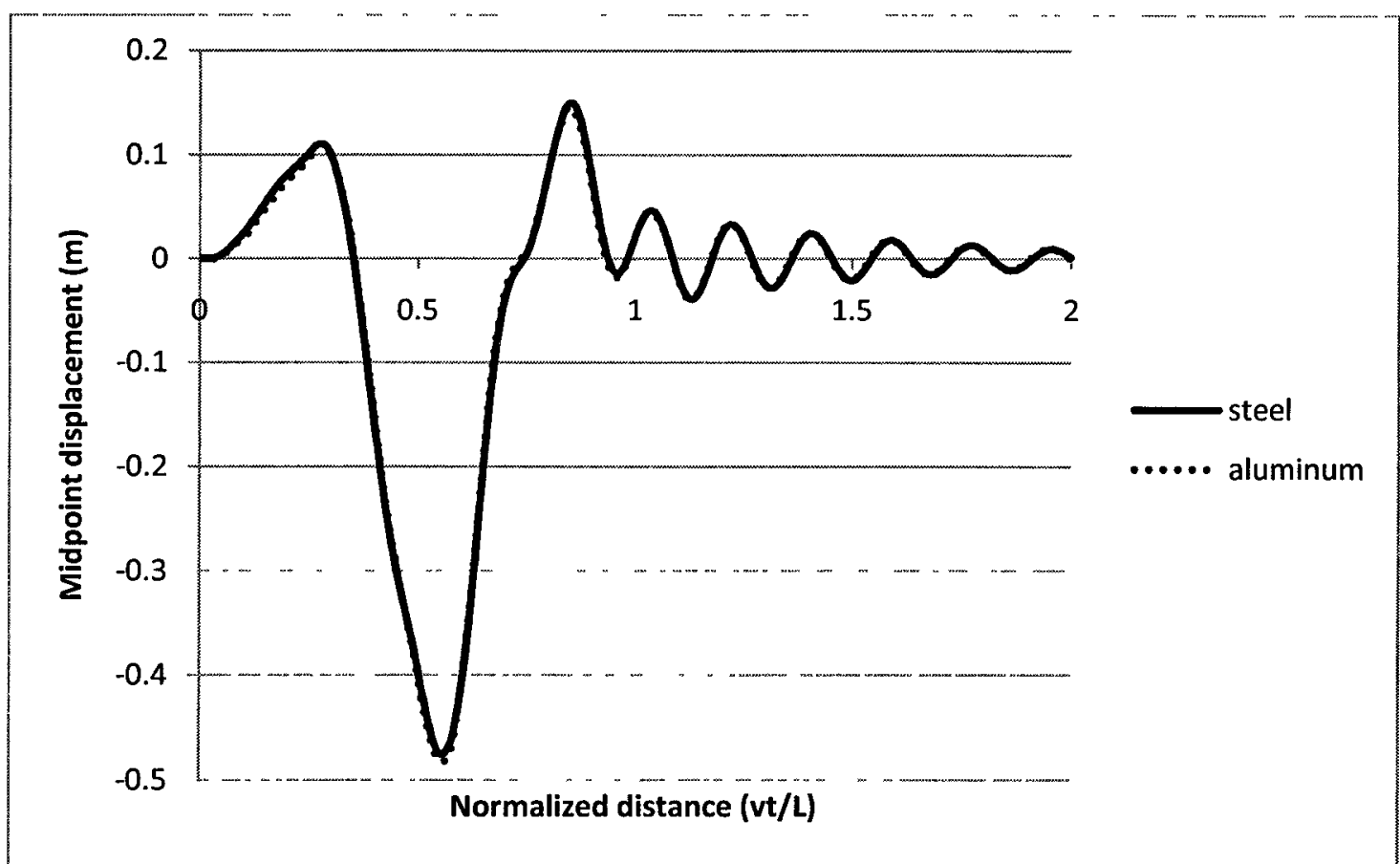

Figure 4.46: Time history of midpoint displacements in hinge-connected steel and aluminum bridges due to 50 -tonne two-axle vehicle loads $(\mathrm{v}=10 \mathrm{~km} / \mathrm{h})$

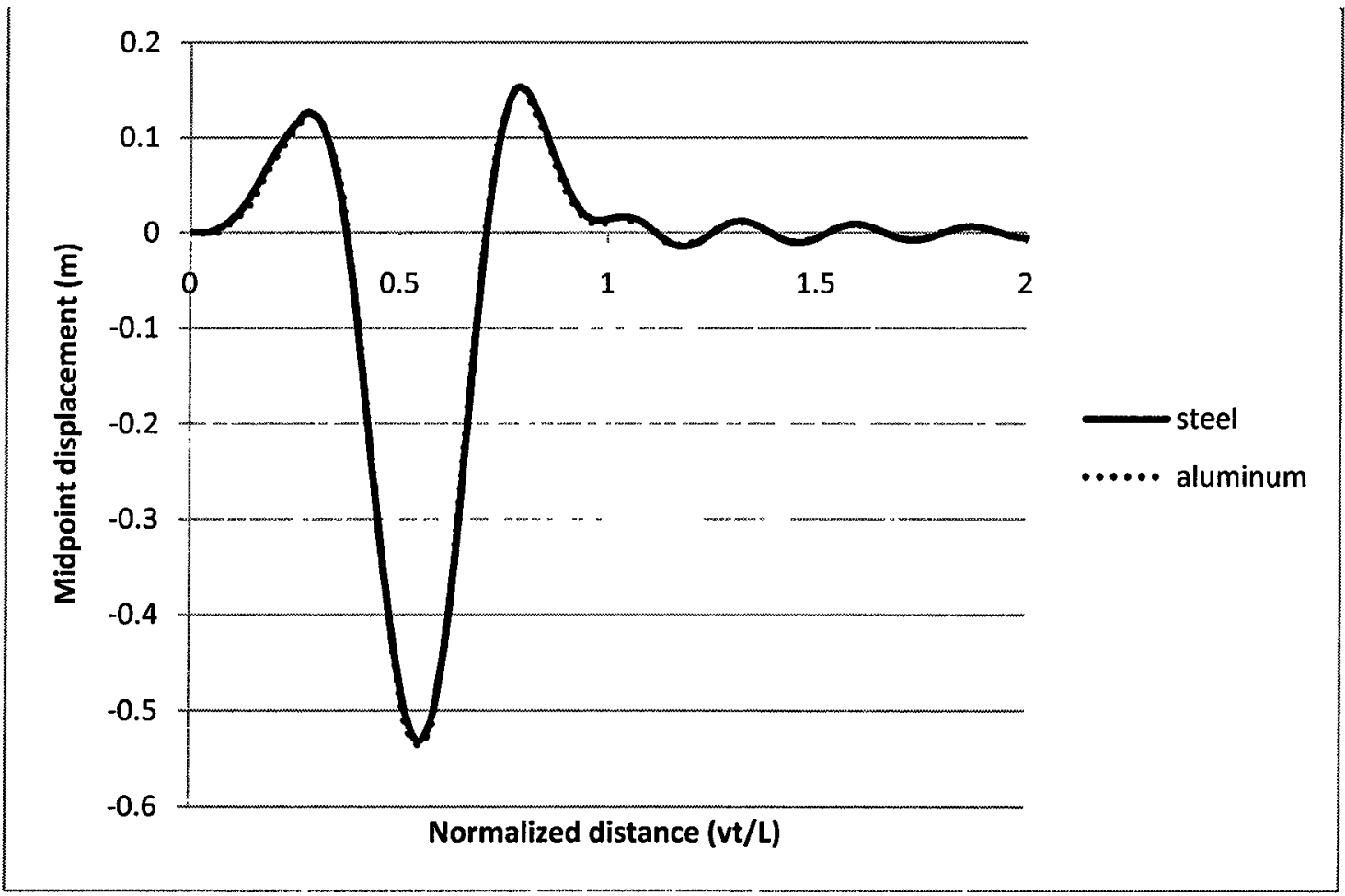

Figure 4.47: Time history of midpoint displacements in hinge-connected steel and aluminum bridges due to 50 -tonne two-axle vehicle loads $(\mathrm{v}=15 \mathrm{~km} / \mathrm{h})$ 


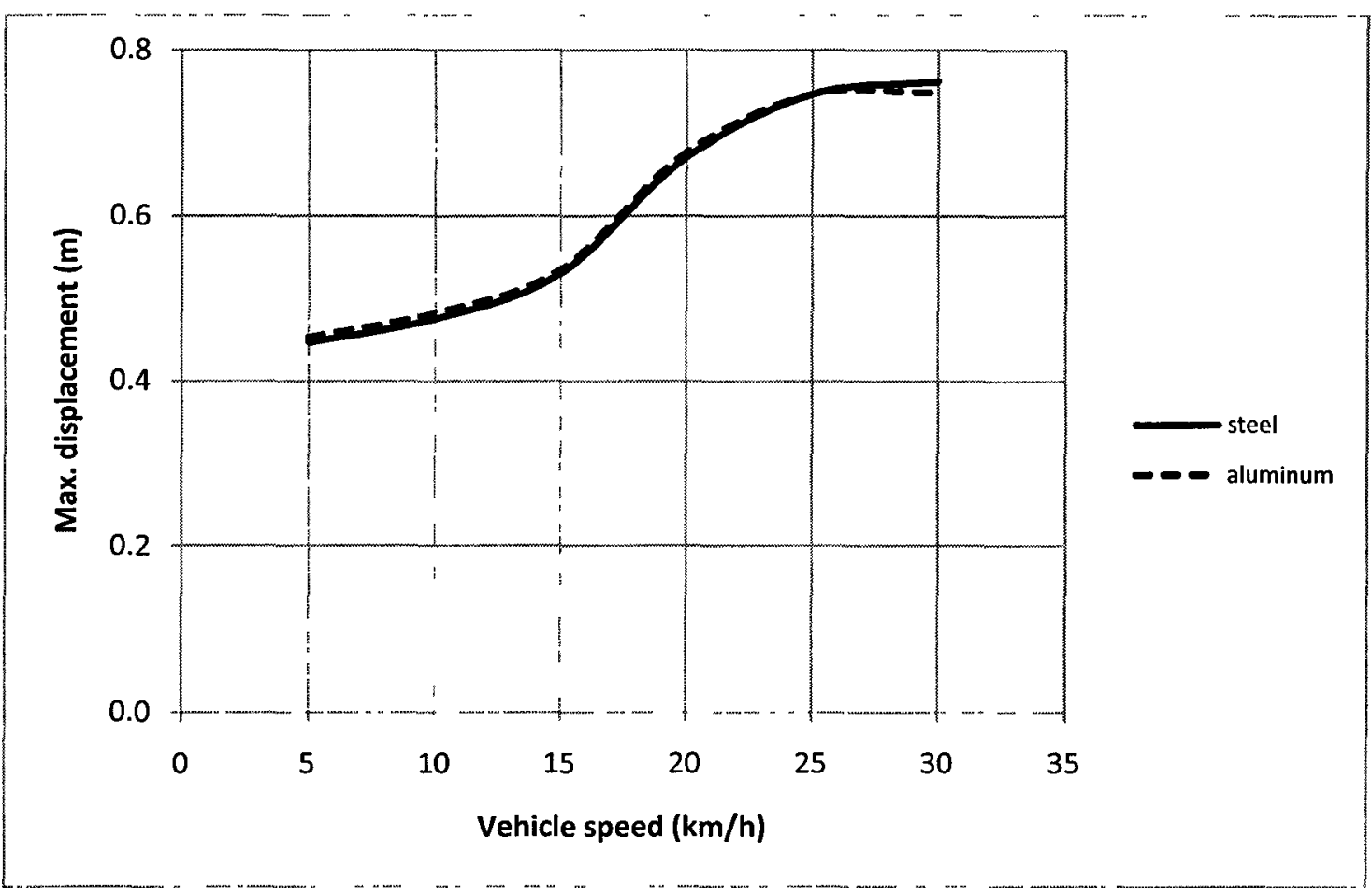

Figure 4.48: Effect of vehicle speed on maximum displacement in hinge-connected steel and aluminum bridges due to 50-tonne two-axle vehicle loads

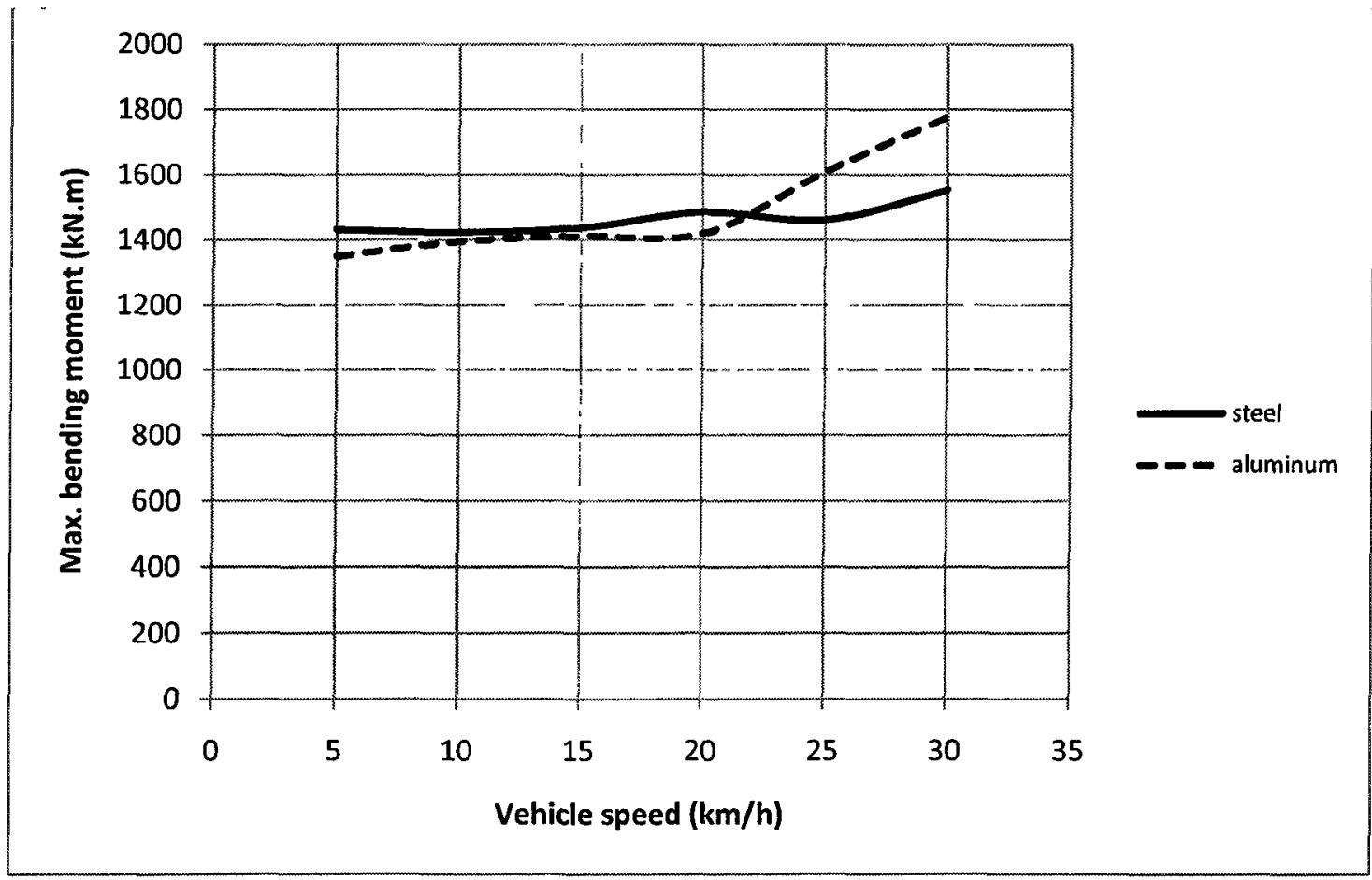

Figure 4.49: Effect of vehicle speed on maximum bending moment in hinge-connected steel and aluminum bridges due to 50-tonne two-axle vehicle loads 


\subsection{Parametric Study for the Transport Capacity and Dynamic Response of Floating Bridges to Successive Vehicle Loads}

The objective of the parametric study presented in this section is to investigate the effect of the separation distance between successive vehicles on the dynamic response of rapid deployment floating bridge. The parametric study is performed for separation distances of 50,40 and $30 \mathrm{~m}$ and for different vehicle speeds and weights in order to investigate and optimize the transport capacity or rate of vehicular flow on the floating bridge. Each vehicle is represented by two-axle model. The displacements and bending moments developed in the bridge due to two successive vehicle loads are divided by the static displacements and bending moments in order to calculate DAFs for displacement and bending moment.

\subsubsection{Dynamic Response of Rigid-Connected Floating Bridge to Two Successive} Vehicle Loads

The dynamic response of rigid-connected floating bridge to two successive vehicle loads is investigated for different vehicle weights, speeds and separation distances. The two successive vehicles are traversing the bridge at different speeds from $5 \mathrm{~km} / \mathrm{h}$ to $15 \mathrm{~km} / \mathrm{h}$ and with separation distances of 50,40 and $30 \mathrm{~m}$. The two vehicles have the same weight of 50,60 or 70 tonnes. The analyses and the results are summarized in Tables 4.18 to 4.26 . 
Table 4.18: Dynamic response of rigid-connected bridge to two successive 50-tonne twoaxle vehicle loads (spacing $50 \mathrm{~m}$ )

\begin{tabular}{|c|c|c|c|c|c|c|c|c|}
\hline $\begin{array}{c}\text { Separ. } \\
\text { dist. } \\
(\mathbf{m})\end{array}$ & $\begin{array}{c}\text { Vehicle } \\
\text { weight } \\
(\mathbf{t})\end{array}$ & $\begin{array}{c}\text { Vehicle } \\
\text { speed } \\
(\mathbf{k m} / \mathbf{h})\end{array}$ & $\begin{array}{c}\text { Max } \\
\mathbf{d e f l} . \\
(\mathbf{m})\end{array}$ & $\begin{array}{c}\text { Static } \\
\mathbf{d e f l} \\
\mathbf{( m )}\end{array}$ & $\begin{array}{c}\text { DAF } \\
\text { for defl. }\end{array}$ & $\begin{array}{c}\text { Max } \\
\text { B.M. } \\
(\text { KN.m) }\end{array}$ & $\begin{array}{c}\text { Static } \\
\text { B.M. } \\
(\text { KN.m) }\end{array}$ & $\begin{array}{c}\text { DAF for } \\
\text { B.M. }\end{array}$ \\
\hline 50 & 50 & 5 & -0.167 & -0.159 & 1.05 & -2322 & -2062 & 1.13 \\
\hline 50 & 50 & 10 & -0.166 & -0.159 & 1.04 & -2345 & -2062 & 1.14 \\
\hline 50 & 50 & 15 & -0.169 & -0.159 & 1.06 & -2384 & -2062 & 1.16 \\
\hline 50 & 50 & 20 & -0.188 & -0.159 & 1.18 & -2514 & -2062 & 1.22 \\
\hline 50 & 50 & 25 & -0.209 & -0.159 & 1.32 & -2618 & -2062 & 1.27 \\
\hline
\end{tabular}

Table 4.19: Dynamic response of rigid-connected bridge to two successive 60-tonne two-

\begin{tabular}{|c|c|c|c|c|c|c|c|c|}
\hline $\begin{array}{c}\text { Separ. } \\
\text { dist. } \\
(\mathbf{m})\end{array}$ & $\begin{array}{c}\text { Vehicle } \\
\text { weight } \\
(\mathbf{t})\end{array}$ & $\begin{array}{c}\text { Vehicle } \\
\text { speed } \\
(\mathbf{k m} / \mathbf{h})\end{array}$ & $\begin{array}{c}\text { Max } \\
\mathbf{d e f l} \\
(\mathbf{m})\end{array}$ & $\begin{array}{c}\text { Static } \\
\mathbf{d e f l} \\
\mathbf{( m )}\end{array}$ & $\begin{array}{c}\text { DAF for } \\
\text { defl. }\end{array}$ & $\begin{array}{c}\text { Max } \\
\text { B.M. } \\
(\text { KN.m) }\end{array}$ & $\begin{array}{c}\text { Static } \\
\text { B.M. } \\
(\text { KN.m) }\end{array}$ & $\begin{array}{c}\text { DAF for } \\
\text { B.M. }\end{array}$ \\
\hline 50 & 60 & 5 & -0.200 & -0.191 & 1.05 & -2787 & -2475 & 1.13 \\
\hline 50 & 60 & 10 & -0.199 & -0.191 & 1.04 & -2815 & -2475 & 1.14 \\
\hline 50 & 60 & 15 & -0.203 & -0.191 & 1.06 & -2862 & -2475 & 1.16 \\
\hline 50 & 60 & 20 & -0.225 & -0.191 & 1.18 & -3019 & -2475 & 1.22 \\
\hline 50 & 60 & 25 & -0.251 & -0.191 & 1.32 & -3142 & -2475 & 1.27 \\
\hline
\end{tabular}


Table 4.20: Dynamic response of rigid-connected bridge to two successive 70-tonne twoaxle vehicle loads (spacing $50 \mathrm{~m}$ )

\begin{tabular}{|c|c|c|c|c|c|c|c|c|}
\hline $\begin{array}{c}\text { Separ. } \\
\text { dist. } \\
(\mathbf{m})\end{array}$ & $\begin{array}{c}\text { Vehicle } \\
\text { weight } \\
(\mathbf{t})\end{array}$ & $\begin{array}{c}\text { Vehicle } \\
\text { speed } \\
(\mathbf{k m} / \mathbf{h})\end{array}$ & $\begin{array}{c}\text { Max } \\
\mathbf{d e f l} \\
\mathbf{( m )}\end{array}$ & $\begin{array}{c}\text { Static } \\
\text { defl. } \\
\mathbf{( m )}\end{array}$ & $\begin{array}{c}\text { DAF } \\
\text { for defl. }\end{array}$ & $\begin{array}{c}\text { Max } \\
\text { B.M. } \\
\text { (KN.m) }\end{array}$ & $\begin{array}{c}\text { Static } \\
\text { B.M. } \\
(\text { KN.m) }\end{array}$ & $\begin{array}{c}\text { DAF for } \\
\text { B.M. }\end{array}$ \\
\hline 50 & 70 & 5 & -0.233 & -0.223 & 1.05 & -3251 & -2887 & 1.13 \\
\hline 50 & 70 & 10 & -0.233 & -0.223 & 1.05 & -3285 & -2887 & 1.14 \\
\hline 50 & 70 & 15 & -0.236 & -0.223 & 1.06 & -3340 & -2887 & 1.16 \\
\hline 50 & 70 & 20 & -0.262 & -0.223 & 1.18 & -3525 & -2887 & 1.22 \\
\hline 50 & 70 & 25 & -0.293 & -0.223 & 1.31 & -3670 & -2887 & 1.27 \\
\hline
\end{tabular}

Table 4.21: Dynamic response of rigid-connected bridge to two successive 50-tonne twoaxle vehicle loads (spacing $40 \mathrm{~m}$ )

\begin{tabular}{|c|c|c|c|c|c|c|c|c|}
\hline $\begin{array}{c}\text { Separ. } \\
\text { dist. } \\
(\mathbf{m})\end{array}$ & $\begin{array}{c}\text { Vehicle } \\
\text { weight } \\
(\mathbf{t})\end{array}$ & $\begin{array}{c}\text { Vehicle } \\
\text { speed } \\
(\mathbf{k m} / \mathbf{h})\end{array}$ & $\begin{array}{c}\text { Max } \\
\mathbf{d e f l} \\
(\mathbf{m})\end{array}$ & $\begin{array}{c}\text { Static } \\
\text { defl. } \\
(\mathbf{m})\end{array}$ & $\begin{array}{c}\text { DAF } \\
\text { for defl. }\end{array}$ & $\begin{array}{c}\text { Max } \\
\text { B.M. } \\
(\text { KN.m) }\end{array}$ & $\begin{array}{c}\text { Static } \\
\text { B.M. } \\
(\mathbf{K N . m})\end{array}$ & $\begin{array}{c}\text { DAF for } \\
\text { B.M. }\end{array}$ \\
\hline 40 & 50 & 5 & -0.183 & -0.173 & 1.06 & -2322 & -1961 & 1.18 \\
\hline 40 & 50 & 10 & -0.184 & -0.173 & 1.06 & -2345 & -1961 & 1.20 \\
\hline 40 & 50 & 15 & -0.194 & -0.173 & 1.12 & -2384 & -1961 & 1.22 \\
\hline 40 & 50 & 20 & -0.197 & -0.173 & 1.14 & -2514 & -1961 & 1.28 \\
\hline 40 & 50 & 25 & -0.225 & -0.173 & 1.30 & -2717 & -1961 & 1.39 \\
\hline
\end{tabular}


Table 4.22: Dynamic response of rigid-connected bridge to two successive 60-tonne twoaxle vehicle loads (spacing $40 \mathrm{~m}$ )

\begin{tabular}{|c|c|c|c|c|c|c|c|c|}
\hline $\begin{array}{c}\text { Separ. } \\
\text { dist. } \\
(\mathbf{m})\end{array}$ & $\begin{array}{c}\text { Vehicle } \\
\text { weight } \\
(\mathbf{t})\end{array}$ & $\begin{array}{c}\text { Vehicle } \\
\mathbf{\text { speed }} \\
(\mathbf{k m} / \mathbf{h})\end{array}$ & $\begin{array}{c}\text { Max } \\
\mathbf{d e f l} \\
(\mathbf{m})\end{array}$ & $\begin{array}{c}\text { Static } \\
\text { defl. } \\
(\mathbf{m})\end{array}$ & $\begin{array}{c}\text { DAF } \\
\text { for defl. }\end{array}$ & $\begin{array}{c}\text { Max } \\
\text { B.M. } \\
(\mathbf{K N . m})\end{array}$ & $\begin{array}{c}\text { Static } \\
\text { B.M. } \\
(\mathbf{K N} . \mathbf{m})\end{array}$ & $\begin{array}{c}\text { DAF for } \\
\text { B.M. }\end{array}$ \\
\hline 40 & 60 & 5 & -0.219 & -0.207 & 1.06 & -2787 & -2353 & 1.18 \\
\hline 40 & 60 & 10 & -0.220 & -0.207 & 1.06 & -2815 & -2353 & 1.20 \\
\hline 40 & 60 & 15 & -0.233 & -0.207 & 1.12 & -2862 & -2353 & 1.22 \\
\hline 40 & 60 & 20 & -0.237 & -0.207 & 1.14 & -3019 & -2353 & 1.28 \\
\hline 40 & 60 & 25 & -0.270 & -0.207 & 1.30 & -3269 & -2353 & 1.39 \\
\hline
\end{tabular}

Table 4.23: Dynamic response of rigid-connected bridge to two successive 70-tonne two-

\begin{tabular}{|c|c|c|c|c|c|c|c|c|}
\hline $\begin{array}{c}\text { Separ. } \\
\text { dist. } \\
(\mathbf{m})\end{array}$ & $\begin{array}{c}\text { Vehicle } \\
\text { weight } \\
(\mathbf{t})\end{array}$ & $\begin{array}{c}\text { Vehicle } \\
\text { speed } \\
(\mathbf{k m} / \mathbf{h})\end{array}$ & $\begin{array}{c}\text { Max } \\
\mathbf{d e f l} \\
(\mathbf{m})\end{array}$ & $\begin{array}{c}\text { Static } \\
\text { defl. } \\
(\mathbf{m})\end{array}$ & $\begin{array}{c}\text { DAF } \\
\text { for defl. }\end{array}$ & $\begin{array}{c}\text { Max } \\
\text { B.M. } \\
(\mathbf{K N} . \mathbf{m})\end{array}$ & $\begin{array}{c}\text { Static } \\
\text { B.M. } \\
(\mathbf{K N} . \mathbf{m})\end{array}$ & $\begin{array}{c}\text { DAF for } \\
\text { B.M. }\end{array}$ \\
\hline 40 & 70 & 5 & -0.256 & -0.242 & 1.06 & -3251 & -2745 & 1.18 \\
\hline 40 & 70 & 10 & -0.257 & -0.242 & 1.06 & -3285 & -2745 & 1.20 \\
\hline 40 & 70 & 15 & -0.272 & -0.242 & 1.12 & -3340 & -2745 & 1.22 \\
\hline 40 & 70 & 20 & -0.276 & -0.242 & 1.14 & -3525 & -2745 & 1.28 \\
\hline 40 & 70 & 25 & -0.316 & -0.242 & 1.30 & -3823 & -2745 & 1.39 \\
\hline
\end{tabular}


Table 4.24: Dynamic response of rigid-connected bridge to two successive 50-tonne twoaxle vehicle loads (spacing $30 \mathrm{~m}$ )

\begin{tabular}{|c|c|c|c|c|c|c|c|c|}
\hline $\begin{array}{c}\text { Separ. } \\
\text { dist. } \\
(\mathbf{m})\end{array}$ & $\begin{array}{c}\text { Vehicle } \\
\text { weight } \\
(\mathbf{t})\end{array}$ & $\begin{array}{c}\text { Vehicle } \\
\text { speed } \\
(\mathbf{k m} / \mathbf{h})\end{array}$ & $\begin{array}{c}\text { Max } \\
\mathbf{d e f l} \\
\mathbf{( m )}\end{array}$ & $\begin{array}{c}\text { Static } \\
\mathbf{d e f l} \\
\mathbf{( m )}\end{array}$ & $\begin{array}{c}\text { DAF } \\
\text { for defl. }\end{array}$ & $\begin{array}{c}\text { Max } \\
\text { B.M. } \\
(\mathbf{K N} . \mathbf{m})\end{array}$ & $\begin{array}{c}\text { Static } \\
\text { B.M. } \\
(\text { KN.m) }\end{array}$ & $\begin{array}{c}\text { DAF for } \\
\text { B.M. }\end{array}$ \\
\hline 30 & 50 & 5 & -0.222 & -0.214 & 1.04 & -2322 & -1917 & 1.21 \\
\hline 30 & 50 & 10 & -0.225 & -0.214 & 1.05 & -2353 & -1917 & 1.23 \\
\hline 30 & 50 & 15 & -0.229 & -0.214 & 1.07 & -2436 & -1917 & 1.27 \\
\hline 30 & 50 & 20 & -0.254 & -0.214 & 1.18 & -2525 & -1917 & 1.32 \\
\hline 30 & 50 & 25 & -0.270 & -0.214 & 1.26 & -2618 & -1917 & 1.37 \\
\hline
\end{tabular}

Table 4.25: Dynamic response of rigid-connected bridge to two successive 60-tonne twoaxle vehicle loads (spacing $30 \mathrm{~m}$ )

\begin{tabular}{|c|c|c|c|c|c|c|c|c|}
\hline $\begin{array}{c}\text { Separ. } \\
\text { dist. } \\
(\mathbf{m})\end{array}$ & $\begin{array}{c}\text { Vehicle } \\
\text { weight } \\
(\mathbf{t})\end{array}$ & $\begin{array}{c}\text { Vehicle } \\
\text { speed } \\
(\mathbf{k m} / \mathbf{h})\end{array}$ & $\begin{array}{c}\text { Max } \\
\mathbf{d e f l} \\
(\mathbf{m})\end{array}$ & $\begin{array}{c}\text { Static } \\
\mathbf{d e f l} \\
\mathbf{( m )}\end{array}$ & $\begin{array}{c}\text { DAF } \\
\text { for defl. }\end{array}$ & $\begin{array}{c}\text { Max } \\
\text { B.M. } \\
(\text { KN.m) }\end{array}$ & $\begin{array}{c}\text { Static } \\
\text { B.M. } \\
(\text { KN.m) }\end{array}$ & $\begin{array}{c}\text { DAF for } \\
\text { B.M. }\end{array}$ \\
\hline 30 & 60 & 5 & -0.267 & -0.257 & 1.04 & -2787 & -2300 & 1.21 \\
\hline 30 & 60 & 10 & -0.270 & -0.257 & 1.05 & -2824 & -2300 & 1.23 \\
\hline 30 & 60 & 15 & -0.274 & -0.257 & 1.07 & -2925 & -2300 & 1.27 \\
\hline 30 & 60 & 20 & -0.305 & -0.257 & 1.19 & -3033 & -2300 & 1.32 \\
\hline 30 & 60 & 25 & -0.324 & -0.257 & 1.26 & -3142 & -2300 & 1.37 \\
\hline
\end{tabular}


Table 4.26: Dynamic response of rigid-connected bridge to two successive 70-tonne twoaxle vehicle loads (spacing $30 \mathrm{~m}$ )

\begin{tabular}{|c|c|c|c|c|c|c|c|c|}
\hline $\begin{array}{c}\text { Separ. } \\
\text { dist. } \\
(\mathbf{m})\end{array}$ & $\begin{array}{c}\text { Vehicle } \\
\text { weight } \\
(\mathbf{t})\end{array}$ & $\begin{array}{c}\text { Vehicle } \\
\text { speed } \\
(\mathbf{k m} / \mathbf{h})\end{array}$ & $\begin{array}{c}\text { Max } \\
\mathbf{d e f 1} \\
(\mathbf{m})\end{array}$ & $\begin{array}{c}\text { Static } \\
\mathbf{d e f l} \\
\mathbf{( m )}\end{array}$ & $\begin{array}{c}\text { DAF for } \\
\text { defl. }\end{array}$ & $\begin{array}{c}\text { Max } \\
\text { B.M. } \\
(\text { KN.m) }\end{array}$ & $\begin{array}{c}\text { Static } \\
\text { B.M. } \\
(\mathbf{K N . m})\end{array}$ & $\begin{array}{c}\text { DAF for } \\
\text { B.M. }\end{array}$ \\
\hline 30 & 70 & 5 & -0.311 & -0.300 & 1.04 & -3251 & -2684 & 1.21 \\
\hline 30 & 70 & 10 & -0.315 & -0.300 & 1.05 & -3294 & -2684 & 1.23 \\
\hline 30 & 70 & 15 & -0.320 & -0.300 & 1.07 & -3414 & -2684 & 1.27 \\
\hline 30 & 70 & 20 & -0.356 & -0.300 & 1.19 & -3540 & -2684 & 1.32 \\
\hline 30 & 70 & 25 & -0.379 & -0.300 & 1.26 & -3670 & -2684 & 1.37 \\
\hline
\end{tabular}

\subsubsection{Discussion of Results}

Figure 4.50 shows the maximum deflection in the bridge due to two successive vehicle loads with separation distance of $50 \mathrm{~m}$ for different vehicle weights and speeds. The maximum deflection in the bridge increases with vehicle weight and speed. The maximum deflections in the bridge are within the allowable limit $(0.783 \mathrm{~m})$ for 50,60 and 70 tonnes vehicle weights and for all the vehicle speeds from $5 \mathrm{~km} / \mathrm{h}$ to $25 \mathrm{~km} / \mathrm{h}$. The same trend was observed for two successive vehicles with separation distance of 40 and $30 \mathrm{~m}$ as shown in Figures 4.55 and 4.60, respectively. The maximum deflections in the case of a separation distance of $40 \mathrm{~m}$ are higher than those in the case of a separation distance of $50 \mathrm{~m}$. Also, the maximum deflections in the case of a separation distance of $30 \mathrm{~m}$ are higher than those in the case of a separation distance of $40 \mathrm{~m}$.

The maximum bending moment developed in the bridge is presented in Figure 4.51 for a separation distance of $50 \mathrm{~m}$. It is observed that the maximum bending moment increases 
with increasing vehicle weight and speed. The same trend is observed for two successive vehicles with separation distances of 40 and $30 \mathrm{~m}$ as shown in Figures 4.56 and 4.61, respectively. The maximum bending moments in the case of a separation distance of 40 $\mathrm{m}$ are higher than those in the case of a separation distance of $50 \mathrm{~m}$.

Figure 4.52 shows the DAF for both deflection and bending moment in the bridge due to two successive 50-tonne vehicle loads with separation distance of $50 \mathrm{~m}$, and travelling the bridge at different speeds. The DAF for deflection increases with vehicle speed ranging 1.05 at $5 \mathrm{~km} / \mathrm{h}$ to 1.32 at $25 \mathrm{~km} / \mathrm{h}$. The DAF for bending moment also increases with vehicle speed ranging from 1.13 at $5 \mathrm{~km} / \mathrm{h}$ to 1.27 at $25 \mathrm{~km} / \mathrm{h}$. This means that an increase in the vehicle speed has a significant effect on the increase in DAFs for both deflection and bending moment. The same trend is observed for two successive vehicles with separation distances of 40 and $30 \mathrm{~m}$ as shown in Figures 4.57 and 4.62, respectively.

Figure 4.53 shows the DAF for deflection due to two successive vehicle loads with a separation distance of $50 \mathrm{~m}$ for different vehicle weights 50,60 and 70 tonnes. The DAFs for deflection are the same for different vehicle weights, however, the deflections increase as the vehicle weight increases. The same trend was observed for two successive vehicles with separation distance of 40 and $30 \mathrm{~m}$ as shown in Figures 4.58 and 4.63, respectively.

Figure 4.54 shows the DAF for bending moment due to two successive vehicle loads with separation distance of $50 \mathrm{~m}$ for different vehicle weights 50,60 and 70 tonnes. The DAFs for bending moment are the same for different vehicle weights, however, the bending moment values increase as the vehicle weight increases. The same trend is observed for 
two successive vehicles with separation distances of 40 and $30 \mathrm{~m}$ as shown in Figures 4.59 and 4.64, respectively. The DAFs for bending moment are higher than those for deflection.

The foregoing results show that increasing the vehicle weight does not affect the DAFs for both deflection and bending moment. As a result, an increase in the vehicle weight can be achieved without increasing DAF for either the deflection or the bending moment. The same is not true of an increase in vehicle speed.

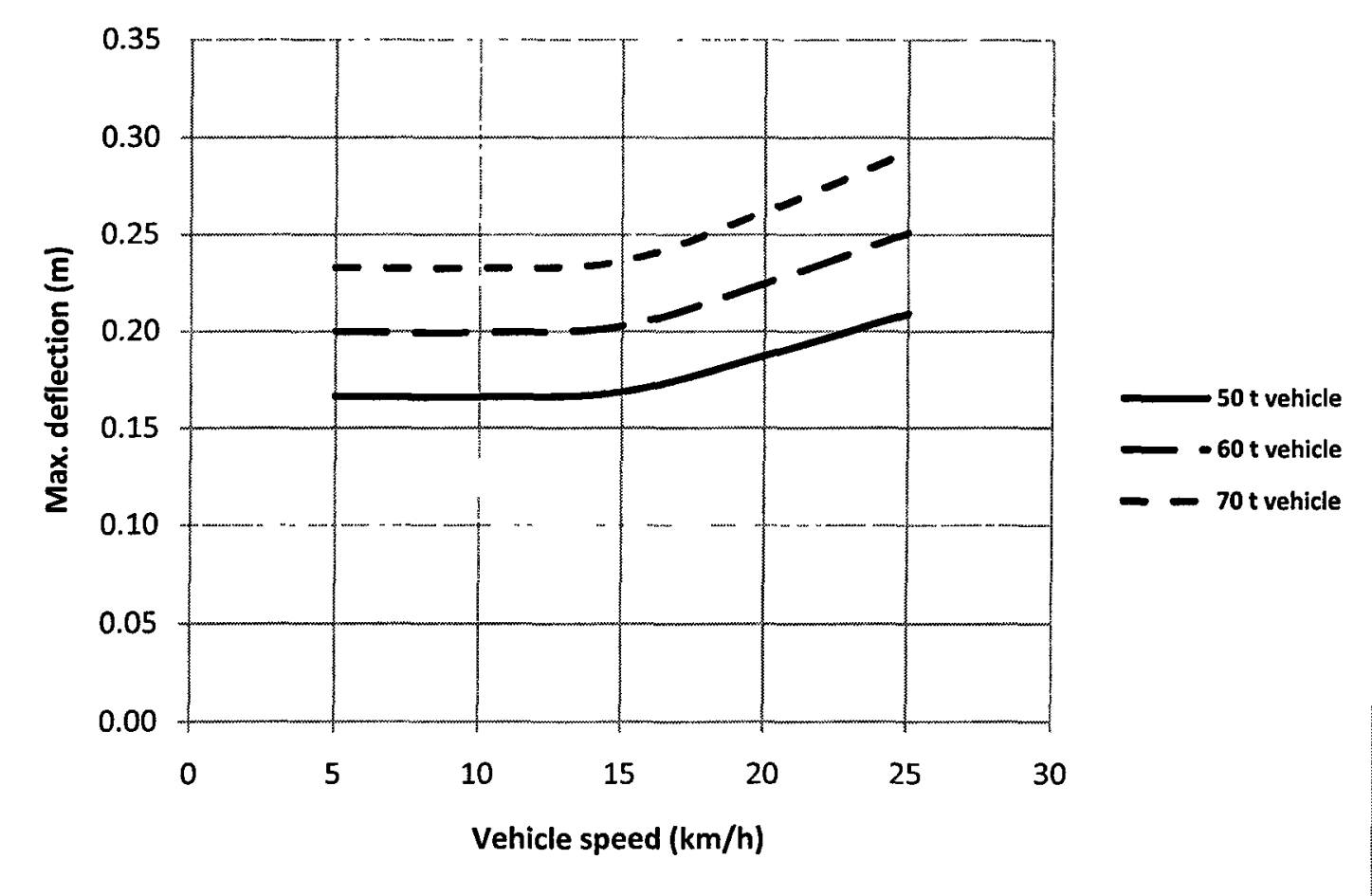

Figure 4.50: Effect of vehicle speed and weight on maximum deflection in rigidconnected bridge (two successive vehicles with spacing $50 \mathrm{~m}$ ) 


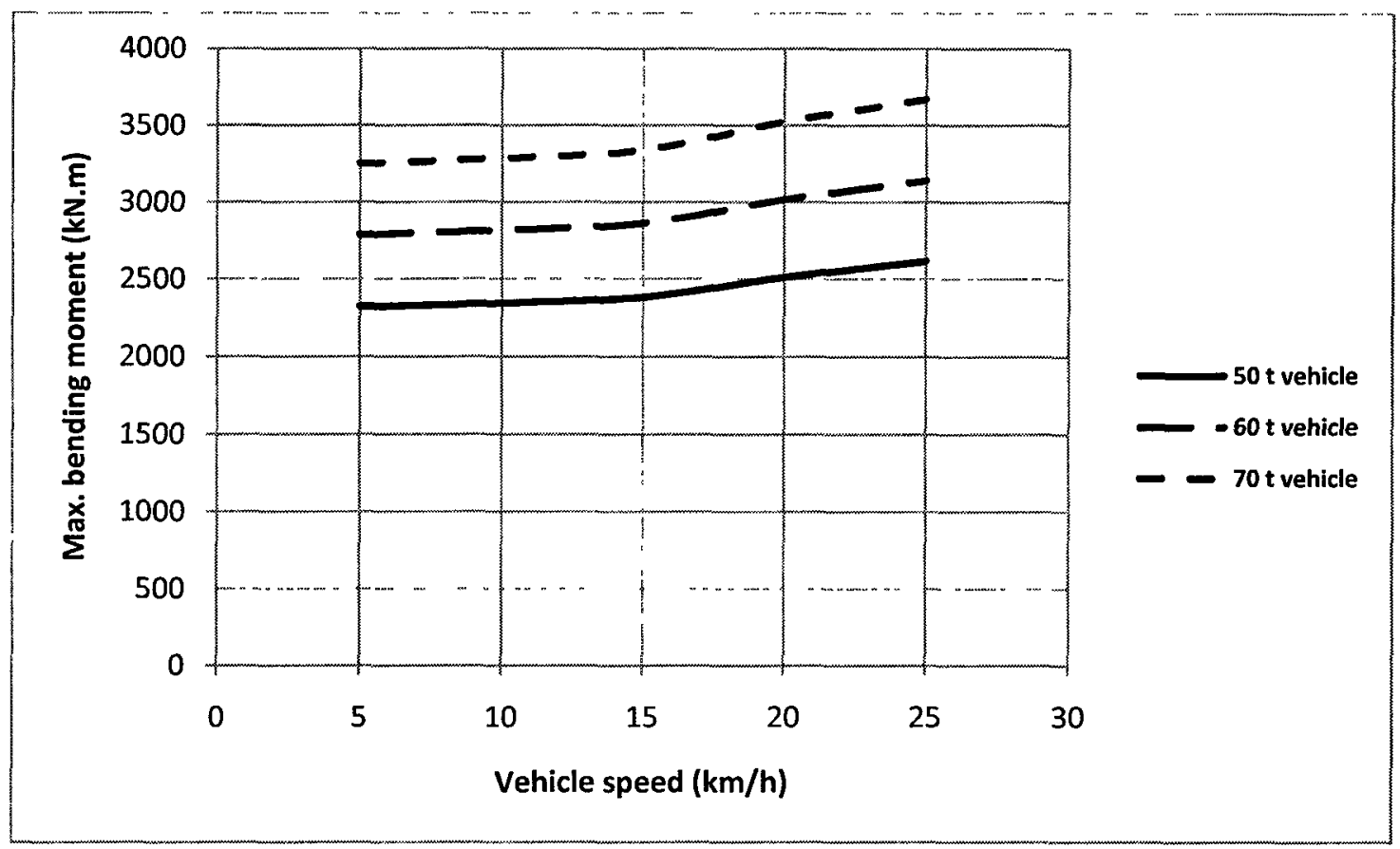

Figure 4.51: Effect of vehicle speed and weight on maximum bending moment in rigidconnected bridge (two successive vehicles with spacing $50 \mathrm{~m}$ )

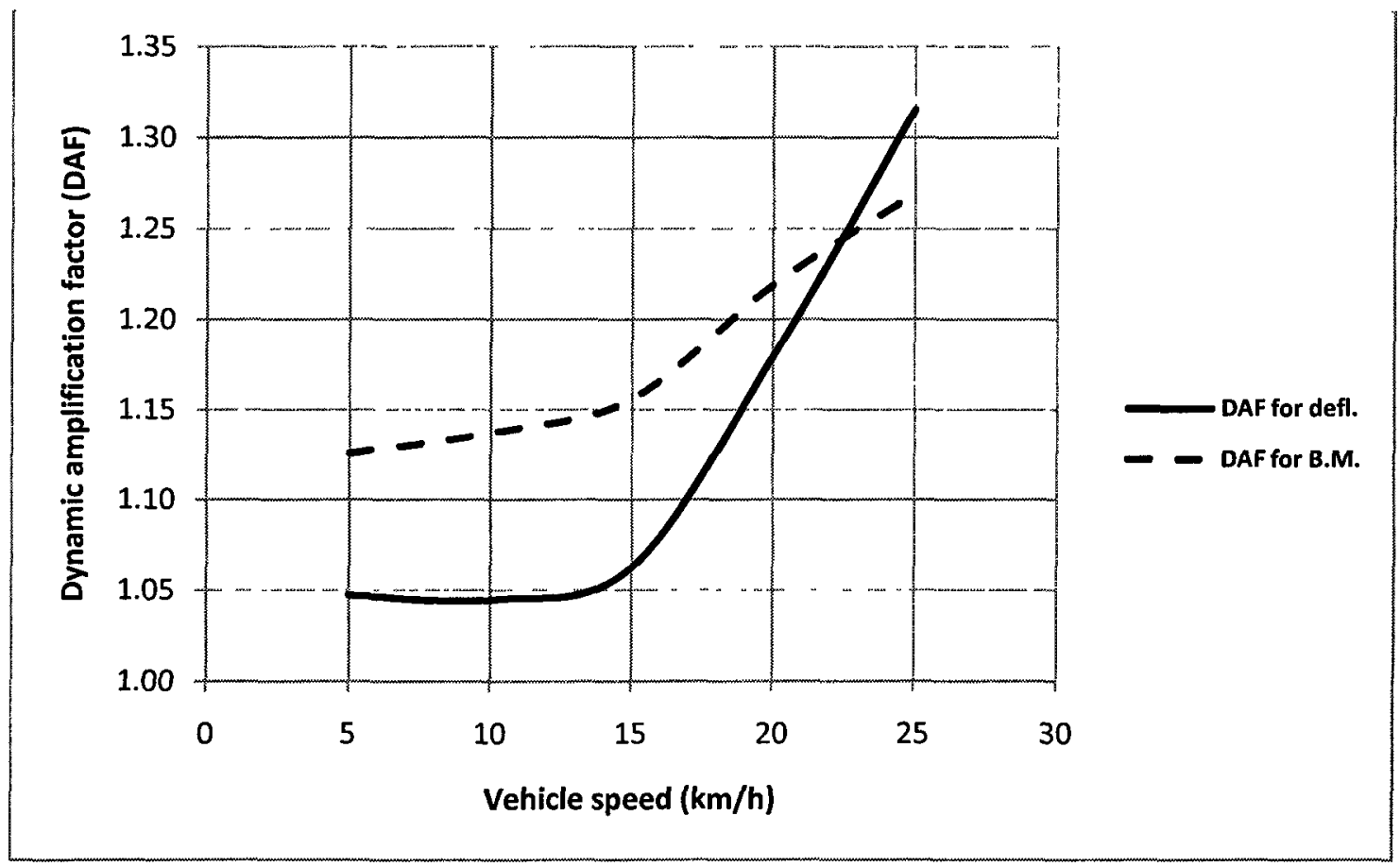

Figure 4.52: DAFs in rigid-connected bridge due to two successive 50-tonne vehicle loads with spacing $50 \mathrm{~m}$ 


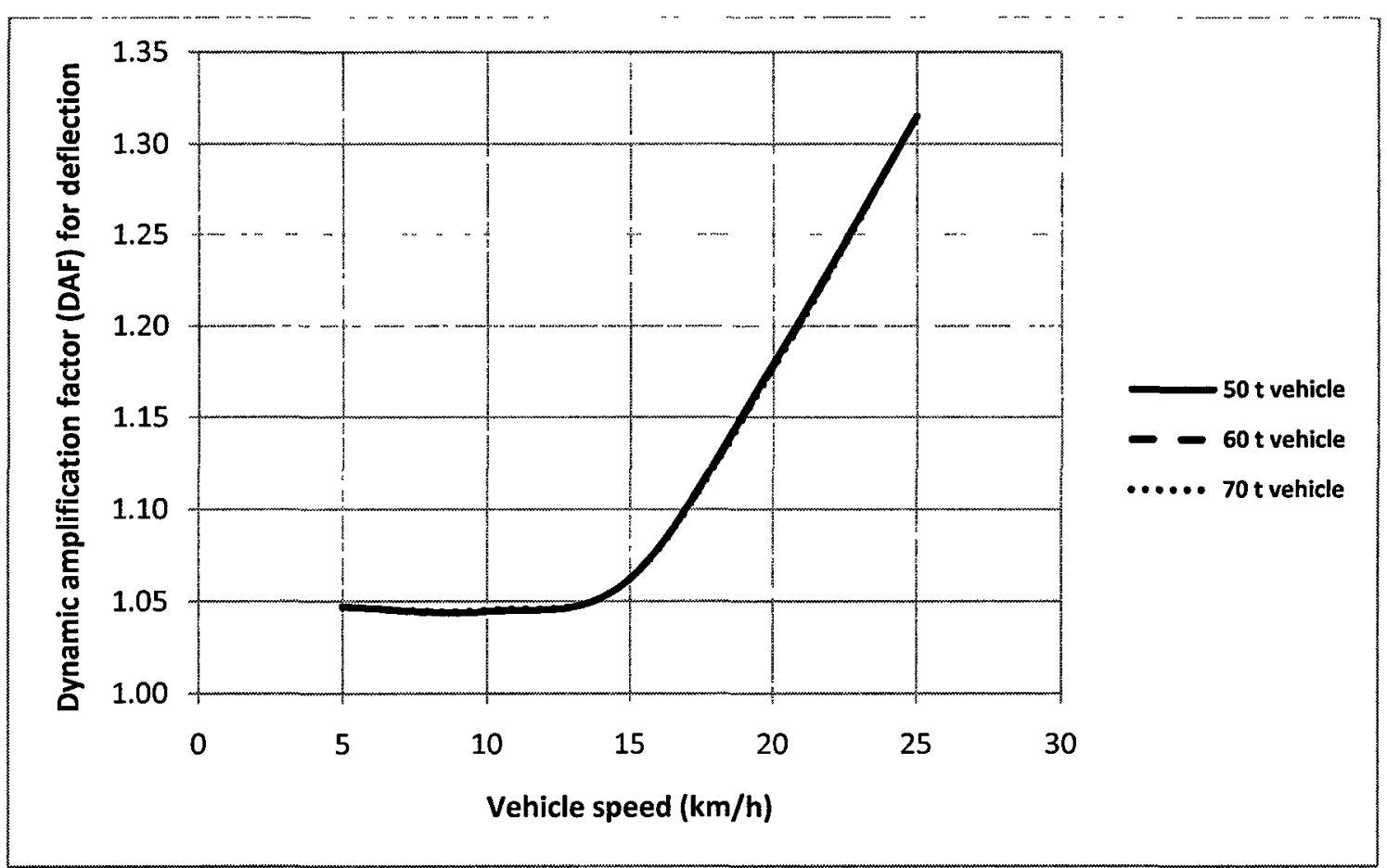

Figure 4.53: DAFs for deflection in rigid-connected bridge due to two successive vehicles with spacing $50 \mathrm{~m}$

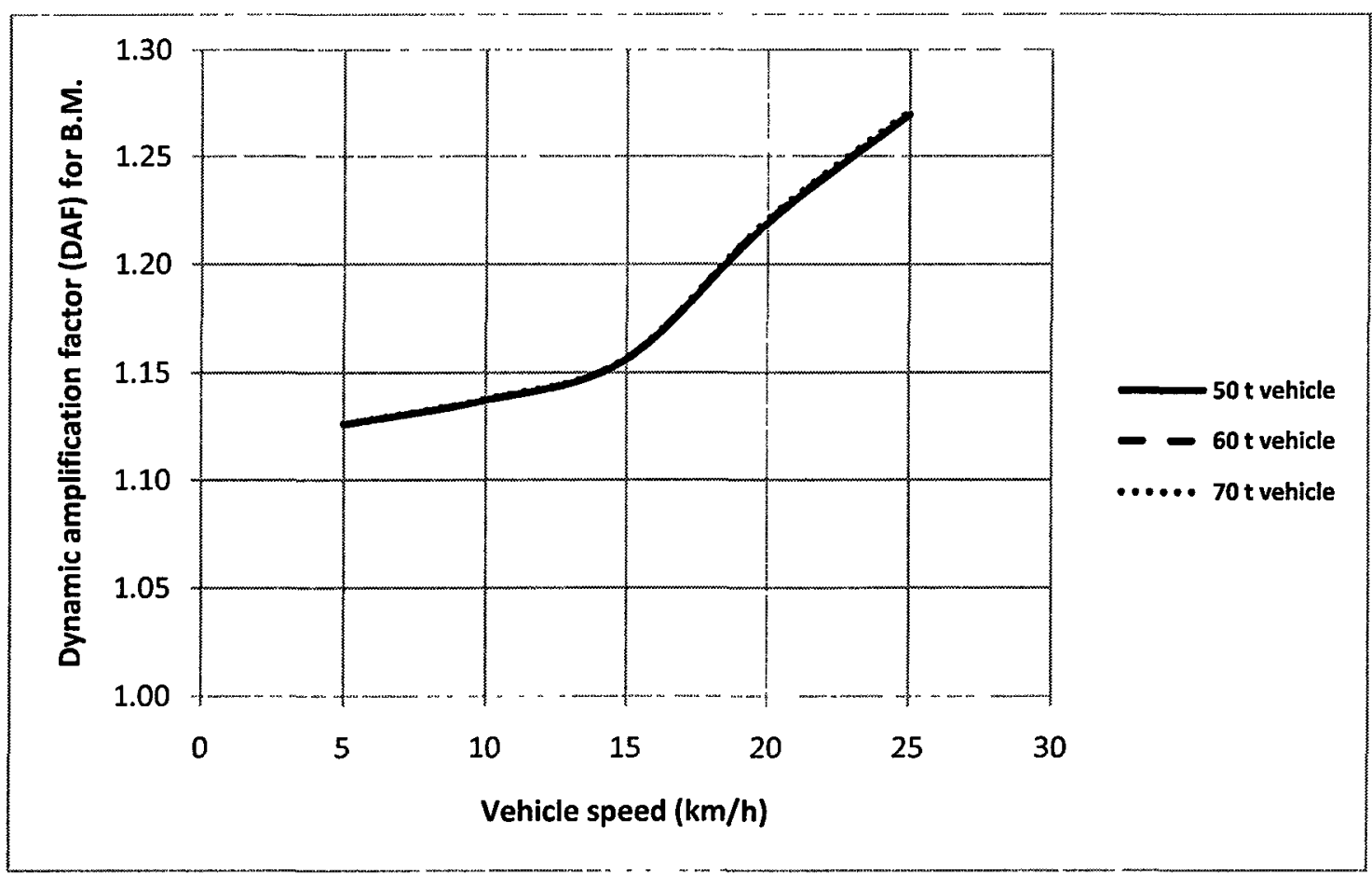

Figure 4.54: DAFs for bending moment in rigid-connected bridge due to two successive vehicles with spacing $50 \mathrm{~m}$ 


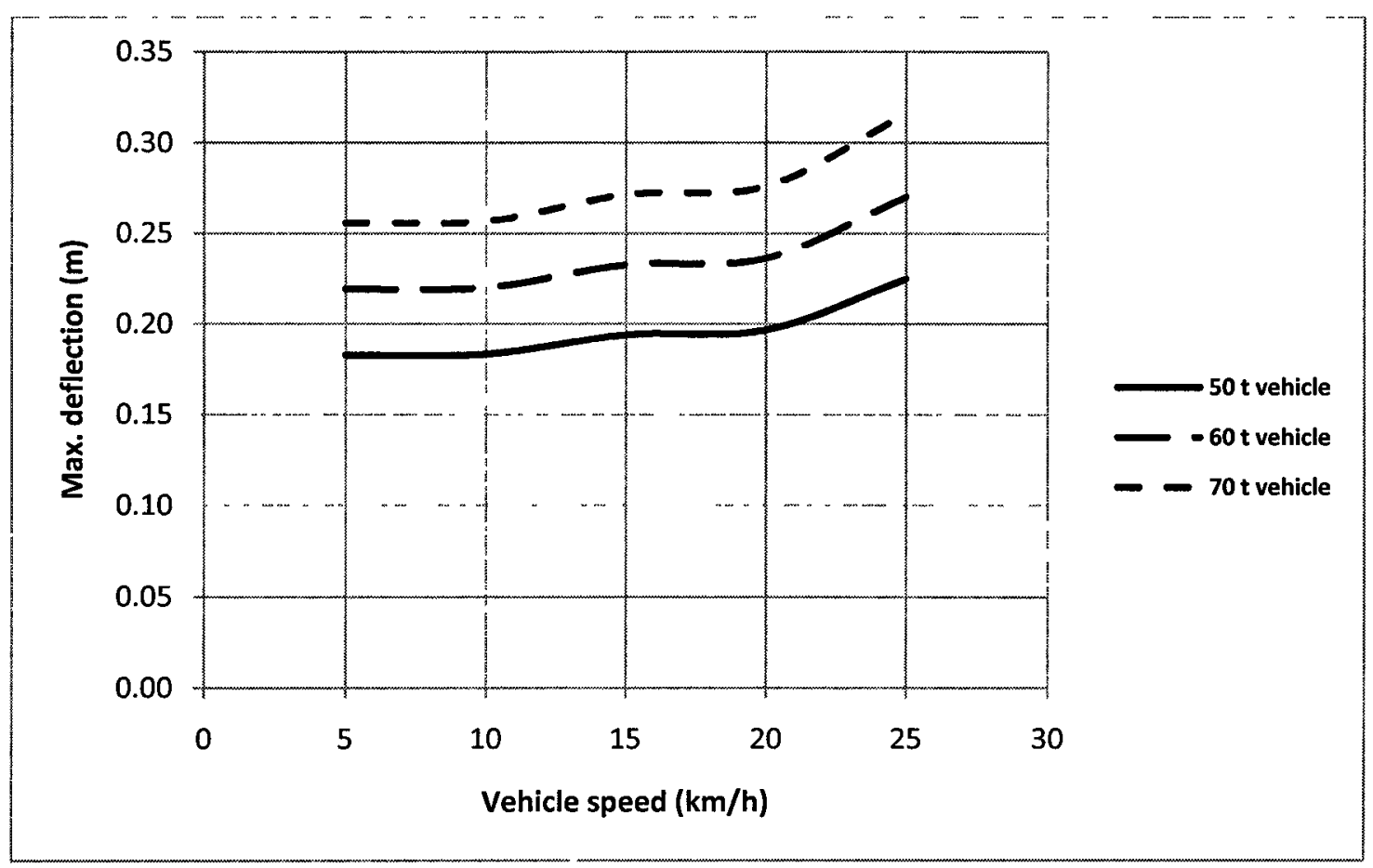

Figure 4.55: Effect of vehicle speed and weight on maximum deflection in rigidconnected bridge (two successive vehicles with spacing $40 \mathrm{~m}$ )

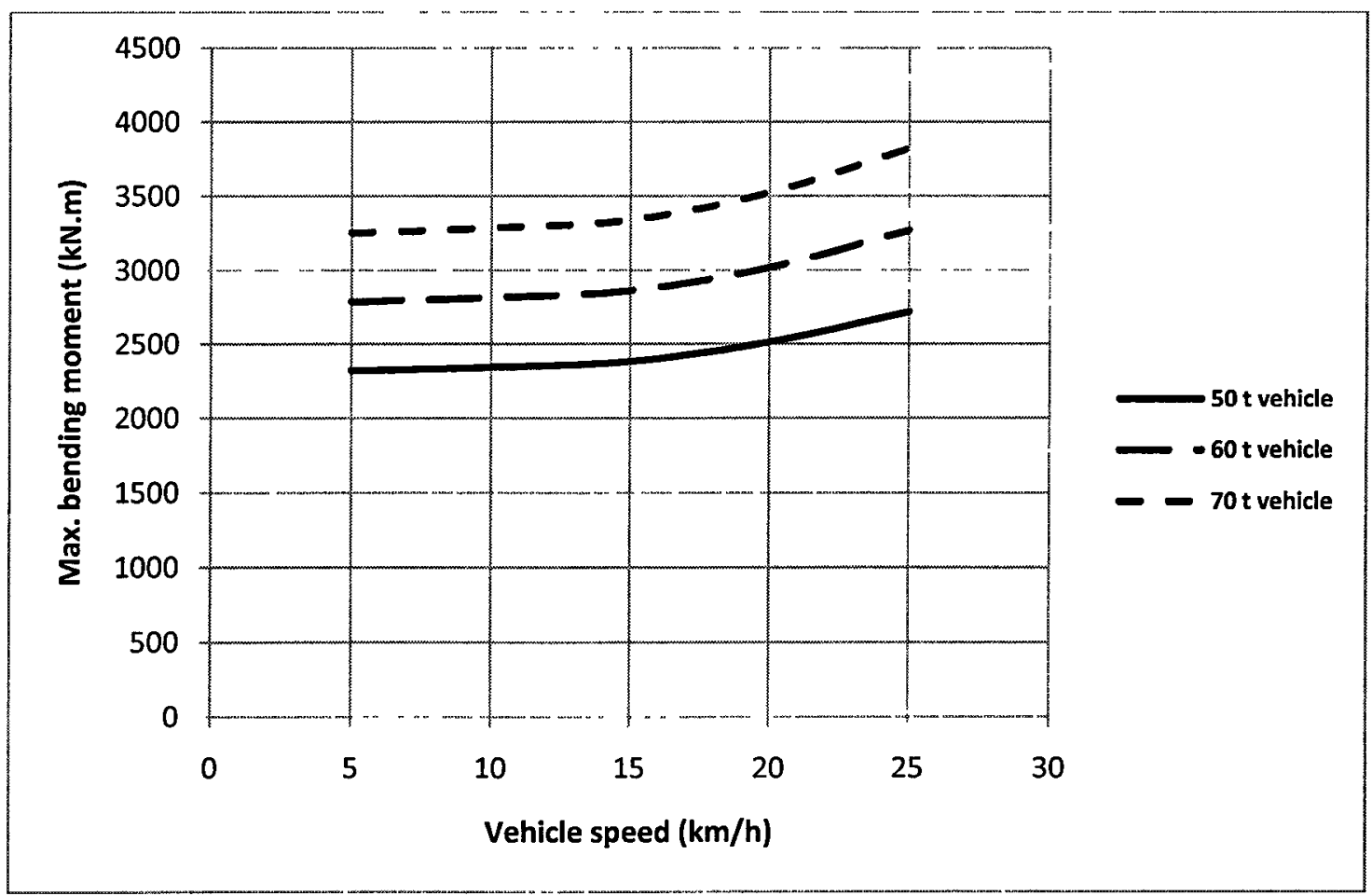

Figure 4.56: Effect of vehicle speed and weight on maximum bending moment in rigidconnected bridge (two successive vehicles with spacing $40 \mathrm{~m}$ ) 


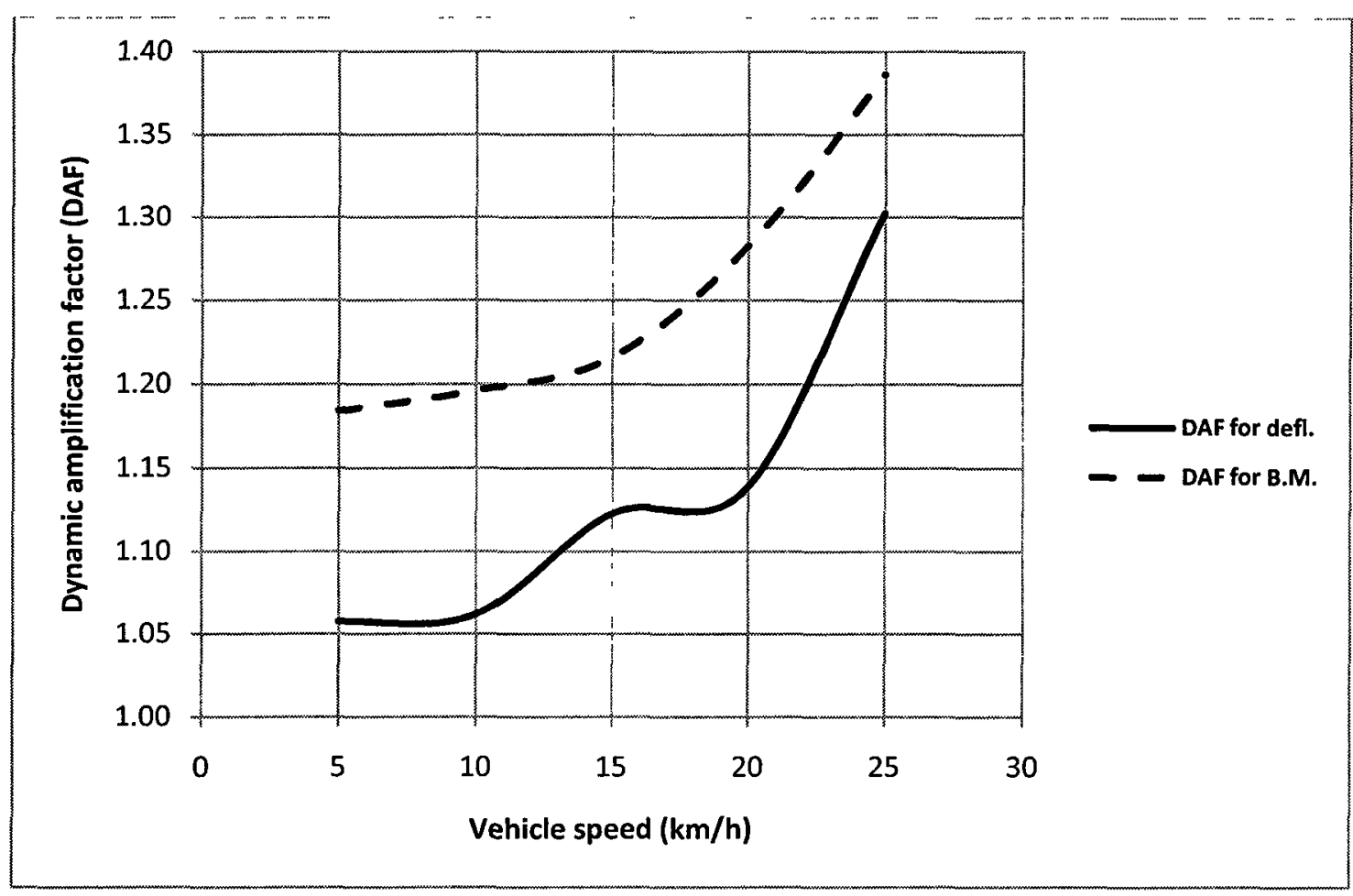

Figure 4.57: DAFs in rigid-connected bridge due to two successive 50-tonne vehicle loads with spacing $40 \mathrm{~m}$

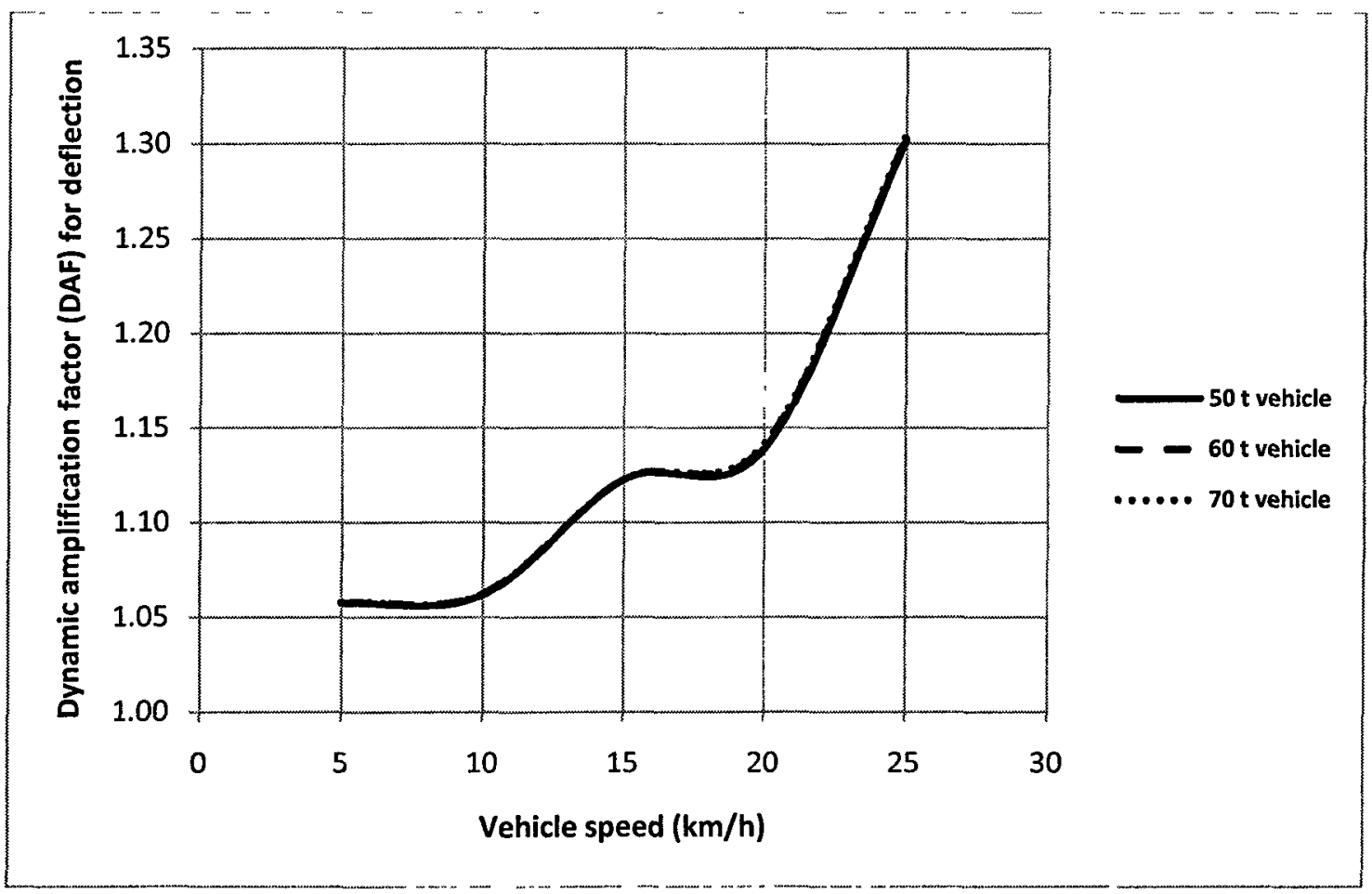

Figure 4.58: DAFs for deflection in rigid-connected bridge due to two successive vehicles with spacing $40 \mathrm{~m}$ 


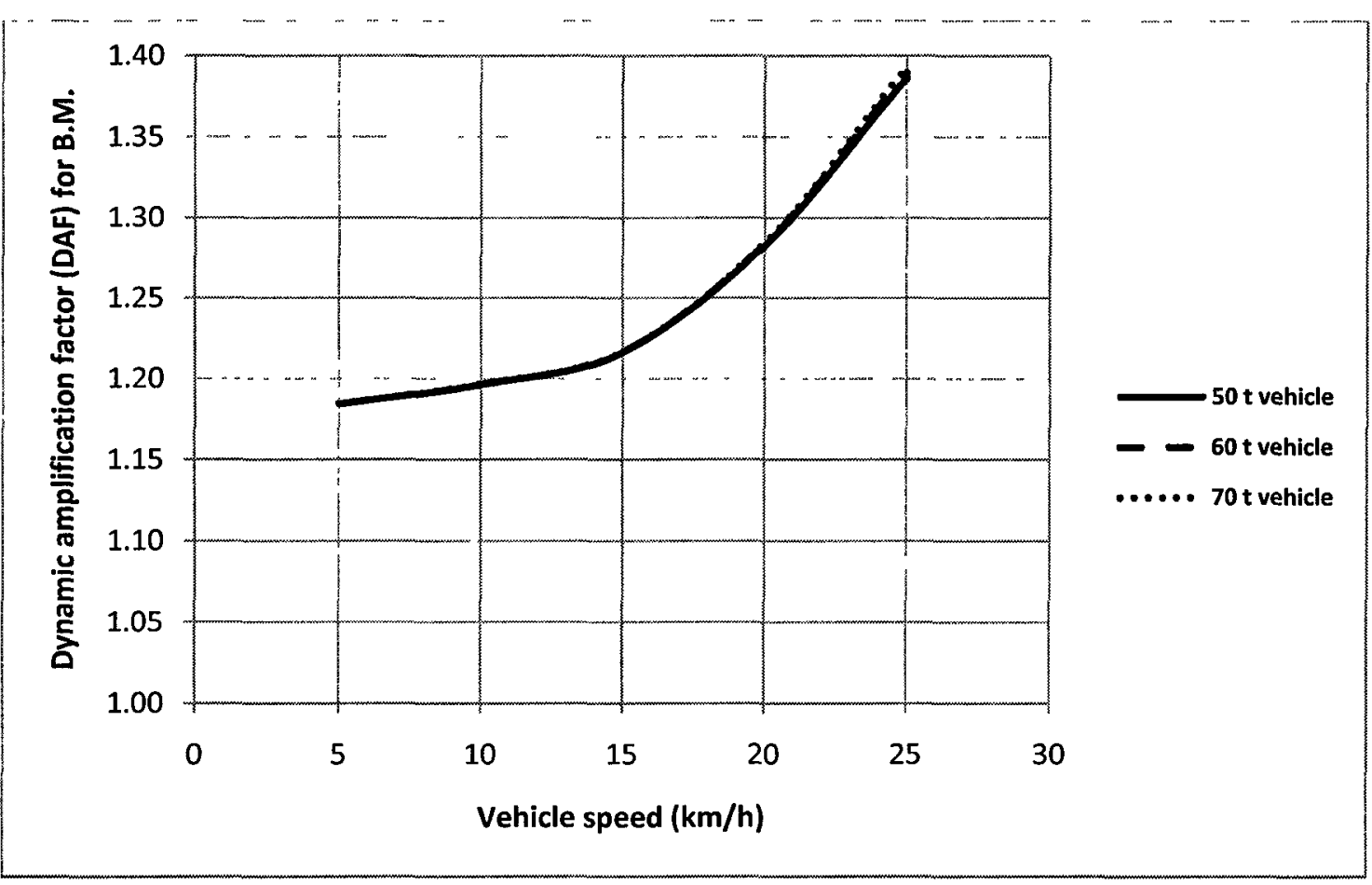

Figure 4.59: DAFs for bending moment in rigid-connected bridge due to two successive vehicles with spacing $40 \mathrm{~m}$

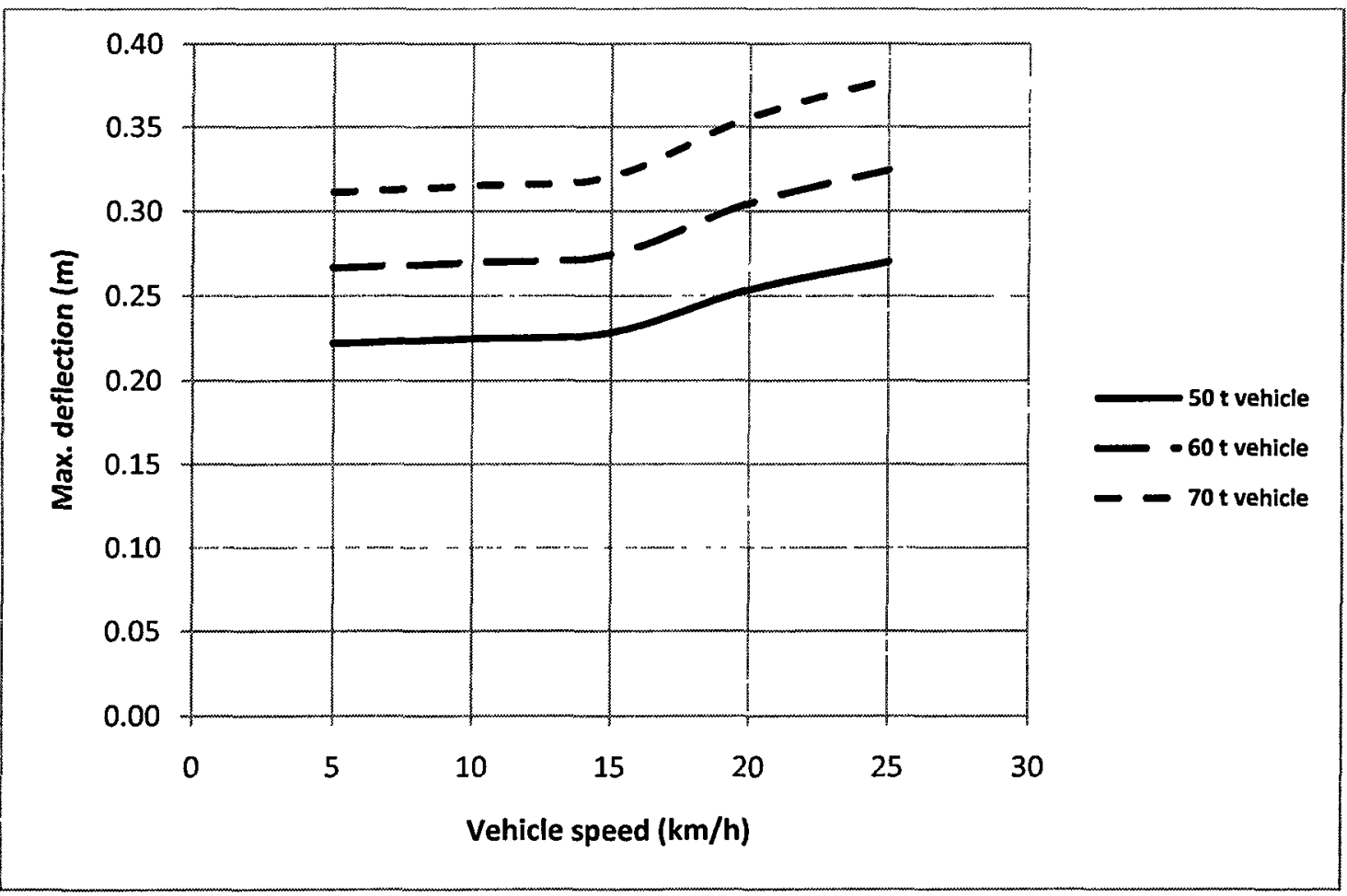

Figure 4.60: Effect of vehicle speed and weight on maximum deflection in rigidconnected bridge (two successive vehicles with spacing $30 \mathrm{~m}$ ) 


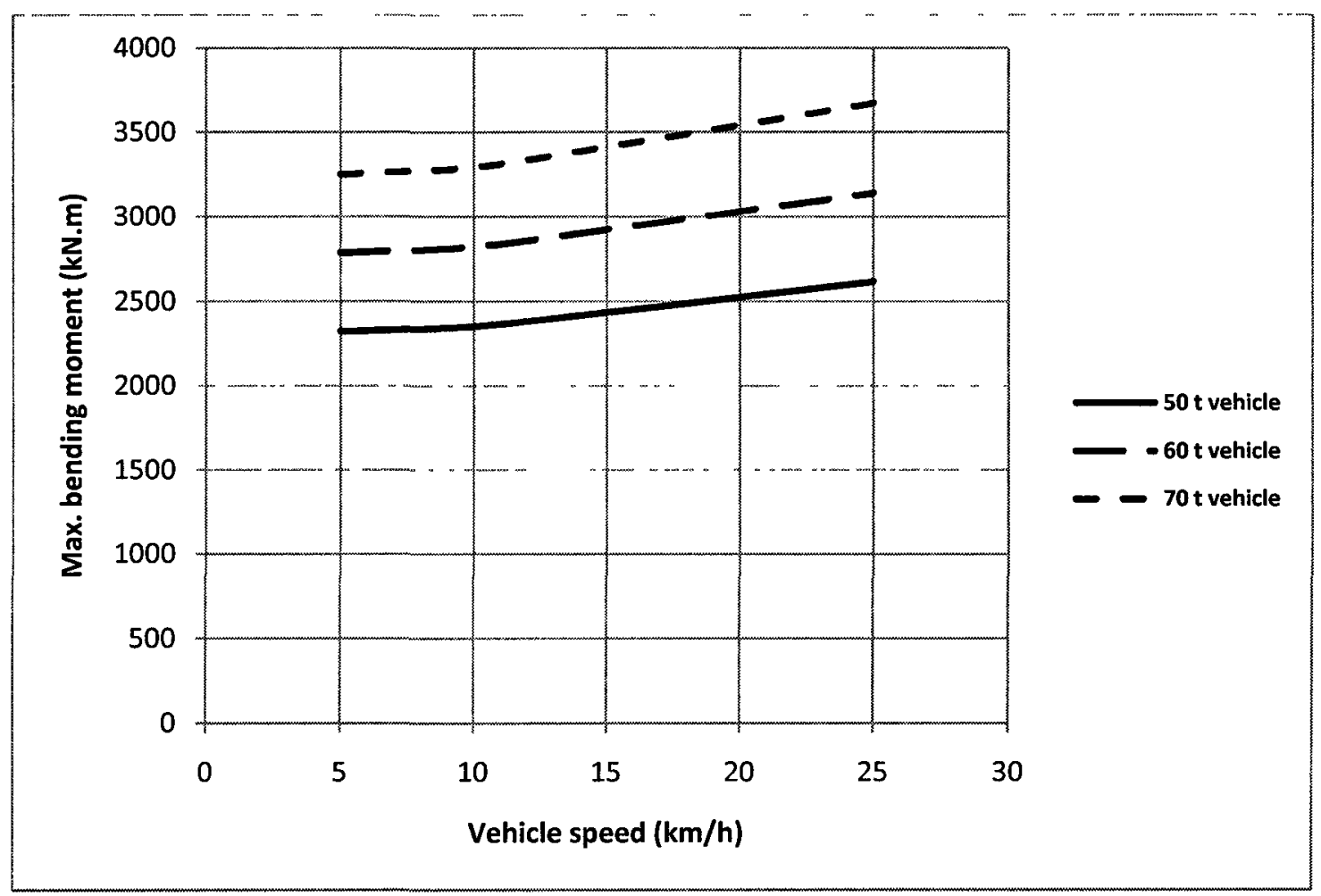

Figure 4.61: Effect of vehicle speed and weight on maximum bending moment in rigidconnected bridge (two successive vehicles with spacing $30 \mathrm{~m}$ )

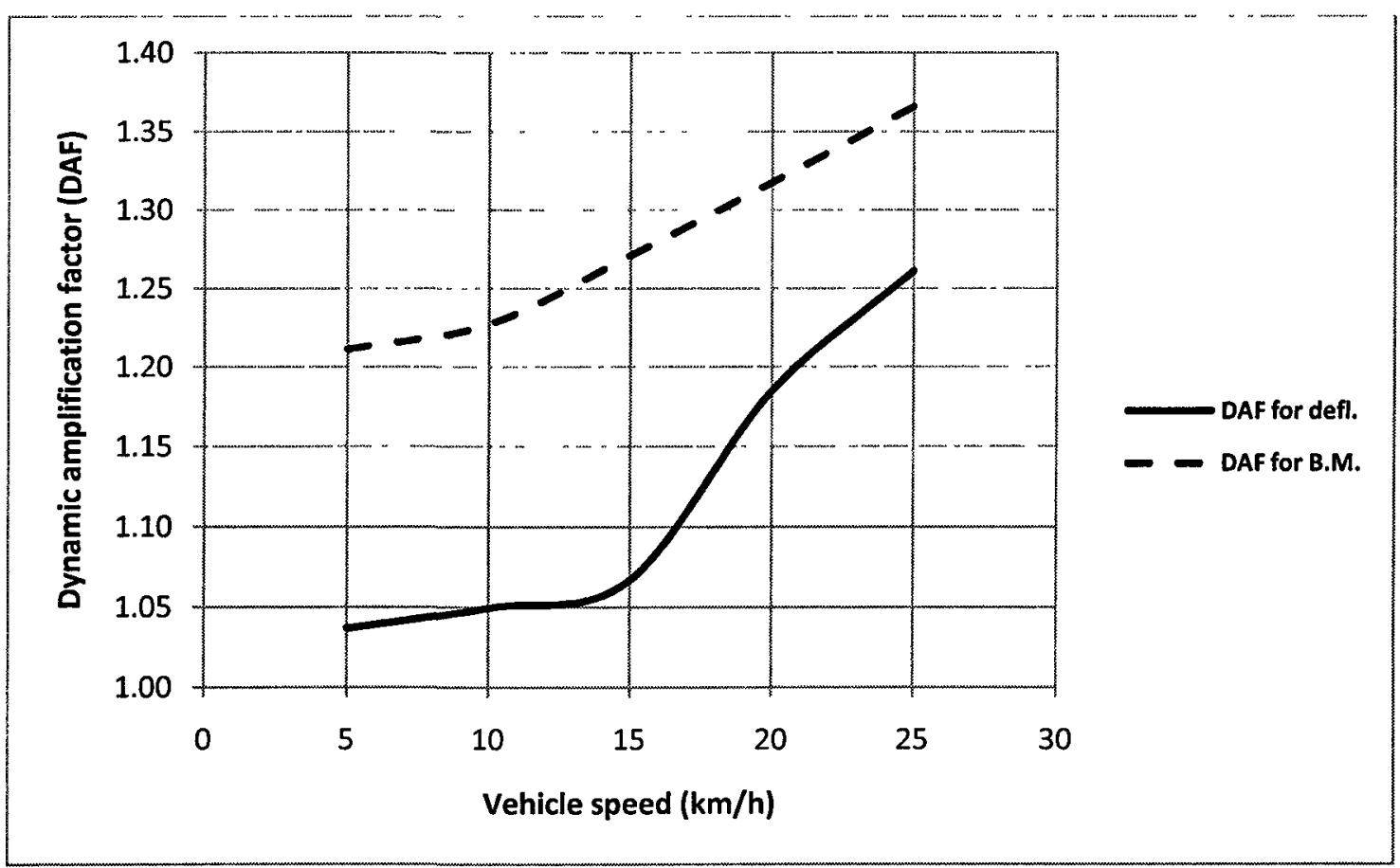

Figure 4.62: DAFs in rigid-connected bridge due to two successive 50-tonne vehicle loads with spacing $30 \mathrm{~m}$ 


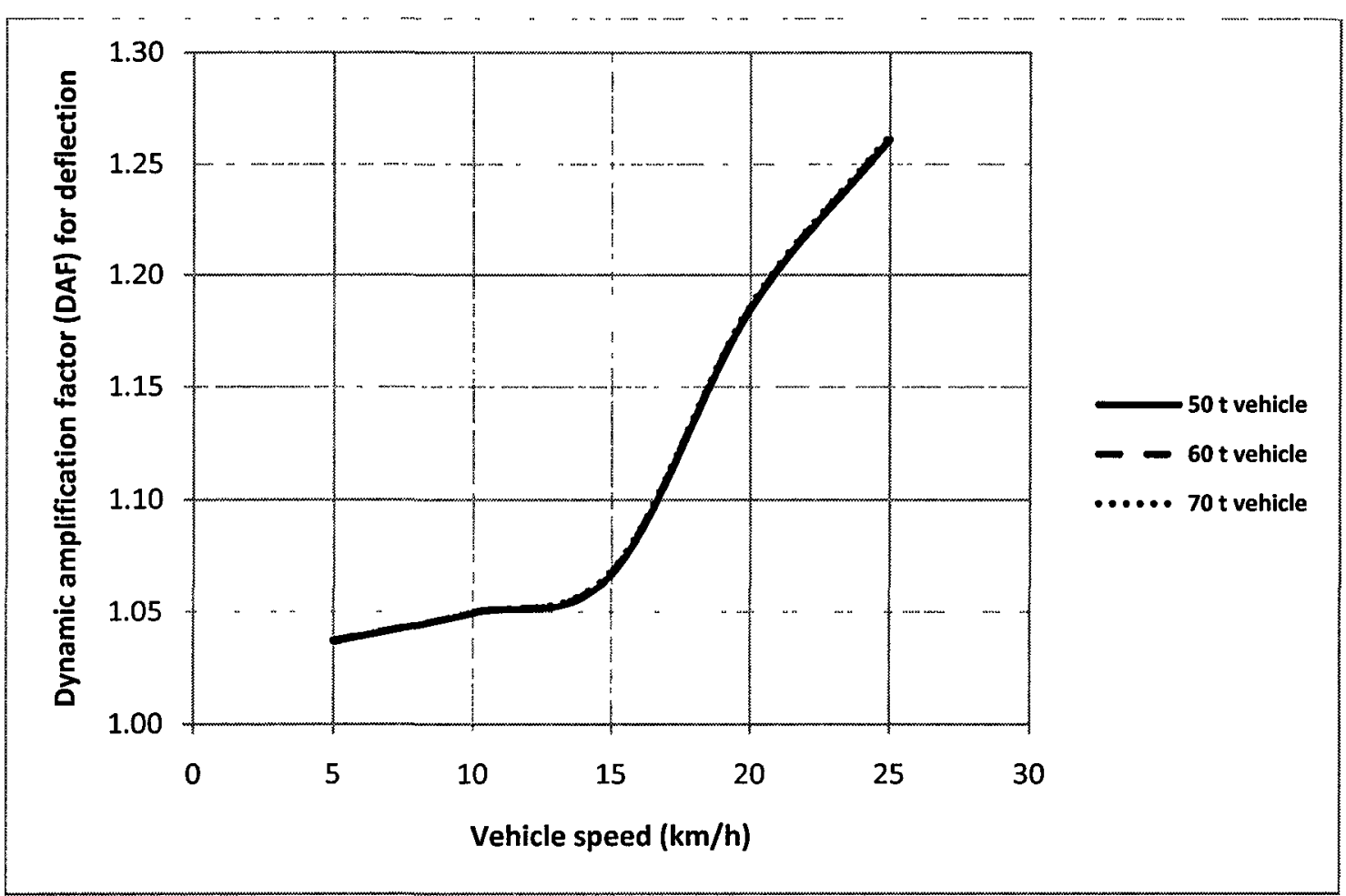

Figure 4.63: DAFs for deflection in rigid-connected bridge due to two successive vehicles with spacing $30 \mathrm{~m}$

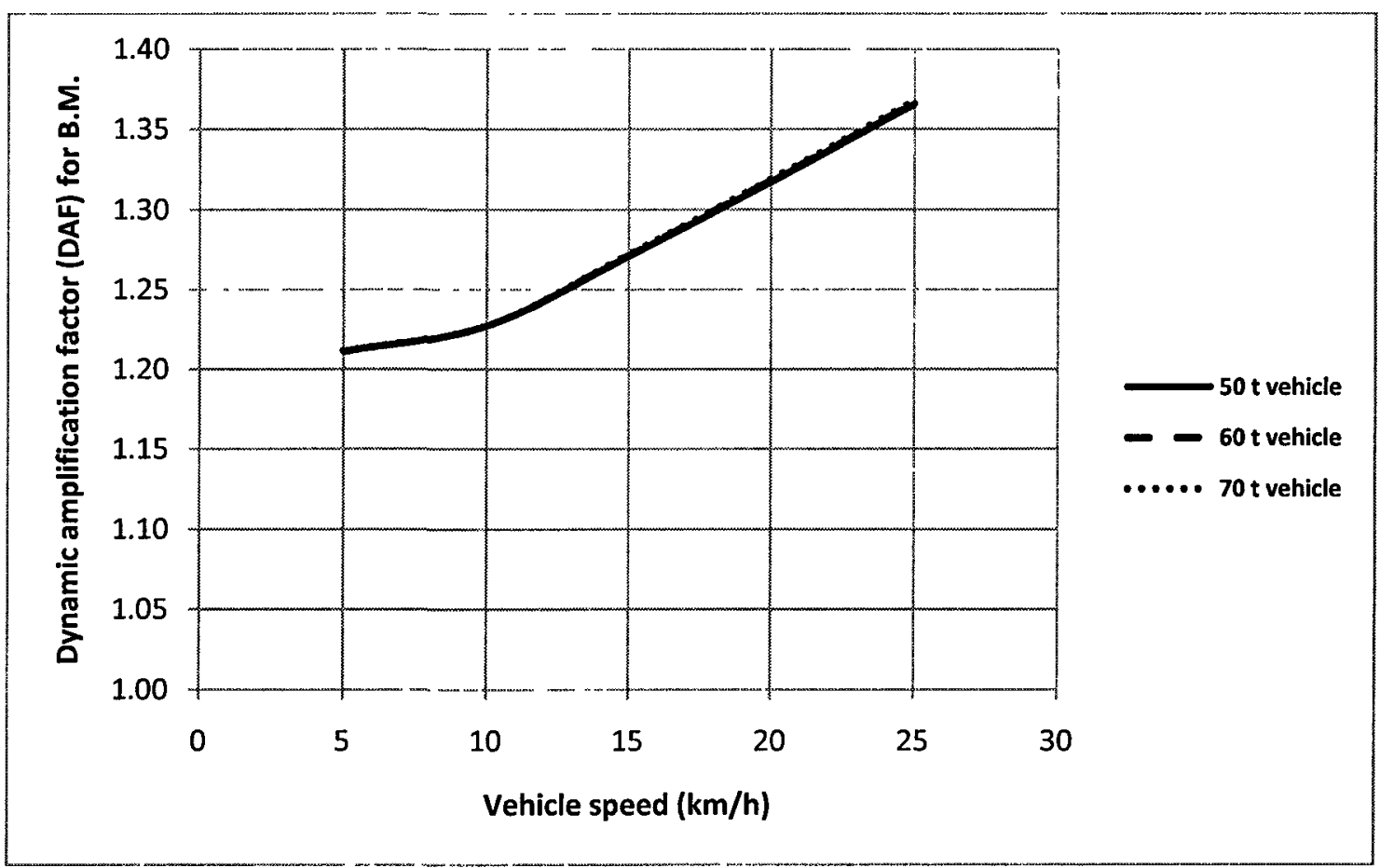

Figure 4.64: DAFs for bending moment in rigid-connected bridge due to two successive vehicles with spacing $30 \mathrm{~m}$ 
4.6.2 Dynamic Response of Hinge-Connected Floating Bridge to Two Successive Vehicle Loads

The dynamic response of hinge-connected floating bridge to two successive vehicle loads was investigated for different vehicle weights, speeds and separation distances. The two successive vehicles are traversing the bridge at different speeds from $5 \mathrm{~km} / \mathrm{h}$ to $25 \mathrm{~km} / \mathrm{h}$ and with separation distances of 50,40 and $30 \mathrm{~m}$. The two vehicles have the same weight of 50,60 or 70 tonnes. The analyses and the results are summarized in Tables 4.27 to 4.35 .

Table 4.27: Dynamic response of hinge-connected bridge to two successive 50-tonne two-axle vehicle loads (spacing $50 \mathrm{~m}$ )

\begin{tabular}{|c|c|c|c|c|c|c|c|c|}
\hline $\begin{array}{c}\text { Separ. } \\
\text { dist. } \\
(\mathbf{m})\end{array}$ & $\begin{array}{c}\text { Vehicle } \\
\text { weight } \\
(\mathbf{t})\end{array}$ & $\begin{array}{c}\text { Vehicle } \\
\mathbf{s p e e d} \\
(\mathbf{k m} / \mathbf{h})\end{array}$ & $\begin{array}{c}\text { Max } \\
\mathbf{d i s p l} \\
(\mathbf{m})\end{array}$ & $\begin{array}{c}\text { Static } \\
\mathbf{d i s p l} \\
(\mathbf{m})\end{array}$ & $\begin{array}{c}\text { DAF for } \\
\mathbf{d i s p l} .\end{array}$ & $\begin{array}{c}\text { Max } \\
\text { B.M. } \\
(\mathbf{K N . m})\end{array}$ & $\begin{array}{c}\text { Static } \\
\text { B.M. } \\
(\mathbf{K N . m})\end{array}$ & $\begin{array}{c}\text { DAF for } \\
\text { B.M. }\end{array}$ \\
\hline 50 & 50 & 5 & -0.452 & -0.417 & 1.08 & -1450 & -1361 & 1.07 \\
\hline 50 & 50 & 10 & -0.510 & -0.417 & 1.22 & -1471 & -1361 & 1.08 \\
\hline 50 & 50 & 15 & -0.540 & -0.417 & 1.29 & -1463 & -1361 & 1.07 \\
\hline 50 & 50 & 20 & -0.671 & -0.417 & 1.61 & -1527 & -1361 & 1.12 \\
\hline 50 & 50 & 25 & -1.098 & -0.417 & 2.63 & -1543 & -1361 & 1.13 \\
\hline
\end{tabular}


Table 4.28: Dynamic response of hinge-connected bridge to two successive 60 -tonne two-axle vehicle loads (spacing $50 \mathrm{~m}$ )

\begin{tabular}{|l|l|l|l|l|l|l|l|l|}
\hline $\begin{array}{c}\text { Separ. } \\
\text { dist. } \\
(\mathbf{m})\end{array}$ & $\begin{array}{c}\text { Vehicle } \\
\text { weight } \\
(\mathbf{t})\end{array}$ & $\begin{array}{c}\text { Vehicle } \\
\mathbf{s p e e d} \\
(\mathbf{k m} / \mathbf{h})\end{array}$ & $\begin{array}{c}\text { Max } \\
\mathbf{d i s p l} . \\
(\mathbf{m})\end{array}$ & $\begin{array}{c}\text { Static } \\
\text { displ. } \\
(\mathbf{m})\end{array}$ & $\begin{array}{c}\text { DAF for } \\
\text { displ. }\end{array}$ & $\begin{array}{c}\text { Max } \\
\text { B.M. } \\
(\mathbf{K N . m})\end{array}$ & $\begin{array}{c}\text { Static } \\
\text { B.M. } \\
(\text { KN.m) }\end{array}$ & $\begin{array}{c}\text { DAF for } \\
\text { B.M. }\end{array}$ \\
\hline 50 & 60 & 5 & -0.544 & -0.501 & 1.09 & -1741 & -1633 & 1.07 \\
\hline 50 & 60 & 10 & -0.614 & -0.501 & 1.23 & -1767 & -1633 & 1.08 \\
\hline 50 & 60 & 15 & -0.651 & -0.501 & 1.30 & -1753 & -1633 & 1.07 \\
\hline 50 & 60 & 20 & -0.807 & -0.501 & 1.61 & -1837 & -1633 & 1.12 \\
\hline 50 & 60 & 25 & -1.325 & -0.501 & 2.65 & -1865 & -1633 & 1.14 \\
\hline
\end{tabular}

Table 4.29: Dynamic response of hinge-connected bridge to two successive 70-tonne two-axle vehicle loads (spacing $50 \mathrm{~m}$ )

\begin{tabular}{|c|c|c|c|c|c|c|c|c|}
\hline $\begin{array}{c}\text { Separ. } \\
\text { dist. } \\
(\mathbf{m})\end{array}$ & $\begin{array}{c}\text { Vehicle } \\
\text { weight } \\
(\mathbf{t})\end{array}$ & $\begin{array}{c}\text { Vehicle } \\
\text { speed } \\
(\mathbf{k m} / \mathbf{h})\end{array}$ & $\begin{array}{c}\text { Max } \\
\text { displ. } \\
(\mathbf{m})\end{array}$ & $\begin{array}{c}\text { Static } \\
\text { displ. } \\
(\mathbf{m})\end{array}$ & $\begin{array}{c}\text { DAF for } \\
\text { displ. }\end{array}$ & $\begin{array}{c}\text { Max } \\
\text { B.M. } \\
(\mathbf{K N} . \mathbf{m})\end{array}$ & $\begin{array}{c}\text { Static } \\
\text { B.M. } \\
(\mathbf{K N} . \mathbf{m})\end{array}$ & $\begin{array}{c}\text { DAF for } \\
\text { B.M. }\end{array}$ \\
\hline 50 & 70 & 5 & -0.636 & -0.584 & 1.09 & -2034 & -1906 & 1.07 \\
\hline 50 & 70 & 10 & -0.719 & -0.584 & 1.23 & -2063 & -1906 & 1.08 \\
\hline 50 & 70 & 15 & -0.764 & -0.584 & 1.31 & -2040 & -1906 & 1.07 \\
\hline 50 & 70 & 20 & -0.945 & -0.584 & 1.62 & -2140 & -1906 & 1.12 \\
\hline 50 & 70 & 25 & -1.554 & -0.584 & 2.66 & 2217 & -1906 & -1.16 \\
\hline
\end{tabular}


Table 4.30: Dynamic response of hinge-connected bridge to two successive 50-tonne two-axle vehicle loads (spacing $40 \mathrm{~m}$ )

\begin{tabular}{|c|c|c|c|c|c|c|c|c|}
\hline $\begin{array}{c}\text { Separ. } \\
\text { dist. } \\
(\mathbf{m})\end{array}$ & $\begin{array}{c}\text { Vehicle } \\
\text { weight } \\
(\mathbf{t})\end{array}$ & $\begin{array}{c}\text { Vehicle } \\
\mathbf{s p e e d} \\
(\mathbf{k m} / \mathbf{h})\end{array}$ & $\begin{array}{c}\text { Max } \\
\mathbf{d i s p l .} \\
(\mathbf{m})\end{array}$ & $\begin{array}{c}\text { Static } \\
\mathbf{d i s p l} \\
(\mathbf{m})\end{array}$ & $\begin{array}{c}\text { DAF for } \\
\mathbf{d i s p l} .\end{array}$ & $\begin{array}{c}\text { Max } \\
\mathbf{B . M} . \\
(\mathbf{K N . m})\end{array}$ & $\begin{array}{c}\text { Static } \\
\text { B.M. } \\
(\mathbf{K N . m})\end{array}$ & $\begin{array}{c}\text { DAF for } \\
\text { B.M. }\end{array}$ \\
\hline 40 & 50 & 5 & -0.456 & -0.428 & 1.07 & -1598 & -1449 & 1.10 \\
\hline 40 & 50 & 10 & -0.465 & -0.428 & 1.09 & -1624 & -1449 & 1.12 \\
\hline 40 & 50 & 15 & -0.527 & -0.428 & 1.23 & -1684 & -1449 & 1.16 \\
\hline 40 & 50 & 20 & -0.895 & -0.428 & 2.09 & -1828 & -1449 & 1.26 \\
\hline 40 & 50 & 25 & -1.266 & -0.428 & 2.96 & -1848 & -1449 & 1.28 \\
\hline
\end{tabular}

Table 4.31: Dynamic response of hinge-connected bridge to two successive 60-tonne two-axle vehicle loads (spacing $40 \mathrm{~m}$ )

\begin{tabular}{|c|c|c|c|c|c|c|c|c|}
\hline $\begin{array}{c}\text { Separ. } \\
\text { dist. } \\
(\mathbf{m})\end{array}$ & $\begin{array}{c}\text { Vehicle } \\
\text { weight } \\
(\mathbf{t})\end{array}$ & $\begin{array}{c}\text { Vehicle } \\
\text { speed } \\
(\mathbf{k m} / \mathbf{h})\end{array}$ & $\begin{array}{c}\text { Max } \\
\text { displ. } \\
(\mathbf{m})\end{array}$ & $\begin{array}{c}\text { Static } \\
\text { displ. } \\
(\mathbf{m})\end{array}$ & $\begin{array}{c}\text { DAF for } \\
\text { displ. }\end{array}$ & $\begin{array}{c}\text { Max } \\
\text { B.M. } \\
(\text { KN.m) }\end{array}$ & $\begin{array}{c}\text { Static } \\
\text { B.M. } \\
(\text { KN.m) }\end{array}$ & $\begin{array}{c}\text { DAF for } \\
\text { B.M. }\end{array}$ \\
\hline 40 & 60 & 5 & -0.549 & -0.513 & 1.07 & -1916 & -1739 & 1.10 \\
\hline 40 & 60 & 10 & -0.559 & -0.513 & 1.09 & -1956 & -1739 & 1.13 \\
\hline 40 & 60 & 15 & -0.633 & -0.513 & 1.23 & -2028 & -1739 & 1.17 \\
\hline 40 & 60 & 20 & -1.083 & -0.513 & 2.11 & -2213 & -1739 & 1.27 \\
\hline 40 & 60 & 25 & -1.526 & -0.513 & 2.97 & -2237 & -1739 & 1.29 \\
\hline
\end{tabular}


Table 4.32: Dynamic response of hinge-connected bridge to two successive 70 -tonne two-axle vehicle loads (spacing $40 \mathrm{~m}$ )

\begin{tabular}{|c|c|c|c|c|c|c|c|c|}
\hline $\begin{array}{c}\text { Separ. } \\
\mathbf{d i s t} . \\
(\mathbf{m})\end{array}$ & $\begin{array}{c}\text { Vehicle } \\
\text { weight } \\
(\mathbf{t})\end{array}$ & $\begin{array}{c}\text { Vehicle } \\
\text { speed } \\
(\mathbf{k m} / \mathbf{h})\end{array}$ & $\begin{array}{c}\text { Max } \\
\mathbf{d i s p l} \\
(\mathbf{m})\end{array}$ & $\begin{array}{c}\text { Static } \\
\mathbf{d i s p l} \\
(\mathbf{m})\end{array}$ & $\begin{array}{c}\text { DAF for } \\
\text { displ. }\end{array}$ & $\begin{array}{c}\text { Max } \\
\text { B.M. } \\
(\text { KN.m) }\end{array}$ & $\begin{array}{c}\text { Static } \\
\text { B.M. } \\
(\text { KN.m) }\end{array}$ & $\begin{array}{c}\text { DAF for } \\
\text { B.M. }\end{array}$ \\
\hline 40 & 70 & 5 & -0.642 & -0.599 & 1.07 & -2235 & -2008 & 1.11 \\
\hline 40 & 70 & 10 & -0.653 & -0.599 & 1.09 & -2287 & -2008 & 1.14 \\
\hline 40 & 70 & 15 & -0.741 & -0.599 & 1.24 & -2376 & -2008 & 1.18 \\
\hline 40 & 70 & 20 & -1.276 & -0.599 & 2.13 & -2597 & -2008 & 1.29 \\
\hline 40 & 70 & 25 & -1.786 & -0.599 & 2.98 & -2633 & -2008 & 1.31 \\
\hline
\end{tabular}

Table 4.33: Dynamic response of hinge-connected bridge to two successive 50-tonne two-axle vehicle loads (spacing $30 \mathrm{~m}$ )

\begin{tabular}{|c|c|c|c|c|c|c|c|c|}
\hline $\begin{array}{c}\text { Separ. } \\
\text { dist. } \\
(\mathbf{m})\end{array}$ & $\begin{array}{c}\text { Vehicle } \\
\text { weight } \\
(\mathbf{t})\end{array}$ & $\begin{array}{c}\text { Vehicle } \\
\text { speed } \\
(\mathbf{k m} / \mathbf{h})\end{array}$ & $\begin{array}{c}\text { Max } \\
\mathbf{d i s p l} \\
(\mathbf{m})\end{array}$ & $\begin{array}{c}\text { Static } \\
\text { displ. } \\
(\mathbf{m})\end{array}$ & $\begin{array}{c}\text { DAF for } \\
\text { displ. }\end{array}$ & $\begin{array}{c}\text { Max } \\
\text { B.M. } \\
(\text { KN.m) }\end{array}$ & $\begin{array}{c}\text { Static } \\
\text { B.M. } \\
(\text { KN.m) }\end{array}$ & $\begin{array}{c}\text { DAF for } \\
\text { B.M. }\end{array}$ \\
\hline 30 & 50 & 5 & -0.438 & -0.401 & 1.09 & -1497 & -1476 & 1.01 \\
\hline 30 & 50 & 10 & -0.476 & -0.401 & 1.19 & -1508 & -1476 & 1.02 \\
\hline 30 & 50 & 15 & -0.523 & -0.401 & 1.30 & -1548 & -1476 & 1.05 \\
\hline 30 & 50 & 20 & -0.788 & -0.401 & 1.97 & -1557 & -1476 & 1.05 \\
\hline 30 & 50 & 25 & -0.788 & -0.401 & 2.02 & -1634 & -1476 & 1.11 \\
\hline
\end{tabular}


Table 4.34: Dynamic response of hinge-connected bridge to two successive 60-tonne two-axle vehicle loads (spacing $30 \mathrm{~m}$ )

\begin{tabular}{|c|c|c|c|c|c|c|c|c|}
\hline $\begin{array}{c}\text { Separ. } \\
\text { dist. } \\
(\mathbf{m})\end{array}$ & $\begin{array}{c}\text { Vehicle } \\
\text { weight } \\
(\mathbf{t})\end{array}$ & $\begin{array}{c}\text { Vehicle } \\
\mathbf{s p e e d} \\
(\mathbf{k m} / \mathbf{h})\end{array}$ & $\begin{array}{c}\text { Max } \\
\text { displ. } \\
(\mathbf{m})\end{array}$ & $\begin{array}{c}\text { Static } \\
\text { displ. } \\
(\mathbf{m})\end{array}$ & $\begin{array}{c}\text { DAF for } \\
\text { displ. }\end{array}$ & $\begin{array}{c}\text { Max } \\
\text { B.M. } \\
(\text { KN.m) }\end{array}$ & $\begin{array}{c}\text { Static } \\
\text { B.M. } \\
(\text { KN.m) }\end{array}$ & $\begin{array}{c}\text { DAF for } \\
\text { B.M. }\end{array}$ \\
\hline 30 & 60 & 5 & -0.527 & -0.481 & 1.09 & -1800 & -1772 & 1.02 \\
\hline 30 & 60 & 10 & -0.572 & -0.481 & 1.19 & -1812 & -1772 & 1.02 \\
\hline 30 & 60 & 15 & -0.632 & -0.481 & 1.31 & -1863 & -1772 & 1.05 \\
\hline 30 & 60 & 20 & -0.951 & -0.481 & 1.98 & -1903 & -1772 & 1.07 \\
\hline 30 & 60 & 25 & -0.940 & -0.481 & 2.01 & -1974 & -1772 & 1.11 \\
\hline
\end{tabular}

Table 4.35: Dynamic response of hinge-connected bridge to two successive 70-tonne two-axle vehicle loads (spacing $30 \mathrm{~m}$ )

\begin{tabular}{|c|c|c|c|c|c|c|c|c|}
\hline $\begin{array}{c}\text { Separ. } \\
\text { dist. } \\
(\mathbf{m})\end{array}$ & $\begin{array}{c}\text { Vehicle } \\
\text { weight } \\
(\mathbf{t})\end{array}$ & $\begin{array}{c}\text { Vehicle } \\
\text { Speed } \\
(\mathbf{k m} / \mathbf{h})\end{array}$ & $\begin{array}{c}\text { Max } \\
\text { displ. } \\
(\mathbf{m})\end{array}$ & $\begin{array}{c}\text { Static } \\
\text { displ. } \\
(\mathbf{m})\end{array}$ & $\begin{array}{c}\text { DAF for } \\
\text { displ. }\end{array}$ & $\begin{array}{c}\text { Max } \\
\text { B.M. } \\
(\text { KN.m) }\end{array}$ & $\begin{array}{c}\text { Static } \\
\text { B.M. } \\
(\text { KN.m) }\end{array}$ & $\begin{array}{c}\text { DAF for } \\
\text { B.M. }\end{array}$ \\
\hline 30 & 70 & 5 & -0.615 & -0.562 & 1.10 & -2103 & -2067 & 1.02 \\
\hline 30 & 70 & 10 & -0.669 & -0.562 & 1.19 & -2117 & -2067 & 1.02 \\
\hline 30 & 70 & 15 & -0.742 & -0.562 & 1.32 & -2183 & -2067 & 1.06 \\
\hline 30 & 70 & 20 & -1.115 & -0.562 & 1.99 & -2209 & -2067 & 1.07 \\
\hline 30 & 70 & 25 & -1.091 & -0.562 & 2.00 & -2308 & -2067 & 1.12 \\
\hline
\end{tabular}

\subsubsection{Discussion of Results}

Figure 4.65 shows the maximum displacement in the bridge due to two successive vehicle loads with a separation distance of $50 \mathrm{~m}$ for different vehicle weights and speeds. The maximum displacement in the bridge increases with vehicle weight and speed. The 
maximum displacement in the bridge is within the allowable limit $(0.769 \mathrm{~m})$ for 50 -tonne vehicles at speeds up to $20 \mathrm{~km} / \mathrm{h}$, and for 60 -tonne and 70-tonne vehicles at speeds up to $15 \mathrm{~km} / \mathrm{h}$. This means that the hinge-connected bridge can support two 50-tonne successive vehicles travelling at a speed up to $20 \mathrm{~km} / \mathrm{h}$, or two 60 -tonne or 70 -tonne successive vehicles travelling at a speed up to $15 \mathrm{~km} / \mathrm{h}$, given that the separation distance between the two vehicles is $50 \mathrm{~m}$.

The maximum displacement in the bridge due to two successive vehicle loads with separation distances of 40 and $30 \mathrm{~m}$ are shown in Figures 4.70 and 4.75, respectively. For separation distances of 40 and $30 \mathrm{~m}$, the hinge-connected bridge can support two 50tonne, 60 -tonne or 70-tonne successive vehicles travelling at speeds up to $15 \mathrm{~km} / \mathrm{h}$.

The maximum bending moment developed in the bridge is presented in Figure 4.66 for a separation distance of $50 \mathrm{~m}$. It is observed that the maximum bending moment increases with vehicle weight and speed. The same trend is observed for two successive vehicles with separation distances of 40 and $30 \mathrm{~m}$ as shown in Figures 4.71 and 4.76, respectively.

Figure 4.67 shows the DAF for both displacement and bending moment in the bridge due to two successive 50-tonne vehicle loads with a separation distance of $50 \mathrm{~m}$, and travelling the bridge at different speeds. The DAF for displacement greatly increases with vehicle speed ranging from 1.08 at $5 \mathrm{~km} / \mathrm{h}$ to 2.63 at $25 \mathrm{~km} / \mathrm{h}$. The DAF for bending moment also increases with vehicle speed ranging from 1.42 at $5 \mathrm{~km} / \mathrm{h}$ to 1.51 at 25 $\mathrm{km} / \mathrm{h}$. This means that an increase in the vehicle speed has a strong effect on the increase in DAFs for displacement and a smaller effect on the increase in DAFs for bending moment. The same trend is observed for two successive vehicles with separation 
distances of 40 and $30 \mathrm{~m}$ as shown in Figures 4.72 and 4.77, respectively. The DAFs for displacement are much higher than those for bending moments.

Figure 4.68 shows the DAF for displacement due to two successive vehicle loads with a separation distance of $50 \mathrm{~m}$ for different vehicle weights: 50,60 and 70 tonnes. The DAFs for displacement are the same for different vehicle weights, however, the displacements increase as the vehicle weight increases. The same trend is observed for two successive vehicles with separation distances of 40 and $30 \mathrm{~m}$ as shown in Figures 4.73 and 4.78 , respectively. Figure 4.69 shows the DAF for bending moment due to two successive vehicle loads with separation distance of $50 \mathrm{~m}$ for different vehicle weights: 50, 60 and 70 tonnes. The DAFs for bending moment are almost the same for different vehicle weights. Only minor increases in the DAF for bending moment are observed with increasing vehicle weight. On the other hand, the bending moment values always increase as the vehicle weight increases. Similar trend is observed for two successive vehicles with separation distances of 40 and $30 \mathrm{~m}$ as shown in Figures 4.74 and 4.79 , respectively which show that the DAFs for bending moment slightly increase with increasing vehicle weight especially at high vehicle speeds. The foregoing results show that an increase in the vehicle weight almost does not affect the DAFs for displacement and bending moment. As a result, an increase in the vehicle weight can be achieved without significantly increasing DAFs for both displacement and bending moment as long as the allowable displacement in the bridge is not exceeded. The same is not true of an increase in vehicle speed. 


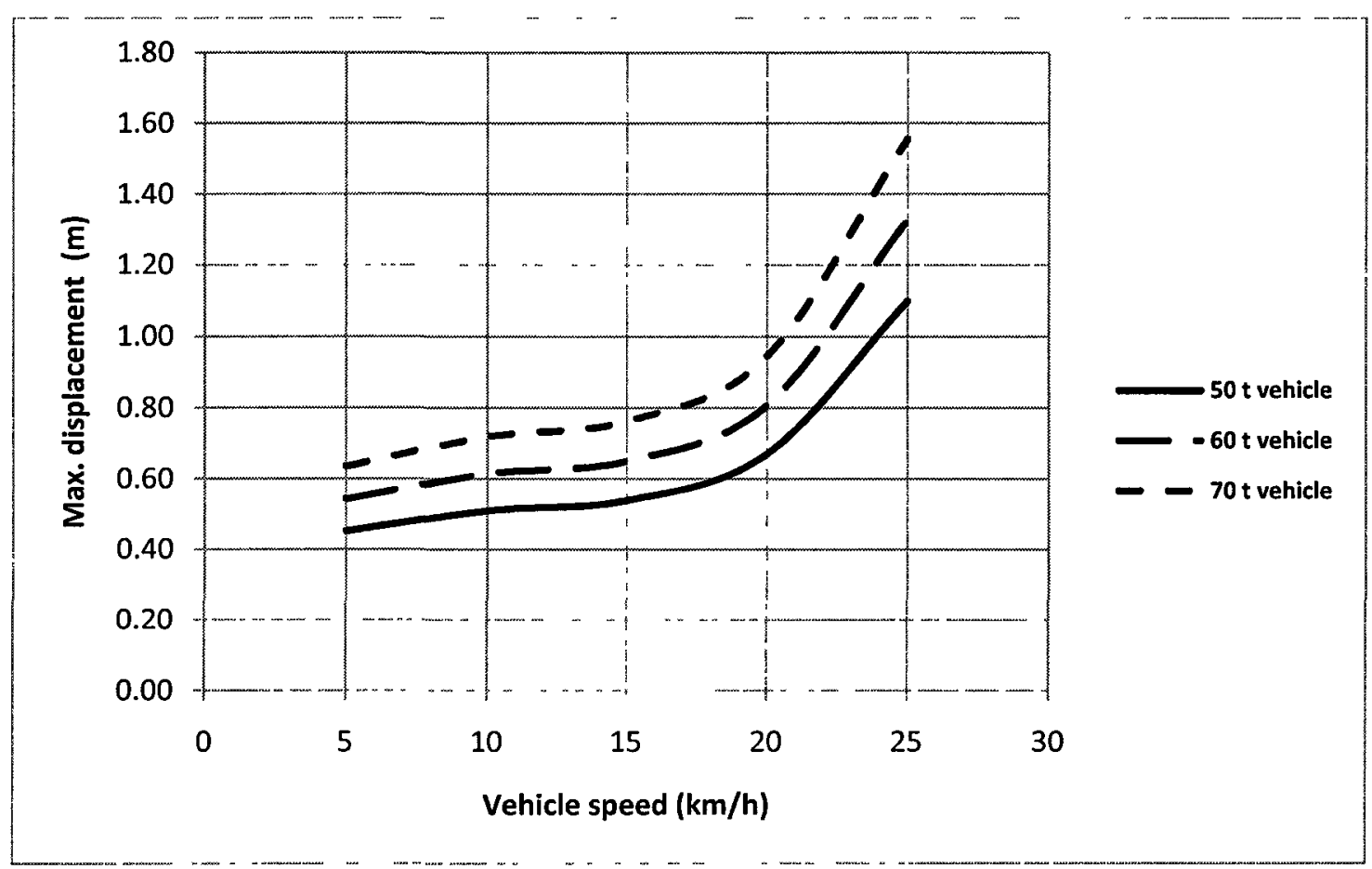

Figure 4.65: Effect of vehicle speed and weight on maximum displacement in hingeconnected bridge (two successive vehicles with spacing $50 \mathrm{~m}$ )

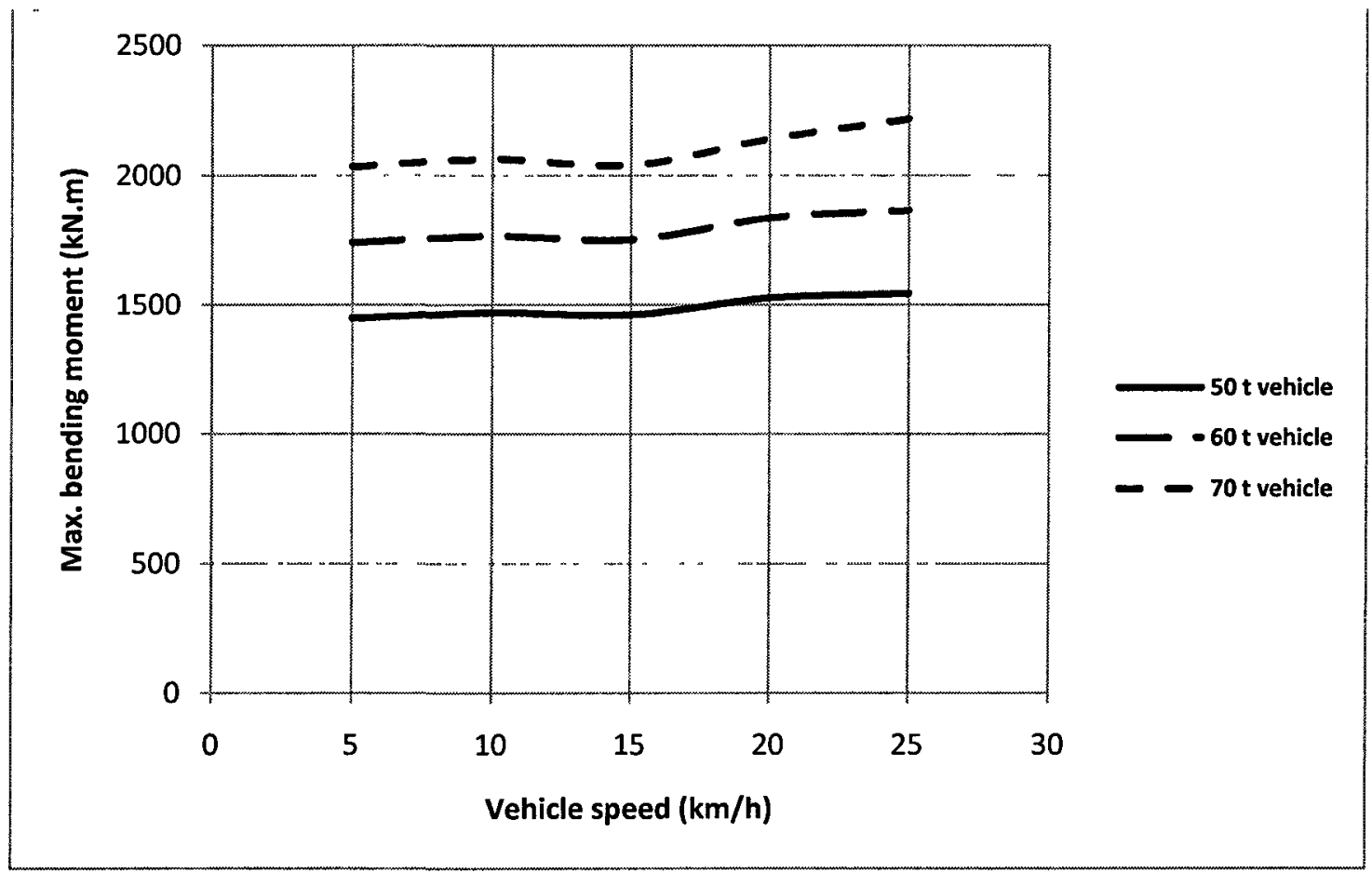

Figure 4.66: Effect of vehicle speed and weight on maximum bending moment in hingeconnected bridge (two successive vehicles with spacing $50 \mathrm{~m}$ ) 


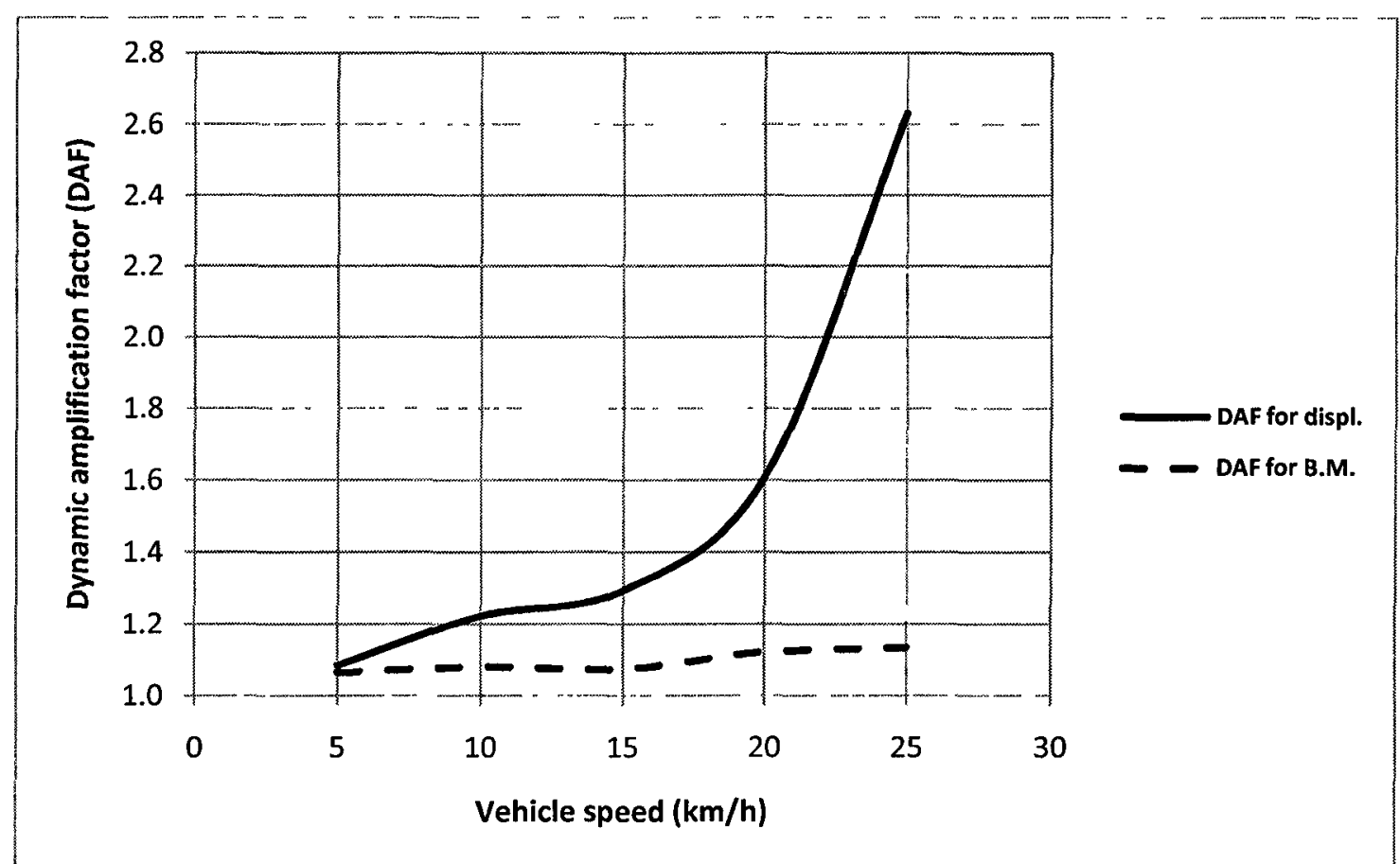

Figure 4.67: DAFs in hinge-connected bridge due to two successive 50-tonne vehicle loads with spacing $50 \mathrm{~m}$

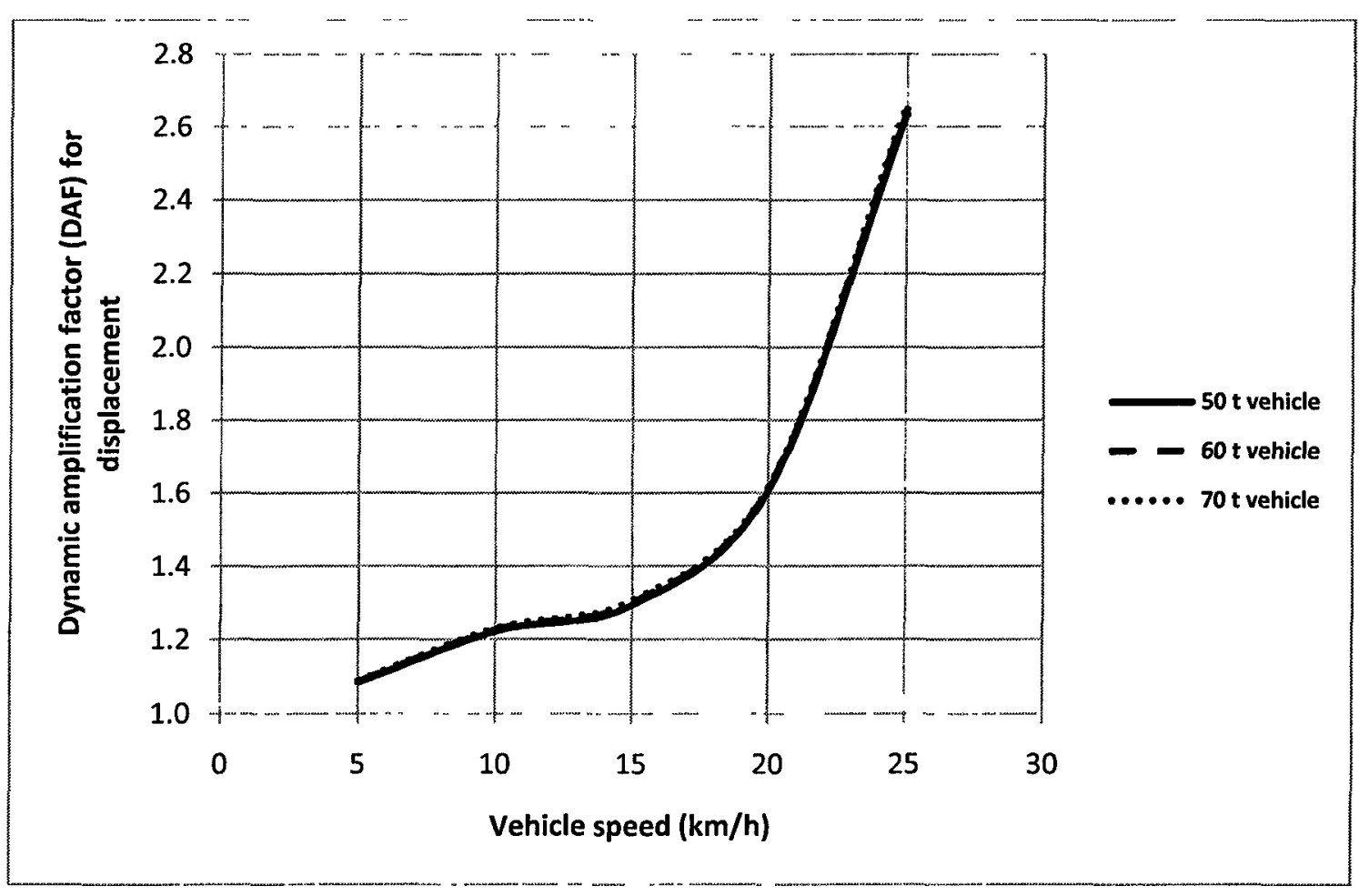

Figure 4.68: DAFs for displacement in hinge-connected bridge due to two successive vehicles with spacing $50 \mathrm{~m}$ 


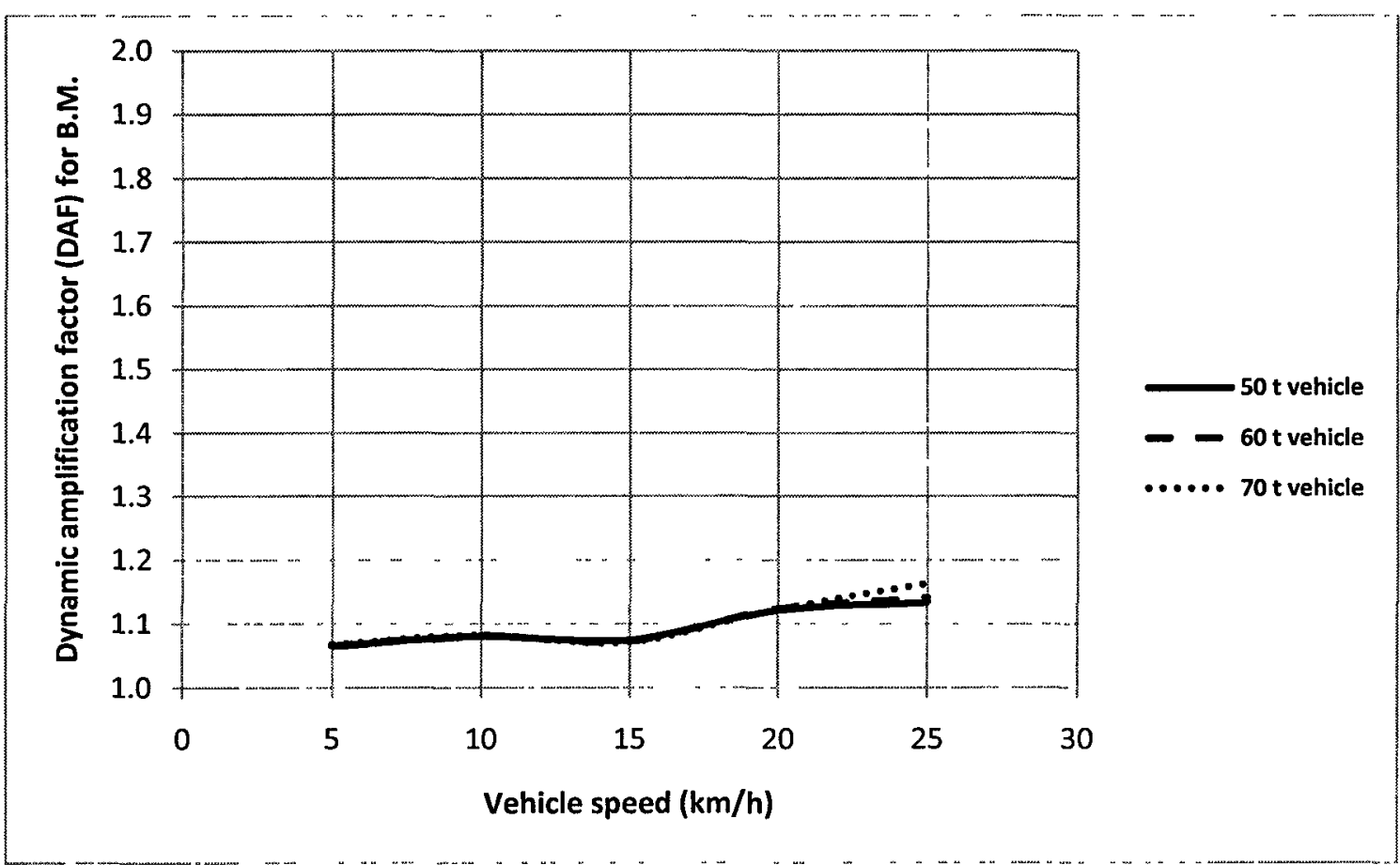

Figure 4.69: DAFs for bending moment in hinge-connected bridge due to two successive vehicles with spacing $50 \mathrm{~m}$

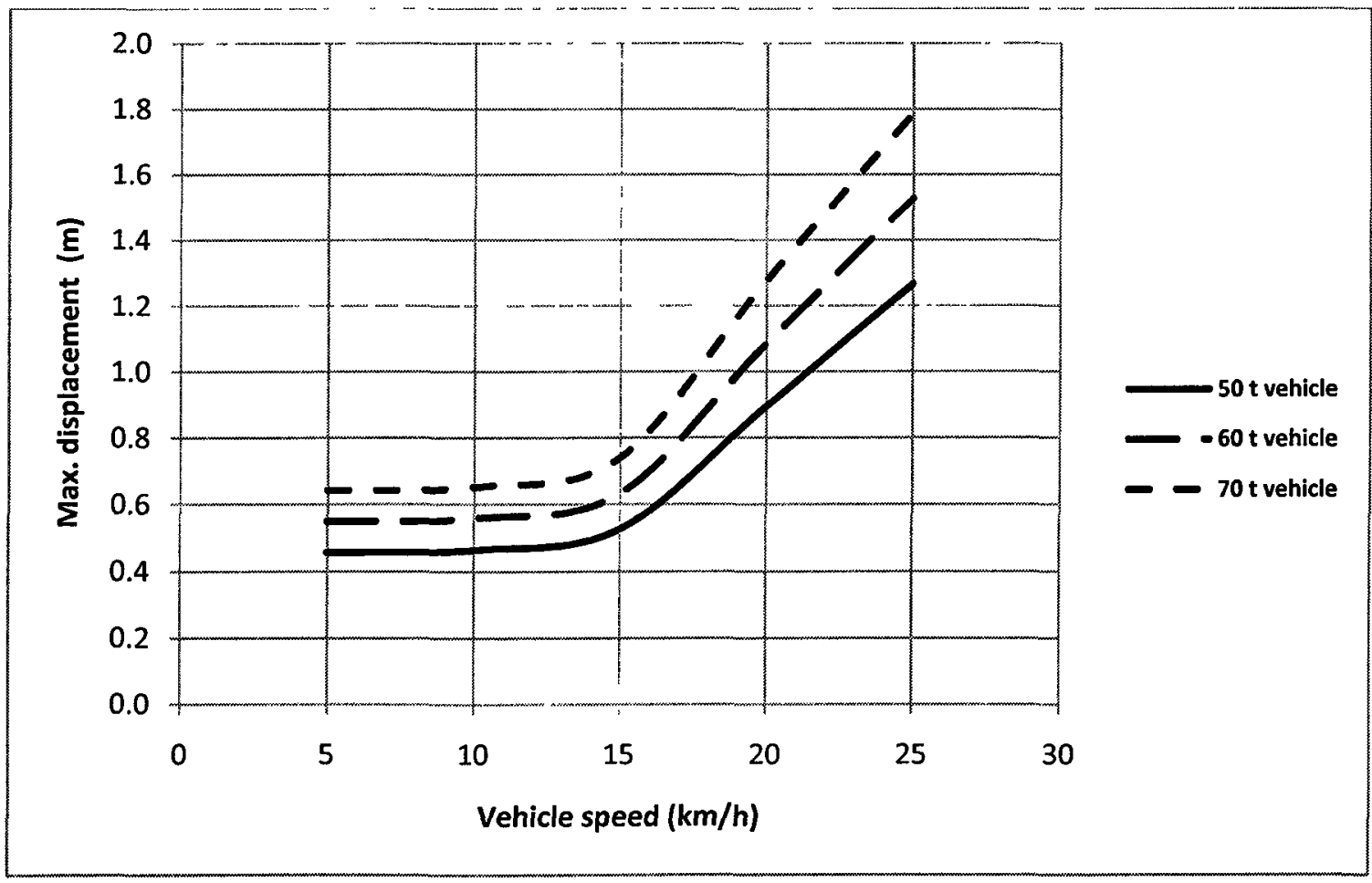

Figure 4.70: Effect of vehicle speed and weight on maximum displacement in hingeconnected bridge (two successive vehicles with spacing $40 \mathrm{~m}$ ) 


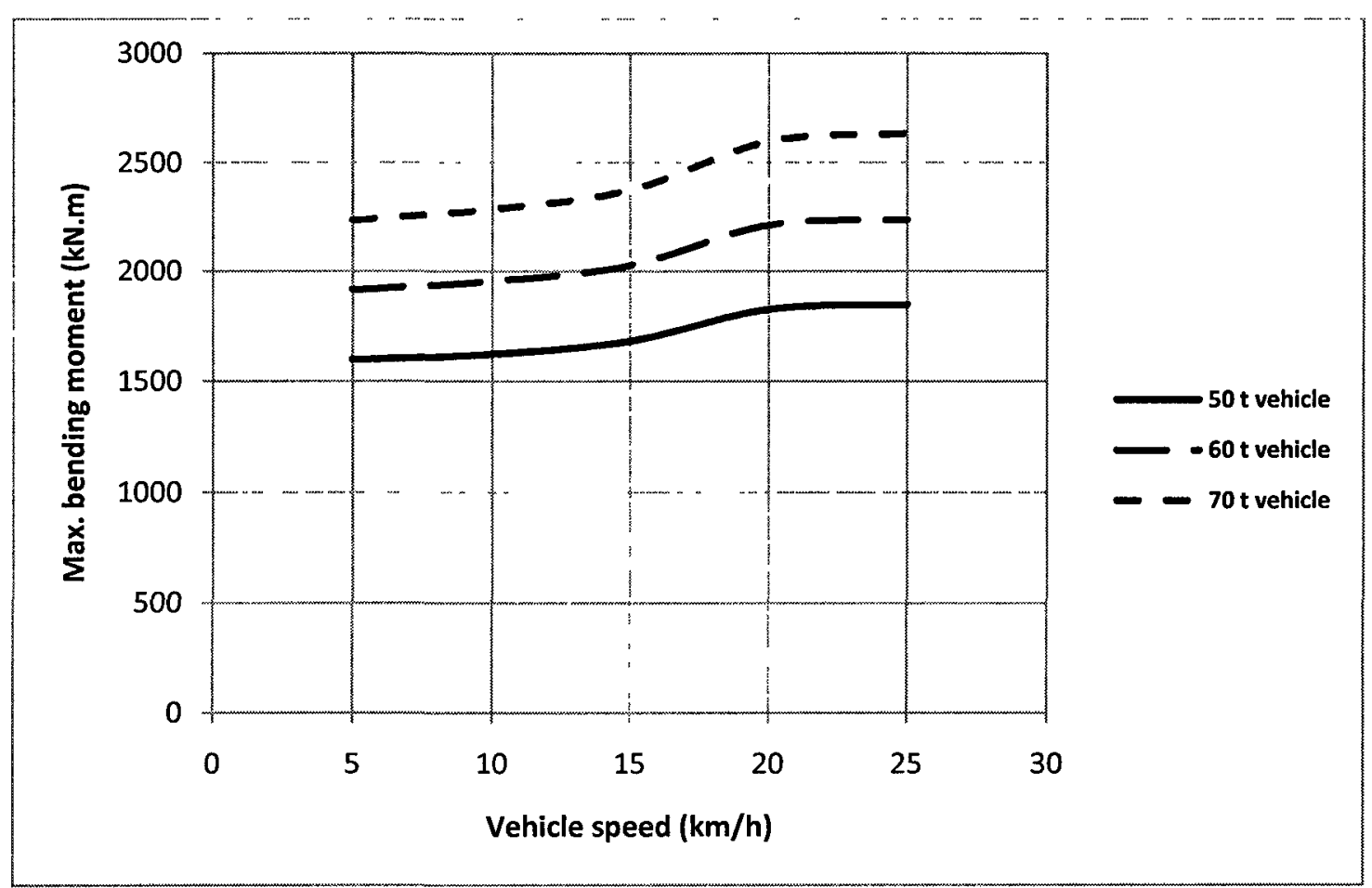

Figure 4.71: Effect of vehicle speed and weight on maximum bending moment in hingeconnected bridge (two successive vehicles with spacing $40 \mathrm{~m}$ )

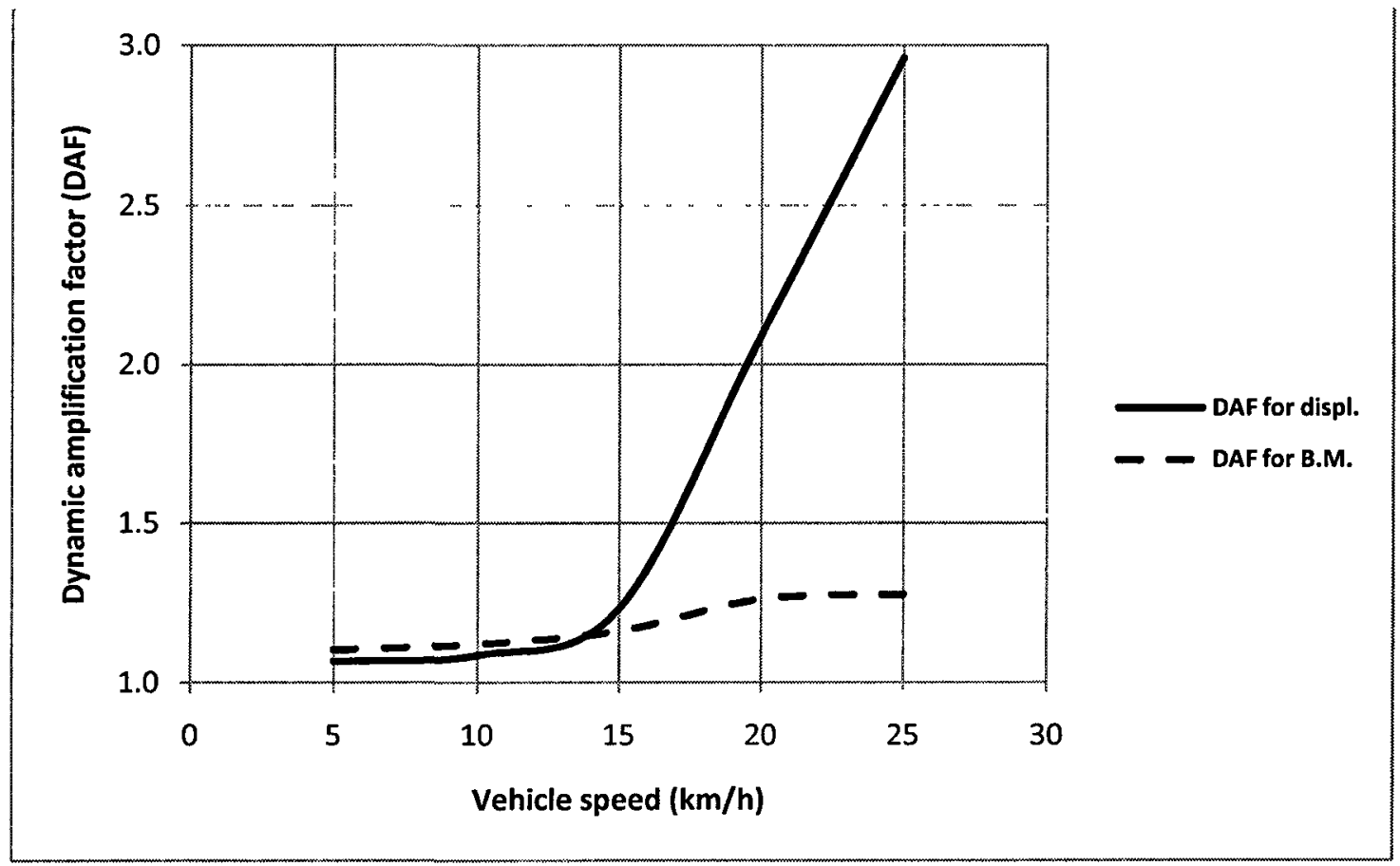

Figure 4.72: DAFs in hinge-connected bridge due to two successive 50-tonne vehicle loads with spacing $40 \mathrm{~m}$ 


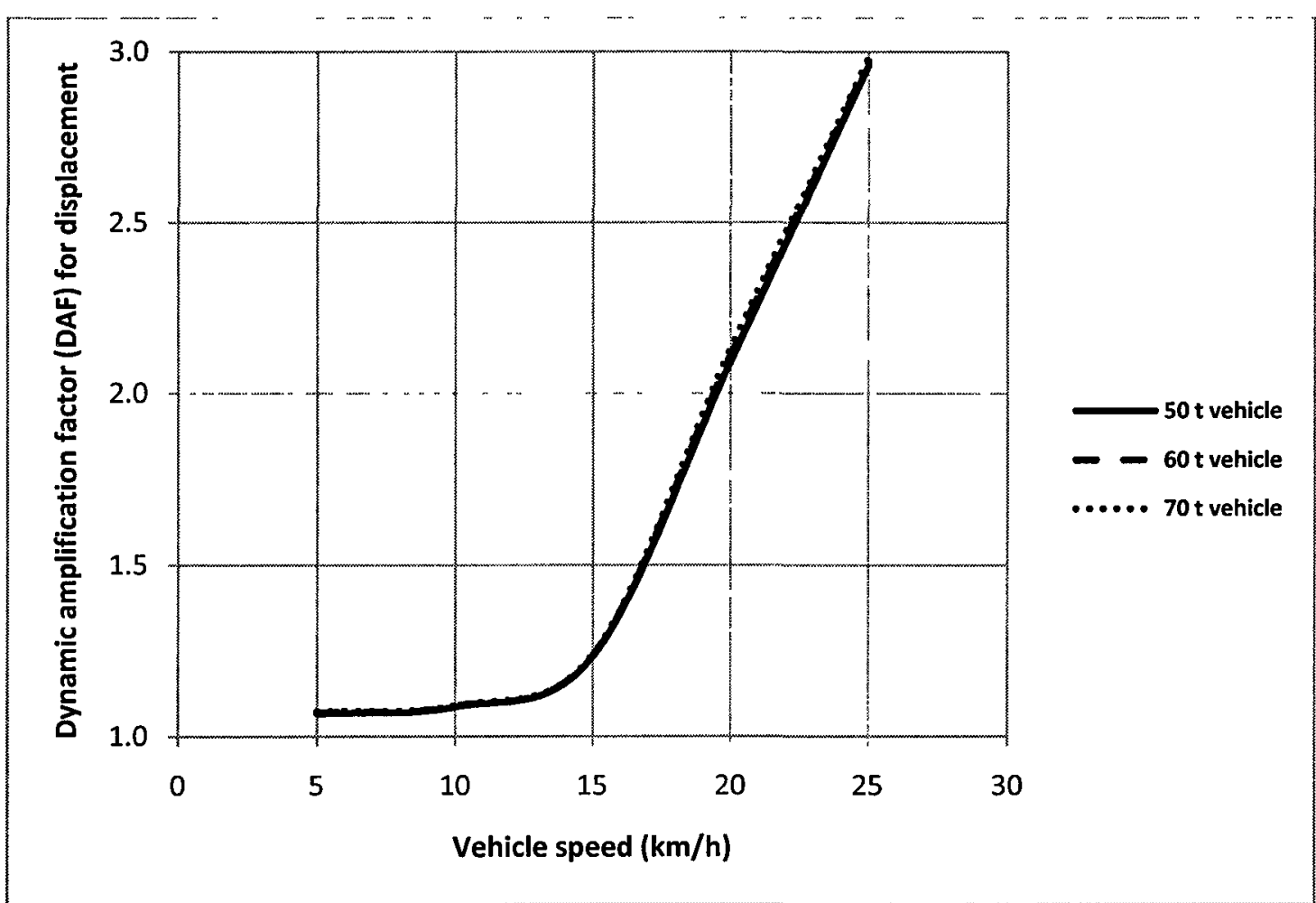

Figure 4.73: DAFs for displacement in hinge-connected bridge due to two successive vehicles with spacing $40 \mathrm{~m}$

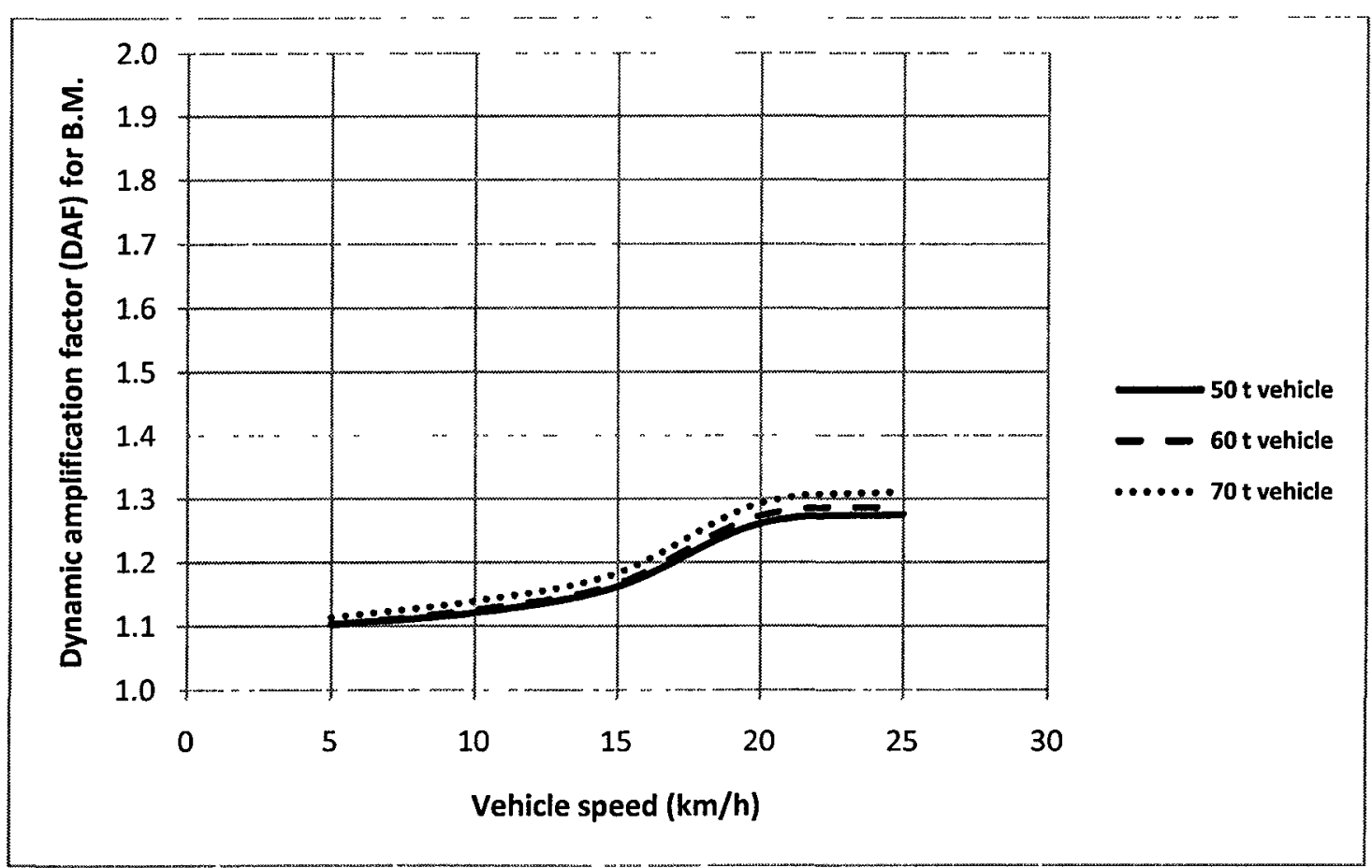

Figure 4.74: DAFs for bending moment in hinge-connected bridge due to two successive vehicles with spacing $40 \mathrm{~m}$ 


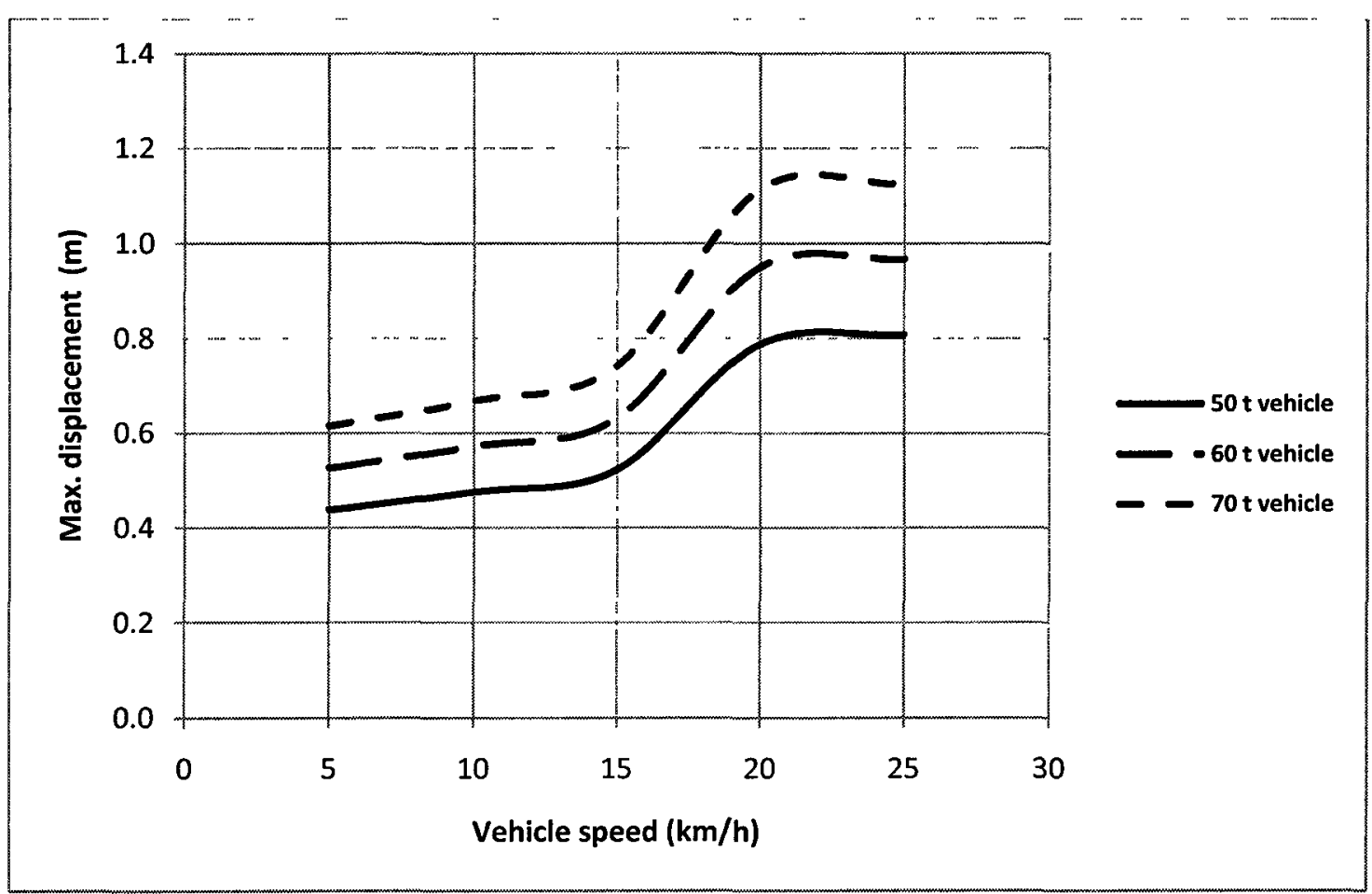

Figure 4.75: Effect of vehicle speed and weight on maximum displacement in hingeconnected bridge (two successive vehicles with spacing $30 \mathrm{~m}$ )

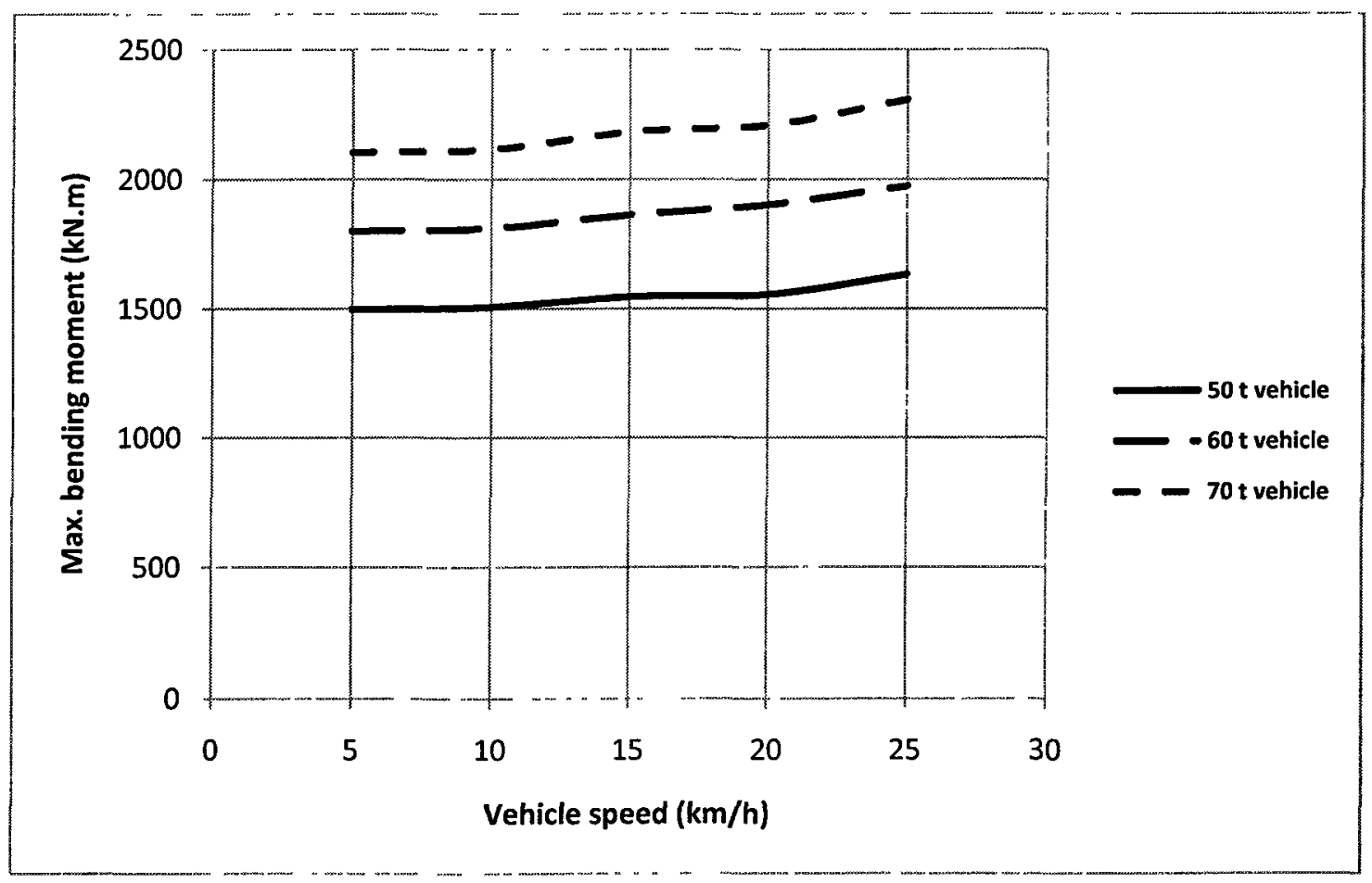

Figure 4.76: Effect of vehicle speed and weight on maximum bending moment in hingeconnected bridge (two successive vehicles with spacing $30 \mathrm{~m}$ ) 


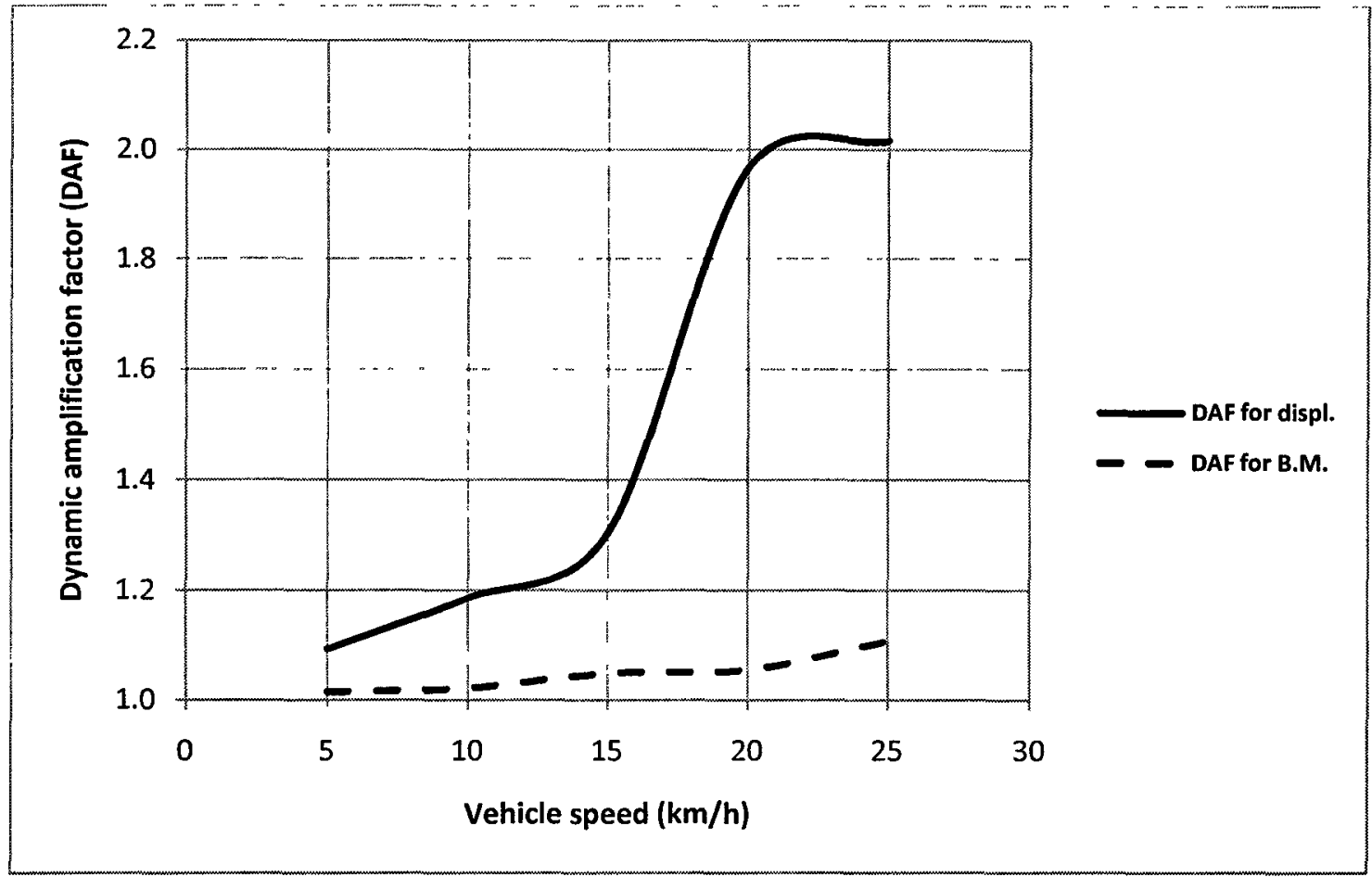

Figure 4.77: DAFs in hinge-connected bridge due to two successive 50-tonne vehicle loads with spacing $30 \mathrm{~m}$

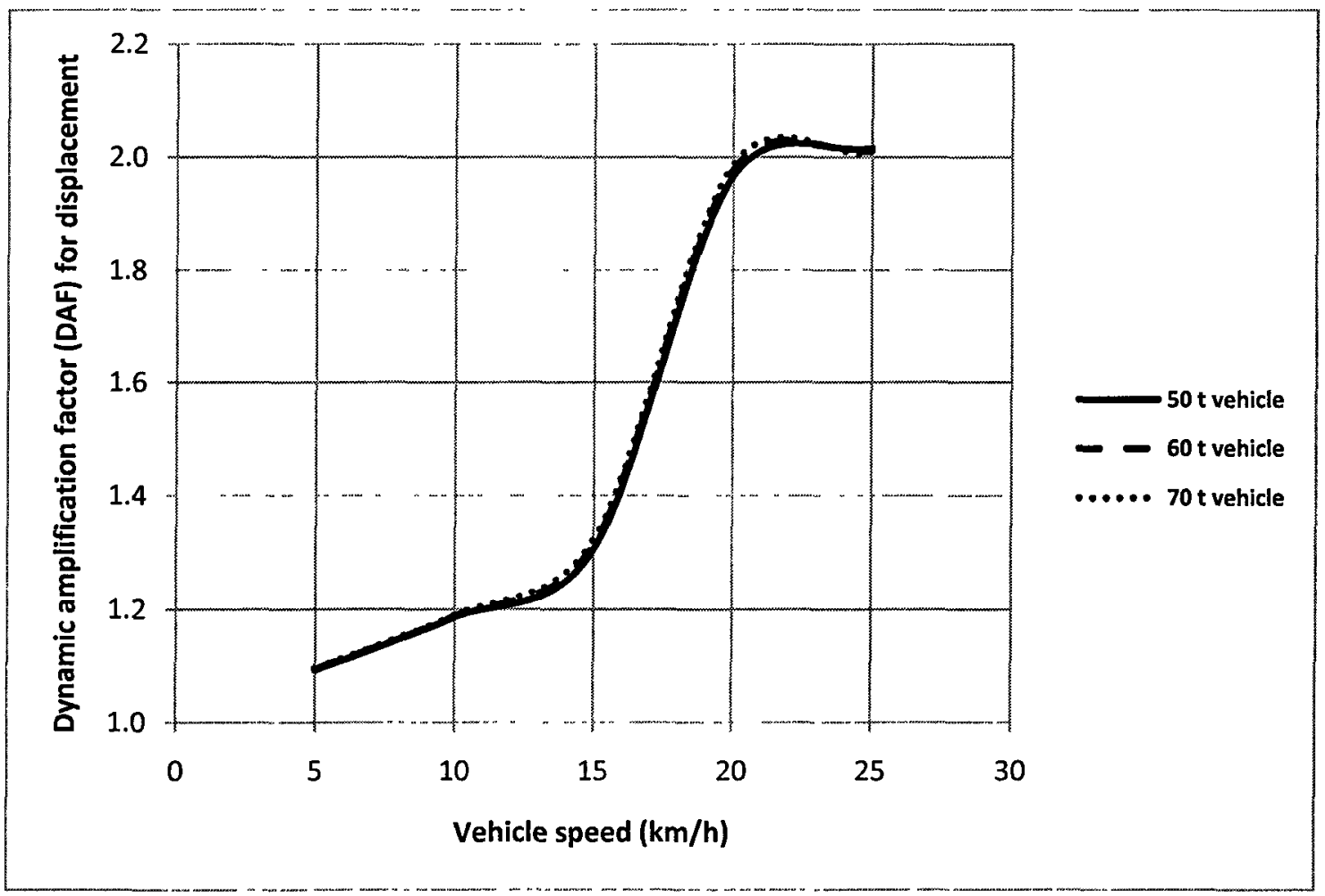

Figure 4.78: DAFs for displacement in hinge-connected bridge due to two successive vehicles with spacing 30 


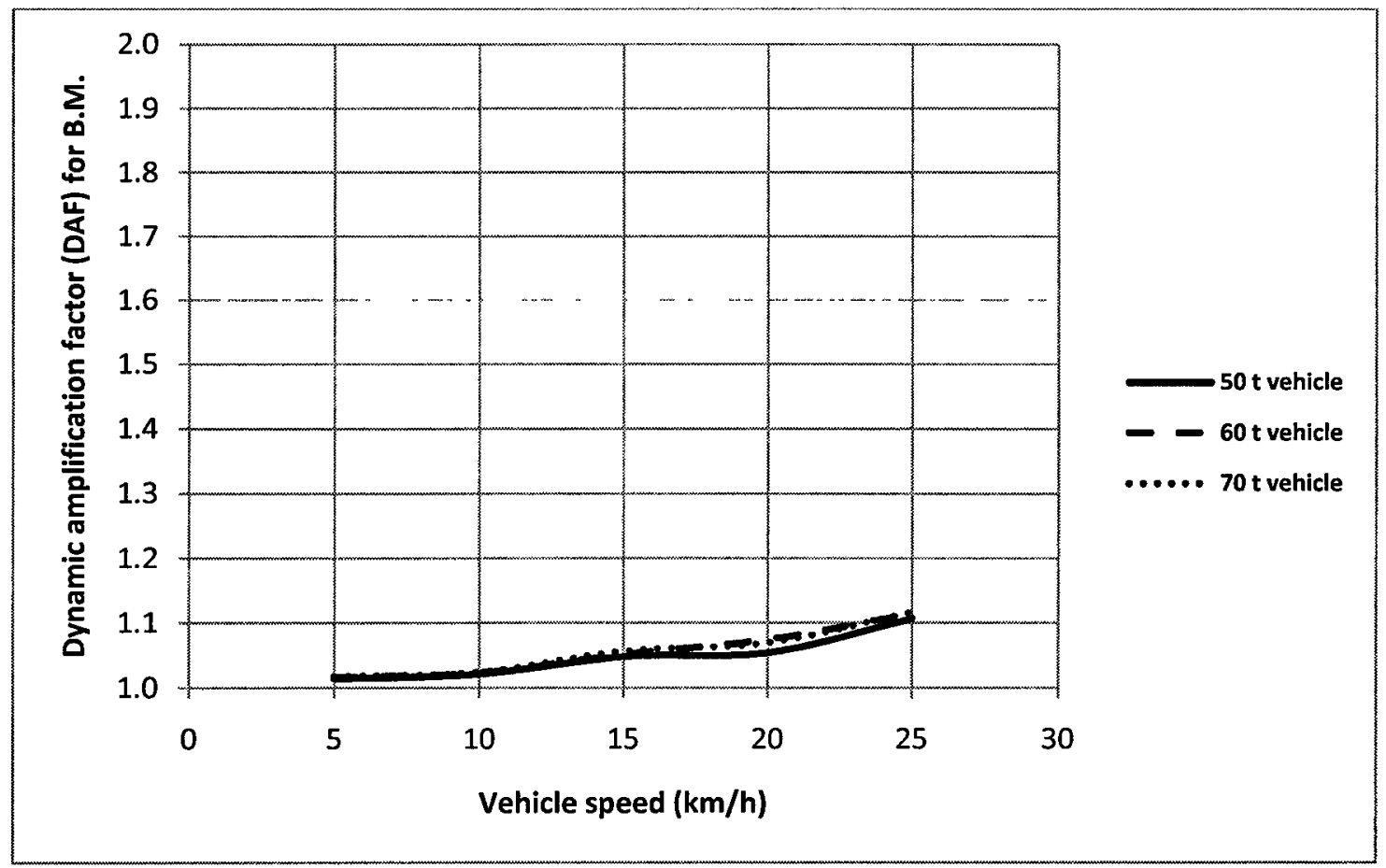

Figure 4.79: DAFs for bending moment in hinge-connected bridge due to two successive vehicles with spacing 30

\subsection{Effect of the Passage of Multiple Vehicles}

In this section, the transport capacity of rigid-connected and hinge-connected floating bridges is investigated by comparing the dynamic responses of these bridges to single vehicle loads with the corresponding responses to two successive vehicle loads with different separation distances, considering different weights and speeds of the vehicles. Each vehicle is represented by a two-axle model.

\subsubsection{Dynamic Response of Rigid-Connected Floating Bridge to Single and Two}

\section{Vehicle Loads}

The midpoint displacements due to two successive vehicles are higher than those due to single vehicle for all vehicle speeds from $5 \mathrm{~km} / \mathrm{h}$ to $25 \mathrm{~km} / \mathrm{h}$, and those displacements 
increase as the separation distance between successive vehicles decreases and the two vehicles get closer to each other. Figure 4.80 and 4.81 show typical time history plots of midpoint displacements at vehicle speeds of $10 \mathrm{~km} / \mathrm{h}$ and $15 \mathrm{~km} / \mathrm{h}$, respectively. Figure 4.80 and 4.81 show that the curve for midpoint displacement due to single vehicle coincides with the curve for midpoint displacement due to two successive vehicles up to the time corresponding to the separation distance between the two vehicles, where only the first vehicle load acts on the bridge. After that time the midpoint displacement differs due to the entrance of the second vehicle on the bridge.

The midpoint accelerations due to two successive vehicles are higher than those due to single vehicle for all speeds up to $25 \mathrm{~km} / \mathrm{h}$ and those accelerations, in general, increase as the separation distance between successive vehicles decreases. Figure 4.82 and 4.83 show typical time history plots of midpoint accelerations at vehicle speeds of $10 \mathrm{~km} / \mathrm{h}$ and 15 $\mathrm{km} / \mathrm{h}$, respectively.

Figure 4.84 shows the maximum deflections in the bridge due to 50 -tonne single vehicle and two 50-tonne successive vehicles with different spacing of 50,40 and $30 \mathrm{~m}$. The maximum deflections in the bridge due to two successive vehicles are higher than those due to single vehicle for all vehicle speeds, and those deflections increase as the separation distance between successive vehicles decreases and the two vehicles get closer to each other. The same trend was observed for 60 and 70 -tonne vehicles.

The maximum bending moments developed in the bridge due to 50-tonne single vehicle and two 50-tonne successive vehicles with different spacing of 50,40 and $30 \mathrm{~m}$ are presented in Figure 4.85. The maximum bending moments of the bridge due to two 
successive vehicles are almost the same as those due to single vehicle for all separation distances at vehicle speeds of 5,15 and $20 \mathrm{~km} / \mathrm{h}$. While at vehicle speed of $10 \mathrm{~km} / \mathrm{h}$ the maximum bending moments of the bridge due to two successive vehicles with separation distance of $30 \mathrm{~m}$ are higher than those due to single vehicle. At vehicle speed of $25 \mathrm{~km} / \mathrm{h}$ the maximum bending moments of the bridge due to two successive vehicles with separation distance of $40 \mathrm{~m}$ are higher than those due to single vehicle. The same trend was observed for 60 and 70-tonne vehicles.

Figure 4.86 shows the DAFs for deflection due to a 50 -tonne single vehicle and two 50tonne successive vehicle loads with different spacing of 50,40 and $30 \mathrm{~m}$. The DAFs for deflection due to two successive vehicles are higher than those due to a single vehicle for all vehicle speeds up to $25 \mathrm{~km} / \mathrm{h}$. The effect of the separation distance between successive vehicles on the DAFs for deflection differs according to the vehicle speed. The DAFs for bending moment are presented in Figure 4.87, which shows that the DAFs for bending moment due to two successive vehicles are lower than those due to single vehicle; those DAFs, in general, increase as the separation distance between successive vehicles decreases and the two vehicles get closer to each other. The same trends were observed for DAFs for 60 and 70 tonne vehicle weights. 


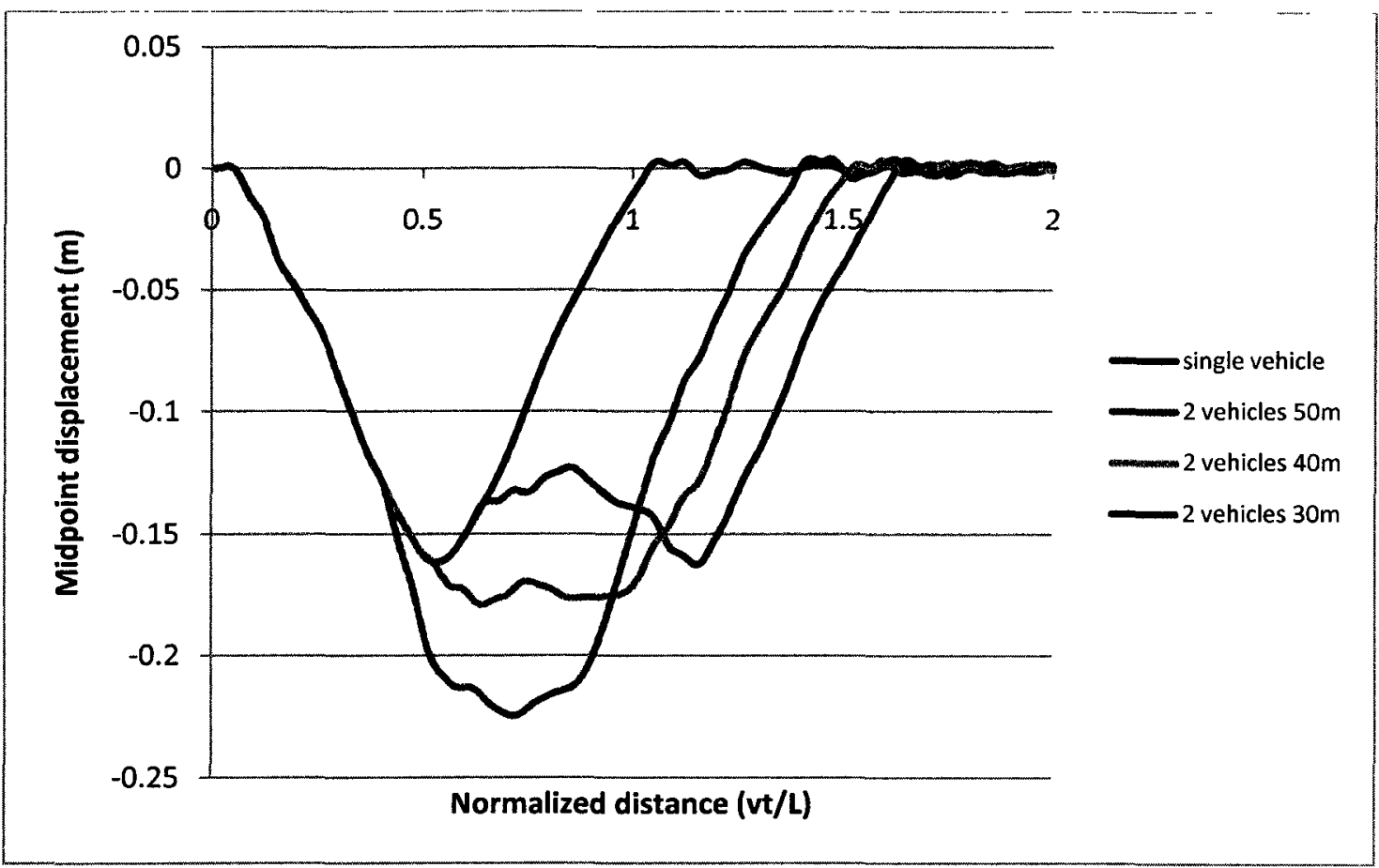

Figure 4.80: Time history of midpoint displacements in rigid-connected bridge due to two successive 50-tonne vehicle loads with different spacing $(\mathrm{v}=10 \mathrm{~km} / \mathrm{h})$

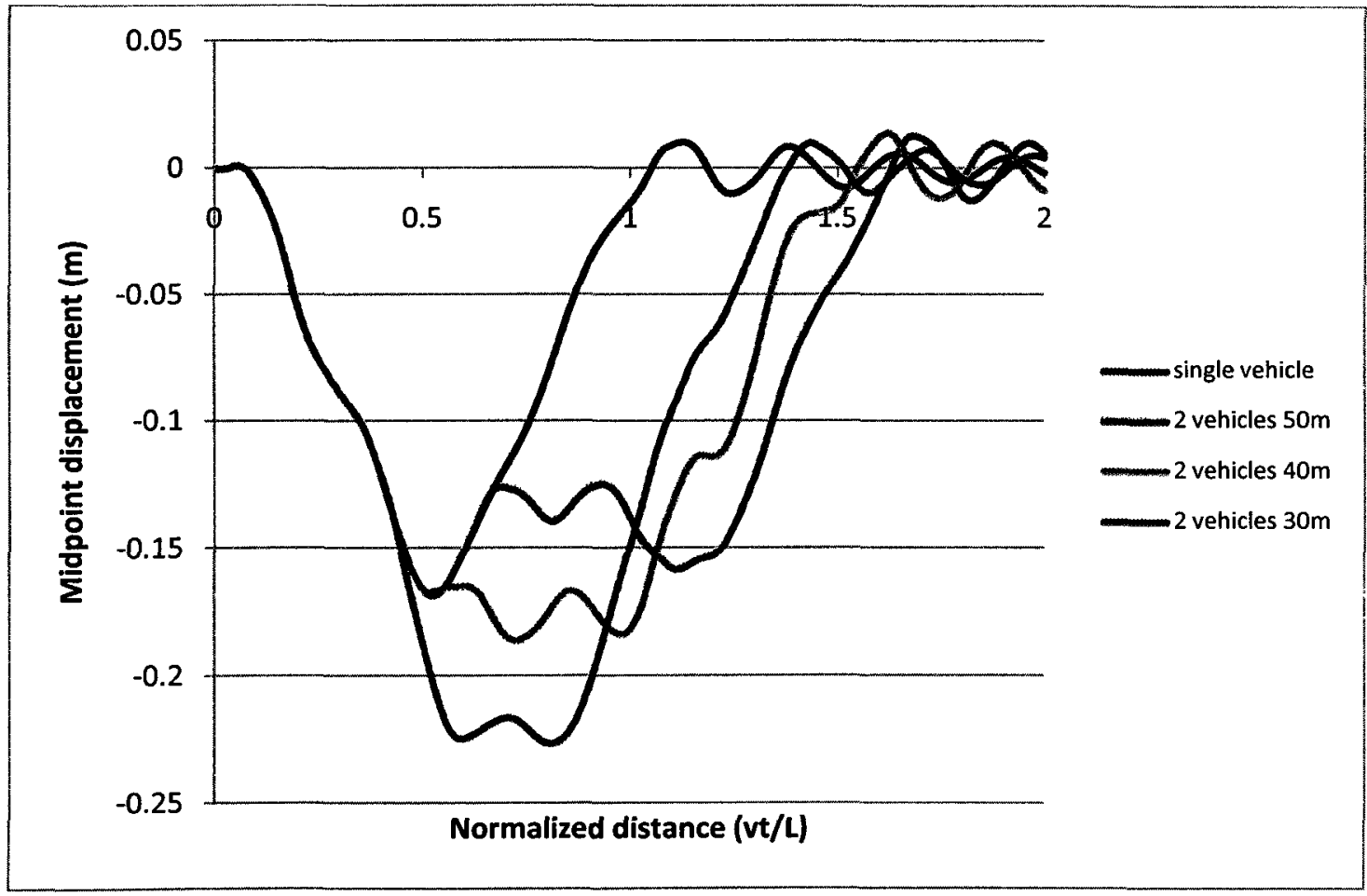

Figure 4.81: Time history of midpoint displacements in rigid-connected bridge due to two successive 50-tonne vehicle loads with different spacing $(\mathrm{v}=15 \mathrm{~km} / \mathrm{h})$ 


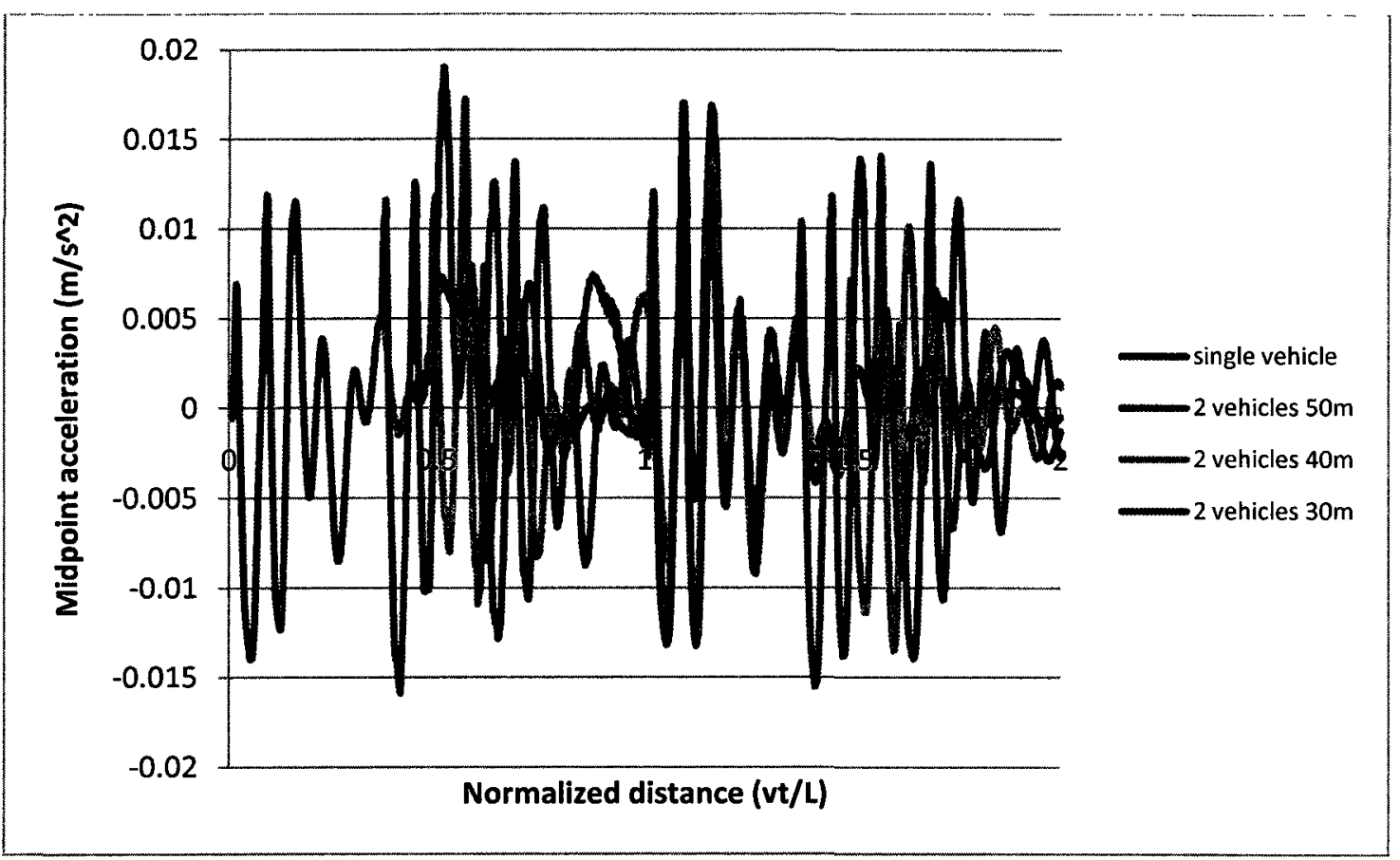

Figure 4.82: Time history of midpoint accelerations in rigid-connected bridge due to two successive 50 -tonne vehicle loads with different spacing $(\mathrm{v}=10 \mathrm{~km} / \mathrm{h})$

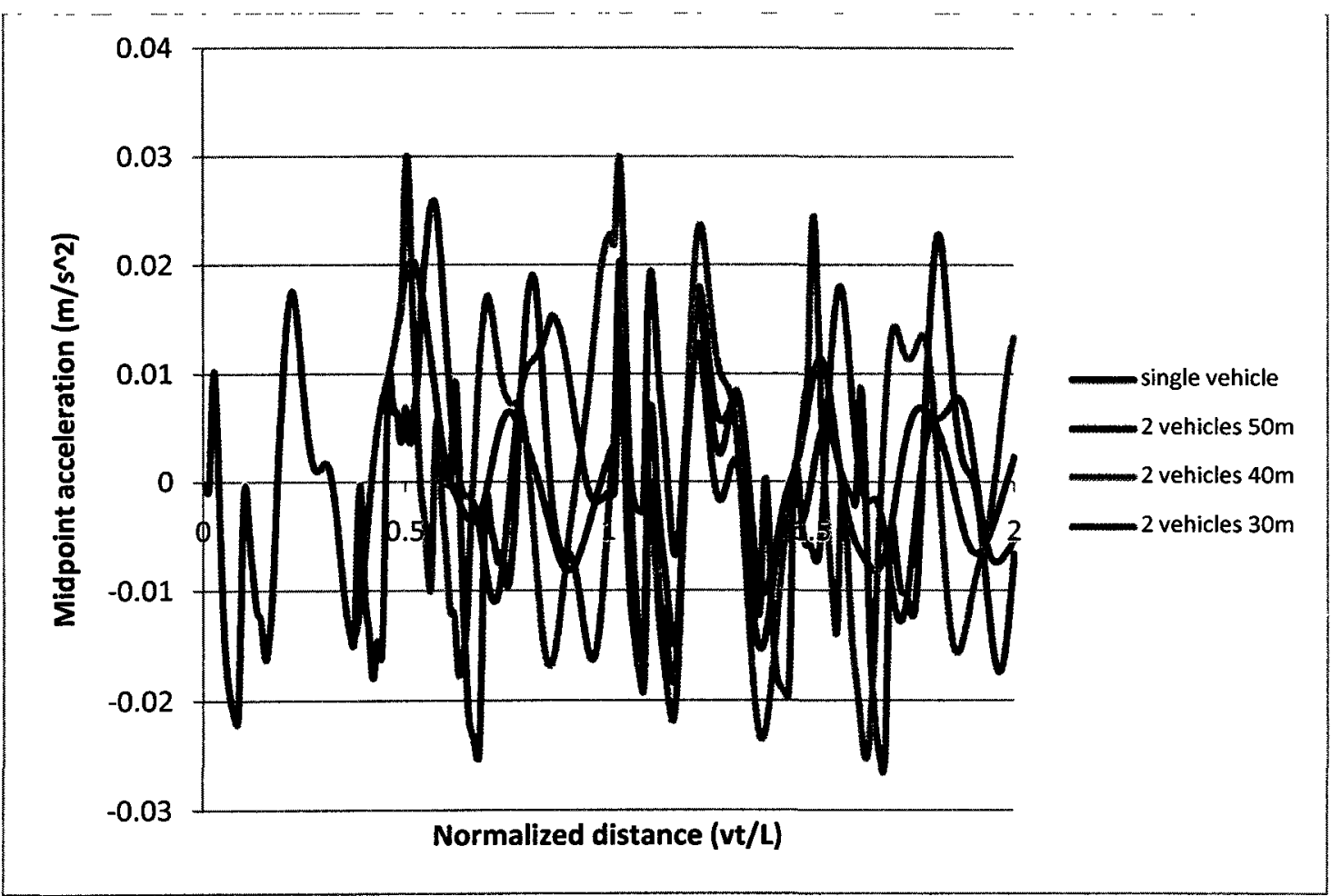

Figure 4.83: Time history of midpoint accelerations in rigid-connected bridge due to two successive 50-tonne vehicle loads with different spacing $(\mathrm{v}=15 \mathrm{~km} / \mathrm{h})$ 


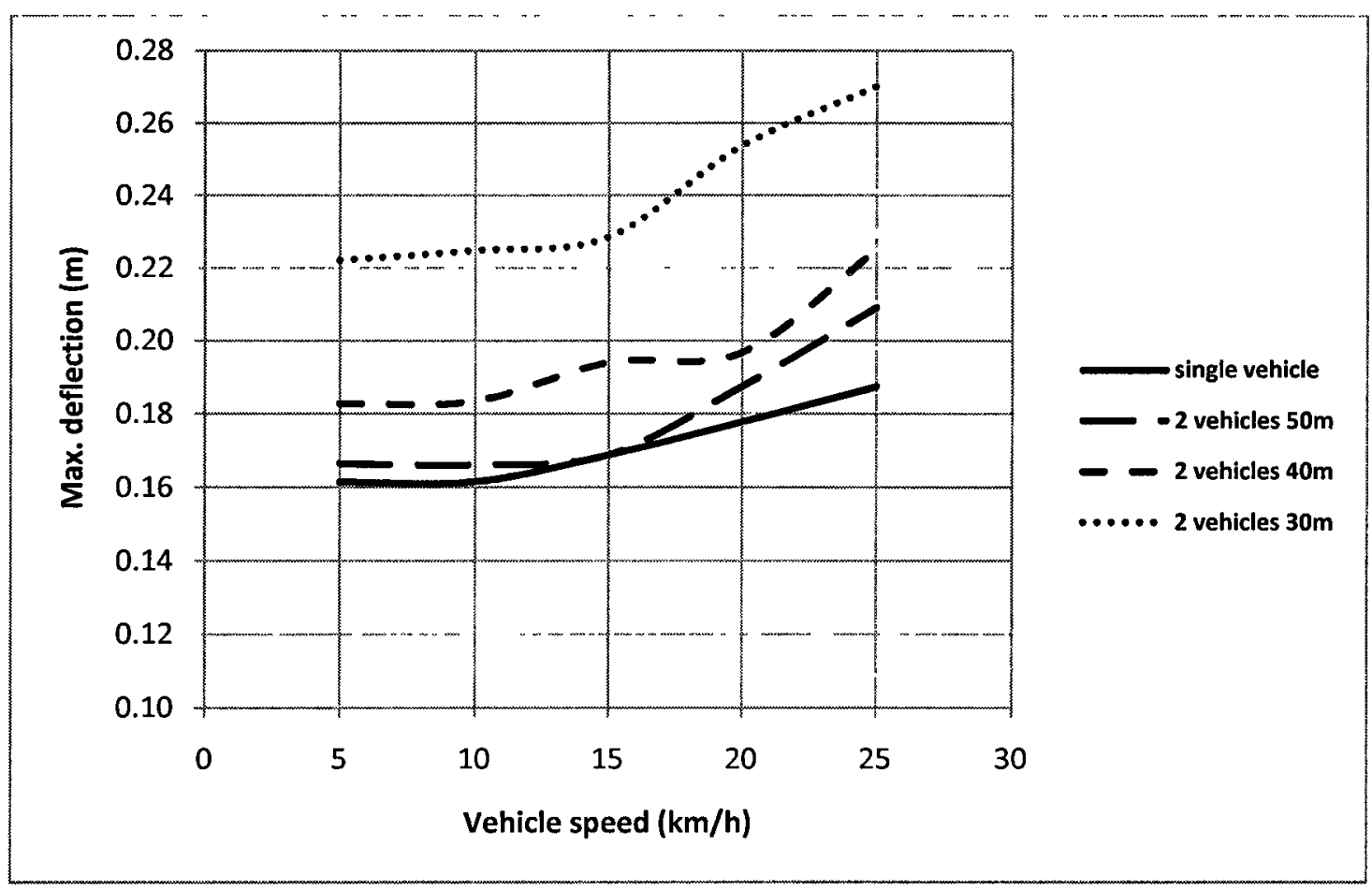

Figure 4.84: Maximum deflections in rigid-connected bridge due to 50-tonne vehicle loads

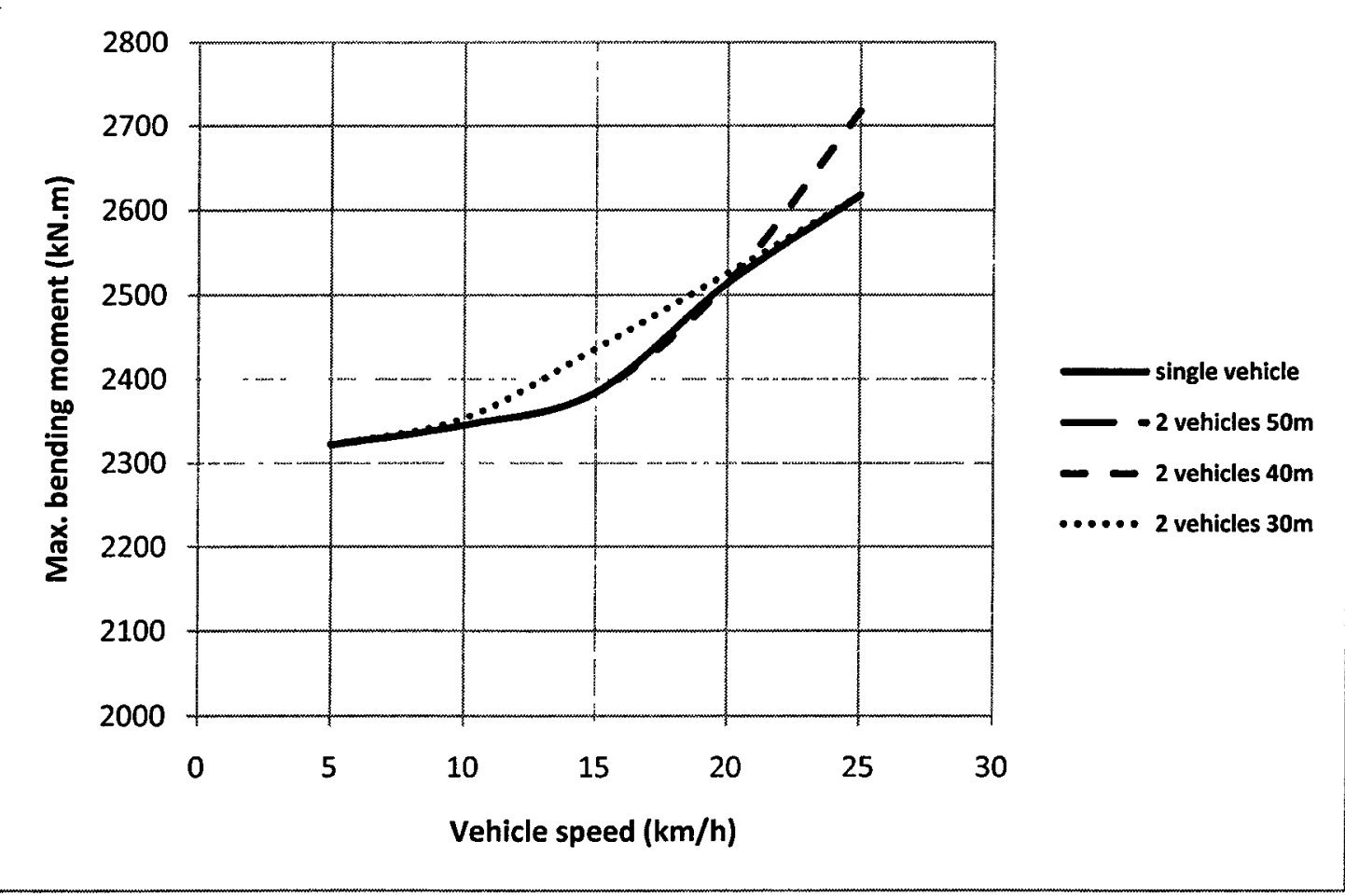

Figure 4.85: Maximum bending moments in rigid-connected bridge due to 50-tonne vehicle loads 


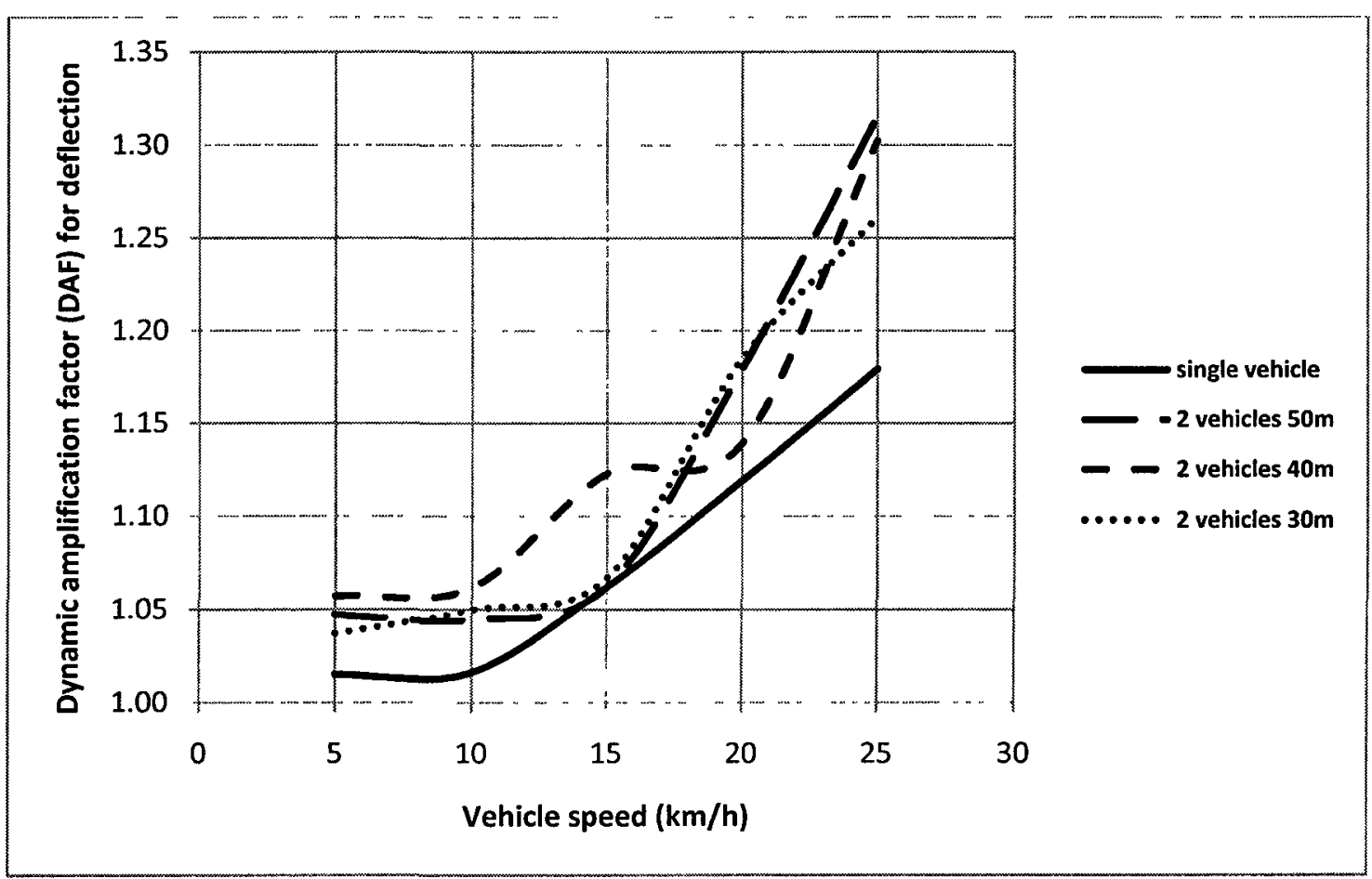

Figure 4.86: DAFs for deflection in rigid-connected bridge due to 50-tonne vehicle loads

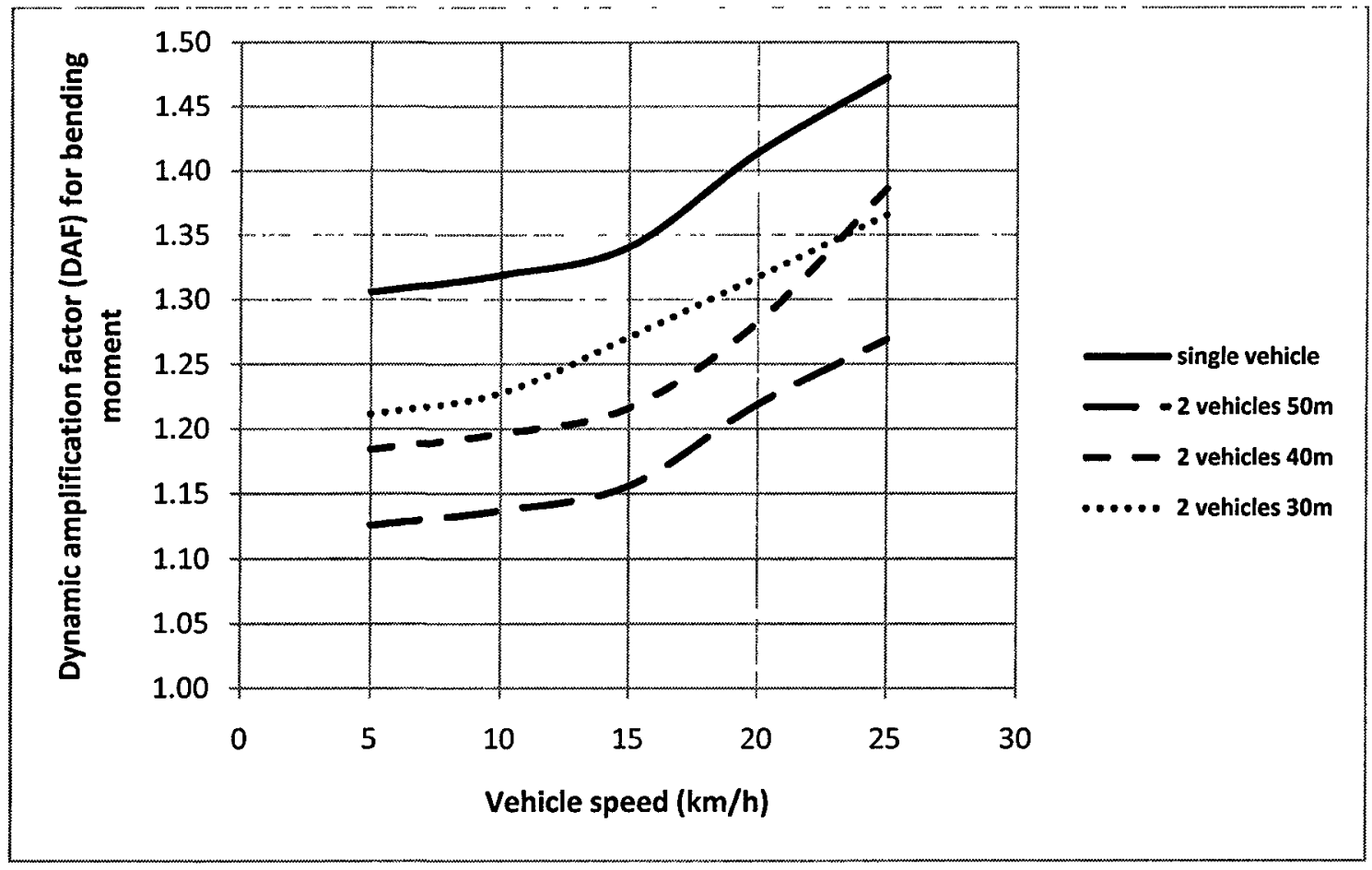

Figure 4.87: DAFs for bending moment in rigid-connected bridge due to 50-tonne vehicle loads 


\subsubsection{Dynamic Response of Hinge-Connected Floating Bridge to Single and Two}

Vehicle Loads

The midpoint displacements due to two successive vehicles are higher than those due to a single vehicle for all vehicle speeds up to $25 \mathrm{~km} / \mathrm{h}$, and the effect of the separation distance between successive vehicles differs according to the vehicle speed. Figure 4.88 and 4.89 show typical time history plots of midpoint displacements at vehicle speeds of $10 \mathrm{~km} / \mathrm{h}$ and $15 \mathrm{~km} / \mathrm{h}$, respectively.

Also, the midpoint accelerations due to two successive vehicles are higher than those due to single vehicle for all vehicle speeds up to $25 \mathrm{~km} / \mathrm{h}$, and the effect of the separation distance between successive vehicles differs according to the vehicle speed. Figure 4.90 and 4.91 show typical time history plots of midpoint accelerations at vehicle speed of 10 $\mathrm{km} / \mathrm{h}$ and $15 \mathrm{~km} / \mathrm{h}$, respectively.

Figure 4.92 shows the maximum displacements due to a 50 -tonne single vehicle and two 50-tonne successive vehicles with different spacing of 50,40 and $30 \mathrm{~m}$. The maximum displacements due to two successive vehicles are higher than those due to single vehicle, except at vehicle speeds of 10 and $15 \mathrm{~km} / \mathrm{h}$ where the maximum displacements due to two successive vehicles with a separation distance of $40 \mathrm{~m}$ are lower than those due to single vehicle. At higher vehicle speeds of 20 and $25 \mathrm{~km} / \mathrm{h}$, a separation distance of $40 \mathrm{~m}$ between the two successive vehicles results in the highest displacement. The maximum displacements for a separation distance of $30 \mathrm{~m}$ are lower than those for a separation distance of $50 \mathrm{~m}$ at vehicle speeds of 10 and $25 \mathrm{~km} / \mathrm{h}$; however they are higher than those for a separation distance of $50 \mathrm{~m}$ at vehicle speed of $20 \mathrm{~km} / \mathrm{hr}$. The same trends were 
observed for the maximum displacements due to 60 and 70 -tonne vehicles. It is seen from Figure 4.94 that the DAFs for displacement due to a 50-tonne single vehicle and two 50tonne successive vehicles with different spacing have the same trends as the maximum displacements. The same trends were also observed for the DAFs for displacement due to 60 and 70-tonne vehicles.

Figure 4.93 shows the maximum bending moments due to a 50 -tonne single vehicle and two 50-tonne successive vehicles with different spacing of 50,40 and $30 \mathrm{~m}$. The maximum bending moments in the bridge due to the passage of two successive vehicles are higher than those due to single vehicle. The maximum bending moments in the bridge due to two successive vehicles with separation distance of $30 \mathrm{~m}$ are higher those in the case of a separation distance of $50 \mathrm{~m}$, but lower than those in the case of a separation distance of $40 \mathrm{~m}$. The same trends are also observed for maximum bending moments due to 60 and 70 -tonne vehicles.

The DAFs for bending moment are presented in Figure 4.95 for a 50 -tonne single vehicle and two 50-tonne successive vehicles with different spacing of 50,40 and $30 \mathrm{~m}$. The DAFs for bending moment due to two successive vehicles are lower than those due to single vehicle and those DAFs decrease as the separation distance between the two successive vehicles deceases and the two vehicles get closer to each other. The same trends were observed for the DAFs for bending moment for 60 and 70-tonne vehicles.

For the hinge-connected bridge, the typical bridge bay has a length of $21 \mathrm{~m}$; those typical bays are hinge-connected to each other. This can explain the reason for having higher dynamic response of the hinge-connected bridge to two successive vehicle loads at a 
separation distance of $40 \mathrm{~m}$ which is almost twice the distance between two intermediate hinges in the bridge. This means that the two vehicle-loads can act over two hinges at the same time resulting in higher dynamic response of the bridge. The dynamic response of the bridge is better in case of separation distance of $50 \mathrm{~m}$ which is almost two and half of the distance between two intermediate hinges in the bridge. This means that when onevehicle loads act over a hinge, the other vehicle-loads act at the middle between two hinges and help in stabilizing the bridge resulting in lower dynamic response. The dynamic response of the bridge is better in case of a separation distance of $30 \mathrm{~m}$ which is almost one and half of the distance between two intermediate hinges in the bridge. This means that when one vehicle-loads act over a hinge, the other vehicle-loads act at the middle between two hinges closer to the first vehicle loads and help in stabilizing the bridge resulting in lower dynamic response. For the hinge-connected floating bridge subjected to the two successive vehicle-loads studied here, it was found better to avoid having the separation distance between the two vehicles such that the two vehicle-loads act over intermediate hinges at the same time otherwise the bridge will have a higher dynamic response. In general, the separation distance between the two successive vehicle-loads affects the phase angle between the two dynamic response waves induced by each of the two vehicle-loads and therefore affects the resultant dynamic response of the bridge due to these two response waves. However, it should be noted that each of these two response waves affects the other. 


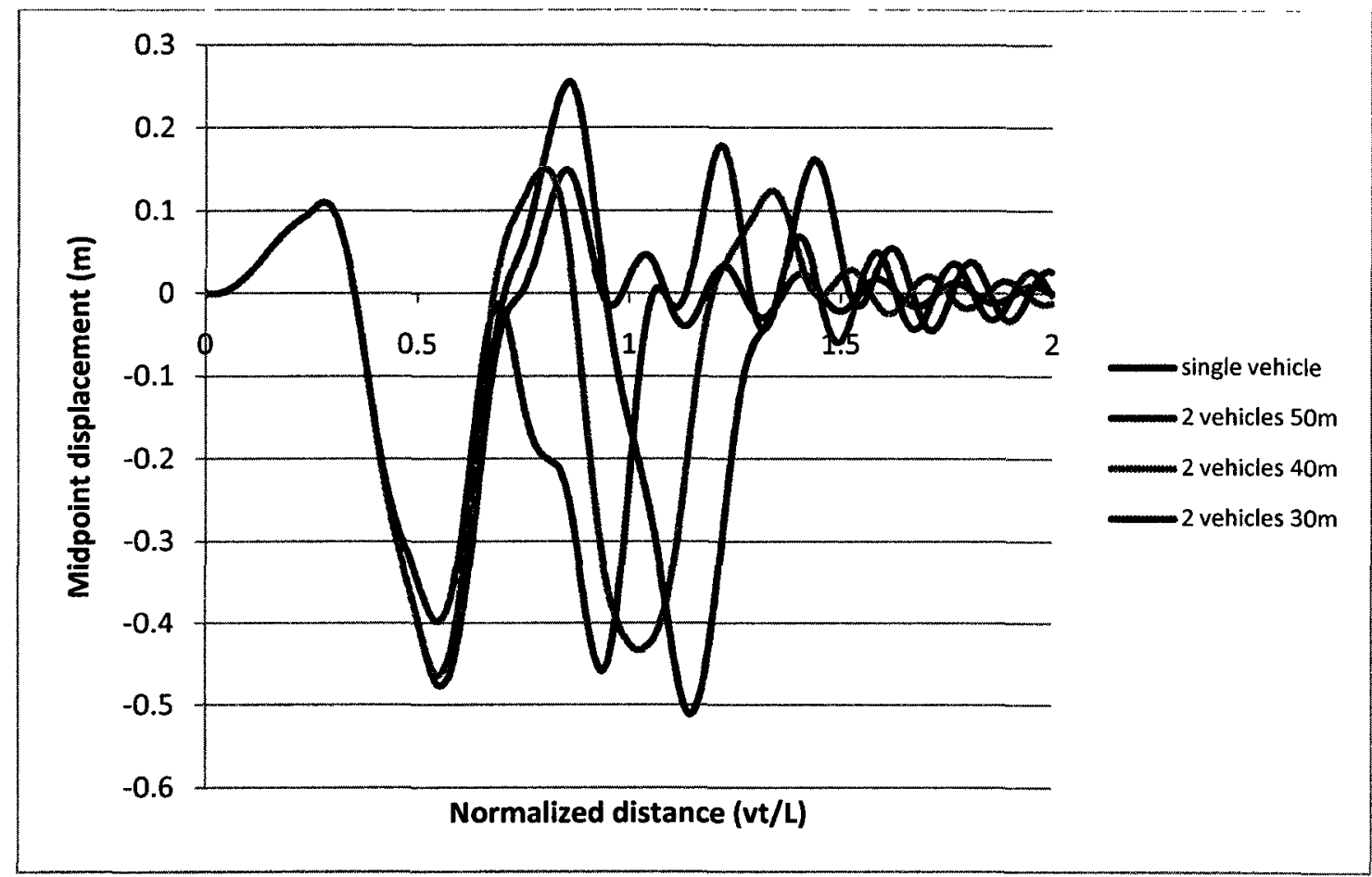

Figure 4.88: Time history of midpoint displacements in hinge-connected bridge due to two successive 50-tonne vehicle loads with different spacing $(\mathrm{v}=10 \mathrm{~km} / \mathrm{h})$

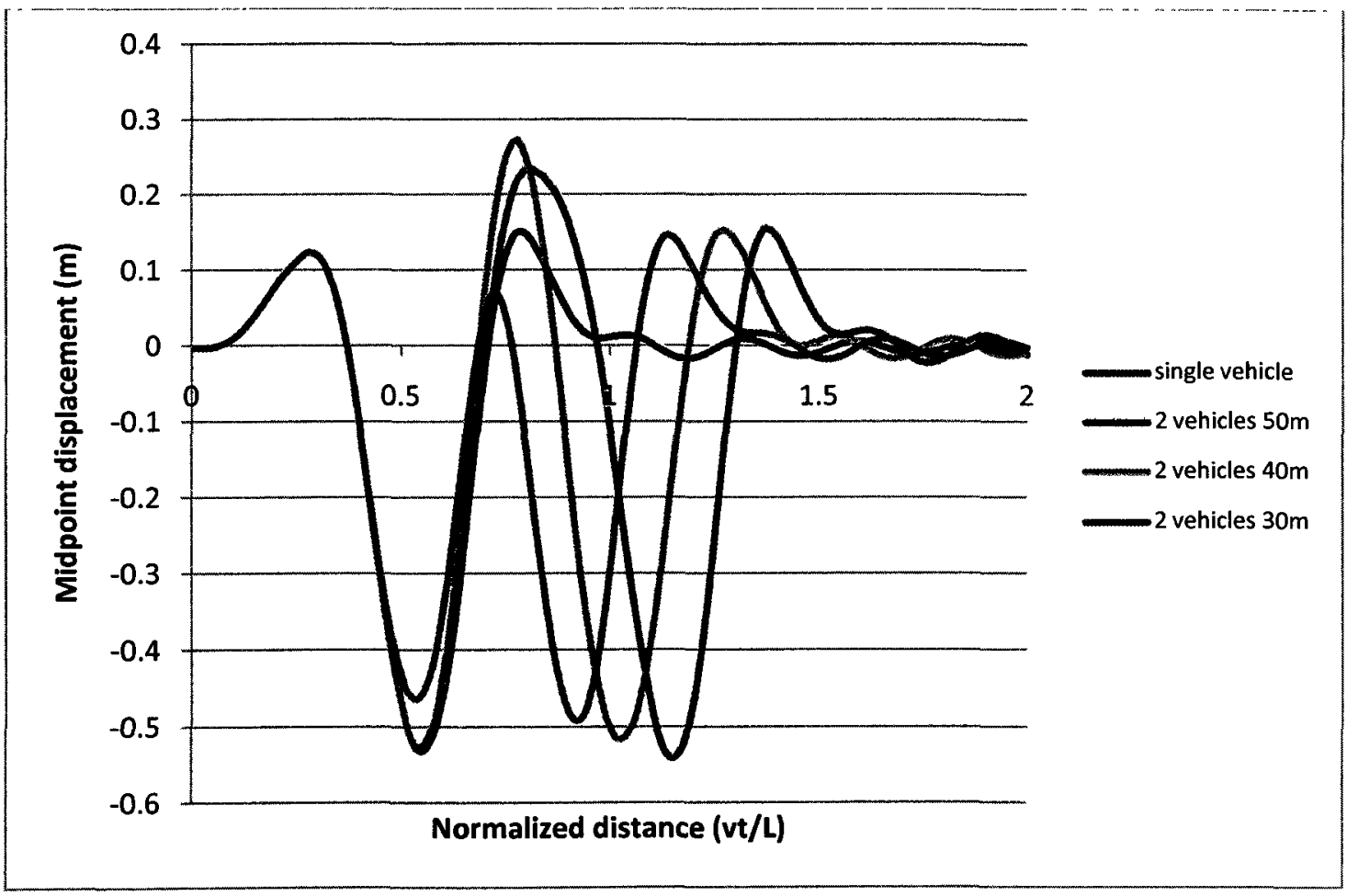

Figure 4.89: Time history of midpoint displacements in hinge-connected bridge due to two successive 50-tonne vehicle loads with different spacing $(\mathrm{v}=15 \mathrm{~km} / \mathrm{h})$ 


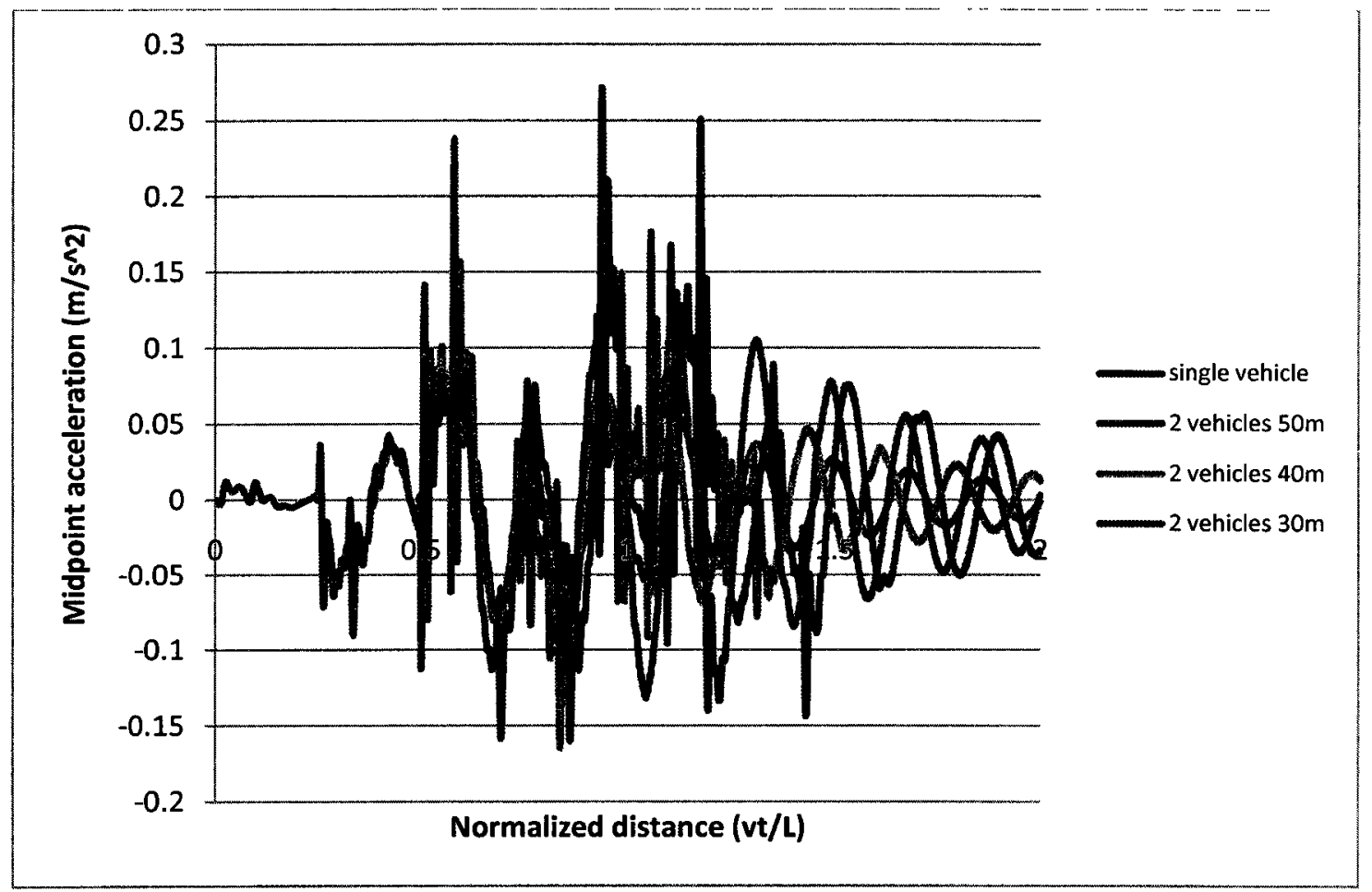

Figure 4.90: Time history of midpoint acceleration in hinge-connected bridge due to two successive 50-tonne vehicle loads with different spacing $(\mathrm{v}=10 \mathrm{~km} / \mathrm{h})$

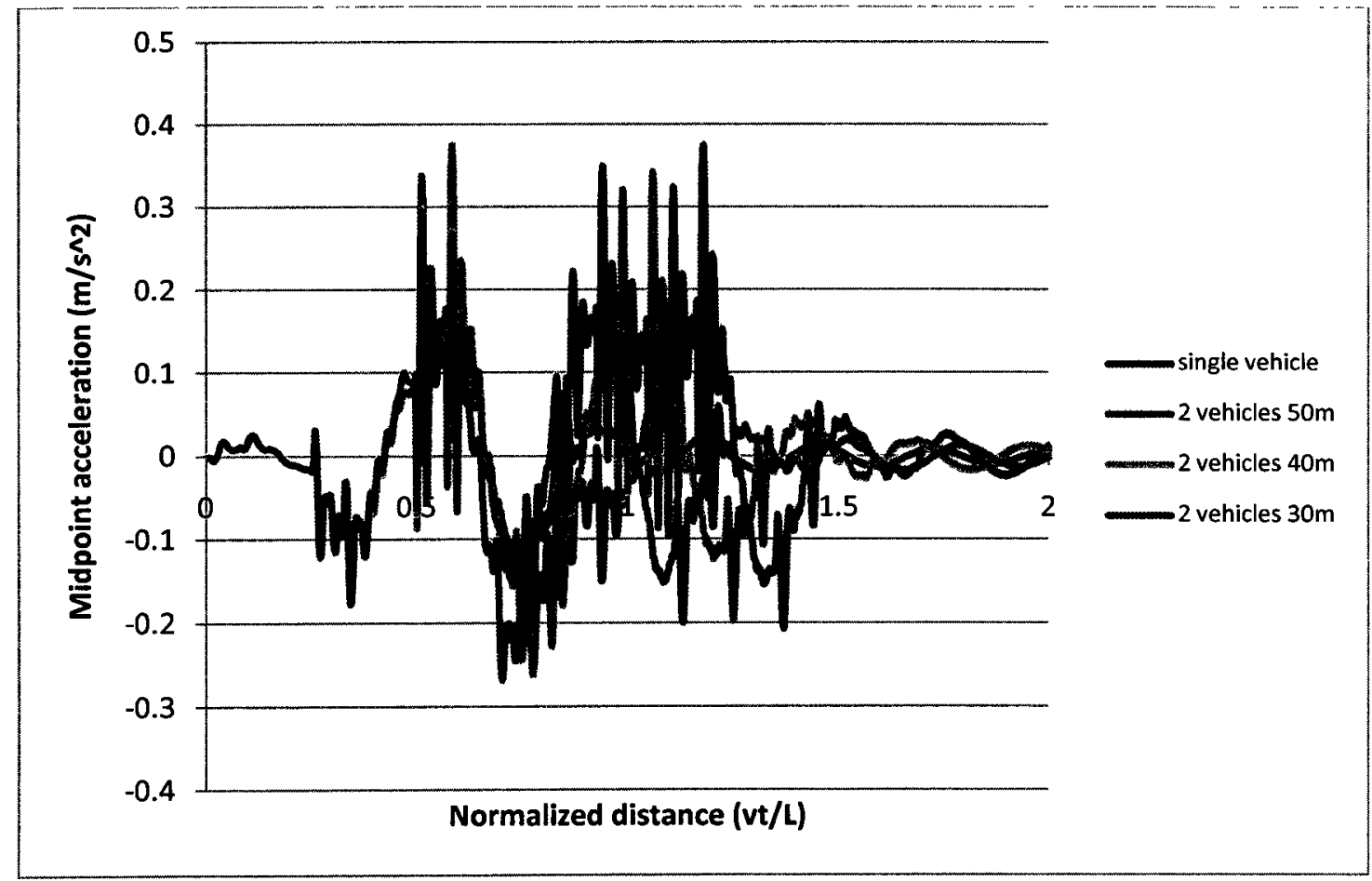

Figure 4.91: Time history of midpoint acceleration in hinge-connected bridge due to two successive 50-tonne vehicle loads with different spacing $(\mathrm{v}=15 \mathrm{~km} / \mathrm{h})$ 


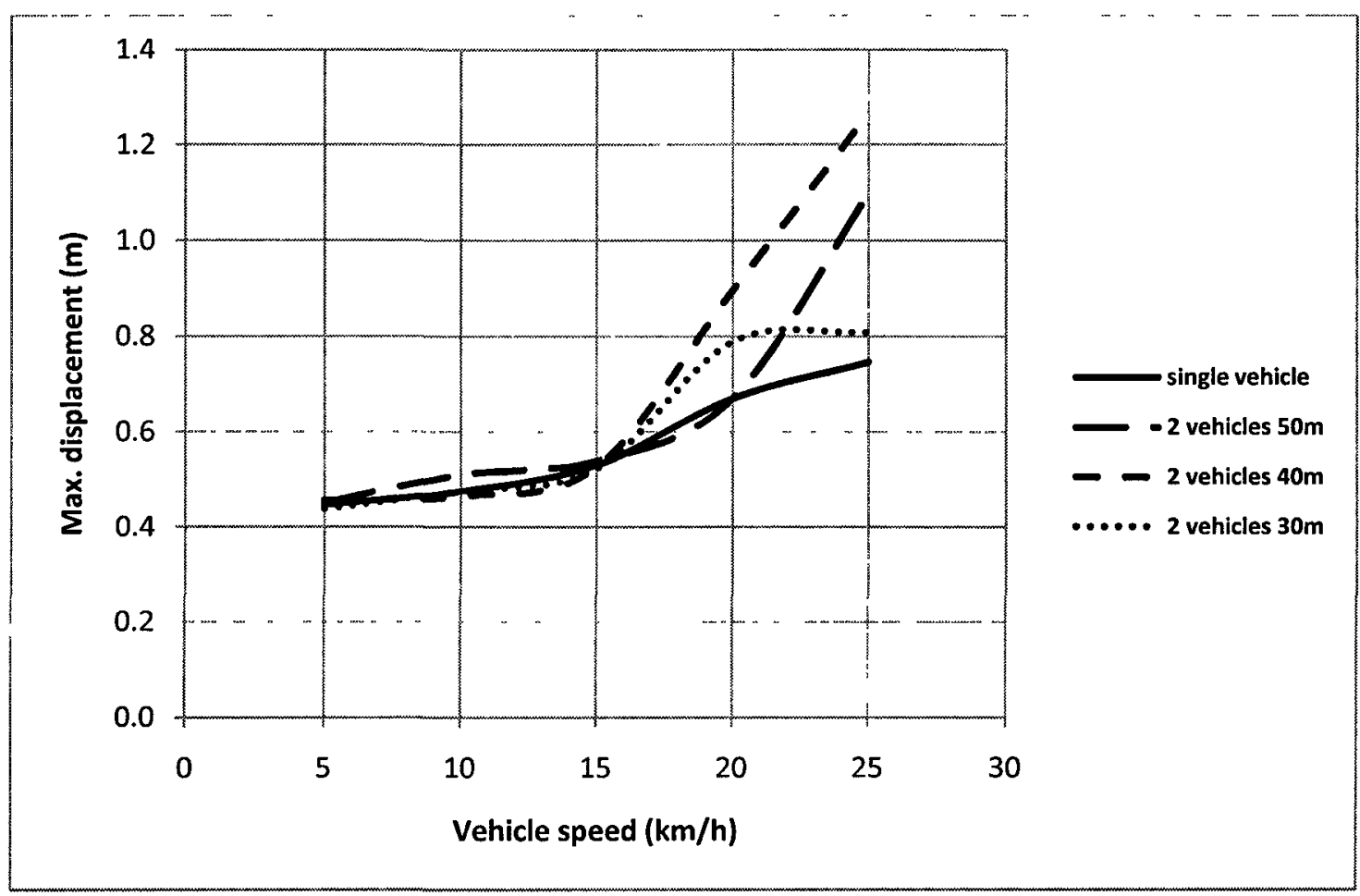

Figure 4.92: Maximum displacements in hinge-connected bridge due to 50-tonne vehicle loads

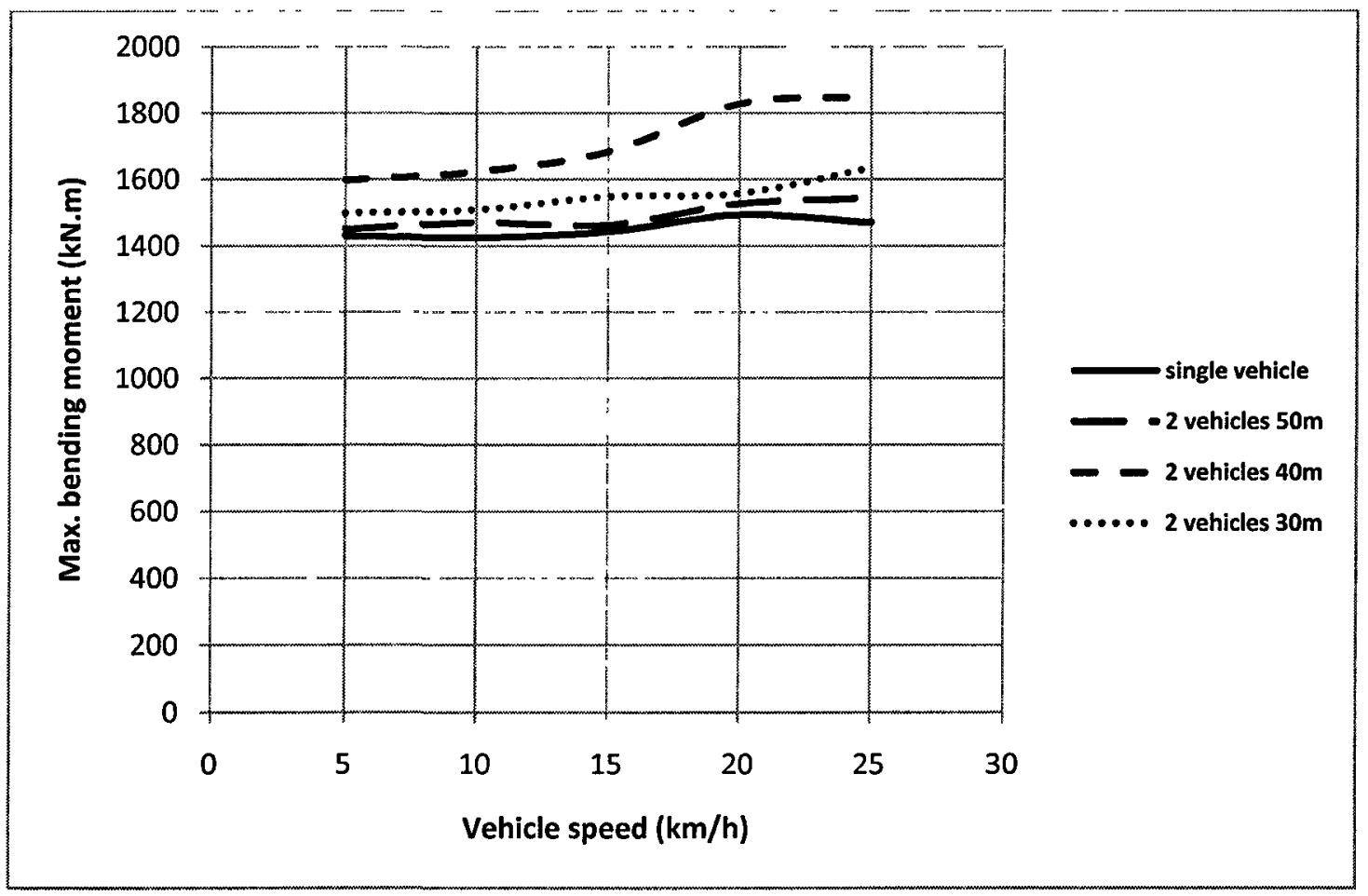

Figure 4.93: Maximum bending moments in hinge-connected bridge due to 50-tonne vehicle loads 


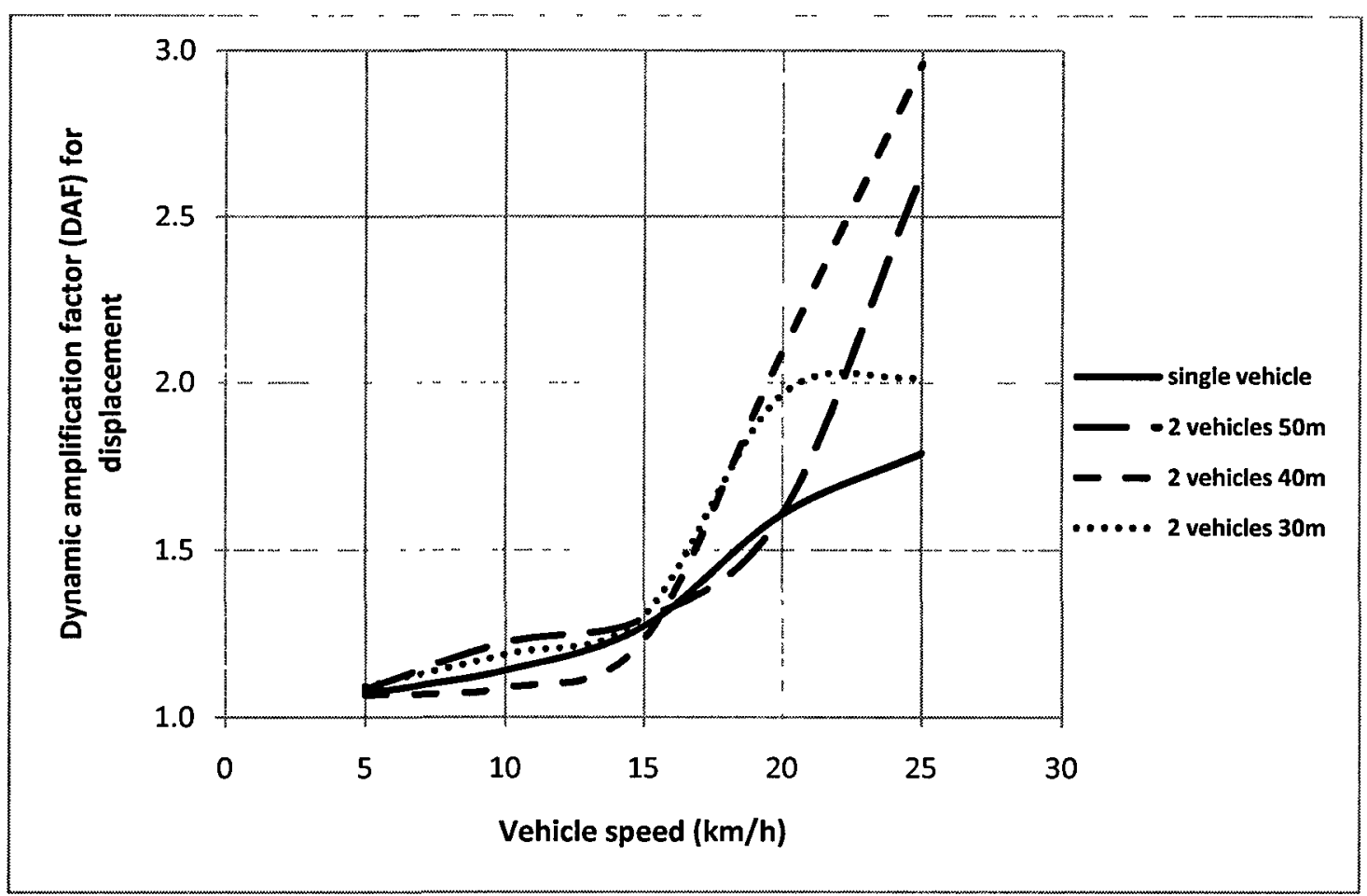

Figure 4.94: DAFs for displacement in hinge-connected bridge due to 50-tonne vehicle loads

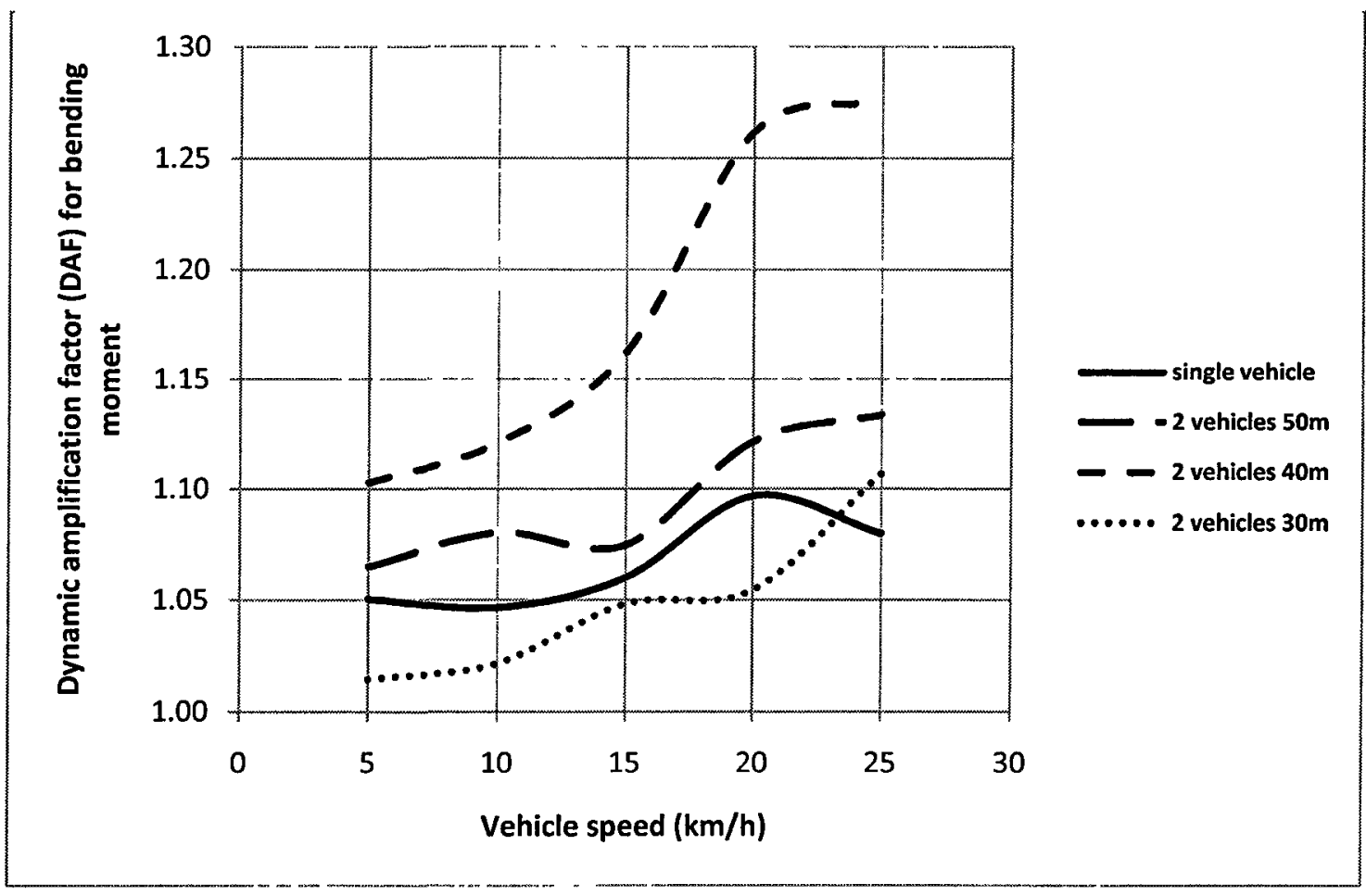

Figure 4.95: DAFs for bending moment in hinge-connected bridge due to 50-tonne vehicle loads 


\subsection{Parametric Study for the Three-Dimensional Interactive Dynamic Response of Floating Bridges to Moving Vehicle Loads and Dynamic Water Current Loads}

The effects of various parameters on the structural behaviour of floating bridges due to water current loads and supporting boat forces have been investigated. The parameters investigated include water current velocity, number of supporting boats and their locations and the magnitude of force each boat exerts on the bridge (see Appendix A for details).

In the present section, the three-dimensional interactive dynamic response of floating bridges to the combined actions of moving vehicle loads and dynamic water current loads along with possible eccentricities of these loads is investigated. The vehicle is represented by a two-axle model. Water current loads are represented as dynamic loads that changes with both time and space coordinates, and are incorporated into the dynamic equations of vehicle-bridge-fluid system. At each time step, water current loads are calculated based on the actual dynamic response of the bridge profile and updated in the global load vector for the next time step. No supporting boats are considered in the present analyses against water current loads. The vertical and lateral responses of the bridge are uncoupled, due to the formulation of the stiffness and mass matrices of the space frame element. Except that the lateral motion of the bridge is affected by the change in water current forces with a change in the submerged depth due the passage of vehicle loads on the bridge. The three-dimensional dynamic response (displacement and acceleration) and internal forces in the bridge due to moving vehicle loads and dynamic 
water current loads are calculated at each position of the vehicle while traversing the bridge, and the maximum values of the displacements and internal forces are determined.

\subsubsection{Three-Dimensional Dynamic Response of Rigid-Connected Floating Bridge to Eccentric Two-Axle Moving Vehicle Loads and Dynamic Water Current Loads}

The three-dimensional dynamic response of $84 \mathrm{~m}$ rigid-connected floating bridge is investigated due to 50-tonne two-axle vehicle loads moving at different speeds varying from $5 \mathrm{~km} / \mathrm{h}$ to $30 \mathrm{~km} / \mathrm{h}$ and dynamic water current loads with two different current velocities: 1 and $2 \mathrm{~m} / \mathrm{s}(3.6-7.2 \mathrm{~km} / \mathrm{h})$. The vehicle is traveling the bridge with a horizontal eccentricity of $0.3 \mathrm{~m}$ with respect to the bridge longitudinal axis. The details of the analyses and the results are summarized in Tables 4.36 and 4.37. The results obtained for the complete three-dimensional structural behaviour of the bridge are shown in Figures 4.96 to 4.100 .

Table 4.36: 3-D dynamic response of rigid-connected bridge to 50-tonne two-axle eccentric vehicle loads and water current loads with current velocity $1 \mathrm{~m} / \mathrm{s}$

\begin{tabular}{|c|c|c|c|c|c|c|c|c|}
\hline $\begin{array}{c}\text { Vehicle } \\
\text { weight } \\
(\mathbf{t})\end{array}$ & $\begin{array}{c}\text { Vehicle } \\
\text { eccent. } \\
(\mathbf{m})\end{array}$ & $\begin{array}{c}\text { Vehicle } \\
\text { speed } \\
(\mathbf{k m} / \mathbf{h})\end{array}$ & $\begin{array}{c}\text { Water } \\
\text { current } \\
\text { velocity } \\
(\mathbf{m} / \mathbf{s})\end{array}$ & $\begin{array}{c}\text { Max ver. } \\
\mathbf{d i s p l .} \\
(\mathbf{m})\end{array}$ & $\begin{array}{c}\text { Max lat. } \\
\text { displ. } \\
(\mathbf{m})\end{array}$ & $\begin{array}{c}\text { Max } \\
\text { B.M. } \\
(\mathbf{K N} . \mathbf{m})\end{array}$ & $\begin{array}{c}\text { Max lat. } \\
\text { B.M. } \\
(\text { KN.m) }\end{array}$ & $\begin{array}{c}\text { Max } \\
\text { torsional } \\
\text { moment } \\
(\mathbf{K N} . \mathbf{m})\end{array}$ \\
\hline 50 & 0.3 & 5 & 1 & -0.161 & -0.004 & -2322 & -59.7 & 140.6 \\
\hline 50 & 0.3 & 10 & 1 & -0.162 & -0.005 & -2345 & -60.2 & 140.3 \\
\hline 50 & 0.3 & 15 & 1 & -0.169 & -0.005 & -2384 & -61.4 & 140.2 \\
\hline 50 & 0.3 & 20 & 1 & -0.178 & -0.005 & -2514 & -61.4 & 139.6 \\
\hline 50 & 0.3 & 25 & 1 & -0.188 & -0.005 & -2618 & -64.4 & 139.3 \\
\hline 50 & 0.3 & 30 & 1 & -0.203 & -0.005 & -2756 & -69.2 & 138.9 \\
\hline
\end{tabular}


Table 4.37: 3-D dynamic response of rigid-connected bridge to 50-tonne two-axle eccentric vehicle loads and water current loads with current velocity $2 \mathrm{~m} / \mathrm{s}$

\begin{tabular}{|c|c|c|c|c|c|c|c|c|}
\hline $\begin{array}{c}\text { Vehicle } \\
\text { weight }\end{array}$ & $\begin{array}{c}\text { Vehicle } \\
\text { eccent. }\end{array}$ & $\begin{array}{c}\text { Vehicle } \\
\text { speed } \\
(\mathbf{t})\end{array}$ & $\begin{array}{c}\text { Water } \\
\text { current } \\
\text { velocity } \\
(\mathbf{k m} / \mathbf{h})\end{array}$ & $\begin{array}{c}\text { Max ver. } \\
\text { displ. }\end{array}$ & $\begin{array}{c}\text { Max lat. } \\
\text { displ. }\end{array}$ & $\begin{array}{c}\text { Max } \\
\text { B.M. }\end{array}$ & $\begin{array}{c}\text { Max lat. } \\
\text { B.M. }\end{array}$ & $\begin{array}{c}\text { Max } \\
\text { torsional } \\
\text { moment } \\
(\mathbf{K N} . \mathbf{m})\end{array}$ \\
\hline 50 & 0.3 & 5 & 2 & -0.161 & -0.018 & -2322 & -239.0 & 142.9 \\
\hline 50 & 0.3 & 10 & 2 & -0.162 & -0.018 & -2345 & -240.7 & 142.6 \\
\hline 50 & 0.3 & 15 & 2 & -0.169 & -0.018 & -2384 & -245.7 & 142.6 \\
\hline 50 & 0.3 & 20 & 2 & -0.178 & -0.018 & -2514 & -245.5 & 142.1 \\
\hline 50 & 0.3 & 25 & 2 & -0.188 & -0.019 & -2618 & -257.4 & 141.6 \\
\hline 50 & 0.3 & 30 & 2 & -0.203 & -0.021 & -2756 & -276.7 & 141.5 \\
\hline
\end{tabular}

\subsubsection{Discussion of Results}

Figure 4.96 shows the maximum vertical displacement in the bridge due to 50 -tonne twoaxle eccentric vehicle loads, where the maximum vertical displacement increases with vehicle speed. Also, the maximum vertical bending moment developed in the bridge increases with vehicle speed as shown in Figure 4.97.

Figure 4.98 shows the maximum lateral displacement in the bridge due to water current loads with current velocity of $1 \mathrm{~m} / \mathrm{s}$. The maximum lateral displacement in the bridge increases with vehicle speed and/or water current velocity. The same trend is observed for maximum lateral bending moment developed in the bridge as shown in Figure 4.99. While the maximum torsional moment about the longitudinal axis of the bridge is almost constant with vehicle speed as shown in Figure 4.100. 


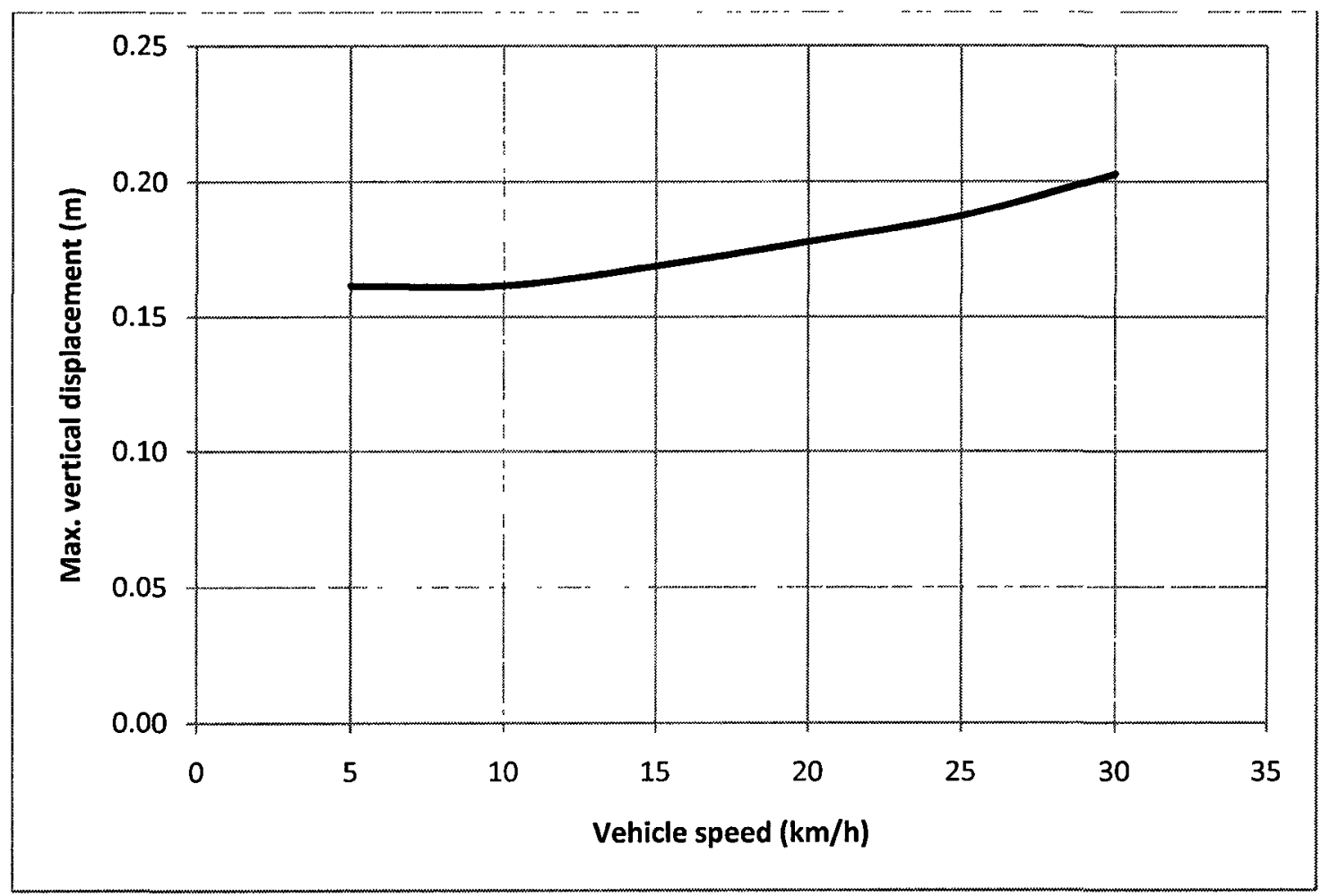

Figure 4.96: Maximum vertical displacements in rigid-connected bridge

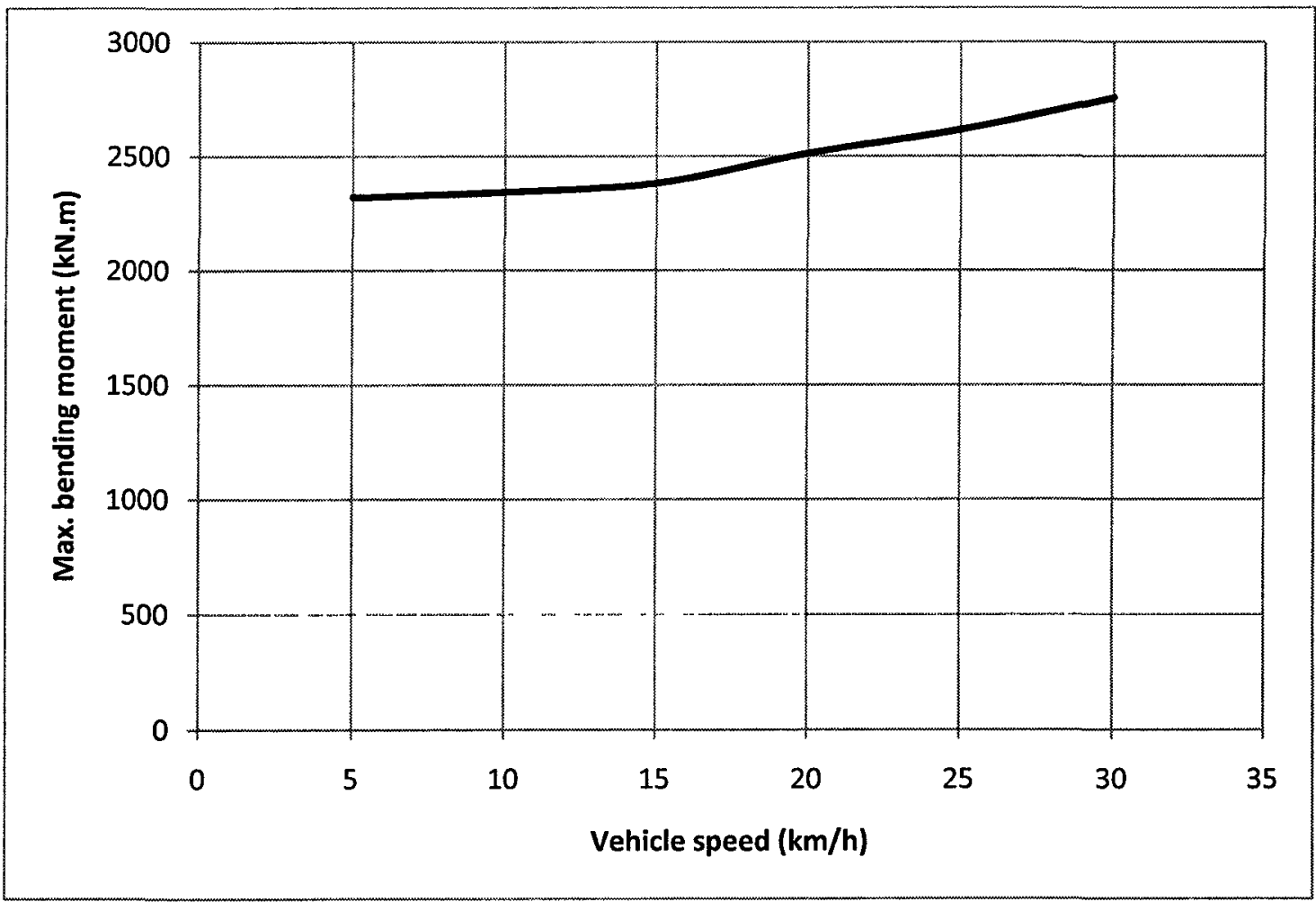

Figure 4.97: Maximum bending moments in rigid-connected bridge 


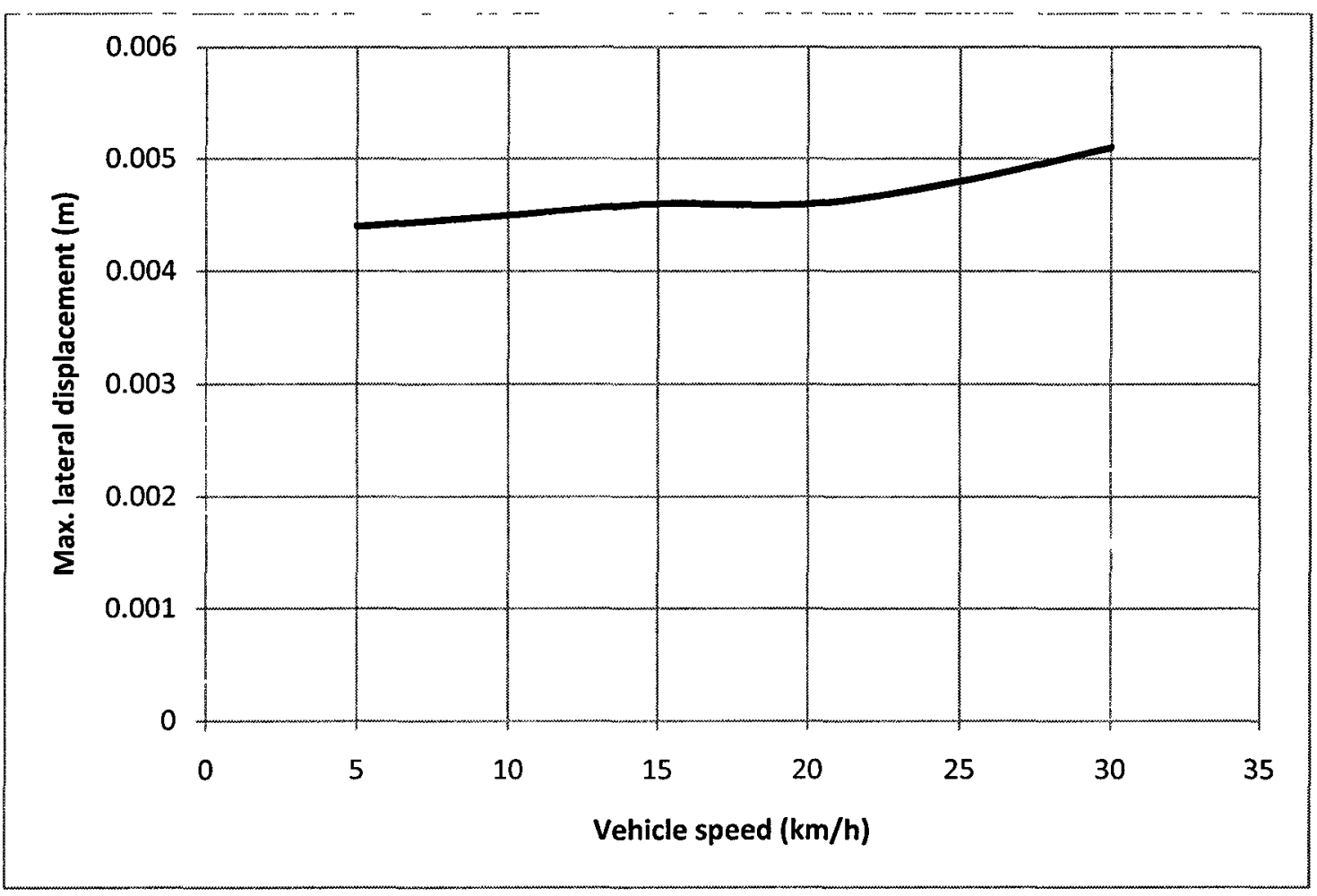

Figure 4.98: Maximum lateral displacements in rigid-connected bridge (current velocity 1 $\mathrm{m} / \mathrm{s})$

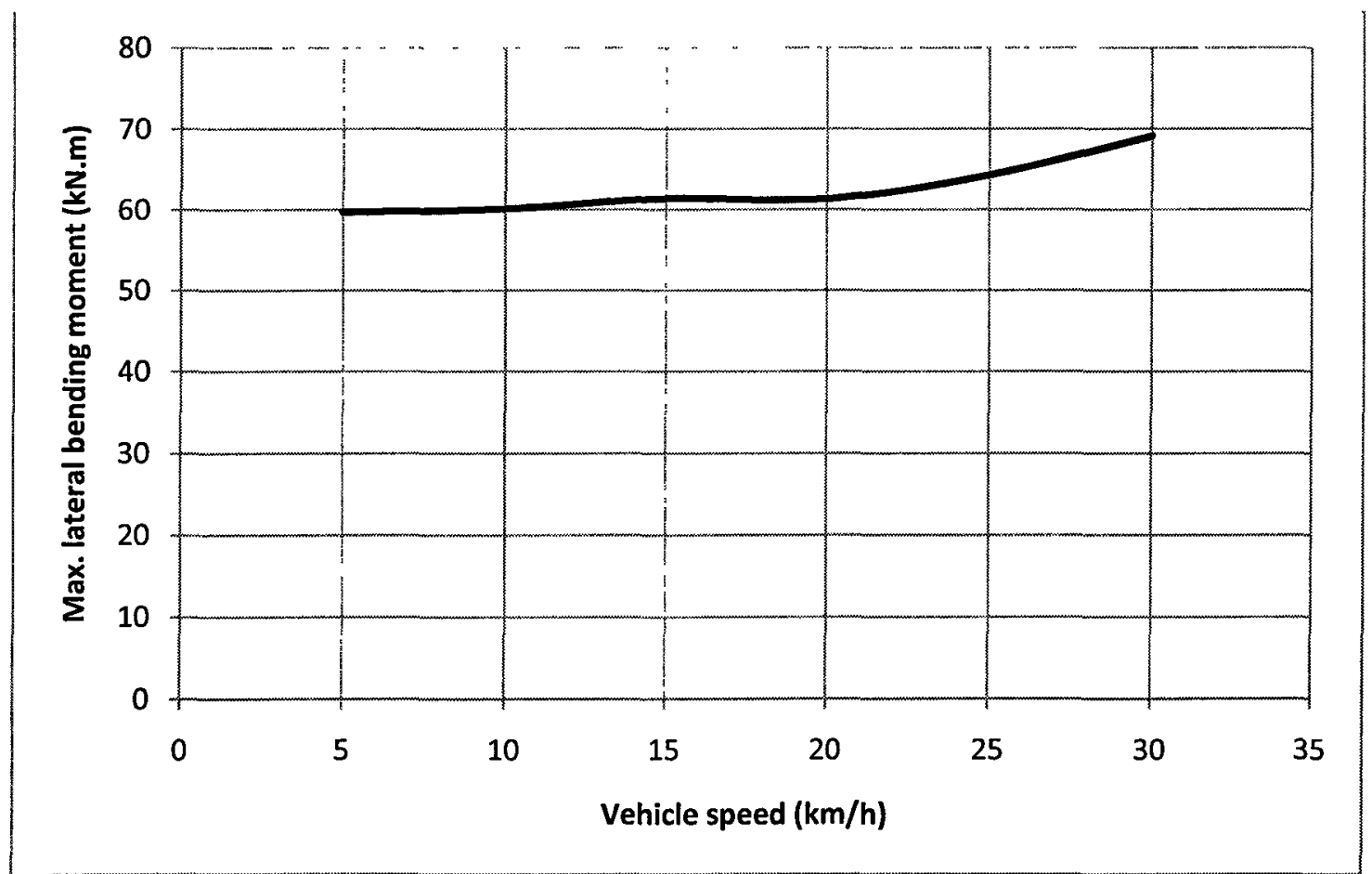

Figure 4.99: Maximum lateral bending moments in rigid-connected bridge (current velocity $1 \mathrm{~m} / \mathrm{s}$ ) 


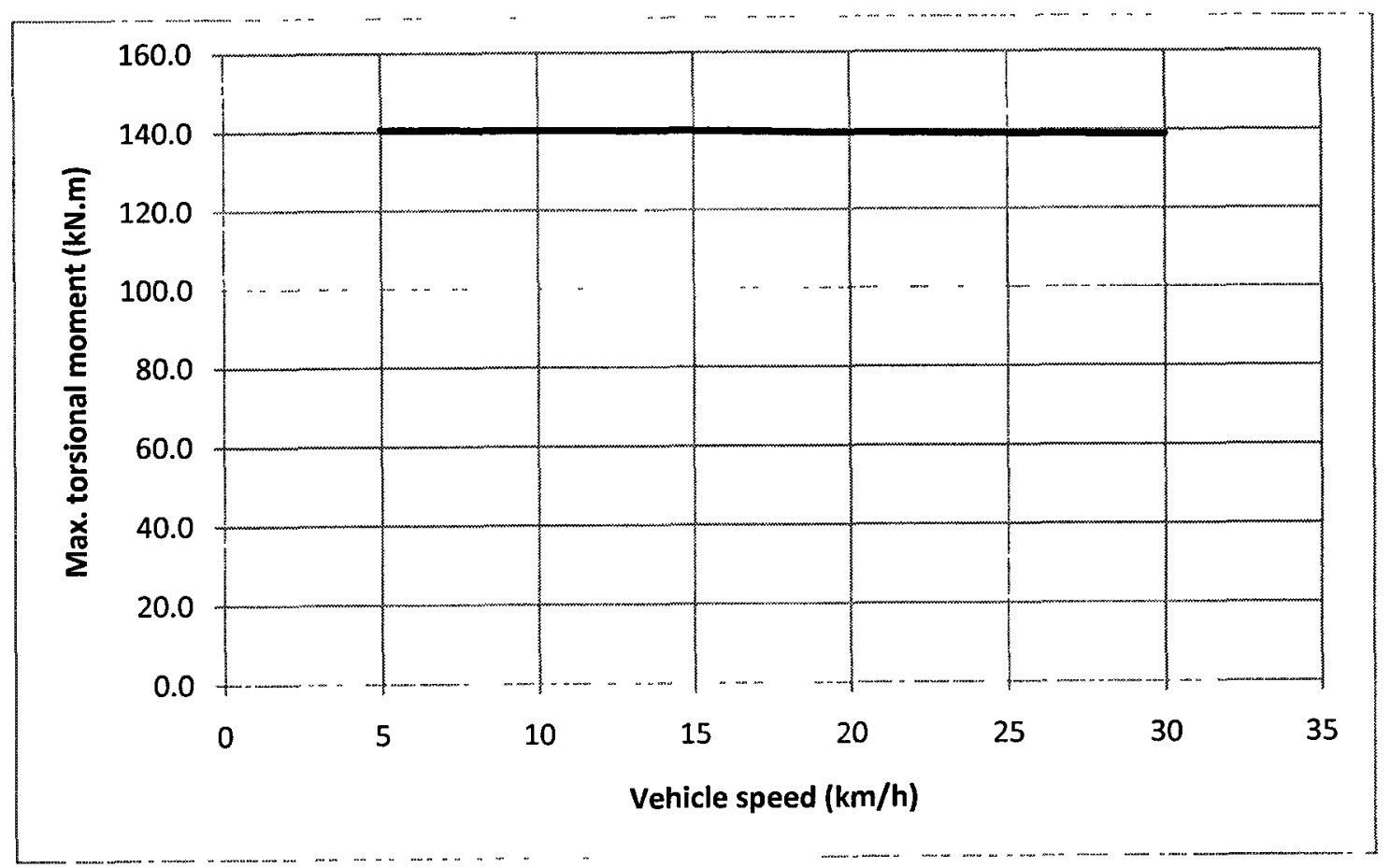

Figure 4.100: Maximum torsional moments in rigid-connected bridge (current velocity 1 $\mathrm{m} / \mathrm{s}$ )

On comparing the maximum lateral displacements and bending moments from tables 4.38 and 4.39 for water current velocities of $1 \mathrm{~m} / \mathrm{s}$ and $2 \mathrm{~m} / \mathrm{s}$, respectively, it is seen that the lateral response of the bridge due to water current loads is proportional to the square of the current velocity, as expected.

\subsubsection{Three-Dimensional Dynamic Response of Hinge-Connected Floating Bridge to Eccentric Two-Axle Moving Vehicle Loads and Dynamic Water Current Loads}

The three-dimensional dynamic response of $84 \mathrm{~m}$ hinge-connected floating bridge is investigated due to 50-tonne two-axle vehicle loads moving at different speeds varying from $5 \mathrm{~km} / \mathrm{h}$ to $30 \mathrm{~km} / \mathrm{h}$ and dynamic water current loads with two different current velocities: 1 and $2 \mathrm{~m} / \mathrm{s}(3.6-7.2 \mathrm{~km} / \mathrm{h})$. The vehicle is traveling the bridge with a horizontal eccentricity $0.3 \mathrm{~m}$ with respect to the longitudinal centre line of the bridge. 
The details of the analyses and the results are summarized in Tables 4.38 and 4.39. The results obtained are shown in Figures 4.101 to 4.105.

Table 4.38: 3-D dynamic response of hinge-connected bridge to 50-tonne two-axle eccentric vehicle loads and water current loads with current velocity $1 \mathrm{~m} / \mathrm{s}$

\begin{tabular}{|c|c|c|c|c|c|c|c|c|}
\hline Span & $\begin{array}{c}\text { Vehicle } \\
\text { weight }\end{array}$ & $\begin{array}{c}\text { Vehicle } \\
\text { speed } \\
(\mathbf{m})\end{array}$ & $\begin{array}{c}\text { Water } \\
\text { current } \\
\text { velocity }\end{array}$ & $\begin{array}{c}\text { Max ver. } \\
\text { displ. } \\
(\mathbf{k m} / \mathbf{h})\end{array}$ & $\begin{array}{c}\text { Max lat. } \\
\text { displ. }\end{array}$ & $\begin{array}{c}\text { Max } \\
\text { B.M. }\end{array}$ & $\begin{array}{c}\text { Max lat. } \\
\text { B.M. }\end{array}$ & $\begin{array}{c}\text { Max } \\
\text { torsional } \\
\text { moment } \\
\text { (KN.m) }\end{array}$ \\
\hline 84 & 50 & 5 & 1 & -0.447 & -0.005 & -1430 & -69.4 & 140.5 \\
\hline 84 & 50 & 10 & 1 & -0.476 & -0.005 & -1425 & -71.2 & 140.6 \\
\hline 84 & 50 & 15 & 1 & -0.531 & -0.005 & -1443 & -73.9 & 139.3 \\
\hline 84 & 50 & 20 & 1 & -0.671 & -0.006 & -1493 & -80.8 & 142.4 \\
\hline 84 & 50 & 25 & 1 & -0.747 & -0.006 & -1470 & -87.6 & 147.3 \\
\hline 84 & 50 & 30 & 1 & -0.762 & -0.006 & -1554 & -90.9 & 146.9 \\
\hline
\end{tabular}

Table 4.39: 3-D dynamic response of hinge-connected bridge to 50-tonne two-axle eccentric vehicle loads and water current loads with current velocity $2 \mathrm{~m} / \mathrm{s}$

\begin{tabular}{|c|c|c|c|c|c|c|c|c|}
\hline $\begin{array}{l}\text { Span } \\
\text { (m) }\end{array}$ & $\begin{array}{c}\begin{array}{c}\text { Vehicle } \\
\text { weight }\end{array} \\
\text { (t) } \\
\end{array}$ & $\begin{array}{l}\text { Vehicle } \\
\text { speed } \\
(\mathrm{km} / \mathrm{h}) \\
\end{array}$ & $\begin{array}{l}\text { Water } \\
\text { current } \\
\text { velocity } \\
(\mathrm{m} / \mathrm{s}) \\
\end{array}$ & $\begin{array}{c}\text { Max ver. } \\
\text { displ. } \\
(\mathrm{m})\end{array}$ & $\begin{array}{c}\text { Max lat. } \\
\text { displ. } \\
\text { (m) }\end{array}$ & $\begin{array}{c}\text { Max } \\
\text { B.M. } \\
\text { (KN.m) } \\
\end{array}$ & $\begin{array}{l}\text { Max lat. } \\
\text { B.M. } \\
\text { (KN.m) }\end{array}$ & $\begin{array}{c}\text { Max } \\
\text { torsional } \\
\text { moment } \\
(\text { KN.m) }\end{array}$ \\
\hline 84 & 50 & 5 & 2 & -0.447 & -0.020 & -1430 & -277.8 & 142.8 \\
\hline 84 & 50 & 10 & 2 & -0.476 & -0.020 & -1425 & -284.7 & 143.0 \\
\hline 84 & 50 & 15 & 2 & -0.531 & -0.021 & -1443 & -295.7 & 141.4 \\
\hline 84 & 50 & 20 & 2 & -0.671 & -0.022 & -1493 & -323.0 & 145.4 \\
\hline 84 & 50 & 25 & 2 & -0.747 & -0.024 & -1470 & -350.4 & 150.5 \\
\hline 84 & 50 & 30 & 2 & -0.762 & -0.025 & -1554 & -363.5 & 149.8 \\
\hline
\end{tabular}




\subsubsection{Discussion of Results}

Figure 4.101 shows the maximum vertical displacement in the bridge due to 50 -tonne two-axle eccentric vehicle loads. It is seen that the maximum vertical displacement increases with vehicle speed. Also, the maximum vertical bending moment developed in the bridge increases with vehicle speed as shown in Figure 4.102. Figure 4.103 shows the maximum lateral displacement in the bridge due to water current loads with current velocity of $1 \mathrm{~m} / \mathrm{s}$. The maximum lateral displacement in the bridge increases with vehicle speed and/or water current velocity. The same trend is observed for the maximum lateral bending moment developed in the bridge as shown in Figure 4.104. As shown in Figure 4.105, the maximum torsional moment about the longitudinal axis of the bridge is almost constant up to a speed of $20 \mathrm{~km} / \mathrm{h}$, and then increases with vehicle speed from $20 \mathrm{~km} / \mathrm{h}$ and up.

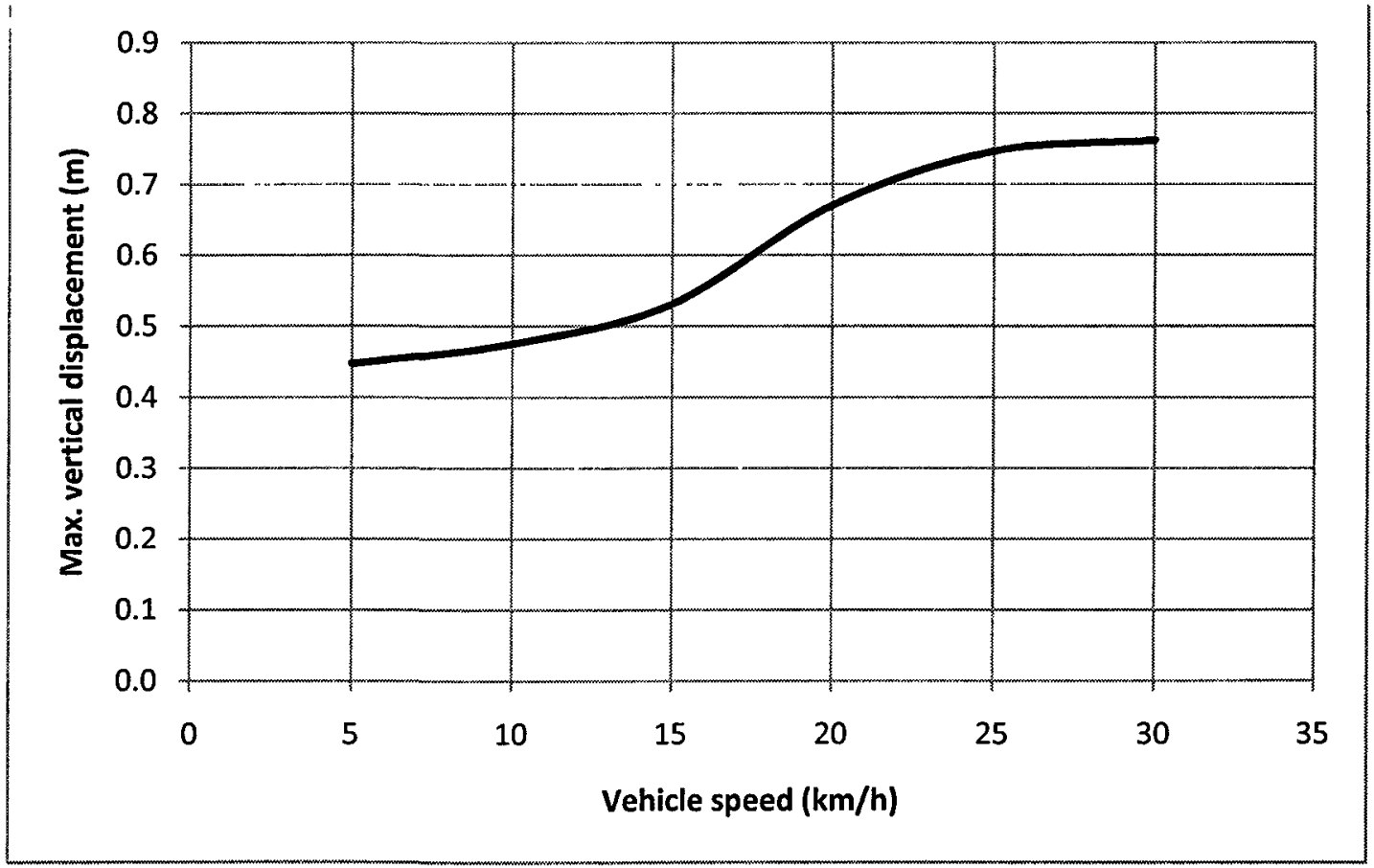

Figure 4.101: Maximum vertical displacements in hinge-connected bridge 


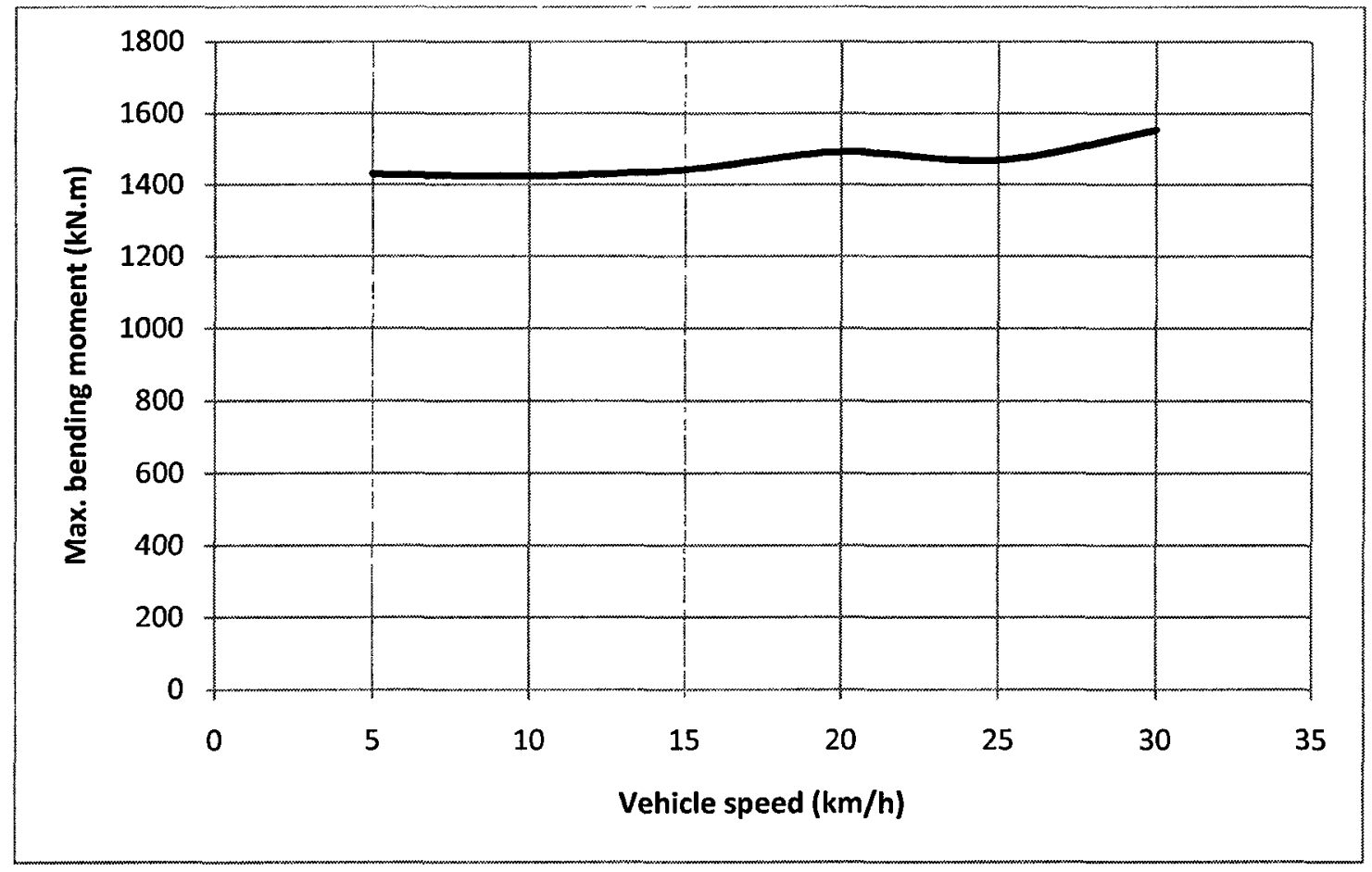

Figure 4.102: Maximum bending moments in hinge-connected bridge

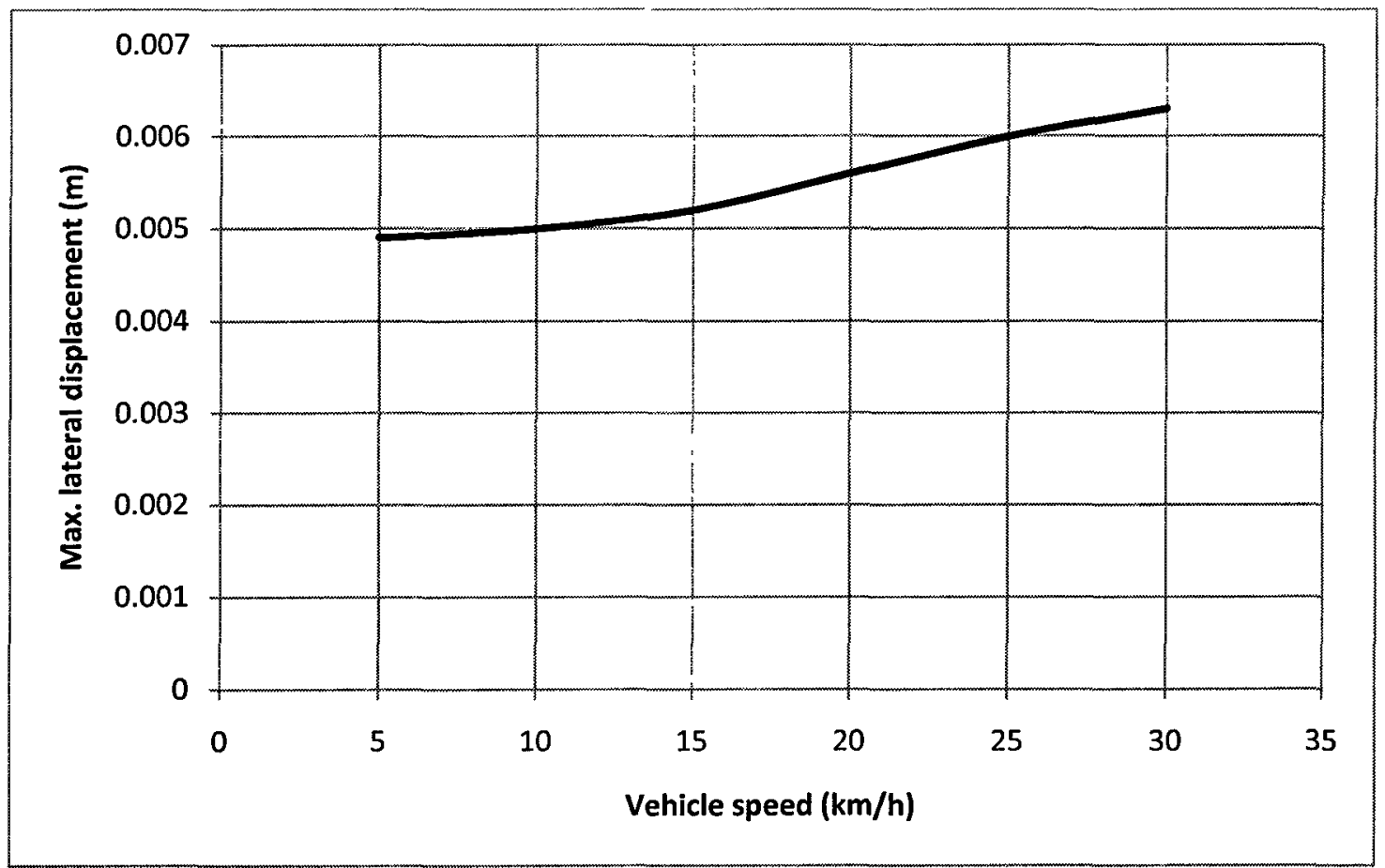

Figure 4.103: Maximum lateral displacements in hinge-connected bridge (current velocity $1 \mathrm{~m} / \mathrm{s}$ ) 


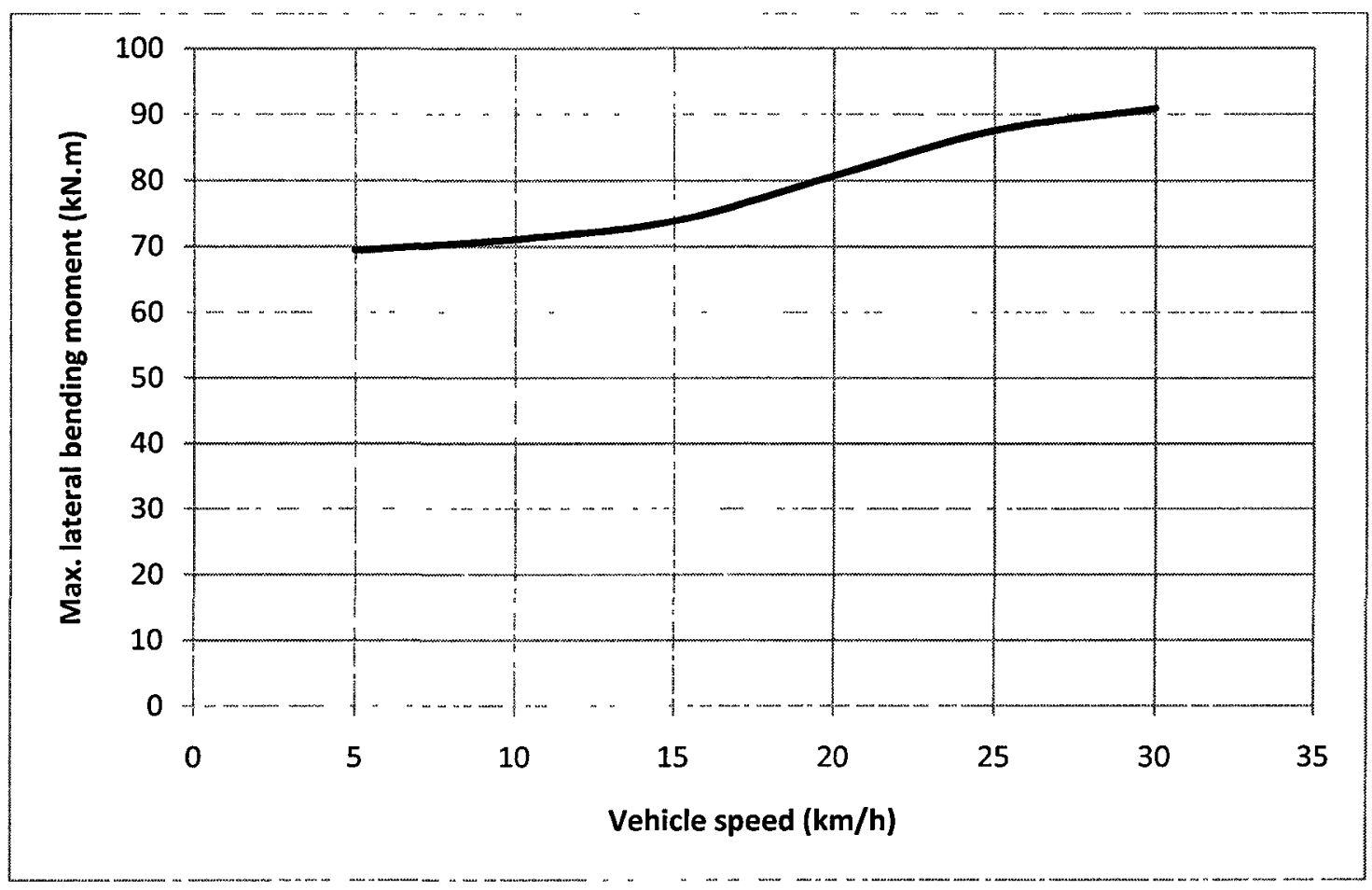

Figure 4.104: Maximum lateral bending moment in hinge-connected bridge (current velocity $1 \mathrm{~m} / \mathrm{s}$ )

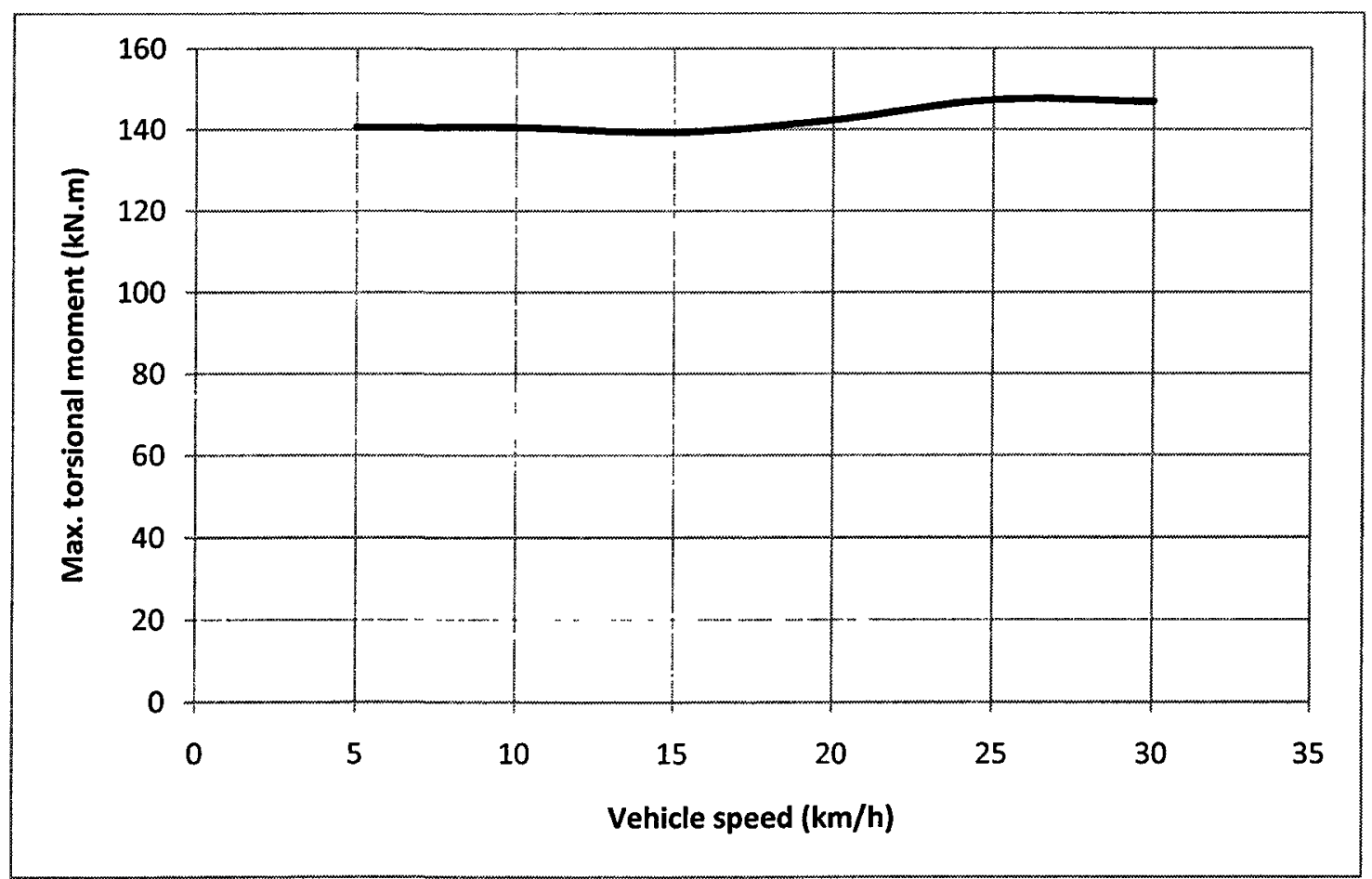

Figure 4.105: Maximum torsional moment in hinge-connected bridge (current velocity 1 $\mathrm{m} / \mathrm{s})$ 


\subsection{Summary}

In this chapter, the dynamic response of rigid-connected and hinge-connected rapid deployment floating bridges to moving vehicle loads was investigated considering different vehicle weights and speeds. An analytical technique for calculating the response of a vehicle-bridge-fluid system was developed and implemented into a finite element program for the dynamic analysis of floating bridges under moving vehicle loads. The dynamic response of floating bridges increases with increasing vehicle weight and/or speed, however increasing the vehicle speed has a stronger effect on the increase in the dynamic response of the bridge. The dynamic response of floating bridges to single-axle vehicle loads was compared to the corresponding response to two-axle vehicle loads. A vehicle with two axles represents a more accurate model of the vehicle, especially in the case of hinge-connected floating bridges. The effect of the vehicle eccentricity on the bridge dynamic response was also investigated. The dynamic response of light-weight floating bridges to moving vehicle loads was also investigated and shows that lightweight materials can be utilized to improve the dynamic behaviour of floating bridges, especially in the case of hinge-connected bridges.

The transport capacity of rapid deployment floating bridges was investigated in terms of the parameters controlling the rate of flow of vehicles on the bridge, namely; the vehicle weight, the vehicle speed and the separation distances between successive vehicles. The dynamic response of rigid-connected and hinge-connected floating bridges to successive vehicles was investigated considering different vehicle weights, speeds and separation 
distances. This can be useful in optimizing the transport capacity of floating bridges to support a certain group of vehicles.

Three-dimensional interactive analysis for the dynamic behaviour of floating bridges due to the combined actions of moving vehicle loads and dynamic water current loads along with possible eccentricities of these loads was investigated. Water current loads were treated as dynamic loads that change with both time and space coordinates, and were incorporated into the dynamic equations of vehicle-bridge-fluid system. At each time step, water current loads were calculated based on the actual dynamic response of the bridge profile, and updated in the global load vector for the next time step to accurately investigate the three-dimensional interactive dynamic behaviour of floating bridges.

The dynamic response of floating bridges to moving vehicle loads is governed by several parameters including the dynamic characteristics of the bridge and the vehicle. Among the most important parameters are the stiffness properties and structural material of the bridge, type of connection between successive pontoons determining the bridge type whether its rigid-connected or hinge-connected floating bridge, vehicle weight, vehicle speed, stiffness and damping coefficients of the vehicle, number of axles of the vehicle and the axle spacing, vehicle eccentricity with respect to the longitudinal axis of the bridge, the number of vehicles traversing the bridge at the same time, and the separation distance between them. 


\section{Chapter: Verification of the Developed Analytical Approach and the Developed Finite Element Program}

In order to demonstrate the capability and reliability of the developed procedure in dealing with vehicle-bridge interaction and determining the dynamic response of the bridge to moving vehicles, some typical examples were studied. The dynamic response of the bridge to moving vehicle loads is determined using the finite element program developed by the author and compared to the results available in the literature.

\subsection{Simply Supported Bridge Subjected to Moving Vehicle Loads}

A simply supported bridge subjected to moving vehicle loads is shown in Figure 5.1. The bridge is represented by a beam model and the vehicle is represented by a sprung mass system, where the sprung mass $m_{v}$ is supported by a dashpot-spring unit of spring constant $k$ and damping coefficient $c$. The sprung mass is further supported by an unsprung mass $m_{t}$. The following data are assumed: Young's modulus $E=2.87$ * $10^{6} \mathrm{kN} / \mathrm{m}^{2}$, Poisson's ratio $v=0.2$, moment of inertia $I=2.9 \mathrm{~m}^{4}$, mass per unit length $m=2303 \mathrm{~kg} / \mathrm{m}$, length of bridge $L=25 \mathrm{~m}$, sprung mass $m_{v}=5750 \mathrm{~kg}$, suspension

spring constant $k_{v}=1595 \mathrm{kN} / \mathrm{m}$, vehicle speed $v=27.78 \mathrm{~m} / \mathrm{s}$, frequency of the bridge $\omega_{1}=30.02 \mathrm{rad} / \mathrm{s}$, frequency of the sprung mass $\omega_{v}=16.66 \mathrm{rad} / \mathrm{s}$. For this particular problem, an approximate analytical solution can be established by considering only the first mode of vibration, where the displacement of the sprung mass is represented by $u_{v}$ and the deflection in the bridge is represented by $u_{b}=z(t) \sin (\pi x / L)$, where $\sin (\pi x / L)$ is the first mode shape of the bridge. If the vehicle is moving with a 
constant speed of $v$ and neglecting the unsprung mass $m_{t}$, the damping in the vehicle $c$, and the damping in the bridge, the equations of motion of the vehicle and the bridge are given by (Biggs 1964; Fryba 1972; Humar 2002):

$$
\left[\begin{array}{cc}
m_{v} & 0 \\
0 & \frac{m_{b}}{2}
\end{array}\right]\left\{\begin{array}{c}
\ddot{u}_{v} \\
\ddot{z}
\end{array}\right\}+\left[\begin{array}{cc}
k & -k \sin \frac{\pi v t}{L} \\
-k \sin \frac{\pi v t}{L} & k \sin ^{2} \frac{\pi v t}{L}+\frac{\pi^{4} E l}{2 L^{3}}
\end{array}\right]\left\{\begin{array}{c}
u_{v} \\
z
\end{array}\right\}=-g\left\{\begin{array}{c}
0 \\
m_{v} \sin \frac{\pi v t}{L}
\end{array}\right\}
$$

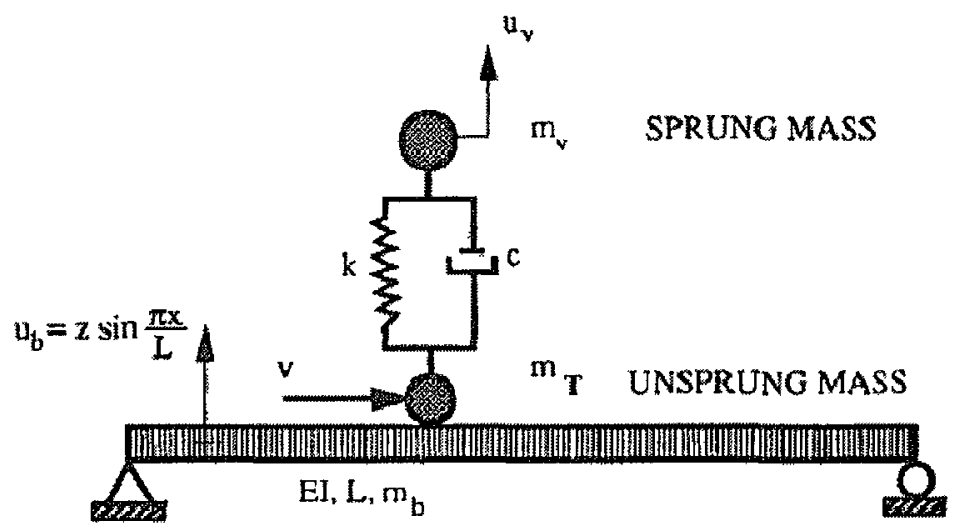

Figure 5.1: A simply supported bridge traversed by a moving vehicle load (Humar and Kashif 1993)

The equations of motion for the vibration of the bridge and the vehicle can be written in another form as follows (Biggs, 1964);

$$
\left\{\begin{array}{c}
\ddot{z} \\
\ddot{u}_{v}
\end{array}\right\}+\left[\begin{array}{cc}
2 \omega_{v}^{2} \frac{M_{v}}{m L} \sin ^{2} \frac{\pi v t}{L}+\omega_{1}^{2} & -2 \omega_{v}^{2} \frac{M_{v}}{m L} \sin \frac{\pi v t}{L} \\
-\omega_{v}^{2} \sin \frac{\pi v t}{L} & \omega_{v}^{2}
\end{array}\right]\left\{\begin{array}{c}
z \\
u_{v}
\end{array}\right\}=\left\{\begin{array}{c}
\left.-2 \frac{M_{v} g}{m L} \sin \frac{\pi v t}{L}\right\} \\
0
\end{array}\right\}
$$

The dynamic response of the midpoint displacement in the bridge obtained by this analytical solution is plotted in Figures 5.2and 5.3 and compared to the dynamic response of the midpoint displacement in the bridge obtained by the developed finite element program. As can be seen, the dynamic response obtained by the developed finite element program agrees very well with the analytical solution. 


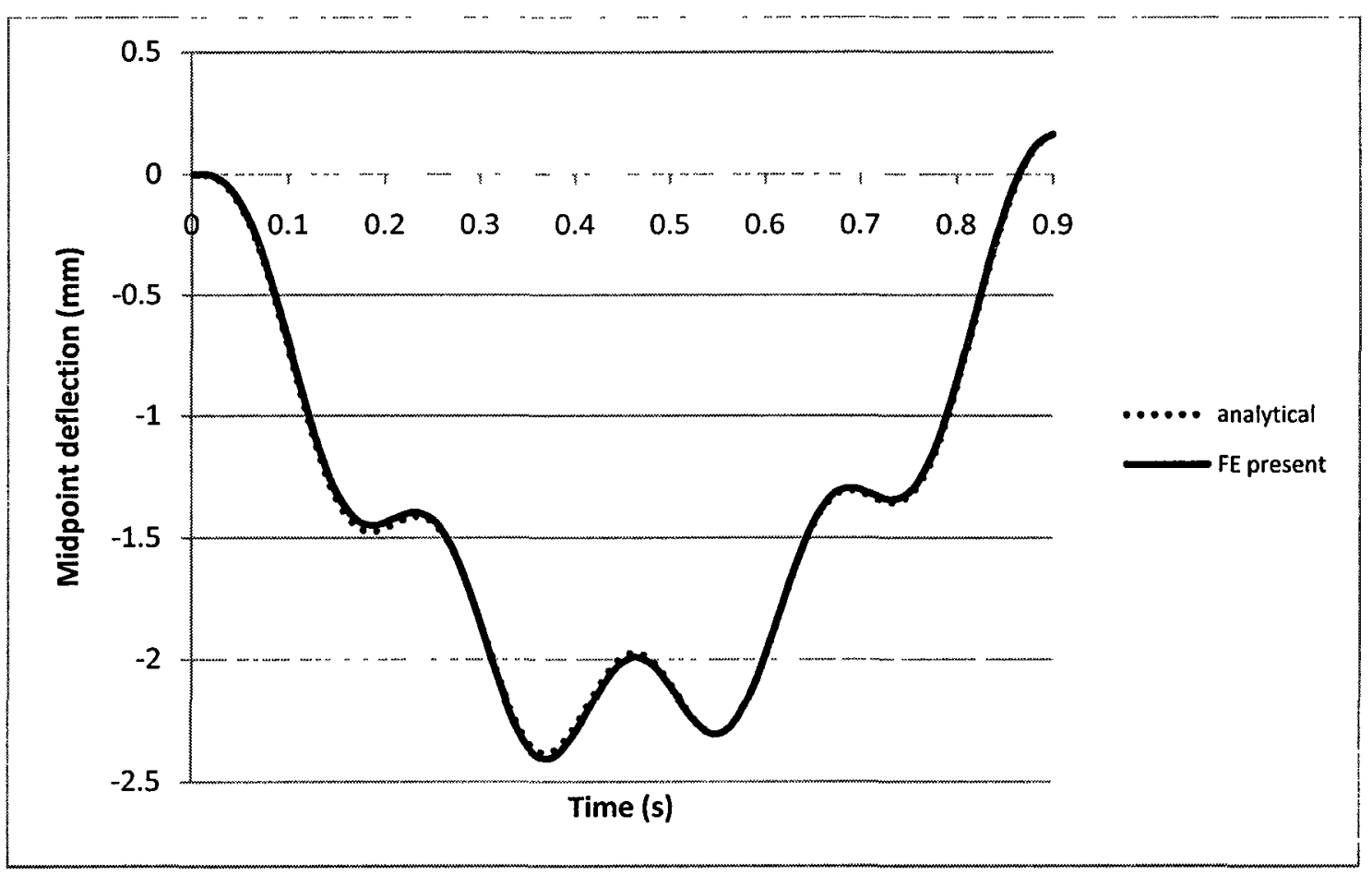

Figure 5.2: Time history of midpoint deflection in a simply supported bridge traversed by a moving vehicle load obtained by different approaches

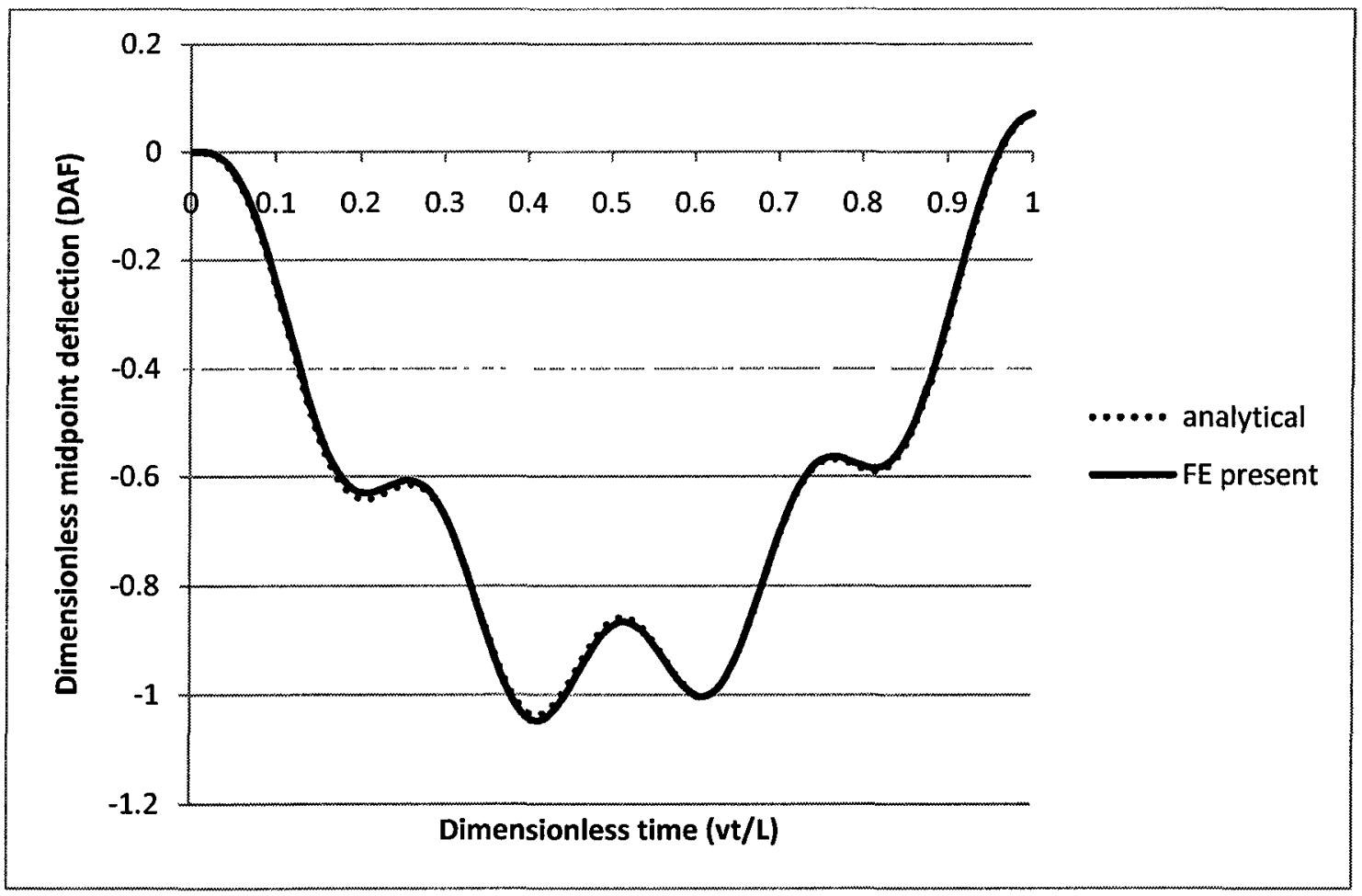

Figure 5.3: Time history of midpoint dimensionless deflection in a simply supported bridge traversed by a moving vehicle load obtained by different approaches 
This particular example for the dynamic response of simply supported bridge to moving vehicle loads exists in many publications and was used to verify the validity and applicability of the developed procedure (Yang and Yau 1997; Yang and $\mathrm{Wu} 2001$; Zhang et al. 2001; Majka and Hartnett 2008).

\subsection{Simply Supported Bridge Subjected to Moving Two-Axle Vehicle Loads}

A simply supported bridge is subjected to moving two-axle vehicle loads represented by a rigid bar supported by two spring-dashpot units as shown in Figure 5.4. The bridge is represented by a beam model and the vehicle is represented by a rigid bar with mass $M_{v}$ and mass moment of inertia $I_{v}$ supported by two spring-dashpot units of spring constant $k_{v}$ and damping coefficient $c_{v}$. Each of the two spring-dashpot units is further supported by an unsprung mass $M_{w}$. The following data are assumed: Young's modulus $E=$ $2.943 * 10^{7} \mathrm{kN} / \mathrm{m}^{2}$, Poisson's ratio $v=0.2$, moment of inertia $I=8.65 \mathrm{~m}^{4}$, mass per unit length $m=36 t / m$, length of bridge $L=30 \mathrm{~m}$, rigid bar sprung mass $M_{v}=540 t$, mass moment of inertia $I_{v}=13800 t \mathrm{~m}^{2}$, suspension spring constant $k_{v}=41350 \mathrm{kN} /$ $m$, damping coefficient $c_{v}=0$, wheel mass $M_{w}=0$, axle spacing $d=17.5 \mathrm{~m}$, vehicle speed $v=27.78 \mathrm{~m} / \mathrm{s}$.

The dynamic responses of the midpoint displacement in the bridge obtained by Yang and

$\mathrm{Wu}(2001)$ and by the developed finite element program are plotted in Figures 5.5 and 5.6, respectively. As can be seen, the dynamic response obtained by the developed finite element program agrees very well with the response obtained by Yang and $\mathrm{Wu}(2001)$. 


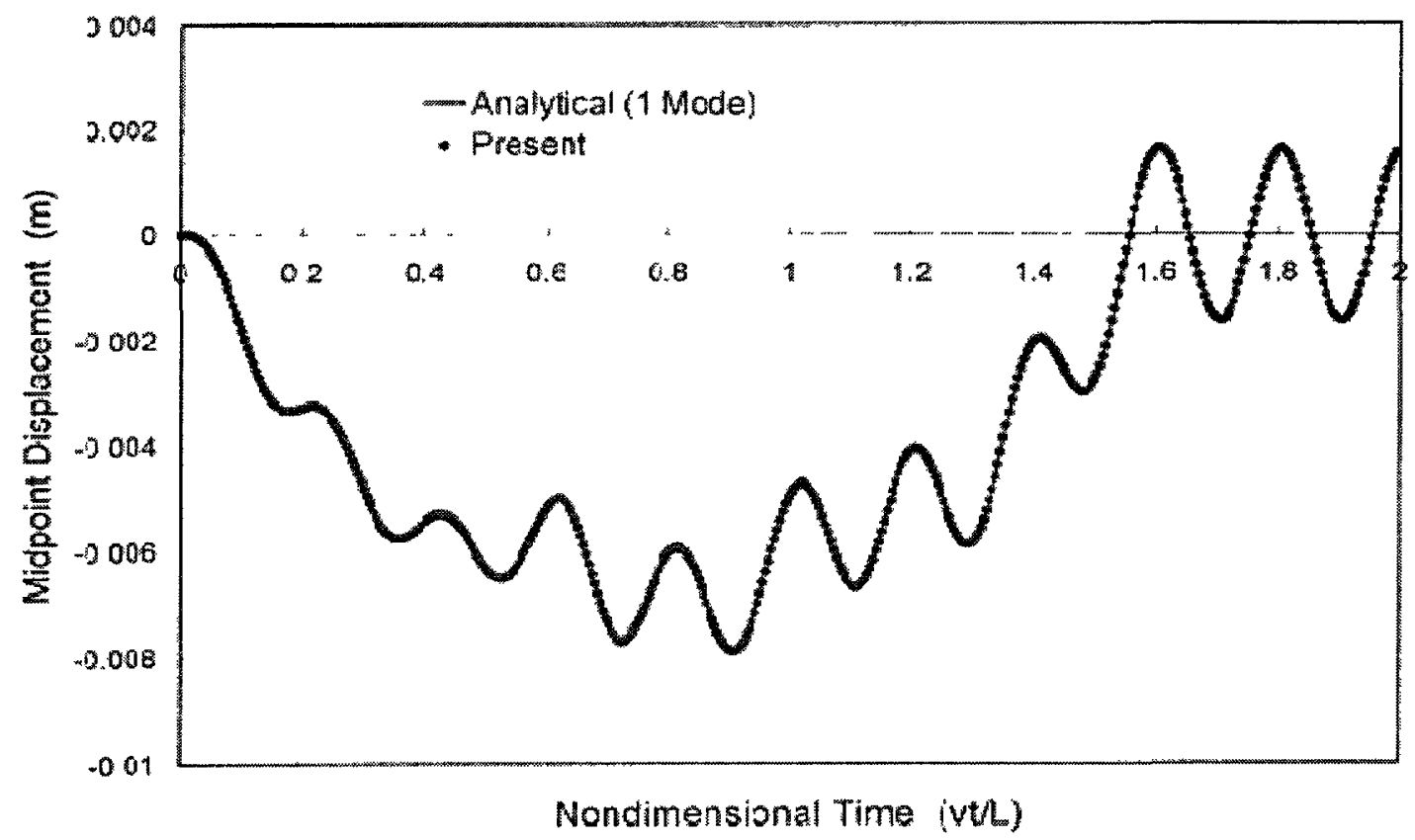

Figure 5.5: Time history of midpoint displacement in a simply supported bridge traversed by a two-axle vehicle (Yang and $\mathrm{Wu} 2001$ )

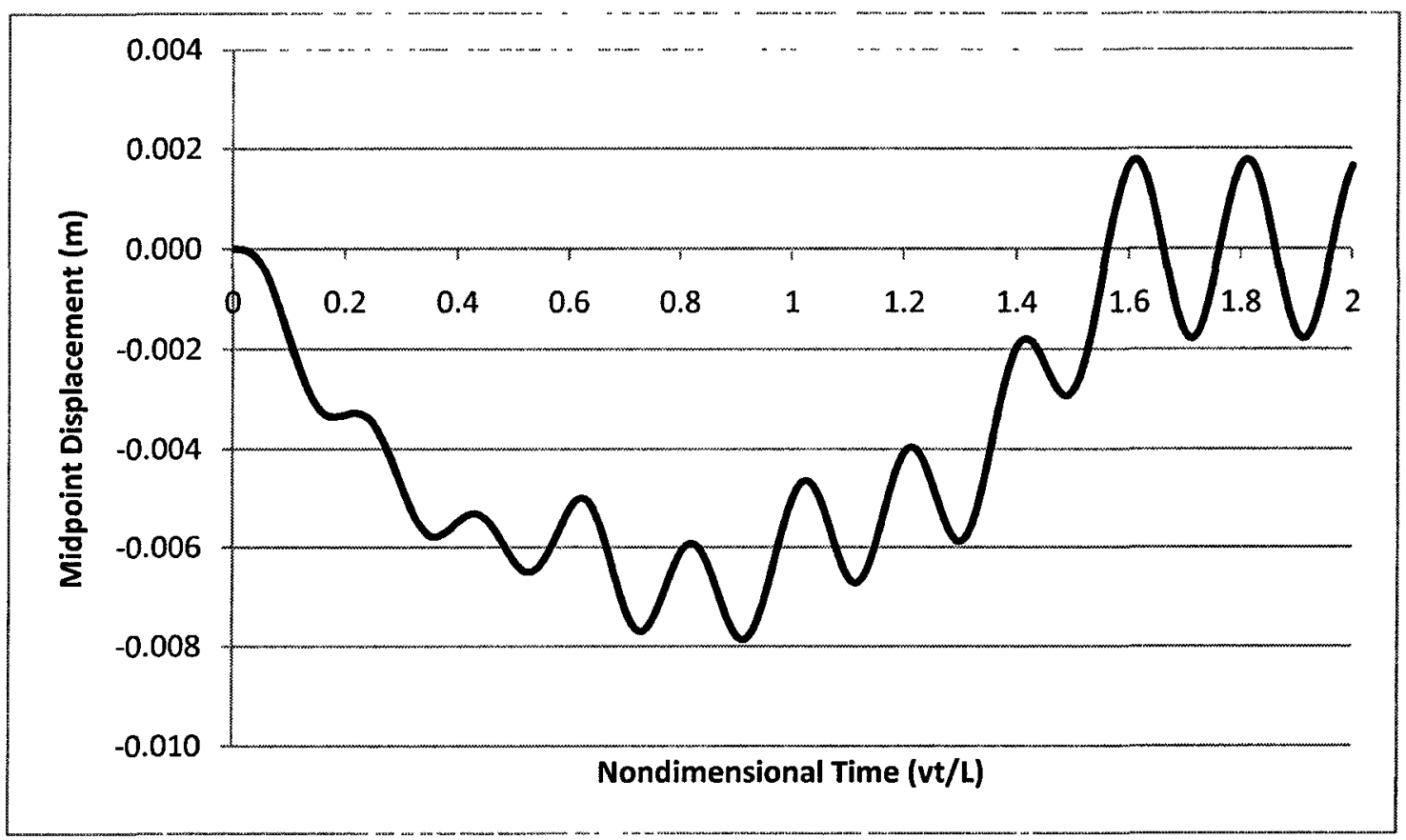

Figure 5.6: Time history of midpoint displacement in a simply supported bridge traversed by a two-axle vehicle (developed FE program) 


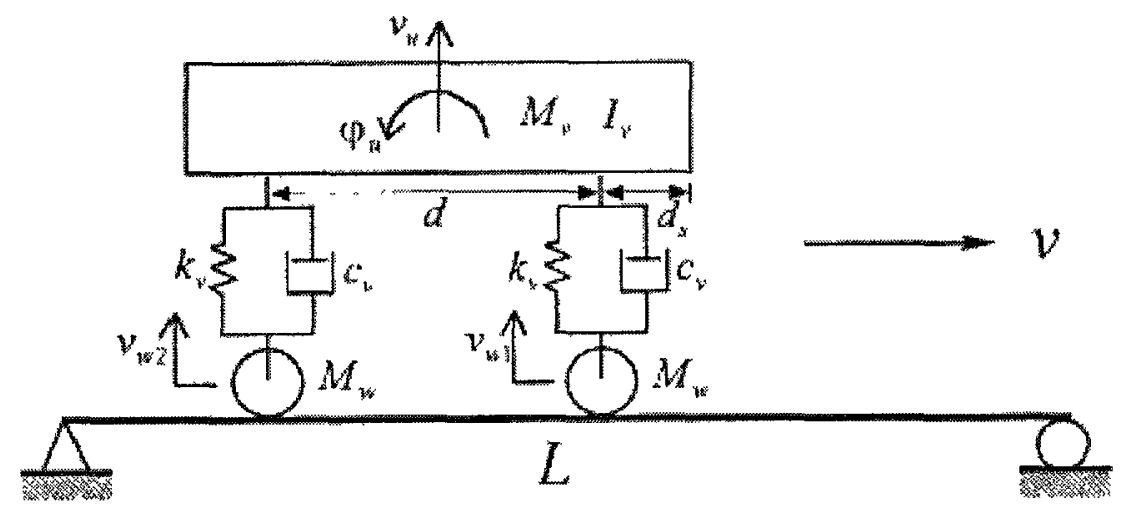

Figure 5.4: Simply supported bridge traversed by a two-axle vehicle (Yang and $\mathrm{Wu}$ 2001)

\subsection{Hinge-Connected Floating Bridge Subjected to Moving Load with Different}

\section{Speeds}

The dynamic response of a hinge-connected floating bridge subjected to moving load with different speeds is investigated here. The combined rigid-body (rolling) motion and elastic torsional vibration of a moored hinge-connected floating bridge is studied. The mathematical model is obtained by simplifying one of the floating bridges in Lake Washington at Seattle, U.S.A and is shown in Figure 5.7. To derive the stiffness matrix and the mass matrix, the bridge is considered to be an elastic beam supported by a number of uniformly distributed springs to simulate the water buoyancy and concentrated springs to simulate the mooring cables.

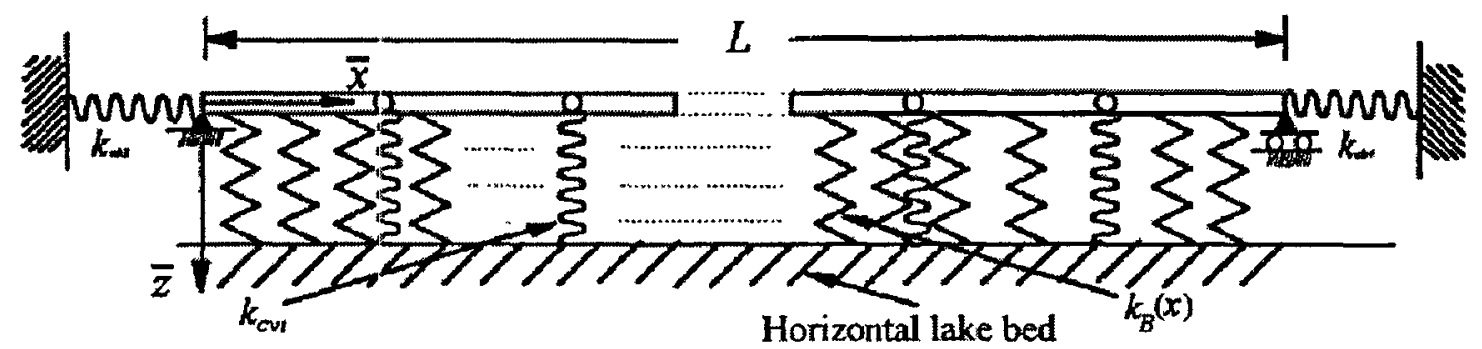

(a) Side view 


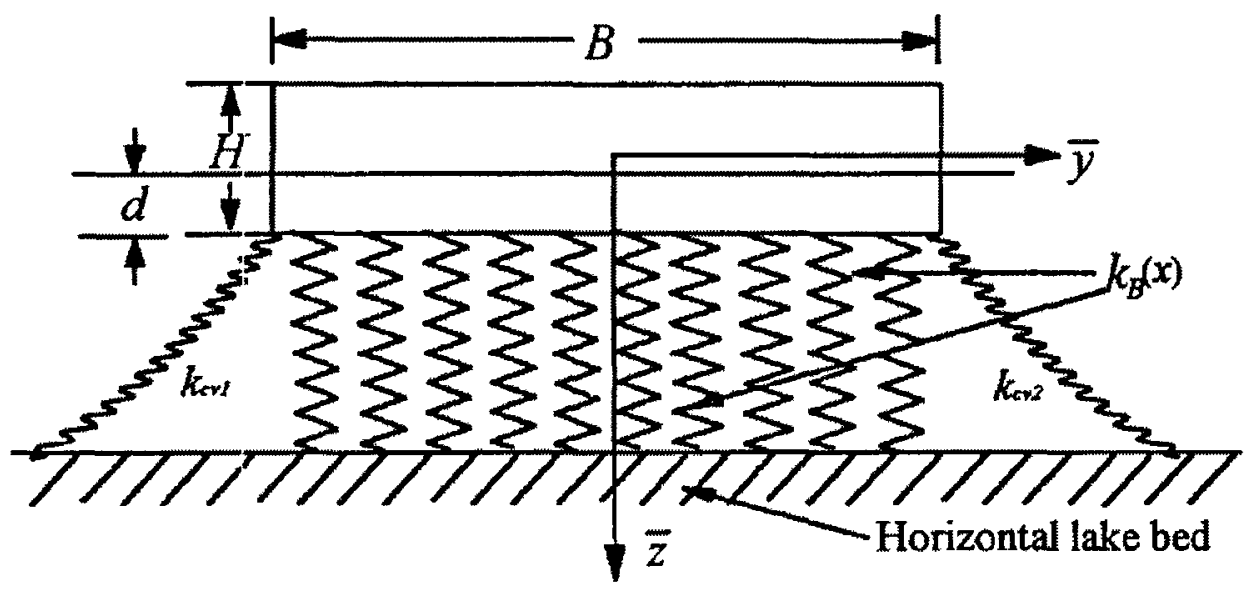

(b) Enlarged left end view

Figure 5.7: Mathematical model of the floating bridge studied by Wu and Shih (1998)

The following data are assumed: total length of the bridge $L_{B}=1800 \mathrm{~m}$, length of each pontoon $L_{p}=100 \mathrm{~m}$, total number of pontoons $N_{p}=18$, length of each beam element $L=25 \mathrm{~m}$, total number of beam elements $N_{b}=72$, total number of hinged joints $N_{h}=17$, width of each pontoon $b=20 \mathrm{~m}$, depth $d=10 \mathrm{~m}$, free draft $d_{f}=5 \mathrm{~m}$, mass density of reinforced concrete $\rho_{c}=2500 \mathrm{~kg} / \mathrm{m}^{3}$, Young's modulus $\mathrm{E}=3.63 *$ $10^{10} \mathrm{~N} / \mathrm{m}^{2}$, Poisson's ratio $v=0.15$, equivalent vertical spring constant of vertical mooring cable $k_{c v}=20624.88 \mathrm{~N} / \mathrm{m}$, longitudinal spacing of mooring cables $\Delta L_{c}=$ $25 \mathrm{~m}$, total number of cables $n=146$, moving load $P_{c}=50 \mathrm{kN}$, eccentricity of load $e_{P}=9 \mathrm{~m}$, moment of inertia $I_{z}=309.15625 \mathrm{~m}^{4}, I_{x}=1230.5 \mathrm{~m}^{4}$, mass per unit length $\bar{m}_{o}=48750 \mathrm{~kg} / \mathrm{m}$, distributed mass due to super-structure $\bar{m}_{u}=2562.5 \mathrm{~kg} / \mathrm{m}^{2}$, total mass per unit length $\bar{m}=100000 \mathrm{~kg} / \mathrm{m}$.

The dynamic response of the midpoint displacement in the hinge-connected floating bridge obtained by $\mathrm{Wu}$ and Shih (1998) due to a load of $50 \mathrm{KN}$ moving with two 
different speeds: $26 \mathrm{~m} / \mathrm{s}$ and $40 \mathrm{~m} / \mathrm{s}$ is plotted in Figures 5.8 and 5.10 , respectively. The dynamic response of the midpoint displacement in the same floating bridge obtained using the developed finite element program due to a load of $50 \mathrm{KN}$ moving with the same speeds of $26 \mathrm{~m} / \mathrm{s}$ and $40 \mathrm{~m} / \mathrm{s}$ is plotted in Figures 5.9 and 5.11, respectively. As can be seen, the dynamic response obtained by the developed finite element program agrees very well with the dynamic response obtained by Wu and Shih (1998) for all different speeds considered.

\subsection{Dynamic Analysis of Floating Bridges Using ANSYS Software Package}

The use of numerical modeling in engineering applications has been growing fast in last few decades. There are now many commercial finite element programs that can be used to analyze different engineering problems with high degree of complexity. Some of these programs are capable of simulating field conditions accurately; therefore they are widely used prior to more expensive field tests to maximize the benefits of both approaches. In this section, a software package known as ANSYS Multi-physics referred herein as ANSYS (ANSYS Inc. 2009) is used to investigate the dynamic response of floating bridges to moving vehicle loads.

The ability of ANSYS to analyze the structural behaviour of floating bridges under moving vehicle loads is checked and the results of the floating bridge dynamic response using ANSYS are compared to the dynamic response obtained using the finite element program developed by the author. The following sections give a brief overview of the finite element technique as applied to the problem at hand and the steps of constructing numerical models to solve these problems. 


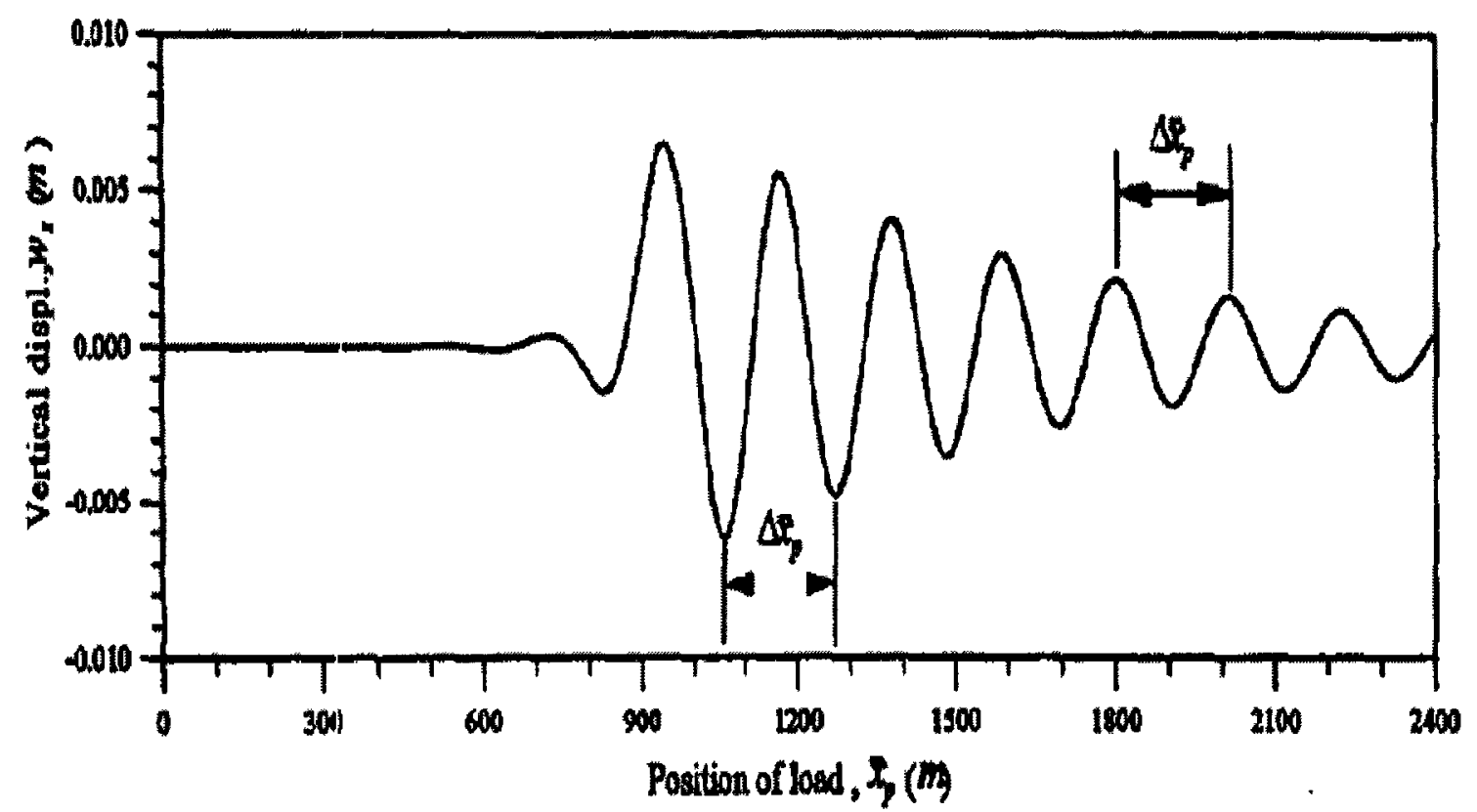

Figure 5.8: The relationship between the moving load position and the central vertical displacement in the hinge-connected bridge $\left(P_{c}=50 \mathrm{kN}, e_{P}=9 \mathrm{~m}, v_{P}=26 \mathrm{~m} / \mathrm{s}\right)(\mathrm{Wu}$ and Shih 1998)

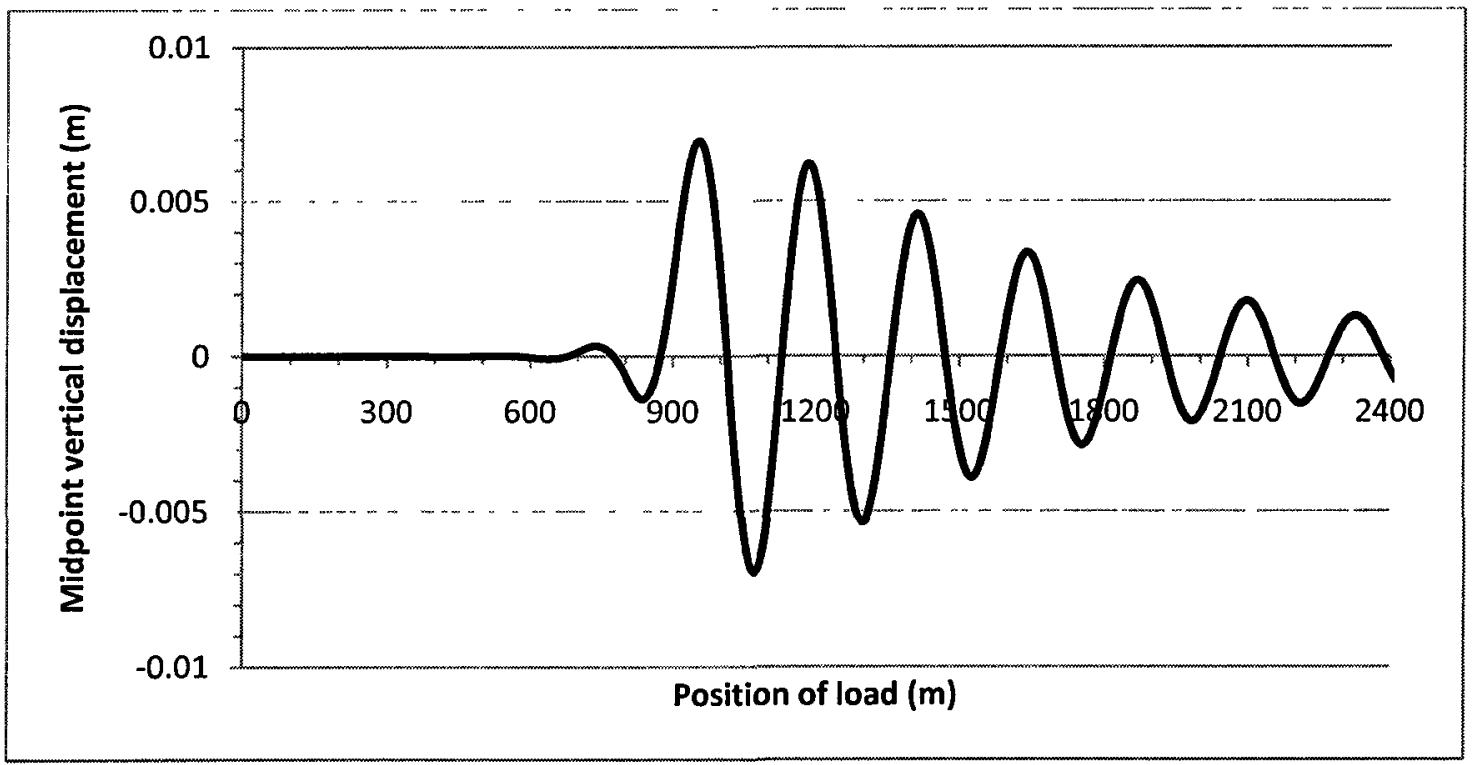

Figure 5.9: The relationship between the moving load position and the central vertical displacement in the hinge-connected bridge $\left(P_{c}=50 \mathrm{kN}, e_{P}=9 \mathrm{~m}, v_{P}=26 \mathrm{~m} / \mathrm{s}\right)$ (developed FE program) 


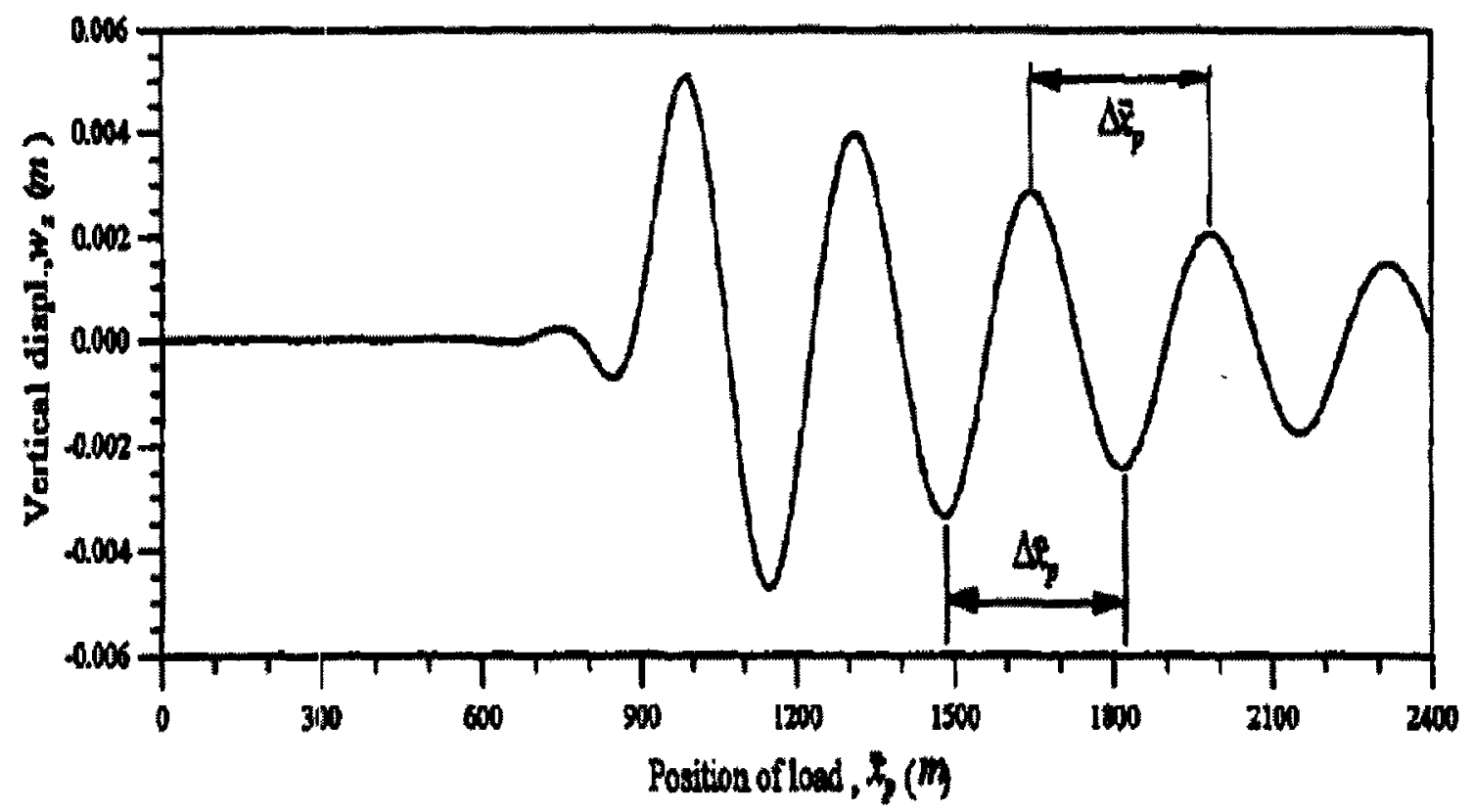

Figure 5.10: The relationship between the moving load position and the central vertical displacement in the hinge-connected bridge $\left(P_{c}=50 \mathrm{kN}, e_{P}=9 \mathrm{~m}, v_{P}=40 \mathrm{~m} / \mathrm{s}\right)(\mathrm{Wu}$ and Shih 1998)

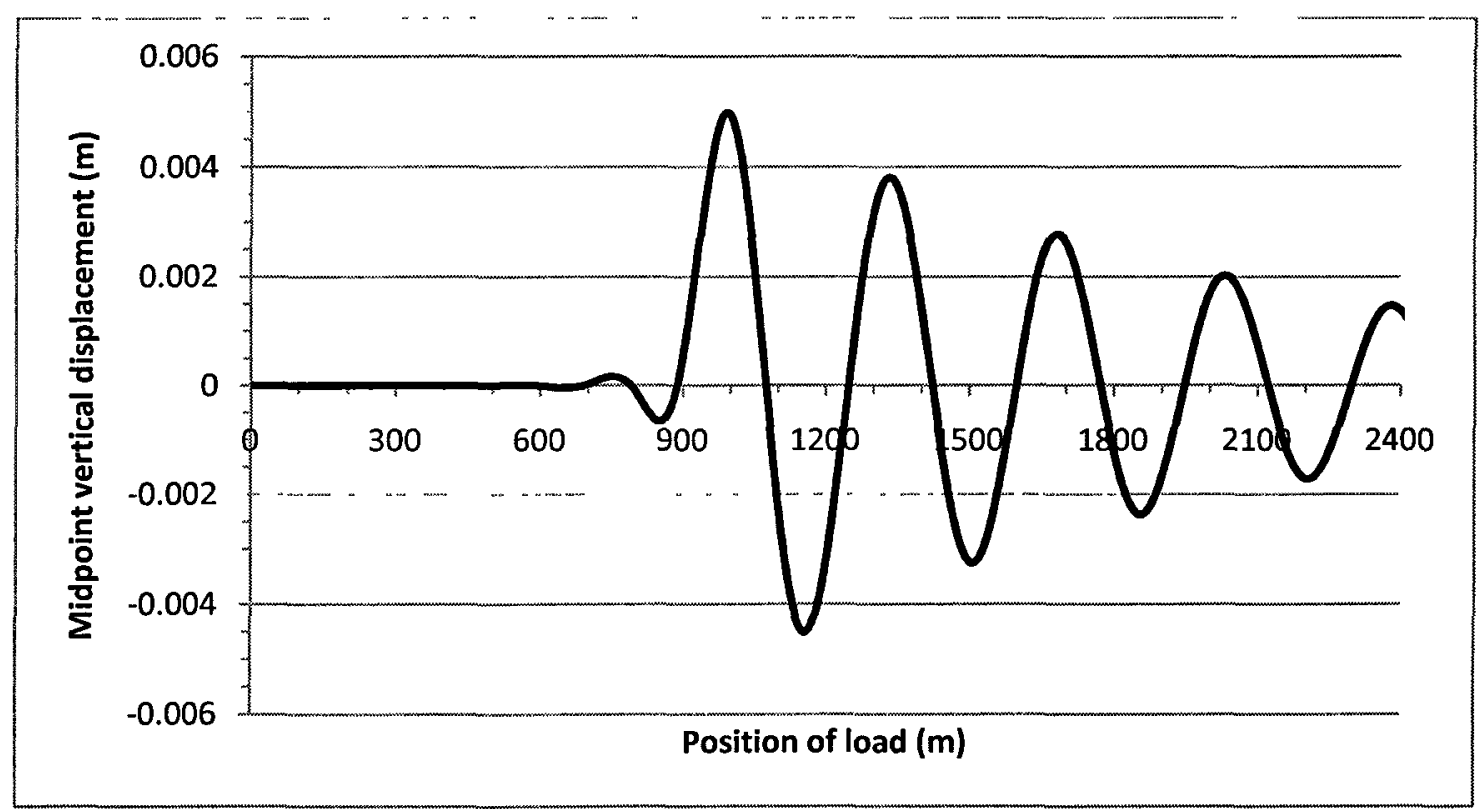

Figure 5.11: The relationship between the moving load position and the central vertical displacement in the hinge-connected bridge $\left(\mathrm{P}_{\mathrm{c}}=50 \mathrm{KN}, \mathrm{e}_{\mathrm{P}}=9 \mathrm{~m}, \mathrm{v}_{\mathrm{P}}=40 \mathrm{~m} / \mathrm{s}\right)$ (developed FE program) 


\subsubsection{ANSYS Element Types}

BEAM4: Structural 3-D Elastic Beam is a uniaxial element with tension, compression, torsion and bending capabilities. The element has two nodes with six degrees of freedom at each node: translations in the nodal $\mathrm{x}, \mathrm{y}$ and $\mathrm{z}$ directions and rotations about the nodal $\mathrm{x}, \mathrm{y}$ and $\mathrm{z}$ axes. The beam must not have a zero length or area. The beam can have any cross-sectional shape for which the moments of inertia can be computed. The element thicknesses are used only in the bending and thermal stress calculations.

COMBIN14: Combination Spring-Damper has longitudinal or torsional capability in 1D, 2-D, or 3-D applications. The longitudinal spring-damper option is a uniaxial tensioncompression element that has two nodes with up to three degrees of freedom at each node: translations in the nodal $\mathrm{x}, \mathrm{y}$, and $\mathrm{z}$ directions. No bending or torsion is considered. The spring-damper element has no mass. The length of the spring-damper element must not be zero, which means that nodes $i$ and $\mathbf{j}$ should not be coincident. The longitudinal spring element stiffness acts only along its length. The element allows only a uniform stress in the spring.

\subsubsection{Dynamic Analysis Due to Moving Load}

In the present study, the floating bridge is represented by beam elements where the beam is simply supported at the two ends and resting on elastic springs representing the underlying water. To simplify the model, the vehicle load is represented by a concentrated load of constant value moving along the bridge and neglecting the stiffness and the damping properties of the vehicle. The vehicle load is a dynamic load that 
depends on time and space coordinates where it changes its position at each time step according to the vehicle speed.

To the best of author's knowledge, there is no ready option in ANSYS to run a load moving at a specific speed and changing its position along the beam at each time step based on that specified speed. While building and running the bridge model in ANSYS, a subroutine was written by the author and added to ANSYS text files to include a do loop that formed a load-step file to represent the moving vehicle load along the beam nodes. The beam element length is chosen based on the specified vehicle speed and time step of the finite element time marching, so that the vehicle load acts at the preceding node at each time step. The bridge is at rest at the beginning and the vehicle load acts on the left support of the bridge. At the second time step, the load is deleted from the left support and added at the end node of the first beam element. At the third time step, the load is deleted from the end node of the first beam element and added at the end node of the second beam element and so on to form the whole load-step file that simulates the vehicle load moving along the bridge length. At the last time step, the vehicle load acts on the right support of the bridge.

For each vehicle speed, a new model has to be created to take into consideration the change of the beam element length and consequently the number of elements. Also, it is difficult to simulate the vehicle load using multiple-axle model and even more difficult to simulate the passage of multiple successive vehicles represented by multiple-axle models (compared to the developed finite element program). To take into consideration the vehicle-bridge interaction which has a significant effect on the dynamic response of the 
floating bridge by considering the dynamic characteristics of the vehicle that represent its stiffness and damping properties, a complicated model has to be built in ANSYS that make the vehicle load acts on a spring-damper unit at each position of the vehicle load instead of acting directly on the bridge nodes. However, the vehicle-bridge interaction is easily taken into consideration in the developed finite element program where the dynamic characteristics of the vehicle are included in the dynamic equation of the bridgevehicle system and constitute one of the input parameters in the developed finite element program. These reasons make the use of ANSYS software package not feasible for the specific problem under consideration for dynamic response of floating bridges to moving vehicle loads, especially for a parametric study comprising a lot of parameters and runs.

\subsubsection{Finite Element Model}

The floating bridge is represented by beam elements BEAM4; the beam is simply supported at the two ends and resting on elastic springs COMBIN14 representing the underlying water. The vehicle load is represented by a single-axle concentrated load of constant value moving along the bridge and neglecting the stiffness and the damping properties of the vehicle.

The bridge has a total length to of $84 \mathrm{~m}$ and subjected to 50 -tonne single-axle vehicle load moving along the bridge at speed of $15 \mathrm{~km} / \mathrm{h}$. The time step for the dynamic analysis was chosen as $0.06 \mathrm{~s}$. Based on the vehicle speed and the time step, the beam element length was chosen as $0.25 \mathrm{~m}$ so that the vehicle load acts at the preceding node at each time step. In this case, the bridge was discretized into 336 beam elements each of $0.25 \mathrm{~m}$ length to form a bridge of $84 \mathrm{~m}$ total length. Boundary conditions for hinged support 
were applied at the two ends of the bridge by preventing the three translational degrees of freedom as well as the rotational degrees of freedom about both $\mathrm{x}$ and $\mathrm{y}$ axes while allowing the rotation about the $\mathrm{z}$ axis. The bridge is also supported on translational elastic springs located at each node of the bridge. The cross-sectional properties of the beam element as well as the translational elastic spring were chosen to match with the case of floating bridges subjected to single-axle moving vehicle loads studied in Section 4.1 of the present thesis for the purpose of comparison. Enlarged view of the left end of the bridge model is shown in Figure 5.12, where the beam element is referred to as element 1 and the translational spring element is referred to as element 3.

A subroutine was written by the author and added to ANSYS text files to include a do loop that formed a load-step file to represent the moving vehicle load along the beam nodes. Figure 5.13 show some time steps from that load-step file where the vehicle load is moving along the bridge and changing its position at each time step starting from the left end of the bridge and ending at the right end of the bridge at the last time step.

\subsubsection{Dynamic Response of Rigid-Connected Floating Bridge to Single-Axle Vehicle Loads Using ANSYS Software Package}

The dynamic response of $84 \mathrm{~m}$ rigid-connected floating bridge to 50-tonne single-axle vehicle load moving at speed of $15 \mathrm{~km} / \mathrm{h}$ is obtained using ANSYS and compared to that obtained using the developed finite element program. The deflections along the bridge length are shown in Figure 5.14 at different time steps where the vehicle load acts at onefourth, one-half and three-fourth of the bridge length, in addition to the right end of the bridge, from top to bottom respectively. 
The time history of midpoint displacement in the bridge due to the passage of 50-tonne single-axle vehicle load is shown in Figure 5.15. The time history of midpoint displacement due to the passage of 50-tonne single-axle vehicle load using both ANSYS and the developed finite element program is shown in Figure 5.16. The results were found to be completely matching for both of them illustrating the reliability and high accuracy of the developed finite element program.

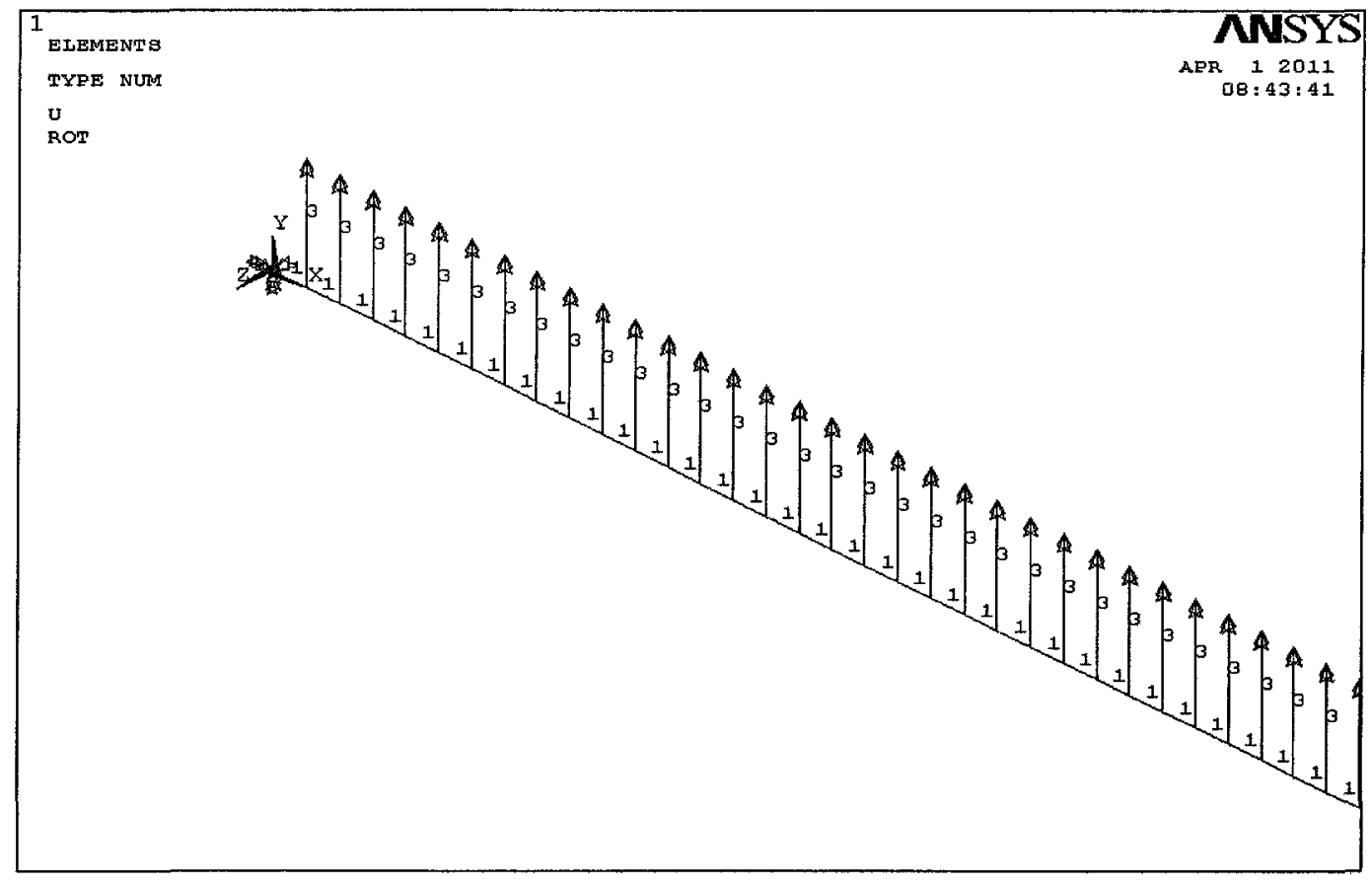

Figure 5.12: Enlarged view of the left end of the floating bridge finite element model 

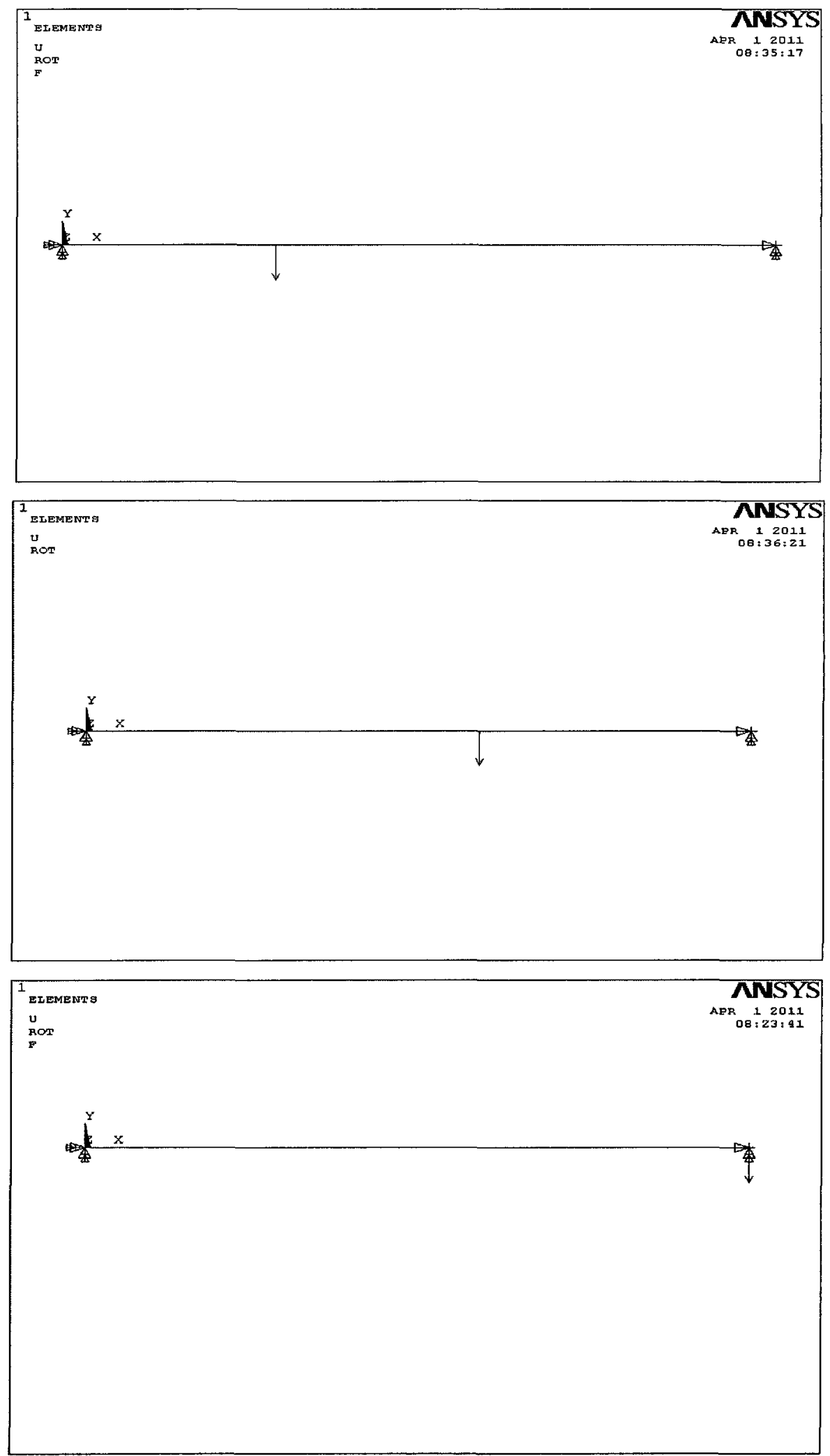

Figure 5.13: Load-step file for the vehicle load moving along the bridge 

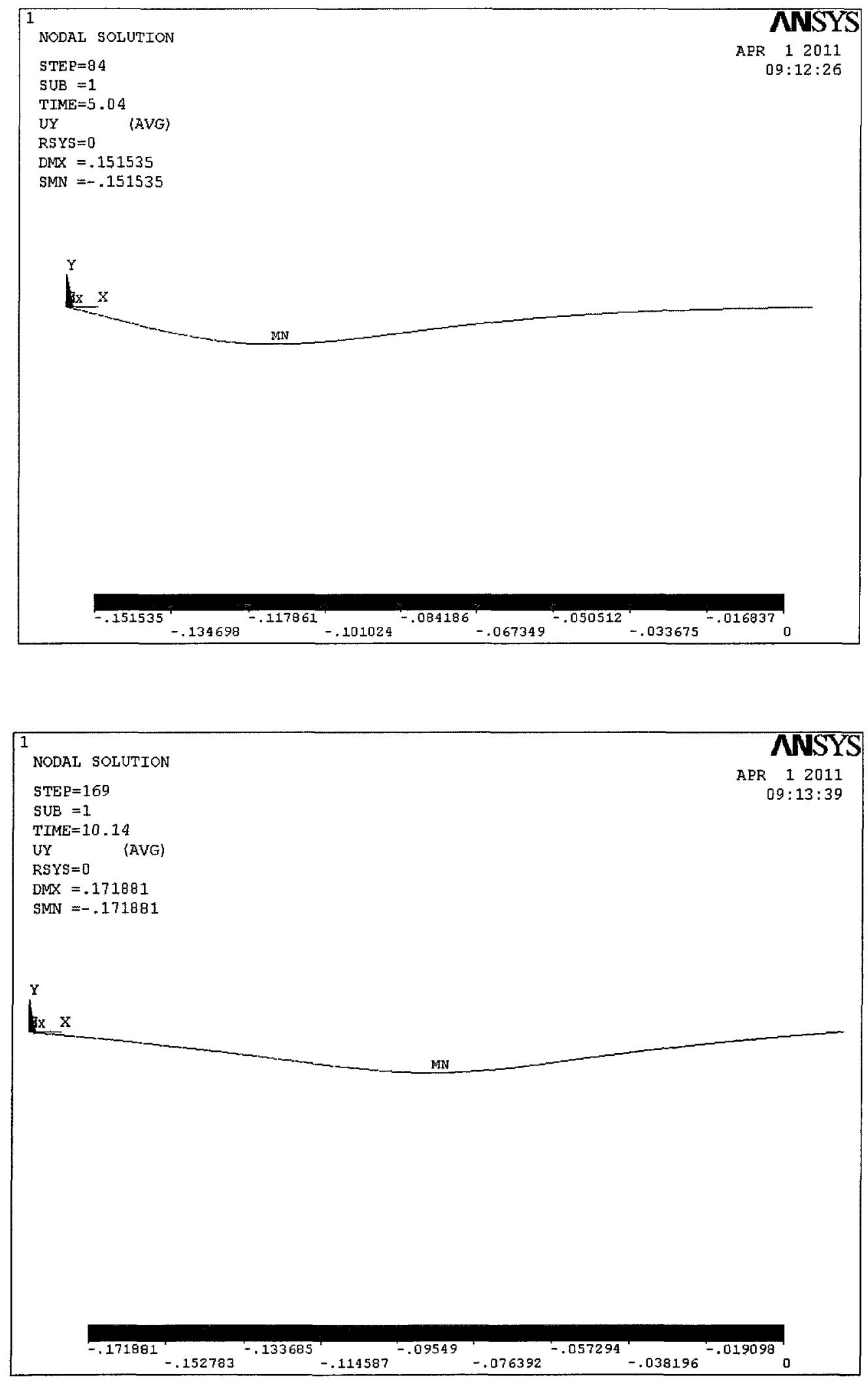

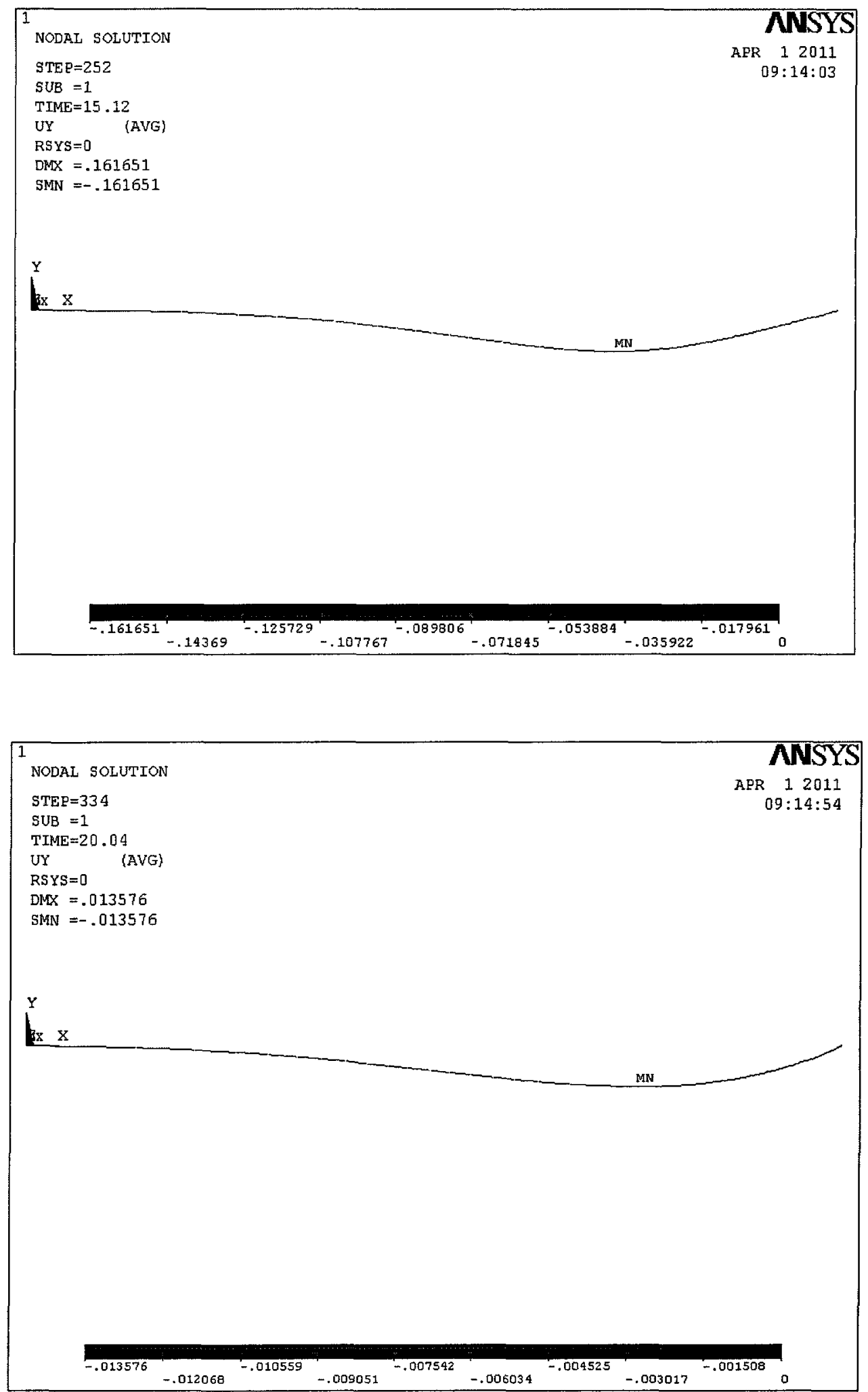

Figure 5.14: Deflection in rigid-connected floating bridge for different position of the vehicle load along the bridge using ANSYS 


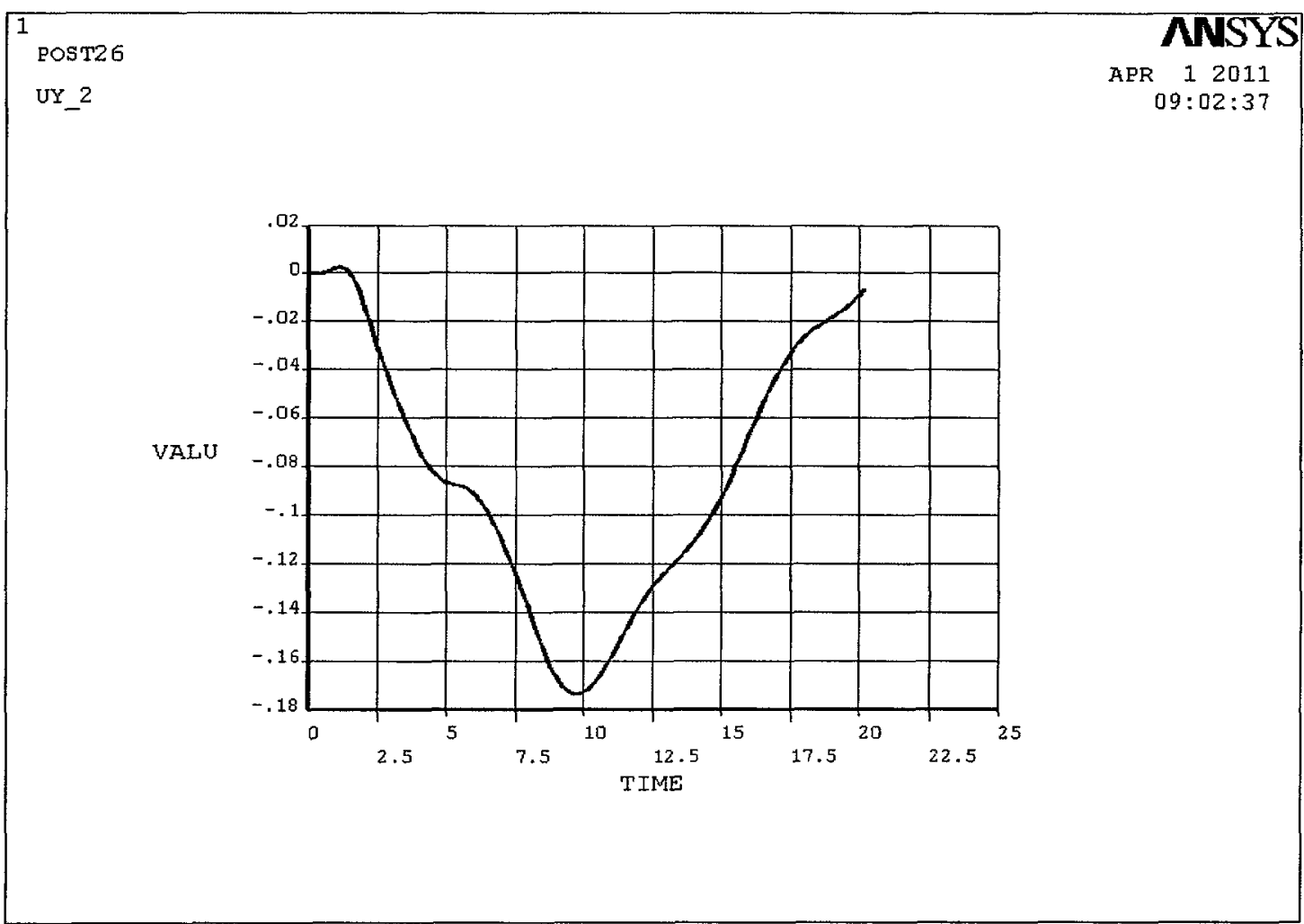

Figure 5.15: Time history of midpoint displacement in rigid-connected floating bridge due to 50-tonne single-axle vehicle loads using ANSYS software package

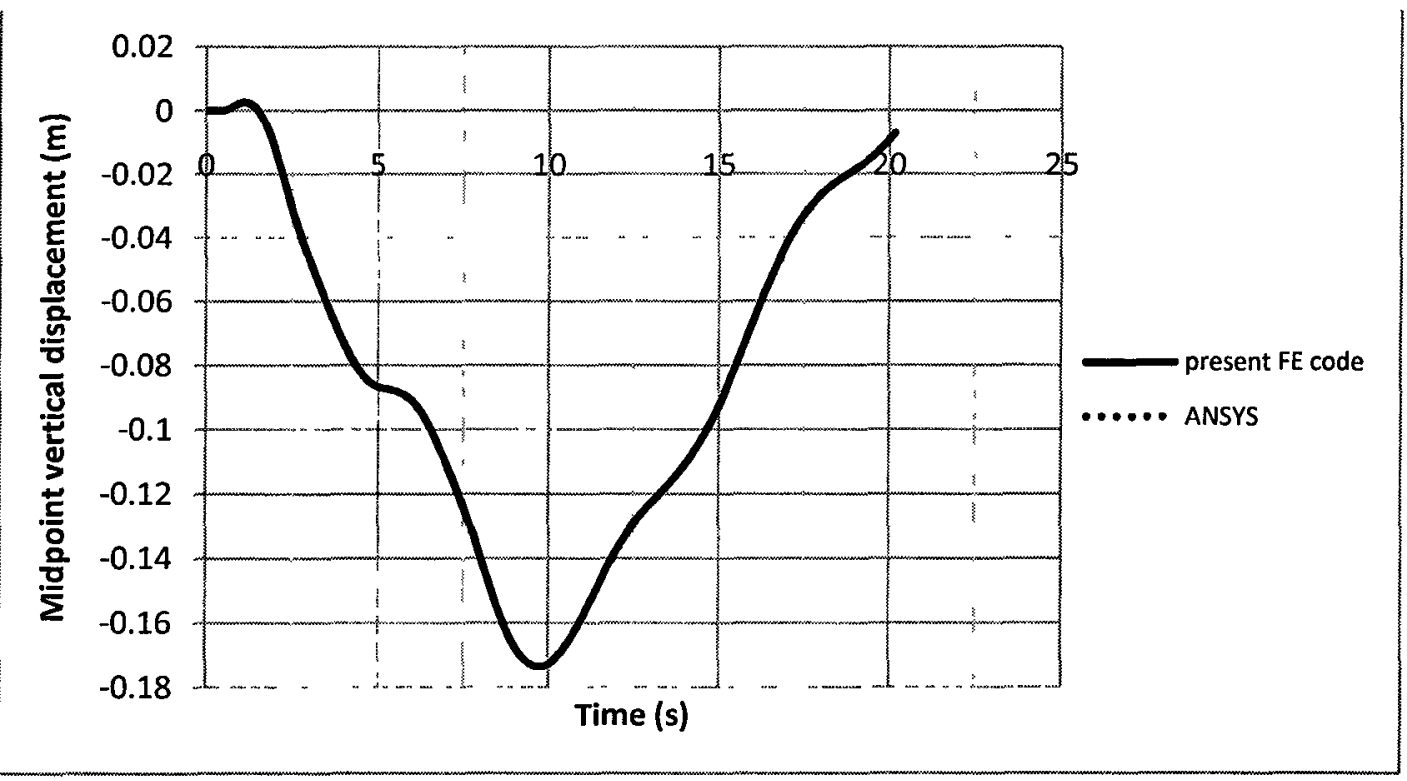

Figure 5.16: Time history of midpoint displacements in rigid-connected floating bridge due to 50-tonne single-axle vehicle loads using ANSYS and the developed FE program 


\subsubsection{Dynamic Response of Hinge-Connected Floating Bridge to Single-Axle Vehicle Loads Using ANSYS Software Package}

The bridge studied here consists of twelve pontoons each of length $7 \mathrm{~m}$, individual pontoons in each group of three are rigidly connected to each other, to form a typical bay of length $21 \mathrm{~m}$. These typical bays are hinge-connected to each other to form a bridge of total length $84 \mathrm{~m}$.

To represent the intermediate hinges located at one-fourth, one-half and three-fourth of the bridge length, an additional node has been added at each of these positions so that the end node of the beam element before the intermediate hinge is different from the first node of the beam element after the intermediate hinge and they are two separate nodes located at the same position. Then, constraints are applied to these two nodes located at the same position such that they have the same translations in $\mathrm{x}, \mathrm{y}$ and $\mathrm{z}$ directions and the same rotations about both $\mathrm{x}$ and $\mathrm{y}$ axes, but are free to have different rotation about the $\mathrm{z}$ axis, thus representing the behaviour of an intermediate hinge at that position. Figure 5.17 shows the bridge with three intermediate hinges, at one-fourth, one-half and three-fourth of the bridge length. An enlarged view of part of the floating bridge including an intermediate hinge is shown in Figure 5.18.

The dynamic response of the $84 \mathrm{~m}$ hinge-connected floating bridge to 50-tonne singleaxle vehicle load moving at speed of $15 \mathrm{~km} / \mathrm{h}$ is obtained using ANSYS and compared to the response obtained using the developed finite element program. The vertical displacement along the bridge length are shown in Figure 5.19 at different time steps 
where the vehicle load acts at one-fourth, one-half and three-fourth of the bridge length, in addition to the right end of the bridge, from top to bottom respectively.

The time history of midpoint displacement in the bridge due to the passage of 50-tonne single-axle vehicle load is shown in Figure 5.20. The time history of midpoint vertical displacement due to the passage of 50-tonne single-axle vehicle load using both ANSYS and the developed finite element program is shown in Figure 5.21. The results were found to be completely matching for both of them illustrating the reliability and accuracy of the developed finite element program.

From the previous sections it can be concluded that the finite element program developed by the author is reliable and accurate in determining the dynamic response of both rigidconnected and hinged connected floating bridges. Also, it has many advantages compared to the available software packages. The developed program is especially suited to the analysis of dynamic response of bridges to moving vehicle loads. It is also quite comprehensive so that all the parameters, such as: bridge length, number of elements, vehicle speed, number of vehicles, number of vehicle axles, dynamic characteristics of vehicle, etc., form simple inputs to the program. This makes the developed finite element program more efficient for parametric studies including a lot of parameters for the dynamic response of a floating bridge to moving vehicle loads.

\subsection{Summary}

In this chapter, the analytical approach and the finite element program developed by the author for the dynamic analysis of floating bridges subjected to moving vehicle loads 
were verified by comparing the response results with several examples available in the literature. The results obtained using the developed finite element program match with the results referred in the literature illustrating the reliability and accuracy of this developed finite element program. The dynamic analysis of floating bridges subjected to moving vehicle loads was also conducted using ANSYS software package where a subroutine was written by the author and added to ANSYS to represent the vehicle moving along the bridge. The results obtained from ANSYS completely match with the results of the developed finite element program. The developed finite element program was found to be more feasible than the ready software packages as all the parameters for the problem under consideration are simple inputs to the program.

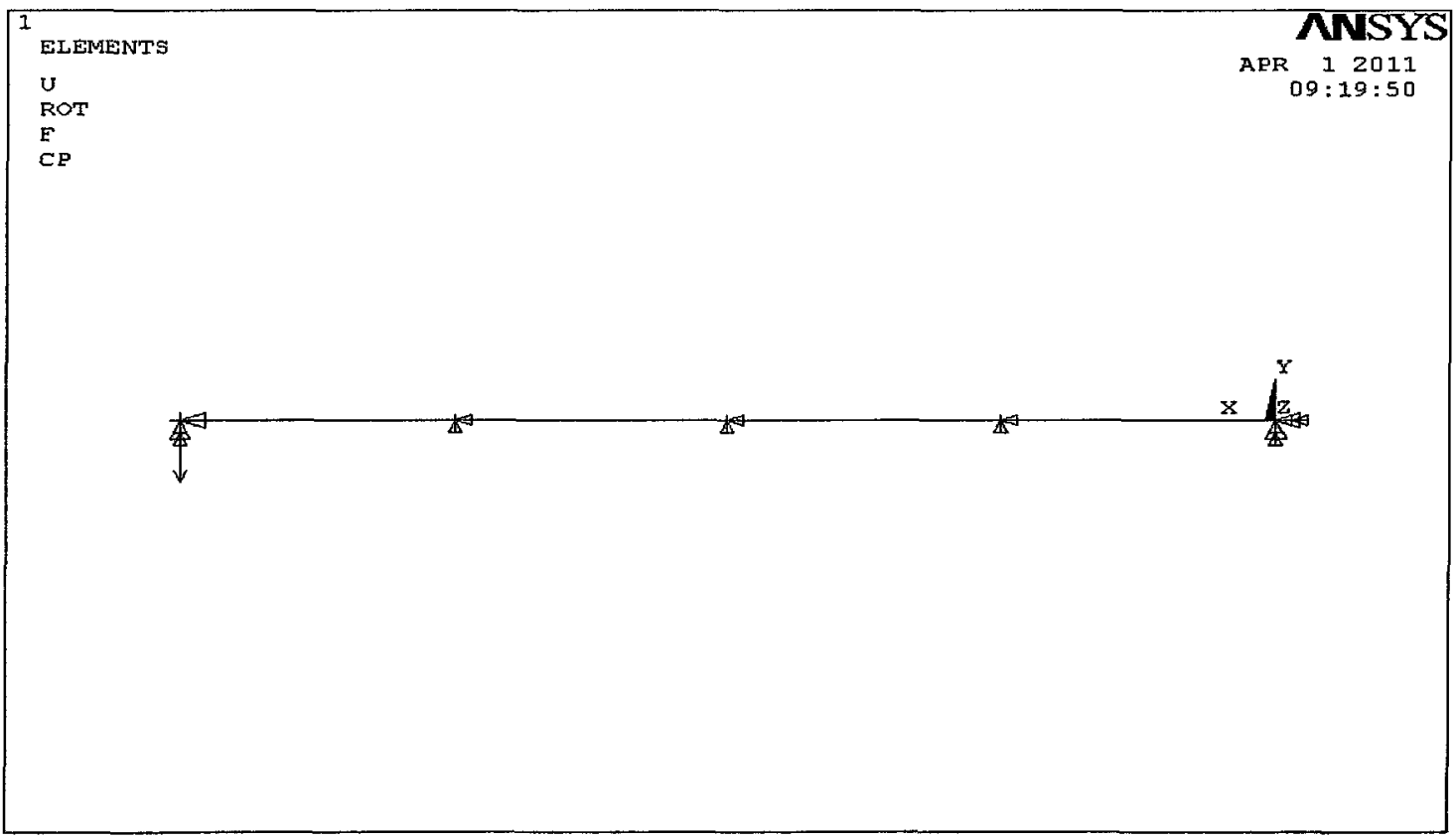

Figure 5.17: Bridge with three intermediate hinges at one-fourth, one-half and threefourth of the bridge length 


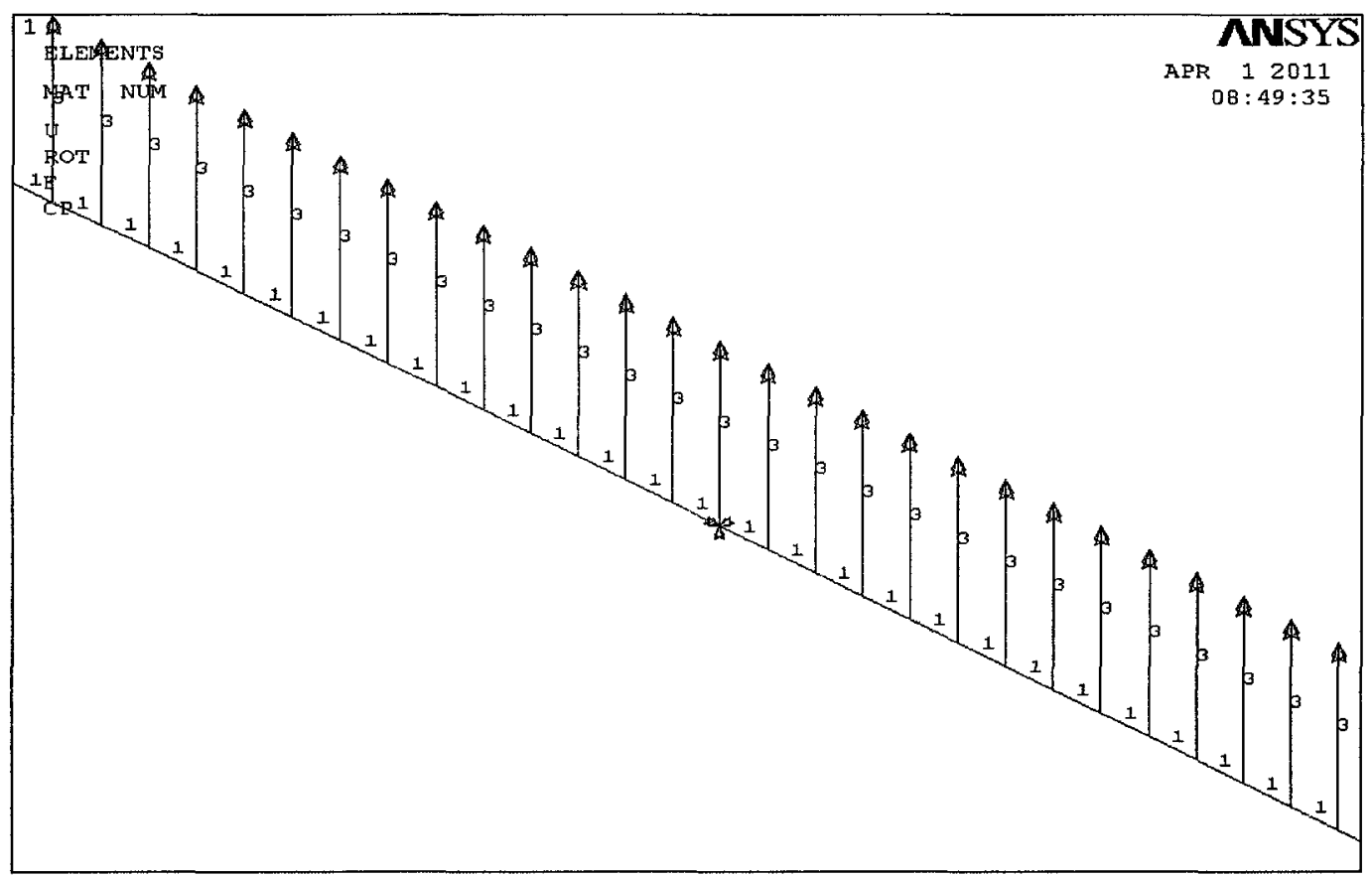

Figure 5.18: Enlarged view of part of the floating bridge including an intermediate hinge

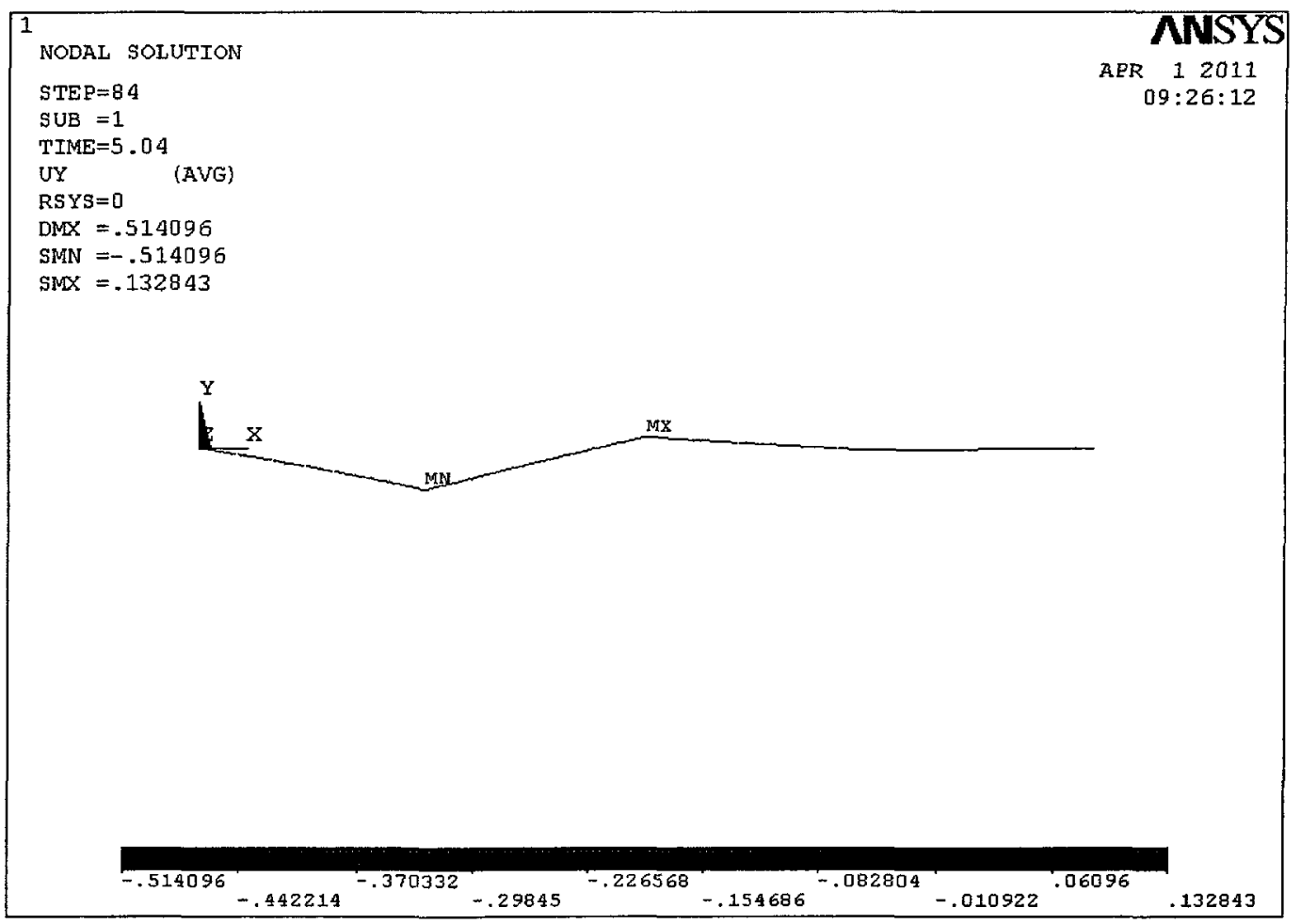



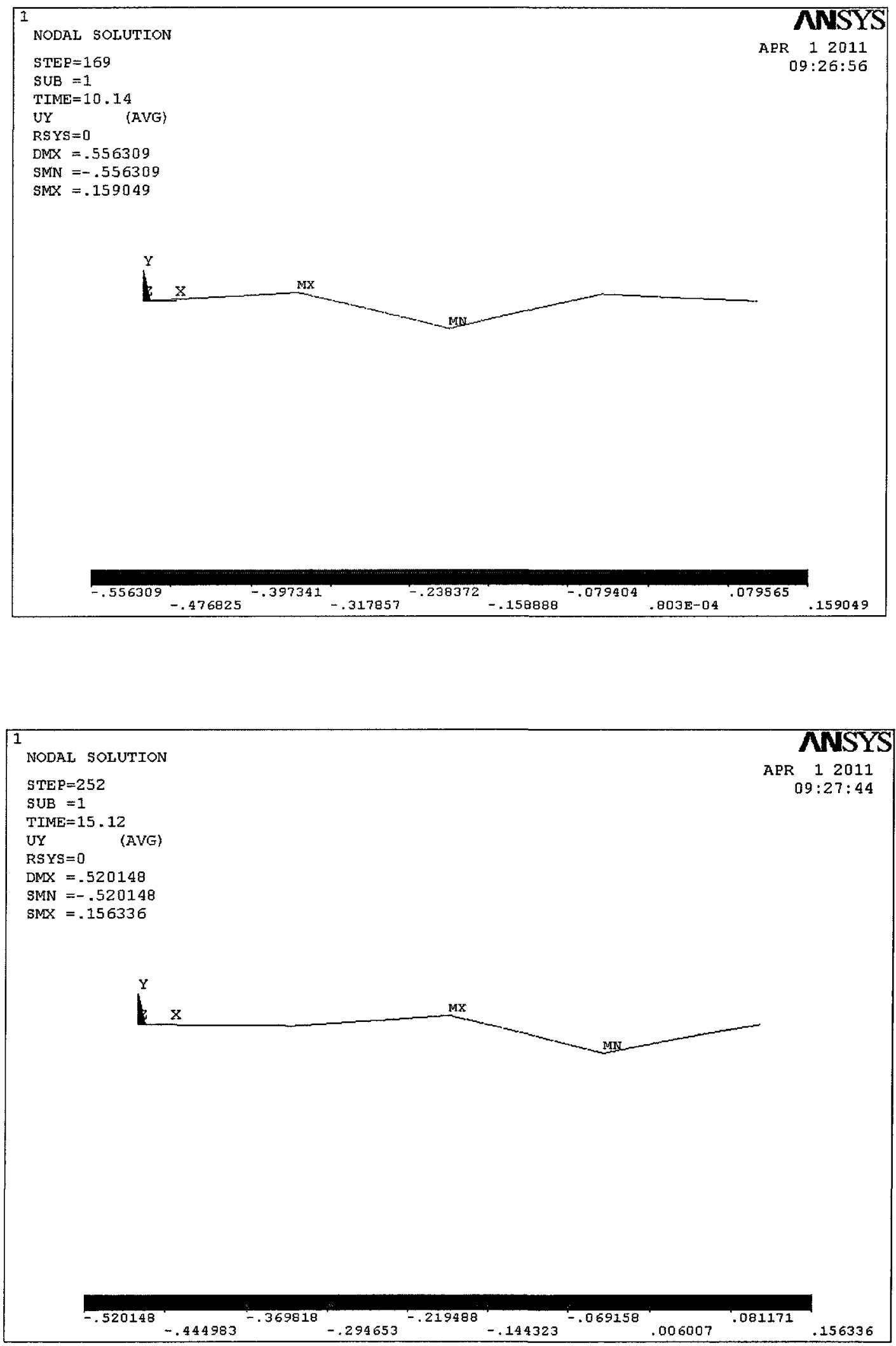


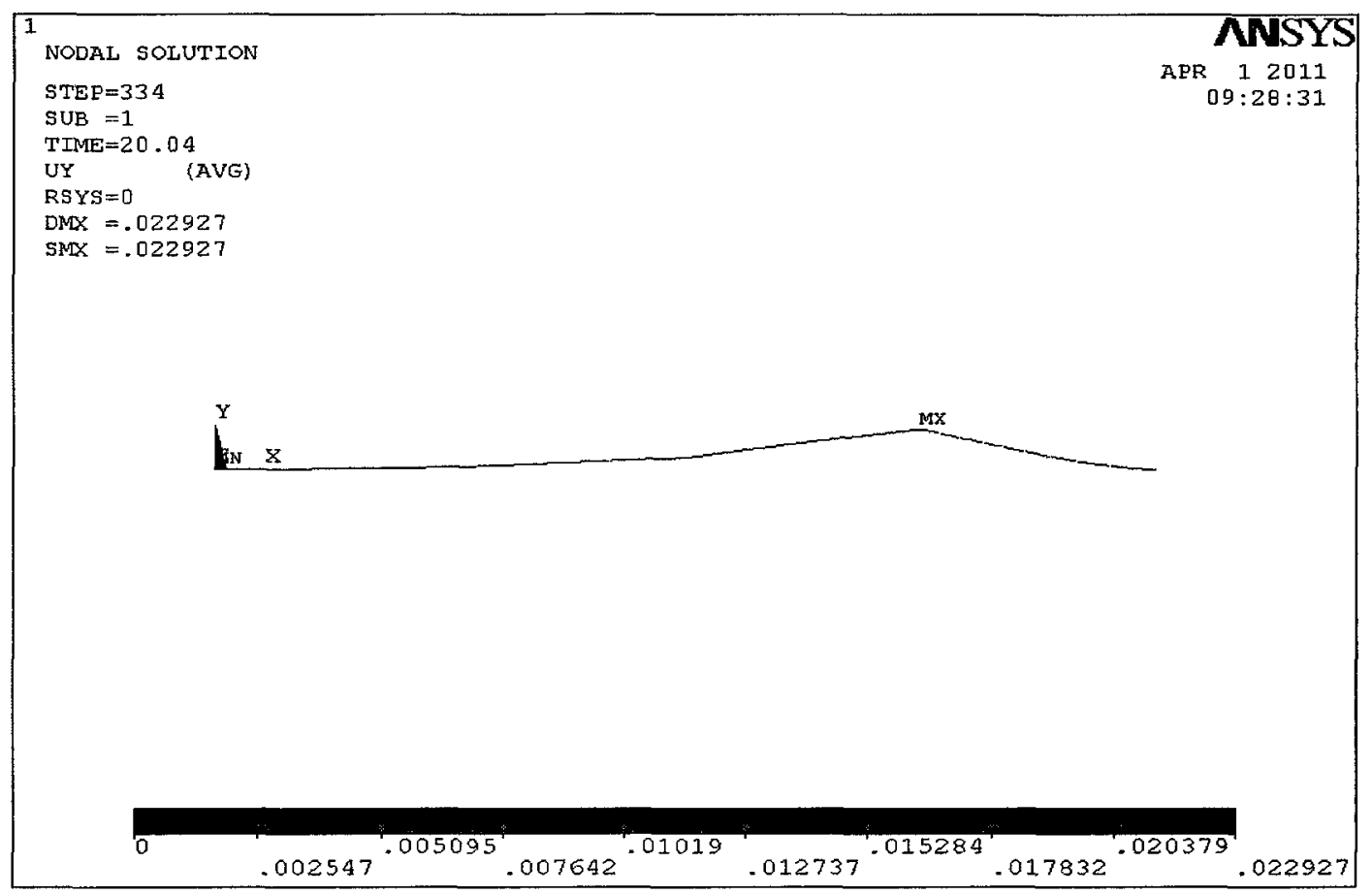

Figure 5.19: Vertical displacement in hinge-connected floating bridge for different position of the vehicle load along the bridge using ANSYS

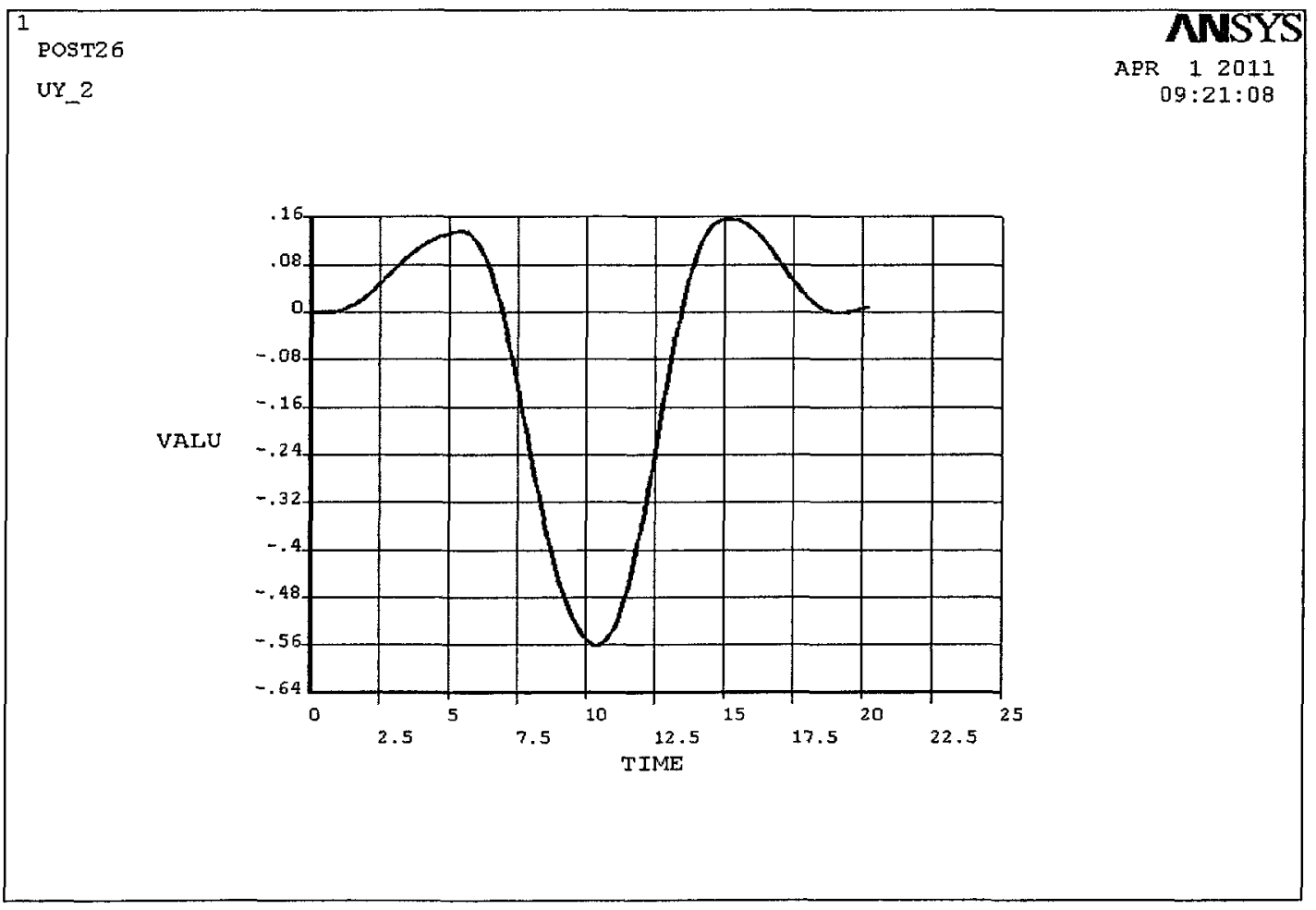

Figure 5.20 Time history of midpoint displacement in hinge-connected floating bridge due to 50-tonne single-axle vehicle loads using ANSYS software package 


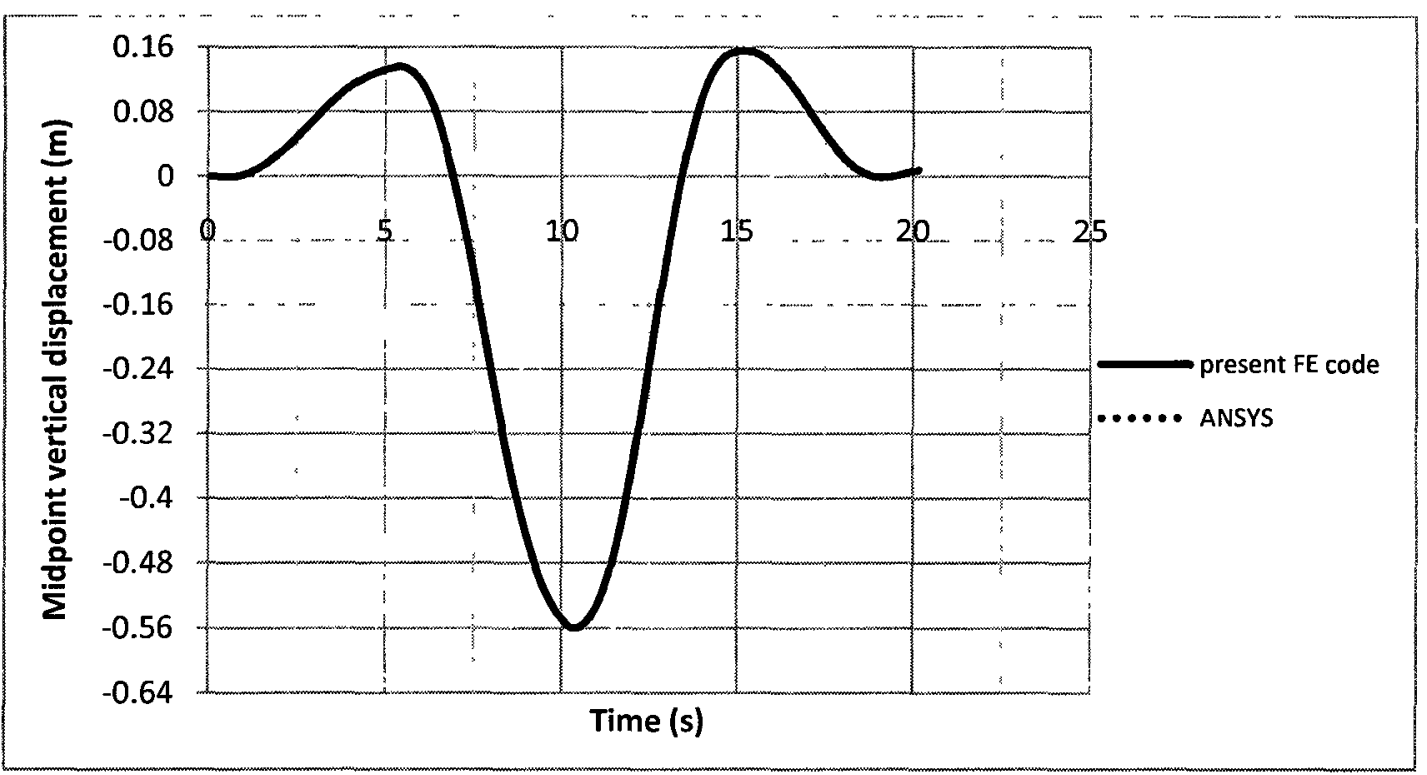

Figure 5.21 Time history of midpoint displacement in hinge-connected floating bridge due to 50-tonne single-axle vehicle loads using ANSYS and the developed FE program 


\section{Chapter: Summary, Conclusions and Recommendations for Future Work}

\subsection{Summary}

The main objectives of the present study are to develop an analytical technique and implement it into a finite element program as a useful tool for the dynamic analysis and assessment of floating bridges and to investigate the dynamic response of rigid-connected and hinge-connected rapid deployment floating bridges to the passage of single and multiple vehicles considering different vehicle weights, speeds and separation distances between successive vehicles. A further objective of the study is to optimize the transport capacity of rapid deployment floating bridges in terms of the parameters controlling the rate of flow of vehicles on the bridge, namely, the vehicle weight, the vehicle speed and the separation distances between successive vehicles. In addition the study investigates the use of light-weight materials for improving the global dynamic response of floating bridges and the three-dimensional interactive dynamic behaviour of floating bridges under the combined action of moving vehicle loads and dynamic water current loads. These objectives have been fulfilled through an organized research plan.

Chapter 1 provided a general introduction to floating bridges, problem definition, objective and scope, research plan and organization of thesis. Chapter 2 presented a comprehensive literature review including the design and analysis of floating bridges, their behaviour under moving loads, dynamic response of conventional bridges to moving loads and vehicle-bridge interaction, and the response of floating bridges subjected to water current and wave loads. Based on the main findings from the literature review, the 
proposal for a technique to be used in the studies reported in the succeeding chapters on the dynamic analysis of floating bridges is presented. In Chapter 3, a technique for the analysis of the response of vehicle-bridge-fluid system is developed and implemented into a finite element program developed by the author for the dynamic analysis of floating bridges under the effect of moving vehicle loads and water current loads. The equations of motion of the vehicle-bridge-fluid system are derived for different vehicle models including single-axle and multiple-axle models and for the passage of single and multiple vehicles across the bridge.

In Chapter 4, the transport capacity and dynamic response of rigid-connected and hingeconnected rapid deployment floating bridges under the passage of single and multiple vehicles is evaluated considering different vehicle weights, vehicle speeds, and separation distances between successive vehicles and considering different vehicle models. The effect of vehicle eccentricity on the dynamic response of floating bridges is also investigated. The dynamic response of light-weight floating bridges to moving vehicle loads and the use of light-weight materials for improving the global dynamic response of floating bridges are investigated. Finally, the three-dimensional interactive dynamic behaviour of floating bridges under the combined action of moving vehicle loads and dynamic water current loads along with possible eccentricities of these loads is investigated. Water current loads are treated as dynamic loads that change with both time and space coordinates and are determined based on the actual dynamic response of the bridge profile, and are incorporated into the dynamic equations of vehicle-bridge-fluid system. 
In Chapter 5, verification of the developed analytical approach and custom finite element program for the dynamic analysis of floating bridges is described through comparison with several examples taken from the literature. This chapter also describes the dynamic analysis of floating bridges subjected to moving vehicle loads using ANSYS software package. Finally, the sixth chapter covers a summary of the research study, main findings and conclusions, and recommendations for future work.

Even though the finite element analysis/program presented in this thesis has adequately modeled the floating bridge more validation is required before it can be used for design/analysis of floating bridges. Due to the cost of experimental testing very few test results are available for a comprehensive validation; nonetheless some validation has been carried out against other studies using either closed form solutions or highly simplistic floating models. Also, the situation where the loss of contact of bridge with water has not been adequately addressed in this thesis.

The following section summarizes the main findings and conclusions from the present research study.

\subsection{Conclusions}

The following conclusions can be drawn from the results obtained in the present research:

- The developed finite element program can be used for the dynamic analysis of floating bridges subjected to different types of vehicle and axle load combinations. The developed model is easy and flexible to use in comparison with other available commercial software packages. 
- The developed model provides excellent tool to investigate the best available options of vehicle weight and speed combinations to optimize the transport capacity of rigid-connected as well as hinge-connected rapid deployment floating bridges.

- The developed model was utilized to establish values for DAF for both displacement and bending moment for the use in the design and evaluation of floating bridges.

- The dynamic response of floating bridges to two-axle vehicle loads is smaller than that to single-axle vehicle loads.

- For rigid-connected floating bridge, the maximum vertical displacements due to two-axle vehicle loads are slightly less than those due to single-axle vehicle loads, while the maximum bending moments due to two-axle vehicle loads are significantly less than those due to single-axle vehicle loads, for all vehicle weights and speeds.

- For rigid-connected floating bridge, the DAF for deflection due to two-axle vehicle loads are slightly lower than those due to single-axle vehicle loads, while the DAF for bending moment due to two-axle vehicle loads are higher than those due to single-axle vehicle loads, for all vehicle weights and speeds.

- For hinge-connected floating bridge, the maximum vertical displacements and bending moments due to two-axle vehicle loads are significantly smaller than those due to single-axle vehicle loads for all vehicle weights and speeds.

- For hinge-connected floating bridge, the DAF for displacement due to two-axle vehicle loads are higher than those due to single-axle vehicle loads, while the 
DAF for bending moment due to two-axle vehicle loads are slightly higher than those due to single-axle vehicle loads, for all vehicle weights and speeds.

- The deflection in the rigid-connected aluminum floating bridge is higher than that in the steel bridge due to the lower modulus of elasticity of aluminum.

- The vertical displacement in the hinge-connected aluminum floating bridge is the same as the vertical displacement in the hinge-connected steel bridge.

- The dynamic response of floating bridges can be improved by utilizing lightweight materials, especially in the case of hinge-connected bridges.

- The maximum deflections in rigid-connected floating bridge due to two successive vehicles are higher than those due to single vehicle for all vehicle speeds, and those deflections increase as the separation distance between successive vehicles decreases and the two vehicles get closer to each other.

- The maximum bending moments developed in the rigid-connected floating bridge due to two successive vehicles are equal to or higher than those due to single vehicle and depending on vehicle speed and separation distance between the two vehicles.

- The maximum displacements in the hinge-connected floating bridge due to two successive vehicles were found to be inconsistent with vehicle speed and the separation distance between two passing vehicles. This conclusion required further investigation especially for shorter separation distances between passing vehicles.

- The maximum bending moments developed in the hinge-connected floating bridge due to two successive vehicles are higher than those due to single vehicle. 
- For the studied case of hinge-connected floating bridge subjected to the two successive vehicle-loads, it is recommended to avoid having the separation distance between the two vehicles such that the two vehicle-loads act over intermediate hinges at the same time in order to avoid higher dynamic response.

- The analysis showed that water current velocity has a significant effect on the deformations and straining actions developed in a floating bridge. The deformations and straining actions increase as water current velocity increases.

\subsection{Recommendations for Future Work}

The work presented in this thesis provides researchers with an insight into the structural behaviour of rapid deployment floating bridges under the dynamic effect of transverse and longitudinal loading conditions. Several areas of research were considered in this research and several conclusions were drawn. However, one of the important finding of this pioneering work is the fact that more research will be needed in the future in order to fully understand the complex problem associated with rapid deployment floating bridges and to be able to realize the advantages and limitations of these important structures. The following areas have been identified for future work:

- While the developed finite element model provides a good starting tool to analyze and investigate several complex dynamic loading conditions, experimental verification and field test data are required in order to improve and fine tune the prediction capabilities of the model.

- The results of the finite element model suggested the important role played by the type of the floating bridge, i.e., rigid-connected or hinge-connected. However, the 
actual modeling and study of the connections or joints used in the bridge and quantifying their contribution to the performance of rapid deployment floating bridges will enhance the overall structural behaviour of these bridges.

- The effects of the forces caused by braking and acceleration of the vehicle on the dynamic response of floating bridges are critical to the safety and smooth flow of the vehicles on the bridge and therefore require further investigation.

- The preliminary analysis of the use of aluminum instead of steel in the fabrication of the pontoons of the floating bridge showed potential benefits in terms of weight and performance. It is recommended that composite materials should be considered in the future investigations of this type of bridges.

Finally, while most of these recommendations can be carried out using the developed model or more advanced versions of the model actual experimental and field data will be needed to build confidence and reliability in the analytical and numerical results of these studies. 


\section{References}

Ali, M.T., Anan, A., "Hydrodynamic interaction for a long array of freely floating 3-D rectangular boxes", Proceedings of the International Conference on Mechanical Engineering 2009 (ICME2009), Dhaka, Bangladesh, ICME09-RT-25, 2009.

Biggs, J.M., "Introduction to structural dynamics", McGraw-Hill Book Company, New York, ISBN 07-005255-7, 1964.

Brennen, C.E., "A review of added mass and fluid inertial forces", Naval Civil Engineering Laboratory, Port Hueneme, California, USA, Report No. CR 82.010, 1982.

Chen, W.F., Duan, L., "Bridge Engineering Handbook", CRC Press LLC, Boca Raton, Florida, USA, 1999.

Chung, H., Chen, S.S., "Hydrodynamic mass", Components Technology Division, Argonne National Laboratory, Argonne, Illinois, USA, Report No. CONF-840647--9, 1984.

Daghigh, M., Koulaei, R.T.P., Seif, M.S., "Mooring system design and optimization for floating bridge of Urmia lake", 21st International Conference on Offshore Mechanics and Arctic Engineering, Oslo, Norway, pp. 613-620, 2002.

Das, S.N., Das, S.K., "Mathematical model for coupled roll and yaw motions of a floating body in regular waves under resonant and non-resonant conditions", Applied Mathematical Modelling, Vol. 29, pp. 19-34, 2005. 
Dusenberry, D.O., Zarghamee, M.S., Liepins, A.A., Luft, R.W., Kan, F.W., "Failure of the Lacey V. Murrow Floating Bridge, Seattle, Washington", Journal of Performance of Constructed Facilities, Vol., 9, No. 1, pp. 4-23, 1995.

Ertekin, R.C., Riggs, H.R., Kim, J.W., Demirbilec, Z., "Hydroelastic analysis of floating bridges in current", Proceedings of the 20th International Conference on Offshore Mechanics and Arctic Engineering, OMAE'01, OMAE2001/OSU-5029, pp. 175-183, 2001.

Farad, M., Laflamme, M., Savard, M., Bennur, M., "Dynamic analysis of existing continuous bridge", Journal of Bridge Engineering, ASCE, Vol. 3, No. 1, pp. 28-37, 1998.

Fleischer, D., Park, S.K., "Plane hydroelastic beam vibrations due to uniformly moving one axle vehicle", Journal of Sound and Vibration, Vol. 273, No. 3, pp. 585-606, 2004.

Fryba, L., "Vibration of solids and structures under moving loads", Noordhoff International Publishing, Groningen, 1972.

Fujikubo, M., Tetsuya, Y., "Structural modeling for global response analysis of VLFS", Marine Structures, Vol. 14, pp. 295-310, 2001.

Georgiadis, C., "Finite element modeling of the response of long floating structures under harmonic excitation", Proceedings of the Third International Offshore Mechanics and Arctic Engineering Symposium, American Society of Mechanical Engineering ASME, Vol. 1, pp. 246-252, 1984. 
Harre, W., "Experimental realization of a pretentious testing task on the field of pioneer bridge structure", Otto-Graf-Journal, Vol. 13, pp. 129-139, 2002.

Henchi, K., Fafard, M., Talbot, M., Dhatt, G., "An efficient algorithm for dynamic analysis of bridges under moving vehicles using a coupled modal and physical components approach", Journal of Sound and Vibration, Vol. 212, No. 4, pp. 663-683, 1998.

Humar, J.L., Kashif, A., "Dynamic response of bridges under travelling loads", Canadian Journal of Civil Engineering, Vol. 20, pp. 287-298, 1993.

Humar, J.L., Kashif, A., "Dynamic response analysis of slab-type bridge", Journal of the Structural Division, ASCE, Vol. 121, pp. 48-62, 1995.

Humar, J.L., "Dynamics of structures", Second Edition, Swets \& Zeitlinger B.V., Lisse, ISBN 9058092453, 2002.

Ikegami, K., Kumamoto, N., Inoue, K., Oka, S., Seto, H., Ueda, S., Maruyama, T., "Elastic response of floating bridge in waves", Proceedings of the 20th International Conference on Offshore Mechanics and Arctic Engineering, OMAE'01, Rio de Janeiro, Brazil, OMAE2001/OSU-5151, pp. 259-267, 2001.

ISSC (2006d), Report of Specialist Task Committee VI.2, "Very Large Floating Structures", In P. Frieze and R. Shenoi (Eds.), Proceedings of the 16th International Ship and Offshore Structures Congress, Elsevier, Southampton, UK, pp. 391-442, 2006. 
Jia, J., Ulfvarson, A., "Structural behaviour of a high tensile steel deck using trapezoidal stiffeners and dynamic of vehicle-deck interaction", Marine Structures, Vol. 18, pp. 1-24, 2005.

Kim, C.W., Kawatani, M., Kim K.B., "Three-dimensional dynamic analysis for bridgevehicle interaction with roadway roughness", Computers \& Structures, Vol. 83, Issues 19-20, pp. 1627-1645, 2005.

Majka, M., Hartnett, M., "Effects of speed, load and damping on the dynamic response of railway bridges and vehicles", Computers and Structures, Vol. 86, pp. 556-572, 2008.

Newman, J.N., "Marine hydrodynamics", The MIT Press Cambridge, Massachusetts, and London, England, VM156.N48, ISBN 0-262-14026-8, 1977.

Olson, R.M., Wright, S.J., "Essentials of engineering fluid mechanics", Fifth Edition, HARPER \& ROW, PUBLISHERS, Inc., 10 East 53d Street, New York, NY 10022, 1990.

Oka, S., Kumamoto, N., Inoue, K., Ikegami, K., Seto, H., Ueda, S., Maruyama, T., "Elastic response analysis method for floating bridges in waves", Mitsubishi Heavy Industries, Ltd. Technical Review Vol. 37, No. 2, pp. 40-44, 2000.

Paik, J.K., Thayamballi, A.K., "A concise introduction to the idealized structural unit method for nonlinear analysis of large plated structures and its application", Thin-Walled Structures, Vol. 41, pp. 329-355, 2003. 
Paultre, P., Proulx, J., Talbot, M., "Dynamic testing procedures for highway bridges using traffic loads", Journal of Structural Engineering, ASCE, Vol.121, No. 2, pp. 362$376,1995$.

Peterson, S.T., McLean, D.I., Anderson, M., Pollock, D.G., "Measurement and evaluation of mooring cable forces during storm events on the evergreen point floating bridge" Transportation Research Board (TRB) 82nd Annual Meeting, pp. 28-36, 2003.

Pontoon bridge, Wikipedia, the free encyclopedia.

http://en.wikipedia.org/wiki/Pontoon_bridge

Qiu, L.C., "Numerical simulation of transient hydroelastic response of a floating beam induced by landing loads”, Applied Ocean Research, Vol. 29, pp. 91-98, 2007.

Qiu, L.C., "Modeling and Simulation of transient responses of a flexible beam floating in finite depth water under moving loads", Applied Mathematical Modelling, Vol. 33, No. 3, pp. 1620-1632, 2009.

Rao, S.S., "The finite element method in engineering", Fourth Edition, Elsevier Science \& Technology Books, 2004.

Rosenthal, D., Connor, R., Peterson, J.C., "Trilateral design and test code for military bridging and gap crossing equipment", USA, 1996.

Sannasiraj, S.A., Sundar, V., Sundaravadivelu, R., "Mooring forces and motion responses of pontoon-type floating breakwaters", Ocean Engineering, Vol. 25, No. 1, pp. 27-48, 1998. 
Segerlind, L.J., "Applied finite element analysis", Second Edition, JOHN WILEY \& SONS Inc., TA347.F5S43, ISBN 0-471-80662-5, 1984.

Seif, M.S., Inoue, Y., "Dynamic analysis of floating bridges", Marine Structures, Vol. 11, pp. 29-46, 1998 .

Seif, M.S., Koulaei, R.T.P., "Floating bridge modeling and analysis", Scientia Iranica, Vol. 12, No. 2, pp. 199-206, 2005.

Shixiao, F., Weicheng, C., Xujun, C., Cong, W., "Hydroelastic analysis of a nonlinearly connected floating bridge subjected to moving loads", Marine Structures, Vol. 18, pp. 85$107,2005$.

Slater, J.E., "A Review of hydrodynamic added mass inertia of vibrating submerged structures", Research and Development Branch, National Defense Canada, Technical Memorandum 84/F, 1984.

Techet, A.H., 2.016 Hydrodynamics (13.012), Open Course, Massachusetts Institute of Technology, version 3, 2006.

Thambiratnam, D., Zhuge, Y., "Dynamic analysis of beams on an elastic foundation subjected to moving loads" Journal of Sound and Vibration, Vol. 198, No. 2, pp. 149$169,1996$.

Wang, C., Shixiao, F., Weicheng C., "Ribbon bridge in waves based on hydroelasticity theory", Frontiers of Architecture and Civil Engineering in China, Vol. 3, No. 1, pp. 57$62,2009$. 
Watanabe, E., Utsunomiya, T., "Analysis and design of floating bridges", Steel Construction, Progress in Structural Engineering and Materials, Vol. 5, Issue 3, pp. 127$144,2003$.

Watanabe, E., Utsunomiya, T., Wang, C.M., "Hydroelastic analysis of pontoon-type VLFS: A literature survey”, Engineering Structures, Vol. 26, pp. 245-256, 2004.

Wu, J.S., Sheu, J.J., "An exact solution for a simplified model of the heave and pitch motions of a ship hull due to a moving load and a comparison with some experimental results" Journal of Sound and vibration, Vol. 192, No. 2, pp. 495-520, 1996.

Wu, J.S., Shih, P.Y., "Moving-load-induced vibrations of a moored floating bridge", Computer and Structure, Vol. 66, No. 4, pp. 435-461, 1998.

Xia, D., Kim, J.W., Ertekin, R.C., "On the hydroelastic behaviour of two-dimensional articulated plates", Marine Structures, Vol. 13, pp. 261-278, 2000.

Yang, Y.B., Yau, J.D., "Vehicle-bridge interaction element for dynamic analysis", Journal of Structural Engineering, ASCE, Vol. 123, No. 11, pp. 1512-1518, 1997.

Yang, Y.B., Wu, Y.S., "A versatile element for analyzing vehicle-bridge interaction response”, Engineering Structures, Vol. 23, Issue 5, pp. 452-469, 2001.

Zhang, Q., Vrouwenvelder, A., Wardenier, J., "Numerical simulation of train-bridge interactive dynamics", Computer and Structures, Vol. 79, pp. 1059-1075, 2001. 
Zhang, J., Miao, G.P., Liu, J.X., Sun, W.J., "Analytical models of floating bridges subjected by moving loads for different water depths", Journal of Hydrodynamics, Vol. 20, No. 5, pp. 537-546, 2008. 


\section{Appendices}

\section{Appendix A: Parametric Study for the Structural Behaviour of Floating Bridges Subjected to Lateral Water Current Loads}

The objective of this parametric study is to investigate the effects of various parameters on the structural behaviour of floating bridges when subjected to water current loads and supporting boat forces. The parameters studied include water current velocity, number of supporting boats and their locations and the magnitude of force each boat exerts on the bridge. The bridge studied here consists of twelve pontoons, each of $7 \mathrm{~m}$ length, rigidly connected to each other in the transverse direction to form a bridge of total length $84 \mathrm{~m}$. The bridge is discretized into 168 elements each of $0.5 \mathrm{~m}$ length. An average submerged depth of the bridge is assumed as $0.5 \mathrm{~m}$.

\section{A.1 Structural Behaviour of Floating Bridge under Water Current Loads}

First, the structural behaviour of floating bridges when subjected to lateral water current loads at various levels of current velocity of $0.5,1,1.5$ and $2 \mathrm{~m} / \mathrm{s}$ with no supporting boats provided is investigated. On comparing the values of the lateral straining actions and displacements in the bridge due to different water current velocities (Figure A.1), it is clear that the lateral straining actions and displacements are all greatly affected by the water current velocity. The straining actions and the displacements along the bridge length are much greater for higher water current velocity. 


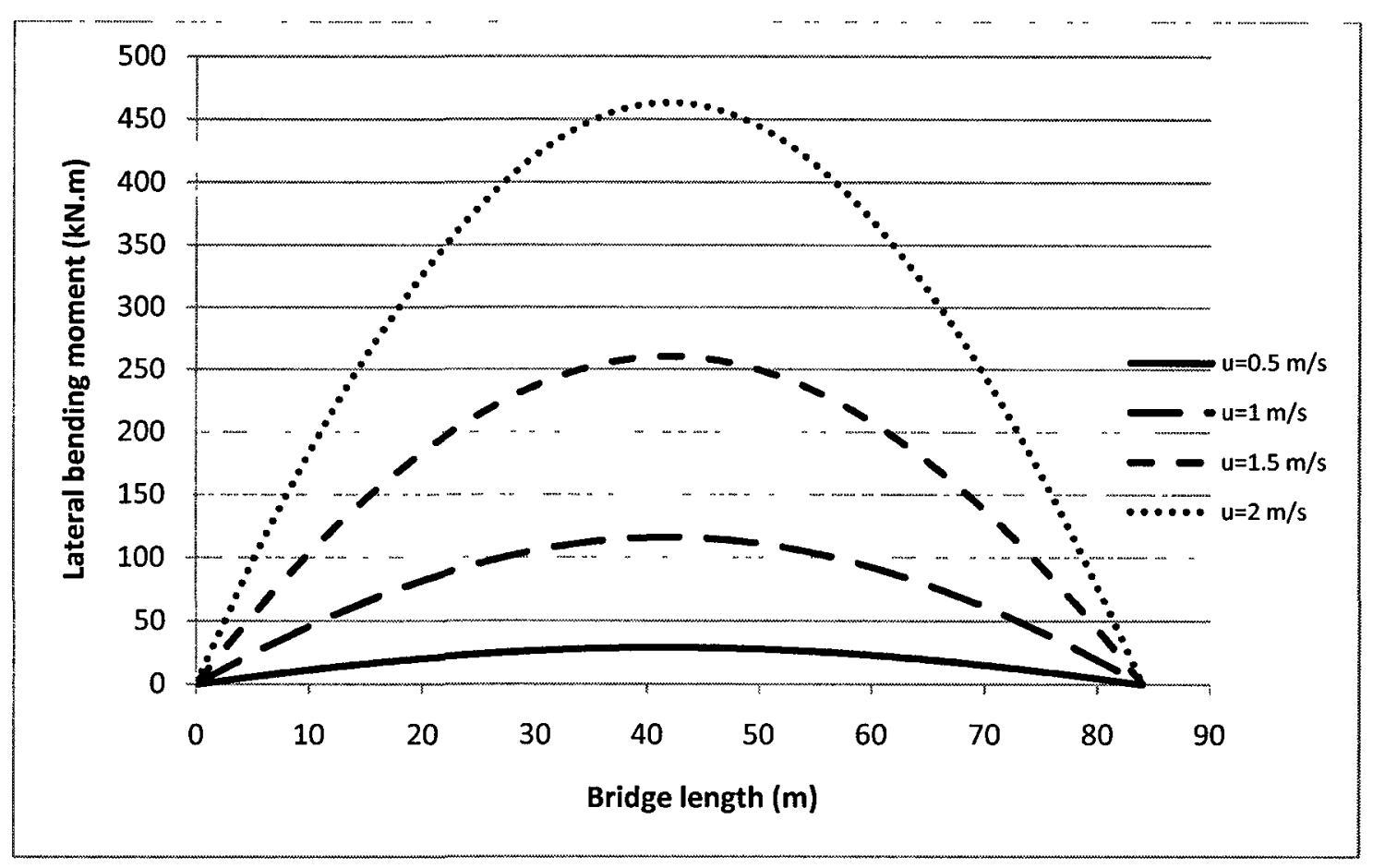

(a) Lateral bending moment

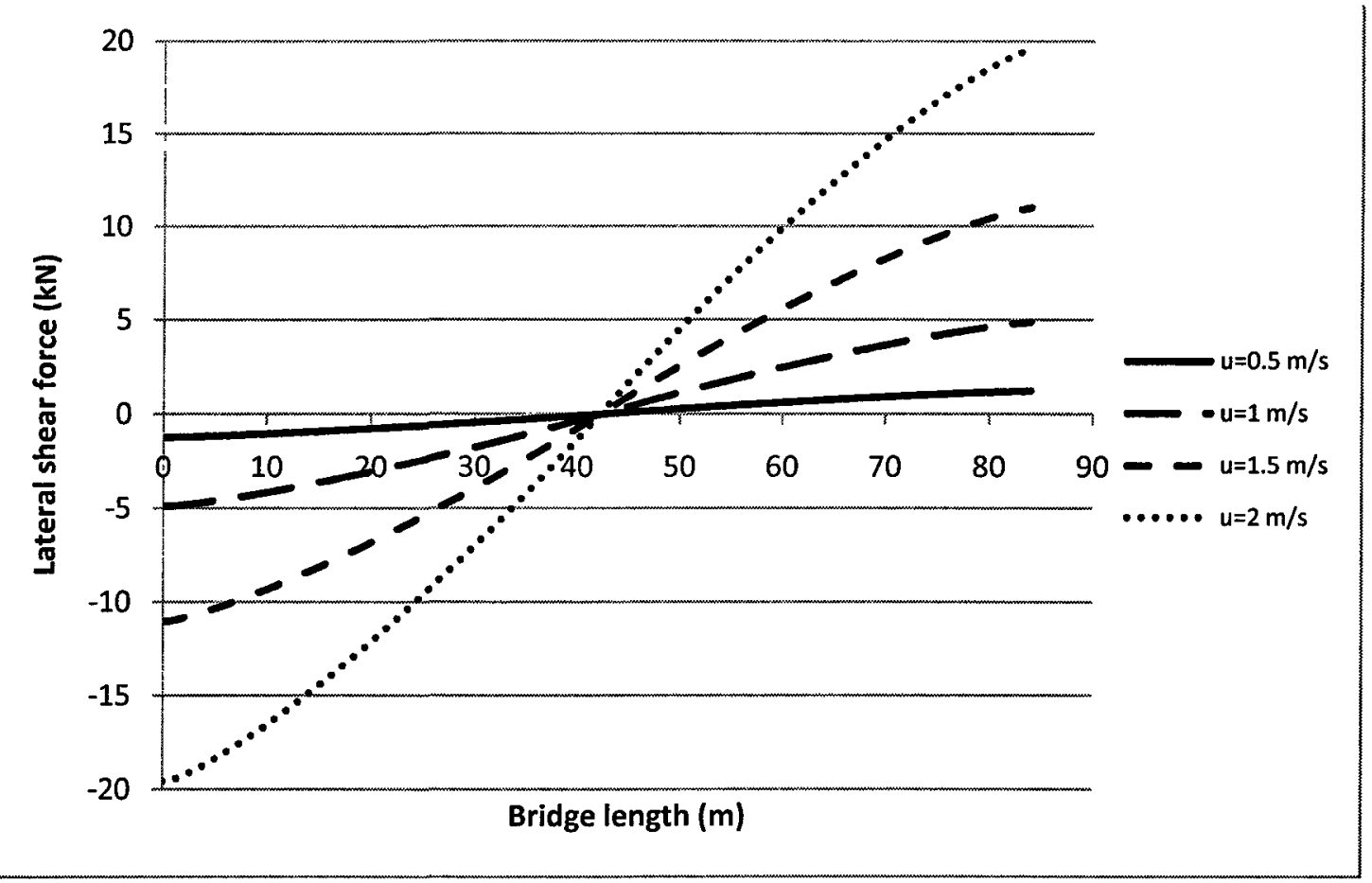

(b) Lateral shear force 


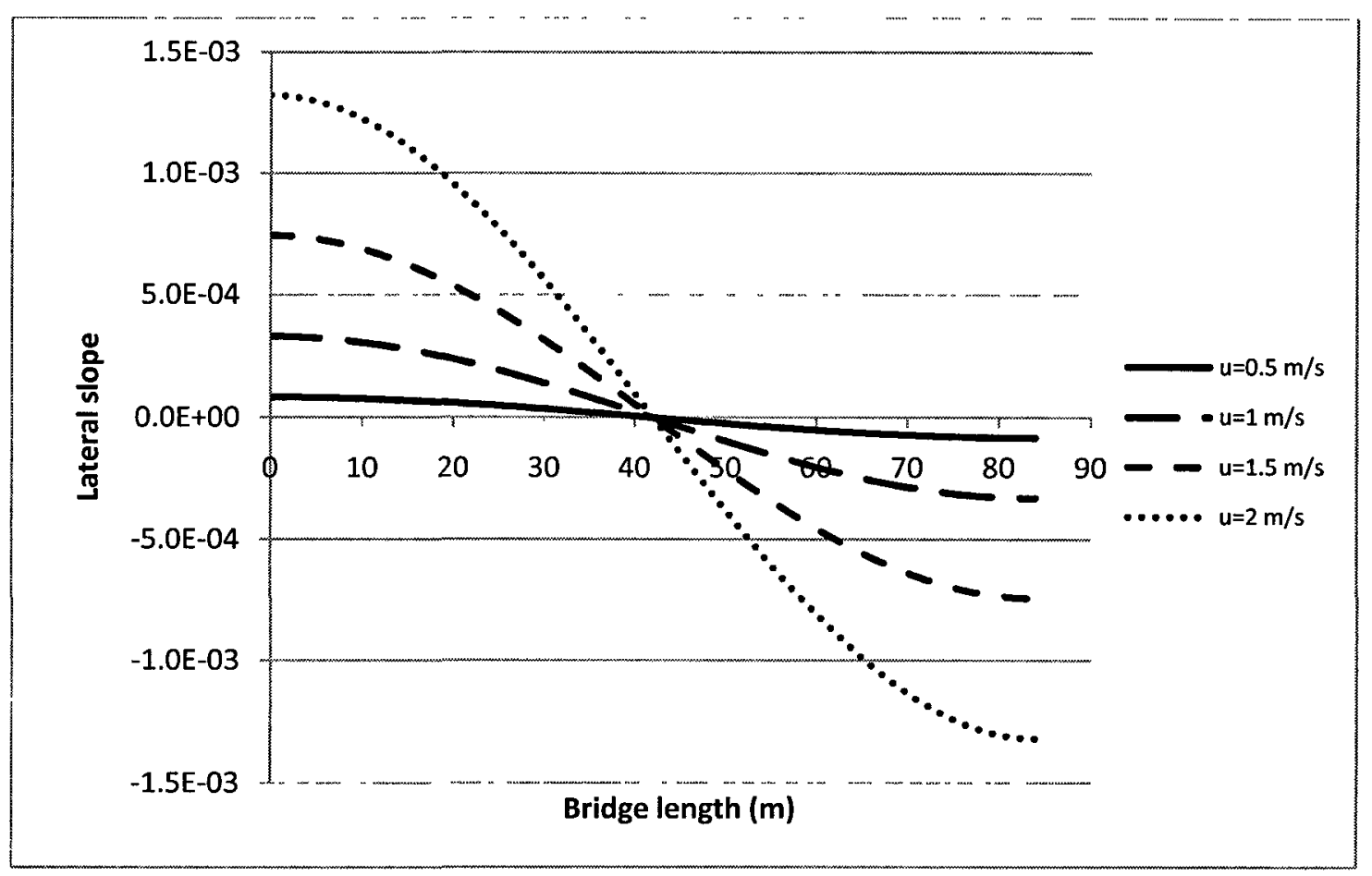

(c) Lateral slope

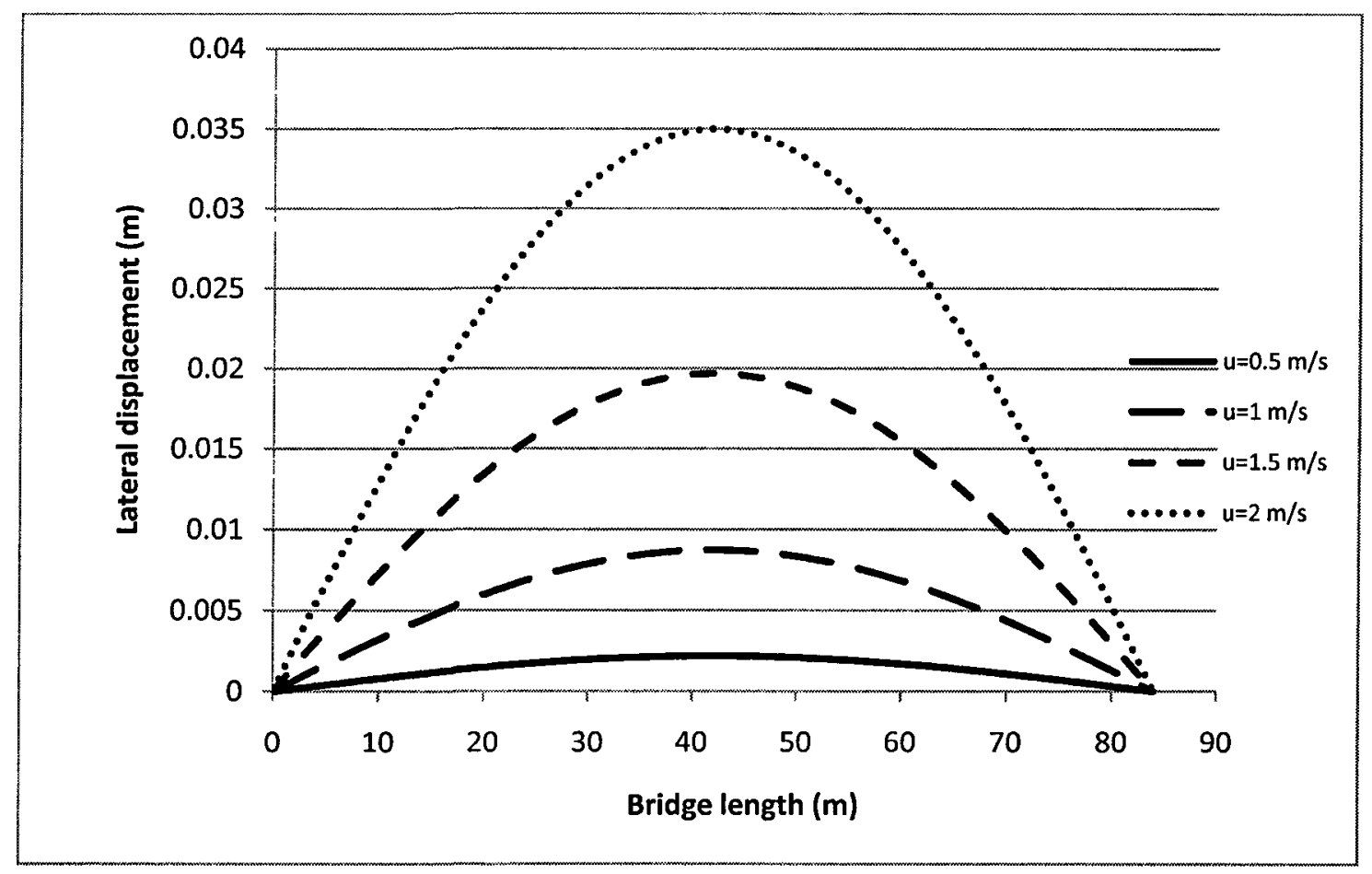

(d) Lateral displacement 


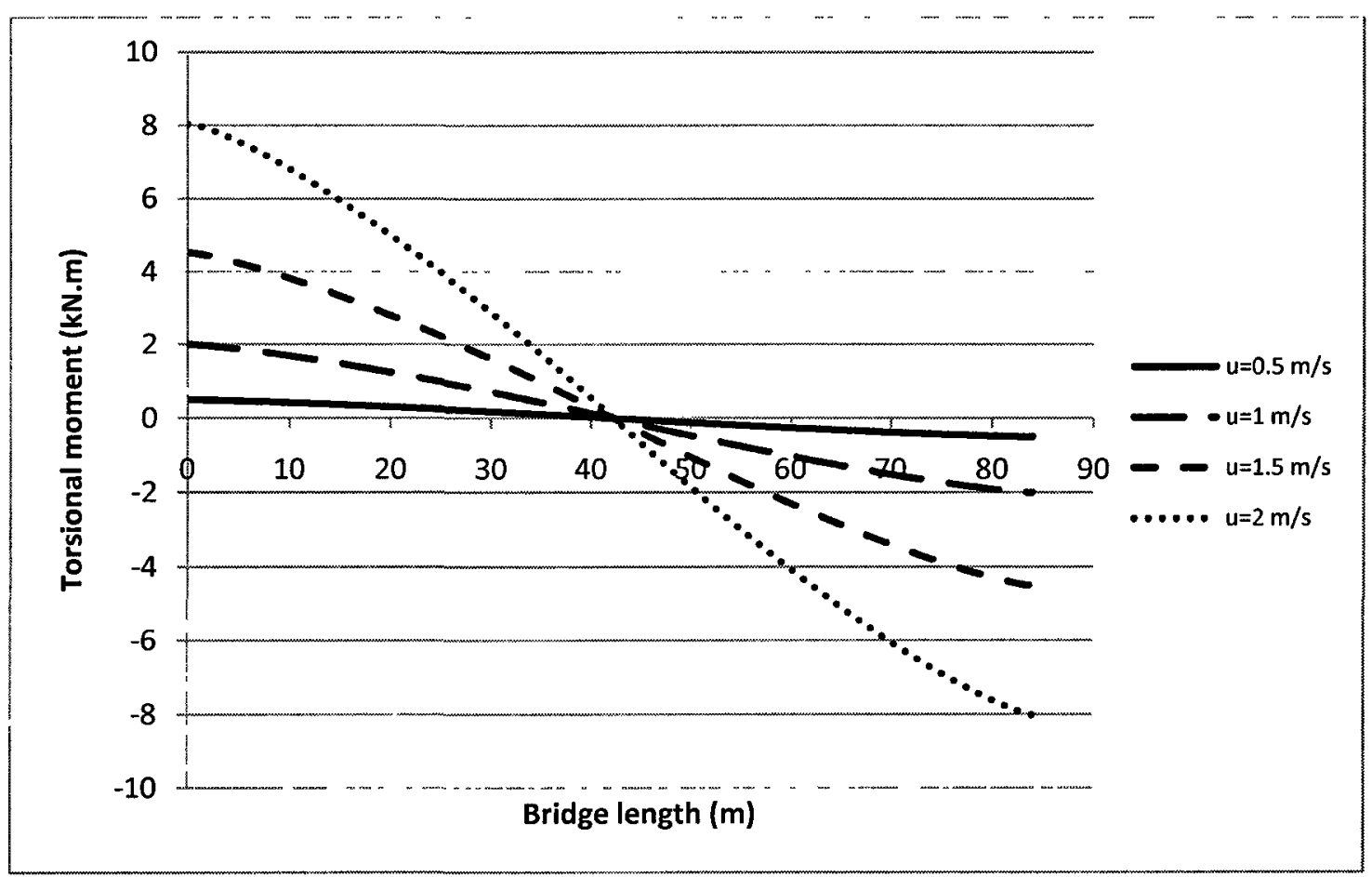

(e) Torsional moment

Figure A.1: Effect of water current velocity on the straining actions and deformations along the bridge (No supporting boats)

\section{A.2 Effect of Supporting Boat Force on the Structural Behaviour of Floating Bridge Subjected to Water Current Loads}

In the second stage, the effect of the magnitude of force exerted by a supporting boat on the structural behaviour of a floating bridge subjected to water current loads is investigated.

Figure A.2 shows the structural behaviour of the floating bridge under the effect of water current with a velocity of $1.0 \mathrm{~m} / \mathrm{s}$. The bridge is supported laterally by one boat acting at the bridge mid-span and exerting a force of several different magnitudes: $0,2,4,6.4,8$, 10 and $12 \mathrm{KN}$. 


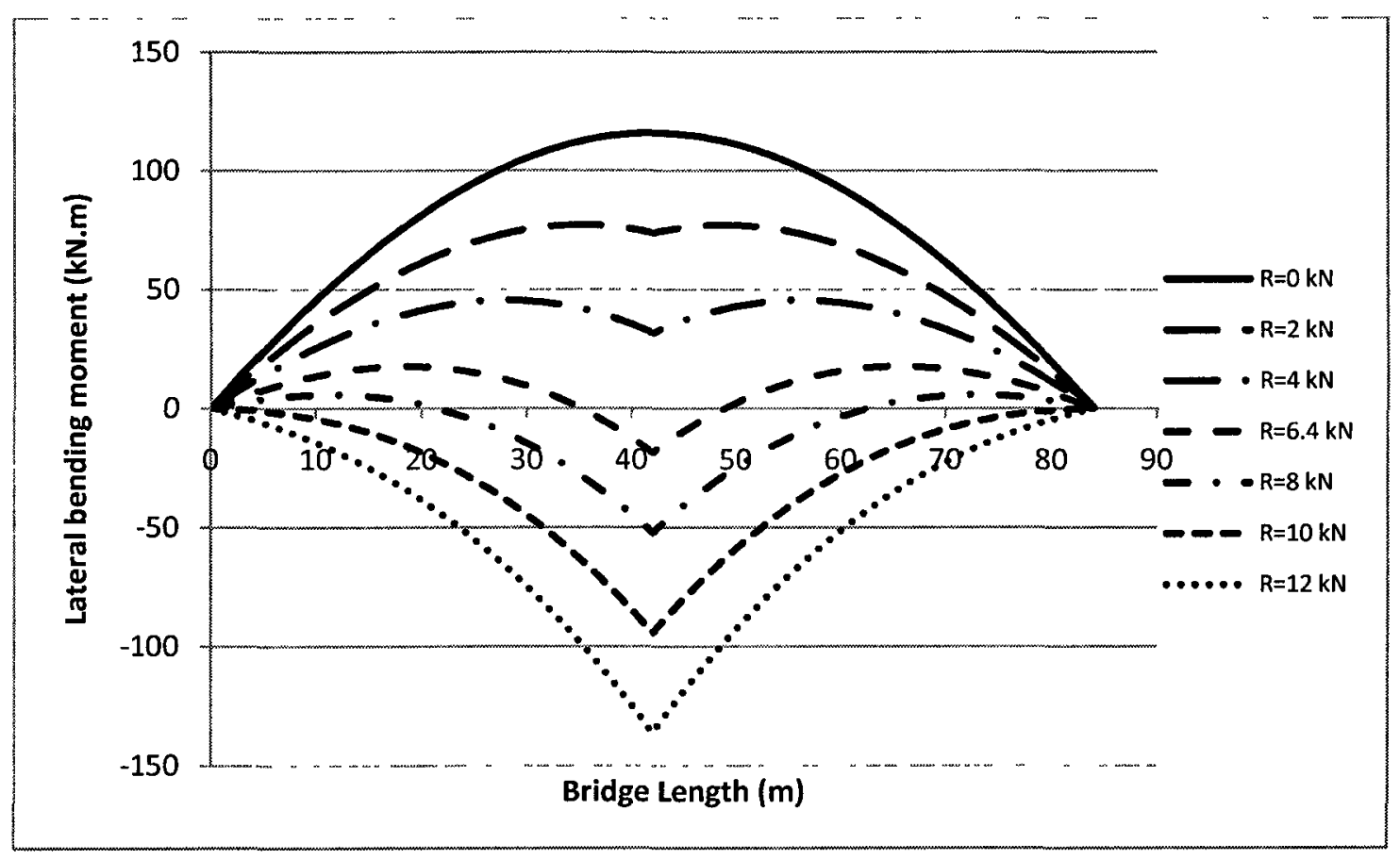

(a) Lateral bending moment

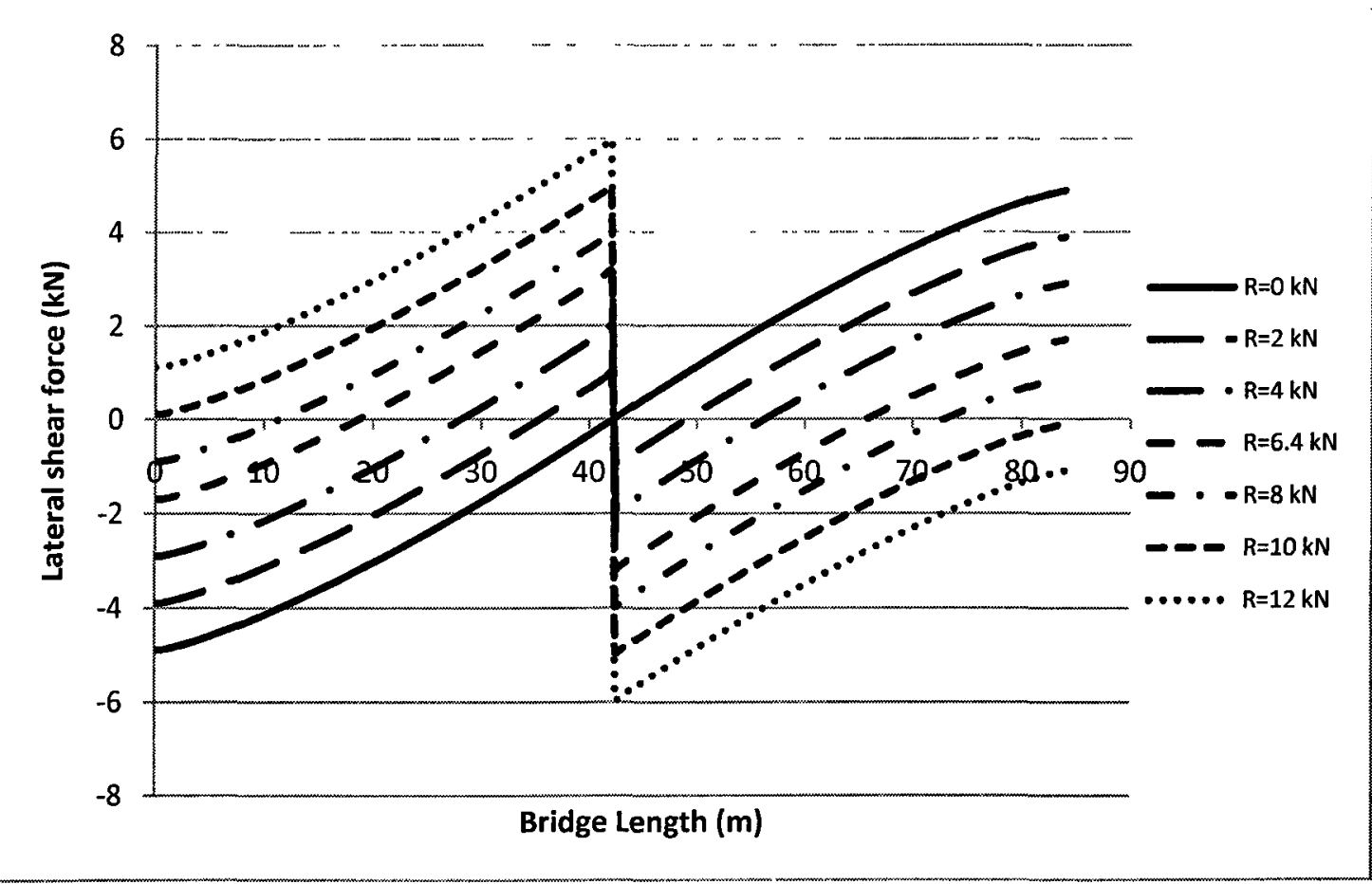

(b) Lateral shear force 


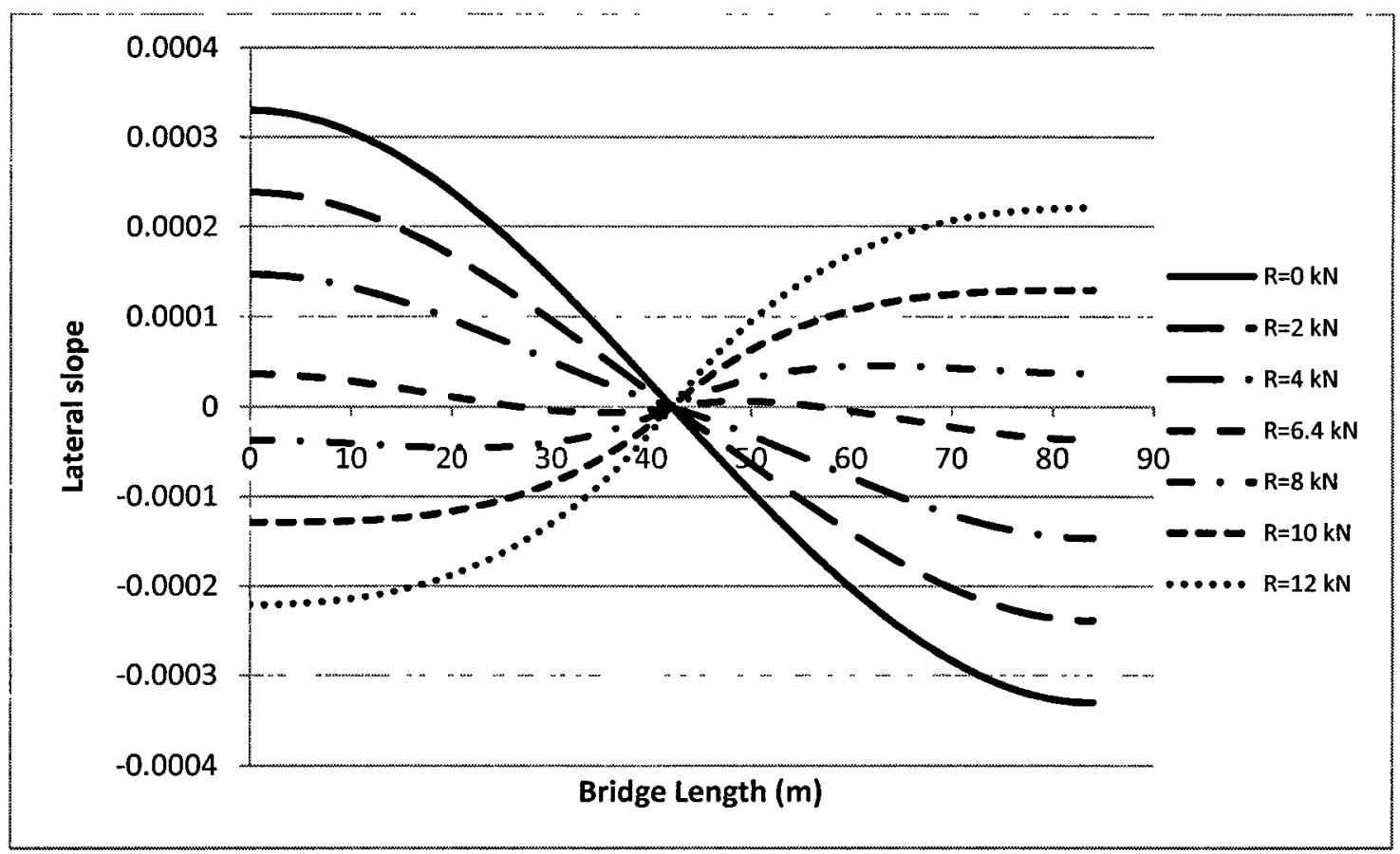

(c) Lateral slope

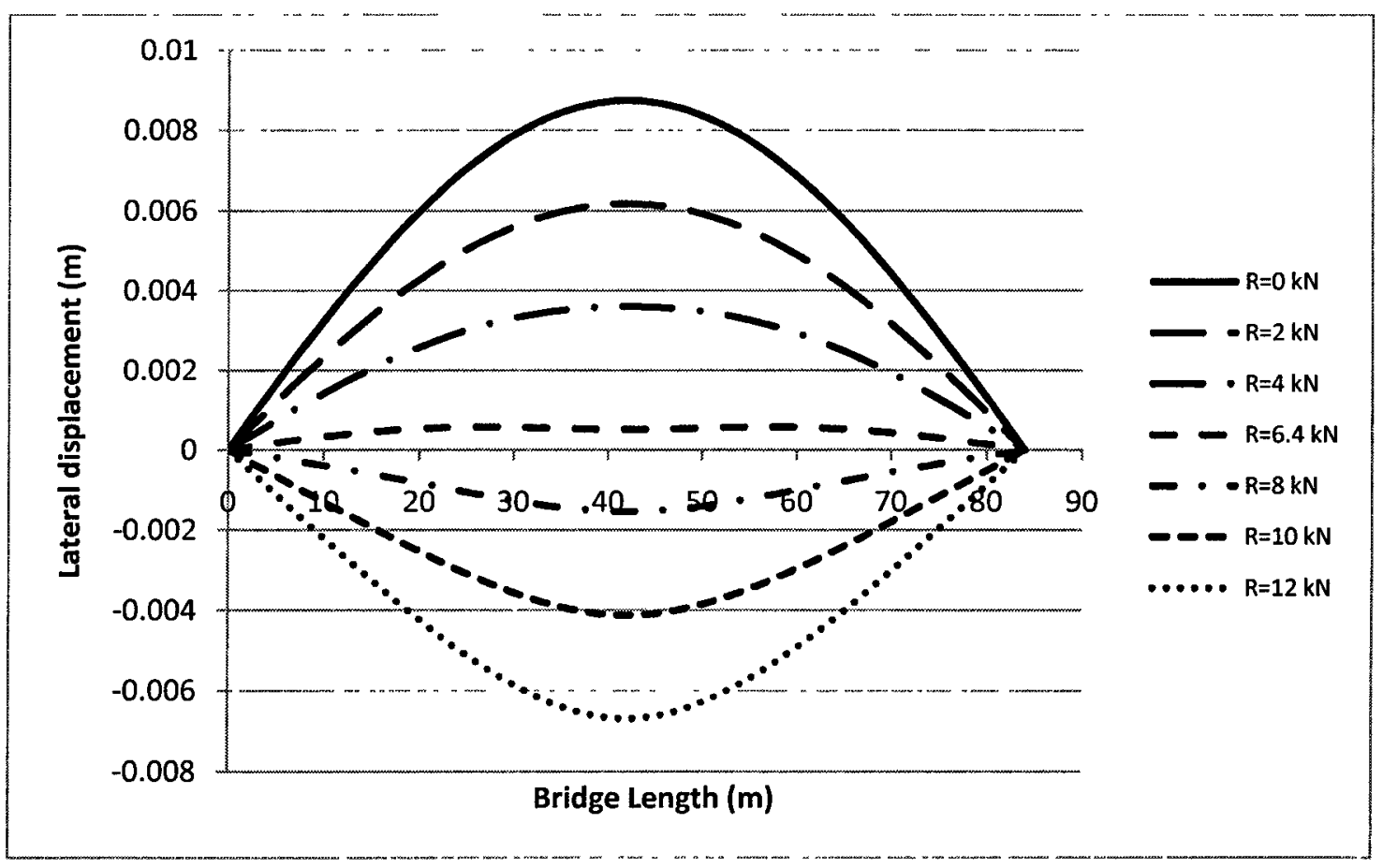

(d) Lateral displacement

Figure A.2: Effect of changes in supporting boat force on bridge structural behaviour

(One supporting boat) 
The bending moment distribution along the bridge length is greatly affected by the magnitude of force exerted by the supporting boat as shown in Figure A.2 (a). The maximum bending moment at the bridge mid-span is about $116,74,32,-19,-52,-94$ and $-136 \mathrm{KN} . \mathrm{m}$ for boat force magnitudes of $0,2,4,6.4,8,10$ and $12 \mathrm{KN}$, respectively.

Figure A.2 (b) shows the lateral shear force distribution along the bridge length. The maximum shear force is about $-5,-4,-3,3,4,5$ and $6 \mathrm{KN}$ for boat force magnitudes of 0 , $2,4,6.4,8,10$ and $12 \mathrm{KN}$, respectively. Similar trend is observed for slope and displacement in the bridge for different magnitudes of supporting boat force as shown in Figures A.2 (c) and (d), respectively.

The foregoing results imply that the structural behaviour of floating bridges under the effect of water current loads is very sensitive to changes in the magnitude of force exerted on the bridge by supporting boats. Insufficient supporting force exerted by the boat could lead to excessive lateral deformations and straining actions in the bridge, while similar excessive deformations and straining actions in the opposite direction may be produced by excessive boat force.

The straining actions and displacements developed in floating bridges due to water current loads can be controlled and minimized by using the optimum number of supporting boats and the optimum magnitude of forces they exert on the bridge.

The optimum magnitude of force a boat should exert on the bridge depends on the water current velocity, the bridge length and the number of supporting boats. In the case of 84 $\mathrm{m}$ floating bridge subjected to water current with a velocity of $1.0 \mathrm{~m} / \mathrm{s}$, a force of 
magnitude $6.4 \mathrm{KN}$ exerted by a single boat acting at the mid-span of the bridge was found to be optimum.

\section{A.3 Structural Behaviour of Floating Bridge under Water Current Loads and Supporting Boat Forces}

In the third stage, the effect of the different numbers of supporting boats and the magnitude of force they exert on the bridge against water current loads on the structural behaviour of the floating bridge is investigated.

Figure A.3 shows the structural behaviour of the floating bridge under the effect of water current loads with water current velocity of $1.0 \mathrm{~m} / \mathrm{s}$. In the first case studied, the bridge is free to sway laterally under the effect of water current loads and there are no supporting boats at all. In the second case, the bridge is supported laterally by a single boat exerting a force of $6.4 \mathrm{KN}$ at the mid-span of the bridge. In the third case, two supporting boats act at one-third and two-thirds of the bridge span exerting a total force of $7.8 \mathrm{KN}$ on the bridge. In the fourth case, three supporting boats act at one-fourth, one-half and threefourth of the bridge span exerting a total force of $8.5 \mathrm{KN}$ on the bridge.

Many analysis runs were carried out to determine the optimum magnitude of force a boat should exert on the bridge. This optimum magnitude of force results in almost equallydistributing the straining actions developed in the bridge between positive and negative so that both of them are as small as possible. The above mentioned magnitudes of supporting boat forces were found to be optimum for each case. 


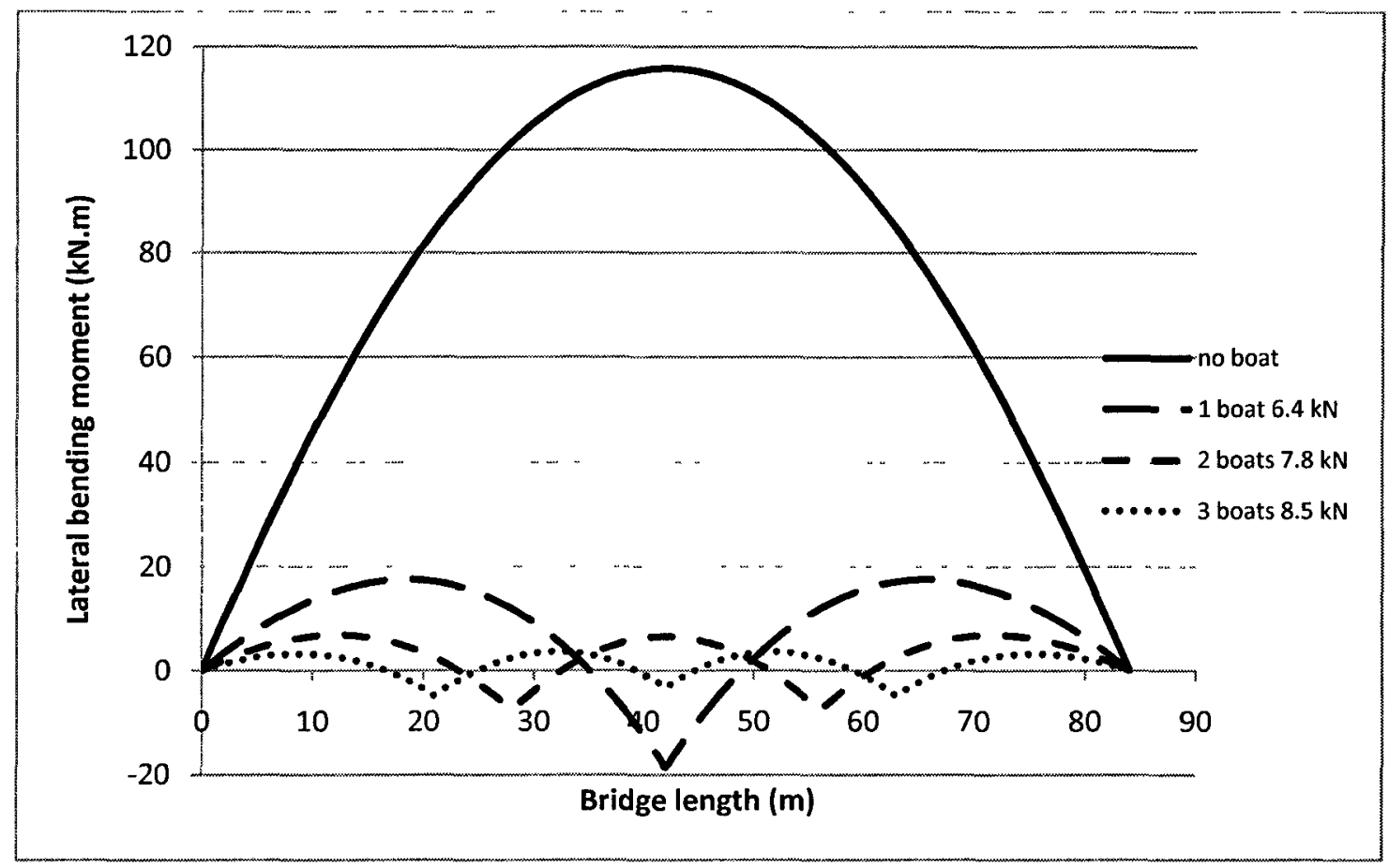

(a) Lateral bending moment

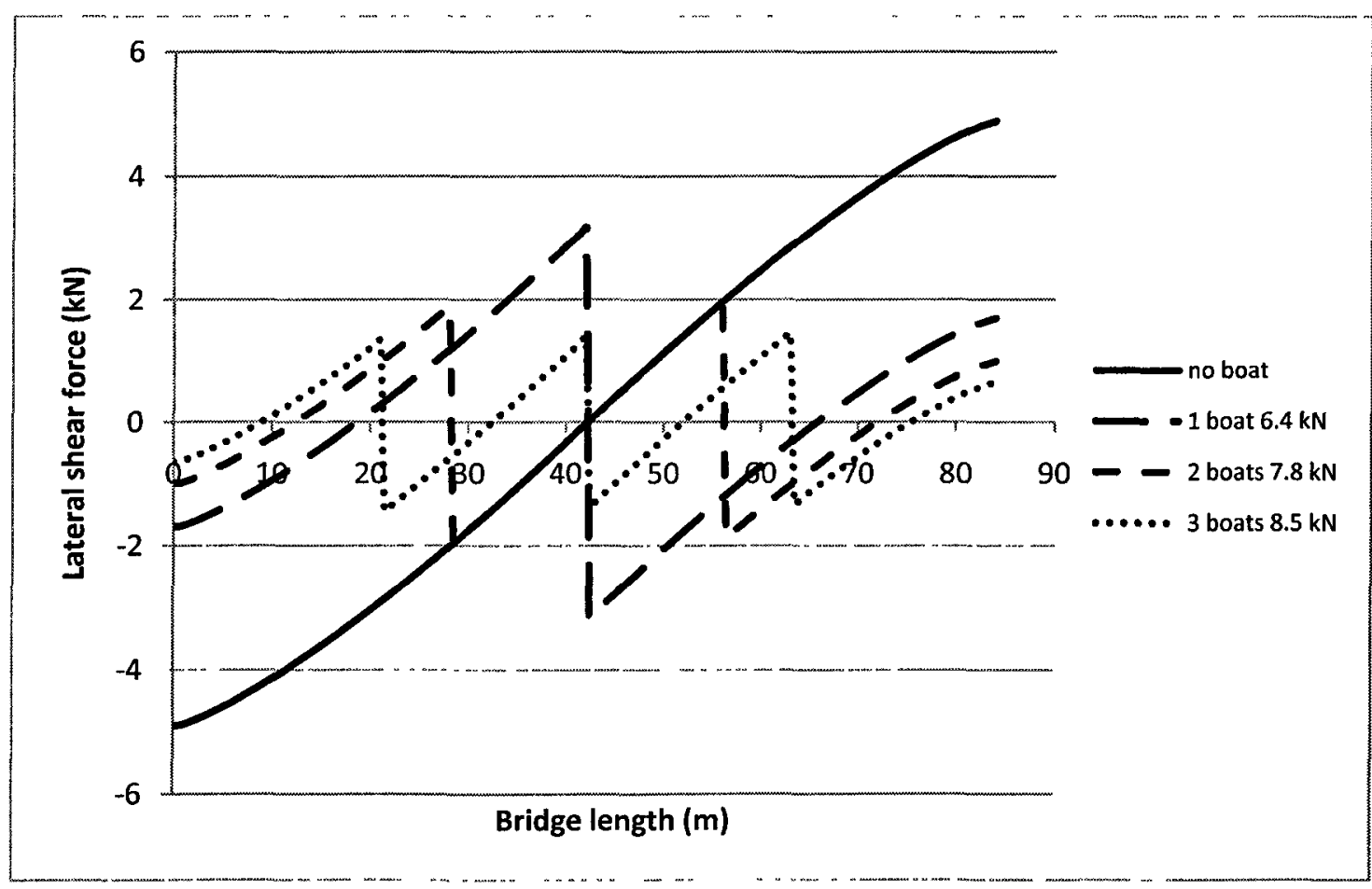

(b) Lateral shear force 


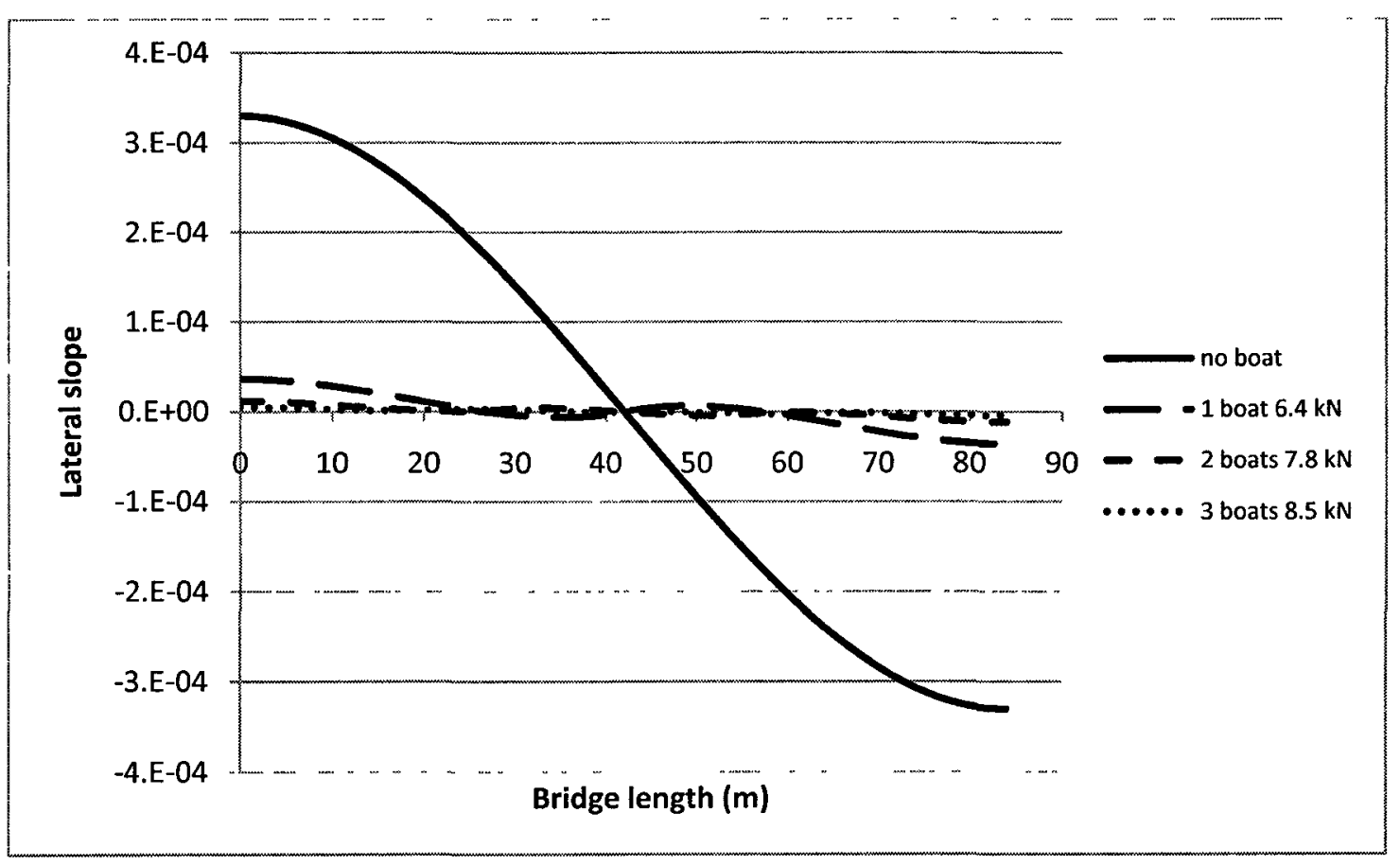

(c) Lateral slope

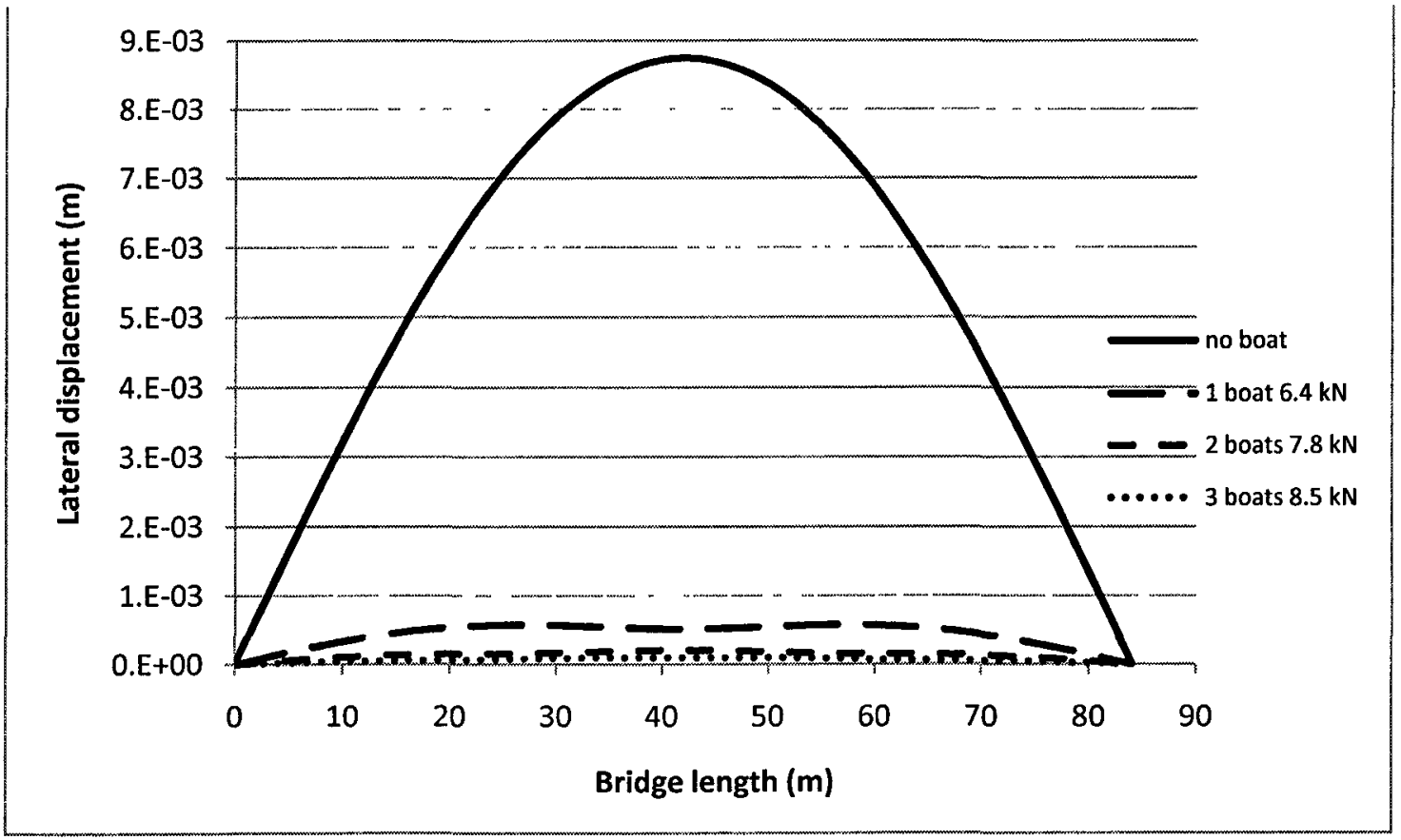

(d) Lateral displacement

Figure A.3: Effect of the existence of supporting boats on the straining actions and deformations along the bridge (Current velocity $1 \mathrm{~m} / \mathrm{s}$ ) 
The lateral bending moment distribution along the bridge length is presented in Figure A.3 (a). The maximum bending moment at the bridge mid-span is $116 \mathrm{KN} . \mathrm{m}$ in the case of no supporting boats. This value is treated as the reference. The maximum bending moment developed in the bridge is reduced to $16 \%, 6 \%$ and $4 \%$ of the reference value as a result of the existence of one supporting boat, two supporting boats and three supporting boats, respectively.

Figure A.3 (b) shows the lateral shear force distribution along the bridge length. The reference maximum shear force developed in the bridge is $5 \mathrm{KN}$ in the case of no supporting boats. The maximum shear force developed in the bridge is reduced to $60 \%$, $40 \%$ and $25 \%$ of the reference value as a result of the existence of one supporting boat, two supporting boats and three supporting boats, respectively.

Figure A.3 (d) shows the resulting lateral displacements along the bridge length. The reference maximum displacement is $8.745 \mathrm{e}-3 \mathrm{~m}$ in the case of no supporting boats. The maximum displacement in the bridge is reduced to $6 \%, 2 \%$ and $1 \%$ of the reference value as a result of the existence of one supporting boat, two supporting boats and three supporting boats, respectively.

The results show that the structural behaviour of a floating bridge under the action of water current loads is greatly affected by the existence of supporting boats, their number and locations and the magnitude of the forces they exert on the floating bridge against water current loads. 


\section{A.4 Summary of Results}

Tables A.1, A.2 and A.3 present a summary of the results obtained from stages 1,2, and 3 of the present study, respectively.

Table A.1: Summary of analysis results for stage 1 of study (No supporting boat forces)

\begin{tabular}{|c|c|c|c|c|c|}
\hline $\begin{array}{c}\text { Bridge } \\
\text { length } \\
(\mathbf{m})\end{array}$ & $\begin{array}{c}\text { Current } \\
\text { velocity } \\
(\mathbf{m} / \mathbf{s})\end{array}$ & $\begin{array}{c}\text { Max. lateral } \\
\text { displacement } \\
(\mathbf{m})\end{array}$ & $\begin{array}{c}\text { Max. lateral } \\
\text { B.M. } \\
\text { (KN.m) }\end{array}$ & $\begin{array}{c}\text { Max. torsional } \\
\text { moment } \\
(\mathbf{K N} . \mathbf{m})\end{array}$ & $\begin{array}{c}\text { Max. lateral } \\
\text { shear force } \\
(\mathbf{K N})\end{array}$ \\
\hline 84 & 0.5 & $2.186 \mathrm{e}-3$ & 28.941 & 0.501 & 1.223 \\
\hline 84 & 1.0 & $8.745 \mathrm{e}-3$ & 115.764 & 2.006 & 4.892 \\
\hline 84 & 1.5 & $19.676 \mathrm{e}-3$ & 260.469 & 4.513 & 11.008 \\
\hline 84 & 2.0 & $34.979 \mathrm{e}-3$ & 463.056 & 8.024 & 19.570 \\
\hline
\end{tabular}

Table A.2: Summary of analysis results for stage 2 of study (Including supporting boat forces)

\begin{tabular}{|c|c|c|c|c|c|c|c|}
\hline $\begin{array}{c}\text { Bridge } \\
\text { length } \\
(\mathbf{m})\end{array}$ & $\begin{array}{c}\text { Current } \\
\text { velocity } \\
(\mathbf{m} / \mathbf{s})\end{array}$ & $\begin{array}{c}\text { No. of } \\
\text { supp. } \\
\text { boats }\end{array}$ & $\begin{array}{c}\text { Total } \\
\text { supp. } \\
\text { forces } \\
(\mathbf{K N})\end{array}$ & $\begin{array}{c}\text { Max. lateral } \\
\text { displacement } \\
(\mathbf{m})\end{array}$ & $\begin{array}{c}\text { Max. } \\
\text { lateral } \\
\text { B.M. } \\
(\text { KN.m) }\end{array}$ & $\begin{array}{c}\text { Max. } \\
\text { torsional } \\
\text { moment } \\
(\text { KN.m) }\end{array}$ & $\begin{array}{c}\text { Max. } \\
\text { lateral } \\
\text { S.F. } \\
(\text { KN) }\end{array}$ \\
\hline 84 & 1.0 & 0 & 0 & $8.745 \mathrm{e}-3$ & 115.764 & 2.006 & 4.892 \\
\hline 84 & 1.0 & 1 & 6.4 & $0.576 \mathrm{e}-3$ & -18.636 & 2.006 & 3.163 \\
\hline 84 & 1.0 & 2 & 7.8 & $0.1983 \mathrm{e}-3$ & -7.638 & 2.006 & 1.957 \\
\hline 84 & 1.0 & 3 & 8.5 & $0.091 \mathrm{e}-3$ & -4.814 & 2.006 & 1.443 \\
\hline
\end{tabular}


Table A.3: Summary of analysis results for stage 3 of study (Including supporting boat forces)

\begin{tabular}{|c|c|c|c|c|c|c|c|}
\hline $\begin{array}{c}\text { Bridge } \\
\text { length }\end{array}$ & $\begin{array}{c}\text { Current } \\
\text { velocity } \\
(\mathbf{m})\end{array}$ & $\begin{array}{c}\text { No. of } \\
\text { supp. } \\
\text { boats }\end{array}$ & $\begin{array}{c}\text { Total } \\
\text { supp. } \\
\text { forces } \\
(\mathbf{K N})\end{array}$ & $\begin{array}{c}\text { Max. lateral } \\
\text { displacement }\end{array}$ & $\begin{array}{c}\text { Max. } \\
\text { lateral } \\
\text { B.M. }\end{array}$ & $\begin{array}{c}\text { Max. } \\
\text { torsional } \\
\text { moment } \\
\text { (KN.m) }\end{array}$ & $\begin{array}{c}\text { Max. } \\
\text { lateral } \\
\text { S.F. } \\
(\mathbf{K N})\end{array}$ \\
\hline 84 & 1.0 & 0 & 0 & $8.745 \mathrm{e}-3$ & 115.764 & 2.006 & 4.892 \\
\hline 84 & 1.0 & 1 & 2 & $6.172 \mathrm{e}-3$ & 77.149 & 2.006 & 3.890 \\
\hline 84 & 1.0 & 1 & 4 & $3.600 \mathrm{e}-3$ & 45.562 & 2.006 & 2.890 \\
\hline 84 & 1.0 & 1 & 6.4 & $0.576 \mathrm{e}-3$ & -18.636 & 2.006 & 3.163 \\
\hline 84 & 1.0 & 1 & 8 & $-1.545 \mathrm{e}-3$ & -52.236 & 2.006 & 3.960 \\
\hline 84 & 1.0 & 1 & 10 & $-6.690 \mathrm{e}-3$ & -136.236 & 2.006 & 5.960 \\
\hline
\end{tabular}

\section{A.5 Summary}

In this appendix, the structural behaviour of floating bridges subjected to lateral water current loads was investigated. The effects of water current velocity, number of supporting boats and their locations and the magnitude of force they exert on the bridge were investigated. The deformations and straining actions developed in floating bridges due to water current loads were controlled and minimized by using the optimum number of supporting boats and the optimum magnitude of forces they exert on the bridge. 


\section{Appendix B: Ribbon Bridge Composition, Construction and Operation}

This appendix presents the description of ribbon bridges, their layout, modes of securing, launch sites, and design of operational requirements. The ribbon bridge is a floating modular asset with an integral superstructure floating on water. Individual bays are joined to form bridges in support of river crossing operations. In military applications, ribbon bridges provide the maneuver commander with a reliable and responsive means to cross wet gap obstacles confronting the march. Ribbon bridge modules are complex structures, generally composed of stiffened plates, deck plates, bottom plates, I-beams, cross frames and bulkheads (see Figure B.4). Each plate, beam or frame has its own function and is characterized by a different geometry and size.

\section{B.1 Ribbon Bridge Description}

While pontoon floating bridges are widely used for civilian purposes, the main use of the ribbon floating bridge is for military purposes and in cases of emergencies and natural disasters. Fast erection of this type of bridges makes it a very important type for the passage of vehicles across waterways during military operations and also for evacuation and rescue in case of emergencies and natural disasters.

The following is a brief background about the ribbon floating bridge, including erection procedure, methods of connecting adjacent pontoons, and the behaviour of the bridge finally in service. The ribbon bridge system consists of three major components:

- Interior bays.

- Ramp bays 
- Supporting boats

In this appendix, focus is placed on the interior bays which are the main component of the bridge providing roadway for the vehicles and marchers to pass over a water barrier.

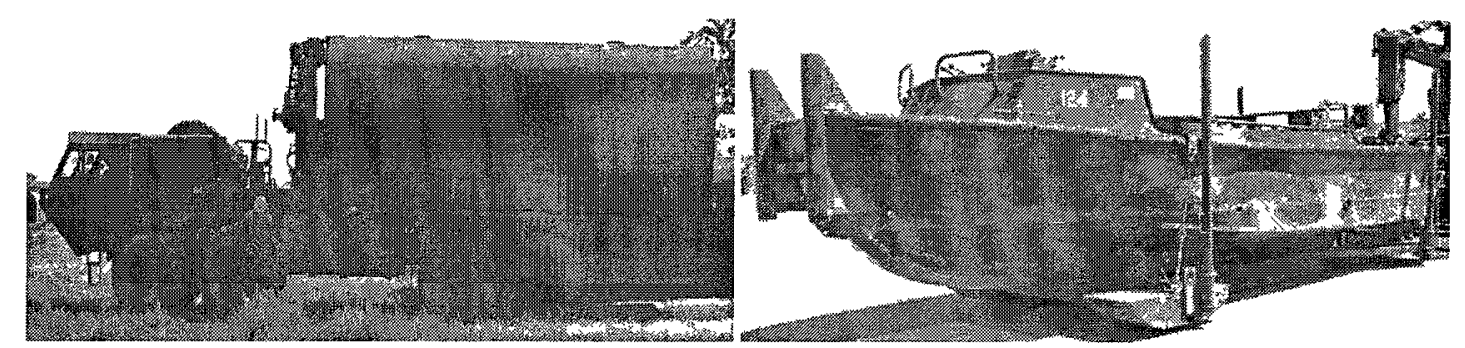

Figure B.1: Bridge transporter and supporting boat (primeportal.net)

Although bridge transporters (Figure B.1) are not a component of the ribbon bridge system; they are required for transporting the floating bridge bays to different locations, as well as for launching and retrieving the bridge bays. A bridge transporter is a truck which provides a self-contained bridge bay and used for transporting, launching and retrieving the bridge bays.

\section{B.1.1 Interior Bay}

The interior bay shown in Figure B.2 is the primary load carrying component of a ribbon bridge. Each interior bay is a four-pontoon folding module (Figure B.3) consisting of two roadway pontoons and two bow pontoons. The interior roadway pontoons are joined to each other and to the adjacent bow pontoons by hinges and pins along their adjacent edges. The roadway is connected to the successive roadway pontoons, thus eliminating the need for separate intermediate pneumatic supports. Two roadway pontoons provide a roadway, connected with each other by joints that prohibit the movements of any point 
with respect to the other except as one unit. The two bow pontoons aid in flotation and provide walkways for personnel on both sides of the roadway. The cross-section of the typical interior bay and its components are shown in Figure B.4.

The roadway consists of 2 prismatic shapes each with width of $2.03 \mathrm{~m}$, length of $6.92 \mathrm{~m}$ and depth of $1.12 \mathrm{~m}$. Each part has four sides, upper surface, lower surface, and bulkhead with each having its own characteristics, shape and function, and in general it is hollow inside.

\section{Interior bay}

\section{Dimensions}

$\begin{array}{lc}\begin{array}{l}\text { Length } \\ \text { foverall) } \\ \text { Length } \\ \text { feffective) }\end{array} & 22 \mathrm{ft} 6.5 \mathrm{in} \\ \begin{array}{l}\text { Width } \\ \text { (lokded) }\end{array} & 22 \mathrm{ft} \\ \begin{array}{l}\text { Whdth } \\ \text { (unfolded) }\end{array} & 26 \mathrm{ft} 6.6 \mathrm{in} \\ \begin{array}{l}\text { Height } \\ \text { (loklded) }\end{array} & 7 \mathrm{f} 7 \mathrm{in} \\ \begin{array}{l}\text { Height } \\ \text { (unlodded) } \\ \text { Weight }\end{array} & 3 \mathrm{ft} 8 \mathrm{in} \\ & 12,000 \mathrm{lb}\end{array}$

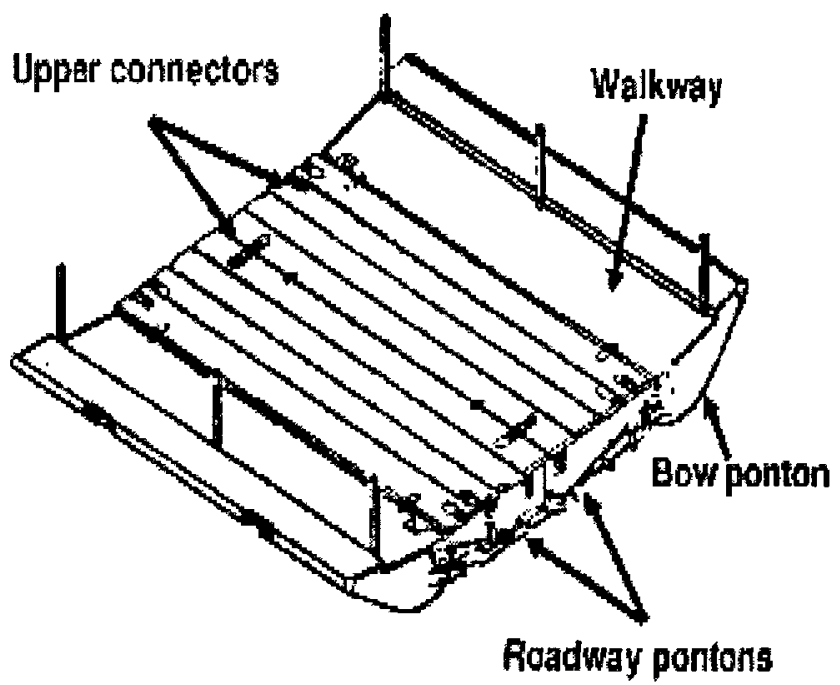

Figure B.2: Schematic layout of the interior bay of the ribbon floating bridge 


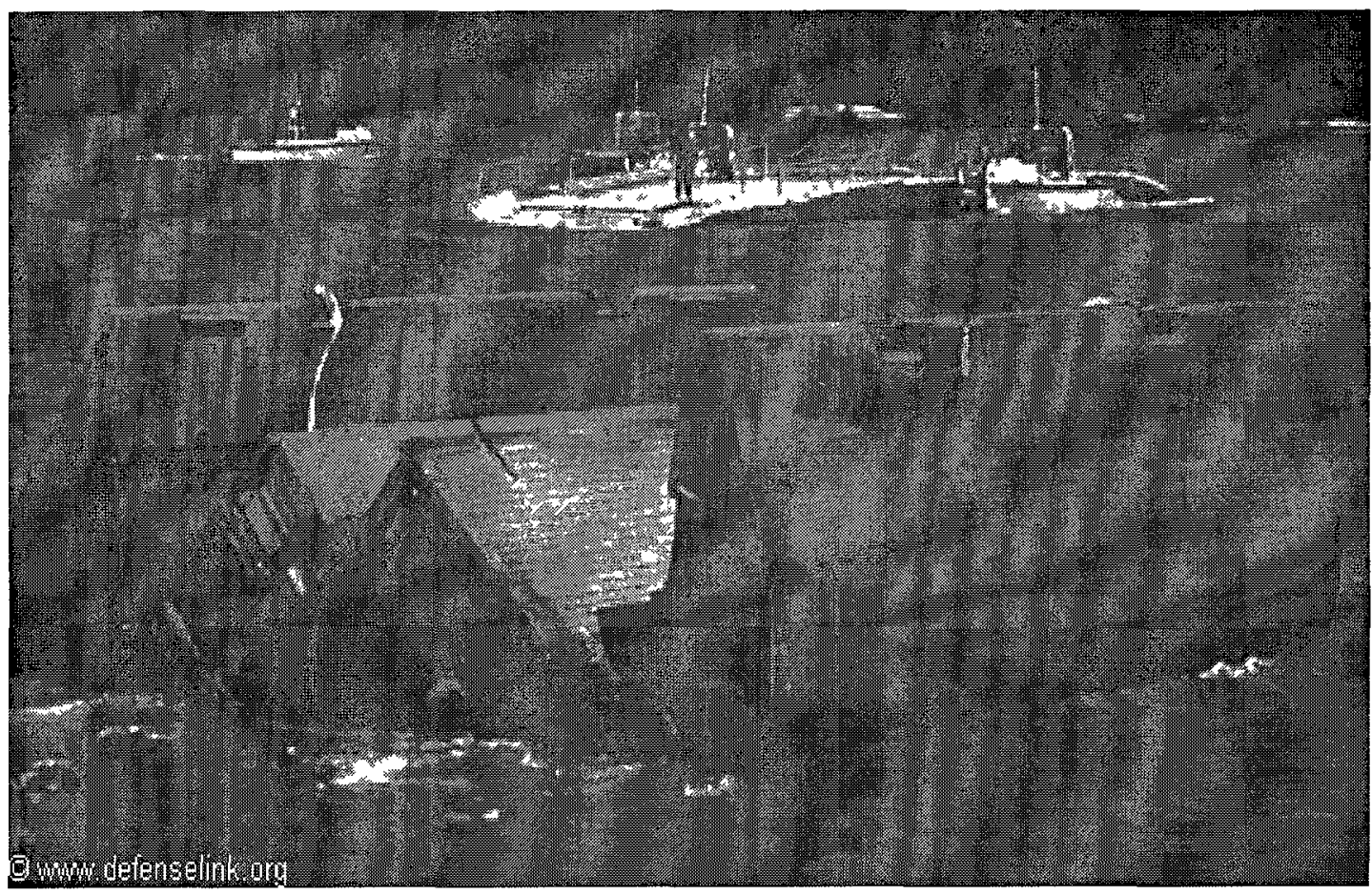

Figure B.3 Overview of the interior bay of the ribbon floating bridge (defenselink.org)
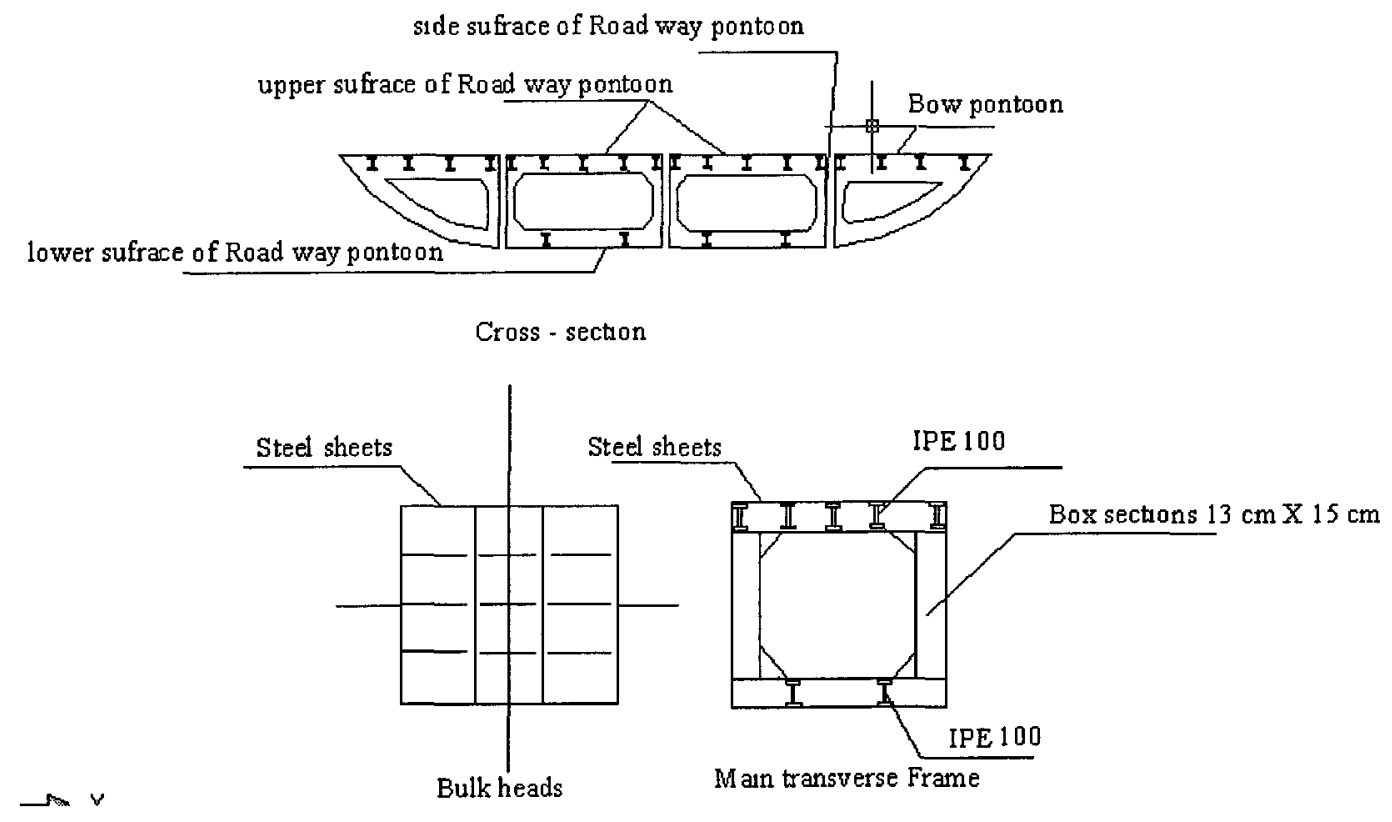

Figure B.4: Cross-section of the interior bay and its components 


\section{B.1.1.1 Road Pontoons}

The typical roadway pontoon consists of an upper surface, lower surface and sides. The structure of the upper surface (Figure B.4) consists of 5 main I-beams of $100 \mathrm{~mm}$ height (I.P.E100) in the longitudinal direction of the pontoon (the roadway direction), at a distance of $450 \mathrm{~mm}$ on centre. These I-beams are supported in the transverse direction with 9 cross frames of steel box sections at a distance of $865 \mathrm{~mm}$ on centre. The cross frames are steel sheets of $3 \mathrm{~mm}$ thickness, shaped as boxes with dimension of $13 \mathrm{~cm}$ width and $15 \mathrm{~cm}$ depth.

The structure of the lower surface consists of 2 main I-beams (I.P.E100) in the longitudinal direction of the pontoon, at a distance of $900 \mathrm{~mm}$ on centre. These I-beams are supported in the transverse direction with the same 9 cross frames of steel box sections supporting the I-beams of the upper surface.

The upper surface and lower surface of the pontoon are covered by steel sheets of $3 \mathrm{~mm}$ thickness. For the sides between the upper surface and the lower surface, the structure of the side face consists of a steel sheet of $2 \mathrm{~mm}$ thickness, supported in the transverse direction with the same 9 cross frames of steel box sections at a distance of $865 \mathrm{~mm}$ on centre. The roadway pontoon is divided by a bulkhead at the midpoint of the pontoon longitudinal direction. The structure of the bulkhead consists of a steel sheet of $2 \mathrm{~mm}$ thickness. 


\section{B.1.1.2 Bow Pontoons}

The typical bow pontoon has a prismatic shape with width of $2.03 \mathrm{~m}$, length of $6.92 \mathrm{~m}$ and depth of $1.12 \mathrm{~m}$. The structure of the bow pontoon is approximately similar to that of the roadway pontoon but with a bow shape at the outer edge.

The upper surface of the bow pontoon consists of 4 main I-beams (I.P.E100) in the longitudinal direction of the pontoon. These I-beams are supported in the transverse direction with 9 cross frames of steel box sections similar to those of the roadway pontoon. The lower surface of the bow pontoon has no I-beams; it consists of the same 9 cross frames of steel box sections. The whole structure is covered by steel a sheet of 3 $\mathrm{mm}$ thickness except for the inner side which consists of a steel sheet of $2 \mathrm{~mm}$ thickness. The bow pontoon is also divided by a bulkhead of $2 \mathrm{~mm}$ thickness at the midpoint of the pontoon longitudinal direction.

\section{B.1.2 Ramp Bay}

The ramp bay shown in Figure B.5 is similar in its structure to the interior bay, except that the bay's shore end is tapered. Ramps are always attached to both ends of a ribbon bridge. A hydraulic system located within the ramp bay permits the ramp to be raised to accommodate bank heights. Two extensions which serve as approach ramps are hinged to the roadway pontoons on the shore side of the ramp bay to allow for ease of loading and unloading vehicles from bridges. 


\section{Dimensions}

\section{Length \\ Length \\ (approach \\ ramp \\ extended)}

Width

(lolded)

Width

(uniolded)

Height

(folded)

Haight

(unfolded)

Weight
$19 \mathrm{tt} .7 \mathrm{ln}$

$25 \mathrm{ft} 4 \mathrm{ln}$

10 6 in

$26 \mathrm{tt} 8 \mathrm{ln}$

7 it 10.1 in

3 ft 7 in

$11,700 \mathrm{lb}$
Ramp bay

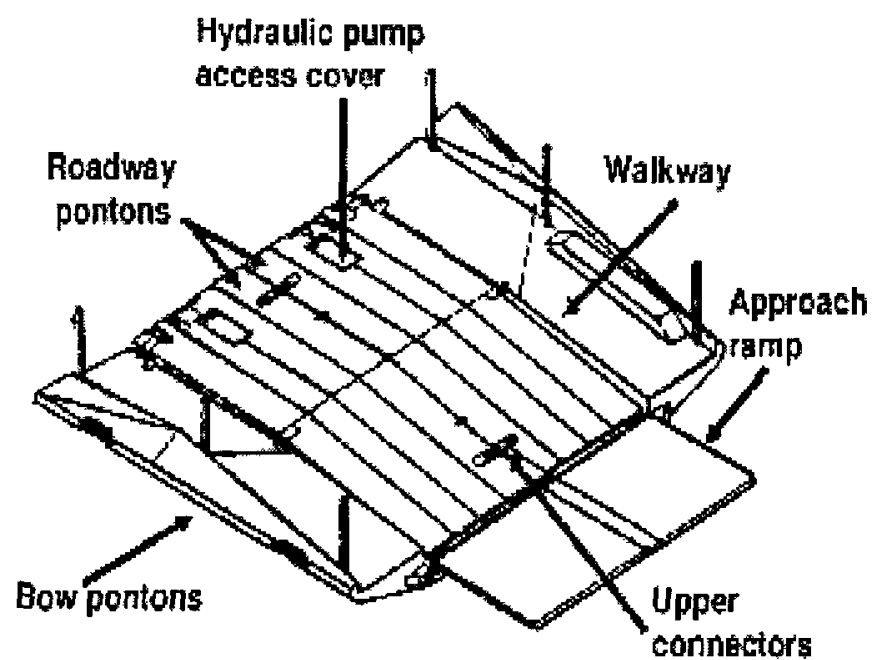

Figure B.5: Schematic layout of the ramp bay of the ribbon floating bridge

\section{B.1.3 Supporting Boats}

Motorized boats (Figure B.1) are required for the assembly and anchorage of pontoon bridges. Boats are also used to support the bridge laterally against water current loads and to reduce the lateral sway of the bridge and the lateral straining actions developed in the bridge. Sometimes a number of pontoons are connected together without any anchorage to shores to form a raft. In this case boats are required for the propulsion of these pontoon rafts from one side to the other side of a water channel while carrying a vehicle or whatsoever. 


\section{B.2 Ribbon Bridge Construction}

\section{B.2.1 Launching Sites}

Site selection depends on several factors such as the height of the banks, the bank slopes, and the depth of the water at the site. Generally, pontoons are launched downstream from bridge or rafting sites to allow for ease of construction and to prevent runaway bridge bays from damaging other bays or injuring personnel.

\section{B.2.2 Securing Bridge Bays}

The securing of bridge bays must be completed as quickly as possible so that the bays may be moved from the launching area to the actual bridge operational site. After the bridge bay has been launched and unfolded, the boats approach from the downstream direction. The front pushing knees of the boats are placed against the downstream bow and centered on the bay. The assistant boat operator secures one bowline to each of the anchoring pins on the downstream bow pontoon of the ribbon bay. The assistant then pulls each line tight and secures it to the bow bollard on its respective side of the boat (see Figure B.6). After the bridge bay is connected to the boat, the bridge crew secures the bay as follows:

1. Engaging the roadway/roadway pontoon upper connectors (dogbones) on the bay. It may be necessary to use the roadway pontoon connector tool when engaging the roadway/roadway upper connectors on the ramp bay.

2. Checking to ensure that the lower lock drive screw turns freely and the connecting pins are fully retracted. 

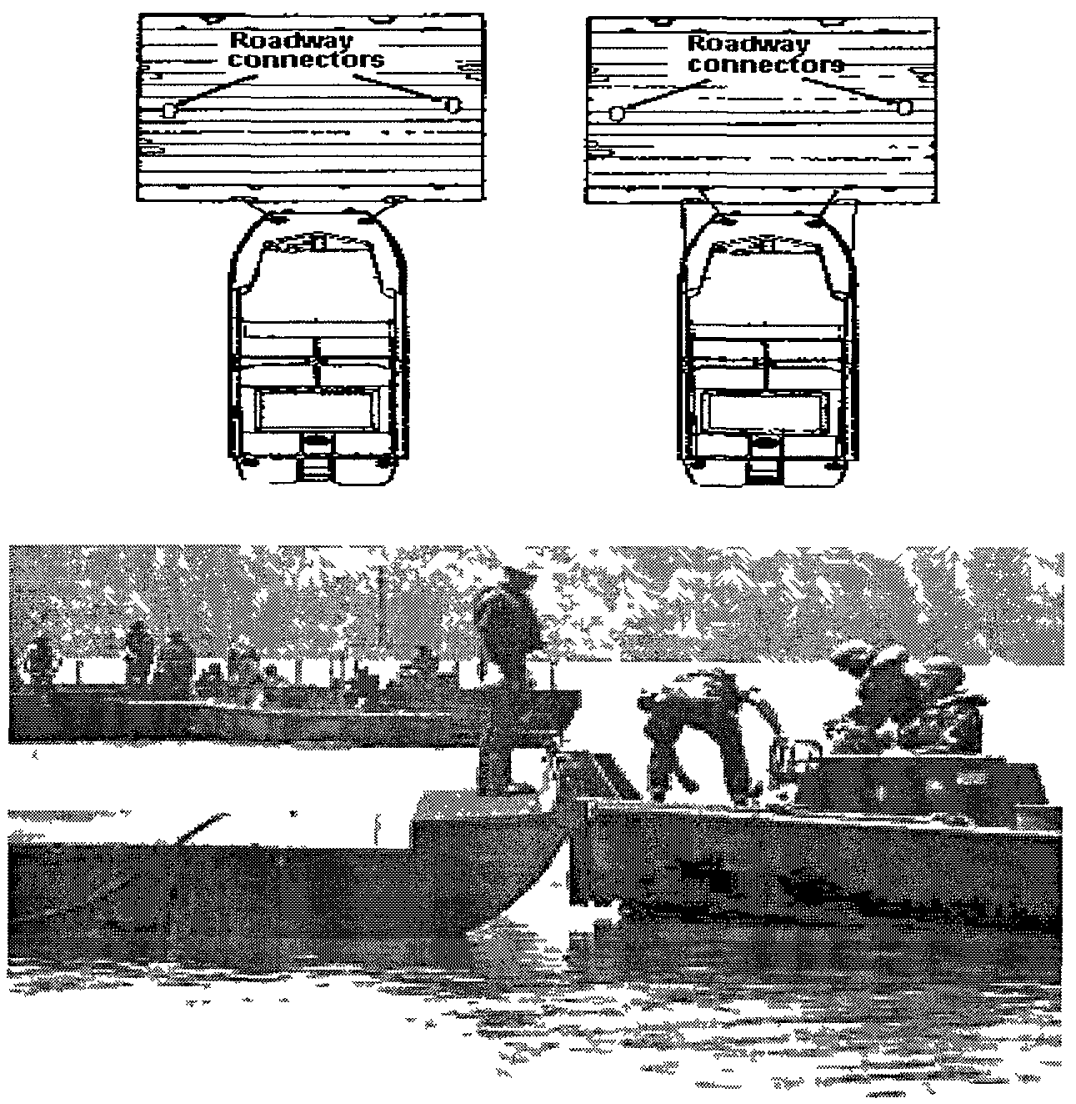

Figure B.6: Layout of securing the bridge bay using boats (primeportal.net)

\section{B.2.3 Interior Bay to Interior Bay Connection}

The interior bay to interior bay connection is made through the following steps as illustrated in Figure B.7:

1. The boat with the interior bay connected approaches the anchored interior bay from the downstream side.

2. When the bays are as close as possible, the bridge centerline crew tosses the tag lines to the boat crew which connects the lines to the bay rope cleats. The bays are then pulled together. Boat hooks may also be used. 
3. The securing crew engages the bridge bay/bridge bay upper connectors.

4. The bridge centerline crew secures the lower lock drive pins by turning the T-bar connecting wrench in the clockwise direction. If connection is difficult, the bridge boat can apply power in forward and reverse to adjust the bay's position. Wrecking bars may also be used to apply an up and down force to the joint by inserting them between the top of the bow walkway of one bay and the bottom of the roadway of the other bay.

5. The boat is disconnected if it is not needed for bridge anchorage or raft propulsion.

\section{B.2.4 Ramp Bay to Interior Bay Connection}

The ramp bay to interior bay connection is made through the following steps as illustrated in Figure B.8:

1. The boat which secured the ramp bay approaches the connected and anchored interior bays from the downstream side.

2. When the ramp bay has been brought as close as possible to the interior bays, the bridge crew secures it using tag lines and boat hooks. The crew next attaches the ramp connection tool hooks to the roadway/pontoon upper connectors of the adjacent interior bay and the ramp bay.

3. The bridge centerline crew aboard the interior bay raises the ramp bay, using the wrecking bar. This is done by inserting the bar into the holes in the ramp bay bow hinge blocks using the interior bay roadway as a pivot point, and applying a downward force to the top end of the bar. 
4. As force is applied to the wrecking bars, the bays are pulled together by ratcheting the ramp bay connection tool. As soon as the bays are together, engage the bay-tobay upper connectors.

5. The lower lock drive pins are then driven by turning the T-bar wrench. If the connection is difficult, the ramp pumps may be pumped to raise the connector yoke while force is applied to the T-bar.

6. The boat is disconnected if it is not needed for anchorage.

\section{Connecting interior bay to interior bay}

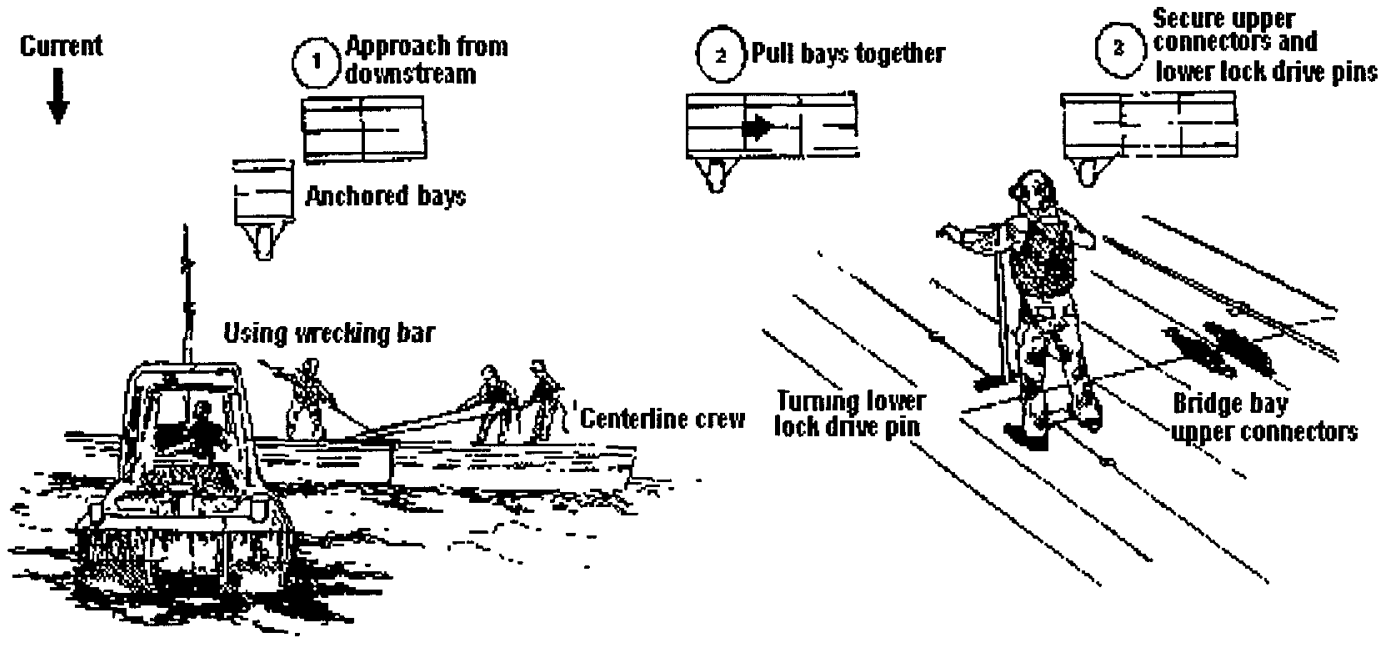

Figure B.7: Interior bay to interior bay connection 


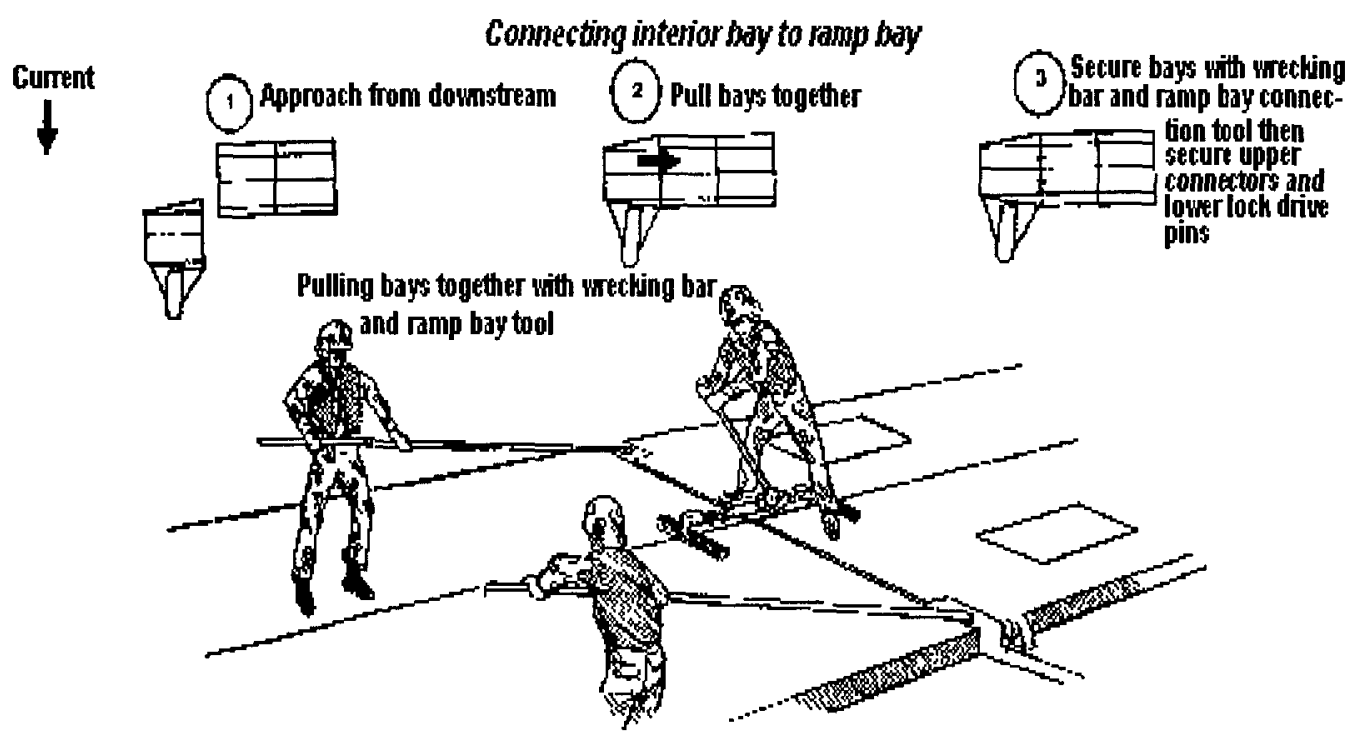

Figure B.8: Ramp bay to interior bay connection

\section{B.3 Ribbon Bridge Operation}

Ribbon bridges are the primary means of river crossing during military operations. When designing ribbon bridges, the quantity of ribbon equipment needed, the required assembly time, and the classification of the bridge are major considerations. The number of ribbon interior bays needed for bridge construction of a given gap can be determined using the following formula:

Number of interior bays $=\frac{\text { River Width ( } \text { in meters })-14}{67}$

Where 6.7 is the effective length in meters of the interior bay pontoon, and 14 is the total length in meters of the two ramp pontoons required at shores (one at each end of the bridge). Additionally, two ramps are required for every ribbon bridge for the approach zone of the bridge. 